\title{
TOWARDS RESTORATIVE SPACES FOR POSTNATAL RECOVERY IN URBAN TERTIARY HOSPITALS
}

\author{
BY \\ LATEEF ADEMOLA LAWAL \\ A thesis \\ submitted to Victoria University of Wellington \\ in fulfilment of the requirements for the degree of \\ DOCTOR OF PHILOSOPHY
}

Victoria University of Wellington

2020 



\title{
TOWARDS RESTORATIVE SPACES FOR POSTNATAL RECOVERY IN URBAN TERTIARY HOSPITALS
}

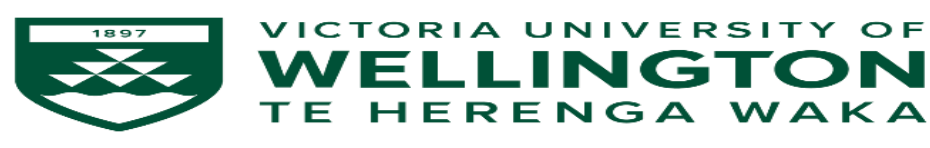

BY

Lateef Ademola Lawal

\author{
A thesis \\ submitted to Victoria University of Wellington \\ in fulfilment of the requirements for the degree of
}

\section{DOCTOR OF PHILOSOPHY}

Research supervision:

Professor Robert Vale

Honorary Research Associate

School of Architecture

Faculty of Architecture \& Design

Victoria University of Wellington

Email: Robert.Vale@vuw.ac.nz
Dr Maibritt Pedersen Zari

Deputy Head of School

School of Architecture

Faculty of Architecture \& Design

Victoria University of Wellington

Email: Maibritt.Pedersen@vuw.ac.nz

(C) Copyright by Lateef Ademola Lawal 2020

All rights reserved 



\begin{abstract}
Modern living and globalisation are increasingly contributing to access to and utilisation of modern healthcare services in both developed and developing countries; from diagnosis, treatments, and even to birth in a specialised healthcare environment. In many resource-rich countries, birth is one of the main reasons for women's hospitalisation. Research suggests that physical environments of healthcare impact on patient/users' health outcomes. Yet decisions about healthcare facilities' design and programming are often made without input from users.
\end{abstract}

A key challenge for patient-centred care in the maternity field is the assumption that healthcare spaces and facilities created for the general "unwell" hospital population can support the emotional, physiological and psychological needs of healthy women who give birth in hospitals. Previous research into the childbirth environment has focused on physical environments and satisfaction with the labour and birth process. But studies examining the impacts of physical environments on women's postnatal recovery experience and wellbeing have been limited. The aim of this thesis is to determine the design factors that influence women's recovery experiences and wellbeing with a view to proposing design guidelines for optimal postnatal environment in large, tertiary hospitals.

A two phase sequential explanatory mixed methods approach was taken to the research which combined a quantitative method (online survey questionnaires) with a qualitative approach (focus group discussions - FGDs). Research was conducted with two user groups, comprising postnatal women and midwives, to examine perceptions of physical environment factors that influence women's recovery experiences and wellbeing in New Zealand hospitals. The results of the questionnaire surveys were followed up in phase two FGDs to provide explanations and descriptions of architectural factors and design features that impacted on women's recovery experiences and psychological wellbeing. The results were compared, synthesised and interpreted to inform the outcomes of this thesis.

The research found several physical environment factors and design features which were significant and a number which were non-significant among the two user groups in all four categories investigated: perception of postnatal room features; interior environment; sensory comfort environment and social comfort environment. The research found factors related to maintenance (e.g. cleanliness), environmental design (e.g. noise/quiet in rooms, and daylighting), 
and interior spatial characteristics (e.g. view to nature, combined home-like and clinical features and privacy) are more important in fostering wellbeing. Strong evidence was found on how building spaces impact on postnatal recovery experience, especially regarding single-bed rooms for emotional support and psychosocial support environments such as communal spaces and outdoor environments.

The original contribution of this thesis is an identification of the environments which have the potential for enhanced recovery experience of women during the postnatal period. This understanding of the architectural design factors and building spaces could help architects, healthcare designers and hospital care providers in applying and promoting restorativeness in large, tertiary hospitals to better help in providing quality postnatal care for women whose hospital stays are crucial to their wellbeing and health prior to going home with their new baby.

Keywords: design factors, postnatal recovery, physical environment, restorative spaces, wellbeing 


\section{Declaration}

I Lateef Ademola Lawal, declare that this thesis is the product of my own work and to the best of my knowledge it contains no materials previously published or which have been accepted for any award of any other degree at Victoria University of Wellington or any other educational institutions, except where due acknowledgement or references to the works of other persons are made in the thesis.

Signature of student:

Date: ...16/03/2020 


\section{Acknowledgements}

I would first and foremost give special thanks to Almighty Allah for His Benevolence and Mercies for seeing me through from the beginning of the doctoral programme to the end.

Thanks are due to the Tertiary Education Trust Fund (TETFund) through the Federal University of Technology Minna, Nigeria for awarding me the TETFund Scholarship; and Victoria University of Wellington through the Victoria Research Trust for awarding me the Victoria Doctoral Submission Scholarship.

I could not fail to acknowledge the immense support and contribution that I received from my supervisors, Professor Robert Vale and Dr Maibritt Pedersen Zari for their knowledge, guidance and moral support in the last three years. I especially single out and appreciate the extra efforts of Professor Robert Vale for his unquantifiable assistance imparting in me priceless knowledge and giving me confidence at every point in the past years. Dr Maibritt Pedersen Zari has been very understanding and cooperative with me, her subtle criticisms contributed in no small measure to this thesis. You both have been a source of my academic strengths. I am grateful to Professor Brenda Vale who provided input on the initial proposal draft that I submitted to Victoria University of Wellington. I give thanks to Dr Robin Skinner, Professor Regan Potangaroa and Associate Professor Morten Gjerde for their interest at various stages of my research.

Special thanks are extended to two great scholars at the Federal University of Technology Minna, Nigeria. Professor Musbau Adewunmi Akanji, the Vice-Chancellor, who graciously approved funding for my $\mathrm{PhD}$ fellowship without which this $\mathrm{PhD}$ degree may not have been possible. I am deeply grateful to Professor Yahaya Ahmed Iyaka, Deputy Vice-Chancellor (Academic) for the immense role he played in my PhD journey. His prayers, encouragements and brotherly warmth and support of friendship contributed in no small measure to making this $\mathrm{PhD}$ a success.

My profound appreciation is offered to the women and midwives who completed my questionnaire surveys which served as the base data for this thesis. I also received immense assistance and cooperation from organisations and healthcare personnel who sacrificed their time for me ensuring that this research went smoothly. In particular, my debt of gratitude goes to the following people: Carolyn Coles, Director of Midwifery; Sinead McGrannachan, Administrator, Women's Health Service; Penny Wyatt; Marina Dzhelali; Linda Massov; Susi Maxwell; Jane Hartfield; Amy Parker; Wendy Devereux; Linda Elvines all of Capital \& Coast District Health 
Board. My appreciation is also extended to Annie McGill and her group of LMC midwives for the support and cooperation I received from them at the beginning of this research. Special thanks to the 14 women in my focus groups whom I cannot name because of ethical implications. Many of them went out of their way ensuring they attended the focus groups in spite of the demands of nursing their babies. Their beliefs in my research spurred me to completing this task.

In the past years, a few of my PhD colleagues made studying in New Zealand really enjoyable and unforgettable. In particular, Rehan Shaikh, Seydar Aydin, Lesley Metibogun, Ged Finch, Zahra Balador and Marzieh Imani. Other are Saima Shaikh, Faeze Yavari, Yukiko Kuboshima, Shuva Chowdhury, Tammy Amasuomo, Jasim Azhar and Nabil Allaf. My appreciations to the administration staff in the School of Architecture for their wonderful welfare support, including Asmaa Bouhalba, Anne Keogh, Kirstin Va'a, and Sarah Wilson. I am grateful to Kelly Atherton of Victoria International.

I am most grateful to all my siblings, in particular my sister, Zulifah Lawal (Nee Kadri) for love and support. I am indebted to a number of individuals who have always shown me affections and kindness.

I am most grateful to Dr Rukaiyat A. Ogunbajo for supporting me with prayers, encouragement and the warmth of her friendship. May Allah reward you with goodness.

Finally, my sincere gratitude to my father, Malam Tiamiyu A. Lawal and my mother, Alhaja Fatima Al Zahra Lawal for their constant prayers and support, which have been my source of strength and accomplishments in life. 


\section{Dedication}

This thesis is dedicated to my beautiful daughter, Nabila Gbemisola and my dear wife, Mulikat Folake; the birth of our daughter is the motivation for investigating the childbirth environment. I had encounters with hospitals when I was growing up as a child. Then, I took only a limited interest in the ways the hospital was structured, particularly its surrounding ambience.

I would like to say that your love, support and prayers throughout the entire process were instrumental in completing this $\mathrm{PhD}$. Despite the enormous distance between us-from Nigeria to New Zealand-you stayed very close and were confident that sooner I should make both of you proud, and indeed, this thesis is for both of you! 


\section{Post-defense acknowledgements}

I am grateful to my two External Examiners, Emeritus Professor Roger Fay and Professor Mardelle McCuskey Shepley. I thank them for their comments and I consider it a great honour that they both were interested in my work. I greatly value Emeritus Professor Roger Fay's comments and the insights which he brought to the task. His insights have made my thesis the better for his inputs and foreshadow, and also illuminate, important potential research paths for the work in the future.

I would also like to thank my Examination Committee chairperson, Dr Cei Maslen for her enthusiasm and support rendered during the oral examination. She is the one who first imformed me of my success with the oral examination. Dr Jan Smitheram served as my Internal Examiner. I greatly appreciate her support and comments.

Finally, I would like to express my greatest gratitude to Professor Robert Vale once again, for his unquantifiable support from the start to finish of my $\mathrm{PhD}$. I would never have finished my thesis without his diligence reading my work. I am also privileged to be the last $\mathrm{PhD}$ student that he supervised at Victoria University of Wellington. 


\section{List of abbreviations and acronyms}

$\mathrm{ABC}$

AMU

ART

BT'T

BUDSET

CCU

CHD

DAC

DHB

EBD

EBD\&R

ED

EFA

FGD

FMU

HAI

HDEC

HVAC

ICU

LDR

LDRP

LMC

LOS

MCAR

MLU

MM

MMR

NHS

NICU

OLU

OVP

PCA

PCC
Alternative Birth Centre

Alongside Midwifery Unit

Attention Restoration Theory

Birth Territory Theory

Birth Unit Design Spatial Evaluation Tool

Critical Care Unit

Center for Health Design

Direct Attentional Capacity

District Health Board

Evidence-Based Design

Evidence-Based Design and Research

Emergency Department

Exploratory Factor Analysis

Focus Group Discussion

Freestanding Midwifery Unit

Hospital Acquired Infection

Health and Disability Ethics Committees

Heating Ventilation and Air Conditioning

Intensive Care Unit

Labour Delivery Room

Labour, Delivery, Recovery and Postnatal

Lead Maternity Carer

Length of Stay

Missing Completely At Random

Midwifery-Led Unit

Mixed Methods

Mixed Methods Research

National Health Service

Neonatal Intensive Care Unit

Obstetric-Led Unit

Open Visitation Policy

Principal Component Analysis

Patient/Person-Centred Care 
PCD

PET

PFCC

PFCD

PICU

PW

SMEAN

SOC

SPR

SPSS

TSD

UN

UNICEF

UK

USA

VUW HEC

WHO
Patient Centred Design

Psycho-Evolutionary Theory

Patient-and Family Centred Care

Patient and Family Centred Design

Peadiatric Intensive Care Unit

Postnatal Ward

Series Mean

Sense of Coherence

Single Patient Room/Single Private Room (used interchangeably in the literature)

Statistical Package for the Social Sciences

Theory of Supportive Design

United Nations

United Nations Children's Fund

United Kingdom

United States of America

Victoria University of Wellington Human Ethics Committees

World Health Organisation 


\section{Table of contents}

Abstract

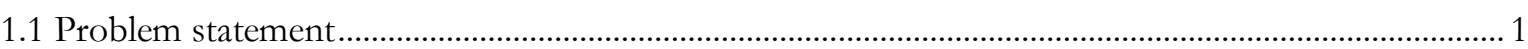

1.1.1 Users experience in designed birth and postnatal environments .................................................. 4

1.2 Research aim................................................................................................................................ 11

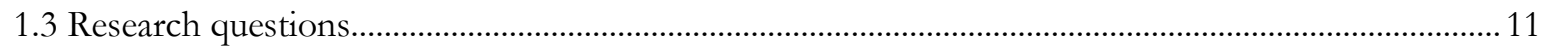

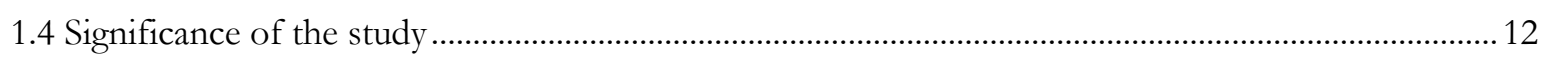

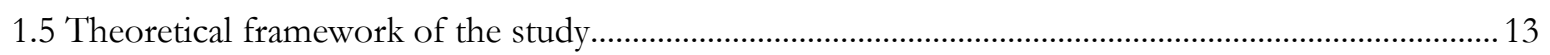

1.6 Research approach - reviews of previous $\mathrm{PhD}$ research methodologies ..............................................17

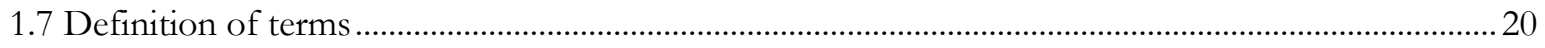

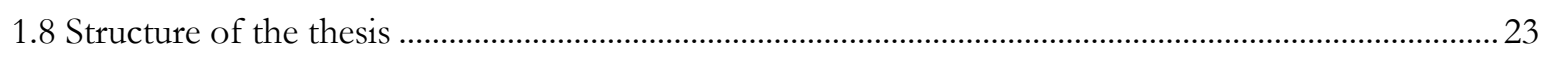

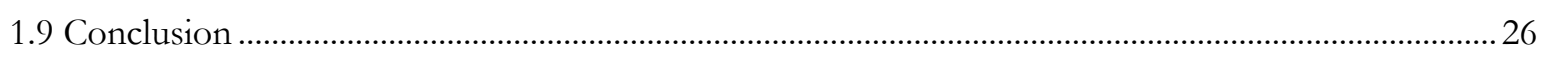

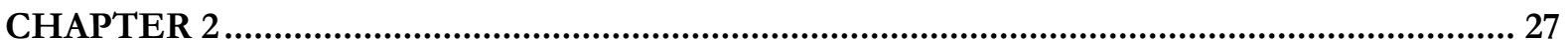

IMPACTS OF HEALTHCARE DESIGN ON HEALTH OUTCOMES ................................. 27

2.1 The healthcare environments and health outcomes................................................................................27

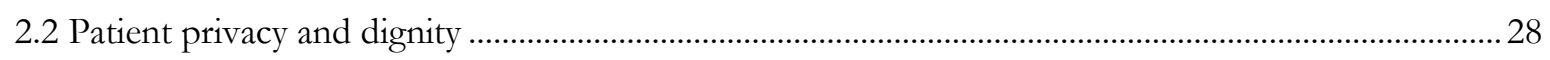

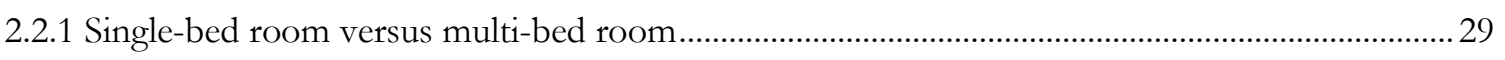

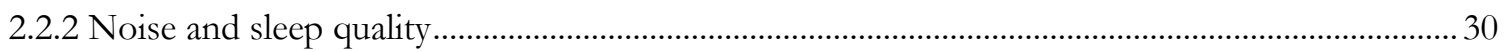

2.2.3 Noise and interventions in the healthcare environments ............................................................ 31

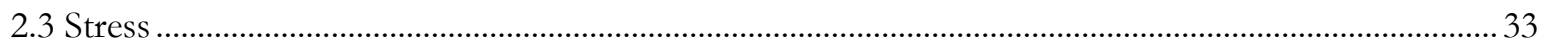

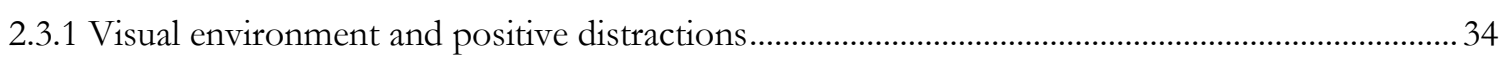

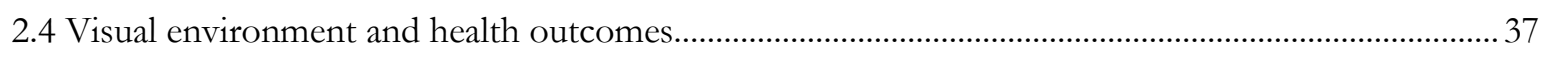

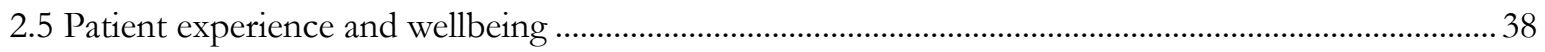

2.6 Family and social support and satisfaction ................................................................................... 40

2.6.1 Family and friends visitation and health outcomes ...................................................................... 41

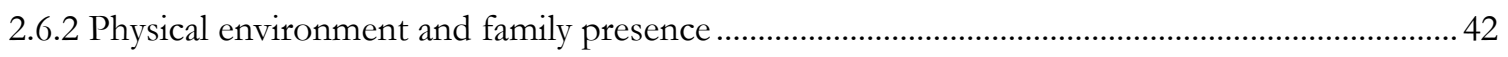

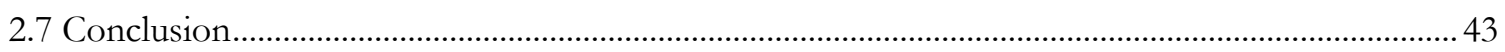


CHAPTER 3

IMPACTS OF CHILDBIRTH ENVIRONMENT ON HEALTH OUTCOMES .

3.1 Historical development of birth environment in high-income countries............................................. 45

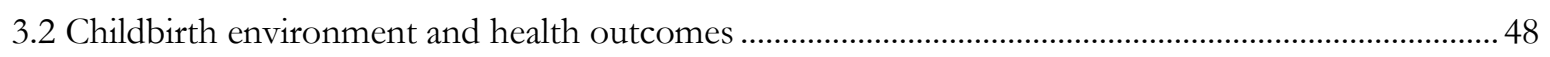

3.3 Birth space design and impacts on women …………............................................................................ 49

3.3.1 Women's wellbeing and birth experience.................................................................................... 51

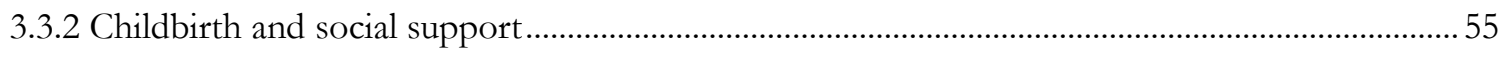

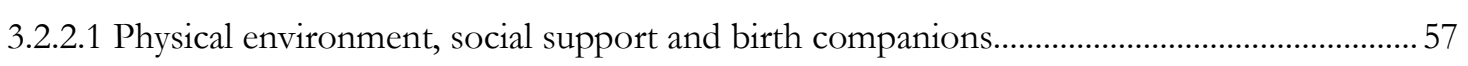

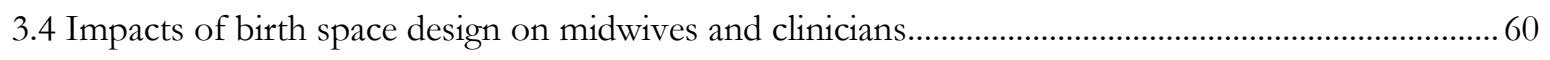

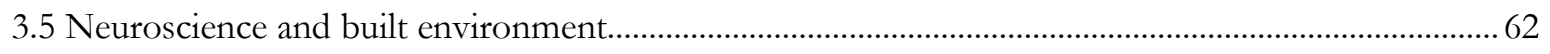

3.6 Research philosophy, phenomenology and lack of fit in this research .................................................71

3.6.1 Research philosophy and pragmatism .......................................................................................... 71

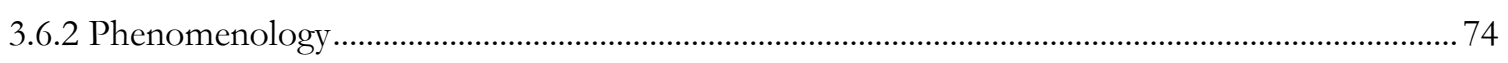

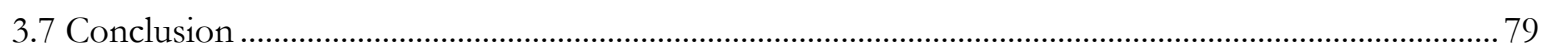

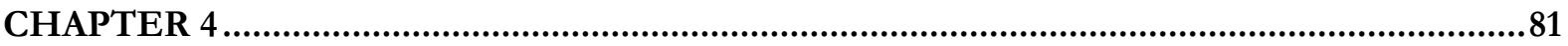

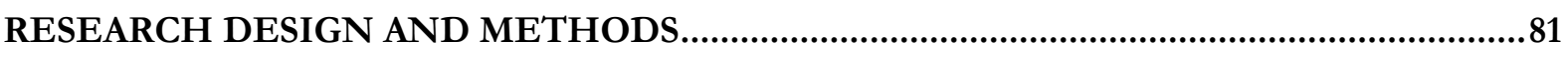

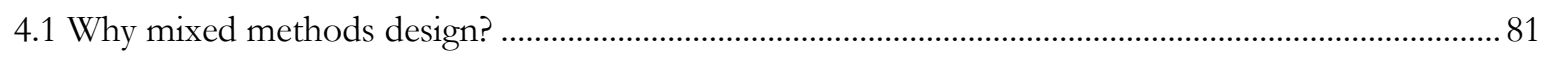

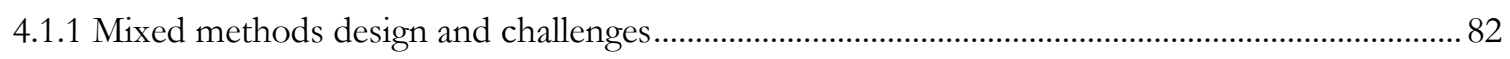

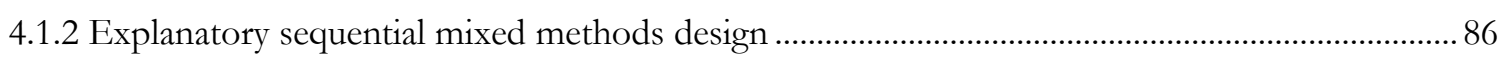

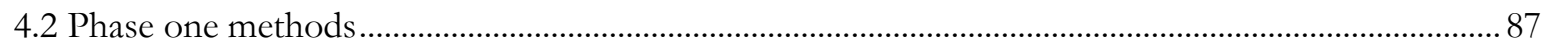

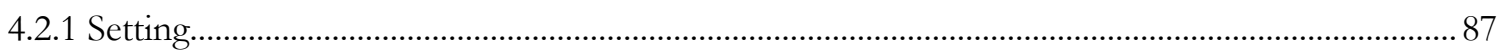

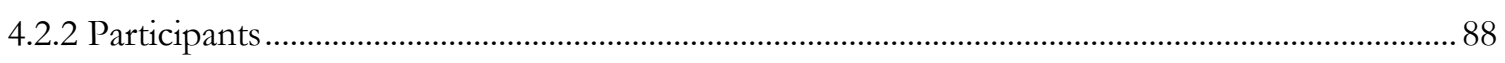

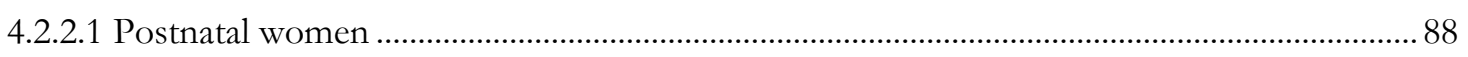

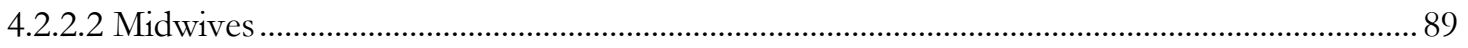

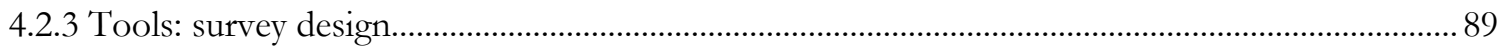

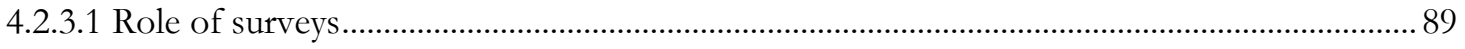

4.2.3.2 Questionnaire development............................................................................................ 91

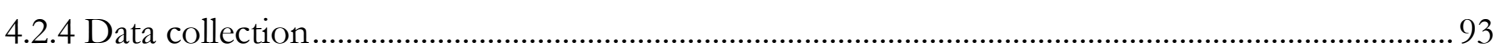

4.2.5 Data management and statistical analysis ..................................................................................... 94

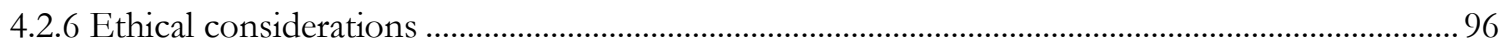

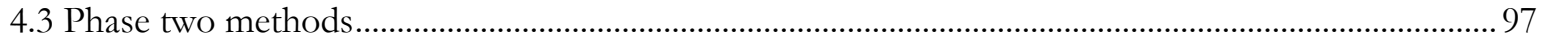

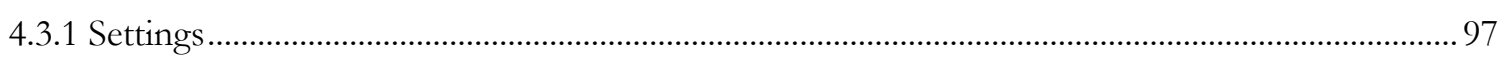

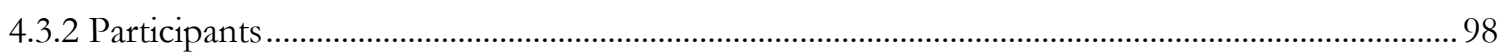

4.3.2.1 Postnatal women and midwives......................................................................................... 98

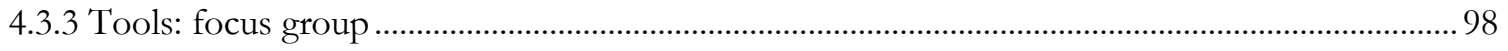

4.3.4 Phase two data collection......................................................................................................... 99

4.3.5 Data management and analysis ............................................................................................... 102

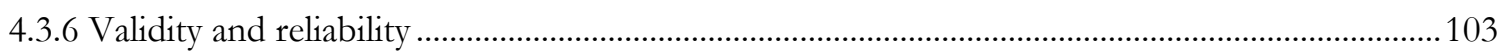


PHASE ONE: WOMEN'S PERCEPTION OF DESIGN FACTORS SURVEY - RESULTS...107

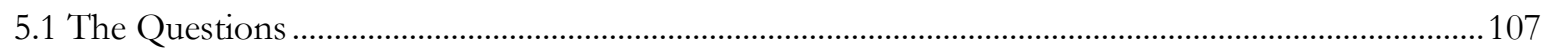

5.2 Summary of results for postnatal room situations ................................................................................ 113

5.3 Women's results perception questionnaire survey …………………………………………………......114

5.4 Perception of impact of physical environment on recovery and psychological wellbeing ................114

5.5 Perception of postnatal room features .................................................................................................... 115

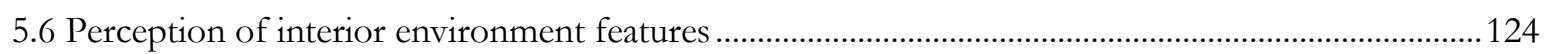

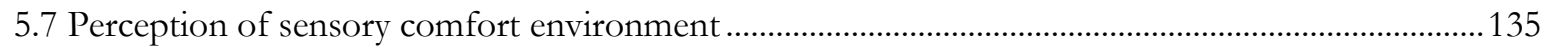

5.8 Perception on social comfort environment...................................................................................... 143

5.8.1 Perception of alternative postnatal environments ...................................................................... 152

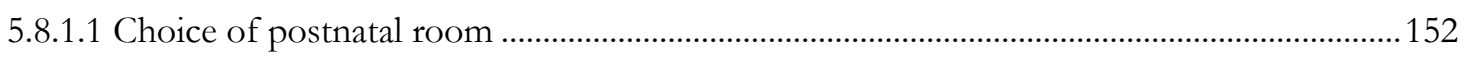

5.8.1.2 Rethinking alternative settings for postnatal care ................................................................153

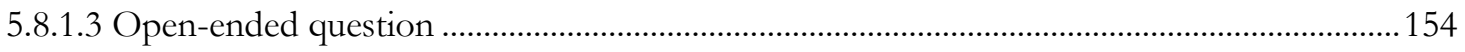

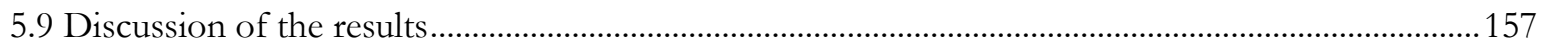

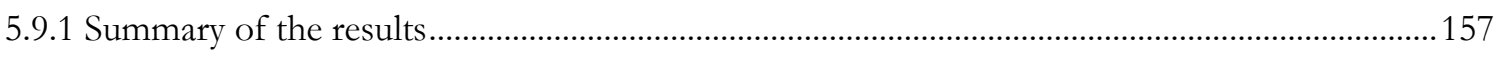

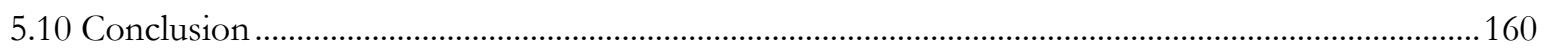

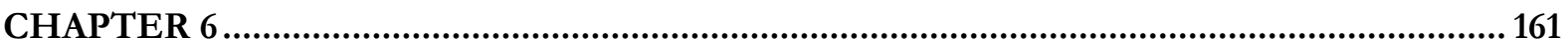

PHASE ONE: MIDWIVES' PERCEPTION OF DESIGN FACTORS SURVEY - RESULTS 161

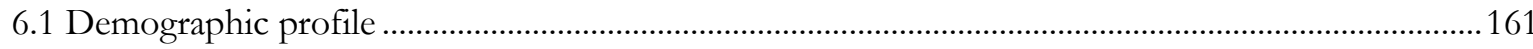

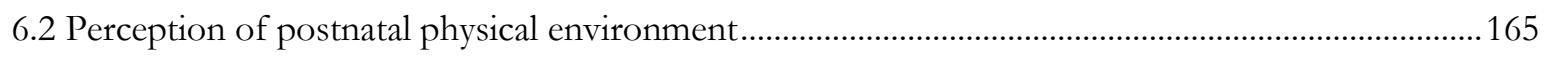

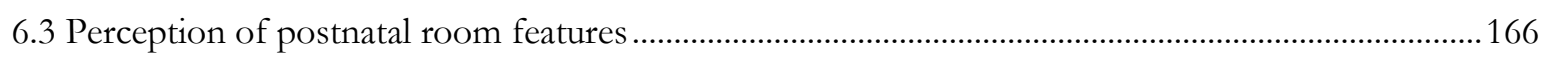

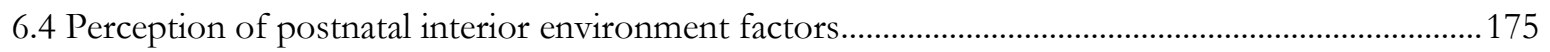

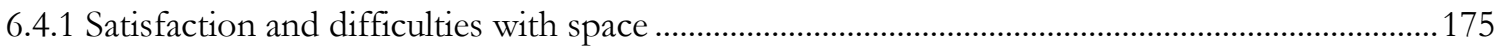

6.5 Perception of sensory comfort environment ………................................................................... 186

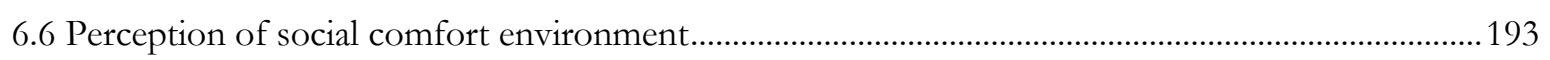

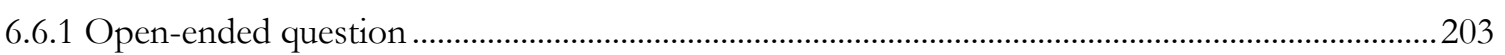

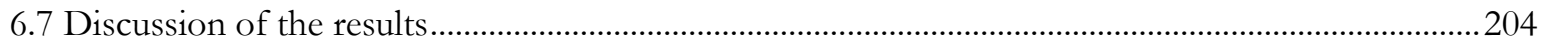

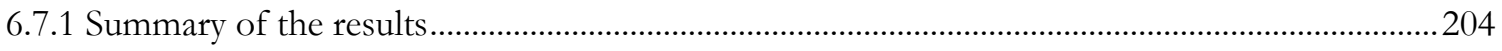

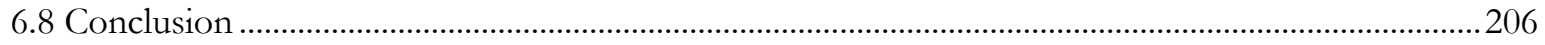

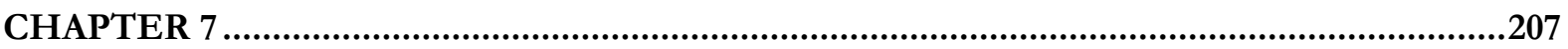

RESULTS OF INFERENTIAL ANALYSIS ON PERCEPTION OF DESIGN FACTORS

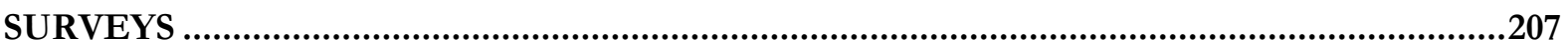

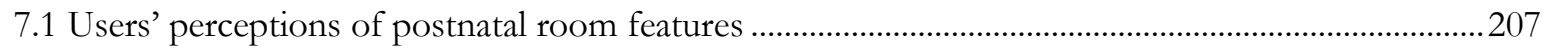

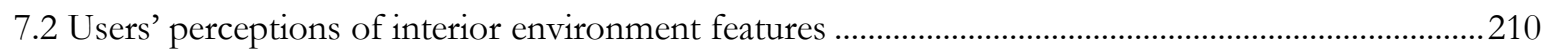

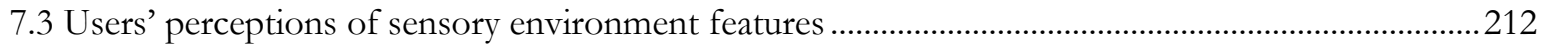

7.4 Users' perceptions of social comfort environment ............................................................................215 
7.5 Exploratory factor analysis

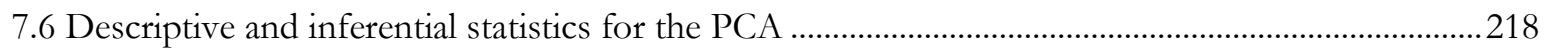

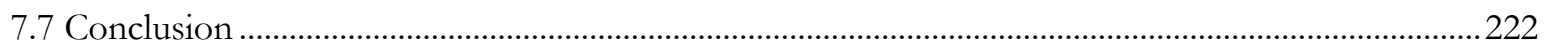

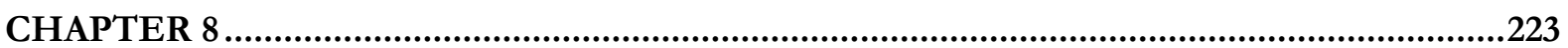

DISCUSSION ON PERCEPTION OF DESIGN FACTORS SURVEYS..............................223

8.1 The similarities: what design factors influence postnatal recovery experiences and wellbeing........225

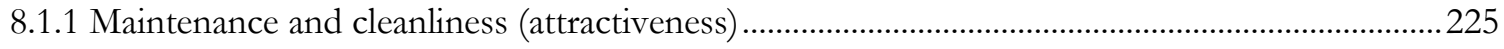

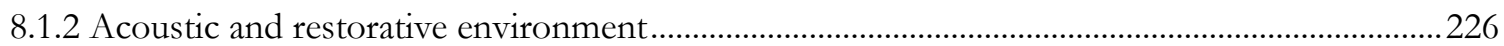

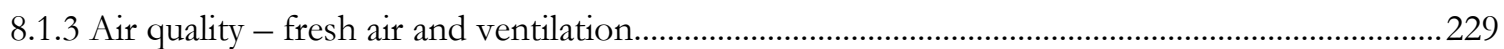

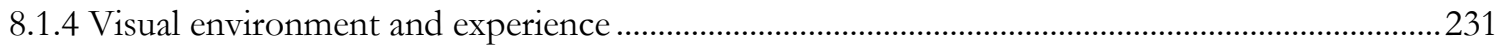

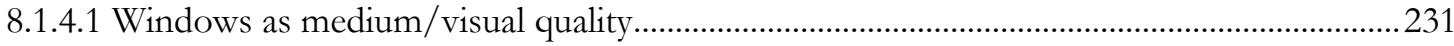

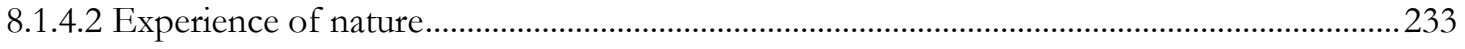

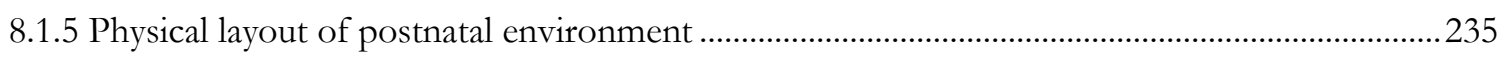

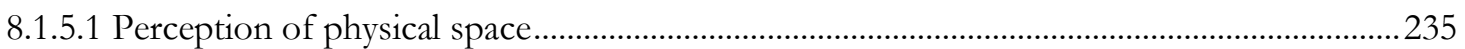

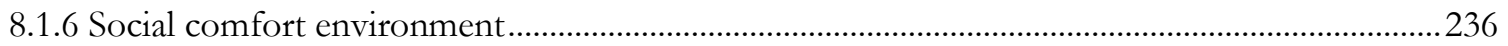

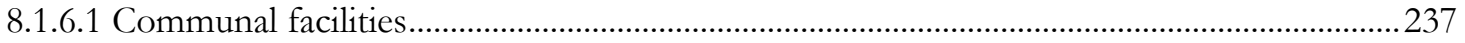

8.1.6.2 Combination of clinical space and familiar home features ................................................238

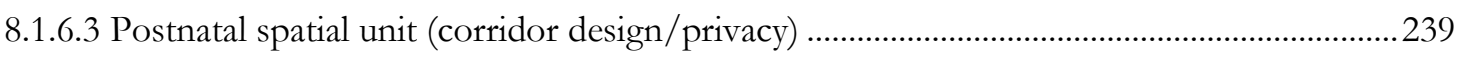

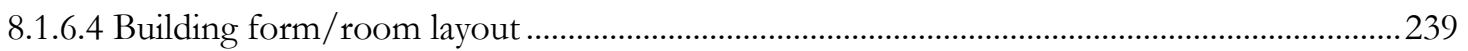

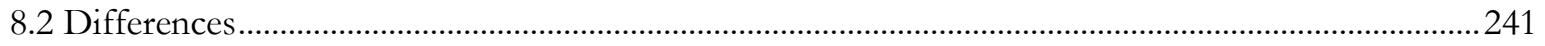

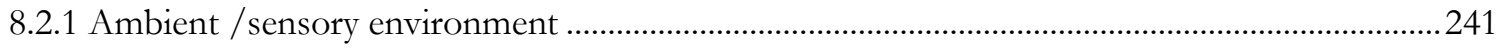

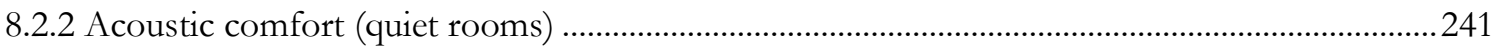

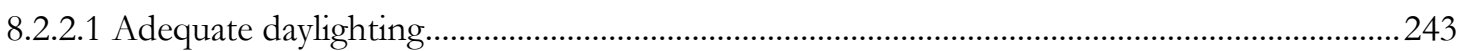

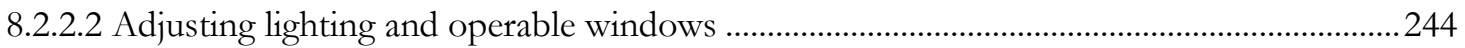

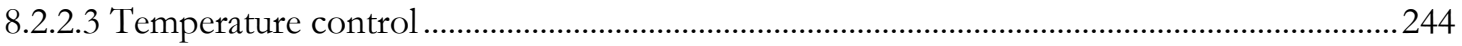

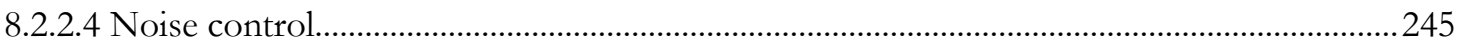

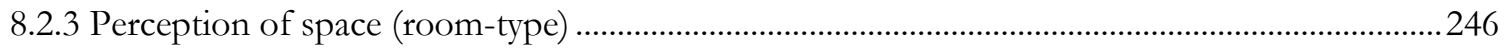

8.2.3.1 Single versus multiple beds accommodation .....................................................................246

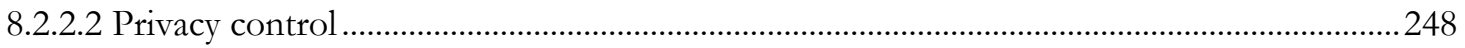

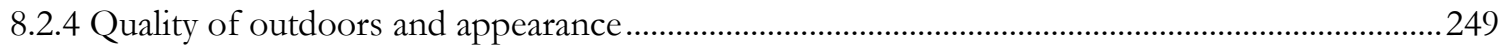

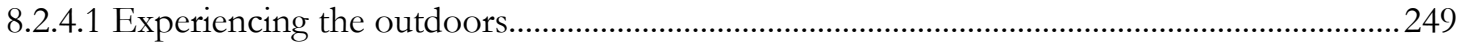

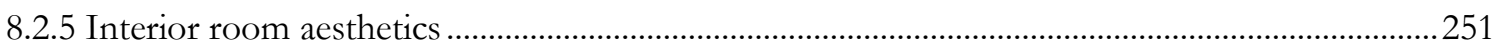

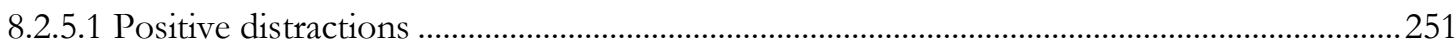

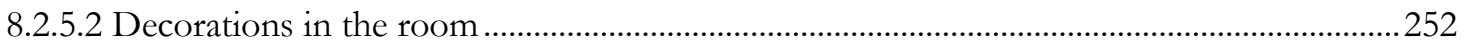

8.2.5.3 Coordinated natural objects (pleasantness) in the room ....................................................252

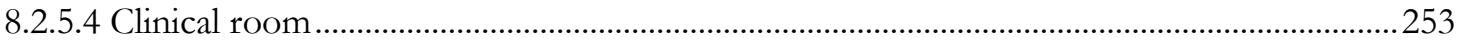

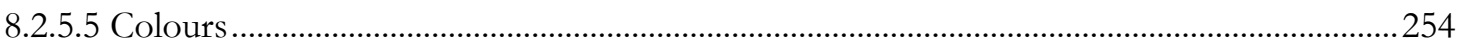

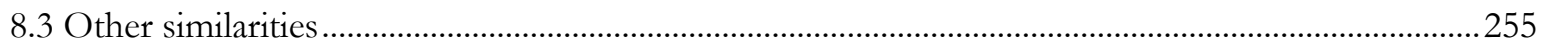


8.3.1.1 Preference for room of choice (personal space) .................................................................255

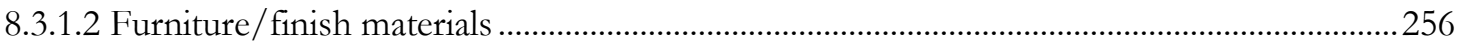

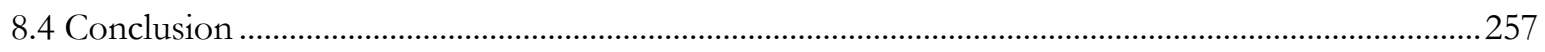

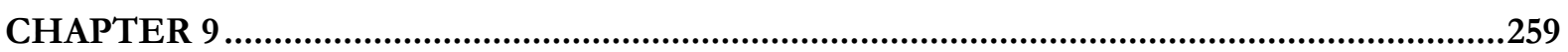

PHASE TWO: FOCUS GROUP FINDINGS AND DISCUSSION......................................259

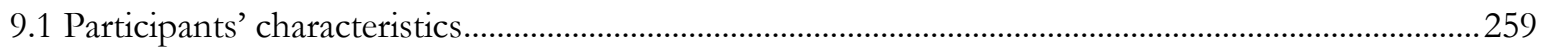

9.2 Category: Recovery experiences in spatial units...........................................................................263

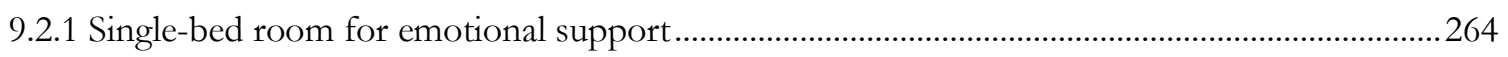

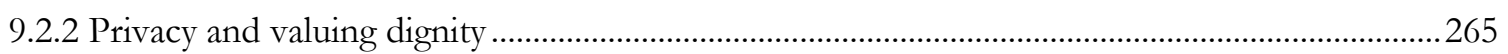

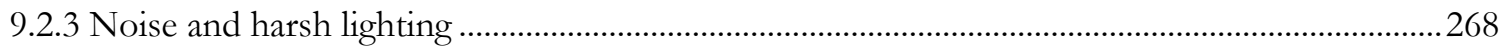

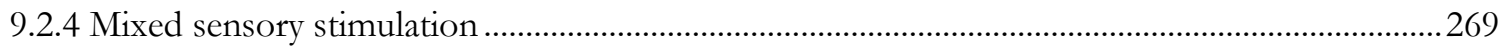

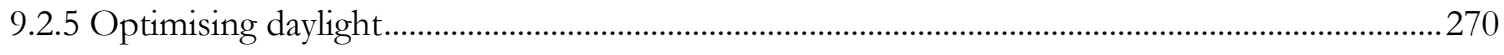

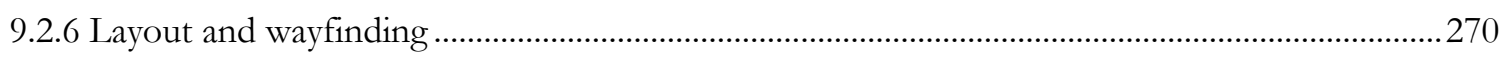

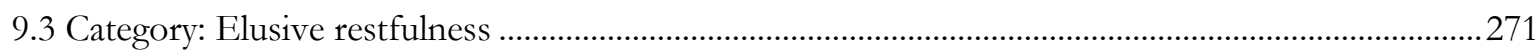

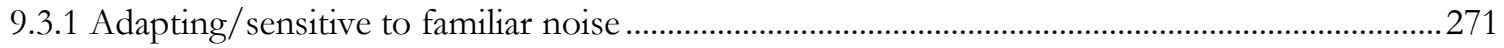

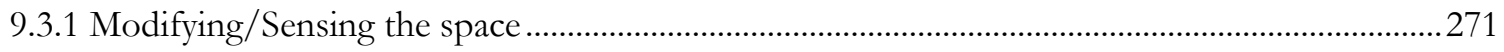

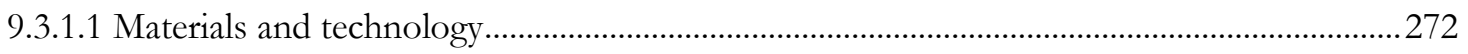

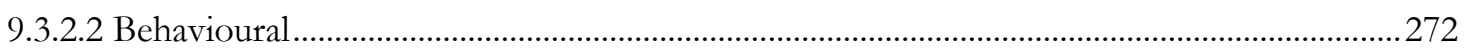

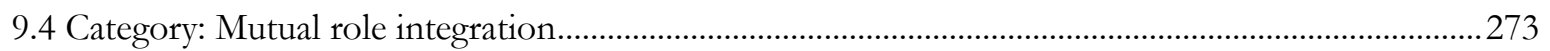

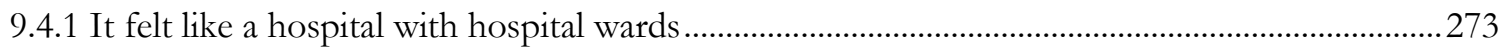

9.4.2 Humanising the space so that we feel we belong here .............................................................274

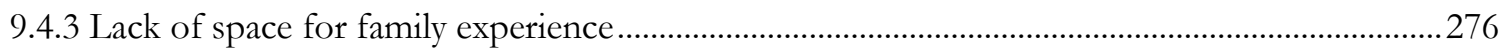

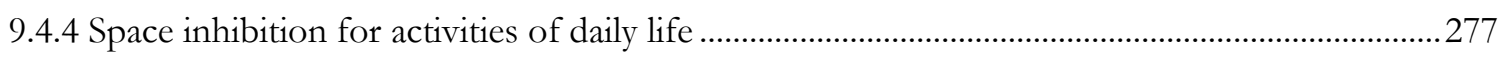

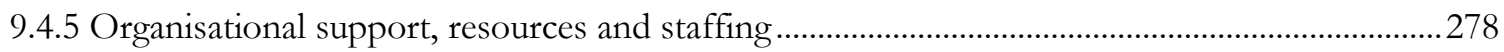

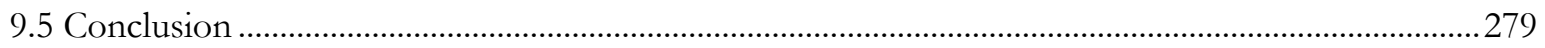

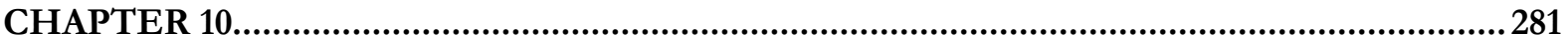

FACILITATING RESTORATIVE SPACES IN URBAN TERTIARY HOSPITAL

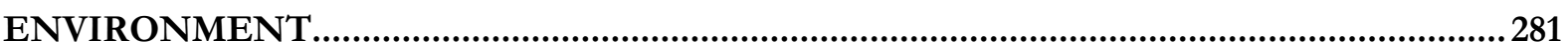

10.1 Conclusions on perception of design factors questionnaire surveys and focus groups .................281

10.2 Discussion of perception of design factors questionnaire surveys and focus groups ....................283

10.2.1 What are the perceptions of design factors influencing women's recovery experience during postnatal hospital stays.

10.2.2 What were the ways in which the design factors and the postnatal spaces affected women's recovery experiences and wellbeing during hospital stays.................................................................285

10.3 Implications for design guidelines to assist quality postnatal care in large, tertiary hospitals ........287

10.3.1 Woman as patient in wellbeing studies - Single-bedroom allocation in healthcare .................287

10.3.2 Visual preference and views to the outdoors - Valuing sensory experience...........................289

10.3.3 Spatial sensitivity and tranquility - restorative design of postnatal spaces .............................291

10.3.4 Access to nature and daylight - designing for outdoor experience ......................................292 
10.4 Design guidelines

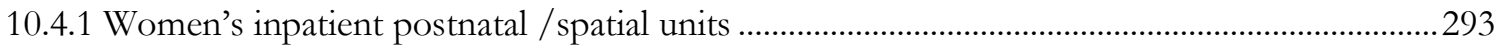

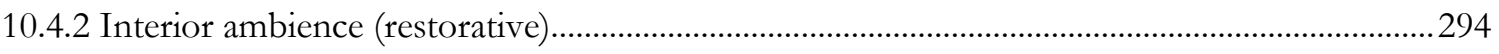

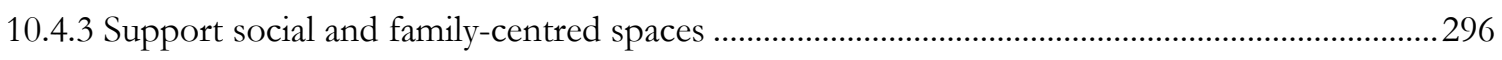

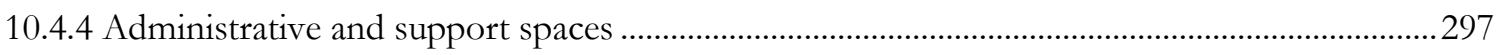

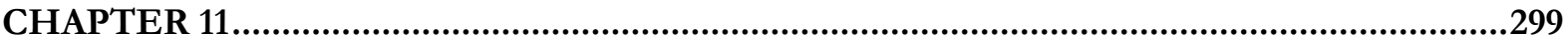

CONCLUSIONS AND FUTURE RESEARCH .............................................................299

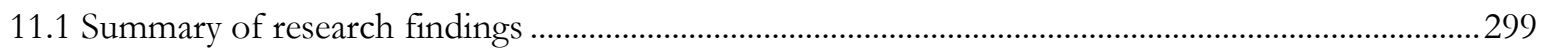



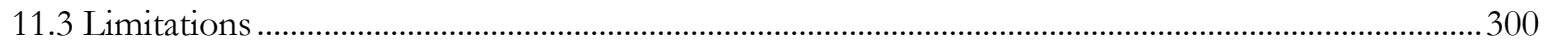

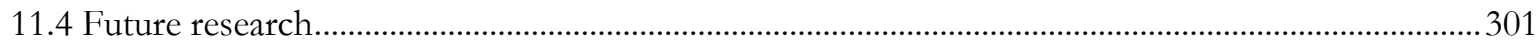

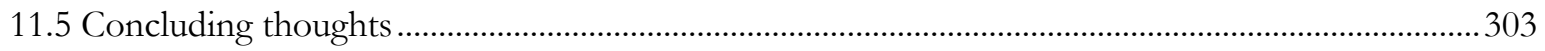

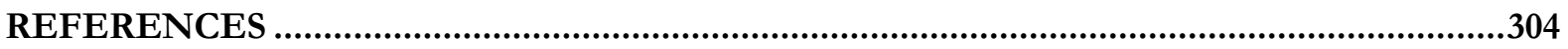

PUBLICATIONS DURING CANDIDATURE ..............................................................344

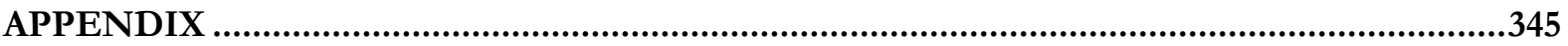

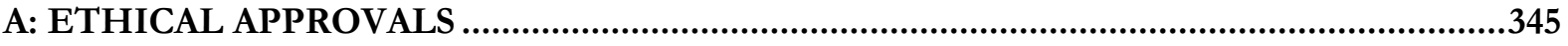

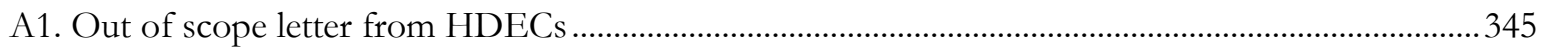

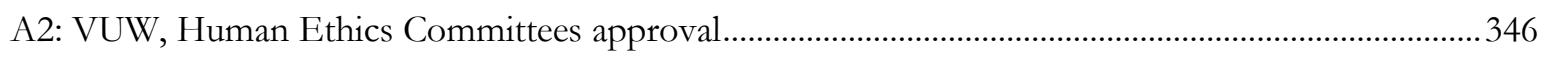

A3: Letter indicating support for phase one of the study from CCDHB .............................................347

A4: Letter indicating support for phase two of the study from CCDHB ............................................348

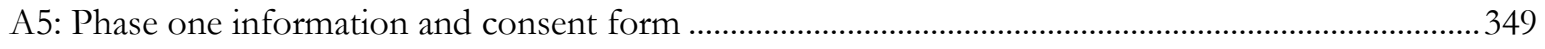

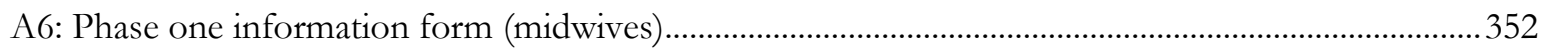

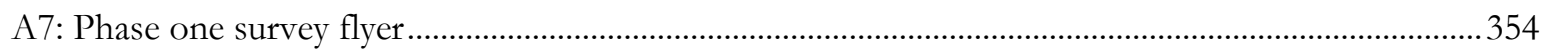

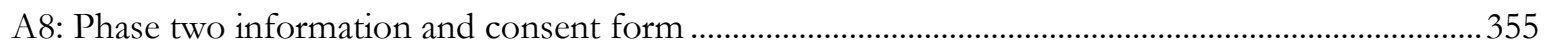

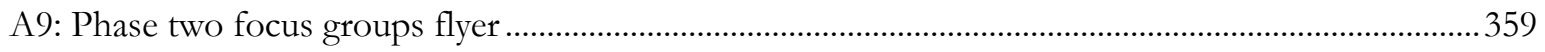

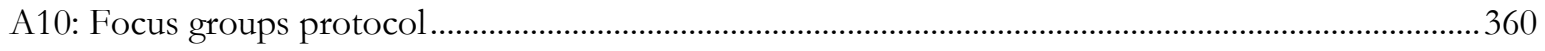

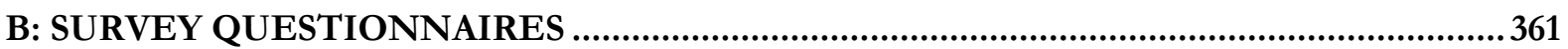

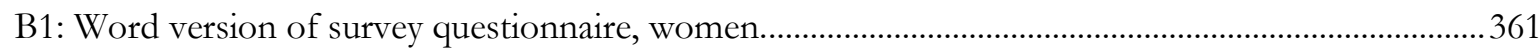

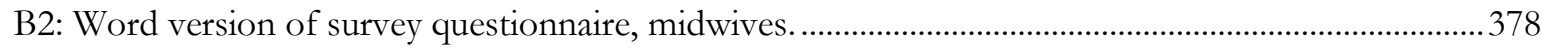

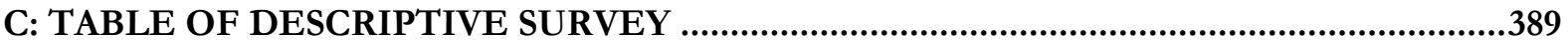




\section{List of tables}

Table 1-1: Overview of postnatal length of stay in resource-rich countries

Table 4-1: Details of recruitment of the focus groups.

Table 4-2: Distribution of the focus group participants by attendance

Table 5-1: Demographic profile of sample participant women $(n=212)$ in phase one survey . 112 Table 5-2: Ranking of different postnatal room situations

Table 5-3: Preference (mean and standard deviation) of respondents for each item on perception of postnatal room features influencing women's recovery and wellbeing (women, $n=212$ )...... 123 Table 5-4: Preference (mean and standard deviation) of respondents on perception of interior environment features influencing women's recovery and wellbeing $(\mathrm{n}=212)$

Table 5-5: Preference (mean and standard deviation) of respondents on perception of sensory comfort environment features influencing women's recovery and wellbeing $(\mathrm{n}=212)$

Table 5-6: Preference (mean and standard deviation) of respondents on perception of social comfort features influencing wellbeing $(\mathrm{n}=212)$.

Table 5-7: The 15 most important items in the Design Factors influencing the women's postnatal recovery experiences and wellbeing of the design perception survey (women, $\mathrm{n}=212$ ) ........... 160

Table 6-1: Demographic details of sample participant midwives $(\mathrm{n}=50)$ in phase one

Table 6-2: Preference (mean and standard deviation) of respondents on perception of postnatal room features contributing to recovery care and women's wellbeing (midwives, $\mathrm{n}=50$ )

Table 6-3: Descriptive factors in interior environment of postnatal spaces that contribute to midwives' work in postnatal care.

Table 6-4: Preference (mean and standard deviation) of respondents on perception of interior environment features contributing to recovery care and women's wellbeing ( $\mathrm{n}=50)$.

Table 6-5: Preference (mean and standard deviation) of respondents on perception of sensory comfort features contributing to recovery and women's wellbeing $(\mathrm{n}=50)$

Table 6-6: Preference (mean and standard deviation) of respondents on perception of social comfort environment contributing to information support and women's wellbeing ( $\mathrm{n}=50) \ldots .202$ Table 6-7: The 15 most important items in the Design Factors influencing the women's postnatal recovery experiences and wellbeing of the design perception survey (midwives, $n=50$ )..........206

Table 7-1: Preference (Independent Samples $t$-tests) of respondents on items in postnatal room features comparing women and midwives responses

Table 7-2: Preference (Independent Samples t-tests) of respondents on items in interior environment features comparing women and midwives responses

Table 7-3: Preference (Independent Samples t-tests) of respondents on items in sensory environment features comparing women and midwives responses

Table 7-4: Rotated component matrix of the questionnaire items (women and midwives)........ 219

Table 7-5: Independent samples T-tests results (women and midwives, $\mathrm{n}=262$ ) ........................ 220

Table 7-6: Independent samples T-tests results (women and midwives $n=262$ ) 
Table 8-1: The summary of most important Physical Design Factors influencing the women's postnatal recovery experiences and wellbeing from perception of design surveys $(n=262) \ldots .224$

Table 9-1: Demographic profile and characteristics of focus group participants in the study $n=$ 14

Table 9-2: Characteristics of settings and duration of the FGDs .................................................261

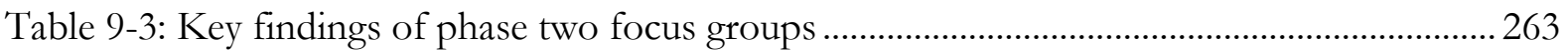

C-1: The mean and standard deviation for all item in the Design Factors influencing women's recovery experiences and wellbeing on perception of design factors survey (women, $n=212$ ) 389 C-2: The mean and standard deviation for all item in the Design Factors influencing women's recovery care on perception of design factors survey (midwives, $\mathrm{n}=50$ )

C-3: Participant responses as a percentage of the sample, means and standard deviations for each item of the perceptions of design factors influencing recovery and wellbeing in women's postnatal design survey $(\mathrm{n}=212)$

C-4: Participant responses as a percentage of the sample, means and standard deviations for each item of the perceptions of design factors influencing recovery and wellbeing in women's postnatal design survey $(\mathrm{n}=212)$

C-5: Participant responses as a percentage of the sample, means and standard deviations for each item of the perceptions of design factors influencing recovery care in midwives postnatal design survey $(\mathrm{n}=50)$

C-6: Participant responses as a percentage of the sample, means and standard deviations for each item of the perceptions of design factors influencing recovery care in midwives postnatal design survey $(\mathrm{n}=50)$ 


\section{List of figures}

Figure 1-1: Conceptual model for recovery experience and wellbeing in this study 13

Figure 4-1: Visual diagram for mixed methods sequential explanatory design procedures adapted from Ivankova and Stick (2007) 87

Figure 5-1: Distribution of respondents giving birth by parity level, age group and by ethnicity

Figure 5-2: Distribution of respondents giving birth at a hospital facility, by age group and postnatal room type...

Figure 5-3: Distribution of respondents' most recent birth, by age group and ethnic group ..... 110

Figure 5-4: Percentage of respondents' education levels, by age group and ethnic group .......... 111

Figure 5-5: Perception on effect of physical environment on wellbeing........................................ 115

Figure 5-6: Respondents' ratings of a single room for postnatal recovery ...................................... 116

Figure 5-7: Respondents' ratings of a restful room for sleep and recovery process....................... 116

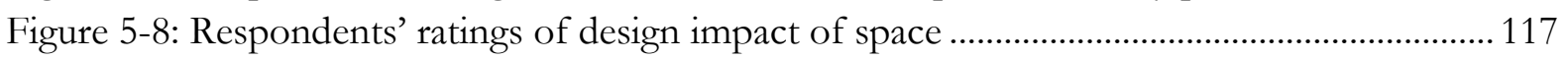

Figure 5-9: Negative skew distribution of ratings of design impact of space .................................. 118

Figure 5-10: Respondents' ratings of a room with colours versus a plain room............................ 118

Figure 5-11: Normal distribution of ratings of a room with colours versus a plain room ..........119

Figure 5-12: Respondents' ratings of a room with views of nature............................................... 120

Figure 5-13: Respondents' ratings of a room with daylight access for recovery ........................... 120

Figure 5-14: Respondents' ratings of ability to walk outside .......................................................... 121

Figure 5-15: Respondents' ratings of shape and natural form for postnatal room design........... 122

Figure 5-16: Respondents' ratings of postnatal room features ....................................................... 123

Figure 5-17: Respondents' ratings on perception of interior environment for postnatal recovery

Figure 5-18: Respondents' ratings on perception of a restful room for recovery .......................... 125

Figure 5-19: Respondents' ratings on perception of a clinical room for recovery ......................... 126

Figure 5-20: Positive skew distribution of ratings of a clinical room for recovery ....................... 126

Figure 5-21: Respondents' ratings on perception of a combination of clinical room and home

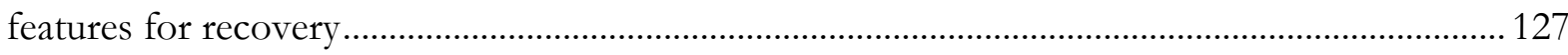

Figure 5-22: A fairly normal distribution of ratings for a combination of clinical and familiar home features for recovery.....

Figure 5-23: Respondents' ratings on perception of decoration with art/images in the room for

recovery

Figure 5-24: A normal distribution of ratings of decoration with natural art, images or painting for recovery

Figure 5-25: Respondents' ratings on perception of bright colours in the room for recovery ... 130 Figure 5-26: A somewhat negatively skewed distribution of ratings of bright colours for recovery

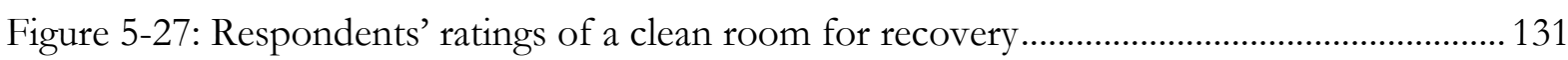

Figure 5-28: Respondents' ratings of a room with furniture made of natural materials .............. 132 
Figure 5-29: Respondents' ratings of a room regarding a protective corner 133

Figure 5-30: Respondents' ratings of a room with door closed for privacy.

Figure 5-31: Respondents' ratings of interior environment design factors for recovery experience

Figure 5-32: Respondents' ratings of adequate daylighting for wellbeing ..................................... 136

Figure 5-33: Respondents' ratings of temperature control for wellbeing ........................................ 136

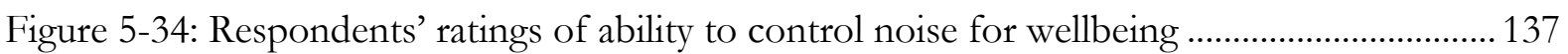

Figure 5-35: Respondents' ratings of quiet room for wellbeing .................................................... 138

Figure 5-36: Respondents' ratings of air quality and room freshness for wellbeing...................... 138

Figure 5-37: Respondents' ratings of additional heating in the room for wellbeing...................... 139

Figure 5-38: Respondents' ratings of operable windows for comfort and wellbeing................... 140

Figure 5-39: Respondents' ratings of natural things in the room for wellbeing ............................ 140

Figure 5-40: A slightly positive skewed distribution of ratings of natural things such as aquariums

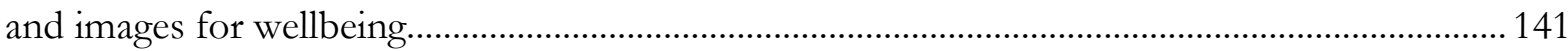

Figure 5-41: Respondents' ratings of hygiene/cleanliness in the room for wellbeing................... 141

Figure 5-42: Respondents' ratings of sensory environment design factors for wellbeing ............ 142

Figure 5-43: Respondents' ratings of frequency of visitation....................................................... 143

Figure 5-44: Respondents' ratings of opportunity to choose room for social comfort ................. 144

Figure 5-45: Respondents' ratings of spatial connection of postnatal room ................................ 145

Figure 5-46: Respondents' ratings of positive distractions for social comfort ............................... 146

Figure 5-47: Respondents' ratings of communal space for information support ........................... 147

Figure 5-48: Respondents' ratings of furniture and décor layout for group learning .................... 147

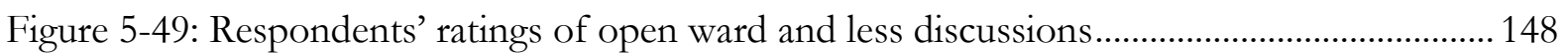

Figure 5-50: Respondents' ratings of a corridor for privacy comfort ............................................. 149

Figure 5-51: Respondents' ratings of family support and space need............................................ 150

Figure 5-52: Respondents' ratings of open postnatal ward and interactions .................................. 151

Figure 5-53: Respondents' ratings of social comfort environment features .................................. 152

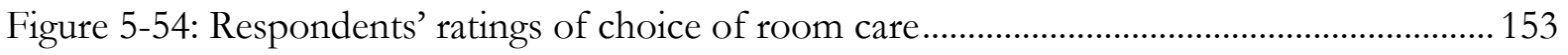

Figure 5-55: Respondents' ratings of alternative settings for postnatal care................................... 153

Figure 5-56: Top fifteen design features influencing women's recovery experience .................... 158

Figure 6-1: Percentage of respondents' model of care, by age group and education level .......... 162 Figure 6-2: Percentage of respondents' (midwives) years of experience, by age group and ethnic

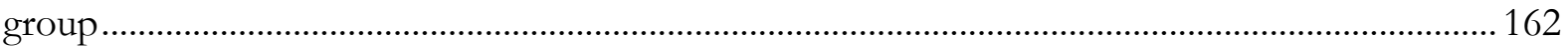

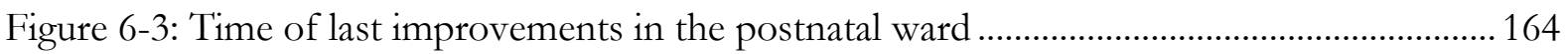

Figure 6-4: Respondents' experience across model of care in the postnatal ward......................... 165

Figure 6-5: Respondents' rating of impact of physical environment on care ................................. 165

Figure 6-6: Respondents' ratings of a single-bed room's impact on recovery ................................ 166

Figure 6-7: Respondents' ratings of a room/ward's impact on sleep and recovery ...................... 167

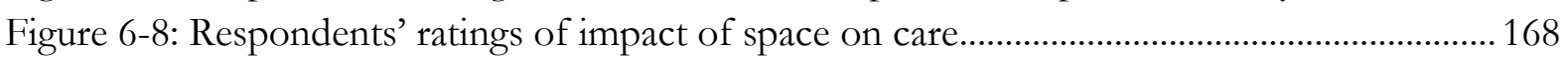

Figure 6-9: Respondents' ratings of room with colours versus plain room for birth recovery... 168

Figure 6-10: A normal distribution of ratings of room with colours and plain room .................... 169

Figure 6-11: Respondents' ratings of room with views of nature for care .................................... 170

Figure 6-12: Respondents' ratings of a room with access to the outdoors for recovery .............. 170 
Figure 6-13: Respondents' ratings of a room with access to daylight for recovery ...................... 171

Figure 6-14: Respondents' ratings of natural finishes in the room for recovery ............................ 172

Figure 6-15: A slightly normal distribution of ratings of natural finishes in the room.................. 172

Figure 6-16: Respondents' ratings of shape and natural form for postnatal room design............ 173

Figure 6-17: Respondents' ratings of postnatal room features ...................................................... 174

Figure 6-18: Respondents' ratings of interior environment on staff job performance ................. 175

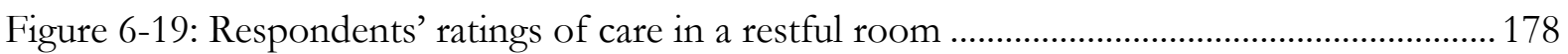

Figure 6-20: Respondents' ratings of clinical space ..................................................................... 179

Figure 6-21: Respondents' ratings of care in a room with clinical and familiar home

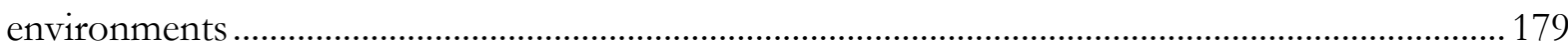

Figure 6-22: Respondents' ratings of care in a room with art or images ....................................... 180

Figure 6-23: Respondents' ratings of care in a room with bright colours ..................................... 181

Figure 6-24: Respondents' ratings of care in hygienic and clean room ......................................... 182

Figure 6-25: Respondents' ratings of care in a room with furniture made of natural materials.. 183

Figure 6-26: Respondents' ratings of privacy in the room with closing of doors .......................... 183

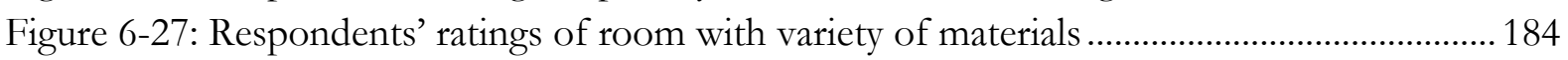

Figure 6-28: Respondents' ratings of interior environment room features.................................... 185

Figure 6-29: Respondents' ratings of adequate lighting for sense of wellbeing.............................. 186

Figure 6-30: Respondents' ratings of temperature control for sense of wellbeing ........................ 187

Figure 6-31: Respondents' ratings of noise control for sense of wellbeing .................................. 188

Figure 6-32: Respondents' ratings of a quiet room for sense of wellbeing ......................................... 188

Figure 6-33: Respondents' ratings of good indoor air quality and room freshness for sense of

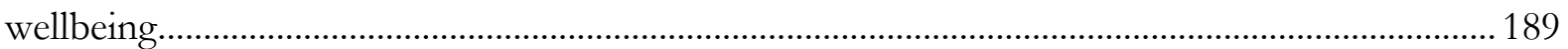

Figure 6-34: Respondents' ratings of hygienic/clean environment on sense for wellbeing ........ 190

Figure 6-35: Respondents' ratings of lighting control on sense for wellbeing............................... 190

Figure 6-36: Respondents' ratings of natural stimuli in the room on sense for wellbeing........... 191

Figure 6-37: Respondents' ratings of sensory comfort environment features............................... 192

Figure 6-38: Respondents' ratings of women situations during advice sessions ............................ 193

Figure 6-39: Respondents' methods of communication for parental skills..................................... 194

Figure 6-40: Respondents' ratings of opportunity to choose room for preference ....................... 195

Figure 6-41: A slightly positive skew distribution of ratings of preference to choose room ...... 195

Figure 6-42: Respondents' ratings of open bay wards decrease opportunity for discussion....... 196

Figure 6-43: A negative skew distribution of ratings of open bay wards on discussion .............. 196

Figure 6-44: Respondents' ratings of spatial connection of postnatal ward with birth rooms ... 197 Figure 6-45: A normal distribution of ratings of spatial connection of postnatal ward with birth

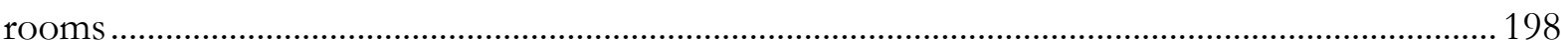

Figure 6-46: Respondents' ratings of a corridor for privacy in postnatal space ............................. 198

Figure 6-47: Respondents' ratings of communal space for education support ............................. 199

Figure 6-48: Respondents' ratings of furniture and décor for group learning support ................200

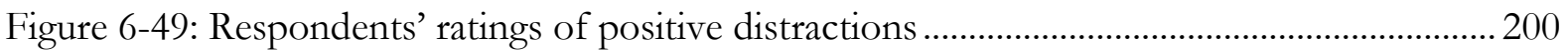

Figure 6-50: Respondents' ratings of interaction in single-bed rooms versus open wards........... 201

Figure 6-51: A negative skew distribution of ratings of interaction in single versus open ward. 202

Figure 6-52: Respondents ratings of social comfort environment features ................................... 203

Figure 6-53: Top fifteen design features influencing women's recovery experience (midwives) 205 
Figure 7-1: Respondents' perception on levels of importance regarding postnatal room features

Figure 7-2: Respondents' perception on levels of importance regarding interior environment features

Figure 7-3: Respondents' perception on levels of importance regarding sensory comfort environment

Figure 7-4: Respondents' perception on levels of importance regarding social comfort environment.

Figure 7-5: Flow chart of selection of design factors and architectural features in phase two ... 222

Figure 10-1: Linking of quantitative attributes to qualitative categories, adapted from (Buck et al., 2009) 286

Figure 10-2: Mixed methods findings linking quantitative and qualitative data 287 


\section{CHAPTER 1}

\section{INTRODUCTION}

Those who aspire to evidence-based design must ensure that more...data on medical outcomes of patients is generated, in order to inform better hospital design.

- Martin Colin (in C.Martin, 2000, p. 1)

The $2^{\text {nd }}$ International Conference on Health and Design, Stockholm, Sweden (2000)

and we should not forget that we are also building with the existing staff

- Ragnhild Aslasken, Chief Architect at Trondheim

Hospital, Norway (in Martin, 2000, p. 1)

\subsection{Problem statement}

For modern humans health and wellbeing have intrinsic connections to the places and spaces in which medical services are delivered. From a visit to a diagnostic centre, to an ambulatory routine check-up and to the transient-yet-forever experience of giving birth in hospitals, healthcare buildings and their physical environment have become an inseparable symbol for promotion and continuation of life and wellbeing. It is therefore critical to understand the factors in the design of hospital buildings that contribute to human health outcomes (Evans \& McCoy, 1998; Rubin, Owens, \& Golden, 1998; Schweitzer, Gilpin, \& Frampton, 2004; Ulrich, Zimring, Joseph, \& Choudhary, 2004; Ulrich et al., 2008).

In this era of increasing concerns regarding the experience of patients, staff, and childbearing women of hospitals and the current state of healthcare spending globally, some type of alignment and correspondence between medicine and architecture is critical for transforming and humanising hospitals (Crouwel, 2006; Verderber, 2006). As Verderber explains, it is concerning that modern hospitals fall down for their lack of appreciation of humanistic concerns in many dimensions (Verderber \& Fine, 2000). To promote a more humanistic healthcare environment, healthcare organisations have the task to improve the image of hospital spaces and create environmental meanings that could have a substantial role aligning with physical, emotional, and psychological wellbeing as key to person-environment transactions (Felippe, Kuhnen, da Silveira, \& Lelli, 2017; 
Ulrich et al., 1991) of different user groups, in particular women who continue to use hospital birth spaces on a daily basis.

Beginning from the 1940s, birth has moved from home to hospitals (Fink, 2011). This has led to hospital spaces for childbirth geared to the dictates of technology and to birth viewed as a medical condition (Foureur, Leap, Davis, Forbes, \& Homer, 2010; Malkin, 1992, p. 237). Improvements in design and in the planning of hospitals for users' experience are often met with challenges of matching costs with empirical evidence and outcome benefits (Ulrich, 2001). In recent times, with the emergence of concepts of patient-centred care, patient experience and satisfaction in domains of healthcare services are important to insure the effectiveness of healthcare organisations' commitments to high quality care (Manary, Boulding, Staelin, \& Glickman, 2013) including opportunities for improvements in the physical environment of care.

In the United States, 12 percent of the aggregate costs, estimated to be 361.5 billion dollars, of all hospital stays is due to birth-related hospitalisations (McDermott, Elixhauser, \& Sun, 2017; Wier et al., 2011) and increasing utilisation of healthcare services by women suggests birth-related healthcare spaces may need to prevent hitherto unrecognised exposure to risks and issues related to women's health and wellbeing prior to going home (Walker \& Wilging, 2000). Despite efforts in the 1960s and 70s by consumer-led movements in the UK and USA, that led maternity hospitals to be instrumental to creating therapeutic environments (Stichler, 2007; Verderber, 2010) aspects of postnatal hospital environments are still being neglected.

In the 1860s, Florence Nightingale laid down precedents for the design of a humanistic care environment for therapeutic purposes including adequate natural ventilation, warmth, noise control, colour and variety and beauty in a patient's room (Nightingale, 1863), which were ignored or slow in their application in design of healthcare environments for many years (Stichler, 2001). This approach of patient/user-centered care has implications in the maternity field, given the current assumption that healthcare spaces and facilities created for the general "unwell" hospital population can support the emotional, physiological and psychological needs of healthy women who give birth in hospitals.

In parallel Williams (1995) argued that there is a need for building and design adaptation to reflect the needs of "general" patients as distinct from the "special" population of the very unwell in both the designing and planning stages of healthcare facilities, ensuring all users' needs are taken on board (van Hoof \& Verkerk, 2013). 
Existing studies have found links between healthcare facilities and patients wellbeing and positive outcomes for staff including supporting environmental needs of women in hospitals (Alvaro, Wilkinson, Gallant, Kostovski, \& Gardner, 2016; Devlin \& Arneill, 2003; Schreuder, Lebesque, \& Bottenheft, 2016). The ensuing field of evidence-based design (EBD), which is an embodiment of ideas shared among architects, healthcare designers, neuro-scientists and medical professionals, has proven that it can be a useful tool to improve hospital architecture that is more responsive and inclusive for the needs of users (Mazuch \& Stephen, 2005; Stichler, 2011; Verderber, 2006; Wagenaar, 2006). Proponents and adherents of EBD share common aspirations that utilising the latest research findings in creating healing environments termed as "smart investments" in design and planning of healthcare facilities, including renovations and refurbishments could assist in making hospitals less stressful, safer and more efficient and could go a long way in benefitting a wide category of users, thereby serving as vital tools in the promotion of patient-centred care programmes (Huisman, Morales, van Hoof, \& Kort, 2012, p. 70; Orr, 1995; Stichler, 2011; Ulrich, 1992).

Many decades of research in EBD have yielded a handful of rigorous studies that provide a basis for the design of healthcare buildings focusing on specific design features for patient outcomes, including single-bed occupancy rather than multi-bed rooms, negative impacts of noise on patients' sleep and recovery, appropriate lighting systems, and reduction of air-borne infections through proper ventilation as well as provision of positive distractions for patients to reduce pain; these studies and more form the knowledge base of the EBD movement (Ulrich et al., 2004; Ulrich et al., 2008). However, none of these studies are inclusive to the birth environment (Foureur, Davis, et al., 2010), which raises the question about the fit and applicability of those studies to the birth environment in general.

Since most existing findings relate to healthcare in the context of "illness" it may be reasonable to infer that healthcare buildings could work differently on people regarding the physiological, emotional and psychological processes of birth and recovery experiences of "well" women giving birth - a significant social and salutogenic event of a woman's life and that of her family (Dahlen, Barclay, \& Homer, 2010; Fink, 2011; Foureur \& Harte, 2017). Ultimately, transferring those EBD findings relating to unwell patients into hospital birth environments may be incompatible with respect to the wellness situations of healthy women who give birth in hospitals. 


\subsubsection{Users experience in designed birth and postnatal environments}

Healthcare buildings are designed to cater for the needs of both users and caregivers, who have to be able to do their work effectively without hindrance. Design and layouts affect experience and the way individuals feel in spaces. Research shows that both women and caregivers are affected by the design of hospital birth facilities with caregivers often struggling in the spaces to be able to do their work (Davis \& Walker, 2010; Hammond, Homer, \& Foureur, 2014). Accordingly since users spend time in hospitals and healthcare providers work long hours in birth spaces, providing care, both groups, women and caregivers, could provide useful and substantial feedback regarding their experiences in the physical environment and may be able to proffer solutions for positive experience of hospital stays for women who go to hospital to give birth.

The ubiquitous presence of nurses and midwives and other healthcare providers in healthcare environments sometimes puts them into a representational capacity for patients regarding design of healthcare facilities because of their experience and this singular ability means they could communicate a variety of ideas, opinions, beliefs and expertise in a manner that can be useful to healthcare designers and architects (Annemans, Van Audenhove, Vermolen, \& Heylighen, 2018; Igarashi, Wakita, Miyazaki, \& Nakayama, 2014; Seibold, Licqurish, Rolls, \& Hopkins, 2010).

Others may collate and synthesise opinions and information that stakeholders in healthcare have supplied and translate them into useful design parameters. An example of such a platform for collation and integration of information was the interdisciplinary amalgam between architectpractitioners, healthcare designers, and midwifery/nursing scholars' and researchers that created the Birthing Unit Design Spatial Evaluation Tool (BUDSET (Foureur, Leap, et al., 2010; Foureur, Leap, Davis, Forbes, \& Homer, 2011; Lepori, 1994; Lepori, Foureur, \& Hastie, 2008) primarily focused on women in achieving a positive normal birth experience (Dahlberg et al., 2016; Stark, Remynse, \& Zwelling, 2016).

Additionally, the concerns of researchers, scholars and midwives in facilitating a physical environment of birth and postnatal care with a focus on wellbeing have increasingly led to the adoption of the principle of the salutogenesis model in transforming hospital birth spaces. The salutogenesis principle conceptualises the physical environment in terms of health promotion in contrast with the pathogenesis found in the biomedical-focused childbirth environment (Foureur \& Harte, 2017). This stems from Antonovsky's (Antonovsky, 1987, 1996) work in which the key premise is the sense of coherence (SOC) which defines a health - disease continuum as the basis for health promotion and directs efforts in both research and action to encompass all persons such 
that they focus on what promotes their wellbeing in physical healthcare environments. This approach, including other theories used in this thesis, is discussed in greater detail in section 1.5.

Research shows environmental satisfaction is strongly linked with overall satisfaction in healthcare settings, just under perceived quality of clinical care. Factors including space, light, views to nature and environmental stressors such as noise and lighting affect women and staff who work in healthcare environments (Foureur, Davis, et al., 2010; Ulrich et al., 2008).

Previous studies on the evaluation of environmental factors in the childbirth environment have focused on labour and birth rooms, leading to specific designs and guidelines for birthing design (Hauck, Rivers, \& Doherty, 2008; Hodnett, Stremler, Weston, \& McKeever, 2009; Jenkinson, Josey, \& Kruske, 2014; Newburn \& Singh, 2003; Shin, Maxwell, \& Eshelman, 2004). However, no studies have fully investigated environmental factors and design characteristics in postnatal environments underscoring the potential need for this study. In particular, a very recent review by Setola et al. (2019) of relevant literature in the childbirth environment shows a lack of studies investigating this all important topic. Additionally design of childbirth facilities affects care providers' ability to provide care which may have consequences on workflows and expectations in the discharge of quality recovery care (Plough et al., 2019).

Consideration of the impact of the postnatal environment on women's recovery experiences highlights a considerable gap in knowledge in the childbirth literature. From design and planning perspectives, postnatal wards are often located in separate areas or on another floor of a hospital where women may need to walk a considerable distance to get to the postnatal facility. This situation could be stressful during the postnatal period and could result in unintended consequences (Stark, 2000). Another crucial factor that arguably has impact on women's hospital experiences and quality care is the considerably shorter postnatal hospital stays after childbirth. American Academy of Paediatrics (AAP) and the American College of Obstetricians and Gynaecologists (ACOG) defined "early postnatal discharge" as a post-delivery stay of less than 48 hours for women who had uncomplicated vaginal births and less than 96 hours for those who had caesarean sections (CS) (American Academy of Paediatrics \& American College of Obstetricians and Gynaecologists, 1992). (Brown, Small, Argus, Davis, \& Krastev, 2002) argued there is a lack of consensus about what constitutes "early discharge" following childbirth as this concept assumes there is a standard length of time for which women have to stay in hospital. Several studies have been published focused on early postnatal discharge/short length of stay in many resource-rich countries. For example, in-hospital length of stay (LOS) in Australia indicates three to four days for a normal vaginal birth and four to five days for a ceaserean birth (McLachlan 
et al., 2009); in Denmark, women are discharged to home within 24 hours or less (Danbjørg, Wagner, \& Clemensen, 2014); and in the UK, (Beake, McCourt, \& Bick, 2005; Beake, Rose, Bick, Weavers, \& Wray, 2010) reported a postnatal hospital stay of 1.5 days for a normal vaginal birth and as late as two to four days approxinately (3.9 days) for ceaserean births. With regards to Canada, it is 2.6 days for a normal vaginal birth and three days for ceaserean sections (Wen, Liu, Marcoux, \& Fowler, 1998). Similar trends of shortening of postnatal length of stay in hospital are seen in countries such as the United States (Fink, 2011), in Sweden (Johansson, Aarts, \& Darj, 2010), and the Netherlands Details of postnatal length of stay (LOS) in resource-rich countries are illustrated in Table 1-1, showing a broad range of what is considered appropriate (Watt, Sword, \& Krueger, 2005).

Researchers have discussed the shortening in length of maternal hospital stay (Brown et al., 2002; Danel, Johnson, Berg, Flowers, \& Atrash, 1997; Datar \& Sood, 2006), however, findings from different studies show maternal hospital stay varies from 12 hours to up to 72 hours (Danbjørg et al., 2014). Longer postnatal hospital stays of more than 24 hours enable nurses/midwives and other healthcare professionals to provide support and education to ensure that mothers have smooth transitions to parenthood (Patterson, 1998). A comfortable setting adequate for relaxation is likely to make the care-giving and support less stressful, more effective and efficient. (Bravo, Uribe, \& Contreras, 2011) conclude there is not yet substansive evidence available to either reject or accept that early postnatal discharge could have detrimental effects on new mothers and newborns due to mixed outcomes, however, hospital characteristics (Danel et al., 1997), which may include the physical environment, cannot be overlooked.

While there is no doubt that maternal mortality is significantly lower in most high income countries (UNICEF \& World Bank, 2015) and childbirth is not regarded as life threatening, there is a need to focus on postnatal women - especially in large tertiary hospitals - to make sure there is an optimum environment to facilitate the rest and recovery experience (Adatia, Law, \& Haggerty, 2014). As Warren, Daly, Toure and Mongi (2006) explain, lack of care during this postnatal period may have consequences including missed opportunities to promote health and wellbeing and information for the care of women and newborns. A physical environment to facilitate rest and recovery is of the essence because most labours are now taking longer than in past decades (Aburas, Pati, Casanova, \& Adams, 2017). Consequently, the fatigue, tiredness and exhaustion arising from labour and birth and the influence of the postnatal hospital environment could play a significant role in women's hospital recovery experiences and subsequent physical and psychological wellbeing. 
Table 1-1: Overview of postnatal length of stay in resource-rich countries

\begin{tabular}{|c|c|c|c|c|}
\hline \multirow[t]{2}{*}{ Author } & \multirow[t]{2}{*}{ Country } & \multicolumn{2}{|c|}{$\begin{array}{l}\text { Postnatal length of stay } \\
\text { (LOS) (hours) }\end{array}$} & \multirow[t]{2}{*}{ Impact of the physical environment } \\
\hline & & $\begin{array}{l}\text { Uncomplicated } \\
\text { vaginal birth }\end{array}$ & $\begin{array}{l}\text { Ceaserean } \\
\text { birth }\end{array}$ & \\
\hline $\begin{array}{l}\text { McLachlan, et } \\
\text { al., (2009) }\end{array}$ & Australia & Around 48 & $\begin{array}{l}96-120 \\
(4-5 \text { days })\end{array}$ & $\begin{array}{l}\text { Increasing pressure on postnatal beds and } \\
\text { spaces for the care of women }\end{array}$ \\
\hline $\begin{array}{l}\text { Shi Wu et al., } \\
\text { (1998); Public } \\
\text { Health Agency } \\
\text { of Canada, } \\
\text { (2009). }\end{array}$ & Canada & Around 48 & $\begin{array}{l}72 \\
(3<4 \\
\text { days })\end{array}$ & $\begin{array}{l}\text { Reduction in staffed beds in Canadian } \\
\text { hospitals }\end{array}$ \\
\hline $\begin{array}{l}\text { Danbjørg, et } \\
\text { al., (2014) }\end{array}$ & Denmark & 24 or less & & $\begin{array}{l}\text { An "asynchronous" environment to meet } \\
\text { needs of new parents, and may include the } \\
\text { physical environemt within hospitals }\end{array}$ \\
\hline $\begin{array}{l}\text { Campbell, et } \\
\text { al., (2016); } \\
\text { OECD, (2014) }\end{array}$ & Netherlands & 24 or less & & \\
\hline $\begin{array}{l}\text { Research New } \\
\text { Zealand. } \\
\text { Maternity } \\
\text { Consumer } \\
\text { Survey, (2014); } \\
\text { OECD, (2014) }\end{array}$ & $\begin{array}{l}\text { New } \\
\text { Zealand }\end{array}$ & $\begin{array}{l}\text { Around } 48 \text { or } \\
\text { less }\end{array}$ & & $\begin{array}{l}\text { Busyness and routine of the hospitals were } \\
\text { among the reasons for leaving hospitals even } \\
\text { though a few women were not yet ready to } \\
\text { leave. Lack of space where partners can stay } \\
\text { overnight }\end{array}$ \\
\hline $\begin{array}{l}\text { Johansson, et } \\
\text { al., (2010) }\end{array}$ & Sweden & Around 48 & & $\begin{array}{l}\text { New parents were excited to leave hospital } \\
\text { settings because they constitute hindrance to } \\
\text { perform important parental lessons. Home } \\
\text { was seen as a familiar environment, where } \\
\text { parents can recover in comfort, feel secure } \\
\text { and safe }\end{array}$ \\
\hline $\begin{array}{l}\text { Boulvain, et al., } \\
\text { (2004) }\end{array}$ & Switzerland & $\begin{array}{l}72-96 \\
(3-4 \text { days })\end{array}$ & $\begin{array}{l}120-144 \\
(5-6 \text { days })\end{array}$ & $\begin{array}{l}\text { Economic constraints and unavailability of } \\
\text { hospital beds, and issue related to postnatal } \\
\text { spaces }\end{array}$ \\
\hline $\begin{array}{l}\text { Beake, et al., } \\
(2005 ; 2010)\end{array}$ & $\begin{array}{l}\text { United } \\
\text { Kingdom }\end{array}$ & $\begin{array}{l}36 \\
(1.5 \text { days })\end{array}$ & $\begin{array}{l}48-96 \\
(2-4 \text { days })\end{array}$ & $\begin{array}{l}\text { The routine of postnatal environment } \\
\text { constitutes an impediment for rest in new } \\
\text { mothers who have had long hours of labour } \\
\text { and experiencing pain including difficult } \\
\text { demands of a newborns. Additionally, lack } \\
\text { of privacy in hospitals influences a short } \\
\text { length of postnatal stay. }\end{array}$ \\
\hline $\begin{array}{l}\text { Braveman, } \\
\text { 1995; Declerq, } \\
\text { 1997; Fink, } \\
\text { (2011) }\end{array}$ & $\begin{array}{l}\text { United } \\
\text { States of } \\
\text { America }\end{array}$ & $12-24$ & $48-72$ & \\
\hline
\end{tabular}

From recovery perspectives, there could be interesting connections with the seminal work by Ulrich. Roger Ulrich, an environmental psychologist and architecture professor, posits that merely exposing hospital patients to a physical environment with rich, pleasant views of nature increased their recovery rates and improved positive health outcomes in comparison with patients who 
viewed blank walls (Ulrich, 1984). If the hospital patients benefited from a pleasant and relaxing environment then women having a maternity hospital stay could also be better off when cared for in a similar postnatal environment, thereby reducing stress and assisting them to focus on the important tasks of parenting and recovery. Aspects of the physical environment such as light, colour, sound, aroma, space and texture have a substantial influence on physical and emotional wellbeing such that their careful application in healthcare settings may serve as "good medicine" (Gappell, 1995).

Existing knowledge regarding postnatal health and hospital environments could be traced to a set of studies by Martell (Martell, 2000, 2001, 2003). These studies have become a reference point for many other studies focused on the area of postnatal care in hospitals generally. Martell (2003), describing postnatal women's perceptions of the hospital environment, found the physical environment such as sensory stimuli, room size, furnishings, and location of room for quietness had impacts on postnatal experiences; Symon, Paul, Butchart, Carr and Dugard (2008b) examined environmental comfort and control in which noise was a barrier to women's hospital experiences; Forster et al. (2006) identified staffing as a contributory factor for the provision of quality postnatal care when they explored the experiences and expectations of women receiving in-patient postnatal care; Beake et al. (2010) found that qualities of the postnatal ward environment were among key factors standing in the way for women's positive experience. In Australia, hospital-based postnatal care has been studied by a group of scholars focused on both private and public hospitals (Rayner, McLachlan, Peters, \& Forster, 2013; Yelland, McLachlan, Forster, Rayner, \& Lumley, 2007). However the study by Rayner, Forster, McLachlan, Yelland and Davey (2008, p. 317) which explored the vision of postnatal care in an "ideal world" was not only interested in the problem but was important because of the quality of the questions that were asked of the midwives as participants. Rayner and colleagues asked: what is the vision of postnatal care in an ideal world regarding the physical layout of the postnatal environment, the organising of care and the resources considered important to achieving optimum postnatal care? This question requires an approach to and contributions from both architecture and midwifery/nursing backgrounds including obtaining users' perceptions of design factors for postnatal facilities in order to inform decision making in design, planning, reorganisation of existing facilities and construction of new build postnatal environments. Pragmatically, efforts aimed at improving maternity services for women's experiences in the immediate postnatal phase have proved difficult and challenging to implement for staff regarding routine, design and layout of postnatal hospitals (Cuncarr \& Skinner, 2011). Ideally, women want to stay close to a hospital that is safe, secure and conducive for rest and 
privacy for both birth and the postnatal period rather than at home (Beake et al., 2005; Beake et al., 2010; Cuncarr \& Skinner, 2011; McLachlan et al., 2009).

If more supportive environments were available they could lessen the fears and anxiety experienced by one-third (186/572) of first-time mothers and eight percent (195/2446) of multiparas transferred to hospitals from home in four Nordic countries (Blix et al., 2016). Even though these were not urgent transfers, they suggest reasons to consider creating better, more inclusive and "friendly" postnatal hospital facilities and spaces. In this regard, healthcare organisations have a duty of care in driving the changes in design and layouts and features of postnatal environments to support needs of women especially in large, tertiary hospitals, which research has found unconducive and unrestful for recovery experiences for new mothers while hindering support from staff (Adatia et al., 2014; Rayner et al., 2008).

International research reveals a number of initiatives and strategies focused on postnatal care provision ranging from emotional supports in the UK (Bhavnani \& Newburn, 2010; MacArthur et al., 2002); increase in individualised care for women in Australia (Morrow, McLachlan, Forster, Davey, \& Newton, 2013; Schmied, Cooke, Gutwein, Steinlein, \& Homer, 2009); progressive forward planning for maternity and neonatal care in Scotland (The Scottish Government, 2017); home care after early discharge in Sweden (Askelsdottir, Lam-de Jonge, Edman, \& Wiklund, 2013); postnatal continuity of care in the women's home or at a community centre in New Zealand (Cuncarr \& Skinner, 2011; Davis \& Walker, 2010), clinical guidelines for women and infants in the USA (Haran, Van Driel, Mitchell, \& Brodribb, 2014); to design of context-specific packages in Sub-Saharan Africa countries (Duysburgh et al., 2015). Clearly these initiatives are indicators of the values placed on women's health and wellbeing after the birth. However, postnatal hospital environments and overall ambience could have a greater role in postnatal recovery care, in particular when poor building design and physical environment have potential links with avoidable maternal fatalities and consequences (PMMRC, 2018). Another key driver affecting quality postnatal care during hospital stays has been found to be addressing building designs and layouts that minimise long travels to reach facilities in the postnatal environment while adequate resources mobilisation specifically for postnatal maternity services could help in creating more supportive environments that also consider more bed provisions, which have been key drivers affecting quality postnatal care during hospital stays (Goodwin, Taylor, Kokab, \& Kenyon, 2018; McLachlan et al., 2009; Redshaw et al., 2011). The alternative of a family suite more like a hotel-setting was also found to offer women the comfort of adequate rest including support from staff, making a contribution to their positive outcomes and wellbeing (Parsons, Mahoney, \& Weathers, 1999). 
Turning to New Zealand, 59,661 women gave birth in 2017 with 96.6 percent of the births occurring in hospital settings, including 29,913 women giving birth in tertiary hospitals. An additional 24,230 and 5,886 women gave birth in secondary and primary facilities (Ministry of Health New Zealand, 2019). Women require adequate care in a supportive environment for their health and wellbeing but the identification of a quiet, stress free and supportive environment for women and new families has been recently identified as a huge gap in the provision of adequate recovery care for the wellbeing of a mother and baby in New Zealand hospitals in the immediate postnatal phase (Maternity Services Consumer Council, 2018).

Without the consideration of the physical environment, provision of quality postnatal care in the immediate postnatal phase may continue to be problematic and women may continue to have perceptions of not being adequately supported in terms of basic needs of individuals and learning parental skills and support during hospital stays (Cuncarr \& Skinner, 2011; Rudman \& Waldenström, 2007) as well as how to enjoy restful days while balancing care of their baby (Kurth et al., 2010). Correspondingly, creating postnatal spaces that are less constraining and more enabling towards an optimum birth satisfaction and relaxation can have potential for lessening the impact of the biomedical focused hospitals which many women use for giving birth including in New Zealand (Davis \& Walker, 2010; Grigg et al., 2017; Grigg, Tracy, Schmied, Daellenbach, \& Kensington, 2015; Keating \& Fleming, 2009; Overgaard, Fenger-Grøn, \& Sandall, 2012). Focusing attention on the physical environment suggests a pathway for how postnatal recovery care could be organised in the future addressing women's wellbeing after birth (Schmied \& Bick, 2014).

More importantly, these existing studies have shed light regarding the problem and laid the foundation for this current study, setting the research in the broad spectrum of evidence-based design to investigate the impact of the physical environment regarding recovery care and physical restoration for women during the postnatal phase. Research is lacking regarding design guidelines for creating an appropriate optimal postnatal hospital environment to meet environmental needs and support of women during the postnatal phase.

In order to gain a better understanding of hospital recovery experience for women and what building spaces and architectural features could provide for more conducive and restorative environments and to facilitate quality postnatal care, this study was undertaken. The focus of the study is on the users of postnatal facilities - women and midwives - but the ultimate aim is to determine how women might be better supported in hospitals by applying concepts and ideas from a range of theories of supportive design to understand how restorative spaces might serve to 
support women and make a difference in assisting midwives in adequately catering for women and babies prior to returning home.

\subsection{Research aim}

The aim of this research is to gain an understanding of the design factors and building spaces influencing women's recovery experiences and to develop evidence-based design guidelines for restorative postnatal spaces in urban tertiary hospitals. The central goal is to offer direction for support environments to facilitate quality postnatal care of women in large, tertiary hospitals.

The study had three objectives:

1. To summarise the state-of-the-art research on the effects of the general healthcare environments on health outcomes and wellbeing with a view to gaining insights about the design factors in the postnatal hospital environment influencing women's recovery experiences.

2. To identify the users' perceptions of design factors in the postnatal hospital environment influencing women's recovery experience and how the physical environment impacts midwifery practice for quality postnatal care.

3. To determine the architectural characteristics and design factors in the postnatal environment and their impacts on recovery experiences of women and wellbeing in the immediate postnatal phase.

\subsection{Research questions}

Evolving from the gap in the interdisciplinary literature, this study was structured around the main research question of "how can restorative and conducive settings facilitate the postnatal recovery experience and wellbeing in large tertiary hospitals?" The study had other sub questions:

1. What are the perceptions of design factors influencing women's recovery experience and wellbeing during postnatal hospital stays?

2. What were the ways in which the design factors and the postnatal spaces affected women's recovery experiences and wellbeing during hospital stays?

3. What are the implications of the findings for design guidelines for postnatal restorative spaces to assist quality postnatal care in large, tertiary hospitals? 


\subsection{Significance of the study}

This study is an applied piece of research that investigates a real-world problem, the provision of quality postnatal care especially in large, tertiary hospitals. As discussed earlier, the physical environment has a potential role in the delivery of quality postnatal care for new mothers who give birth at tertiary levels of care.

The research uses findings from both women and midwives to study restorative environments for recovery experiences in hospitals, its results will form valuable inputs for healthcare design scholars, architecture-for-health practitioners, midwifery researchers and practitioners, and healthcare providers in specific ways:

First, the significance of this thesis is that it shifts the current focus from birth and labour roomsrelated research to recovery of new mothers in the postnatal environment, enabling a better understanding of design characteristics and building spaces that may influence physical restoration and wellbeing of women in the immediate postnatal phase in which there is a gap in knowledge. The findings from this thesis, which can be taken as a first exploratory attempt at studying the physical environment and design factors of the postnatal hospital environment present opportunities for future research in both the healthcare design research and midwifery/nursing research disciplines.

The second significance of this thesis is that the findings will be useful for a wide range of applications in order to be better able to deliver efficient and quality postnatal care for new mothers prior to leaving hospitals. For midwives, improved organisation of postnatal wards and spaces would enable more effective, functional, and quality caregiving for women.

The third significance of this thesis concerns how the findings would assist architects, healthcare designers and healthcare organisations in making improvements in the postnatal hospital environment in three areas - in changing how an existing building is used, in upgrading the existing spaces, and in constructing new postnatal units. The application of the design guidelines and research knowledge would likely be beneficial in supporting the physical, emotional, physiological and psychological health and wellbeing of women while using hospitals as transient spaces prior to home. 


\subsection{Theoretical framework of the study}

Explanatory theories are fundamental to bridging the gaps between design, environments, and humans (Moore, 1984). The relationships between environmental stress, restoration, and psychological wellbeing have been well studied mainly in the environmental psychology and architecture fields particularly under the banner of person-environment transactions. Theoretical underpinnings from the Birth Territory Theory, Attentional Restorative Theory (ART), PsychoEvolutionary Theory (PET), the Salutogenic Theory, and Theory of Supportive Design construct the knowledge base in this thesis (see Figure 1-1).

Women are affected by the physical environments in which birth occurs, particularly in hospital environments. The Birth Territory Theory (Fahy, Parratt, Foureur, \& Hastie, 2011; Fahy \& Parratt, 2006), proposes "terrain" and "jurisdiction" as two main concepts to predict how the physical environment affects women and babies during the stages of childbearing during the labour, birth and also postnatal phases.

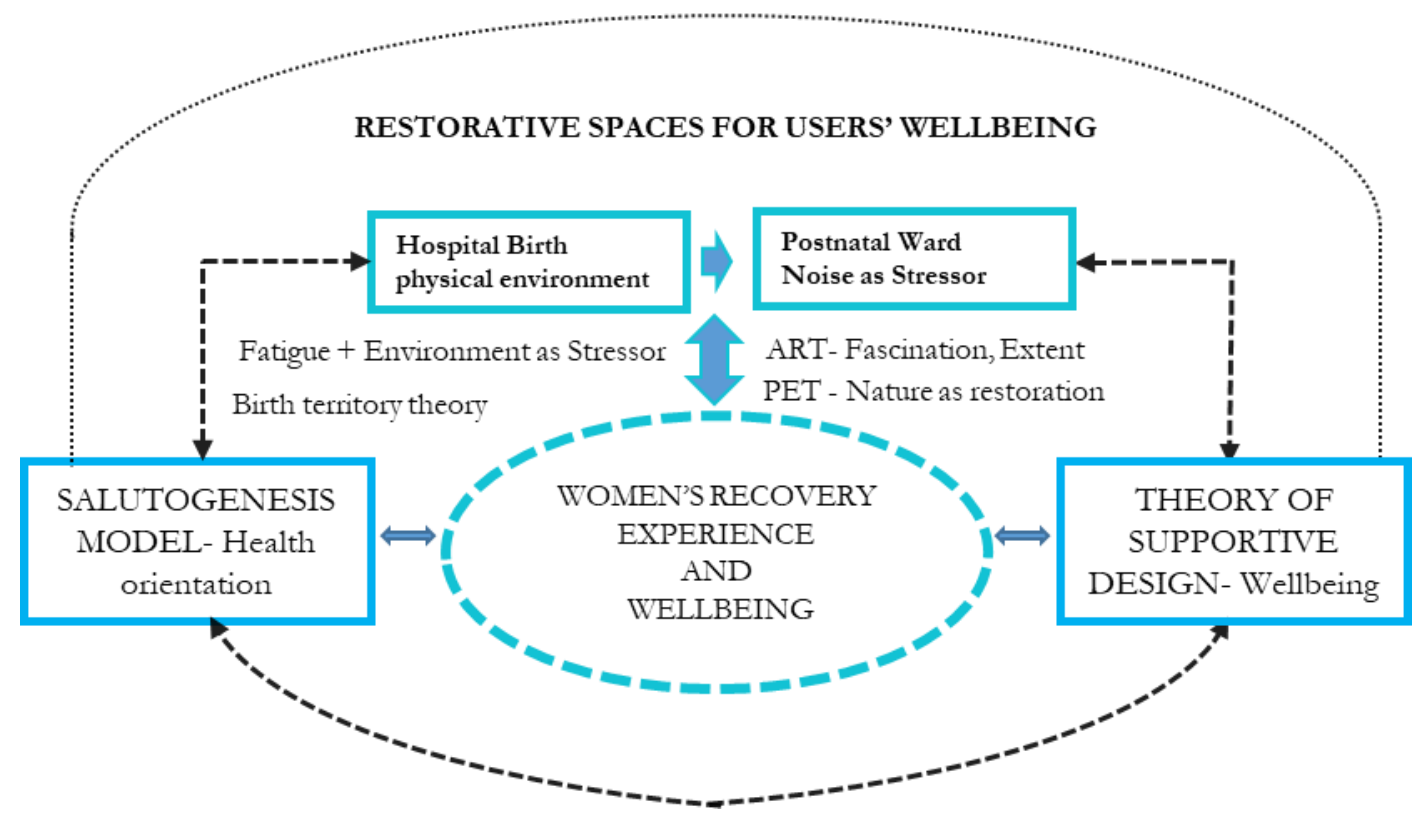

Figure 1-1: Conceptual model for recovery experience and wellbeing in this study

Succinctly put, the theory espouses how the birth environment operates and how the concept of jurisdictive power applies in the birth settings (Hastie, 2011). By definition, a terrain is constructed as the physical features and geographical area of the women's birth environment. According to the 
theory, ideal birth terrain is conceptualised as a "sanctum" in which the woman has a homelike environment to optimise privacy, comfort and control. On the other hand a "surveillance room" is characterised by a clinical environment to facilitate the observation of the woman for the ease of the staff. In the process of giving birth to a life, to a greater or lesser degree some levels of pain and stress are anticipated. To reduce the impact of stress while giving birth, the Birth Territory approach could be relevant in the design of both the birth and postnatal environments to eliminate or reduce the impact of stressful encounters. Logically, moving to the postnatal environment can be regarded as a way of moving to a sanctuary for birthing women away from the jurisdiction of a more medically-focused environment. The environments, interactions and freedom associated with a sanctuary in the postnatal phase could help physical restoration, in particular given the wellness situations of the woman in the birth settings (Foureur, Davis, et al., 2010; Foureur \& Harte, 2017; Van den Berg, Hartig, \& Staats, 2007). A supportive postnatal environment that incorporates environmental features allowing a "sanctum" function could aid restoration of women and could optimise recovery experiences.

In Attention Restorative Theory (ART) (Kaplan \& Kaplan, 1989; Kaplan, 1995) the restoration process can be understood as restoration of cognitive functioning after mental fatigue or directed attentional fatigue (DAF); in other words, an antecedent state of prolonged use of directed attention that could lead to mental fatigue. The Psycho-Evolutionary Theory (PET) (Ulrich, 1983; Ulrich et al., 1991), posits the importance of affective functioning when humans face a threatening or challenging situation. According to ART, individuals can restore a diminished capacity when they encounter fascination, an involuntary model that is effortless and limitless such that when a person is immersed in it, it provides opportunity to rest the attentional- dominance scenarios. The process of stress recovery also means that a person whose psychological resources have been depleted needs a psychological distance away from the tasks which are depleting resources- being away-and recovery could be sustained when the environment is coherent, ordered and rich in scope or extent. The last stage of the process is the recognition of the fit of the person's inclinations and the environmental support for the intended activities or compatibility (Kaplan \& Kaplan, 1989; Kaplan, 1995). Conceptually, both ART and PET theories have convergence that humans have a biological inclination for responding more to natural settings such as vegetation or water features as opposed to most built environments (Kaplan \& Kaplan, 1989; Ulrich, 1984, 1999).

The event of birth suggests that women are more likely to over-use directed attentional capacity that may lead to mental fatigue, tiredness and exhaustion. At the same time, environmental stimuli in the birth and postnatal environments also contribute to stress. Stressful situations include 
frequent noise, busyness and routine in postnatal environments, crying babies, and ultimately sleep deprivation, anxiety and lack of restfulness that could be severe and interfere with the wellbeing of new mothers. Consequences of stress include negative emotions and heightened autonomic arousal.

Recent conceptualisation of natural environments in healthcare research (Chi, Gutberg, \& Berta, 2020), since it is unlikely to be possible to achieve being away and extent while in hospital, arguably by no means equates to what the authors of ART suggested particularly for women who are in hospitals to give birth. However, some design features such as single-bed rooms, single-family rooms, exposure to daylight and outdoor contact may be beneficial for stress reduction and reducing the impact of noise, while providing privacy for meditation, enhancing positive experiences and wellbeing of users including staff members (Beauchemin \& Hays, 1996; Chaudhury, Mahmood, \& Valente, 2005; Choi \& Bosch, 2013; Nejati, Shepley, Rodiek, Lee, \& Varni, 2016; Pati, Freier, O’Boyle, Amor, \& Valipoor, 2016). Perception of the value of healthcare environments that are restorative is growing especially in interior spaces for women's and staff wellbeing (Pati, Harvey Jr, \& Barach, 2008; Schreuder et al., 2016; Shin et al., 2004)

The Theory of Supportive Design proposes that the qualities of healthcare physical-social environments contribute to stress reduction and promote wellbeing of patients and women in hospital birth facilities. In particular, Roger Ulrich (1991) assumes that healthcare physical and social environments address stress and foster wellbeing if they focus on a sense of control, access to social support and access to positive distractions in physical surroundings. Encounters with birth and subsequent postnatal recovery in health facilities influence stress levels (Buckley, 2015; Tanja-Dijkstra, 2011). The three elements of the theory, access to social support, positive distractions and sense of control, when present in hospital rooms, suggest lower stress levels for patients and in this case women (Andrade \& Devlin, 2015). Sense of control in hospital rooms was associated with stress reduction among individuals who expressed desirability for control (Andrade \& Devlin, 2016).

Women and their partners or family members are more likely to have a greater sense of control when they are either in single-bed rooms or family suites which could help in stress reduction and facilitate social comfort and wellbeing (Ulrich, 1991). Comfortable environments that encourage the bonding of new mothers and babies with their families could permit physical and psychosocial benefits that could be useful in restoration and wellbeing of women. 
The Salutogenic Theory hinges on factors that actively promote health-giving and wellbeing as opposed to a focus on illness or pathogenesis (Antonovsky, 1996). The central idea is that a supportive environment could make a difference when someone is in hospital. In 1991, Antonovsky looked at identifying health-giving factors in contrast to disease-causing factors. At the heart of the Salutogenic Theory is what is termed as a "sense of coherence" (SOC) which emphasises that a person with greater health resources has an appropriate coping approach in tackling stressful events. The principal elements promoted by the Salutogenic Theory which relate to SOC emphasise the importance of the three components of comprehensibility, manageability, and meaningfulness that may be applied in the design of supportive environments especially in postnatal hospital environments. A strong sense of coherence correlates to higher scores on the three components (Dilani, 2015) which are described below.

Comprehensibility is the ability to recognise the things in the environment such that it may be possible to develop feelings and experiences of them. As Golembiewski (2010) notes, in architectural parlance, readability, that is, reading the environment to make meaning of it, equates to comprehensibility. As sensing and responding to the environment usually occurs in the subconscious whether planned or unplanned (Hastie, 2011), women in a postnatal hospital environment would need a supportive environment that is not disorientating and less stressful to assist in their recovery process (Bailey \& Timmons, 2005).

When a woman is in control of the postnatal environment and can adjust the room features, furniture and equipment, this access to resources for coping depicts the sense of manageability (Antonovsky, 1987). There are however situations where the birth and postnatal environments are detrimental to a woman's intentions, amplifying the power element of the birth territory (Fahy, Foureur, \& Hastie, 2008; Fahy et al., 2011). In a postnatal environment, provision of single-bed rooms for individualised care for women who aspire to it may serve to promote manageability in the hospitals. Such arrangements could benefit women to be able to focus on themselves and their baby while promoting recovery.

Meaningfulness is derived when a person is motivated to find meaning in internal or external environments that sustain coping. A postnatal hospital stay should afford implicit or explicit opportunity for interaction and experience of the environment with installation of supportive visual features and facility for social support for recovery experiences and satisfaction (García Marcos et al., 2014; Golembiewski, 2010). 
Given the antecedent condition of the process of labour and birth for women and environmental stressors such as noise in the postnatal environment, consideration of both the Theory of Supportive Design and the Salutogenic Theory suggest that supportive environments can be approached and applied in the design of postnatal hospital environment for optimum recovery experiences and wellbeing and can be useful for creating design guidelines.

\subsection{Research approach - reviews of previous PhD research methodologies}

Finding a suitable methodological approach is a critical part of the research investigation. The process of finding one in this thesis was not an easy or straightforward task.

Due to limited existing research focused on the investigation of the physical environment's impact on the recovery of women in postnatal hospital environments, this thesis reviewed research design approaches previously used by other researchers in the general study of birth environments. Similar $\mathrm{PhD}$ studies which used quasi-experimental design (Stark, 1999), quantitative experimental design (Aburas, 2014) and mixed research synthesis methods (Havill, 2012) were reviewed. Aside from these, a work by Felippe et al. (2017) which assessed restorative hospital environments was reviewed.

Among the four studies, Stark (1999) and Felippe et al. (2017) closely aligned with this thesis in terms of their focus both on restorative intervention and restorative hospital environment contexts. The first case adopted a three-stage design measurements approach taking the focus from labour and birth rooms up to 7 days post-birth. Using a quasi-experimental approach, Stark (1999) conducted her experiment on restorative intervention with 57 women and measured four concepts: psychosocial tasks, directed attentional capacity, restorative intervention and labour pain. Data collection took place within the period of 1-7 days post-birth enabling the researcher to describe how the participants were affected by attentional demands - a concept from the ART as one of the theories framing this study - during pregnancy with physical environment appearing to play a role in the functioning of women.

Because women in the study were encouraged - on their own and perhaps less observed - to engage in 120 minutes of restorative activities in a natural environment and keep a log, the approach raises many methodological and moral issues. Although quasi-experimental design to a large extent facilitated data collection in the study, its potential flaws include the researcher's inability to keep track of previous restorative activities of women thereby raising issues with the results (Stark, 1999, pp. 145-146). The reliance on quantitative methods alone limits opportunity 
for women who are eager to tell their birth stories, inclusion of a focus group would provide stronger views regarding women's health (Savage, 2001; Stark, 1999). Consequently, using focus groups in this thesis allowed for triangulation of both quantitative and qualitative methods with expert validation. This study did not use a similar quasi-experimental method due largely to lack of natural environments in many healthcare settings, although the reintegration of the natural world back into the mainstream healthcare environment has been an ongoing process (Campagnol \& Shepley, 2014; Gerlach-Spriggs, Kaufman, \& Warner, 1998; Marcus \& Sachs, 2014; Shepley, 2012).

In the second case, Felippe et al. (2017) evaluated the relationships between environmental meaning and affective stress restoration and physical characteristics of pediatric rooms. A direct observation of the hospital built environment using photographic and video elicitation techniques (see below) and self-administered questionnaires complemented with architectural plans were used to collect data in four Italian hospitals. However, the study was focused on paediatric patients aged 8 years and above (Felippe et al., 2017), which limited the scope and was a different context from postnatal recovery of women in hospital environments.

The work of Havill (2012) is a synthesis of past research findings on the impact of the birth places on women's birth satisfaction and wellbeing in seven regions comprising Asia, Europe, SubSaharan Africa, Middle East, North America, Latin America and the United Kingdom. The study used mixed research synthesis methods with most of the studies focused on labour and birth rooms. The study is a meta-synthesis analysis of the literature and not an empirical study. Regardless, her conclusion provided useful insights and the basis that aspects of physical environment can be separately studied in order to shed more light on what women want from birth environments and the relationships of the design characteristics with other components for positive birth outcomes (Havill, 2012) and indeed recovery experiences.

Furthermore, early attempts in search of suitable methods for this thesis also considered photoelicitation. Photo-elicitation is a technique introduced by an American photographer and researcher John Collier (1957) who suggested that photographic recording of observations could stimulate recollections of events and possibly capture emotional and behavioural experiences. Collier (1957) argued that photo-elicitation offered a valuable tool to study factors in the physical environment that were too complex for reliable direct observation, or when a comparative analysis over time was anticipated. 
The technique of photo-elicitation was an expedient solution brought about by the lack of consensus among research teams on categories of a housing quality survey. Collier (1957) and his research team used photo-elicitation to investigate how families from different cultural backgrounds adapted to their new environment and to new forms of work in an urban setting. The results led to the conclusion that information content with photographs can be both simple and detailed in contrast to verbal interviews which were less structured and disconnected. Essentially "photographs can be stimulating and can help to overcome the fatigue and repetition often encountered in verbal interviews" (Collier, 1957, p. 857).

Traditionally, photo-elicitation is a commonly used approach in anthropology and sociology to assess environmental satisfaction to uncover experiences of patients for positive salutogenic outcomes (Pati, Freier, et al., 2016). This approach has expanded in other healthcare environments such as in birth settings, although with variations such as use of a video photo-elicitation to uncover and illuminate the mechanisms by which hospital birth spaces impact on midwives and their practice (Hammond, et al., 2014).

At the early stage of this thesis it was reasoned that consenting participants - women in particular - would be asked to create their own worlds of photographic impressions of the ideal postnatal environment for their recovery experiences. The images so created, supplemented with selected audio-recordings of nature sounds and images of nature would form the basis of the focus group discussions to elicit embodied experience about which restorative spaces may be likely beneficial for postnatal recovery experience. According to (Collier, 1957) integrating photographs at a different point in the course of investigation with other subject material might generate "broader and freer expression" (Collier, 1957, p. 858).

Although photo-elicitation offers a good fit for this thesis to compensate for the short postnatal hospital stay (Bravo et al., 2011; Fink, 2011; Goodwin et al., 2018), the practicalities and ethicalities of its adoption are concerns. Concerns range from whether photographs should focus on the hospital in which a woman's birth occurred, content of photographs, issues of timing, preoccupation with newborns and parenting to sharing and coordinating of the photographic material with the investigator. Ethical concerns relate to the anonymity and consent of the hospital in case photo images of the hospital are supplied, if photographs include other people (Harper, 2002) and more importantly because birth is a very sensitive intimate experience (Harte, Leap, Fenwick, Homer, \& Foureur, 2014). Invariably getting around all these tasks is time consuming and even assuming all the processes are hitch-free, interviews using photo-elicitation could take an excessively long time (Collier, 1957). 
Considering all the methods and research designs described above and taking into account their strengths and weaknesses, a mixed methods design was used for this thesis research. The main reasons that informed its selection are described in section 4.1. The research design was largely defined by the primary focus of achieving the research objectives. The design, a two-phase explanatory sequential mixed methods approach, consisted of online survey questionnaires to collect data from 212 women and 50 midwives between October and November 2017. The criteria comprised women who gave birth in a secondary or tertiary hospital including women who used a primary birth unit but were transferred to a tertiary level hospital for postnatal care between July 2016 and November 2017 (see the information sheet in Appendix A5) plus midwives who work in hospitals and have attended women in the postnatal wards prior to the survey. In phase two, focus groups comprising 14 key informants were assembled in four parallel sessions to shed light on the findings from phase one. The composition of the focus groups was drawn from the initial respondents who filled out the online surveys. Constant comparative analysis developed by (Corbin \& Strauss, 2015; Strauss \& Corbin, 1998) guided the stages of coding and the analysis process.

\subsection{Definition of terms}

The following terms and their definitions are used throughout in this thesis.

1. Alongside midwifery unit: A birthing unit located in the grounds of the same hospital as an obstetric unit and staffed with midwives to provide care to women in the labour, birth and postnatal period. This facility is planned to have access to an obstetric or neonatal unit on site in the event that women may require physical transfers to the obstetric unit.

2. Doula: The doula is a person trained to assist the woman in labour and the couple during labour and birth and presumably in the postnatal period. A doula provides physical, emotional and psychological support, and supports the woman with information in decision making.

3. Single room maternity care: A maternity care room where birthing families are admitted from inception of intrapartum and stay in the same room throughout the postnatal period. The concept of single room maternity had its roots in South Africa, referred to as the single unit delivery system (SUDS) (Notelovitz, 1978) however, postnatal recovery took place in another room. It is informed by the philosophy of birth as a natural physiologic process with family-centred care, which allows the baby to stay with the family throughout the hospital stay. SRMC encourages mother-fatherinfant bonding and promotes choice and preference of space for privacy and dignity of the family. 
4. Couplet-care: The model of recovery care that makes the use of a single room maternity room in which the woman and her baby recover in the same room, to facilitate parent-infant bonding, support both mother and infant's wellbeing and enhance positive hospital experiences. Coupletcare is more readily used in North America with the goal similar to "rooming-in" a well-baby model of care in New Zealand (also in Germany and elsewhere) in which newborns constantly remain throughout the postnatal hospital stay.

5. Labour, delivery and recovery (LDR) room: A hospital room where the woman stays in one room for the duration of labour and remains in the same room for birth and for the preliminary recovery. Thereafter, the woman is moved into a different room for postnatal recovery until discharge.

6. Labour, delivery, recovery and postnatal (LDRP) room: A type of model of care/room that is specifically focused on recovery in which the woman is admitted into one single room from intrapartum and throughout the postnatal period until discharge.

7. Free-standing midwifery-led unit (or primary birth unit): A community-based maternity unit, usually staffed by midwives to provide continuity of care model from pregnancy, labour, birth, and postnatal period. In New Zealand, FMLU is known as a primary birth unit and provides midwifery-led care for women of low obstetric risk as an alternate birth option to obstetric mode of delivery.

8. Noise: An unwanted, negative, environmental characteristic that contributes to sleep deprivation, poor sleep, and unrestfulness of a woman in a postnatal ward and may affect the experiences of recovery and wellbeing. Noise also includes crying of babies, visitors' presence and their chatting, and frequent awakenings due to medical assessments by care providers. It may also comprise a lack of control over lighting.

9. Normal birth: The birth that involves a spontaneous vaginal delivery of baby without medical interventions. A well-designed birth environment, with homely features and décor appeal, may contribute to promoting a physiologic normal birth for childbearing women.

10. Oxytocin: A hormonal secretion that is released into a woman's uterine systems to facilitate the process of labour, and to promote optimum labour outcomes. It is also a chemical substance used to alleviate labour pain and to start the birth process, which is known as induction or augmentation of labour. The philosophy of the release of oxytocin during a labour and birth is 
described by Fahy et al. (2008) and Buckley (2015) as being essential to facilitate a normal physiological birth.

11. Obstetric unit: The part of the hospital where labour and birth procedures are undertaken by a team of medical doctors and midwives tending to the childbearing women. In New Zealand, obstetric units are at the third-tier levels of hospitals, accessible to secondary and primary birth units in every District Health Board (DHB) in the event of transfers or birth complications. Midwives provide care to all women in an obstetric unit, whether they are low or high-risk and are core professionals for women with straightforward pregnancies during labour and birth. Facilities such as postnatal wards and neonatal units may be located close to an obstetric unit or in a separate part of the hospital.

12. Perception of space: The individual's innate or expressed response to their immediate physical environment as being essential for comfort, relaxation, experience and wellbeing particularly in healthcare built environment. The ability or capacity to perceive the physical surroundings regarding their physical layouts, elements of the design and impacts on persons inhabiting the space.

13. Natural art: An intervention comprising artistic impressions through paintings, images and decorations related to nature such as water bodies, trees and plantings, greenery or other natural landscapes, sequenced to produce a calming effect for a birthing woman in postnatal wards, her family and friends. Natural art may foster physical, emotional and psychological wellbeing of patients beyond the ideas of a purely medical-focused environment.

14. Recovery: The state of positive, physical and psychological feelings of wellness in which a childbearing woman returns to normal following the birth, other than the purely physiological or medical outcomes. Recovery can be aided and supported by introducing physical environment factors described by Nightingale - ventilation, lighting, warmth, hygiene and cleanliness, quiet, variety and beauty; nourishment and quality of care.

15. Wellbeing: The state of possessing positive moods manifesting in mental, physical, psychological and spiritual wellness particularly when the patient/client has exposure to therapeutic and supportive healthcare environments. This can be facilitated through design elements, objects and rich surroundings to assist the childbearing woman to address social needs and to promote recovery experiences and convalescing during postnatal hospital stay. 
16. Openness: The degree of enclosure that defines a space. It may refer to an open room design that can accommodate several beds, usually a long open space. Traditionally, it refers to bay wards or pods consisting of bays of four or more beds. In this thesis, openness of wards refers to a unit of four-bed to six-bed rooms.

17. Postnatal unit/ward: The area of the birth environment in a hospital where new mothers recover after the birth. The unit may comprise a range of single-bed rooms and double-bed/shared rooms with in-built bathrooms or shared toilets. In New Zealand, postnatal units have facilities such as a small counter for staff hand-over and to contain medical equipment in case there is a need for its use.

18. Postnatal woman: A woman who has undergone the processes of labour, birth and recovery following the birth in a hospital or at home. In New Zealand, women have access to the continuity of care model up to six weeks postnatally.

\subsection{Structure of the thesis}

This thesis contains eleven Chapters as below:

\section{Chapter one:}

Introduces healthcare environments and the challenges they may pose for health and wellbeing, states the research problem, the research aim and guiding research questions. Several concepts and theories related to the pursuits of healing environments, restorative, and therapeutic environments for hospital experience are described, thus setting the stage for the remainder of the thesis.

\section{Chapter two:}

Presents the current state of the art in a literature review on the healthcare built environment and the impact on patients' health outcomes and hospital experience, and on staff and significant others' wellbeing. It reviews the effect of environmental noise on stress and its effects on sleep quality, recovery and overall satisfaction in healthcare environments as well as the impact of singlebed versus multi-bed occupancy rooms, privacy and dignity, and control and autonomy over the physical environment. In addition, the role of family support in care, as well as forms of positive distractions, are reviewed. 
Illustrates how birth was moved from home to hospitals in the early 20th century, altering the environment for birth, and then how increased consumerism led to the creation of alternative birth settings in middle and high-income countries. This review also focuses on the childbirth environment, factors that facilitate birth experiences of women, social support and how design of hospital rooms affects caregiving and midwifery practice. A brief discussion regarding neuroscience and the built environment is highlighted. In addition, the role of philosophy, particularly phenomenology, as a methodological approach is introduced, which also provides a rationale for its lack of fit in this study. Correspondingly, chapters two and three situate this research within a tradition and knowledge base of healthcare design research including architecture, landscape design, environmental psychology, applied social and behavioural sciences and nursing/midwifery.

\section{Chapter four:}

Describes the research design, procedures and research methods. The settings, procedures and methods for data collection and analysis are extensively described. An explanatory sequential mixed methods approach is introduced comprising two key phases of quantitative and qualitative research methods. In phase one, the quantitative core strand focused on questionnaire surveys is presented as the technique to investigate the perceptions of the design factors among two users of postnatal facilities, women and midwives. In phase two, the qualitative supplementary strand comprising four focus groups is discussed.

\section{Chapter five:}

Presents the results of the phase one quantitative method question: what are the perceptions of design factors influencing women's recovery experience and wellbeing during postnatal hospital stays? In this chapter, results of women's perception of the four main aspects of design factors; postnatal room features, interior environment features, sensory comfort environment, and social comfort environment are presented. Descriptive statistics for the items in the questionnaire survey plus analysed inferences based on the survey responses are described. The top 15 design factors in terms of relevance to recovery experiences are presented including a brief discussion of the results. 


\section{Chapter six:}

Presents the results on perceptions of the design surveys for midwives. The top 15 design factors/features based on their perceived importance are enumerated. The results on perceptions of the design factors surveys for women and midwives in phase one provide the basis for the inferential statistics analysis and discussion in chapter seven.

\section{Chapter seven:}

Presents the inferential statistics of the results on perceptions of the design surveys between women and midwives, which enable responses to be compared among the two user groups and also makes it possible to see trends in the responses based on the questionnaire surveys and overall aim of phase 1.

\section{Chapter eight.}

In chapter eight, a discussion of the results of descriptive and inferential statistics reported in the previous chapters is presented. The chapter contains three sections. The first section identifies the design factors where no significant differences were found pertaining to the mean scores among the two user groups. Section two presents a discussion on the items/design factors which showed statistically significant differences. Section three discusses other design attributes with similarities based on their moderate levels of acceptance. Linking the results of both descriptive and inferential statistics to the pertinent literature provides a basis from which to draw conclusions on the ranking of the design characteristics. The results build on to further explanation in the phase two qualitative focus group discussions in the following chapter.

\section{Chapter nine:}

Describes the findings of the qualitative focus groups aligned to the second question: what were the ways in which the design factors and the postnatal spaces affected women's recovery experiences and wellbeing during hospital stays? The three main categories that emerged from the focus groups are described and discussed.

\section{Chapter ten:}

Presents the integration and interpretation of the results of inferential statistics on the perceptions surveys and findings of the focus groups. The main categories and their related sub-categories are further linked back to the design characteristics and factors from phase one. 
The discussion provides an understanding of and insights into the architectural design factors and building spaces influencing women's recovery experiences in large, tertiary hospitals.

\section{Chapter eleven:}

Draws the conclusions for the thesis and consists of an overview of research findings, implications for postnatal hospital facilities in tertiary hospitals, study limitations and future research on and around the topic of wellbeing and hospital experience.

\subsection{Conclusion}

This chapter has outlined the challenges posed by healthcare buildings for the promotion of wellbeing and humanistic experiences. Hospitals are increasingly being utilised by "healthy" women for whom giving birth and subsequent postnatal recovery are a part of their normal physiological life events. Previous research in the childbirth field has extensively focused on labour and birth-related environments in hospitals. The issue of physical environment for recovery after the birth has been under-researched yet it is argued that a supportive environment is important to facilitate the goal of adequate and quality care for women in the immediate postnatal phase.

Although trends in evidence-based design, alongside patient-centred care, offer promise for improvements in healthcare environments, available studies may not be a good fit and have limited specific application to postnatal areas because of their uniquely "wellness" situation compared to an "unwell" general hospital population. Following the background studies, and identifying gaps in knowledge regarding postnatal hospital environment for recovery and physical restoration, the research aim and guiding research questions were developed to understand physical design factors and architectural characteristics influencing recovery experience and wellbeing of women and proposing evidence-based design guidelines for the postnatal environment. The next two chapters review the effects of the healthcare built environment on patient outcomes, staff satisfaction and wellbeing. These literature surveys provide useful guides for evaluating the current knowledge about how hospital birth spaces impact women and their caregivers in the childbirth environment. 


\section{CHAPTER 2}

\section{IMPACTS OF HEALTHCARE DESIGN ON HEALTH OUTCOMES}

In chapter 1, the physical environment is demonstrated to have a connection with health outcomes for patients, staff members and visitors. This chapter provides a literature review of the impacts of healthcare built environments on healthcare outcomes and wellbeing of healthcare users.

\subsection{The healthcare environments and health outcomes}

Research into healthcare built environments and their impacts on health outcomes has primarily focused on physiological health, medication use, perception of health status and physical outcomes (Codinhoto, Tzortzopoulos, Kagioglou, Aouad, \& Cooper, 2008). Alongside these is the increasing awareness of patients' and users' experiences and wellbeing during hospitalisation hence the importance of patient-centred design.

The patient experience has gained prominence in healthcare delivery research (Steele, Jones, Clarke, \& Shoemaker, 2015) expanding beyond the focus of clinical outcomes toward the idea of the physical environment combining with the skills of healthcare staff in delivering patients' overall experience, satisfaction and wellbeing. (LaVela \& Gallan, 2014; Suess \& Mody, 2017; Zborowsky, 2014).

As discussed in Chapter 1, the shift to patient-centred care and the role of physical surroundings is based on evidence-based design (EBD) which approaches design and development of healthcare facilities using best findings in healthcare research (Malkin, 2003; Stichler, 2018). EBD aims to make healing and restorative environments that emphasise links between healthcare users and health providers that can bring fruitful integration of needs regarding hospital experiences and wellbeing of users (Huisman et al., 2012). More than a thousand studies made available by the Center for Health Design (CHD) ${ }^{1}$ demonstrate the multidisciplinary orientation of researchers,

${ }^{1}$ The CHD website hosts a collection of peer-reviewed and recent research papers on a range of topics in healthcare design research https://www.healthdesign.org/knowledge-repository 
practitioners and organisations committed to improvements in design of buildings and environments for patient experience, safety and care (Malkin, 2008).

Understanding therapeutic milieus for improved patients' and users' health outcomes requires has led to healthcare facilities that are appreciative of the users' satisfaction with the hospital experience. Factors that promote this in the literature are congruent with patient privacy and dignity, hygiene and cleanliness, audio and visual environment and comfort regarding interior design leading to improved patient health outcomes, family and staff satisfaction (Devlin \& Arneill, 2003; Huisman et al., 2012; Laursen, Danielsen, \& Rosenberg, 2014; Lorenz, 2007; Timmermann, Uhrenfeldt, \& Birkelund, 2015; Ulrich et al., 2008).

The following review of the literature is organised into four areas: 1) patient privacy and dignity; 2) visual environment and health outcomes; 3) patient experience and wellbeing and 4) patient and family support and satisfaction.

\subsection{Patient privacy and dignity}

Patient privacy and dignity are an essential component of healthcare that has been highlighted as important by both patients and governments (Department of Health, 2001; Matiti, Cotrel-Gibbons, \& Teasdale, 2007). Patient privacy and dignity are tantamount to the worth of an individual expressed as the "quality or state of being honoured or esteemed" (Hofmann, 2002, p. 89). It could be argued that having respect for and valuing patient dignity in hospital not only corresponds to knowing personal information will be held in trust but also that the patient has feelings of being in safety regarding medical procedures. The issue of privacy and confidentiality has generated increased interests from the perspectives of patients and staff members in Emergency Departments (ED) and in Intensive Care Units (ICUs) (Brown \& Gallant, 2006; Karro, Dent, \& Farish, 2005; Kesecioglu, 2015). A study carried out in oncology wards found that patients chose to withhold vital information from care providers because other patients might hear their conversations (Larsen, Larsen, \& Birkelund, 2014) and absence of such privacy can jeopardise dignity (Baillie, 2009). The physical layout of healthcare facilities affects promotion of dignity by nurses and care providers, for example enclosed nurses' stations while allowing nurses to concentrate on tasks, hampered communication with their patients (Shattell et al., 2015). When 
staff promote dignity through privacy and interactions patients feel satisfied and valued (Baillie, 2009).

Advances toward patient-centredness have promoted discourse around kinds of accommodation as means for ensuring privacy and confidentiality of patients for improved positive hospital experience and satisfaction. In the childbirth environment context, the notion of patient/womancentredness has received increasing attention from researchers. For example, Bailey and Howe (1993) compared two best concepts of single-room maternity care (SRMC), known as the labour, delivery, recovery and postnatal (LDRP) room, and labour, delivery and recovery (LDR) rooms. The SMRC concept has been found to be effective in many respects ranging from improved satisfaction of care for women and families, improved job satisfaction for staff, to restoring the sense of human caring (Bailey \& Howe, 1993; Janssen, Klein, Harris, Soolsma, \& Seymour, 2000; Spradlin, 2009). Below is a review of studies focused on single-bed versus multi-bed room occupancy including measures to provide for acoustic comfort.

\subsubsection{Single-bed room versus multi-bed room}

The choice of a single-bed or multi-bed room has attracted considerable interest among healthcare researchers. A single-bed room comes with the advantage for a patient of being sole occupant in the room, together with a physician, which promotes privacy and confidentiality. By comparison, multi-bed or semi-private rooms allow for other patients' presence in the same room. However, the need for company and preventing isolation is a feature of multi-bed rooms (Pease \& Finlay, 2002). A review focused on single versus multiple occupancy rooms concludes that single private rooms help in controlling hospital-acquired infections (HAIs), support flexibility of usage and have increased positive impacts on patients (Chaudhury et al., 2005). Aside from privacy and dignity, a single-bed room was found to have moderate effect on satisfaction with care, reduces noise levels and enhances sleep, while showing conflicting results with infection rates (van de Glind, de Roode, \& Goossensen, 2007). A study of nurses' perceptions of care in a single private room (SPR) after a move from an old unit found a $6 \mathrm{~dB}$ reduction in noise levels in the SPR and reduction in nosocomial infection with staff feeling that SPR design had more benefit than an open room (Walsh, McCullough, \& White, 2006).

In terms of birth experiences and satisfaction, accommodating childbearing women in a single room maternity care unit rather than the traditional rooms can improve emotional and psychological health outcomes. For example, Janssen et al. (2000) used questionnaires to measure levels of satisfaction among 205 women who stayed in a single room maternity care unit (SRMC) 
and 212 women in traditional rooms in Vancouver, British Columbia. They found that single rooms were correlated with significant women's satisfaction and fostered continuity of care. Other benefits associated with single-bed rooms include prevention of errors, and permitting increased presence of family members (Choi \& Bosch, 2013; Williams \& Gardiner, 2015). Despite the seeming benefits linked to single-bed occupancy rooms, there are concerns that some patients may experience loneliness in a single-bed room and they should be provided the option of shared rooms (Pennington \& Isles, 2013). The debate is far from being conclusive coupled with limited research about the impact of single patient rooms on recovery and health outcomes. Effects of single-bed room on staff wellbeing are least explored (Ulrich, Berry, Quan, \& Parish, 2010).

\subsubsection{Noise and sleep quality}

Noise refers to an unwanted sound that can cause sleep deprivation and disturbance, it has been shown to have a detrimental effect on health. Excessive noise can cause confusion, restlessness in patients, increased blood pressure, elevated heart rate and lower patient satisfaction and has serious effects for critically ill patients both physically and psychologically as well as causing increased stress and annoyance for staff (Kamdar, Needham, \& Collop, 2012; Lei et al., 2009; Morrison, Haas, Shaffner, Garrett, \& Fackler, 2003; Short, Short, Holdgate, Ahern, \& Morris, 2011; Zaharna \& Guilleminault, 2010). Noise can induce headaches, prolong wound healing and recovery, increase sensitivity to pain and have harmful effects on staff (Biley, 1994, 1996) as well as being disturbing to patients (Akansel \& Kaymakçi, 2008).

Routine activities of individuals and caregivers have been cited as responsible for noise ranging from discussions of patients' treatment, to carts delivering food or supplies to hard-soled footwear Environmental conditions including noise in inpatient rooms can potentially affect patients' sleep, recovery and other health consequences (Dube et al., 2008; Monsen \& Edéll-Gustafsson, 2005; Tembo \& Parker, 2009). World Health Organisation (WHO) guidelines state that for good sleep, the sound level should not exceed $30 \mathrm{~dB}$ for continuous background noise with individual noise of not more than $40 \mathrm{dBA}$ during the day and $35 \mathrm{dBA}$ at night for healthcare settings (Berglund, Lindvall, Schwela, \& World Health Organization, 1999).

Studies about noise and sleep disturbances have been focused on intensive care units (ICUs), critical care units (CCUs) and paediatric ICUs. Examples include studies focused on daytime decibel levels measurements in patient rooms (Pope, 2010), the effect of intensive care unit noise on patients (Akansel \& Kaymakçi, 2008), sound levels and patient experience (Johansson, Bergbom, Waye, Ryherd, \& Lindahl, 2012). Busch-Vishniac et al. (2005) examined hospital noise 
levels in five departments of John Hopkins hospital in accordance with the WHO guidelines (Berglund et al., 1999). They found that the observed sound levels exceeded the WHO recommendations by at least 10 to $20 \mathrm{dBA}$ with sound levels great enough to affect sleep.

Oliveira, Gomes, Nicolau, Ferreira and Ferreira (2015) assessed three composite environmental factors of light, sound and temperature and measured their impact on sleep in five paediatric wards in a tertiary hospital. The authors used a questionnaire administered by caregivers to assess children's sleep and found sound levels and temperature exceeded the recommended values in the different wards. The environment had negative influence on sleep quality and increased restlessness. Effects of high noise levels have also been reported in other studies which found that mean levels of between 55-66 dB were experienced during the daytime in patients' rooms, with maximum levels reaching 80 dB (Akansel \& Kaymakçi, 2008; Bailey \& Timmons, 2005). Therefore the acoustic environment of healthcare settings, as part of the physical environment may be far from ideal and could have serious health implications for patients and for staff wellbeing. While excess noise can be detrimental to health and wellbeing and may thwart recovery, one line of enquiry in the UK suggested that noise in the clinical envirormnet conveys social meaning, which is necessary in the day-to-day interactions and functioning as humans. Indeed, Brown, Rutherford and Crawford (2015, p. 1514) argued that clinical environments have a "soundscape" that embodies noises "about the activities of other people, the rhythms of the day and the nature of the auditory community of the hospital."

\subsubsection{Noise and interventions in the healthcare environments}

Healthcare environments should provide the patient opportunity for sleep after medical procedures. Higher hospital noise levels are negative contributors affecting communication, stress levels, sleep disorder and aggressive behaviour (Short et al., 2011). The acoustic environment can be a stressor for patients who may need to spend time in hospital following painful medical procedures or for routine monitoring of treatments, when sleep becomes an important physiological process necessary to repair and restore body functions (BaHammam, 2006). Environmental noise-reduction strategies to improve sleep are reviewed below. They fall into three areas comprising 1) noise source control, 2) sound absorption and 3) noise blocking such as acoustic walls in single-bed rooms in addition to behaviour change measures. These approaches have been shown to potentially benefit both patients and staff in terms of improved sleep and wellbeing. 
Blomkvist, Eriksen, Theorell, Ulrich and Rasmanis (2005) used a two-step approach that first utilised a remodelled ceiling tile and in the second, sound absorbing ceilings in the entire ward. Using questionnaires to collect data at three shifts (morning, afternoon, and night), they found that reverberation times and speech intelligibility improved when the ceiling tiles were changed from sound reflecting tiles to sound absorbing tiles and also improved acoustics had positive impact on the work environment.

Chang, Pan, Lin, Chang and Lin (2006) conducted an experiment using a noise-sensor light alarm in the Neonatal ICU. They reported that the noise-sensor light alarm significantly reduced sound level and peak noise in the NICU and this could mitigate stress of noise for neonates having medical issues. More recently, a similar line of enquiry by Ukegjini, Kastiunig, Widmann, Warschkow and Steffen (2020) collected information on the impact of attempting to reduce noise on surgeons' stress using a sound-activated device and found that a visual noise warning device in an operating room correlated to reductions both in the noise levels and surgeon stress.

Gabor et al. (2003) evaluated the effect of noise and impact on sleep between mechanically ventilated patients and healthy patients in the ICU. They concluded that patients had improved sleep in a single patient ICU room due to lower noise levels. The operating machines, tools and buildings were the major contributors of noise in the operating room (Tsiou, Efthymiatos, \& Katostaras, 2008). Similarly, noise can also have adverse physical and physiological effects on medical staff and can potentially increases mistakes by healthcare personnel (Murthy, Malhotra, Bala, \& Raghunathan, 1995).

Recently, Johansson et al. (2018) tested an intervention consisting of a refurbished two-bed ICU patient room (using a wall-to-wall suspended ceiling and a low frequency absorber) and a traditional two-bed room as a control. The researchers found a difference in the room acoustics with a reverberation time lower and speech clarity higher in the experimental room than in the control room, with some concerns are important for consideration in future studies.

In terms of behavioural change, Long and Stover (2014) introduced what they dubbed "a culture of quiet" in one hospital that aimed to provide a more healing environment for the patients and staff alike. The modus operandi adopted was purely a behavioural and moral one which required that staff were solicited to be voluntary "quiet champions" working as a group or on an individual basis. Three aspects were noted 1) avoiding conversations either in front of patient rooms or in nurse stations when dealing with patients' vital records; 2) volunteers to be comfortable to control noisy situations and monitor sound equipment; and 3) get visitors to be aware of their presence in 
a healing environment. The authors indicated that such interventions helped noise reduction and improved the patient experience including staff satisfaction (Long \& Stover, 2014, p. 45).

Hinkulow (2014) also reported a similar approach termed the Hospital's Ultimate Silence for Healing (HUSH) as a nursing action for mental respite and quiet restful environment. The success of HUSH has led to its acceptance in a hospital-wide implementation with implications for future research on the effects of a quiet restful environment on mental health and wellbeing of patients and staff (Hinkulow, 2014).

An earlier study by Gardner, Collins, Osborne, Henderson and Eastwood (2009) indicated that quiet time intervention in an acute care setting can affect noise level and patient sleep/wake patterns with enhanced therapeutic benefit. A recent review of evidence on quiet time, particularly focused in the ICUs indicates that quiet time interventions can improve patients' sleep quality, promote restfulness and may engender a healthier workplace environment as well as enhanced patient and family satisfaction (Lim, 2018). The contributions of quiet time interventions in the healthcare environment for improved sleep quality and health outcomes suggest their consideration towards improving the acoustic environment for healing.

\subsection{Stress}

A healthcare environment that is disorienting can be a source of stress for patients, staff and visitors. The inherent complexities of hospital buildings make them susceptible to patients, particularly first time visitors, missing their way. Stress can manifest in both painful health-related symptoms and procedures and physical healthcare facilities that invade privacy and prevent social connection (Ulrich, 1992). Stress can negatively manifest in emotional, physiological, and behavioural forms resulting in poor health outcomes (Ulrich, 1991). In addition, empirical evidence has shown that complex hospital layouts can be frustrating for patients and families, lead to fatigue and create physical and psychological stress for patients and staff members (Pati et al., 2008; Wang et al., 2013). Research has found that using a practical design experience that introduced a dedicated service corridor in a healthcare setting improved nurses' stress and satisfaction levels (Wang et al., 2013). Not only are patients exposed to the consequences of stressed healthcare environments, staff "burn-out" is largely due to high workload and work environment (Ulrich, 1992; Ulrich et al., 2004).

Research has found exterior wayfinding and disruptions in the interior elements could mislead, make predictions difficult and trigger stress for patients, families and people (Carpman, Grant, \& 
Simmons, 1985; Devlin, 2014; Evans \& McCoy, 1998; Passini, Rainville, Marchand, \& Joanette, 1995). Research suggests that an overload of information constitutes stress for patients with regard to wayfinding (Carpman et al., 1985).

The appearance and types of room a patient is allocated could impact stress levels. When patients are attended in single-bed rooms there is the possibility that they may feel more relaxed and may respond better to basic information from care providers. Discussions about single-bed rooms situate them as better options for relieving or restoring vitality from stress. Single-bed rooms are associated with lower stressors (Gabor et al., 2003), improved privacy (Olsen \& Sabin, 2003), lower noise levels (Kol, Aydin, \& Dursun, 2015), and they promote communication among patients, families and nursing staff (Choi \& Bosch, 2013; Curtis \& Northcott, 2017; Taylor, Card, \& Piatkowski, 2018). The physical healthcare environment (both interior design and exterior) needs to be bolstered in its affordances to elicit capacity for coping against stress to improve patients' hospital experience and staff morale and wellbeing.

Incorporating supportive design elements, positive distractions and environmentally rich surroundings could serve to relieve stress and may facilitate well being and provide support for coping (Andrade \& Devlin, 2015; Campagnol \& Shepley, 2014; Ulrich, 1991, 1999). Measures proposed to mitigate or reduce stress include improving the visual environment, natural daylight and use of positive distractions (exposure to nature and other forms) (Pati et al., 2008; Ulrich, 1984).

\subsubsection{Visual environment and positive distractions}

The appearance of healthcare facilities can contribute to patient experience. An enhanced aesthetically pleasing environment promotes better evaluations by patients of their satisfaction levels (and invariably too, higher reimbursements to hospitals) (Suess \& Mody, 2017). Research about improvements in visual environments includes lighting and positive distractions. Lighting consists of both natural lighting and artificial lighting while positive distraction refers to inherently environmental attributes imbued with dinstinctive aesthetic appeal, positively altering mood and [which] provide more restoration from stress (Ulrich et al., 1991).

Evidence supports the use of positive distractions to reduce stress and anxiety. Fenko and Loock (2014) measured the subjective pleasantness and arousal of different scents and music. The authors conducted their experiment in a waiting room and collected data of levels of anxiety in four situations: without scent and music; with lavender scent; with instrumental music; and with both 
scent and music. They found that ambient scent and music helped in reducing patients anxiety however, they should be applied minimally. Another study looked at the influence of positive distractions on children in waiting areas. Pati and Nanda (2011) presented five distraction features in the waiting areas of two clinics. Data collection was focused on attention, behaviour and activities engaged in by the children. The authors concluded that the introduction of positive distractions to patients were associated with calmed behaviours and better satisfaction with wait periods.

In a study conducted in a postanaesthesia care unit (PACU) Fredriksson, Hellström and Nilsson (2009) tested to see whether patients prefer listening to music compared to ordinary sound. Their results found a significant link with listening to music implying that music can provide a more healing environment by lowering stress levels. Diette, Lechtzin, Haponik, Devrotes and Rubin (2003) explored the combined effects of nature scene murals with nature sounds to which patients listened before, during and after the medical procedure. Patients who underwent bronchoscopies reported significantly less pain suggesting that nature scene murals and sounds served as potential distraction therapy. However, research has yet to link positive distractions, in particular music, with the effect on noise levels/intensity for likely detrimental health outcomes.

Lighting is a vital feature for navigating safely in spaces and in performance of tasks in healthcare environments. Research has demonstrated the impact of adequate daylighting for recovery and rehabilitation of patients and for general wellbeing of staff (Ulrich, 2001). Empirical evidence suggests windows and natural light and having access to natural views improve health outcomes for patients and staff alertness. Absence of a view outside has adverse psychological effects emphasising the importance of windows, natural lighting and views in hospital design decisions (Pati et al., 2008; Ulrich, 1984; Verderber, 1986). Visual access to nature can be achieved through outdoor gardens, indoor plants and views of nature through windows. Nature-related images can provide a proxy for the visual environment that could promote stress reduction for patients, staff and visitors.

Because of perceived influence of the physical environment on health and wellbeing, research into nature and surrounding "greenness" crosses multidisciplinary boundaries between environmental psychology, horticulture, landscape architecture and architecture. Many studies have taken their lead from the work of Ulrich (1984) which proved that views to nature aided recovery, reduced stress and lowered pain. From horticulture and landscape architecture, Lohr and Pearson-Mims (2000) examined the role of plants in the perception of pain. They found that individuals having plants in their room exhibited a higher tolerance of pain than those who had no plants in their 
room. The authors added that a calming and cheerful room interior will likely engender positive feelings. Park and Mattson (2009) performed a randomised clinical trial with surgical patients and collected information related to length of hospital stay (LOS), physiological measures, anxiety and fatigue. The patients reported viewing plants during recovery had significant positive influence on health outcomes. Another study found that participants with exposure to indoor plants reported less stress than those without (Dijkstra, Pieterse, \& Pruyn, 2008). These findings have demonstrated the potentials of indoor plants in alleviating stress and lowering of anxiety. However a few of these studies were not done in hospital situations with patients and staff which is necessary to draw valid conclusions.

Another aspect of visual environment that is found a useful antidote against stress is the complementary use of lighting and colour. Dalke et al. (2006) explained that when lighting and appropriate colours are introduced on the exterior and interior spaces of healthcare spaces they assist in 'signposting' of important areas, ease navigation and improve wayfinding. The eye also needs adequate lighting to be able to see well (McCullough, 2009) as implicated in the findings that medication errors were reduced with higher lighting levels (Buchanan et al., 1991; Ulrich \& Zimring 2008).

One study tested the links between exposure to natural daylight, pain medication use and cost on post-operative patients. Eighty-nine patients were assigned to single-occupancy rooms located on either the bright or dim side of the hospital unit. It was found that patients exposed to increased amounts of natural sunlight had less perceived stress, less pain, and took less analgesic medication (Walch et al., 2005). Furthermore, Alimoglu and Donmez (2005) studied the effect of daylight exposure on nurses' job burnout and satisfaction. They noted that exposure to daylight for a few hours was linked to lower stress levels and higher job satisfaction among nursing staff. In terms of length of stay (LOS) after episodes of depressive illness, patients who stayed in brightly lit rooms spent an average of 16.9 days while patients in dull rooms spent 19.5 days (Beauchemin \& Hays, 1996).

While a study conducted in an ICU with seven rooms with windows and five without found that the presence of a window had no effects on the outcomes for critically ill patients (Wunsch, Gershengorn, Mayer, \& Claassen, 2011), there is sufficient evidence that natural daylight improves health outcomes for patients and staff wellbeing, however, one study found indoor daylight levels above the recommended standards. This could expose patients to visual discomfort, thus attention must be paid to the effect of the glazing materials on daylight performance (Alzoubi \& Al-Rqaibat, 2015). In specific cases where access to natural light is not available, exposure to adequate bright 
artificial lighting in the daytime may compensate but at night-time, the light in patients' rooms should be dimmed enough to ensure good sleep (Ulrich et al., 2008). The decisions taken in a hospital design should give attention to interior design, furniture and windows in patient rooms as essential factors that influence indoor daylight performance (Alzoubi, Al-Rqaibat, \& Bataineh, 2010).

\subsection{Visual environment and health outcomes}

Geue et al. (2010) in a review of studies focused on painting or drawing based art therapy interventions for cancer patients found that art therapy resulted in improved mental health of patients. Monti et al. (2012) found in paediatric care the parental perception of the hospital environment improved after natural landscape pictorial interventions were provided suggesting that pictorial intervention may create a welcoming therapeutic environment and reduce stress for parents whose child is hospitalised. A study examining what type of art image has potentially stressreducing effects on children in hospitals found that representational nature art was the most preferred art image suggesting that art intervention in paediatric patients may likely serve as positive distraction for stress reduction and perception of pain (Eisen, Ulrich, Shepley, Varni, \& Sherman, 2008). The perceived pleasing aesthetic of the hospital environment increased the feelings of connection of children and helped to reduce emotional stress regarding hospitalisation (Bishop, 2012). The findings suggest that art impacts on patient experience and on self-reported mood, stress and overall satisfaction. Patients may respond positively to other types of art in addition to nature art.

Aside from art, a collection of specific nature images showed potential benefit to lower sensory pain however, there was no indication of the "most" therapeutic image among the collection (Vincent, Battisto, Grimes, \& McCubbin, 2010). Karnik, Printz and Finkel (2014) used an online survey to evaluate the effects of a collection of arts effects on patient mood, stress and comfort. However, presentation of emotional abstract art images was found to cause aggressive behaviours and anxiety for patients in a corrective facility (Ulrich, 1991). In brief, there is evidence that pictorial art, nature art and images of natural landscapes can potentially serve as positive distractions and may have beneficial health outcomes for patients. However, there is also a need for caution that not all art images may be suitable in all healthcare spaces. Considerably more studies are in paediatric settings than in other care settings which future studies could consider. 


\subsection{Patient experience and wellbeing}

Patient-centredness deals with the notion that patients should have enhanced hospital stays in addition to care being provided. This could translate to approaches that make hospitals less stressful and should improve the wellbeing of patients. Empirical evidence has found that environmental "adequateness" factors of interior design, quality of architecture, privacy, and the ambient environment are strong predictors of hospital experience and can enhance patients' satisfaction (Harris, McBride, Ross, \& Curtis, 2002). There is a "maturing" of healthcare landscapes as they shift from the post-war focus on functional efficiency to an appreciation of balance of function with aesthetics (Horsburgh, 1995, p. 735).

A trend seeking alignment of hospitals with a wellness focus has resulted in increasing efforts to "recreate" healthcare settings using the principle of patient-centred design for hotel-like hospital rooms for improved patient wellbeing (Suess \& Mody, 2017). Research is interchangeably referred to as patient-centred design (PCD), healing environments or therapeutic milieu addressing topics that include the ambient features, social features, staff and patient perceptions, architectural change and multidisplinary contributions (Connellan et al., 2013).

Schweitzer et al. (2004) focused on best practices in healthcare environments that fully embraced almost all of the aspects mentioned above. Their review recognised the effect that ambiance of space exerts on users and that efforts are needed to increase the pace of research that should contribute to impact healthcare outcomes and wellbeing of patients and significant others. Research by Stichler (2001) suggested the inclusion of views of nature, natural light, soothing colours, therapeutic sounds and single-patient rooms that offer more space around the bed are important for family interaction and enhance the healing process and patient wellbeing. Reed (1995) discussed several approaches and opportunities that can be taken to lessen the impact of healthcare's institutional image, comprising support for sensory diversion involving lighting, artwork and soothing sounds; for comfort and human interaction for patients' hospital experience and wellbeing. Patients and staff tend to benefit from daylighting, artwork and music including sensory stimulations and positive distractions when provided in healthcare environments.

Numerous studies have established the impacts of layouts on patients and staff wellbeing. A very recent study by MacAllister, Zimring and Ryherd (2019) explores the relationships between patient room layout and patients satisfaction and experience in a large hospital. Utilising information from 8,336 patients the study found significant links between spatial layout and patient satisfaction. Specifically, higher performing rooms were those located at a medium distance from the nurses' 
station, with the patient facing the entry door. In another study, Quan, Joseph and Ensign (2012) compared two types of rooms; one with positive distractions and control over the environment and another traditionally designed room. The study found that the first was rated higher than the second, suggesting that environmental pleasantness and control improved the patient experience and reduced stress, though the study did not find improved staff satisfaction owing to small sample size for staff. In a review of studies focused on environmental stimuli on psychologically mediated outcomes, there is general agreement in the literature that sunlight/daylighting, odours, seating arrangements and windows were associated with patient wellbeing (Dijkstra, Pieterse, \& Pruyn, 2006).

Studies have reported the contributions of enhanced therapeutic environments in moving from an old facility to a new facility. Alvaro et al. (2016) found improvements in health outcomes, satisfaction and interactions among staff and patients were perceived greater at the new facility compared to the old facility. Siddiqui, Zuccarelli, Durkin, Wu and Brotman (2015) conducted an experiment to evaluate changes in patient experience and satisfaction regarding a renovated hospital. The authors found patients felt positive about pleasing surroundings and comfort but had different rating of the hospital experience while more clinicians felt greater satisfaction after a relocation to the new hospital facility. Conversely, poor physical-social environment features were found to have severe consequences on care quality and could hinder the realisation of personcentred care (Alexiou, Degl' Innocenti, Kullgren, \& Wijk, 2016).

Moving to a new health facility may result in adverse effects manifesting in staff stress and disatisfaction. This is particularly demonstated in one study that found a new hospital adopting the single-patient model increased workload for the staff even months after the move (Maguire, Burger, O'Donnell, \& Parnell, 2013). Although the goal remains to facilitate the promotion of patient-and family-centred care (PFCC), this may discourage clinicians and other staff. However patientcentred design with such features as single rooms, natural light and comfort considerably promote patient-and family-centred care (PFCC) (Bromley, 2012; Douglas \& Douglas, 2005; Kotzer, Zacharakis, Raynolds, \& Buenning, 2011). Empirical evidence indicates that patients in single-bed rooms are exposed to less noise, have better privacy and confidentiality and have lower stress levels and better communication with care providers and satisfaction with care. Given the benefits of the single-bed room, it has been widely embraced in the USA and France, while the UK, the Netherlands and Norway are increasingly moving toward the single-bed room as a standard of care and it has been recommended for improved safety and patient wellbeing (Detsky \& Etchells, 
2008; NHS Estates, 2005; Trochelman, Albert, Spence, Murray, \& Slifcak, 2012; Verderber \& Todd, 2012).

\subsection{Family and social support and satisfaction}

Family and social support have increasingly emerged as important in care provision. Patients usually expect to have the presence of families and friends to provide emotional and psychological support before, during and after care. Such needs for social support have been acknowledged as a psychosocial factor that influences health outcomes (Berkman, Glass, Brissette, \& Seeman, 2000; Cohen, 1988) with emotional support having substantial influence for families in care (Tarkka, Paavilainen, Lehti, \& Åstedt-Kurki, 2003). Shiovitz-Ezra and Litwin (2012) evaluated the links of social networks with engagement in physical activity. The authors referenced the effect of social networks on health outcomes (Berkman et al., 2000) and found that (older) people embedded in diminished social networks were prone to indecent social behaviours, reduced physical enagagements and decreased use of medications (Shiovitz-Ezra \& Litwin, 2012). This implies that families may be important in decisions and in a supervisory capacity with compliance of patients' needs regarding medication.

The preeminence of family as a structure of social support during illness transcends cultures. In an unfamiliar institutional setting, families and others support can be great assets for coping with stress (Ulrich, 1991, 1992). Additionally, an orientation towards a religious/spiritual form of support with usage of faith-based personnel or groups is claimed to have psychological and spiritual benefits and may optimise a healing experience in hospitals (Saad \& de Medeiros, 2016). The authors identified five reasons for an investment in religious-spiritual support programmes. Increasingly, the practice of religious-spiritual support has found its way into mainstream healthcare, the connection between spirituality and healing appearing the norm rather than the exception in hospitals around the world (Bediako et al., 2011; Ellis, Thomlinson, Gemmill, \& Harris, 2013; Hilbers, Haynes, \& Kivikko, 2010; Selman et al., 2013).

Furthermore, healthcare settings that facilitate social support have been linked to ameliorating stress in patients, familiies and improving staff morale and satisfaction (Ulrich et al., 2010). A high level of social support may reduce the likelihood of severe psychological distress (Park, 2007). Ulrich (1991) hinted that studies undertaken in settings other than in healthcare facilities underscore the importance of social support, therefore it seems logical to suggest that social support acts as a buffer against stressors and enhances wellbeing. The social component of the Theory of Supportive Design (TSD) draws on the importance of amenities and features for 
fostering social interaction and cohesion. Building on their previous work on perceived quality of physical and social environment on patients' satisfaction (Andrade, Lima, Pereira, Fornara, \& Bonaiuto, 2013), conducted an experiment to test if the hospital environment fosters perception of social support (Andrade \& Devlin, 2015). The authors found that hospital rooms with more presence of social support were associated with reduced stress for patients. Overall there are few studies focused on social support in healthcare facilities.

\subsubsection{Family and friends visitation and health outcomes}

One of the most tangible aspects of social support is having family, friends and close relations around to provide empathy and emotional support, which may improve health outcomes. It is common to find families and friends by the bedsides of patients only during visiting hours largely because there is a lack of provision to accommodate significant others in care.

Visitations to healthcare settings often are governed by restrictive policies causing isolation, stress and anxiety for both patients and families (Cleveland, 1994; Halm \& Titler, 1990; Stover, 2002). Multiple studies underscore a need for reappraisal of visitation policy to accommodate needs of families of critically ill patients. Review of studies on visitation in critical care revealed the desire for a more open visitation policy (OVP), from patients' families and healthcare providers; while a less restrictive visitation policy is advocated by nursing staff. In addition, post-intervention surveys indicate higher patient and family satisfaction (Roland, Russell, Kathy Culpepper, \& Sheila Cox, 2001). A flexible OVP may promote more family presence and in return benefit patients. Shulkin et al. (2014) monitored the implementation of a 24 hours open visitation policy in an acute care facility and a rehabilitation unit of a hospital. Comparision of patient satisfaction scores before and after the OVP suggests improvements of patient and family experiences. Patient satisfaction level increased while no new complaints from patients or families were reported. Additionally, the staff acknowledged the positive impact of the OVP. Kozub, Scheler, Necoechea and O'Byrne (2017) collected survey information that asked staff questions (i.e. if they experienced stress, have difficulty in reducing their anxiety, managing conflicts with patients/families etc.) following the adoption of an OVP. They found that nurses' overall stress level was decreased and perception of patients' or families' anxieties decreased.

Pineda et al. (2012) examined the relationships between two NICU room types comprising openbay and single-private rooms (SPR) and parent visitation and maternal health. Parents of infants in the SPR demonstrated more hours of visitations over the first 2, 3 and 4 weeks of life compared to parents of infants in the open-bay rooms. However, it was reported that mothers of infants in 
the SPR reported more stress. Other researchers have reported that provision of rooms for overnight stay for families, soothing colours, family facilities, TVs in the waiting rooms, internet provision, comfortable seating and storage spaces were strong indicators of improved health outcomes for patients and satisfaction of families (Jongerden et al., 2013; Ulrich et al., 2004; Ulrich et al., 2008).

\subsubsection{Physical environment and family presence}

A growing body of research has found links between the physical environment and support for presence of family and visitations in healthcare environments. Choi and Bosch (2013) compared the extent of family presence and family-patient and staff interaction in a patient and familycentered ICU and a traditional ICU with with no family zone. The family-centered ICU had larger spaces and comfortable accommodation for visitors. The unit which had spaces for family was associated with higher family presence and higher family-patient interactions as opposed to the traditional ICU. Maintaining a close physical distance to the family members and a comfortable environment emerge as key to influence the overall wellbeing of family members in the hospital arena (Kutash \& Northrop, 2007).

Multiple studies suggest units designed/transformed using the patient and family-centred design (PFCD) concept may facilitate family involvement and satisfaction for families. Rippin, Zimring, Samuels and Denham (2015) compared two ICUs to examine the effects of PFCD on staff-family interactions. In spite of challenges of meeting the needs of different occupants in a complex clinical environment, the PFCD unit was associated with higher interactions, which were family driven. Besides the challenges found with more staff workload and stress (Maguire et al., 2013) that may arise from increased interactions, the findings suggest dedicated family units housing single-bed rooms with larger spaces support more family presence and promote interactions with clinicians. A very recent study looked at whether dedicated family space affects family presence at night before and after moving into an ICU with in-room family space. The study found a statistically significant increase in patient rooms with visitors after the move to the ICU unit with family space as well as full utilisation of space by families (Huynh, Owens, \& Davidson, 2019).

In addition to a designated family-centred design with accommodation for interaction, Andrade and Devlin (2015) included a garden having shade and benches as part of social support. Gardens with abundant trees and benches may offer settings for patients and family members to walk into and retreat into, to facilitate meditation and recuperation away from the indoor hospital 
environment. Marcus (2007) notes that aside from gardens being able to mitigate stress, they provide a relaxed setting for interaction.

Existing studies have been concentrated in the ICUs with little research in other healthcare settings which implies more studies are needed in other healthcare settings. Added to this is the relatively short period of consideration of family involvement in healthcare settings compared to the longterm focus on accommodation of functions and service delivery in healthcare facilities.

\subsection{Conclusion}

This chapter has presented the review of the literature on the general healthcare environment and impacts on patients' and significant others' health outcomes. Based on the evidence on the potential benefits of well-designed buildings for patients in healthcare settings, while not all of the findings reported may be applicable in the postnatal environment, there are indications that some significant research outcomes regarding the physical healthcare environment suggest they could be vital in the recovery process and wellbeing of women. Three of these physical design factors, comprising single bed accommodation, patients' sleep and noise and quality of lighting and appropriate levels of stimulation in the room stand out as highly relevant and needing further research especially for their impacts on physical restoration, psychological recovery and wellbeing of women in the immediate postnatal phase. The next chapter reviews the literature with specific focus on the childbirth environment and the effects of the physical environment on birth experience and satisfaction for women and midwives. 


\section{CHAPTER 3}

\section{IMPACTS OF CHILDBIRTH ENVIRONMENT ON HEALTH OUTCOMES}

In chapter 2 , the review of literature showed physical environment can have significant impacts on patient-centred care and health outcomes. Patient-family-centred care has been a promising approach for humanising healthcare services and revitalising hospital settings including design of the birth environment for wellbeing of new mothers and performance of care providers. This chapter provides a literature review of the impacts of childbirth settings on experiences of wellbeing of women as well as care providers, in addition to a review of studies focused on effects of hospital birth spaces on social support.

\subsection{Historical development of birth environment in high-income countries}

Historically, prior to advances in medical technology, most mothers gave birth at home. This important social event was considered as a normal event where family members and trusted friends supported women throughout the birth process in a familiar environment (Jackson \& Bailes, 1995; Phillips, 1999). In the United States for example, birthing practice was largely attended by women until the middle of the twentieth century (Mathews \& Zadak, 1991). In the true sense of it, birth is primarily a wellness life event rather than illness (Fink, 2011).

By the 1940s, hospitals became the norm and women were attended to in medically-equipped settings under the guidance of physicians. In the nineteenth century, "lying-in" maternity hospitals were already built but only served the indigent and un-married expectant women (Wertz \& Wertz, 1989), although prior to this time maternity hospitals had faced difficult challenges due to a widespread child-bed fever otherwise known as puerperal fever. However, by the 1880 s advances in science and technologies made possible the elimination of puerperal fever from hospitals which resulted in new medical skills for the hospital-based obstetricians (Wertz \& Wertz, 1989). The development gave hospital an improved image that allowed many women across different social strata to choose it as a safer, cleaner and more comfortable alternative to their homes (Wertz \& Wertz, 1989). Correspondingly, the percentage of women having hospital births surged from 5 percent in 1920 to almost 80 percent by 1945 in the United States (Phillips, 1999; Wertz \& Wertz, 1989). 
A similar trend was paralled in Germany, which showed similar historical developments of hospitalisation for childbirth. Nickel (2011) noted that during the period of industrialisation, the rate of childbirths increased. At the turn of the 20th century, a large majority of births took place in the home, and the same trend was seen in rural areas until the middle of the 20th century. Generally speaking, the obstetric unit (OU) has today become the primary setting in particular in the middle and high-income countries (Overgaard et al., 2012). For example, in Canada, almost all (97.9\%) women give birth in hospital settings (Public Health Agency of Canada, 2009). Between 2007 and 2008, around 650,000 women gave birth in England with the majority of births in hospitals (The NHS Information Centre, 2009).

Nickel (2011, p. 015) continued to advance possible reasons responsible for the move of birth to the hospitals:

As general access to hospital care increased and with the specialisation of medical disciplines, birth were relocated more and more to the hospitals. Today in Germany, home births, at less than two percent, play a very minor role; in the Netherlands the rate is around 30 per cent. It is not known what factors are responsible for for the popularity in Germany of hospital births; it may be assumed that with falling birth rate there is a parallel increase in the demand for safe births on the part of parents-to-be.

In parallel to the above, authors in the United States have indicated several other factors contributing to increase in hospital births. Firstly, the impact of World War II had an effect on hospitalisation for childbirth as women moved from home support to be closer to the military camps where their husbands were stationed. Additionally, financial incentives and dwindling support systems for care generally played significant roles in more women giving birth in hospitals, with resultant effects on the shortage of maternity beds and healthcare personnel (Fink, 2011). Other factors included the surge in the number of hospitals; the development of modern nursing; the emergence of specialised medical education and a corresponding need for more women to undergo medical student experience (Mathews \& Zadak, 1991), all resulting in hospital births from increasing through the first half of the 20th century. However, increasingly, trained nurse midwives were gradually replacing the lay midwives in the management of birthing processes. Once this was facilitated, through greater prominence of nurse midwives and their involvement in many social programmes, nurse midwifery was in a position to provide services to drive the alternative birthing movement. 
The late 1960s and 1970s was the time the natural childbirth movement gained traction with collaborative efforts of both the consumerism and feminism movements. These movements had one common objective: to gain control and have a say in decision-making regarding the so-called paternalistic environment. Their efforts yielded immensely positive results in which women were beginning to choose their obstetric provider and birth location (Phillips, 1999). Accordingly, this gave women the freedom to decide what they wanted in terms of their birthing experience, on their own terms and more importantly, in their own territory and jurisdiction.

A salient feature in the 1970 s movement was the increased campaigns on public education, awareness and making informed choice to the extent that consumers "researched" physicians and place to give birth and more importantly, requested rooming-in and insisted on fathers' being at the birth (Mathews \& Zadak, 1991; Phillips, 1999). Consequently, home births attended by nurse midwives increased, which almost simultaneously ushered in many alternative birth centres rather than the conventional/traditional hospital setting (Fink, 2011). An alternative birth setting is defined as "any medically supervised, non-conventional birthing setting located within a hospital, or may be a Free-Standing Birth Center (FSBC) separate from a hospital" (Mathews \& Zadak, 1991, pp. 45-46). The driving force behind this change was the need for family-centred maternity care, which saw the evolution of labour, delivery and recovery (LDR) plus sometimes, postnatal/postpartum (LDRP) designs of maternity units with nursing staff trained to provide both maternal and newborn care (Phillips, 1999).

Given these developments, hospitals across the United States and elsewhere began renovating their traditional labour and delivery units to the new labour-delivery-recovery (LDR) units or to the new single-room maternity care design (LDRP). These new alternative birthing units, emphasising women centred care, demonstrated what the psychological effect and economic impact that a well-designed, health care environment can have on patient, family and providers (Stichler, 2007). The redesign of maternity units into LDRs/LDRPs which began in the late 1960s and 1970s continued into the 1980s and 1990s. Many hospitals either remodeled old maternity units or have built new units in which case, an LDR or LDRP model would probably be settled for (Zwelling \& Phillips, 2001). Today, alternative birth centres/settings (ABC) such as freestanding midwifery units (FMUs) are widespread in several countries including in the Netherlands (Hermus et al., 2015), New Zealand (Dixon et al., 2012), the United Kingdom, the United States, Italy, Germany, Brazil and Norway, in some of which childbirth policies aim to provide women with a choice of birthplace (Overgaard et al., 2012). 
In terms of architecture and structure, alternative birth settings offer a single room for labour, delivery and recovery with minimal interference by healthcare professionals, and a home-like environment with carpeting, artwork, television and refrigerators (Mathews \& Zadak, 1991) and may include comfortable chairs and beddings unlike the traditional hospital settings. Additionally, the self-contained birth and recovery room permits the mother, infant and family members to stay together (Bailey \& Howe, 1993; Spradlin, 2009).

In conclusion, the emergence of alternative birth settings in middle- and high-income countries demonstrates a symbolic determination for a change from the obstetric model of care that had held sway in the paternalistically orientated environment. The alternative birth settings movement was holistic, anchored in the views that childbirth is natural rather than the pathological treatments given to it. Given this, ultimately the mother may exercise control over non-medical decisions, although there are still in existence policies that prescribe who can attend the mother, and when and how many people may be present at a time and how the infant remains with the mother after birth (Mathews \& Zadak, 1991).

\subsection{Childbirth environment and health outcomes}

Whether a woman's birth occurs in a hospital or at home is a topic of interest in the healthcare and childbirth fields. Birth in many resource-rich nations increasingly takes place in hospital due in part to a feeling that hospital is the safest place and women could receive help if complications arise (Coxon, Sandall, \& Fulop, 2014; Kuck, 1972). In the Netherlands, women with low risk pregnancies have the opportunity to choose homebirth or birth in a hospital (Bolten et al., 2016; Wiegers, van der Zee, Kerssens, \& Keirse, 1998), with an increasing number of women in other resource-rich countries preferring homebirth due to perceived control and sense of emotional support through relationships with midwives and perceptions of home as a relaxing environment (Boucher, Bennett, McFarlin, \& Freeze, 2009; Jouhki, 2012; Lindgren \& Erlandsson, 2010; Zielinski, Ackerson, \& Low, 2015). However, research suggests choosing to give birth at home comes with some negative consequences as well as benefits for women's birth experiences and health outcomes (Lindgren, Rådestad, \& Hildingsson, 2011; Zielinski et al., 2015).

Multiple studies suggest that a woman's birth experiences and wellbeing in hospital settings are multidimensional and could be influenced by factors including control and empowerment, satisfaction with place of birth, medical interventions, social support, and design of birth space (Dahlberg \& Aune, 2013; Davis et al., 2011; Foureur, Davis, et al., 2010; Goodman, Mackey, \& Tavakoli, 2004; Green \& Baston, 2003; Hodnett et al., 2009; Janssen, Carty, \& Reime, 2006; 
Lundgren, 2010; Waldenström, Borg, Olsson, Sköld, \& Wall, 1996). Many studies in the childbirth environment are primarily focused on the labour and birth spaces environment and its impact on women's childbirth experiences and satisfaction.

The following sections present a review of empirical studies focussing on the effects of the birth environment on women's birth experiences, satisfaction with care and birth outcomes including the impacts of design of birth spaces on staff job performance.

\subsection{Birth space design and impacts on women}

The physical healthcare environment has been shown to have substantial impacts on patient health outcomes and wellbeing and satisfaction of staff (see chapters 1 and 2). The widespread use of hospitals for birth using similar hospital faciliites means that the maternity units may influence childbirth experience and wellbeing for women. Low-stressed birth environments are beneficial for mother and baby (Buckley, 2015) and stress reduction for women in the physical environment may have a positive impact in achieving women-family-centred maternity care (Simkin, 1986). Wood, Mignone, Heaman, Robinson and Roger (2016) conducted semi-structured interviews to understand the underlying reasons for women to make the choice for an out-of-hospital birth. The findings suggest that design of the facility plays an important role, suggesting that the design of birth environments influences women's childbirth experiences and satisfaction.

The freedom to move during labour has an impact on women's outcomes. Lepori (1994) studied the traditional institutional birth settings, birthing centres, and labour delivery and recovery rooms (LDRs) in the context of freedom of movement. In the case of institutional settings, movement is seen as directional or a straight path, and in the home, it is seen as spiral or concentric suggesting that the woman is more in control of the birth process. The features of an LDR suggest an inclination toward a medicalised focus with the labour bed in the centre of the room that could exert a different way of behaviour on the woman. The bed in the room in maternity units was identified as a hindrance for upright birth positions for women and the psychological effects of preparing the bed with hospital supplies and gown were negative (Gould, 2002). Beds in the birthing environment largely convey different messages for women and midwives, and are capable of influencing women's childbirth experiences and satisfaction with clinicians' practice (Townsend, Fenwick, Thomson, \& Foureur, 2016). In addition to these, Hodnett (1989) contends that an optimal birth environment with few unfamiliar procedures or personnel, alongside personal expectations of control, encourages freedom of expression, ultimately improving the birth experience. 
Because the design of childbirth environments may facilitate normal labour and birth as well as improved birth outcomes for women, some researchers have used evidence from surveys of birthing women, midwives and architects to produce an audit tool dubbed the Birth Unit Design Spatial Evaluation Tool (BUDSET) (Forbes, Foureur, Leap, \& Homer, 2008; Foureur, Leap, et al., 2010; Foureur et al., 2011). The BUDSET had four domains comprising fear cascade, facility, aesthetics and support, each one with their respective sub categories. Validation of the BUDSET, (Sheehy, Foureur, Catling-Paull, \& Homer, 2011) was undertaken in two settings; one a major hospital and midwifery research center in Australia using a survey for agreement on the BUDSET items plus in-depth interviews. The authors found that the domains of facility and support were rated valid by most participants while the fear cascade and aesthetic were less strong. While the BUDSET was verified to be valid for evaluating birthing environments, more recent research suggests it may not be appropriate for diverse groups and cultures (Menke, Jenkinson, Foureur, \& Kildea, 2019). In addition, the BUDSET did not focus on the postnatal environment, meaning that design factors influencing recovery experiences of women may have been receiving least attention in the research.

With respect to impact of space specifically focused on women postnatally and celebration of birth Wray (2012) found that the culture of the postnatal ward was at variance with the expectations of women and being in the postnatal ward undermines the notions of celebration of birth. An earlier study concluded that post-birth recovery is contigent upon a good hospital stay, however for many women this does not happen (Wray, 2006).

As hinted previously in Chapter 2, research related to the concept of single-room maternity care exists, to a greater degree in the United States and less elsewhere. Spradlin (2009) described the implementation of a couplet care programme (see definition of terms for description) at a Women's Center in the United States for women who have had a ceaserean birth by offering couples/postnatal families a designated couplet care room. She reported that the couplet care room promotes the opportunity for quiet time to achieve bonding in the first few hours of the infant's transition to the world; early establishment of breastfeeding; encouraging skin-to-skin touching by nurses and assisting temperature regulation for the infant. In addition, there is overall patients'/clients' satisfaction at the hospital owing to this model of care. Similarly, combining both LDR and LDRP units has been shown to be effective in meeting individual women's birthing preference and experience (Bailey \& Howe, 1993). Given that nursing/midwifery and childbirth literature has numerous accounts of negative and unmet expectations due in part to the physical environment during the postnatal hospital stay, it is reasonable to consider how design of space 
and specific design guidelines might inform such space and environment. One study in the United States conducted a post-occupancy evaluation at a women's health centre, which provides new building protoptypes comprising single-room LDRP maternity care. Using questionnaire, interview and behaviour mapping methodologies, the researchers found that the LDRP unit had positive evaluation in all ramifications although some specific suggestions were offered with corresponding design guidelines (Shepley, Bryant, \& Frohman, 1995).

Gaining insights into important design guidelines may contribute improvements for healthcare environemnts. In this connection, architects have used evidence-based findings for improvements in childbirth facilities and childbirth care for women and clinicians. Case studies of maternity/birth units include the maternity waiting village in Kasungu District, Malawi which accommodates expectant mothers nearing their term. The centre comprises modular vernacular sleeping units clustered around courtyards, offering privacy and comfort, whilst being optimised for daylight and ventilation thus mitigating the spread of infectious diseases (Murphy \& Mansfield, 2017). Additionally, the MASS $^{2}$ group with Ariadne Labs have studied three birth centres and nine hospitals in the USA and reported that the findings reveal several ways by which design may facilitate or hinder clinicians in the care of women with regard to enabling a flexible approach to patient needs and distribution of workload. The findings also suggest an association between facility design and caesarean section outcomes (Ariadne Lab \& MASS Design Group, 2017).

Leydecker (2017) indicated the value of incorporating a natural approach that offers the mothersto-be an atmosphere of wellbeing and homeliness rather than the sterility of hospitals in the design of the maternity unit at Elisabeth Hospital, Essen, Germany. Design revolves around scenery of river valleys, to elicit perceptions of forms, patterns and colours that all together create an atmosphere of wellbeing and homeliness for expectant mothers, their families and hospital staff.

\subsubsection{Women's wellbeing and birth experience}

Becoming a mother goes beyond only a psychosocial transition, it is a health transition (Walker \& Wilging, 2000, p. 230). Conception of health encompasses the physical, mental, physiological and

\footnotetext{
2 A design group founded in 2008 by Michael Murphy and Alan Ricks, with a philosophy that form follows facts. MASS designed and completed The Maternity Waiting Village in 2015 in partnership with the University of North Carolina - Malawi, Malawi Ministry of Health, the Presidential Initiative for Safe Motherhood, the Bill and Melinda Gates Foundation, and the Autodesk Foundation. Further reading can be found at: https://www.livinspaces.net/projects/architecture/kasungu-maternity-waiting-village-malawi-mass-design-group/
} 
psychological, with the birth of a baby as a powerful life event which has implications for a woman's health and wellbeing (Hildingsson, Johansson, Karlström, \& Fenwick, 2013). The design of the birth environment has been shown to be important for safe and satisfying experiences and improved health outcomes for a birthing woman in hospital (Foureur, Leap, et al., 2010; Jenkinson et al., 2014). Understanding of women's health promotion regarding the physical healthcare environment could contribute to women's wellbeing outcome prior to going home.

The following is a review of research evidence on the effects of the childbirth environment on women's birth experience, satisfaction, and wellbeing.

The interior design features of the birth environment can communicate visual messages, with some researchers finding evidence of the techniques of positive distractions in the interior environment. Studies that offered women a set of interior design elements indicated that interior design and well-designed hospital birthing settings can contribute to women's feeling of "hominess" through personal control over visual access and exposure to the outdoors (Shin et al., 2004). A study by Bowden, Sheehan and Foureur (2016) explored images of birth rooms in developed countries to anlayse the messages and the visual discourses being communicated about childbirth.. Several images were collected on the Internet that comprised images from obstetric units, both alongside midwifery and freestanding midwifery units (AMU) and (FMU) in six countries - New Zealand, Australia, Canada, Europe, UK and the US. The findings revealed three types of birth room images: the technological, the homelike and the hybrid domesticated birth room. The technological birth room with the labour bed and medical equipment had a powerful impact that portrayed childbirth as potentially risky and unsafe, influencing women's attitudes, choices and behaviour and having a negative impact on wellbeing in the space.

Having elements of domesticity is an important consideration for optimal birth unit design. Mondy, Fenwick, Leap and Foureur (2016) examined and described the concept of domesticity in birth spaces with different domestic features. Video ethnography and interviews, including filming of the labours of six Australian women, both in a tertiary hospital and in a birth centre of a tertiary hospital, provided the data. Video footage served to compare and contrast the birth experiences of all the women in the study. The findings revealed women in conventional hospital birth rooms acted and interacted with the environment in a passive way due in part to absence of domesticity of the spaces making the women less able to adapt to the space. In the birth centre with domestic environments and spaces, the design and furnishings and features of the space encouraged activity and a feeling of attachment in the space. 
Hauck et al. (2008) described women's experiences of labour assigned to a "Snoezelen room." A Snoezelen room is a specialised indoor environment which exposes users to several sensory stimulations. Benefits include distraction, environmental control, relaxation and choice of complementary therapies, and safety in an archetypal non-clinical atmosphere. The research found that the effect of distraction impacted women in a positive way. Hodnett et al (2009) explored a modified labour room referred to as an "ambient room" with specific types of auditory, visual and tactile stimuli. The ambient room received positive evaluations from the women and caregivers including less use of oxytocin by women in the room.

Aside from the usage of images in the birth rooms, blocking the view of medical equipment with visual art is another design consideration for the birthing environment. Duncan (2011) conducted a carefully controlled study in the labour and delivery rooms to determine whether an introduction of visual art can induce improved physiological and healthcare outcomes for women in labour, and as a means for cost savings. The researcher used different techniques of applying art in the labour and delivery room to hide the emergency birthing equipment. The researcher found that there was a significant reduction of labour by 2.1 hours whereas a control group showed an average of 7 hours of labour suggesting that introducing appropriate visual art in the labour and delivery room could offer positive distraction for women in labour.

Empirical evidence indicates women desire features in the physical birth environment to assist in the labour and delivery process. In a series of studies by the NHS in the UK that investigated the views of women about the design and facilities in maternity units and what women thought birth spaces should provide, (Newburn \& Singh, 2003, 2005) found nine out of ten women thought the physical surroundings can affect how easy or difficult it is to give birth. Women identified a number of aspects including control of temperature, a pleasant place to walk, a non-clinical room with home-like features, privacy and support for snacks and drinks. They indicated having a clean room and ability to stay in the same room, and walk around in addition to having en-suite bathrooms, adjustable bed and chair for the birth partner were important for their birth experiences. Building upon two earlier studies, the authors found very similar results (some met and unmet in birth facilities) and concluded the place where a woman gives birth is important to her comfort and may have impact on her birth experience and wellbeing (Singh \& Newburn, 2006).

Maintaining privacy of a woman during labour and birth impacts on the experience of birth. Rados, Kovács and Mészáros (2015) assessed the role of privacy and intimacy and perceived stress due to lack of privacy during labour and birth. The researchers found a higher sense of privacy was associated with less stress for mothers and vice-versa. An atmosphere for privacy could promote 
a satisfying birth experience and better health outcomes for mothers. Research shows that women in labour and birth desire many features in the birth rooms that may be contradictory such as privacy and having a window or a pleasant view of the outside. However, an inappropriate surrounding such as an open window can be perceived as a lack of privacy and could cause distress and discomfort for women in labour (Aburas et al., 2017; Rados et al., 2015). In this regard, optimising control of daylight for women can be a useful design consideration for women having lack of access to natural lighting or at a distance from windows in multiple-bed rooms. A new research investigation by Canazei et al. (2019) studied the effects of adjustable bedroom lighting and found that mothers exposed themselves and their babies to higher daytime and lower evening light levels under dynamic light compared to standard light indicating that an adjustable dynamic light may improve indoor light exposure in a maternity ward.

Another feature for an enhanced birth experience is the use of water that offers positive distractions in labour and birth rooms for therapeutic effects. Some researchers have found evidence that water immersion or use of a birthing pool enhances positive birth experience and wellbeing of mothers. In the UK, birthing pool use was associated with a high frequency of spontaneous birth particularly for first-time mothers although during water birth due diligence is necessary to guide the baby to the surface (Burns, Boulton, Cluett, Cornelius, \& Smith, 2012).

In Italy, Henderson et al. (2014) conducted a wide ranging study which indicated that 95.6 percent of women who used a birthing pool had a spontaneous delivery, with a higher percentage $(63.9 \%)$ occurring in water, with an absence of adverse maternal and neonatal outcomes. In New Zealand, Maude and Foureur (2007) explored the stories and experiences of women who used water for labour and birth, at home and in hospital. The authors indicated that birthing in warm water offered an all-encompassing experience of warmth, leading to feeling relaxed and comforted while offering a sense of privacy. Beyond these, women used water to alleviate fear of pain and childbirth and as a means of coping against pain.

However, some researchers have expressed skepticism about the usage of water birth despite newfound affinity for it. Johnson (1996) reviewed a large body of evidence regarding birth under water and reported that research was required into the benefits and risks of birth under water. Appropriate monitoring that does not interfere or alarm the mother will benefit investigation of birth under water in addition to use of safety sensor devices and technology to safely and effectively monitor fetal heart rate without obstructing the mother's position. Another review of empirical studies on the use of water birth concluded that water birth may be associated with complications (such as drowning, neonatal waterborne infectious disease, cord rupture and possible death) that 
may not be visible in land-based birth, although the rates of these complications are likely to be low (Pinette, Wax, \& Wilson, 2004).

In terms of what might be considered "at the edge" of physical environment-related factors, (Taavoni, Sheikhan, Abdolahian, \& Ghavi, 2016) investigated the effects of birth ball and perineal heat therapy on labour pain. They found that mean pain severity score in the heat therapy group was lower than the control group at 60 and 90 minutes following interventions. Both nonpharmocological means were very positive and could serve as low risk treatment for labour pain.

Patient-woman-centred care in the birthing sector cannot be isolated from a supportive childbirth environment for the overall wellbeing of women and infants. A very recent review of literature of research into childbirth settings focused on identifying building spaces to support labour and birth care concluded the importance of considering the physical environment in maternity care. The review suggests a need for architectural-focused research to broaden knowledge in creating appropriate recommendations and guidelines to effectively and efficiently support women during and after the birth (Setola et al., 2019).

\subsubsection{Childbirth and social support}

The arrival of a new baby is a special event and an important time for family to celebrate and support the new mother regarding personal needs and care of the infant. These tasks, if birth occurs at a hospital, require the support of many people including clinicians and midwives as well as family members. Efforts at humanising childbirth experience in keeping with consumer needs (patients' and visitors'), are the impetus for hospitals to embrace family-centred care whose goal is to recognise the psychosocial needs to facilitate quality of care in the maternity services for the childbearing families (De Labrusse, Ramelet, Humphrey, \& Maclennan, 2016; Harris et al., 2002; Hodnett, 1989).

Social support for birth starts from pregnancy, with antenatal education classes providing opportunity for building of relationships with caregivers and midwives. Benefits of the mutual relationship between women and their midwives appear in routine assessments and subsequent supervisory roles during birth even if families are around new mothers. Social support in the birth process is important to emotional, physical and psychological impacts on women's birth experience and their overall wellbeing.

Research focused on mothers' perceptions of social support from nursing staff and factors associated with the support found that social support from nursing staff was perceived as moderate 
among the mothers who requested support from nursing staff, while affirmational support was perceived as the highest (Salonen, Oommen, \& Kaunonen, 2014). A Swiss study with sixty women revealed discrepancies between received support and expected support. Interactions with caregivers, breastfeeding and hospital environment constituted the higher stress events in the first few days of birth, suggesting increased efforts may be necessary from care providers to provide new mothers with adequate assistance (Razurel, Bruchon-Schweitzer, Dupanloup, Irion, \& Epiney, 2011).

Fathers' birth experiences and the amount of support provided by midwives have implications for the birth experience of women. Hildingsson, Cederlöf and Widén (2011), investigating fathers' experiences in relation to midwifery care found that midwife support and adequate information about the progress of labour were the strongest factors associated with a positive birth experience. Additionally, a maternity care team that provides support for women and families, including doulas (trained non-medical companions), can foster an enhanced, safe-satisfying birth (Zielinski, Brody, \& Low, 2016). Research suggests that when women reported a negative birth experience due in part to a range of factors including pain of birth, high use of analgesics and longer hospital stay, the confidence in the midwife was instrumental to influencing positive birth experiences for firsttime mothers (Larsson, Saltvedt, Edman, Wiklund, \& Andolf, 2011). A body of empirical evidence found that women would like healthcare providers to maintain continuous support and presence throughout childbirth as a key element of care to enhance coping ability and avoid feelings of loneliness and fear (Van der Gucht \& Lewis, 2015).

Anticipating more support from healthcare providers during childbirth may pose a challenge and lead to unmet expectations due to the routine and workload of healthcare staff that may be beyond their control. This may affect the childbirth experience of women. For example, Yelland et al. (1998) assessed women's views about their care, support and satisfaction with postnatal hospital stay in three maternity hospitals in Australia and found that overall satisfaction with care was low. A few women left hospital when they felt there was a lack of support and assistance with personal needs and catering for the baby.

Due to the increasing awareness of social support needs, a study developed and tested an instrument to measure women's perceptions of social support provided during childbirth. They found two subscales including emotional support and tangible support which could be used to address the needs of birthing women, partners and their families. However, inclusion of a larger sample from different maternity settings and in particular, women with different cultural backgrounds, may benefit the refinement of the instrument (Dunne, Fraser, \& Gardner, 2014). 


\subsubsection{Physical environment, social support and birth companions}

The physical environment of birth can offer support for the normal and physiologic birth process (Stark et al., 2016). The event of birth benefits from the presence of partners and family members to assist and ease women in the birth process. Some first-time mothers may experience fears, anxiety and pain that could be stressful in part due to their perceptions of the event and how to deal with the event and coping with pain could be a crucial factor to improve birth experience (Larsson et al., 2011; Mercer \& Ferketich, 1988). Involving fathers as birth supporters may be an essential factor for attainment of a positive birth experience (Johansson, Rubertsson, Rådestad, \& Hildingsson, 2012). In critical situations, the assurances of family and network of support contribute to boosting the sense of trust and emotional strength of women including the support offered through a familiar and safe environment (Aune et al., 2015). There is evidence that negative feelings of women in labour may be lessened by support through a range of physical therapeutic strategies such as touching, soothings and interactions with labouring women (Stenglin \& Foureur, 2013).

It was suggested that increased levels of involvement of fathers with newborns and labouring women may compensate for the new mothers when they had less satisfaction with the birth experience, due in part to lack of environmental features (Westreich et al., 1991). This implies that the physical environment may influence behaviours and activities in the birth environment. The provision of both a supportive environment and supportive people can help women manage labour and the design of the environment may further help to foster a sense of mastery and maternal autonomy (Carr, 1994). A distinction between a supportive childbirth environment and a non-supportive childbirth environment is that the former involves active engagement of support persons and nurses who are both physically and psychologically closely attending the woman. By contrast, the non-supportive environment is one in which the support persons' role is diminished, providing little or no physical or psychological support, and the supporters are physically withdrawn from the woman (Carr, 1994).

The physical environment of childbirth appears to have contributed very little to support women through the experiences of both birth and the postnatal recovery phase in many maternity facilities. An Irish study found women often felt alone and unsupported; by extension this is the reason why some may not choose to have another child in hospital because of their birth experience (Larkin, Begley, \& Devane, 2012). A form of support that has continually had less focus is the support offered to women by doulas during birth. Pascali-Bonaro and Kroeger (2004) trace the 
contribution of doulas' care and support as necessary because labour and birth are both a psychosocial and physiological process. It was contended that the doula helps in creating a positive environment for women in labour, their partners and families as well as midwives and other healthcare providers. Harte, Sheehan, Stewart and Foureur (2016) studied both inhibiting and facilitating design factors impacting the experiences of childbirth supporters. The authors found that childbirth supporters experiences were complex, yet there was very little understanding of their needs, and consequently they were not facilitated by the physical space (Harte et al., 2016). They suggested some design guidelines to enable childbirth support roles to be achieved. However, such guidelines were exclusive only to labour and delivery rooms and not to postnatal spaces.

A growing number of studies have shown that fathers' presence and support during childbirth can enhance new mothers' birth experience. In Sweden, fathers wanted to have an "allowing atmosphere" to be seen as a labouring couple/dyad and when isolated they feel helpless which can put their supportive role of their partner at risk (Bäckström \& Wahn, 2011, p. 69). In Singapore, a study of 16 first-time fathers found that fathers appreciated the social support received, in particular, tangible and intangible support from family and friends and information and help given to support the new mothers. Healthcare professionals need to guide and make available information to prepare fathers for any unforeseen changes (Poh, Koh, Seow, \& He, 2014). Aside from these, supporting women who have experienced forced migration and settling in a new environment can be challenging for the health and wellbeing of women. Riggs et al. (2016) conducted interviews with thirty Afghan men and women who had a baby in Australia and found that Afghan men supported their wives in several ways during pregnancy and postnatal care: accompanying their wives to appointments, and providing language and transport support. The authors concluded that childbirth-related care needs to be responsive to the social and psychological needs of families of refugee background to enhance family health outcomes.

However, a study conducted in Nepal with 13 husbands/partners revealed that presence of a man in the labour rooms is "culturally discouraging", but regardless of the uncomfortable situations many of the husbands were satisfied to have provided emotional and physical support to their wives through physical and verbal experessions at her bedside (Sapkota, Kobayashi, \& Takase, 2012). A study in a surburb of Tanzania exploring the postnatal experiences of first-time fathers, that echoes other studies about cultural beliefs about fathers' role in childbirth, found first-time fathers were keen about fatherhood and showed a concern for the support of the mother and infant. However, the health sector would need to be more responsive regarding fathers' concerns for family health and needs for support (Mbekenga, Lugina, Christensson, \& Olsson, 2011). 
The support received during the stressful event of birth may produce positive feelings and improved birth experience. A study by Ford and Ayers (2009) that attempted to clarify birth stressors and support received during birth collected data from 137 women who rated their perceived control, mood and anxiety. It was found that the levels of support provided by staff had a greater effect on women's emotional reactions than stressful events. Further, supportive care was responsible for perceived control while lowering anxiety and negative mood.

Social support during the postnatal period can be a major buffer against postnatal depression. Studies in the postnatal phase have found the beneficial importance of supporting women in times of recovery and acquisition of parental skills. Negron, Martin, Almog, Balbierz and Howell (2013) gathered four focus groups comprising thirty-three ethnically diverse women as participants. The authors suggest recognition of support needs and expectations of new mothers would benefit mothers' recovery after childbirth.

Nurturing an infant is an important feature in the postnatal period that requires a supportive maternity environment. New mothers are expected to initiate and establish breastfeeding prior to going home (Carling, Demment, Kjolhede, \& Olson, 2015; Hallowell, Froh, \& Spatz, 2017). One study found that experiences of women with breastfeeding could be challenging and related to higher degrees of tension, insecurity and anxiety (Holmberg, Peterson, \& Oscarsson, 2014). Breastfeeding requires an environment that is peaceful, relaxing, private and free from interruptions for optimal results. Reseachers have studied the impact of the physical environment for facilitating breastfeeding. Morrison and Ludington-Hoe (2012) observed the frequency and duration of interruptions and maternal perceptions of the influence of interruptions on breastfeeding experience in an LDR unit. The researchers observed the door to the rooms of 30 mothers for 12 hours on postnatal day one. A high number of interruptions were observed, which mothers perceived as interrupting breastfeeding. Zadoroznyj, Brodribb, Young, Kruske and Miller (2015) found a similar result of inadequate breastfeeding support, which constitutes an issue for women irrespective of birth environment. Gaboury, Capaday, Somera and Purden (2017) described the impact of physical environment and on mothers's and fathers' goals in the postnatal period. The study found both fathers and mothers had the same objective of developing parenting competence while in the hospital, although the routine of the unit and physical environment were a hindrance to the parents' goals. The facilitating role of the environment is especially important in the quest for woman/family centred care. 


\subsection{Impacts of birth space design on midwives and clinicians}

Just as general hospital built environments may influence patients and healthcare providers regarding health outcomes, safety and overall wellbeing, hospital birth room design may have influence on midwives and their practice in hospitals. Caring for women during the birthing processes requires a midwife navigating within defined spatial-physical boundaries using her privileged role in creating a facilitative environment for mother and baby (Hastie, 2011). It is contended that experiences and perceptions of the physical environment could have links with complex neurobiological interactions that may trigger the release of oxytocin, hence shaping midwifery practice (Hammond, Foureur, Homer, \& Davis, 2013). Midwifery practice entails that its practitioners work in different birth settings including at home, exposing them to work-related stress, anxiety, pain and burn-out with environmental-related factors playing a contributory role (Geraghty, Speelman, \& Bayes, 2019). Contextually, space and place are essential features in any work environment necessary for tasks to be carried out however, research in the childbirth environment is beginning to examine the ways they shape midwifery practice research (Hammond et al., 2014).

Hospital birthing environments may influence the birth experiences of women, including interfering with midwives' work. A study of the impacts of birth environment in Australia and in the UK on the midwives who practice in a variety of birth settings found that midwives recognised the importance of creating ambience in different settings such as noise reduction, lighting and privacy creating a domestic rather than sterile character. It was reasoned that ambience and the creation of a space that is conducive to labour might facilitate a woman's positive labour experience as well as being an important part of midwifery practice (Davis \& Homer, 2016, p. 410). Knowledge and skills of midwives can be useful for creating a facilitative environment to support mothers birthing out-of-hospitals. One study that comprised 20 midwives: 14 of whom assisted at births in midwifery homes in Japan - similar in character to birth centres - and six independent midwives assisting at home births, found that the midwives created a physical environment that facilitates autonomy for the mother and family and a physical environment that facilitates birth. Labour progresses smoothly through the maintenance of a warm room temperature where the mother is kept comfortable in an environment that facilitates movement. In addition, the environment facilitated for natural and indirect lighting giving the opportunity for the mother to relax while the midwives modulate/regulate the light to increase concentration for the mother. 
Hammond et al. (2014) explored the relationship between the birth environment and the practice of midwifery using face-to-face photo-elicitation interviews with sixteen midwives who worked in a birth centre on the site of the hospital and delivery suite. The analysis of the photo-elicitation interviews suggests that the design and aesthetics of hospital birth rooms convey messages to midwives that elicit emotional and cognitive responses that could shape the activities and attitudes of individual midwives. Research has found friendliness, functionality and freedom were key design features that could support midwifery practice in hospital settings. The findings indicated friendly rooms alleviate stress and increase midwives' feelings of safety; functional rooms allow options to be tailored to the needs of women and freedom entails a flexible and responsive midwifery practice in the settings. These design characteristics may enhance support for midwives and may facilitate effective care provision (Hammond, Homer, \& Foureur, 2017).

Facilitating an environment for normal birth has been the pursuit in midwifery practice and for maintaining a healthy birthing experience (Kruger \& McCann, 2018). A study exploring the views of the midwives regarding the factors that facilitate or impede the attainment of normal birth in a hospital's obstetric-led unit (OLU) found several barriers including a risk-averse culture of birth, time pressures and women's expectations, while creating an intimate and supportive space where the midwife could work with other midwives and support the labouring woman and the midwife's desire to promote a normal birth were factors for facilitating a normal birth. Midwives who were keen on promoting a normal birth felt unsupported (Carolan-Olah, Kruger, \& Garvey-Graham, 2015). Promoting a normal birth in hospital birth settings is a contentious issue between obstetricians and midwives largely due to the culture of the birth environment that has promoted birth as a medical condition with midwives feeling uncertainty about when to exercise the authority of their scope of practice (del Rosario Ruiz \& Limonero, 2014; Keating \& Fleming, 2009; Kruger \& McCann, 2018).

Another study focused on a number of alongside midwifery units (AMUs) to explore midwives' experiences of working in the units in promoting a sense of wellbeing and re-normalising concepts of birth. It found AMUs were designed to facilitate and to represent a restoring and supporting of birth as a normal physiological process including a social life transition. The findings indicated that AMUs used domestic features as metaphors of home represented in the design and the imagery of the birth rooms with homely touches including a domestic-style double bed and where possible concealing the medical equipment from view. The authors believed such spaces had a function for wellbeing of midwives who practice in them (McCourt, Rayment, Rance, \& Sandall, 2016). 
Communication with patients and family members by healthcare staff is a key aspect in the healthcare delivery system. In the context of the birthing environment, communication usually goes hand in hand with note-keeping consisting of documentation of vital statistics, monitoring of the process and periodic recording of labour progress of a childbearing woman. Documentation and note-keeping usually take place in the nurses'/midwives' unit, meaning that furniture and furnishings of the space may contribute to its effectiveness. A study which evaluated quality of midwifery and looked at documentation of sixty-one women in labour found basic note-keeping dropped between the middle and the end of the 12 hour shift, with fatigue possibly having a contributory role in poorer documentation towards the middle and the end of the shifts. Recommendations were that scheduled breaks may be beneficial to midwives particularly in the final third of their shifts (Bailey, Wilson, \& Yoong, 2015). Additionally, such scheduled breaks should prioritise a restorative environment with homely seats and décor where midwives could go and relax as well as during documentation, with a space to facilitate note-taking including appropriate facilities to encourage documentation.

Returning to section 3.4, the notion of neuroscience is implicitly indicated in considerations of how the built environment shapes experience and perceptions of space, memory and cognition. Neuroscience is concerned with complex physiological and neurological processes, manifesting through consciousness, thought-process, and cognition with the brain acting as the domiciliary receptor and processor of information. Also, there has been a rapidly growing body of research focusing on the links between neuroscience and architecture. This will be briefly addressed in the next section.

\subsection{Neuroscience and built environment}

In the pursuit of a suitable methodological approach for this research, the researcher read widely on different approaches, and found neuroscience research approaches that can be utilised for understanding human experience in the built environment. Although there is much to read and already a lot has been written on the intersections between neuroscience and architecture, however neuroscience research approaches to the study of the built environment are still in their infancy. This section briefly presents the potentials of this new knowledge base and ideas through a discussion of publications about neuroscience and built environment, and advances reasons for the lack of fit of a neuroscience approach in this current study.

The human brain is the most sophisticated structure of the human body. Its strategic location on the frontal and upper portions of the human body - arguably confers it a greater capacity for 
performing the most-complex, supervening role of how human beings think, act and/or behave, perceive, learn and remember places, respond to external stimuli, movements (fight or flee encounters), excitement or sadness - the whole range of human activities. Architecture is questing for understanding human experience in the built environment. Neuroscience appears to offer a robust platform for a field of architectural research into human consciousness and experience in relation to the built and landscape environments. Further, in 2003, the Academy of Neuroscience for Architecture (ANFA) was founded in San Diego, the United States, to explore ways to link research between neuroscience and architecture. Over the past years, there has been an increasingly growing momentum with many publications and conferences focused on design of spaces and places, and human experience in the built environment.

One of the influential books on intersections between the neuroscience and architecture research fields is by Eberhard (2009b). John Paul Eberhard, an architect and one of the founders and pioneer president of the ANFA, died on May 2, 2020. Alongside important figures such as Harry Francis Mallgrave, Juhani Pallasmaa, architect Frank Gehry and others, he helped to sow the seeds of research between neuroscience and architecture. His book highlighted some $70+$ research hypotheses as potential areas for possible scholarship for doctoral and post-doctoral students across both the disciplines of architecture and neuroscience. Specifically, five areas identified for studies in the brain's systems include:

1) Sensation and perception (how do we see, hear, smell, taste, etc.?)

2) Learning and memory (how do we store and recall our sensory experiences?)

3) Decision making (how do we evaluaute the potential consequences of our actions?)

4) Emotion and affect (how do we become fearful or excited? or what makes us feel happy or sad?

5) Movement (how do we interact with our environment and navigate through it?)

Research into human-built environments which are responsive and perceptive of the human brains and minds received the impetus with the work of Goldhagen (2017), Welcome to Your World: How the Built Environment Shapes Our Lives. Goldhagen taught architecture and landscape at Harvard University's Graduate School of Design and is an architecture critic. This book described, in detail, the less than adequate state of the human-built environment, the choices and actions that could be made in support of a well-designed, properly constructed environment as it affects and supports human health, cognitions, and social relations. Goldhagen (2017) argues about misplaced priorities and judgements of architecture and building and affirms that the design of all built 
environments is so especially profound that the preeminence of safety and functionality must not only be the urgent priorities.

Goldhagen (2017) writes:

All kinds of design elements influence people experiences, not only of the environment but also of themselves. Good design ... create[s] coherent places that have a powerful effect on people. Urban spaces, landscapes, and buildings... profoundly influence human lives. They shape our cognitions, emotions and actions, and even powerfully influence our wellbeing. They actually help constitute our very sense of ourselves, our sense of identity (Goldhagen, 2017, p. 23).

Karakas and Yildiz (2020) state that advances in science and technology, together with improved ways of measuring and understanding human experience, have expanded the knowledge on human-built environment interaction. These developments have been instrumental for research on the interaction betweeen neuroscience and architecture. This evolving field of study may be the domain of a new generation of applied social and behavioural scientists, engineers and architects within the next few decades (Eberhard, 2009b).

(Eberhard, 2009a, p. 753) declares in one of his treatises, on the misconception about what architecture is about:

Most neuroscientists think of architecture as a profession concerned with asthetic beauty ... But, architecture is more than aesthetics. Well-designed buildings need to respond to the functional needs of the occupants, and users need to be provided with adequate lighting, well-modulated heating and cooling systems, structural soundness, and public safety provisions.

Eberhard (2009a) argues that it is through the efforts to expand the horizon for neuroscience that a new knowledge base would be developed for architecture, and this may be useful for both disciplines in more fruitful research ways.

John Eberhard writes:

... We would then know how the design of classrooms can support the cognitive activities of students, how the design of hospital rooms can enhance the recovery of patients, and how the design of offices and laboratories can facilitate interdisciplinary activities of neuroscientists, and so forth (Eberhard, 2009a, p. 753). 
Consciousness and cognition are two relatively important paradigms in neuroscience underlying an understanding of human experience and response to the built environment. Consciousness is the "binding" context for understanding how we experience architecture. Eberhard (2009b) states that it seems obvious that we need to be conscious for an experience to take place, even if that experience is one that emanates through dreams or memories. In the last few years, there has appeared a growing number of works that discuss the relation of neuroscience to the scholarly and professional worlds with respect to consciousness, cognitions and human experience, with emerging terms that sometimes overlap (Edelstein, 2015; Goldhagen, 2017; Hauptmann \& Neidich, 2010; Hollander \& Foster, 2016; Sussman \& Hollander, 2014). Such terms include cognitive architecture, cognitive neuroscience, neuro-architecture and cognitive neuropsychology. In its broadest sense, neuroscience is part of cognitive sciences concerned with the nervous system with a specific focus on the human brain. Also, within the neuroscience field is an emerging field termed neuroaesthetics that relates to the biological basis for aesthetic experiences in the built environment. As Chatterjee and Vartanian (2014) state, this domain of knowledge cuts across traditional areas of cognitive neuroscience such as perception, emotion, semantics, and decisionmaking.

On the other hand, cognitive science embodies neuroscience, neuro-architecture and cognitive architecture (Karakas \& Yildiz, 2020). Neuro-architecture is a nascent and emerging field concerned with the interactions of the neural systems (brain and body) and the built environment. Edelstein (2015) states that the term neuro-architecture has long been used to denote the form of the brain and function, and increasingly architects have embraced the term to describe how the form of architecture may better serve human functions and generate delight. This new emerging research field consists of an amalgam of biomedical science, neuroscience, architecture and environmental psychology. Edelstein (2015) argues that the neuro-architecture process is based on the knowledge of the interconnected systems and feedback from the brain, which enables an understanding of the biological bases of sensory perceptions, emotions and cognitions that may be incorporated into design guidelines. In one sense, how people perceive and experience the built environment is clearly the function of consciousness, sub-consciousness, and unconsciousness, which are innate, idiosyncratic and inextricably linked to humans' evolutionary past (Goldhagen, 2017; Karakas \& Yildiz, 2020).

No doubt, the human brain plays a significant and often complex role in the way we perceive, in cognitions and the nature of experience in the built environment. The human brain is divided into three major parts: the forebrain, midbrain and hindbrain. The forebrain comprises the two outer 
hemispheres of the cerebrum, divided into four lobes with specific functions (Edelstein, 2015). It is responsible for thinking and processing sensory input, which make up the left and right cerebral hemispheres. It is covered by the cerebral cortex and subcortical structures. Nested under the cortex are five diverse structures that partake in higher level thinking including the hippocampus and the amygdala, which is in charge of memories and emotional regulation (Sussman \& Hollander, 2014). The hippocampus is responsible for navigation including creating memories of events (Pallasmaa, Mallgrave, \& Arbib, 2013; Sternberg \& Wilson, 2006). The middle brain controls eye movement. The hindbrain extending up to the spinal cord, is in charge of activities considered lower-order and automatic: such as breathing, heart rate, blood pressure and maintaining balance and equilibrium (Sussman \& Hollander, 2014). These neural processes coordinate and direct our impulses, reactions and actions in the built environment, particularly our experiences.

Goldhagen (2017) introduced seven main ideas: 1) the sorry places we live, 2) blindsight: experiencing the built environment, 3) the bodily basis of cognition, 4) bodies situated in natural worlds, 5) people embedded in social worlds, 6) designing for humans, and 7) from blindsight to insight: enriching environments, improving lives. Within these ideas, Goldhagen espouses the relationship of our bodies to both the social and natural worlds, which have potential for human experience especially, through enriched built environments.

In chapter 2 of the book, (Goldhagen, 2017) makes clear the distinction between "experience" and "cognition" in that experience is grounded/imbued in our sensory perceptions and internalised in thoughts, which in totality determine our judgements of the information that comes to us from being in the world. This means that experience is idiosyncratic, personal and amorphous. Cognition relates to the processes by which people come to understanding, learn, interpret and organise sensory, social, physical and internally generated data. In this sense, cognition is understanding of situations and knowledge differentiated from intuitions, hypotheses or guesses.

Goldhagen (2017, p. 83) argues that to properly explore the relations of cognition and its role in the experience of the built environment, a shift of focus is necessary in three ways:

1) What our minds think is largely shaped and profoundly influenced by the human body that we have;

2) That our human bodies are shaped by the environments in which we live and have evolved, suggests that much of our internal cognitive lives takes place outside of language and below the level of our conscious awareness; and 
3) These factors transform our understanding of how humans live in the world by making us less the imperially sovereign agents over our experiences that we often believe ourselves to be, and more environmentally embedded beings that a bird's-eye view of any human settlement would suggest.

To paraphrase what Goldhagen (2017) says about human experience in the built environment, she states that the story of our relationship to the built environment is revelatory; rich, multilayered, and mediated by the changing rhythms of the day and the operations of human memory, temporary and complex. Experiencing the built environment is little more than processing of sensory cues and impressions momentarily, however the effort and process taken to store cognitions is crucial.

In a very recent research article linking neuroscience research and architecture (Karakas \& Yildiz, 2020) found five main categories based on the outcomes of experimental studies including 1) restorative and stress-reducing effects of the environment; 2) aesthetic judgement and/or appreciation of the physical environment; 3) navigation and wayfinding; 4) visual engagement, attention and imageability; and 5) characteristics of human experience focused on phenomenological positive/negative user experiences, experiential intensity and sensory experiences. However, these studies used a wide range of measurement techniques to understanding brain functioning such as functional magnetic resonance imaging (FMR), magnetoencephalograms (MEGs), and positron emission tomography (PET). The psychophysiological measurement techniques used to evaluate the central nervous system (CNS) can be grouped into electroencephaography (EEG) and event-related brain potentials (ERPs). EEG works on transdermal readings of electrical signals through the scalp while ERP is basically derived from EEG recordings. Another measurement technique is electromyography (EMG), which measures peripheral nervous system through the electrical activity generated by skeletal muscles. EMG can detect facial movements that are not observable from looking at the face itself (Go "kcay \& Yildirim, 2011).

The increasing application of a range of digital measurement technologies, although interesting, presents a challenge for a research project that is time-bound. Also the cooperations of the research participants are more likely to be hindered regarding the nature of this type of research in the postnatal hospital environment where the rest and recovery of new mothers are crucial after the birth. Ethical considerations may have an effect on the use of these measurement techniques with hospital inpatients. Coupled with these, the neuroscience approach cannot be ventured into, when the contours of the field being applied to architecture, that is to understand human experience and 
perception, are still evolving, with emergent research fields coming on board. Neuroscience research also requires expertise in analysing rigorous experimental studies involving the brains and minds of participants (Eberhard, 2009a; Karakas \& Yildiz, 2020) to avoid errors and risks due to misinterpretation and unsuitable applications (Edelstein, 2015).

Understanding the perceptions and cognitions and human experiences of the built world especially in healthcare facilities is of particular interest to architects and researchers. Two notable exemplars exist that appear to challenge what a hospital is in terms of the human experience. The design of these two hospital buildings qualify as research connecting neuroscience and architecture, which are reviewed. The first is Rehab Basel. As the name suggests, it is a highly specialised centre for neuro-rehabilitation (brain damage) and paraplegia (spinal cord injuries) founded in 1967 as the Swiss paraplegics centre. Gutmann (2011) states that rehabilitation relates to treatments for the abatement or remedy of chronic physical or psychological illness with the intention of reducing the impact of impairment and developing the capabilities and coping skills necessary for the person to function well in his/her environment. Rehab Basel is set in wide expansive, organic grounds with well-trained medical and nursing staff to support a range of patient's illnesses - behavioural and physiological - through a tailored programme to achieve the greatest independence and mobility in a therapeutic setting. At the centre, a defining goal was the need to balance physiotherapy treatments with a psychoneuro-immunological (PNI) environment (Gappell, 1995; Shepley, 2006).

Gappell (1995) defines PNI as the art and science of creating environments that prevent illness, hasten healing, and foster a sense of wellbeing. Carefully designed spaces and places at Rehab Basel allow the in-patient to find peace and harmony, which in some sense represents a significant landmark of architecture in healing and recovery experience. At Rehab Basel for example, the built environment plays a significant role during physiotherapy interventions with abundant airy spaces. The space is well-lit and inviting and helps the patients to find a sense of peace and enthusiasm at a difficult time in their lives(REHAB Basel, n.d.). Gappell (1995) claims that full-spectrum light is responsible for prophylactic control of viral and staph infections and contributes improvements in physical working capacity through a decrease in heart and pulse rate, reduction in blood pressure, and increase in oxygen intake.

The neuroscientist Richard J. Wurtman writes that the effects of light are moderated by a multisynaptic neuronal system involving the brain, spinal cord and sympathetic nervous system. The author stresses that it seems clear that the light environment may provide a health measure for the prophylaxis for appropriate body functioning (Wurtman, 1975). Presence of rich nature at 
Basel, with its wooded surrounding landscapes, reflecting the rhythms of day and night, supports inpatients for emotional and psychological recovery including nourishing the mind and body. Walkable outdoors enhancing views to greenery contributes to a rich nature contact experience. In addition, animal therapy (companion animals serve as a positive distraction) for the patients plays a valuable role in Basel's holistic rehabilitation programme. Also, the Living Practice Ward (LVP) allows ways of connecting inpatient stay and life at home. Home elements and features allow inpatients to trial a wide range of activities of daily life as part of therapy, which give the patients a new daily structure. Recreation, art and music therapies are a range of services that inpatients have exposure to during the course of rehabilitation. The bold and interesting architecture including the relaxing image of Rehab Basel $^{3}$ makes it readily suitable for a consideration for future research studies employing neuroscience principles.

The second exemplar is the Sarah Network of rehabilitation hospitals in Brazil designed by the Brazilian architect, João Filgueiras Lima. One of the world largest neuro-rehabilitation and neuropsychology networks, the Sarah Network comprises 1,000 beds across nine hospital units in Brasília, Brazilia-Lago Norte, São Luis, Rio de Janeiro, Belo Horizonte, Fortaleza, Salvador, Belém, and Macapá. With with 1.6 million patients annually, the Sarah Network has a reputation of performing over 400,000 medical consultations, 1.2 million diagnostic examinations and 11,000 surgical procedures annually (Braga, 2018). In 1980, the Sarah Network (Campagnol \& Shepley, 2014) opened to the public its first rehabilitation centre in Brasilia. The driving ideology for the hospital's programme was the importance and creation of therapeutic, humanised environments that promote the healing process of patients who have been impaired and have difficulty moving from their beds.

The design of the Sarah Network played a significant role in the healing and recovery journeys of patients. In their paper on the Sarah Network, Campagnol and Shepley (2014) explain how the combined fruits of architectural and medical domains resulted in the Sarah Network hospital

https://www.rehab.ch/en/home.html

The design of Rehab Basel emphasises much use of organic forms for creating therapeutic environments as well as natural materials. Its set of buildings carefully integrates and assimilates seamlessly into the landscapes, and preserves the ecological capital of the community. The success of the design is an attestation that built environment can be created to preserve the cultural, social and political landscape integrity of the region. 
design through the Brazilian architect, João Filgueiras Lima simply known as Lelé and his friend, orthopedic surgeon Aloysio Campos da Paz:

Areas previously occupied by ... sophisticated equipment, areas that are necessarily confined places, could then become open spaces. Lelé designed light beds similar to gurneys that could be reclined as well as circulate throughout the hospital, increasing patients' mobility throughout the building and their access to gardens, open spaces, and interaction with other patients and family(Campagnol \& Shepley, 2014, p. 207).

The Sarah Network of hospitals showcase many vibrant and rich settings for the promotion of healing and recovery experiences for patients, for example, the design of the light bed-gurney that allows transportation and mobility of the patients and connections from the inside to outside including water views from the rehabilitation rooms. In addition, the wards are physically and visually integrated to the garden terraces promoting indirect access to nature and experience. These aspects of therapeutic environments and many more were parts of the Sarah Network of hospitals distributed through the nine hospitals in Brazil, providing a great sense of optimism to the patients and their families in their recovery journeys. The enabling and very positive environments of all the nine hospital centres ${ }^{4}$ in Brazil represent significant contributions of architecture and offer the potential to stimulate positive medical outcomes for patients and wellbeing of significant others.

The study (Campagnol \& Shepley, 2014) based on the Sarah Networks of hospitals is exclusively focused on positive distractions - utilising a wide range of therapeutic factors such as daylighting, and natural ventilation, solaria, indoor and outdoor pools, reflective pools and views of nature in the wards and rooms and pleasant artwork - as part of rehabilitative treatments for promoting positive behavioural outcomes for the patients. Positive distractions refer to environmental attributes or conditions which alleviate stress and takes the focus away from medical conditions. Companion animals, music, laughter, some art, and nature viewing and/or experience all fit the label of positive distractions. However, the effectiveness of the Sarah Network hospital design has not been analysed (Campagnol \& Shepley, 2014).

\footnotetext{
${ }^{4}$ https://ipbis.org/the sarah network.html; http://www.sarah.br/en-us/a-rede-SARAH/nossasunidades/
} 
This means that the patients' perceptions of the built environments and their hospital experience are lacking, as are informed decisions about environmental features that minimise negative physiological, cognitive and emotional effects (Sternberg \& Wilson, 2006). Access and support for collaborative research to better advance neuroscience and architecture are important in settings such as the Sarah Network of hospitals and Rehab Basel and similar healthcare facilities elsewhere may be available for research adopting a neuroscience research lens.

\subsection{Research philosophy, phenomenology and lack of fit in this research}

In this section, a statement of the research philosophy guiding this present study is presented to assist its interpretation with regards to its stance on matters of ontology (which can be defined loosely as the study of being and physical existence) and epistemology (which is about the sources, scope and limits of knowledge). Numerous research design techniques exist for addressing different research tasks, however, situational context and practicability often dictate what the researcher can do or not. This research adopts a mixed methods design due to many reasons that will be briefly introduced in the following sections and detailed later in chap

\subsubsection{Research philosophy and pragmatism}

The built environment poses an array of complexities and contradictions; its design, process, interaction with users, perception and meanings that its architecture conveys to users and its impact on the surrounding landscapes. To approach this topic , that is the healthcare physical environment, there is a need to answer questions about philosophy, including a recognition of different research paradigms, as a premise for understanding the research process which may include biases such as personal history, ethics and beliefs that the researchersmay introduce into the enquiry (Creswell, 2013). In simplest terms, philosophy can be regarded as the consideration of higher order problems and how we attempt to solve them. The approach that is undertaken in solving everyday problems has an underlying reference to an individual's philosophy (Birks, 2014). The branch of philosophy concerned with the study of knowledge is known as epistemology and it embodies several schools of thought, which date to ancient times (Birks, 2014). In brief, there are rationalists who contend that some forms of knowledge can be acquired simply through reasoning; and there are the empiricists who contend that all knowledge is dependent on observations, experiments and other forms of sensory experience (Birks, 2014). 
Groat and Wang (2013) describe schools of thought as broad theoretical perspectives that have increasingly influenced many research fields including architectural research. Creswell (2013) explains that it is the initial, abstract ideas and beliefs that inform the research process, and choosing one paradigm over another could influence how the research questions are framed (Groat \& Wang, 2013). Ontology relates to "the nature of reality", for the social sciences this description is developed to encompass discussions of what exists and what it looks like as well as the units of which it is composed and how they interact. Epistemology considers views about the most rational ways of investigating the nature of the world and to ascertain what will be considered to count as scientific knowledge (Mingers, 2004) including how such knowledge claims are justified (Creswell, 2013). Within the realms of philosophy, epistemology is concerned with the study of knowledge and the process of "how one knows, what one knows, and how one knows what one knows what he or she does not know"(Davey, 2011, p. 14). Its overarching focus is on education; that is to say, what researchers do in uncovering phenomena. It should be emphasised that the present research aligns with the feminist epistemological stance, albeit not as the philosophical paradigm taken to this research, regarding lives and experience of women following birth. Therefore, the feministic standpoint epistemology recognises the "fusion of knowledge and practice" (Brooks, 2007, p. 55).

Constructing knowledge about postnatal hospital environment requires positioning postnatal women and nurse midwives as caregivers in which their conscious and unconscious perception of the physical environment has an impact on the mind and may contribute to healing and restorative environments (Lepori et al., 2008). Brooks (2007) argues that until the experiences of women is made to be the primary source of research investigation only then can knowledge that accurately reflects and represents women be realised.

Because this research is a piece of applied research which investigates a real-world problem - the postnatal recovery experience of postnatal women in large, tertiary hospitals in particular, how the physical environment influences individuals or groups. Polit and Beck (2017) state applied research is solution-oriented to existing problems that could be in the service of greater immediate application for evidence-based practice in healthcare environment. Hence the research philosophy which underpins this piece of work carries the conviction that information about hospital experience and wellbeing can be readily gained from users of healthcare facilities whose interactions in space can generate important outcomes for the research. Winkel, Saegert and Evans (2009) argued that some phenomena may be intrinsically linked to personal experience such as the relationship of perceived social support to subjective wellbeing. This aptly fits into this study as 
the physical design factors represent, primarily, a means to measure the subjective wellbeing and recovery experiences of new mothers in the immediate postnatal phase.

Furthermore, the current research sits within the pragmatist approach, which uses an ontology focused on concrete experiences of what women do as part of their daily life activities (Brooks, 2007). Within the healthcare research domain, a key premise for research couched in a pragmatic stance is that it allows researchers to engage with the complexity of health, healthcare and the physical environment in which studies are conducted (O'Cathain, 2009). The pragmatic philosophical approach assigns importance to what works at the time of the investigation and does not depend on the dualism between the reality independent of the mind or within the mind. Additionally, pragmatist researchers confine themselves to the "what" and "how" of the research based on the intent and purposes of the research problem (Creswell, 2013). In essence the pragmatic philosophical approach lends itself to multiple methods and sources of data collection, and may include tools from both positivist and interpretive/naturalistic paradigms in order to fulfil the practical realities of the research goal (Creswell, 2013; Guba \& Lincoln, 1994).

The epistemological approach in this study is pragmatic and utilises two strands of quantitative/objective and qualitative/subjective methods for data collection. Correspondingly, this present research sits within the continuum of what Groat and Wang (2013, p. 76) argued to be "intersubjective" as a justification for its "interstitial position between the positivist emphasis on objectivity and the constructivist emphasis on subjectivity. This tradition recognises multiple, distinct perspectives and the importance of socially shared action and knowledge."

(Groat \& Wang, 2013, p. 78) elaborate this point further:

Ontologically, it assumes that although there are multiple diverse viewpoints regarding sociocultural realities, it is nevertheless possible to achieve shared understandings of those realities. In contrast to the objective segment of the continuum, the intersubjective perspective assumes that it is neither possible nor necessarily desirable for research to establish objectivity within a value-free stance.

The mixed methods design approach was a good fit for this research because the study aim was to identify the design factors in the hospital environment influencing maternal recovery experiences and women's wellbeing during the immediate postnatal phase. Therefore, the adoption of mixed methods design was not only relevant for this present research because it is a combination of qualitative and quantitative research techniques but it enables the dual functions of gaining insights quickly (through surveys) into the environmental factors which support recovery experience in the 
hospital and that explications of these design factors are possible using the constructivitist paradigm, through the service of the qualitative approach (focus group).

\subsubsection{Phenomenology}

Phenomenology constitutes the exploration and description of phenomena, where "phenomena" refer to things or experiences as human beings experience them. A phenomenological inquiry generally comprises any object, event, situation or experience that a person can see, perceive, understand, or live through (Seamon, 2000).

Historically, phenomenology is a philosophical tradition that can be traced to the works of the 20th German philosophers, in particular, Edmund Husserl, which was subsequently expanded upon by Heidegger. The goal of phenomenology is an investigation and explanation of a phenomenon as is experienced, without any reliance on theories or preconceived jugdments, imaginations or intuitions about their explanation. Schwandt (2007) states that phenomenology is a complex, multifaceted philosophy that defies simple characterisation because of its lack of a unified philosophical standpoint due largely to the eclectic view points of its founding fathers. For example, the transcendental phenomenology of Edmund Husserl (1859-1938), the existential forms of Maurice Merleau-Ponty (1908-1961) and Jean-Paul Sartre (1905-1980), and the hermeneutic phenomenology of Martin Heidegger (1889-1976). Usher and Jackson (2014) affirm that phenomenology is considered to be both a philosophy and methodology with much of the earlier phenomenological work being philosophical in intent rather than research motivated.

As Creswell (2013) describes it, phenomenological enquiry aims to find the common meaning for several individuals of their lived experiences of a concept or phenomenon. In other words, the researcher aims to grasp the essential underlying meaning of experience, where experience embodies an outward appearance and inward consciousness based on memory, image and meaning (Creswell, 2013). Schwandt (2007) clarifies the goal of phenomenological inquiry which focuses on the description of the lived experience from the point of view of those who lived it. As such, the purpose of phenomenology is concerned with how to reduce individual experiences with a phenomenon to a description of the universal essence (Creswell, 2013). While stressing the importance of descriptions to some degree, Seamon (2000) argues that the aim is to deploy the descriptions as a basis to uncover underlying commonalities among the subjects that mark the essence of the meaning of the lived experiences through interpretation (Creswell, 2013).

As Juhani explains lived experience: 
The lived world is closer to the reality of dream than a scientific description... to distinguish the lived space from physical and geometrical space, we can call it existential space. Lived existential space is structured on the basis of meanings and values reflected upon it by an individual or group, either consciously or unconsciously; existential space is a unique experience interpreted through the memory and experience of the individual. [....] The experiential lived space is the object and context of both the making and experiencing of art as well as of architecture (Pallasmaa, 2005, existential space).

Although the term lived experience is often synonymously ascribed to research, it has much significance for phenomenology. According to van Manen and Adams (2010), the notion "lived experience" originates from the German "Erlebnis", or experience as we live it, which is a particular type of experience. Phenomenologist researchers are committed to understanding what our experiences in the world are like; experience should be investigated as it actually occurs and on its own terms (Smith, Flowers, \& Larkin, 2009). In other words, the central focus of phenomenology is the way people exist in relation to their world (Seamon, 2000).

Following Husserl is the basic principle guiding an inquiry referred to as "epoche" or "bracketing", requiring the researcher to put aside or suspend any preconceived judgements and assumptions about the "natural attitude" in order to uncover the essential information whilst avoiding altering of information about the phenomenon of interest (Creswell, 2013; Groat \& Wang, 2013). This process of bracketing out the outer world about the phenomenon is important "in order to see it [essences] clearly" (Laverty, 2003, p. 23) Phenomenology seeks to understand how people develop meaning about a phenomenon as it is being experienced firsthand, without the interference of the researcher. According to Shirazi (2014) phenomenology intends to return to "what is directly given," where the "given" is not scientific presupposition, but rather what is intrisincally given in our intuition. Shirazi (2014) continued by emphasising that by so doing, we will be able to return to the "phenomena" and seize the essence of things." Although the phenomenological tradition has since been influential in architectural research, it is a system of inquiry that is challenging to situate among other schools of thought, due to its relatively unique conceptualisation of "subjectivity" (Groat \& Wang, 2013, p. 95).

A significant advantage of the architectural field towards a phenomenological approach lies in the basic principle that consciousness is often directed towards an object and reality is intrinsically linked to one's consciousness (Creswell, 2013) and may apply to the physical environment (Groat \& Wang, 2013). Within the realms of consciousness, perception of a phenomena is possible as 
humans can grasp what is real (Laverty, 2003; Moran \& Mooney, 2002). Giorgi (1997) clarifies consciousness as an agent for awareness as it presents itself to those involved in it. Two key elements are vital to consciousness; intuition and experience. Intuition refers to ordinary types of awareness while experience is the intuition of real objects in space and time. Intuition is the wider form of perception and experience is the narrower.

Given this assertion, phenomenology shares close important connections with architectural research as opposed to other qualitative approaches with exclusive focus on people's interactions which appear removed from the physical environment context (Groat \& Wang, 2013). With regards to the architectural field and attraction towards phenomenology, (Douglas, 2014, p. 85) states that architectural phenomenology is a study of how complex relationships constitute our being in places, engaging with matter, substance and experience and about how our physical and psychological experiences are of being "above and below" and "near and far" rather than heights and coordinates based on the Cartesian grid.

Based on different texts of scholars two strands of phenomenological approaches are recognised: hermeneutical (van Manen, 1990) and empirical, transcendental or psychological phenomenology (Moustakas, 1994) and within the environment design fields and consistent with the above Seamon (2000) introduced what he calls the first-person phenomenological inquiry alongside the more common phenomenological research types.

Hermeneutical phenomenology is concerned with the theory and interpretation of texts which may be any material artefacts having symbolic meaning with human experience such as a painting, a song, public documents and so forth. A useful example of hermeneutic phenomenological research can be seen in the work of Norwegian architect Thiis-Evensen (1987) who proposes a universal language of architecture by focusing on experiences of floor, wall and roof as phenomena, which he claims are the relationship between "inside" and "outside" and with all the three elements collectively and differently creating multifacted essence of "intricate sets of tensions between architecture elements and experience"(Seamon, 2000, p. 168).

Moustakas (1994) transcendental phenomenology focuses on scientific investigation when the knowledge sought is arrived at through descriptions "that make possible an understanding of the meanings and the essences of the experience." One defining characteristics of this method is the focus on concept of epoche (bracketing). According to Moustakas (1994, p. 85) epoche entails setting aside prejudgements, biases, and preconceived ideas about things which are "to be known naively and freshly through a purified consciousness." A distinction between hermeneutical and 
transcendental phenomenology is that it becomes hermeneutical when its approach gets interpretive rather than more descriptive as is the case with transcendental phenomenology. Although van Manen and Adams (2010) contend there is a thin fine line between the two, for as Heidegger indicates, that all description is by and large an interpretation.

In terms of first-person phenomenological inquiry, Seamon (2000, p. 165) explains that "the researcher uses her own firsthand experience of the phenomenon as a basis for examining its specific characteristics and qualities." Seamon shows how this approach can be used in phenomenology especially as a starting place from which the researcher can bring to awareness her preconceived notions and biases about the experience being investigated so that that the researcher's biases do not have a place in the interpretion of the phenomenon. The first-person phenomenological approach appears to have some commonalities with the participant-observer method of research in that the researcher's own experience is involved in the phenomenon. The major advantage of this approach is that it provides rich and important insights about the phenomenon of interest and could be inspirational for design professionals. On the other hand, this technique's drawback lies with the methodological concerns as the research needs to "bracket" preconceived judgements and notions to arrive at an understanding of the "essence" of the experience beyond individual subjectiveness(Groat \& Wang, 2013). Another point is its explicit focus on a lived experience of one person leaving out the views of many others.

Existential-phenomenological inquiry focuses on the specific experience of particular groups or individuals involved in actual places or actual situations. The premise behind this type of phenomenological inquiry is that when individual descriptive accounts are carefully analysed and considered collectively, meaningful themes will be revealed.

There are four steps in the process that should be considered for conducting existentialphenomenological research as described by Seamon (2000) as follows:

1) identifying the phenomenon in which the phenomenologist is interested;

2) gathering descriptive accounts from respondents regarding their experience of the phenomenon;

3) carefully studying the respondents' accounts with the aim of identifying any underlying commonalities and patterns; and

4) presenting results, both to the study respondents and to fellow researchers in the form of scholarly presentation. 
In the words of Edmund Husserl (1970), we can only know what we experience. When focusing on perception about the user's experience of the built environment, attention and awareness must sometimes co-exist, athough our responses in the physical environment vary with ever-changing amounts of attention because of preoccupation with other activities (Peri Bader, 2015). Therefore, the task for the researcher is to, first of all, take into account the research participants (the postnatal women and midwives) their consciousness and perception of the physical postnatal environment, emotions and situations, in addition to readiness to get to understand the lived experience of recovery in postnatal space

Having considered phenomenology in general, it will be useful at this point to highlight why a phenomenological approach was not utilised for this present study.

As mentioned above, the purpose of existential phenomenological research is to describe the experience of specific individuals or groups who have in common a specific experience and situation in actual place. For this research, the phenomenon is recovery experience in hospitals. Because the reference to the things and facts themselves, as Husserl might say, is the phenomenon of hospital environment, hence the research participants must have actual experience and intuition (Moustakas, 1994, pp. 46-47). However, hospitals are places where the patients including the childbearing women and their families have minimal control over the environmental factors. In addition, most users of healthcare facilities and spaces generally pay less attention to their architectural surroundings with attendant consquences that the architectural objects may result in fleeting awareness or indifference or oblivion than to conscious appreciation (Peri Bader, 2015).

Although phenomenological inquiry could be a method by which spaces, places, and individual experiences of the built environment may be studied, however its fit in this research is limited for many reasons. Some of these reasons have already been highlighted in Section 3.6.1 above, but more specifically for the use of phenomenology as a methodology, the pertinent ones will briefly, be mentioned here. Firstly, the subjects (postnatal women) whose lived experience of a situation is fundamental in phenomenological inquiry, continue to experience shortening length of postnatal hospital stay (see Chapter 1 for the detail of studies). Trends and its increasing impact have been documented (Bravo et al., 2011; Moore, 2003; Patterson, 1998; Watt et al., 2005; Wen et al., 1998), and therefore postnatal women could have had increasingly short-lived experiences in a physical environment that ought to be researched as a lived experience of hospital stay as a phenomenon.

Secondly, the busyness of the postnatal environment would likely have a pivotal role in making it difficult to establish meaningful interactions with the research participants whose opinions largely 
count with regards to the essence of physical environment in postnatal childbirth recovery. Thirdly, ethically, recruitment of research participants clearly renders the choice of a phenomenological approach inappropriate for this research. Most of the research participants would need adequate rest and to recover following the birth. This situation and the likely dispositions of research participants toward in-depth interviews (for data collection in phenomenological research) may not lead to a satisfactory response.

Although phenomenology could be a useful approach for enquiring into the lived experiences of hospital stays of postnatal women and of midwives, and may result in interesting and detailed accounts of the essence of the physical environment for the recovery of women, but it requires extra work in terms of conducting in-depth interviews with women when indeed they may be already burdened with the tasks of nurturing of newborns or be struggling with their physical recovery. Despite these limitations, the phenomenological approach is a promising technique that could be utilised in other future research as a means of expanding this research further.

\subsection{Conclusion}

This chapter began by sketching the historical development of birth environment in both middle and high-income countries, the nature of events that led to hospitalisation of childbirth and earlier subsequent efforts to "regain control" of the childbirth environment through the evolution of alternative birth settings. Following these historical perspectives comes the review of the literature on the childbirth environment and impacts on childbearing women's experience of birth, satisfaction with care and wellbeing. Birth unit design may hinder or interfere with caregivers in providing adequate care of women during labour and birth. A significant point to note in many of the studies reviewed is their focus on the labour and delivery rooms and how design of birth spaces influences the behaviour of women during the birth process. Findings suggest midwives could create inviting, initimate supporting environments through elements of homely features to promote normal positive births which in turn may enhance midwifery practice. However, research focusing on the post-birth period and corresponding spaces for the physical restoration of women and how these may function for midwives in quality care has been less studied. A small number of studies has pointed to a lack of research into hospital postnatal environments to support women prior to going home. This research is an applied research study to fill a gap in the real-world situation.

The last section of the chapter described the philosophical underpinnings for the present study. First, the role of neuroscience as it relates to architecture regarding how human experience is 
shaped in the built environment was discussed. This is followed by the exposition of research paradigms and phenomenology. In each of these later discussions reasons for lack of fit in this research were discussed. The chapter that follows moves on to describe the research design and approaches taken to understand the design factors in hospital built environments that could influence women's recovery and wellbeing. The goal is to have an understanding of supportive environment/spaces for adequate and quality postnatal recovery care from healthcare providers. 


\section{CHAPTER 4}

\section{RESEARCH DESIGN AND METHODS}

This study utilised an explanatory sequential mixed methods approach to facilitate data collection in two phases. Phase one collected quantitative data and phase two qualitative data to explain further the results from phase one. In this chapter the methodological approach and its relevance for this study are discussed. Next, the methods for data collection for each phase are described sequentially. The phase one methods discussion concludes with ethical considerations. In phase two, the settings for the focus groups are presented, then the procedures of data collection and analysis are discussed. Lastly validity and reliability for the study are discussed.

\subsection{Why mixed methods design?}

The aim of the study was to investigate how postnatal hospital environments can be restorative and conducive for postnatal recovery care and wellbeing of women in large, tertiary hospitals. For a question this broad, it was necessary to simplify it by shifting focus to the architectural characteristics and design features in the postnatal hospital environment influencing women's recovery experience and how these features might impact on wellbeing. A mixed methods approach was taken to the research for a number of reasons including the following:

\section{Limited research exclusive to physical environment for postnatal recovery}

Much of the previous research into physical environments for childbirth has been on the factors that women considered important for labour and birth in the delivery rooms, but limited information exists regarding the physical environment for women's recovery during the postnatal phase - which is often in quite different kind of spaces in hospital buildings.

\section{Challenge of access to participants in the research setting}

The research was studying the immediate postnatal period, a critical time in the lives of women with need for rest, sleep and to begin the process of recovery along with their preoccupation with child rearing. Engaging research participants - including midwives who have considerable workload - in hospitals seemed impracticable if not impossible, plus the short length of a typical postnatal stay rendered it difficult to undertake any meaningful engagements with participants. In 
view of these reasons, a mixed methods approach was considered appropriate to combine both the quantitative and qualitative data for greater understanding of the research problem.

\section{Interdisciplinary drive of the investigation}

To deepen the discourse on healing environments particularly involving users of healthcare facilities, this study needed to cross disciplinary boundaries. The concepts of evidence-based design, patient-centred environments, the salutogenic design model and the patient-centred care approach arguably have been approached from a mostly single disciplinary focus while the complex healthcare and design fields arguably require more holistic multiple methodological means. In order to better bring out the values in these ideas, a mixed methods design approach allows the use of constructs from interdisciplinary perspectives. When studying the physical healthcare environment related to person-environment relation transactions where users' opinions, perceptions and attitudes are sought, interdisciplinary methods from other fields are considered important (Joroff \& Morse, 1984).

In addition, this study is exploratory and preliminary. It did not utilise architectural precedents as a method due in part that the needs of women post-birth differ from specific needs of patients in other healthcare facilities, although there could be an overlap of environmental factors. It takes the position that design factors in the postnatal hospital environment influencing women's experience of recovery and wellbeing need to be first understood, rather than limiting research to a few existing architectural precedents/healthcare facilities, which are focused on different domains of recovery and healing (see Section 3.5). While these and other healthcare facilities could provide heuristic perspectives, understanding the specific purposes of different facilities is fundamental to effective design because each design and feature not only needs to support the overall goals of the facility but also the requirements of the individuals who occupy it (Kopec, 2006). What leads to good outcomes for patients in other healthcare environments may not apply in the postnatal environment.

\subsubsection{Mixed methods design and challenges}

The mixed methods (MM) approach is a method that has been in use in the 1940s in the social and behavioural sciences and environmental psychology disciplines although the approach became widespread around the end of the 20th century. In the 1970s, researchers have used what is called a multi-method technique to evaluate the physical environment (Patterson \& Passini, 1974). The MM approach has been termed a "third methodological movement" taking its position alongside 
the dominant quantitative and qualitative methodological designs (Tashakkori \& Teddlie, 2010b, p. ix). The MM approach was birthed to complement the two existing traditional research approaches drawing from the strengths and reducing the shortcomings of both in single research projects or across studies (Johnson \& Onwuegbuzie, 2004). As (Creswell, 2010, 2015) points out, mixed methods is an approach in which the researcher not only collects quantitative and qualitative data strands, but also involves the connection, integration or linking of the two strands to facilitate conclusions, thereby taking advantages of the strengths of both approaches to answer research problems.

Qualitative research approaches emphasise the existence of multiple realities and explore a concept of interest using a small sample of key informants to obtain detailed insights about the nature of the investigation. By comparison, quantitative research approaches are concerned with making causal relationships utilising larger samples to test hypotheses and make predictions enabling generalisability of the findings. As the emergent third movement, the mixed methods approach acknowledges both subjective and objective ways of knowledge production.

Polit and Beck (2017) note that combining quantitative and qualitative data techniques in a study is straightforward, however definitions of MM research are not. As Morse and Niehaus (2009) describe it, mixed methods is the fusion of one or more methodological strategies drawn from a second method, in a single research study, to compensate access of some aspects about the phenomenon of interest that cannot be accessed if the first method alone was used (Morse \& Niehaus, 2009).

Following a review of contributions from the leading scholars and researchers in MMR, Johnson, Onwuegbuzie and Turner (2007) put forward a definition of mixed methods.

\footnotetext{
Mixed methods research is the type of research in which a researcher or team of researchers combines elements of qualitative and quantitative research approaches (e.g., use of qualitative and quantitative viewpoints, data collection, analysis, inference techniques) for the purposes of breadth and depth of understanding and corroboration (Johnson et al., 2007, p. 123).
}

Pragmatically, a mixed methods approach allows for both explanatory and interpretative research to be conducted in one single study. This comes with many benefits particularly given the multidimensional factors that influence health Morgan (1998). Given all the indices involved in health outcomes including the role of the physical environment, the use of a mixed methods approach 
enables a researcher to get answers to both exploratory and confirmatory questions (Teddlie \& Tashakkori, 2006, p. 22). This process of confirming or verifying in mixed methods aptly fits the objectives of this study; that is to say, to provide in-depth insights about the design factors in the postnatal environment influencing recovery experiences for women. Measuring variables in the physical environment is carried out initially to provide information about design factors on the one hand and this is followed up with confirmatory research in a bid to derive explanation of how important are the design features to recovery experiences. Additionally, a ground for architecture research utilising other approaches, such as MM, is premised on the basis that architecture research needs to embrace methods that are inclusive and integrative of deductive and inductive methods that might present fruitful outcomes for the research investigation (Robinson, 1990). However, Robinson cautions that the sub-disciplines of architecture (art history, engineering, environmentbehaviour sciences) do not integrate due in part to divergent notions of what architecture may represent to these fields (Robinson, 1990).

Although MM has been expanding in the past decades it is mired in a paradigm debate (Morgan, 2007), and value derivation of its choice as a research approach (see Creswell, 2011a; Creswell \& Plano Clark, 2018). This contention comes from the underlying methodological and epistemological implications of merging qualitative and quantitative approaches, otherwise called the incompatibility of methods thesis. According to Morgan (2007) "different paradigms create different kinds of knowledge, the attempt to use this strong version of incommensurability repeatedly fails at every level except for debates about the nature of reality and truth" (Morgan, 2007, p. 64). Further, Morgan notes that despite the “... claim that methodological problems in the social sciences could be addressed through an ontology-driven version of the philosophy of knowledge, this belief system remains disconnected from practical decisions about the actual conduct of research" (Morgan, 2007, p. 64).

This position has been rejected by the mixed methods research community they are rather advancing methodological eclecticism as an alternative to the view point of the incompatibility thesis (Teddlie \& Tashakkori, 2010). A key defining attribute of methodological eclecticism is that the researcher is at liberty to select from a range of methods, to combine them, using the best forms of judgement in a manner that the selected tools or techniques are suitable enough to answer the researcher's questions (Teddlie \& Tashakkori, 2010). In so doing, researchers selecting the mixed methods approach including methodological eclecticism become "connoisseurs of methods" and become competent and skillful in similar research activities (see Teddlie \& Tashakkori, 2010; Teddlie \& Tashakkori, 2012, p. 777). Moreover, it is argued that the mixed methods approach 
strongly emphasises humanistic conceptualisation of the research process in greater measure than the two monolithic methodological movements, which renders the incompatibility issues inconsequential (Tashakkori \& Teddlie, 2010a). With such strong views expressed in the quest for mixed methods research, proponents have thought it fit to carry on reassessing and reviewing it to uncover new advances in procedures and embracing dialogues in order for it becoming increasingly viable as the third methodological movement (Creswell \& Plano Clark, 2018; Teddlie \& Tashakkori, 2012). Consequently, proponents of the MM approach have no doubts that when both quantitative and qualitative data are collected and are integrated they give clearer insights about the research problem than if either method is used independently (Creswell, 2012).

In this study, the overarching research question was: how can postnatal hospital environments be restorative and conducive for postnatal recovery care and wellbeing in large, tertiary hospitals? This nature of research question hinges on interdisciplinary orientations and lies at the intersections of other discipline boundaries. Consequently, a mixed methods design is needed to guide the research design and connect understanding on multiple levels regarding how the healthcare built environment impacts on its immediate users (Joroff \& Morse, 1984).

Information regarding the physical environment is crucial to support ongoing health of women involved in the physiologically normal activity of giving birth (Foureur \& Harte, 2017) and restoration, and may be served best using multiple methodological approaches allowing credible outcomes (Rashid, 2013). Rashid (2013) argues that improving patients' outcomes focused on human-design interactions requires a holistic approach that considers multifaceted dimensions of emotion, physiology and psychology to understand patients' - and in this case, women's experiences in the healthcare environment.

Leech and Onwuegbuzie (2009) explain that the myriads of mixed methods designs could be challenging to researchers, however several typologies of mixed methods design have been developed, which can offer guidance and direction for the researcher to accomplish their research objectives (Leech \& Onwuegbuzie, 2009; Morse \& Niehaus, 2009; Nastasi, Hitchcock, \& Brown, 2010; Teddlie \& Tashakkori, 2006).

Researchers have argued that a mixed methods approach combining quantitative and qualitative approaches is considered more "realistic" and "productive" as a vital tool for answering research questions and offering potential application for healthcare research designs (Marcus \& Sachs, 2014, p. 16). The MM approach can be suitable to addressing the complexity of conducting research in healthcare settings (Devlin \& Arneill, 2003). This complexity has led to researchers adopting 
pragmatism as a guiding factor for choosing MM when there are perceived inadequacies of quantitative methods to address the nature of research in the healthcare environment ( $\mathrm{O}^{\prime} \mathrm{Cathain}$, Murphy, \& Nicholl, 2007). In this current study quantitative methods utilising questionnaire surveys are complemented by the qualitative focus groups.

In stressed hospital environments, MM has been found useful in examining the restorative components of staff break areas on nurses' satisfaction and stress reduction, which has assisted them to serve the patients and nurses better (Nejati, Rodiek, \& Shepley, 2016; Nejati, Shepley, et al., 2016). Similarly an understanding of the physical design features in the interior environment that may be beneficial to recovery experiences requires an holistic approach to gain adequate insights into the design features in the interior postnatal environment that support postnatal recovery needs.

\subsubsection{Explanatory sequential mixed methods design}

A sequential explanatory mixed methods design is a type of research design in which the core component project is completed before the supplemental projects are conducted (Morse \& Niehaus, 2009). Both data collection and analysis must be completed before the start of another strand referred to as a supplemental component. According to Morse and Niehaus (2009) the pacing of the study can be set at the proposal stage or as it emerges in a manner that the phases of the project can provide logical conclusions. One disadvantage of this approach is the issue of limited time to complete two phases in which one phase informs the next. In this study the quantitative strand (questionnaire survey, phase one) was started and then followed by the supplementary qualitative component (focus groups, phase two). The quantitative method results serve as the base information which phase two follows up for explanation, thereby providing indepth information (Creswell, 2012, 2015). Specifically, a two-phase explanatory sequential mixed methods design was selected in this study (see Figure 4-1). 
Phase 1

women and midwives

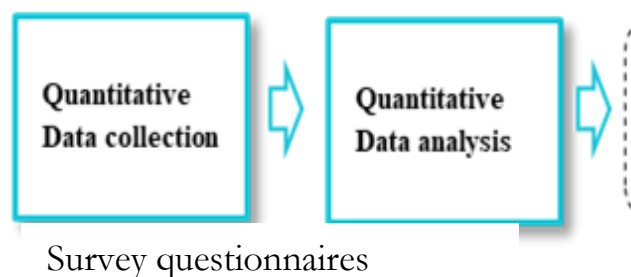

Phase 2

women and midwives
FOCUS

GROUPS

PROTOCOL

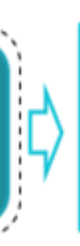

Focus group discussions

\section{Point of interface}

Figure 4-1: Visual diagram for mixed methods sequential explanatory design procedures adapted from Ivankova and Stick (2007)

The approach is useful when results are not so clear cut making explanation difficult (Polit \& Beck, 2017). Recently, some authors in the childbirth environment field have adopted MM approaches to evaluate birthing environments (Grigg et al., 2017; Grigg et al., 2015). The point of interface in the present study took place at the commencement of phase two, when the results of questionnaire surveys inform and are followed up in the phase two focus groups. While the two phases of the study were conducted sequentially, emphasis was not given to either quantitative or qualitative data, although it was found that the qualitative focus groups offered great depth for arriving at the final outcomes of the study.

\subsection{Phase one methods}

The methods used to conduct this study were driven by a pragmatic approach aimed at achieving the purpose of the research in the light of the challenges that have been highlighted in this chapter. The methods are described here sequentially, following the procedures mentioned in the introductory portion of this chapter.

\subsubsection{Setting}

The selected site for this research is a tertiary hospital where 3,245 mothers gave birth to 3,305 babies in 2017 (The Women's Health Service (WHS) \& Capital \& Coast District Health Board (CCDHB), 2017) and accommodates women from other lower-tier hospitals. This hospital is one of five major Regional hospitals in New Zealand. The Women's Clinics area is located on Level 4 North of the hospital within a large complex which houses the women's and children's wing of the hospital. The 41-bed maternity facility, located in the middle floor with the postnatal wards above, was renovated and completed in 2009 to meet the woman-centred approach to care. The 
birth/delivery suites consist of 12 single LDR rooms and a 39 bed postnatal ward comprising single and shared/double rooms. The bathrooms were either shared or built in. Close to these rooms is a 36 bedded Neonatal Intensive Care Unit (NICU) situated on level 4, the same floor as the birth/delivery suite and the postnatal ward. The hospital also included a surgery suite, administrative and support spaces. This hospital site was chosen for this study because it was renovated and transformed from its old 1970s' birthing unit design utilising Lepori's Birth Rooms concepts and supported by Professor John Gray from Victoria University of Wellington (Lepori et al., 2008).

\subsubsection{Participants}

The study population consisted of two user groups, the first comprising women who underwent postnatal hospital stays in large, tertiary hospitals in New Zealand together with women who gave birth in either primary birthing units or secondary hospitals but who were attended in the selected tertiary hospital for their postnatal care.

The other user group comprises midwives employed by District Health Boards (DHBs) who have been working in the birth/delivery suites and postnatal wards of large, tertiary hospitals and community-based Lead Maternity Carers (LMCs) midwives who are contracted with the Ministry of Health in New Zealand hospitals. The latter group provides care to a caseload of women from early pregnancy to labour and delivery and up to the end of the six week postnatal period. They work in different tiers of hospital care: primary, secondary and tertiary levels requiring navigation across different hospital spaces. The hospital based midwives maintain a rostered shifts basis whereas the community-based LMC midwives depend on when a client goes into labour. The LMC midwives cater for a caseload of women in the community and are usually organised into a group sometimes based on their orientation and philosophy of birth. All respondents for the phase one survey remained anonymous to the researcher.

\subsubsection{Postnatal women}

Nonprobability consecutive sampling was used to recruit participants. Consecutive sampling is a technique used to invite participants who meet eligibity criteria to participate in a study over a fixed time period, thus limiting risk of bias (Polit \& Beck, 2017). Eligibility criteria included first-time and multiparous mothers whose most recent birth was between one day and no longer than two years ago at the time of this study. It was taken as given that those who would be completing the survey understood the English language. 


\subsubsection{Midwives}

A snowball sampling approach was used for the recruitment of midwives. Snowball sampling is a type of nonprobability sampling, also known as chain-referral or contact-tracing. It is an approach anchored on building relationships between participants and researcher to enhance access (Atkinson \& Flint, 2001; Sadler, Lee, Lim, \& Fullerton, 2010). While snowball sampling is recommended for hard-to-reach populations, while midwives are accessible and available, it served also as a means for women - who are attended routinely by midwives in hospitals - to become aware of the study and acted as a link to other midwives who work in different hospitals in New Zealand in general.

On a particular day, a midwife living in the community was introduced to the researcher and thereupon invited other co-midwives to a meeting where the aim of the study was discussed. Ultimately, this approach facilitated contacts with many hospital-based midwives who were much interested in this type of research especially research undertaken from the standpoint of a person with a background in architecture. While selection bias has been identified as one of the weaknesses of snowball limiting the validity of the sample (Atkinson \& Flint, 2001) midwives in this study were not formally recruited; in the sense that the survey questionnaire was voluntary. Initial participants facilitated and encouraged their colleagues in their meetings towards participation in the online survey questionnaire. Importantly, a good number of midwives were positive about the study. Eligibility for midwives to participate was that they must have been working or have worked in tertiary hospitals particularly in postnatal wards as midwives.

\subsubsection{Tools: survey design}

Phase one of this research adopted a survey questionnaire as technique. The goal of phase one was to answer the research question: What are the user perceptions of design factors influencing women's recovery experiences during postnatal hospital stays? To answer this question, women and midwives as users of postnatal facilities (as care receivers and care givers respectively) were identified.

\subsubsection{Role of surveys}

The quantitative method places emphasis on discrete, specific concepts and can be used to gather information based on numeric data, with a focus on generalisability of results to a larger population. Therefore, the researcher used a survey approach for phase one to assist the gathering of 
information (Morse \& Niehaus, 2009), particularly to compensate for short postnatal hospital stays, making it suitable to reach participants anonymously without biasing their responses (Creswell, 2012).

Using a survey questionnaire allows for greater coverage of information on different topics and opinions or perceptions to be collected from a large number of people in a timely fashion (Groat \& Wang, 2002, 2013). In addition surveys are considered suitable to generate fast, valuable, and cost effective ways of collecting large amounts of both quantitative and qualitative data (Zeisel, 2006). Much of the research in environmental psychology and other allied fields has utilised surveybased research design permitting studies where several different variables are investigated at once (Lindberg, Tran, \& Banasiak, 2016).

A key issue in using a survey questionnaire is that potential occupants in the physical environment understand what they want and can express this to the researcher (Sommer, 1983, p. 75). Sommer warned that when people are turned off from their physical surroundings and ignored from the decision-making process they may not provide useful responses, as found in a hypothetical situation in which people were asked about civic improvements. This could lead to withholding of valid and useful responses as a consequence that people feel their opinions did not matter. As a result, the need to ask the right questions is essential to enhance informed answers (Sommer, 1983). Several researchers in healthcare design have found value in a survey-based approach to collect potential information from patients, care providers and significant others in order to facilitate information for the promotion of healing, restorative and family-centered hospital design (Felippe et al., 2017; France et al., 2009; Kotzer et al., 2011; Lawson \& Phiri, 2000).

Although surveys have the potential for uncovering a range of information on a subject, the potential weakness lies with challenges of obtaining the in-depth results for which the survey was undertaken (Groat \& Wang, 2002). Common criticisms about survey-based research stems from the claim that answers to questions are not reliable (Beam, 2012) and the argument that the outcomes of the process, the data in and of itself, is self-reported information, reporting what people think rather than what they do (Creswell, 2012). However, such perceived drawbacks could be reduced through multiple approaches such as follow-up focus groups. In this study subsequent focus group interactions, observations and gestures were considered suitable to address the inadequacies of survey-based research. Regardless of its shortcomings, the use of survey questionnaires has widespread application in the social science research and healthcare science fields. 


\subsubsection{Questionnaire development}

Due to lack of a suitable instrument specifically to examine the impact of hospital environments on women's recovery experiences and wellbeing, a survey instrument had to be developed. At the same time, the short postnatal hospital stay, including the demand of taking on the tasks of parenting, made hospital an unsuitable place to meet women. Consequently, an online survey questionnaire was deemed appropriate to reach women and midwives who constitute the user groups in postnatal hospital environments.

To develop the survey instrument involved a four stage approach. First, items and variables related to the physical healthcare environment and its effects on health outcomes and wellbeing were derived following an extensive review of the evidence-based design literature carried out between March 2016 and April 2017. Because the current research pertains to the postnatal physical environment and its impact on the population of women who use it with very limited precedents to turn to, the survey instrument utilised the findings from literature on the impact of healthcare physical environments on health and wellbeing outcomes complemented with some findings in labour and birth rooms studies. This literature was covered in Chapters 2 and 3.

Second, in mid-2017, three meetings were held with five midwives as caregivers of women. Further, a series of consultations with leaders and experts in midwifery who work as hospital-based midwives was undertaken to share their ideas about their experiences working as midwives in the childbirth environment in particular catering to women in postnatal environments. Consequently, a few items measuring social comfort and interior preferences were crafted based on discussions with midwives and hospital managers/administrators.

Third, through the support of an LMC midwife, the researcher twice visited and inspected the postnatal spaces/rooms in the hospital site selected for the study when no occupants were present. Video recordings provided insights into the nature of the spaces and facilities provision in the existing postnatal spaces. When necessary, questions were asked of the tour-guide midwife to further assist in the formulation of survey questions.

Fourth, a draft survey questionnaire was developed based on the findings from the previous stages which was then reviewed for clarity of wording and validity of contents by four core hospital midwives and two experts from the Women's Health Service at the selected hospital site. Subsequently, two versions of the questionnaire were developed from the draft; one for women and the other for midwives. Both survey questionnaires were similar with the exception of a few 
aspects of particular relevance to each population. Administering largely the same questions was to enable comparisons of the results of the two groups for credible insights about the architectural design factors and features in the postnatal hospital environment considered beneficial for women's recovery experiences and wellbeing.

The draft survey questionnaire was then pilot tested with a small sample of women to determine comprehensibility and clarity of the items and specific attributes that the survey instrument was set to achieve. Questions and feedback were used to improve the content validity of the instrument, which produced a revised instrument with improved validity. Cronbach alpha for most of the question categories for reliability was 0.7 (Nunnally, 1978). At this stage aspects regarding safety and security were dropped from the survey questionnaire because of their lower reliability scores.

The survey questionnaires adopted two response style options based on a 5 point Likert-type scale. The first is: "no, strongly disagree" scored as 1, "no, disagree" scored as 2; "neither agree nor disagree" scored as 3; "yes, agree" scored as 4; and "yes, strongly agree" scored as 5. The second option employed "not at all", "minimally important", "somewhat"," moderately important", and "very important" scored in the same manner. Formats and techniques for setting-up online questionnaires were reviewed and relevant styles of questions were chosen such as a unipolar scale method enabling the researcher to conceptually gauge participants' perceptions in the ascending order of gradation of the survey items (Dillman, Smyth, \& Christian, 2014). The questionnaires also included open-ended questions allowing the participants to communicate their ideas to enhance the design of the postnatal hospital environment. The questionnaire also had sections where demographic information of the participants can be collected (see survey questionnaires in Appendix B).

The survey questionnaire is divided into four main parts.

Part 1: General demographic characteristics of the respondents;

Part 2: Respondent's perceptions of the postnatal ward facilities provision;

Part 3: Ranking of physical and environmental design factors in terms of impact on postnatal recovery and wellbeing;

Part 4: Open-ended questions to deepen information about physical environment for recovery. 


\subsubsection{Data collection}

On the 9th of October 2017, online survey questionnaires were started. The Qualtrics software platform (Qualtrics, 2017) was used to design and to administer the questionnaire surveys. The survey questionnaires included 48 questions comprising both closed and open ended, and organised into five sections that includes (1) demographic information, (2) perception of the room, (3) interior design features, (4) sensory comfort, and (5) social comfort. For the postnatal women's survey, a nurse-midwife distributed the questionnaires via an online survey link to six hundred and five $(n=605)$ postnatal women who attended breastfeeding classes in 2016 and 2017. The online survey was completed by 229 women, which represents about thirty-eight percent of the sample. For the midwives' survey, a site contact person distributed the questionnaires to the entire membership list of about 200 midwives who worked in postnatal hospital environments through a different survey link.

Exclusions comprised postnatal women who had their babies at home or in a primary birth unit (see Appendix A5 - 6), and midwives who had no prior experience of working in the postnatal wards. Moreover, incomplete surveys related to all the included questions by the respondents were excluded. The handling of incomplete responses in the surveys is described in detail later in this thesis.

As the process of the survey was ongoing, the researcher periodically monitored the progress and documented the number of participants who had completed the survey while hoping for a smooth process of the exercise using the on-line technology. The first two weeks of starting the survey recorded low turnout as there were few respondents. However, in the third week up until November 2017 there were significant improvements as by then many respondents had completed the surveys. A total of 229 postnatal women filled in and returned their survey responses. 58 midwives also completed the relevant version of the questionnaire. Data collection for phase one lasted from October to the first week in December 2017.

This high turnout rate by the respondents indicates the use of a Web -based survey application had a considerable success rate compared to pencil and paper surveys (Kaplowitz, Hadlock, \& Levine, 2004). Using this survey approach also afforded recruitment of participants for the phase two qualitative focus groups. If a participant contacted the researcher via the researcher's email provided in the survey questionnaire, that was taken to be consent to participate in phase two of this study. Recruitment of participants for focus groups will be discussed in more detail later. 


\subsubsection{Data management and statistical analysis}

Having concluded the data collection for phase one of the study in December 2017, the completed survey responses were imported into SPSS Statistics software version 24. A computer storage file was created where all information regarding the survey data was kept and assessed during data analysis. Preliminary checks were manually conducted on the data collected to identify any missing data and possible outliers in the datasets.

Datasets were first examined for any missing data from the individuals' responses using the explore option in the SPSS. The initial screening revealed a few areas of missing data both for women's and midwives' datasets. Missing data might be due to a participant omitting a particular question or not completing some parts of the questions or entirely abandoning other portions of the survey questions. As Creswell (2012) points out, decisions about missing data need to be reported for ethical purposes. In the current study, the researcher utilised the missing data techniques to ensure that the data is rid of missing values and some outliers found in the datasets. First, datasets were evaluated for any "missingness" to determine whether some participants were missing a lot of data in the datasets. To do this, a different variable was created called missing data and then descriptive frequency was executed to determine the frequency of those missing data. Following the descriptive statistics, and manually screening the data, it was found that seventeen participants in the women's dataset failed to answer any question and had no or very slow progress in the survey questions. Consequently, the affected participants were deleted from the analysis. Dancey, Reidy and Rowe (2012) suggest that participants could be excluded from analysis if they missed or omitted one or many questions.

Furthermore, a total of 25 cases in the women's dataset were retained and screened. Missing completely at random (MCAR) applies to the datasets because a few respondents only omitted or could not answer part of the questions for some reasons. The series mean (SMEAN) was then employed to replace the missing data only for the twenty-five cases. To ensure that the replaced means are not different from the initial variables in the datasets, a paired samples $\mathrm{T}$ - test was conducted. The results generally for all variables yielded a zero value meaning there was no difference in the old variables and the new ones. The same procedure was employed in the midwives dataset. In the case of midwives, five participants were initially deleted from the dataset for failing to complete any question or were just non-responsive. Subsequently the above procedures were applied to replace missing values in only three cases (see detailed analyses and results on missing data in Appendix C). 
Descriptive statistics on the items, percentages, means and standard deviations were performed using SPSS. Lee, Lee and Lee (2013) discuss two basic types of statistics comprising descriptive and inferential statistics. Descriptive statistics involve a measurement of central tendency in the data using mean and median and measure of dispersion, such as standard deviation for the spread of scores. This type of statistics is useful to summarise numerical information (Lee et al., 2013). Inferential statistics deals with the use of sample data to reach general conclusion (Lee et al., 2013), which ensures that errors are eliminated that could result from a set of sample data compared to the actual population. Following descriptive analyses to identify design factors in the postnatal hospital environment, an inferential statistics study was performed to compare the mean scores on perceptions of design factors between women and midwives. Independent-samples T- tests were undertaken to establish the mean of independent groups to what results would be like for the total population (Creswell, 2012). In order to do that, two hypotheses were proposed:

$\mathrm{H}_{0}$ (Null): There are no significant differences in the mean scores on perceptions of design factors between women and midwives. In other words, women and midwives have equal perceptions on design factors.

$\mathrm{H}_{1}$ (Alternate): There are significant differences in the mean scores on perceptions of design factors between women and midwives.

Furthermore, the Levene's Test for equality of variances was used to examine the variances in the two groups by considering the F statistics and the level of significance p-value. Equal variances are assumed, if $\mathrm{p}>0.05$ on the one hand, indicating that the outcomes are normally distributed among the two independent groups (women and midwives) therefore the assumption of equal variance is satisfied.

On the other hand, Welch t' tests are reported whenever the condition of normality is violated; that is, when $\mathrm{p} \leq 0.05$. Additionally, the effect size enables the "measurement of the absolute magnitude of a treatment effect, independent of the size of the samples used" (Gravetter \& Wallnau, 2011, p. 231). The measurement of the effect size is Cohen $d$, which can be calculated using the formula: $M_{2}-M_{1} / \sigma$, in which $\mathrm{M}=$ difference in the means between the groups; and $\sigma$ $=$ standard deviations of the groups (Gravetter \& Wallnau, 2011). The effect sizes of .20, .50, .80 and $1.5+$ are considered small, medium, large and very large respectively. A significant level of 0.05 has been taken as a generally acceptable level of confidence mostly used in behavioural research and was adopted in this present study. 


\subsubsection{Ethical considerations}

This study was granted ethical approval following an ethical clearance from the Health and Disability Ethics Committees (HDECs), which consider and approve health research in New Zealand. This study was classified under category "A" of the Human Ethics Committees (HEC) of Victoria University of Wellington, therefore it was mandatory that ethical clearance had to be obtained from the HDEC. This research fits into HDEC's classification of an Observational/Non experimental study. This means that the research does not have any implicit or explicit involvement in manipulating samples or using any human tissues or any medically intrusive procedures. In other words, there is little risk that participants would be affected in any way.

HDECs are established under section 11 of the New Zealand Public Health and Disability Act 2000 to "provide independent ethical review of health research and innovative practice in order to safeguard the rights, health and wellbeing of consumers and research participants and, in particular, those persons with diminished autonomy"(Health and Disability Ethics Committees, 2016, p. 1).

Accordingly, this study was reviewed and determined to fall outside the scope of HDEC review. The HDECs then provided the letter which served as the first ethical clearance (see Ethical approval in Appendix A) and upon which ethical approval was granted by the Human Ethics Committees of Victoria University of Wellington (Ref: 24397). Additionally, localised ethical clearances from the concerned DHBs were obtained at each phase of the research.

All participants in the study were assured of anonymity and confidentiality. Using an online based survey in phase one of this study provided by the Qualtrics software platform (Qualtrics, 2017) under the licenses of VUW, New Zealand further protected and ensured the anonymity of participants. Participants were self-selecting and they remained anonymous to the researcher.

If survey takers met eligibility criteria (see Information and consent form in Appendix A5), consent was requested in the on-line survey to see whether they would like to participate through a "Yes" or "No" response. If a participant decided to choose a No response, the survey would automatically end at that stage; likewise a Yes response implied that a survey taker gets to proceed with survey questions. Informed consent was implied when a person submitted the survey.

Although some items of background information were requested for the survey, names of participants were not included in the results segment. All electronic files are stored in a passwordprotected computer. Stored data are regularly checked to ensure both the safety and integrity of the data and access to the data is limited only to the researcher and the supervisory team. All 
questionnaire surveys, focus groups recordings and transcripts were held for the length of the research and destroyed at the completion of the research and the thesis submission.

The findings of this study have been and will be disseminated to the profession through academic conferences and publications in peer-reviewed journals. Some focus group participants requested copies of the findings when published, their respective e-mails provided opportunity to contact them.

\subsection{Phase two methods}

Phase two concerns the qualitative strand of the mixed methods approach used to follow-up and to explain the quantitative results on perceptions of design factors surveys in phase one. The methods used to conduct the Focus Group Discussions (FGDs) are described and ethical issues are highlighted.

\subsubsection{Settings}

The phase two component (focus group discussions) of this study was conducted at three locations in New Zealand. The first setting was at a 500 bed tertiary hospital providing a range of services for children's health and medical and maternity services. This site accommodated the Graduate School of Nursing \& Health of a tertiary institution in New Zealand from which one of its meeting rooms was made available for the FGDs. The room was selected due to its convenience of access from public transport services in the city. Although all three settings for the focus group were open to participants, only midwives selected this setting, it was their workplace and they could find it easier to respond to on-call work and emergency situations. It had facilities to make tea and coffee and had washrooms.

The other two separate settings were office spaces located at a university. The first had seating for eight to twelve participants. Its location, tucked away in a corner from the frequent corridors of activities, coupled with provision of washroom facilities, made it suitable for the focus groups. The second setting was a larger space, although within the vicinity of other activities, the fact that it is dedicated for meetings fitted the purpose of the FGDs. These two settings met the purpose of the focus group members primarily because they were closer to the homes of participants with excellent access to public transport. 


\subsubsection{Participants}

\subsubsection{Postnatal women and midwives}

The sample for phase two consisted of nine women and five midwives from the initial phase one sample questionnaire surveys. Prior to data collection for the phase one survey, the researcher had the privilege to deliver a five minute presentation to the expecting parents regarding the healthcare physical environment and postnatal recovery, which helped to create awareness for this study. The brief interaction with the expecting women was devoid of information pertaining to the survey questionnaires and did not form part of the talking points to the effect that it could compromise the integrity of the questionnaires and bias from the researcher. Ultimately, the goal of the interaction was largely an awareness about how the physical environments of healthcare could impact emotional and psychological wellbeing, as well as influence the recovery experiences of new mothers and their families in the immediate postnatal period.

Phase one survey questionnaires offered the benefit that participants, postnatal women and midwives, could indicate their willingness to participate in phase two. The survey takers were asked to check a box if they would like to participate further in the research. This way, potential participants were recognised and their email contacts were collated. Criterion sampling was used to select both women and midwives who agreed to a follow-up and demonstrated ability to likely contribute well (Hamilton, Sandelowski, Moore, Agarwal, \& Koenig, 2012) in the focus groups. The process of recruitment of participants is shown in Table 4-1 which shows the stages of participants' recruitment.

\subsubsection{Tools: focus group}

Focus groups served as the method for data collection for phase two of this research. Attention was given to the anonymity and confidentiality of the focus group informants by using a pseudonym to identify them when describing their responses, that is to say, FGD1W1 means focus group discussion one, woman number one and so forth. The description of the informants is provided in detail in Chapter 9. 
Table 4-1: Details of recruitment of the focus groups

$\begin{array}{lccccc}\text { Respondent } & \begin{array}{c}\text { Participated } \\ \text { in the survey }\end{array} & \begin{array}{c}\text { Included } \\ \text { in analysis }\end{array} & \begin{array}{c}\text { Volunteered } \\ \text { for phase 2 }\end{array} & \begin{array}{c}\text { Returned } \\ \text { emails }\end{array} & \begin{array}{c}\text { Attended } \\ \text { focus groups }\end{array} \\ \text { Women } & 229 & 212 & 41 & 21 & 9 \\ \text { Midwives } & 58 & 50 & 19 & 8 & 5 \\ \text { Total } & 287 & 262 & 60 & 29 & 14\end{array}$

All information and data sets pertaining to this study have been held and securely stored in a locked file and access restricted to the researcher throughout the study.

\subsubsection{Phase two data collection}

Data were collected from four focus group discussions between May, June and August 2018. FGDs were conducted at the three selected sites. All participants had the choice of two venues with booked times for the focus groups as can be seen in the focus groups flyer in Appendix A).

Initially, sixty participants (41 women and 19 midwives) signed up for the phase two study, however many of them were unable to fit into the booked times that did not suit everyone's needs and conveniences. Some women had concerns of catering for their baby while discussions were to take place plus other issues like facilities to change their baby, while for midwives increasing high workloads and unpredictability of calls (labour may happen without notice) made them busy and sometimes unable to attend.

On May 29, 2018, the first focus group was held. The duration of FGDs ranged from 80 minutes to 95 minutes with an average of 83 minutes, with the exception of the one held in May, which lasted for 52 minutes.

Powell and Single (1996) highlighted the need for focus groups especially when the current knowledge of a subject is limited and elaboration is important. A focus group thrives on ideas, beliefs and perceptions. An example is the opportunity it offers focus group members to come to self-disclosure about issues or events by contributing ideas or experiences (Krueger \& Casey, 2000, 2009). In research such as this study, focus groups offer opportunity for forms of interaction with women and among women (Madriz, 2000) providing greater insights about their perceptions and opinions toward certain environments (Powell, Single, \& Lloyd, 1996; Zeisel, 2005) and especially to better understand the physical environment's impact on women's recovery and wellbeing. Knowledge generated through the use of focus groups as a method is considered to strengthen 
the results of quantitative surveys in which statistical associations require clarification and elaboration (Powell \& Single, 1996). As pointed out by Zeisel, focus groups are best suited to test, refine and modify the analysis (Zeisel, 2006, p.128).

In the current study, results of perception of design factors surveys yielded several design features requiring further explanations and verification (Polit \& Beck, 2017). According to Powell et al. (1996), results generated by questionnaires may at times be in sharp conflict between the opinions of healthcare providers and those expressed by the clients - in this case postnatal women - who are the targets of the study. Evidence of widespread use of focus groups as a method in healthcare research includes their use for examining optimum physical environments (Gardiner, Brereton, Gott, Ingleton, \& Barnes, 2011), women's life experiences after childbirth (Larkin et al., 2012; Olsson, Lundqvist, Faxelid, \& Nissen, 2005; Parvin, Jones, \& Hull, 2004), views of postnatal care by midwives (Cattrell, Lavender, Wallymahmed, Kingdon, \& Riley, 2005) and improvements in hospital-based postnatal care (Schmied et al., 2009).

Although focus groups have strengths, there are concerns about the process of producing interactions, the role of the moderator and the role of group influence on the data produced (Curtis \& Redmond, 2007; Morgan, 1996), nonetheless they have a wide acceptability in social sciences and health sciences research.

Existing studies have found both activities and design of tertiary hospital postnatal units largely worked against the expectations of postnatal goals (Gaboury et al., 2017; Morrison \& LudingtonHoe, 2012). Therefore, focus groups can provide opportunity for women and healthcare providers by exploring "designerly" ways to alleviate the busyness of hospital physical environments in order to support women regarding postnatal care in hospitals and afterwards (Brodribb, Zadoroznyj, \& Dane, 2012; Larkin et al., 2012).

Although a common practice would be six to ten focus group members, Morgan strongly argued using smaller groups which should produce better and more structured outcomes than with many participants but less structured discussions (Morgan, 1996).

As can be seen from Table 4-2, each FGD was composed of three to four members: an all women group $(n=3)$ represented in the first and last FGDs; and an all midwives group $(n=4)$ in the second FGD, and the remainder was a blend of women and a midwife. 
Table 4-2: Distribution of the focus group participants by attendance

\section{Focus groups Date of focus groups Attendance by group Attendance} session

\begin{tabular}{|cccc}
$\mathbf{1}$ & $29 / 5 / 18$ & Women & 3 \\
$\mathbf{2}$ & $20 / 6 / 18$ & Midwives & 4 \\
$\mathbf{3}$ & $21 / 6 / 18$ & 3 women and 1 midwife & 4 \\
$\mathbf{4}$ & $16 / 8 / 18$ & Women & 3 \\
\hline
\end{tabular}

Focus groups use a small number of participants who share common experiences but who may have divergent views which they could communicate through interactions. Authors writing about focus groups such as (Krueger \& Casey, 2000, 2002), Powell and Single (1996) and Curtis and Redmond (2007) have extensively discussed the procedures for conducting focus groups such as moderating skills and creating a comfortable environment, and approaches to be used to ask questions in focus groups (Krueger, 1994). In this study, the researcher served as a moderator and engaged two assistant moderators in the taking of notes.

\section{Conducting the focus group discussions}

Facilitating an environment for discussion is crucial before and during focus groups. At the start of each focus group discussion, the researcher created an atmosphere for the participants to feel welcome and introduced the purpose of conducting the focus groups as part of his $\mathrm{PhD}$ research. The participants were encouraged to freely express areas of agreement and disagreement regarding any question, these mixtures of consensus and dissent are what make focus groups stand out (Morgan, 1996). The objective behind focus groups in this study is that a wide perspective can be understood, in a manner differing from consensus-seeking but rather to generate a diversity of knowledge on a particular topic (Ruppenthal, Tuck, \& Gagnon, 2005).

Each focus group member was invited to use the first letter of their name to assist verbatim transcription of interactions (a tag facilitated easy identification of each participant whenever they spoke). Each focus group discussion began with questions "What is your general feeling about the postnatal hospital environment when you were there"? and "Tell me about your positive experiences you have had with the postnatal hospital environment where you were attended or worked"? These initial sets of questions were asked of participants to set the mood for the discussions (see focus groups protocol in Appendix A). The role of the researcher, as a moderator, was to pose the questions and to pay attention to responses from focus group members. Other 
times, when necessary, probes were introduced when the researcher felt that the responses to a question required more clarification and elaboration or could generate further insight about the research questions.

All discussions were audio-recorded using a TASCAM DR-05 Linear PCM Recorder, each audio recording file was labelled and transcribed verbatim by the researcher. Transcripts of all FGDs were checked for accuracy through several readings and re-readings of the transcript materials until the transcripts reflected the context and content of the audio files. Integrating notes and jottings of the assistant moderators for tones of voice, facial expressions or gestures of participants during the FGDs as nonverbal communication data were included where necessary in the transcripts, this is frequently disregarded in focus group reports (Onwuegbuzie, Dickinson, Leech, \& Zoran, 2009). Finally, the transcript materials were stored securely in a password-protected computer managed and accessed only by the researcher.

\subsubsection{Data management and analysis}

Constant comparative analysis was used to analyse the focus groups data. The constant comparative method can be traced to the work of Glaser and Strauss (Glaser \& Strauss, 1967; Strauss, 1987). Its overarching goal was in the development of grounded theory research (Leech \& Onwuegbuzie, 2008; Onwuegbuzie et al., 2009) although this is now increasingly being expanded to accommodate other types of research goals rather than theory building (Corbin \& Strauss, 2008, p. ix ).

According to Strauss and Corbin (1998) constant comparative analysis comprises three important sequential stages. The first stage of data analysis uses the open coding process which involves data reduction, breaking data into smaller units by identifying codes or preliminary categories from the data. In the second stage, axial coding involves the reorganising of all the identified categories and relating them to subcategories dimensionally in such a manner to form an explanation of the phenomenon or concepts. In the third stage, selective coding involves developing broad themes based on the different categories created.

Each item of focus group data was analysed, one at a time, taking the deductive approach to interpret and deduce "what is going on" in the data (Strauss \& Corbin, 1998, p. 137) and inductively by the empirical data in the FGDs to facilitate structure for the analysis (Burnard, Gill, Stewart, Treasure, \& Chadwick, 2008). The first initial process involves data reduction in which data are broken into codes or concepts as described by (Corbin \& Strauss, 2008, 2015). The first round of 
the initial coding process produced several codes. These codes describe a range of beliefs, perceptions, experiences, and concepts about the postnatal hospital environment. In addition, as the coding process was ongoing, several memos were written, which reflect on the meanings of the text to facilitate interpretations on what the data was about. Following the initial coding process, some of the concepts that were generated in each focus group analysis, where applicable, were used in subsequent focus groups to understand differences or commonalities regarding concepts/categories dimensionally and their properties. This enabled theoretical sampling as well as to expand or refine the properties and dimensions of concepts (Corbin \& Strauss, 2008, 2015).

During analysis, the purpose of the phase two qualitative focus groups was kept in focus: What were the ways in which the design factors and the postnatal spaces affected women's recovery experiences and wellbeing during hospital stays? After the analysis was completed, written memos for each FGD were compared ensuring that incidents that are conceptually similar are placed under the same categories (Corbin \& Strauss, 2008; Strauss \& Corbin, 1998). Consequently, categories that are similar in contexts with the design factors in phase one were grouped together with a label attached. This process was repeated resulting in the formation of the initial categories. The data display of each content/grouping (initial categories) was revisited and reviewed for the development of main categories. In order to be able to track the original data source, each of the newly created categories was coded.

The researcher interpreted the findings based on the categories developed with regards to the aim of phase two and the overall research questions. The findings from the phase two qualitative focus groups are supported with extracts of text and quotations from the focus group discussions.

\subsubsection{Validity and reliability}

Two approaches were undertaken to validate the research findings corresponding to the two phase research design approach adopted in this current study. In phase one, after the initial formulation of the survey questionnaire, it was reviewed by five registered midwives and two primiparous women and then adjusted to accommodate revisions that were suggested. Following this, the survey questionnaire was reviewed by three experts at the Women's Health Services at the selected hospital in New Zealand and their feedback was incorporated in the survey questionnaire, hence giving it much improvements.

The most often cited authority regarding validity and reliability is Hammersley (see Hammersley, 1987). In line with Hammersley's thinking, in the phase two qualitative research, Silverman's 
(2013) strategies of increasing validity of findings were used focused on five steps: engaging in the refuting principle, constant comparative method, comprehensive data treatment, searching for deviant cases, and making appropriate tabulations.

\section{Refuting principle}

The refuting principle is concerned with disproving assumptions against data in the course of the research process by the researcher. As discussed previously there is a lack of existing precedents regarding physical design factors that influence women's recovery experience while in hospitals. As such, no priori assumptions were made. The nature of the investigation, to some extent, is about a discovery and gaining insights regarding the design features and architectural characteristics that support wellbeing. However, the choice of explanatory mixed methods provides ample opportunities for cross-checking of the data collected in phase one with the phase two FGDs. In addition, the researcher paid due diligence to the expressed opinions, perceptions and views on every question, which were verified in other remaining focus groups, thereby facilitating checking for convergence and divergence in ideas, concepts and categories.

\section{Constant comparative method}

The constant comparative method is an approach which compares one case against another. As stated previously, in order to achieve constant comparative method in this study, each focus group was conducted separately and analysed following their transcriptions. Each analysis from each focus group was carefully compared with the remainders of the analyses of the focus groups. Categories/concepts that were generated from the analysis of a particular focus group were individually compared based on each participant's responses and collectively as a group.

\section{Comprehensive data treatment}

Comprehensive data treatment is concerned with the inclusion of all cases into the analysis process. The unit of analysis in this study is taken as each focus group, whereby all the women's and midwives' responses in the focus groups are included in the analysis regardless of their dissent or consent on specific questions. A salient feature of focus groups is the necessity for the researcher to listen and pay due diligence to every detail of what every participant in the focus group has to say.

In order to achieve comprehensive data treatment, the participants in the focus groups took turns to answer the questions that were asked of them and contributed dynamic interactive viewpoints 
on a number of the aspects for which the quantitative results needed further explanation. The process produced data and information that included the views, comments, and opinions of the participants, which were generally taken on board to facilitate explanation in this study.

Similarly, the researcher took note of every word, phrase, sentence and non-verbal communication (in the jottings of the assistant moderators) of participants. The combined expressions and thoughts were interpreted in-depth and presented accurately to reflect what the data were about. Lastly, at the end of all the analyses, reports and narratives were considered as a whole rather than in isolation.

\section{Searching for deviant cases}

Deviant cases pertain to inclusion and discussion of cases that do not fit in the pattern. As stated previously in Section 4.10, there is a consensus of opinions among several authors that the strengths of the focus group lies with its abilities to accommodate deviant/dissenting viewpoints.

The selected samples of women and midwives in the FGDs to lesser or greater degrees gave different divergent opinions, beliefs, opinions and perceptions regarding postnatal facilities. For example, while most of the women and a few midwives supported the notion of the provision of single-bed room occupancy, a particular midwife strongly objected to this on the grounds of safety and the responsibility of care entrusted to the midwives. In this regard, identification of this "special case" (and others) represents a part of the discussion in the findings of the FGDs that indicate levels of deviance among the participants. From the findings of this study, excerpts from the discussions that relate to those deviant cases were included and placed alongside other comments that portray alternative positions. However, the findings of the FGDs also had aspects in which women and midwives in the study were completely in agreement.

\section{Making appropriate tabulations}

Appropriate tabulations deals with the use of quantitative figures if they are appropriate for the study. As was pinpointed earlier, the analytic process in this current research was a blend of deductive and inductive approaches allowing conceptualisation of data from the initial focus groups material. As Strauss and Corbin (1998) argued, assumptions about the nature of life and the understanding that a researcher takes to an investigation are guiding factors upon which interpretations are made based on data. They further stressed that the process of conceptualising what is going on and making interpretation thereof is deduction. 
Consequently, in this present study, the narratives from several analytic processes during the FGDs were presented in tabular formats highlighting the main categories and concepts including their respective sub-categories. Furthermore, all the transcript materials of the FGDs were transcribed and analysed without discarding or leaving out minute details, keeping to the guidelines offered by Silverman (2013), although member checking was not done due to the challenges of bringing all the members together and because of the high workload of midwives. Consequently, the researcher devised a set of questions on all the aspects of the FGDs' findings and presented it to six midwives for ranking based on their perceptions of relevance or not. Four of the midwives were a part of the FGD informants and one was a senior member of the Midwifery department. The last person was a midwifery academic in the childbirth environment field. Overall, the key informants agreed that the findings of the focus groups reflected the discussions and can make a potential difference in postnatal building spaces for the recovery experiences of women thereby further confirming that the categories and sub-categories generated in the course of data analyses were in consonance with the research objectives, strengthening the understanding that the research question has been addressed.

\subsection{Conclusion}

Chapter four of this thesis has described the research design and discussed the methods taken to conduct both parts of the two-phase study. It provided a detailed discussion about mixed methods as a methodological and theoretical approach that suggested their adoption, including the techniques adopted in the phase one quantitative core research. Next, it went into the phase two qualitative focus groups as a supplementary component, including the settings, sample, analysis and procedures. In the next chapters, the results of the users' perceptions of design factors quantitative strand are presented, followed by the findings of the qualitative focus groups in phase two. 


\section{CHAPTER 5}

\section{PHASE ONE: WOMEN'S PERCEPTION OF DESIGN FACTORS SURVEY - RESULTS}

This Chapter discusses the results from the phase one quantitative survey questionnaires. For clarity the women's survey results will be presented in this chapter, followed by the midwives' results in the next chapter.

Descriptive statistics are calculated using the mean and standard deviation for the continuous variables. As previously mentioned (see Chapter 4), these sets of statistics are suitable for the measure of central tendency and spread of the data.

Analysis of open-ended questions employed two approaches. The first deals with qualitative methods by examining the texts and dividing them into units, and then into separate sentences from which codes are assigned and categories created. The second involves using respondents' comments and the frequency of repeated words and ranking the comments and presenting them in a table. This approach was considered satisfactory (Pasha, 2013) and has recently been used by other researchers to analyse text responses in a survey-based research (Devlin, Andrade, \& Carvalho, 2016; France et al., 2009).

\subsection{The Questions}

The first set of questions in the survey include demographic characteristics of respondents comprising age, education level, ethnicity/race, most recent birth of participants, and parity level. In addition, respondents were asked place of birth of baby, and type of room in which postnatal care was provided. Data collection about the place of baby's birth includes settings such as large, tertiary hospitals, secondary or primary birth units/centres. Inclusion of parity levels ensured that divergent experiences about perceptions of physical design factors were obtained across a range of hospitals in New Zealandd.

\section{Demographic profile}

Out of the 212 respondents who successfully accessed the on-line survey questionnaire (see Table $5-1$ ), approximately $38 \%$ fell within the age range of $30-34$ years; $34.4 \%$ were in the $35-39$ age 
range, roughly $18 \%$ of the respondents were in the age range of $25-29$, while $6.1 \%$ of the respondents fell in the age range of 40 plus. Only $3.3 \%$ of participants $(n=7)$ were in their early twenties.

\section{Parity level}

Parity level explains the number of times a woman has given birth (see Table 5-1). Most of the respondents in the sample had their baby for the first-time $88.2 \%$ ( $\mathrm{n}=187$ ); Seventeen respondents (8\%) had given birth twice and six respondents (approx. 3\%) were multiparous (see Figure 5-1). At the time of the survey (October-December 2017), approximately $36 \%$ of the respondents' births had occurred between 1 and 6 months ago.

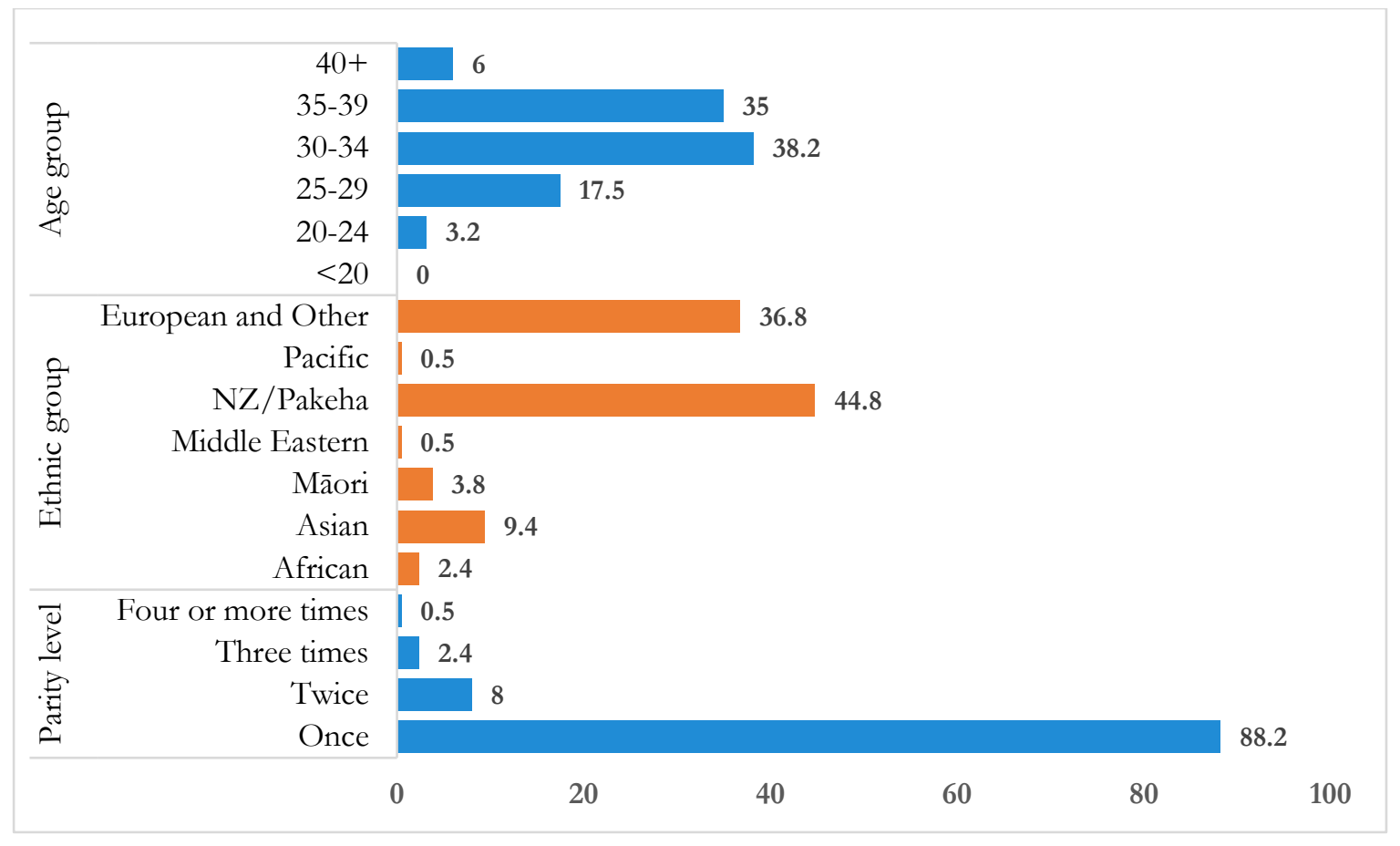

Figure 5-1: Distribution of respondents giving birth by parity level, age group and by ethnicity

\section{Place of birth}

Place of birth indicates the location in which birth occurs (see Table 5-1). Most of the respondents $(n=207)$ have given birth in hospitals. Of those who gave birth in hospitals, $88.2 \%$ were first time mothers with $77.8 \%(\mathrm{n}=165)$ whose births occurred in a large, tertiary hospital. $9.4 \%$ of the total sample who gave birth in primary birth units were first time mothers; and $10.4 \%$ in midwife-led units on the site of a hospital were first time mothers. 


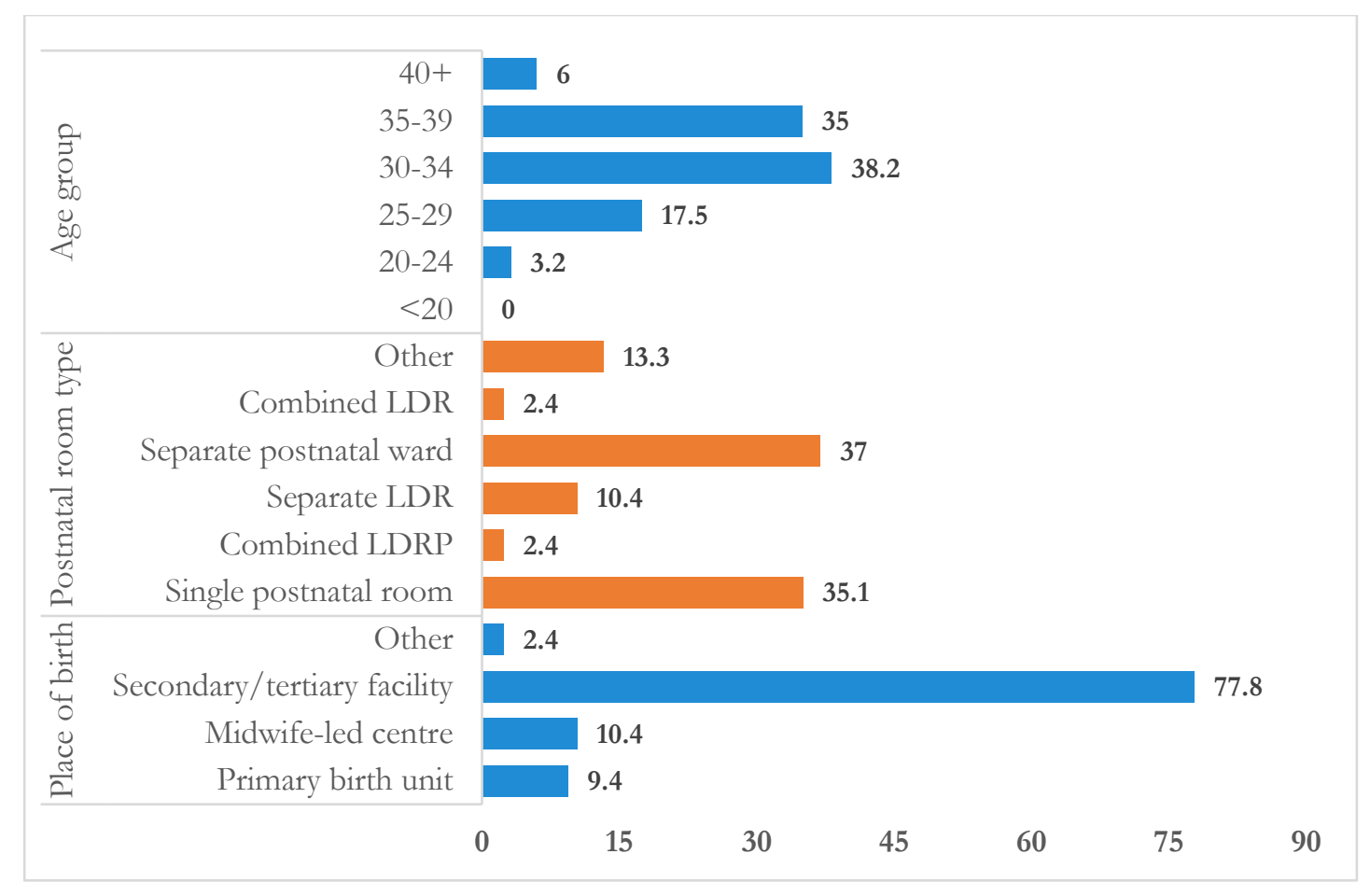

Figure 5-2: Distribution of respondents giving birth at a hospital facility, by age group and postnatal room type

The result reflects the trends in hospital births common in Western countries where almost all births occur in hospitals (Hodnett et al., 2009). Around 10\% of the participants gave birth in midwife-led units on the site of hospitals and $10 \%$ in primary birthing units. Lastly, $1.9 \%$ of the respondents fell into the "other" category of locations that could not be accounted for or that fall outside the given labels.

\section{Most recent birth}

In order to best recall the experiences in the postnatal hospital environment, it was important that the sample reflected a mix of women whose births occurred relatively recently prior to the survey. $14.6 \%$ of the respondents had given birth less than 1 month and up to 3 months ago $(n=31)$. Combined, roughly one third $(n=68,33 \%$ ) of the respondents births occurred within the past 1 to 6 months (see Table 5-1). Approximately $64 \%$ of participants had given birth between the last 7 months and more than 1 year ago prior to the time of survey (see Figure 5-3). 


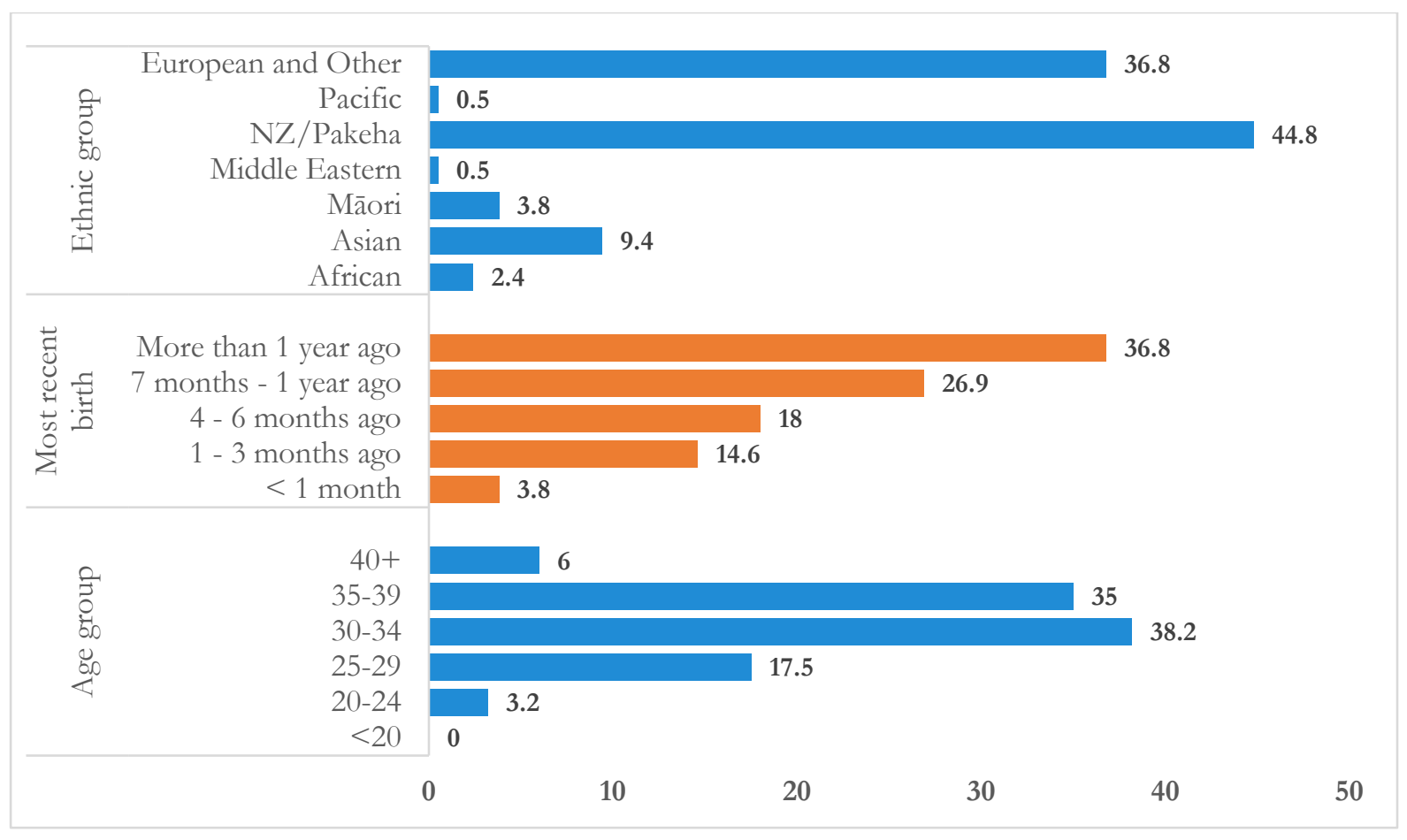

Figure 5-3: Distribution of respondents' most recent birth, by age group and ethnic group

\section{Education background and ethnicity}

Many of the respondents were from a New Zealand/Pakeha $(45.1 \% \mathrm{n}=97)$ background closely followed by respondents from the European background who constituted $31.2 \%$ of the sample. In terms of education, both of these groups had the highest numbers of university and postgraduate qualifications; NZ/Pakeha, university (44.4\%) and postgraduate (39\%); and European, university (28.7\%) and postgraduate (35.3\%). Participants from an Asian ethnic background were 10.7\%; Māori constituted 4.7\% and 3.3\% were from an African background with the vast majority having completed or in the process of completing postgraduate qualifications $(83.3 \%)$. The Asian nationality in the sample had university $(15 \%)$ and postgraduate $(7.3 \%)$ qualifications respectively. Other ethnic backgrounds also accounted for 6.5\% (see Table 5-1). 


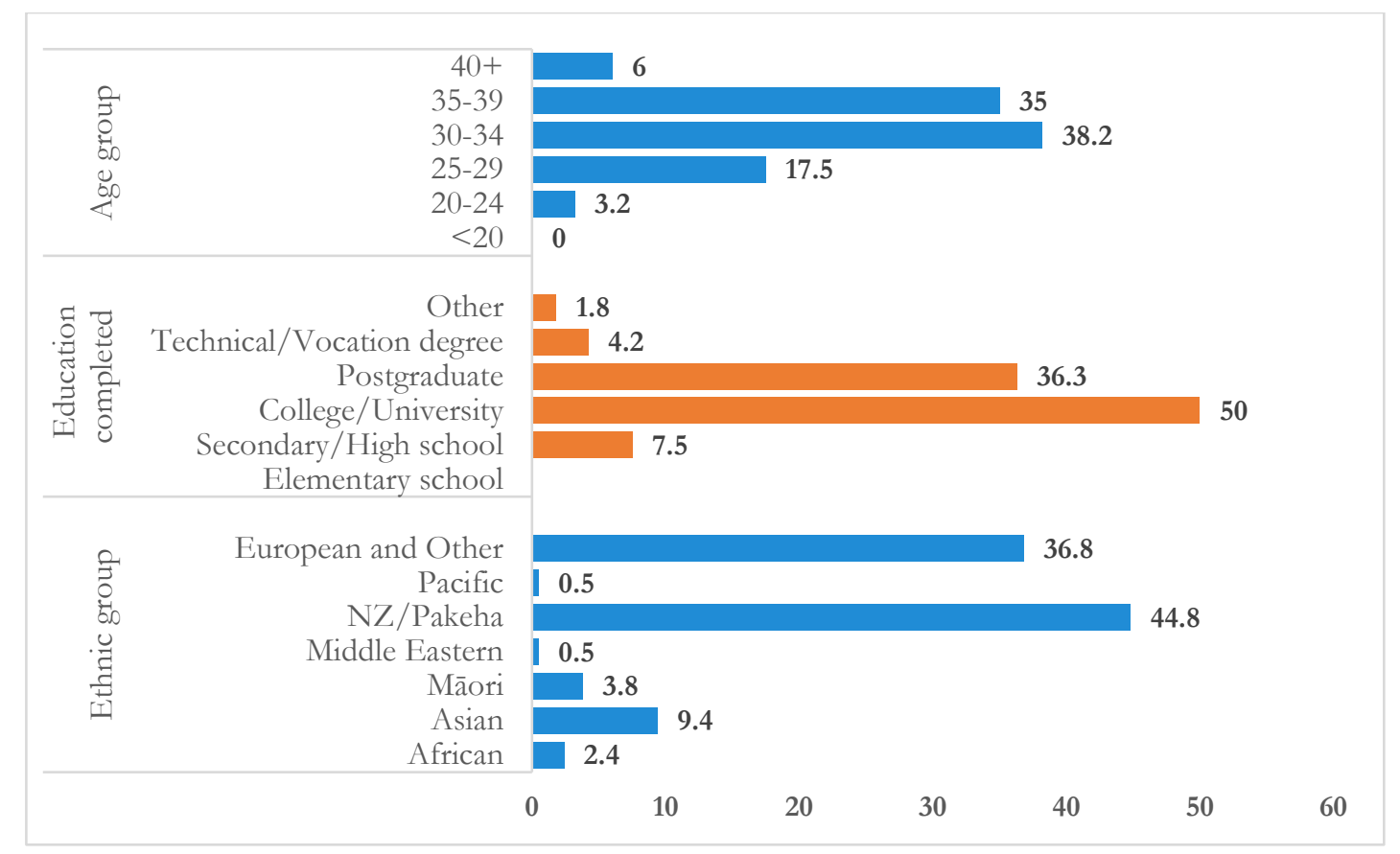

Figure 5-4: Percentage of respondents' education levels, by age group and ethnic group

Overall, a large proportion of the respondents had received higher education qualification. 50\% of the respondents had completed university $(n=106)$. Thirty-seven percent $(n=77)$ had postgraduate qualifications. Sixteen respondents had a secondary/high school certificate $(8 \%$ approx.), while nine respondents $(4.2 \%)$ had completed either technical or vocational education while two respondents $(0.9 \%)$ fell under the category of "other". Table 5-1 below shows the overall demographic profiles of the survey respondents. 
Table 5-1: Demographic profile of sample participant women $(\mathrm{n}=212)$ in phase one survey

Characteristics

\begin{tabular}{|c|c|c|c|}
\hline \multirow[t]{7}{*}{ Age (yr) } & $<20$ & 0 & - \\
\hline & $20-24$ & 7 & 3.3 \\
\hline & $25-29$ & 37 & 17.5 \\
\hline & $30-34$ & 80 & 37.7 \\
\hline & $35-39$ & 73 & 34.4 \\
\hline & 40 and above & 13 & 6.1 \\
\hline & Missing & 2 & 0.9 \\
\hline \multirow[t]{5}{*}{ Parity level } & once & 187 & 88.2 \\
\hline & twice & 17 & 8 \\
\hline & Three times & 5 & 2.4 \\
\hline & Four and above & 1 & 0.5 \\
\hline & Missing & 2 & 0.9 \\
\hline \multirow[t]{5}{*}{ Place of birth } & Primary unit/birth centre & 20 & 9.4 \\
\hline & Midwife-led centre on site of hospital & 22 & 10.4 \\
\hline & Large/Tertiary hospital & 165 & 77.8 \\
\hline & Other & 4 & 1.9 \\
\hline & Missing & 1 & 0.5 \\
\hline \multirow{6}{*}{$\begin{array}{l}\text { Postnatal care room } \\
\text { type }\end{array}$} & Single postnatal room & 74 & 35.1 \\
\hline & $\begin{array}{l}\text { Combined Labour, Delivery, Recovery } \\
\text { and Postnatal (LDRP) room }\end{array}$ & 5 & 2.4 \\
\hline & $\begin{array}{l}\text { Separate Labour, Delivery and Recovery } \\
\text { rooms }\end{array}$ & 22 & 10.4 \\
\hline & Separate postnatal ward & 78 & 37 \\
\hline & $\begin{array}{l}\text { Combined Labour, Delivery and } \\
\text { Recovery (LDR) room }\end{array}$ & 5 & 2.4 \\
\hline & Other & 28 & 13.3 \\
\hline \multirow[t]{6}{*}{ Most recent birth } & Less than 1 month & 8 & 3.8 \\
\hline & $1-3$ months ago & 31 & 14.6 \\
\hline & 4-6 months & 37 & 17.5 \\
\hline & 7 months -1 year & 57 & 26.9 \\
\hline & $>1$ year & 78 & 36.8 \\
\hline & Missing & 1 & 0.5 \\
\hline \multirow{7}{*}{$\begin{array}{l}\text { Highest level of } \\
\text { education completed }\end{array}$} & Elementary school/Primary school & - & - \\
\hline & Secondary/High school & 16 & 7.5 \\
\hline & College/University & 106 & 50 \\
\hline & Postgraduate & 77 & 36.3 \\
\hline & Technical/Vocational degree & 9 & 4.2 \\
\hline & Other & 2 & 0.9 \\
\hline & Missing & 2 & 0.9 \\
\hline \multirow[t]{9}{*}{ Ethnicity } & African & 5 & 2.4 \\
\hline & Asian & 20 & 9.4 \\
\hline & European & 64 & 30.2 \\
\hline & Māori & 8 & 3.8 \\
\hline & Middle Eastern & 1 & 0.5 \\
\hline & NZ/Pakeha & 95 & 44.8 \\
\hline & Pacific Island & 1 & 0.5 \\
\hline & Other & 14 & 6.6 \\
\hline & Missing & 4 & 1.9 \\
\hline
\end{tabular}




\subsection{Summary of results for postnatal room situations}

In order to see what participants thought about the different types of postnatal rooms in combination with some perceived room characteristics, it was necessary to perform a simple cross tabulation. The results of cross tabulation revealed that the single-occupancy room was considered most restful (44.4\%), most attractive $48.6 \%$ ), and most quiet $(31.1 \%)$. The results also reveal it lacks natural features and is unfamiliar.

On the other hand, the combined Labour, Delivery, Recovery and Postnatal (LDRP) room is regarded as the busiest, most uninviting and noisy room. At the same time, a separate postnatal room/ward provides natural features, is more home like and ranked very high for being quiet (see Table 5-2 ). The initial results suggest an idea of the range of room situations which may influence and could be beneficial to recovery experience and wellbeing during hospital stays.

Table 5-2: Ranking of different postnatal room situations

\begin{tabular}{|c|c|c|c|c|c|c|c|}
\hline \multirow{2}{*}{$\begin{array}{l}\text { Characteristics of } \\
\text { postnatal room }\end{array}$} & \multicolumn{6}{|c|}{ Types of postnatal rooms or wards } & \multirow[t]{2}{*}{ Total } \\
\hline & $\begin{array}{l}\text { Single } \\
\text { postna } \\
\text { tal } \\
\text { room }\end{array}$ & $\begin{array}{l}\text { Combined } \\
\text { (LDRP) } \\
\text { room }\end{array}$ & $\begin{array}{l}\text { Separate } \\
\text { LDR } \\
\text { room }\end{array}$ & $\begin{array}{c}\text { Separate } \\
\text { postnatal } \\
\text { room }\end{array}$ & $\begin{array}{l}\text { Combined } \\
\text { (LDR) } \\
\text { room }\end{array}$ & Other & \\
\hline \multirow[t]{2}{*}{ Restful } & 12 & 1 & 2 & 8 & 1 & 3 & 27 \\
\hline & $44.4 \%$ & $3.7 \%$ & $7.4 \%$ & $29.6 \%$ & $3.7 \%$ & $11.1 \%$ & $100 \%$ \\
\hline \multirow[t]{2}{*}{ Busy } & 0 & 5 & 0 & 0 & 1 & 1 & 7 \\
\hline & $0.0 \%$ & $71.4 \%$ & $0.0 \%$ & $0.0 \%$ & $14.3 \%$ & $14.3 \%$ & $100 \%$ \\
\hline \multirow[t]{2}{*}{ Attractive } & 18 & 0 & 2 & 10 & 0 & 7 & 37 \\
\hline & $48.6 \%$ & $0.0 \%$ & $5.4 \%$ & $27.0 \%$ & $0.0 \%$ & $18.9 \%$ & $100 \%$ \\
\hline \multirow{2}{*}{ Uninviting } & 1 & 5 & 0 & 0 & 0 & 0 & 6 \\
\hline & $16.7 \%$ & $83.3 \%$ & $0.0 \%$ & $0.0 \%$ & $0.0 \%$ & $0.0 \%$ & $100 \%$ \\
\hline \multirow[t]{2}{*}{ Comfortable } & 11 & 0 & 6 & 11 & 1 & 3 & 32 \\
\hline & $34.4 \%$ & $0.0 \%$ & $18.8 \%$ & $34.4 \%$ & $3.1 \%$ & $9.4 \%$ & $100 \%$ \\
\hline \multirow{2}{*}{ Noisy } & 0 & 2 & 1 & 1 & 0 & 0 & 4 \\
\hline & $0.0 \%$ & $50.0 \%$ & $25.0 \%$ & $25.0 \%$ & $0.0 \%$ & $0.0 \%$ & $100 \%$ \\
\hline \multirow{2}{*}{$\begin{array}{l}\text { Having natural } \\
\text { features }\end{array}$} & 4 & 1 & 2 & 9 & 0 & 1 & 17 \\
\hline & $23.5 \%$ & $5.9 \%$ & $11.8 \%$ & $52.9 \%$ & $0.0 \%$ & $5.9 \%$ & $100 \%$ \\
\hline \multirow[t]{2}{*}{ Quiet } & 14 & 0 & 5 & 17 & 2 & 7 & 45 \\
\hline & $31.1 \%$ & $0.0 \%$ & $11.1 \%$ & $37.8 \%$ & $4.4 \%$ & $15.6 \%$ & $100 \%$ \\
\hline \multirow{2}{*}{ Unfamiliar } & 5 & 1 & 3 & 4 & 0 & 4 & 17 \\
\hline & $29.4 \%$ & $5.9 \%$ & $17.6 \%$ & $23.5 \%$ & $0.0 \%$ & $23.5 \%$ & $100 \%$ \\
\hline \multirow{2}{*}{$\begin{array}{l}\text { Lacking natural } \\
\text { features }\end{array}$} & 4 & 2 & 1 & 1 & 0 & 4 & 12 \\
\hline & $33.3 \%$ & $16.7 \%$ & $8.3 \%$ & $8.3 \%$ & $0.0 \%$ & $33.3 \%$ & $100 \%$ \\
\hline \multirow[t]{2}{*}{ Home-like } & 5 & 0 & 1 & 7 & 0 & 1 & 14 \\
\hline & $35.7 \%$ & $0.0 \%$ & $7.1 \%$ & $50.0 \%$ & $0.0 \%$ & $7.1 \%$ & $100 \%$ \\
\hline \multirow[t]{2}{*}{ Total } & 74 & 17 & 23 & 68 & 5 & 31 & 218 \\
\hline & $33.9 \%$ & $7.80 \%$ & $10.6 \%$ & $31.2 \%$ & $2.3 \%$ & $14.2 \%$ & $100 \%$ \\
\hline
\end{tabular}




\subsection{Women's results perception questionnaire survey}

Before proceeding with the data analysis in greater detail, it should be emphasised that a few of the items in the survey questions overlap in other aspects, hence these were moved from their original places in the questionnaire and brought to the appropriate categories for analysis purposes. The affected items include "having family members and partner present during postnatal care which increases the need for a large space" which was moved from "postnatal room features" and taken to "social comfort environment" category (see question number 7 in the women's questionnaire in Appendix B). In addition, the item “.. be able to walk outside and experience the outdoor surroundings" was taken from "perception on interior comfort environment" and added to the "postnatal room features" category (see question number 3.11, 3 in the Women's survey questionnaire in Appendix B).

Responses to the two questions asking women about an alternative postnatal setting (see questions 3.33, 3.34, and 3.35 in the questionnaire in Appendix B) and one open-ended question were analysed to see if women given the opportunity to think "outside of the box" reported or suggested an optimum environment for their recovery experiences and wellbeing. The responses to the two questions and one open-ended question are discussed in Sections 5.8.1.1 - 5.8.1.3.

\subsection{Perception of impact of physical environment on recovery and psychological wellbeing}

Most of the respondents thought the physical postnatal environment had an impact on their postnatal recovery experiences. The large majority of the respondents $93.9 \%(n=199)$ rated the physical postnatal environment as very important, nearly $5 \%$ of the participants $(4.7 \%, \mathrm{n}=10)$ ranked it neither important nor unimportant; and three $(1.4 \%)$ respondents disagreed that it was important (see Figure 5-5). The results reflected that the quality of the physical environment was considered important for the recovery experience and wellbeing of women. 


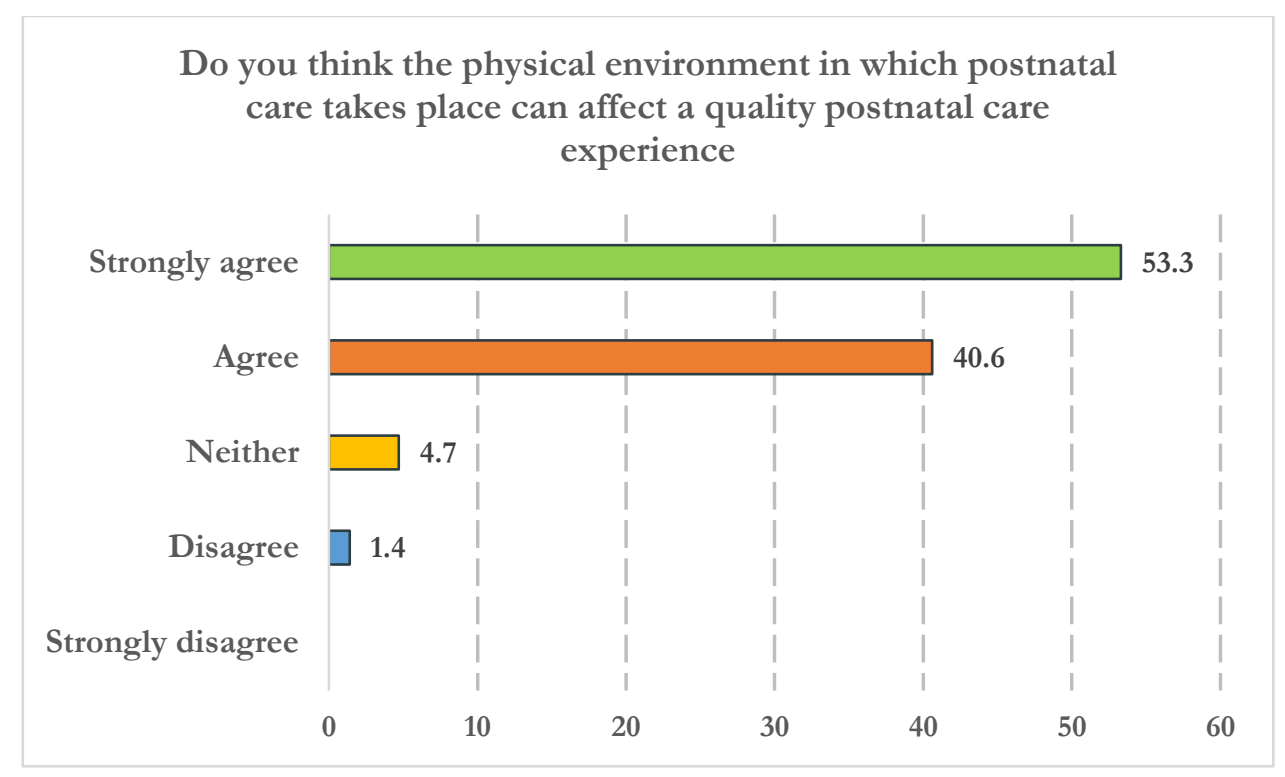

Figure 5-5: Perception on effect of physical environment on wellbeing

\subsection{Perception of postnatal room features}

A total of eight design factors/features on the survey questionnaire (see Appendix B) were rated by the respondents. This section discusses the results of perception of postnatal room features.

\section{What did you think about the room?}

This section of the survey questionnaire builds upon the previous question on perception of physical environment in general. This section focuses on specific aspects of postnatal room features.

\section{Q3.1: A single room is better for a woman's postnatal recovery care}

Most of the respondents thought a single-occupancy room would support women's recovery and wellbeing. Two hundred and nine respondents (98.6\%) the largest group, rated a single-occupancy room as agree or strongly agree respectively. Three (1.4\%) respondents reported neither agree nor disagree. It should be noted that no respondent selected the disagree label (see Figure 5-6).

The result indicated the importance of a single-occupancy room in postnatal healthcare environment. 


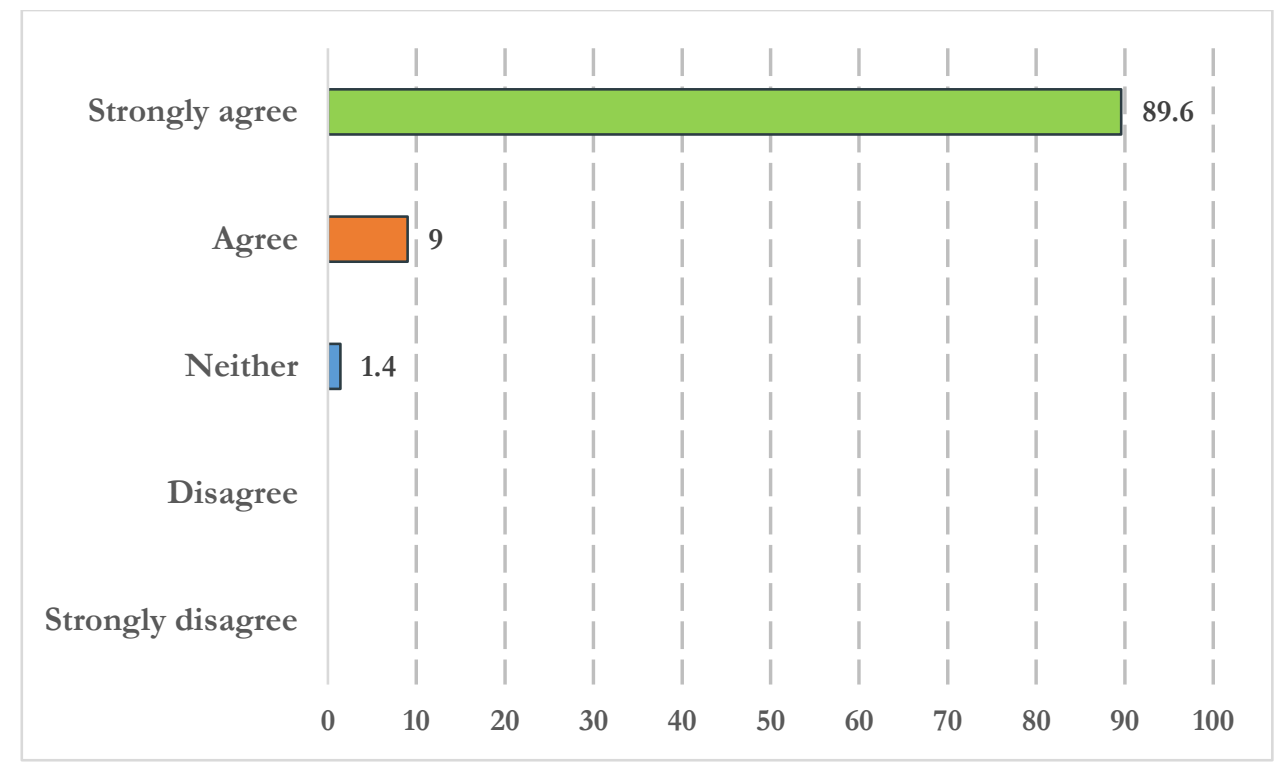

Figure 5-6: Respondents' ratings of a single room for postnatal recovery

\section{Q3.2: A room or ward that is quiet is good for sleep and generally helps in the recovery process}

Most of the respondents perceived that a room which was quiet would support sleep and the recovery process. One hundred and seventy-four (82.1\%) respondents ranked strongly agree, twenty-nine $(13.7 \%)$ respondents selected agree; while four $(1.9 \%)$ of the respondents either ranked strongly disagree or disagree. In addition, five respondents $(2.4 \%)$ selected neither agree nor disagree (see Figure 5-7).

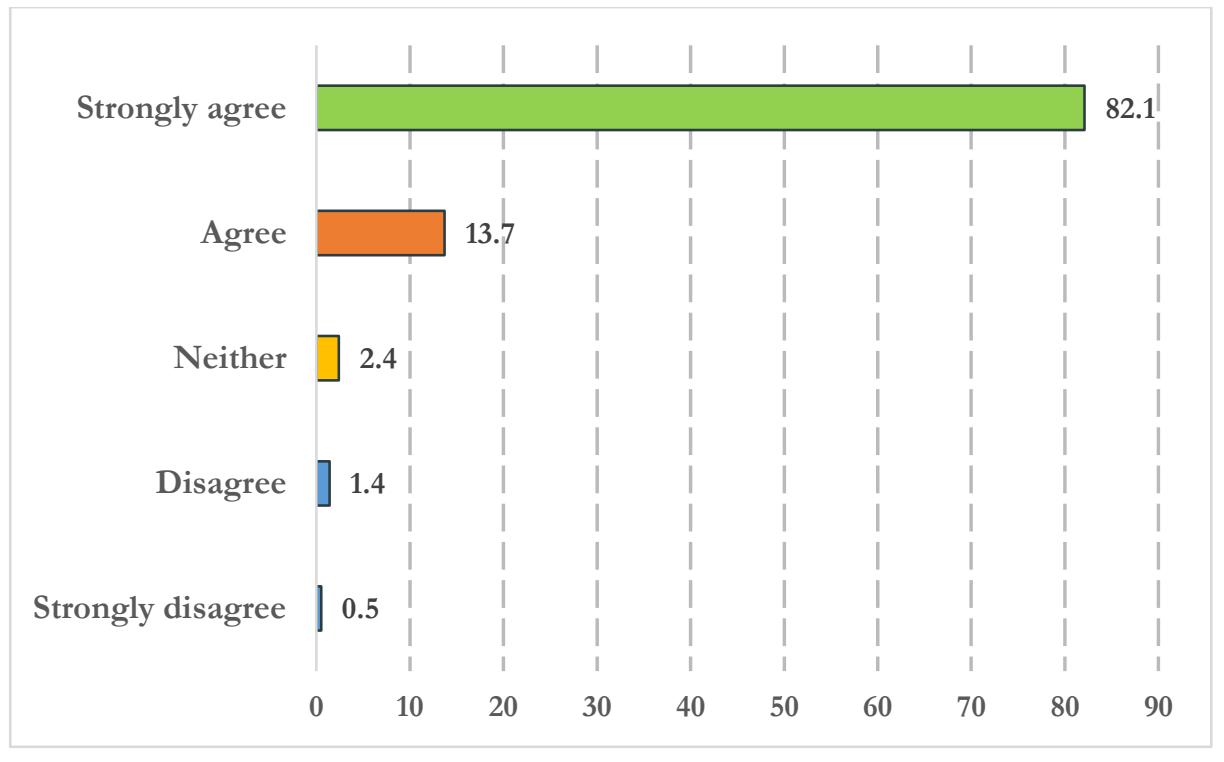

Figure 5-7: Respondents' ratings of a restful room for sleep and recovery process 
The result indicated the value placed on a tranquil environment for the recovery phase of childbirth. The respondents who expressed a contrary opinion are in the minority $(n=9)$ compared with the largest group of respondents who considered that a quiet room was important for recovery and wellbeing.

\section{Q3.3: The postnatal room generally has less impact on how postnatal care is experienced} by women

This question was reverse coded. The perception about the design of the space was rated as strongly disagree by eighty-six (40.6\%) respondents, ninety-two (43.4\%) ranked disagree, and sixteen respondents ranked neither agree nor disagree. On the other hand, eighteen $(8.5 \%)$ of the respondents reported either strongly agree or agree (see Figure 5-8).

The histogram in Figure 5-9 revealed the responses were negatively skewed suggesting that more respondents disagreed that the space has less impact on their recovery experience. The largest group $(84 \%)$ of respondents perceived that space would have impact on their recovery care. The respondents who ranked otherwise are in the small minority of the respondents. Consequently, the result suggests that the quality of the postnatal physical space/environment has an impact on postnatal care and on women's recovery experiences.

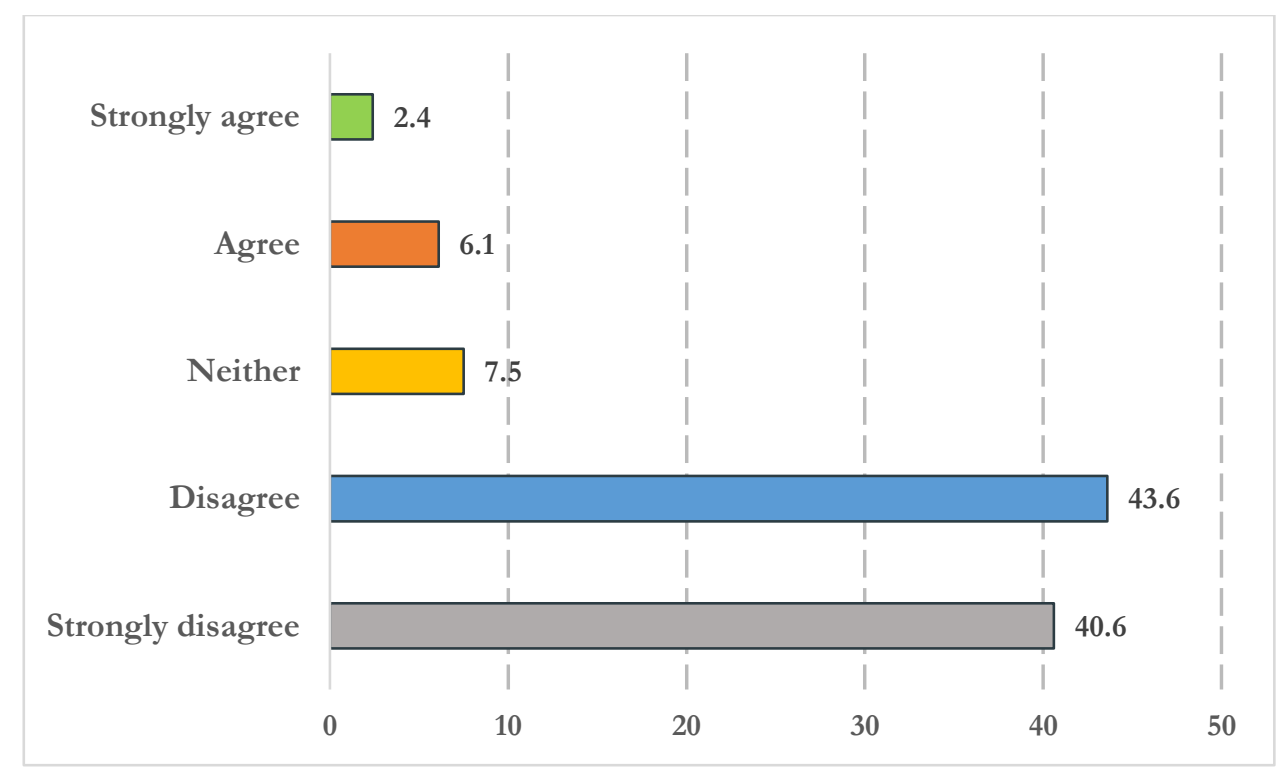

Figure 5-8: Respondents' ratings of design impact of space 


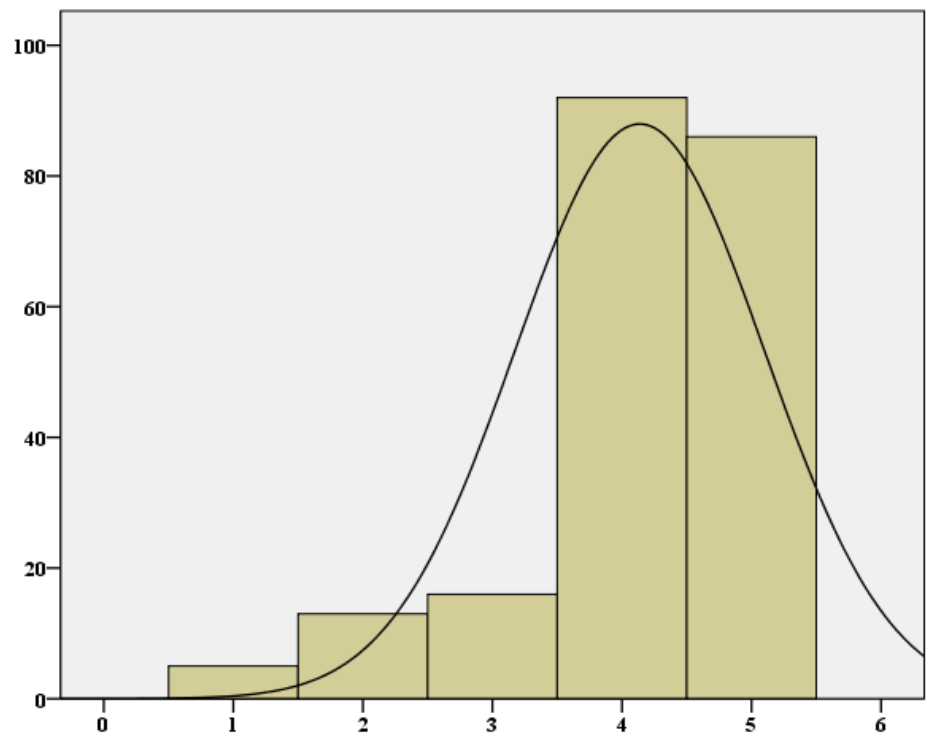

Figure 5-9: Negative skew distribution of ratings of design impact of space

\section{Q3.4: A room with colours is better for recovery than a very plain room}

Respondents who felt that a room with colours is better for birth recovery than a plain room were somewhat in the minority. Seven respondents $(3.3 \%)$ rated a room with colours as strongly agree, fifty one $(24.1 \%)$ respondents selected agree while $58.5 \%(n=124)$ of the respondents rated it as neither agree nor disagree. Twenty-eight $(13.2 \%)$ and two respondents selected disagree and strongly disagree (see Figure 5-10).

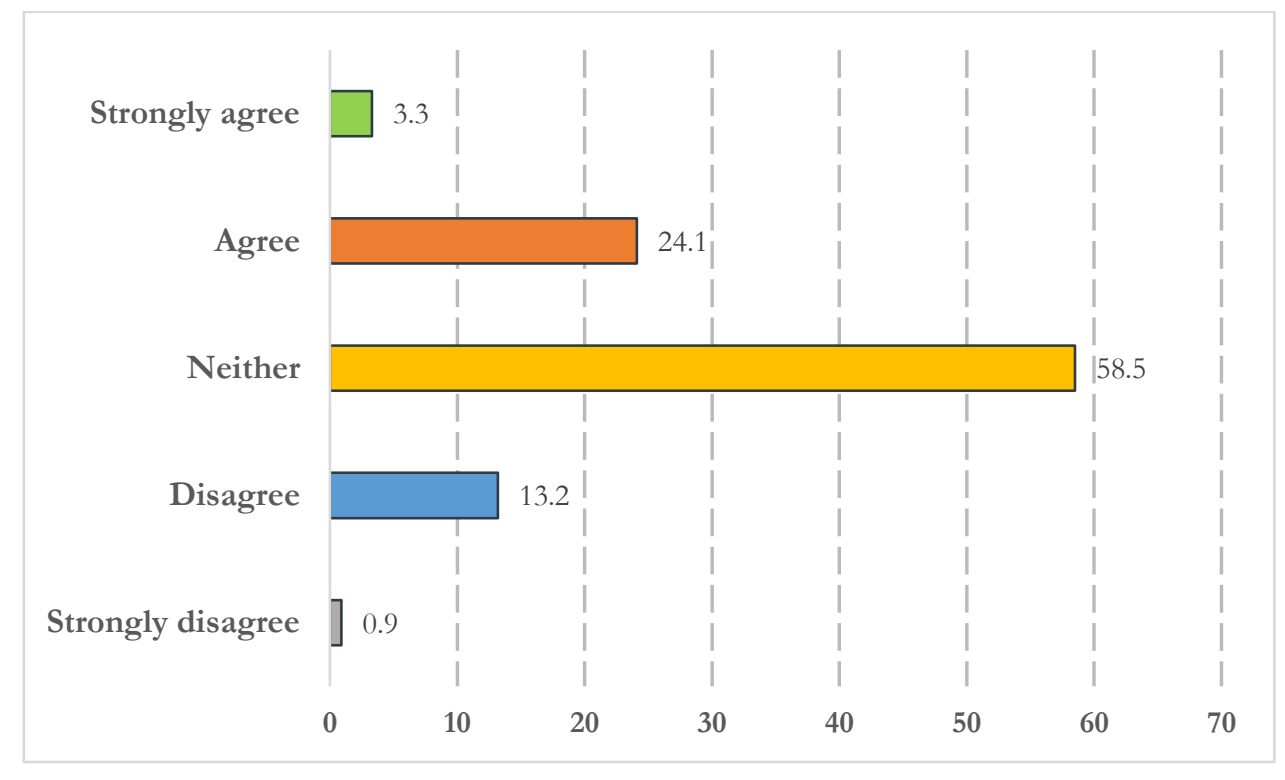

Figure 5-10: Respondents' ratings of a room with colours versus a plain room 


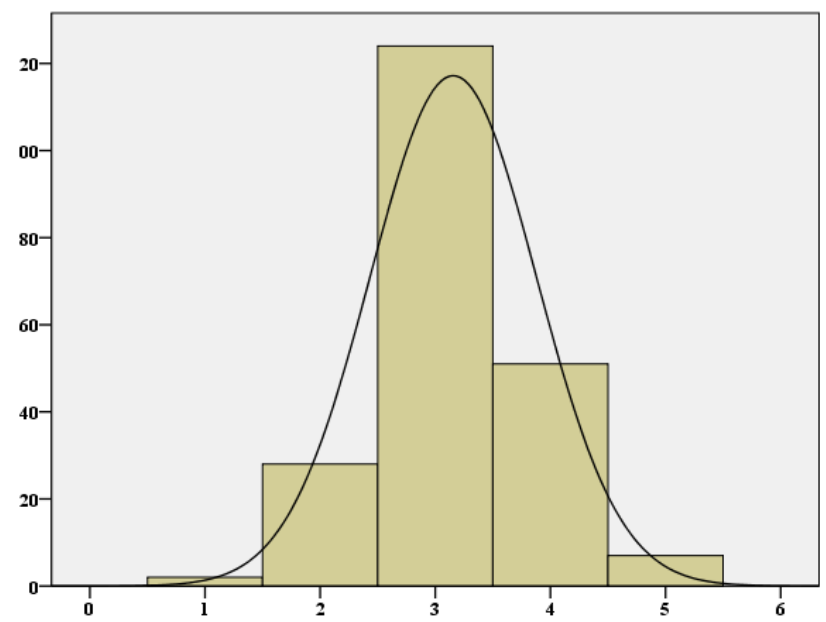

Figure 5-11: Normal distribution of ratings of a room with colours versus a plain room

The result shows a slightly normal distribution among the respondents. The result suggests that colour preferences are more likely to elicit different opinions among people in general. Choice of colours reflects the nature of an individual which varies along the dimensions of gender, age groupings, seasons and cultures. In the next section under the room interiors features, a question was asked of the respondents about colours specifically in order to gain more understanding of colour as a concept and for possible application of colour in the postnatal hospital environment.

\section{Q3.5: Most women prefer to stay in a postnatal room that overlooks a garden, or has pictures of natural scenery on the walls}

Most of the respondents thought a room that has views onto a garden or nature scenery on the walls supported the birth recovery experience. One hundred and fifty nine (75\%) respondents combined rated a room that has opportunity of views on to a garden as strongly agree and agree, five respondents $(2.5 \%)$ as disagree while forty-eight respondents $(22.6 \%)$ selected neither agree nor disagree (see Figure 5-12).

The results indicated that views to nature were seen as an important aspect for the recovery experience. A possible explanation for respondents who reported neither agree nor disagree could be that the rooms in which they were attended had no access to views of nature or there was not opportunity to see the view. Another possible reason could be due to the short length of postnatal stay which reduce the chance to see what is in the outside environment, while a small minority of respondents who disagreed may be likely to have other concerns not related to the physical environment. 


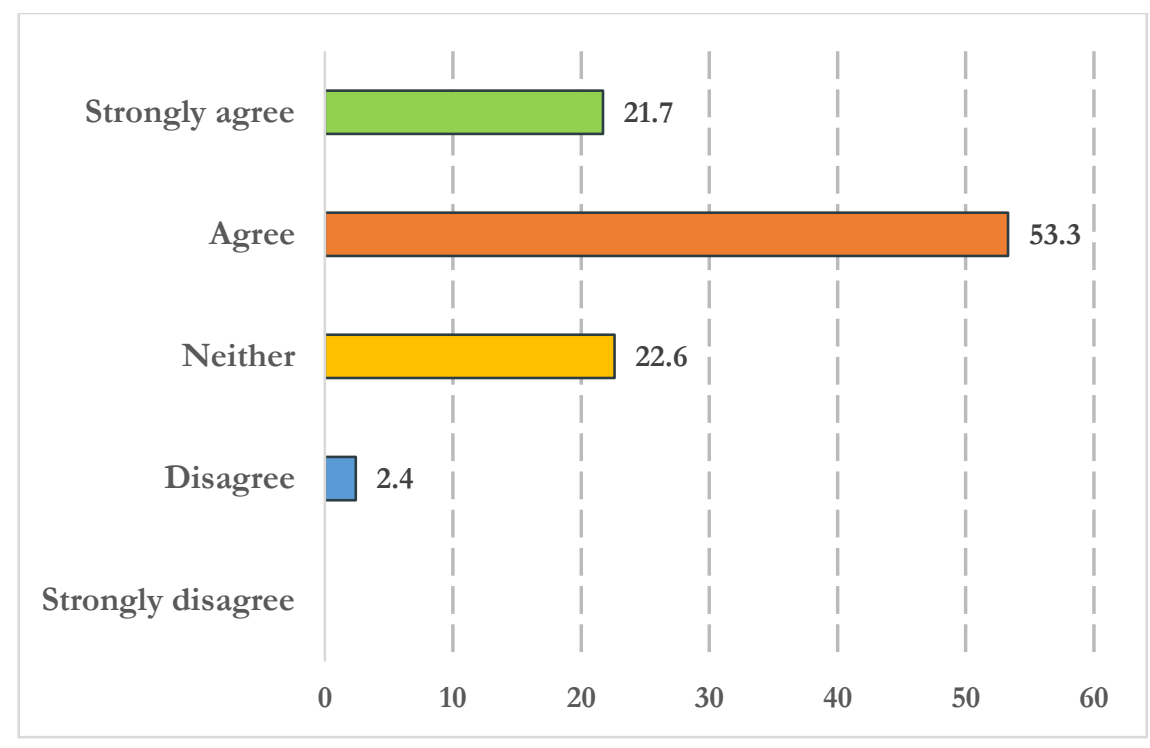

Figure 5-12: Respondents' ratings of a room with views of nature

\section{Q3.6: A postnatal room that lets in natural daylight is better for recovery care}

Most of the respondents reported that natural daylight in the room was better for recovery. A postnatal room with access to natural daylight was rated agree by ninety $(42.5 \%)$ respondents, by one hundred and eleven (52.4\%) respondents as strongly agree while ten respondents (4.7\%) rated neither agree nor disagree. Only one respondent selected disagree (see Figure 5-13).

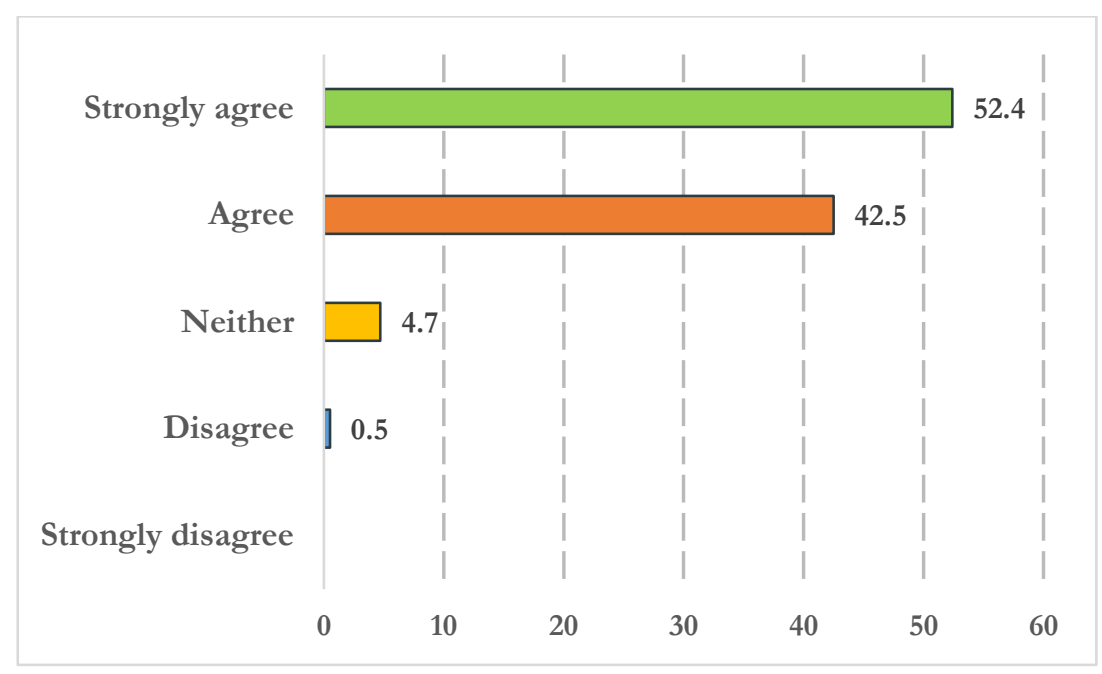

Figure 5-13: Respondents' ratings of a room with daylight access for recovery 
The result reflected the importance of the restorative value of daylight in the healthcare environment. Daylight in the room can have beneficial effects for women to adjust to the rhythmic sensations of day and night.

Q3.7: How important do you think it is to be able to walk outside and experience the outdoor surroundings?

Respondents rated fairly highly their perceptions of walking to experience the outdoor surroundings was. The opportunity to be able to walk to outdoor was ranked as very important by sixty-five respondents (30.7\% in both cases) and another sixty-five respondents rated it moderately important. Fifty-three (25\%) respondents reported somewhat important, nineteen minimally important while ten respondents rated not at all important (see Figure 5-14).

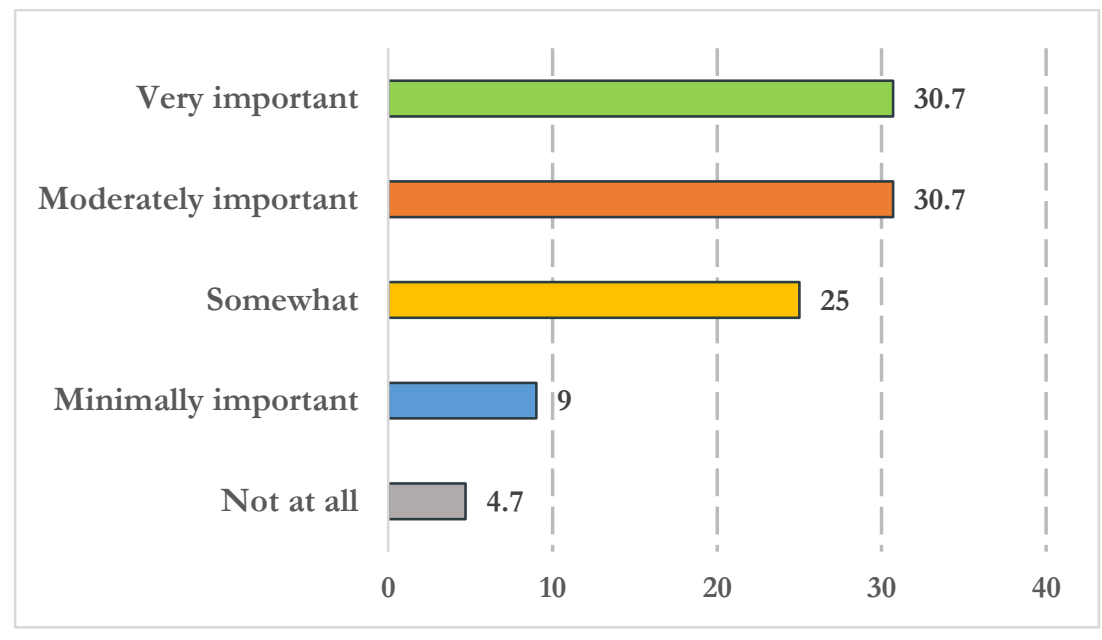

Figure 5-14: Respondents' ratings of ability to walk outside

The result suggested ease of access to the outdoor environment was considered of value in the recovery process. A plausible explanation for a few respondents who reported neither agree nor disagree maybe concerning pain and consequently lack of mobility to go outside. In addition, the hospital for this study required the use of elevators in order to have contact to the outside surroundings.

Q3.8: Shapes and forms like those of natural objects such as flowers, flowing water, etc. can make a more beautiful postnatal room design

The question examined the impact of shapes and forms for creating an appealing postnatal room design. Most of the respondents perceived that natural forms and shapes of objects (flowing water, 
flowers) would make for the design of a more attractive postnatal environment. Thirty-seven $(17.5 \%)$ respondents, selected agree, one hundred and seventeen $(55.2 \%)$ rated strongly agree while fifty-four (25.5\%) respondents selected neither agree nor disagree (see Figure 5-15).

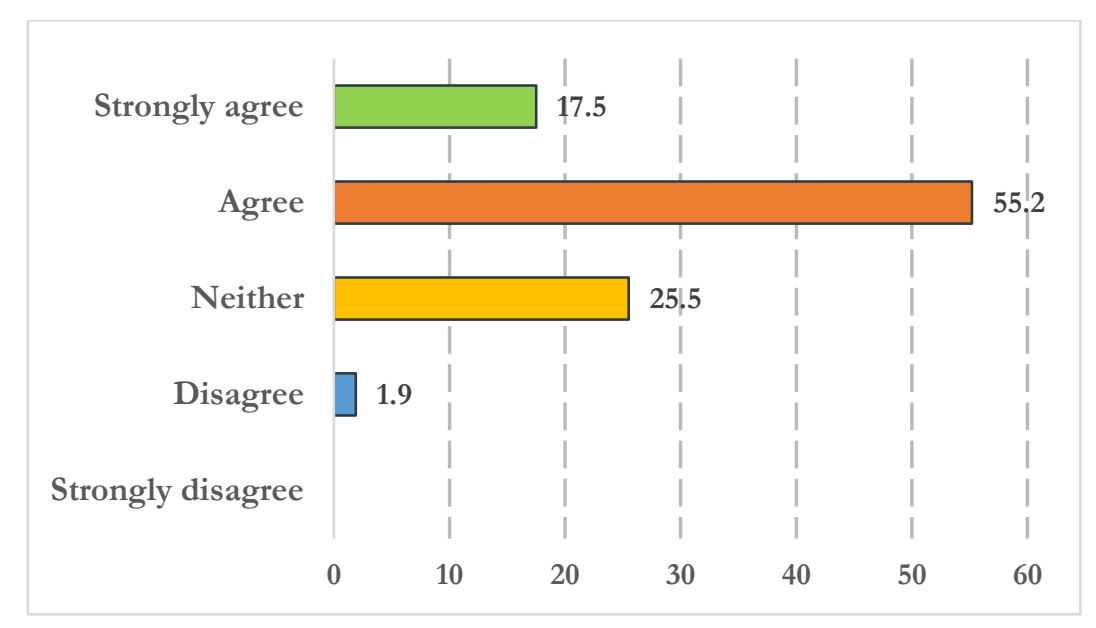

Figure 5-15: Respondents' ratings of shape and natural form for postnatal room design

The result reflected the qualities of a well-designed healthcare environment and the perceptions that women held regarding its impact in their recovery experiences. The few respondents who reported different views maybe concerned about other aspects such as wanting to be in single room accommodation. Another proposition could be that awareness of spatial layout/form could be confounded by other factors (Menke et al., 2019) such as the attitude and supportive role of the midwives, aligning to sources critical of layout and ambience of the physical environment (Seibold et al., 2010). Regardless, the result indicated a considerable acceptance of this design feature for the postnatal environment design. Table 5-3 summarises the results of the descriptive statistics analyses on perceptions of postnatal room features influencing women's recovery experiences and wellbeing. 
Table 5-3: Preference (mean and standard deviation) of respondents for each item on perception of postnatal room features influencing women's recovery and wellbeing (women, $\mathrm{n}$ $=212$ )

\begin{tabular}{llc}
\hline Survey question & M & SD \\
\hline A single room is better for a woman's postnatal recovery care & 4.88 & 0.37 \\
A room or ward that is quiet is good for sleep and generally helps in the recovery & 4.75 & 0.61 \\
process & & \\
Women appreciate a postnatal room in which natural daylight can easily enter & 4.47 & 0.61 \\
The postnatal room generally has less impact on how postnatal care is experienced & 4.14 & 0.96 \\
by women (reverse coded) & & \\
$\begin{array}{l}\text { Most women prefer to stay in a postnatal room that overlooks a garden, or has } \\
\text { pictures of natural scenery on the walls }\end{array}$ & 3.94 & 0.73 \\
$\begin{array}{l}\text { Shapes and forms (natural objects such as flowers, flowing waters, etc.) for } \\
\text { postnatal room design/layout }\end{array}$ & 3.88 & 0.70 \\
$\begin{array}{l}\text { A postnatal room with access to outdoor surroundings is better for care } \\
\text { A room with colours is better for recovery than a very plain room }\end{array}$ & 3.74 & 1.13 \\
\hline
\end{tabular}

Note: $M=$ mean; $S D=$ standard deviation

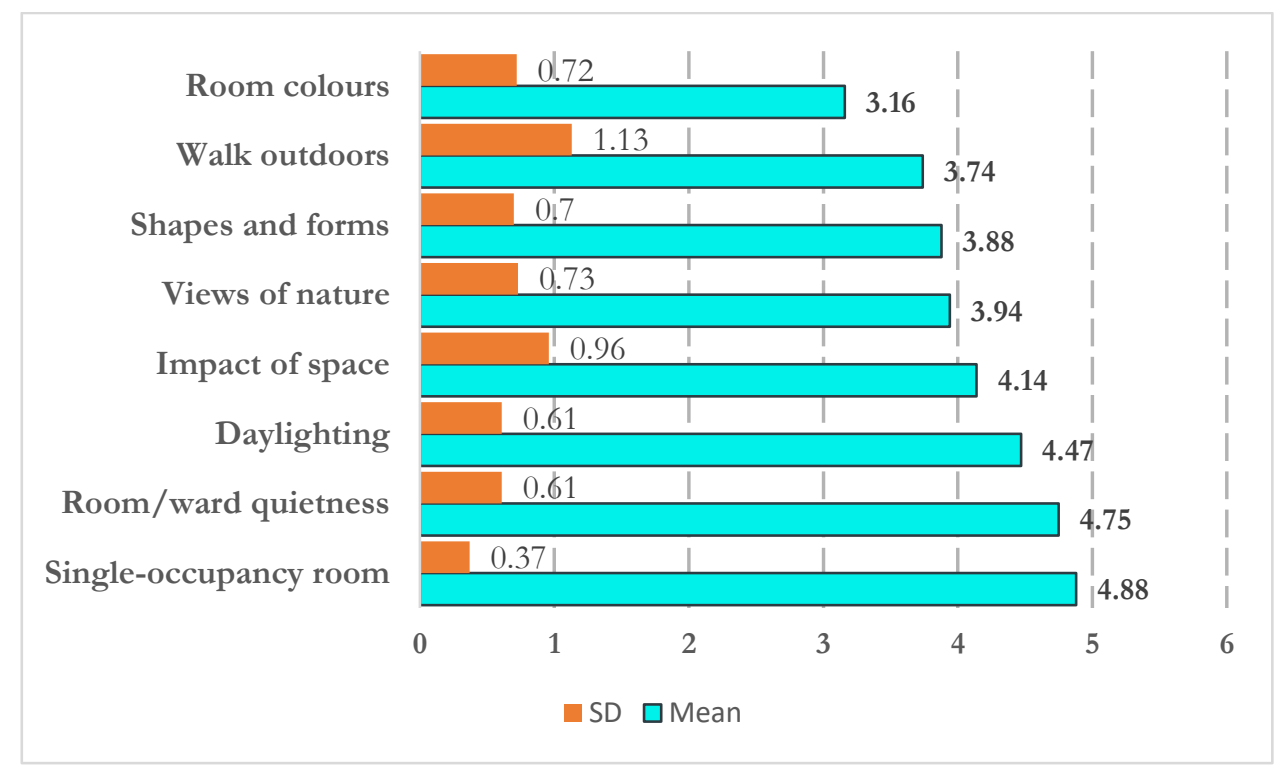

Figure 5-16: Respondents' ratings of postnatal room features

\section{Summary}

Analyses and the results of descriptive statistics for eight aspects on perception of postnatal room design features have been discussed. Two items, a single-occupancy room and a room that is quiet had the highest mean scores. The other factors of natural daylight and overall impact of the space on recovery received mean scores higher than 4 . In addition, a room with views to a garden and shapes and forms for postnatal room design also had higher mean scores of well above 3. A room with colours versus a very plain room had the lowest mean score of 3.16 (see Figure 5-16). 


\subsection{Perception of interior environment features}

This section discusses nine questions focused on interior environment features. As previously stated in Section 4.4 this segment of the questionnaire asked the respondents to select from a range of response options including "not at all", "minimally important", "somewhat", "moderately important" and "very important" (see questions in the questionnaire in Appendix B). However, this set of answer choices does not apply to the lead-in question that will be analysed next. The aim of this was to keep to a consistent set of answer responses for the lead-in questions in the survey generally (see section 5.3.2 and question number 3 in the women questionnaire in Appendix B).

\section{Q3.9: Do you think the interior environment affects postnatal recovery?}

As lead-in to the main survey questions, respondents were asked about the impact of the surroundings on how they recover after giving birth. Most of the respondents thought the interior environment would affect postnatal recovery. Forty seven $(22.2 \%)$ respondents ranked the interior environment for recovery as agree, One hundred and twenty eight (60.4\%) respondents selected strongly agree, while twenty-six (12.3\%) ranked neither agree nor disagree. On the other hand, eleven respondents selected disagree (see Figure 5-17).

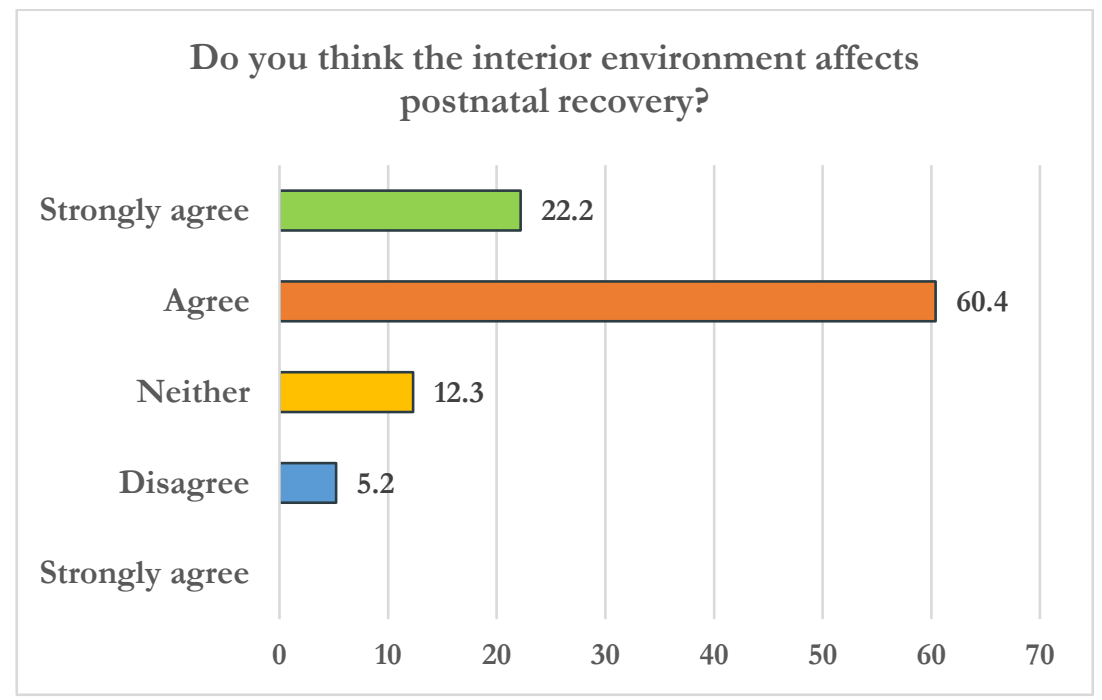

Figure 5-17: Respondents' ratings on perception of interior environment for postnatal recovery 
Given that more than eight out of ten respondents gave a positive response, the result indicated that the quality of the interior physical environment was considered to be of value to postnatal recovery and physical recovery experiences.

\section{Q3.10: During postnatal care how important do you think it is to:}

\section{Q1 Have a restful room}

Most of the respondents thought a restful room would impact on recovery experience. One hundred and ninety three of the respondents $(91 \%)$ rated a restful room as very important, fifteen (7.1\%) rated it moderately important, while only four respondents $(1.9 \%)$ ranked it as somewhat important (see Figure 5-18).

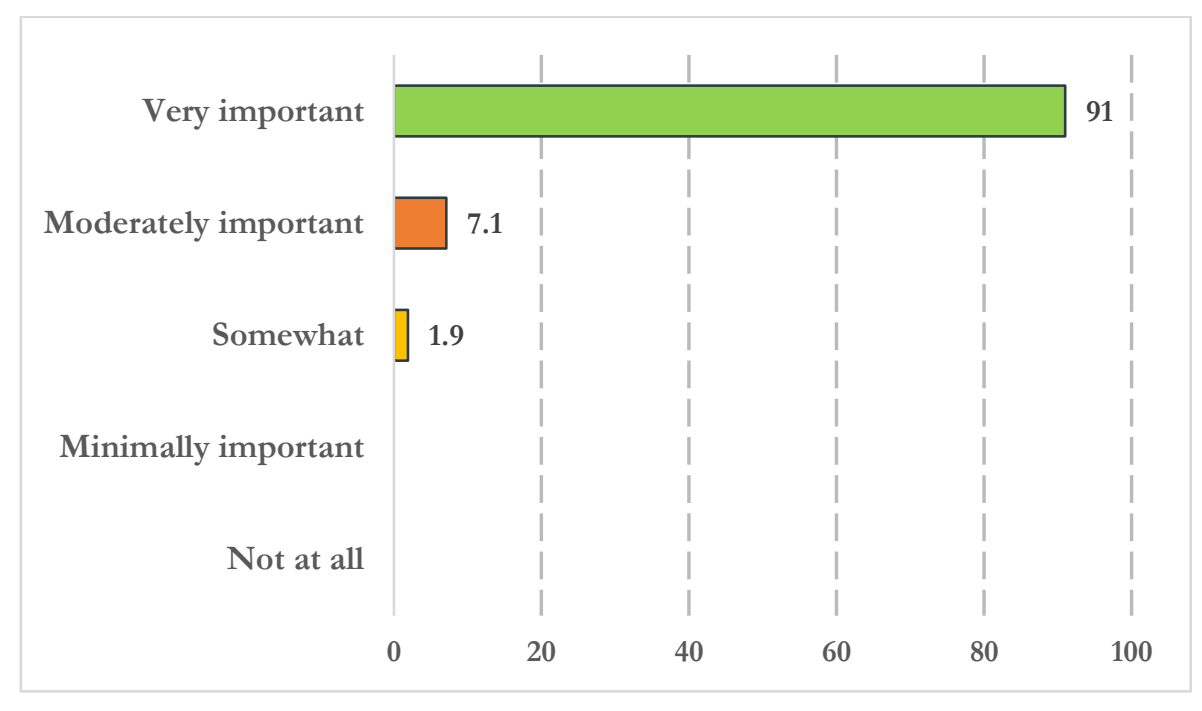

Figure 5-18: Respondents' ratings on perception of a restful room for recovery

The result indicated the perceived importance of a peaceful and tranquil space for recovery and wellbeing of occupants in healthcare physical environment.

\section{Q2 Be looked after in a clinical-looking postnatal space}

Respondents' perception of a clinical-looking space was rated much less favourably. A clinicallooking postnatal space was ranked by fifty-two respondents $(24.5 \%)$ as not at all important, and as minimally important by eighty-seven (41\%) respondents. In addition, thirty eight respondents ranked it as somewhat important (17.9\%). In contrast, seventeen $(8 \%)$ and eighteen $(8.5 \%)$ respondents reported it very important and moderately important (see Figure 5-19). 


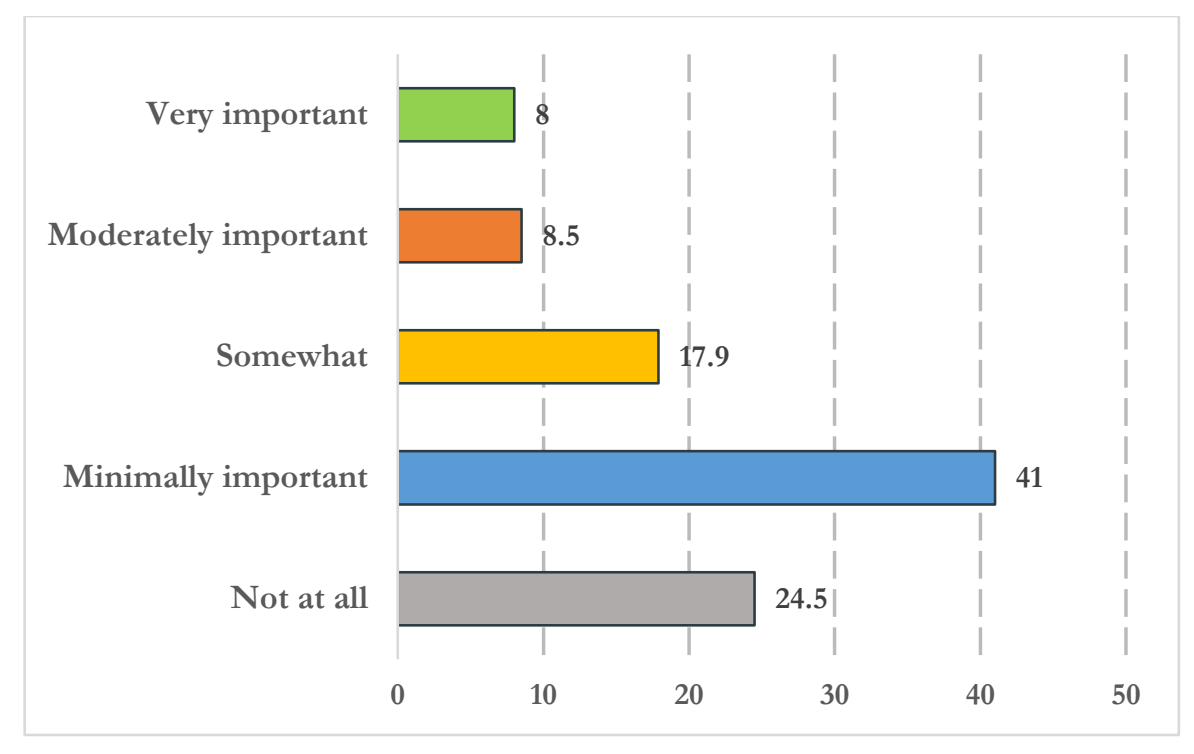

Figure 5-19: Respondents' ratings on perception of a clinical room for recovery

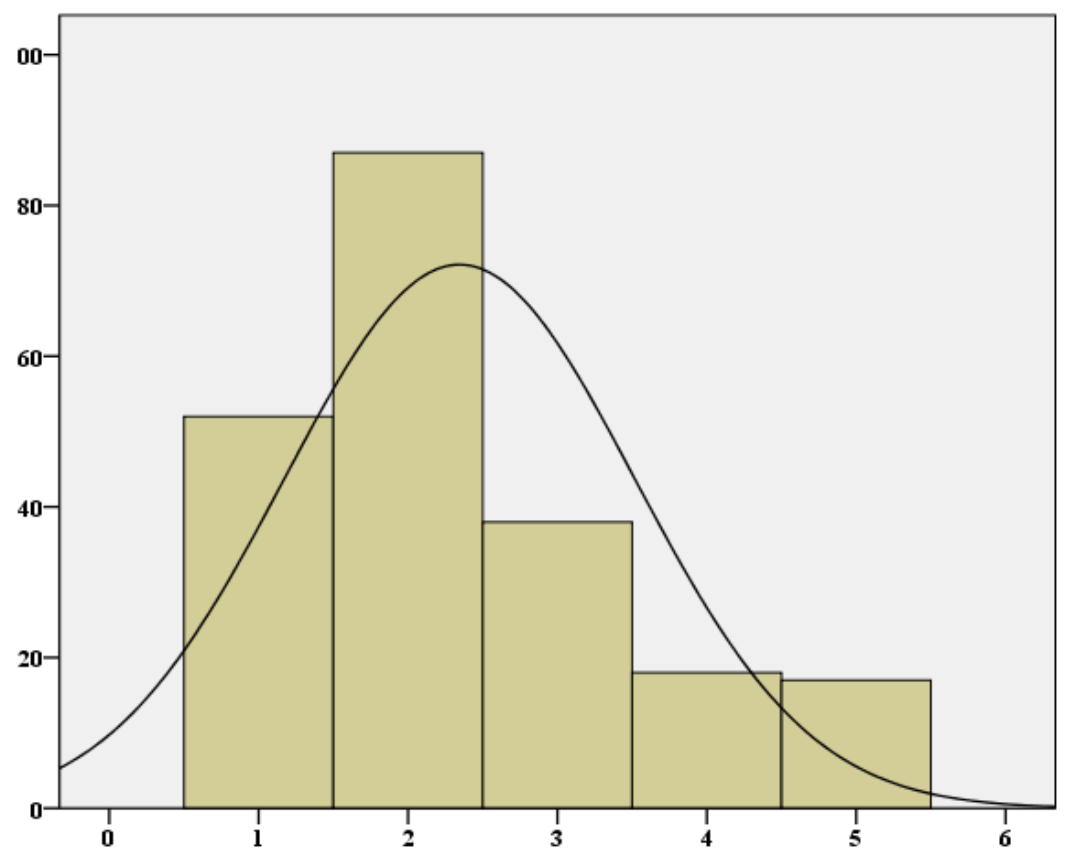

Figure 5-20: Positive skew distribution of ratings of a clinical room for recovery

As can be seen in Figure 5-20, the distribution of the ratings of a clinical-looking room is normal. The distribution is relatively positively skewed towards a fairly large number of the respondents who perceived a clinical room not to be important.

Overall, roughly two-thirds $(\mathrm{n}=139,65.5 \%)$ of the respondents thought a clinical looking space was not important during the recovery phase while just about $17 \%(n=35)$ thought it would have 
an impact on recovery. The responses suggest underlying factors concerning the bio-medical environment and whether having medical equipment in full view is ideal during the postnatal phase.

Q3 Be looked after in a room which has a combination of clinical and familiar home features

Respondents' perception of being attended in a room with a combination of clinical and familiar home features space was ranked fairly high. The perception of being in a room with a combination of clinical and familiar home features was ranked as very important by fifty-two $(24.5 \%)$ respondents, moderately important by seventy $(33 \%)$ respondents and somewhat important by fifty $(23.6 \%)$ respondents. On the other hand, thirty-three (15.6\%) and seven (3.3\%) respondents ranked it minimally important and not at all important (see Figure 5-21).

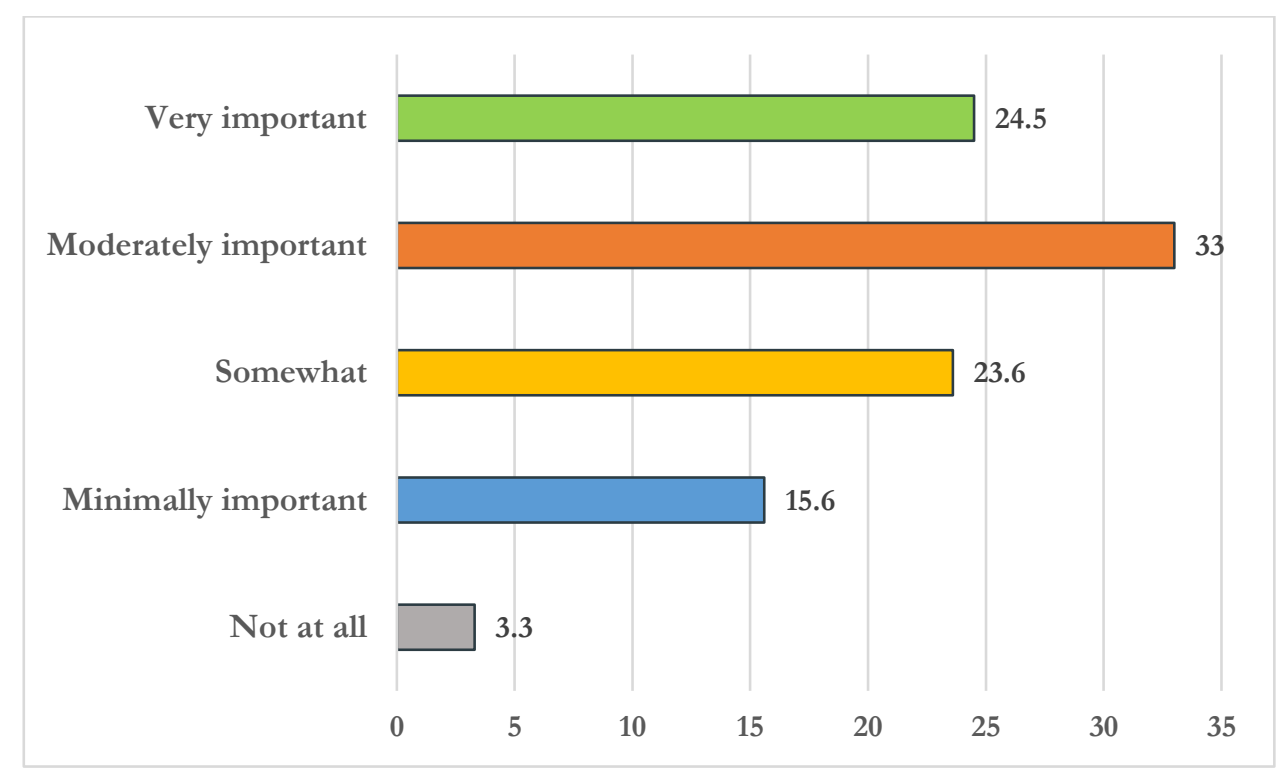

Figure 5-21: Respondents' ratings on perception of a combination of clinical room and home features for recovery 


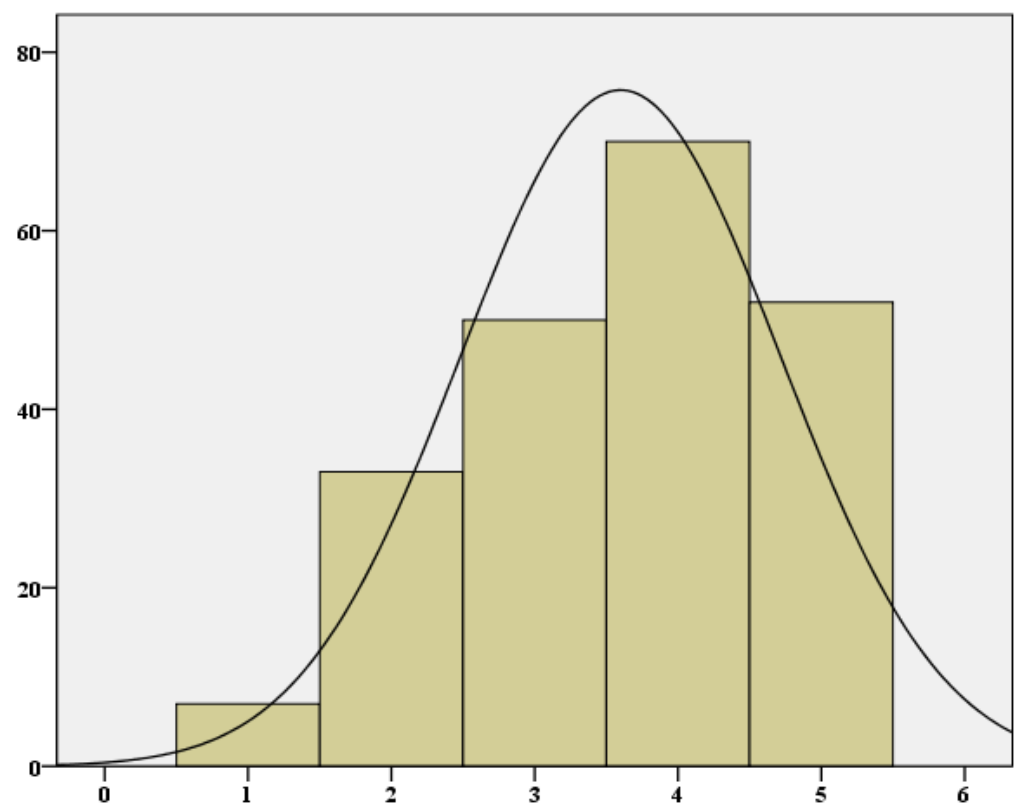

Figure 5-22: A fairly normal distribution of ratings for a combination of clinical and familiar home features for recovery

As can be seen in Figure 5-22, there was a fairly normal distribution among the respondents skewed towards being important. It is reasonable to suggest that although clinical equipment may be necessary, the addition of more familiar home environment features is considered desirable in a room for postnatal recovery for wellbeing.

\section{Q4 Be in a room or ward that is decorated with natural art, images or paintings}

Respondents' perception of being looked after in a room or ward decorated with natural art, images or paintings was rated moderately. The perception of a room with natural art was selected as not at all important by eighteen (8.5\%), by fifty-one as minimally important, and as somewhat important by seventy-nine (37.3\%) respondents. On the other hand, this feature was ranked as very important by fifteen $(7.1 \%)$ and as moderately important by forty-nine $(23.1 \%)$ respondents respectively (see Figure 5-23) 


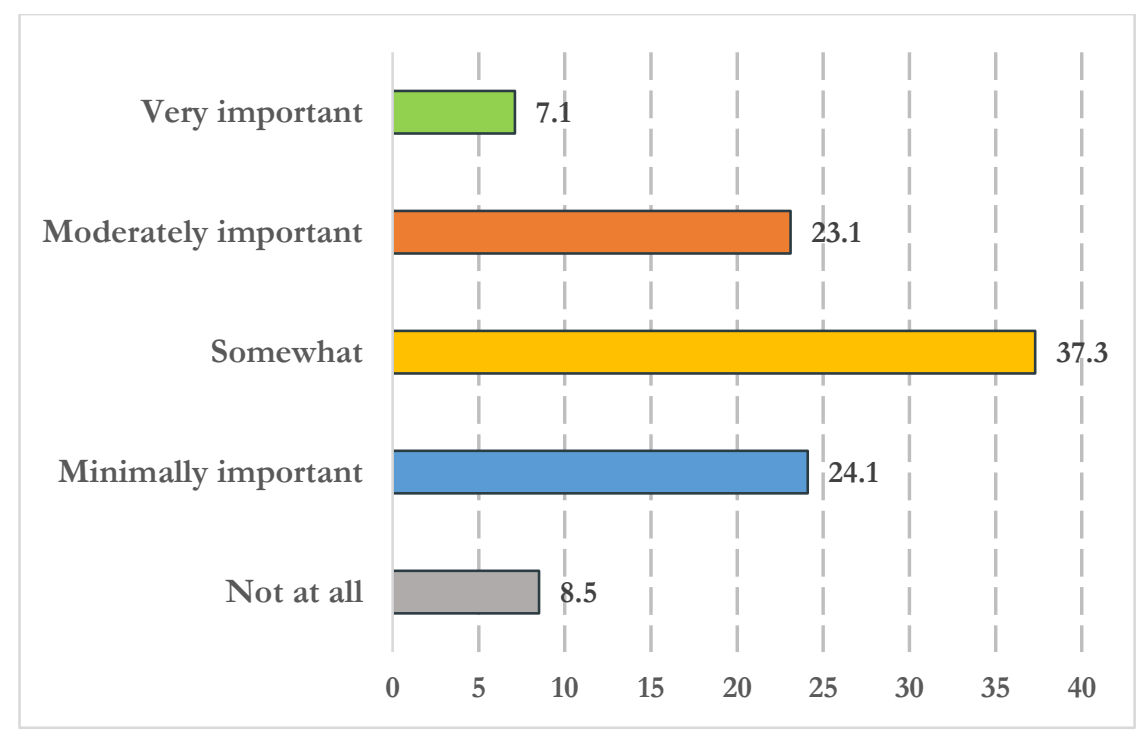

Figure 5-23: Respondents' ratings on perception of decoration with art/images in the room for recovery

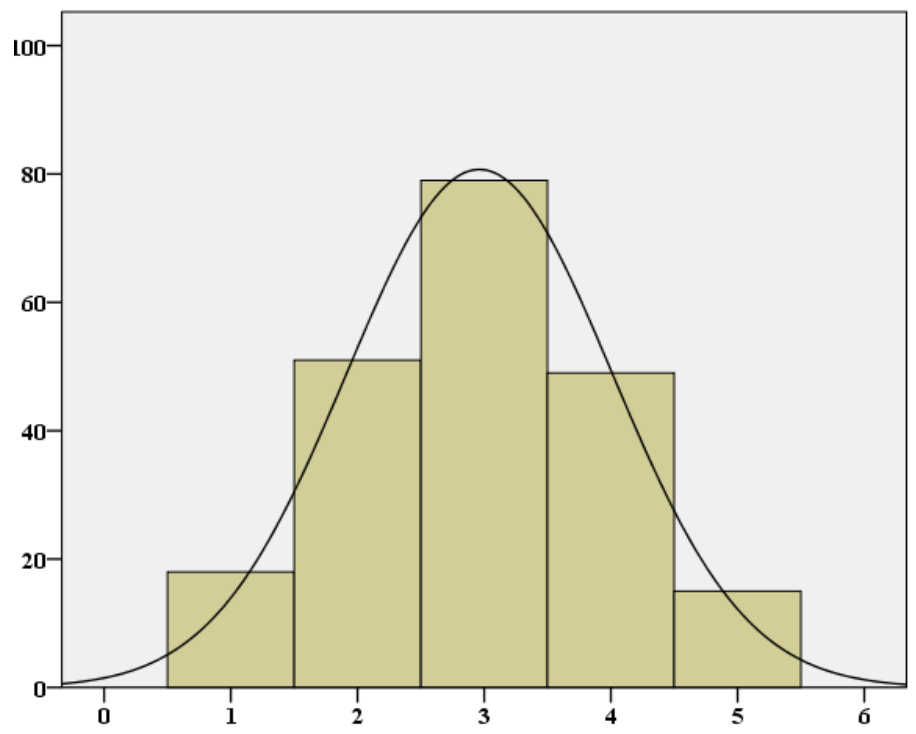

Figure 5-24: A normal distribution of ratings of decoration with natural art, images or painting for recovery

The result reflected a uniform distribution of opinions. Respondents may not necessarily reject having a room or ward decorated with natural art or images or paintings since it could have positive effects on the atmosphere and could enhance a feeling of calm and restorative environment, but they do not see it as a priority. 


\section{Q5 Have bright colours in the room}

As was highlighted (see question number 3.4 under the heading of postnatal room features), colour was revisited as an interior-related component of a room. In this section, the question was specific to bright colours in the room; unlike the previous question about a room with colours versus a plain room. Respondents' perception of having bright colours in the room was rated as not at all important by fifty-three (25\%) respondents, as minimally important by eighty-four (39.6\%), and as somewhat important by fifty-eight (27.4\%) respondents. By contrast, bright colours in the room was rated as moderately and very important by seventeen (8\%) respondents combined (see Figure $5-25)$.

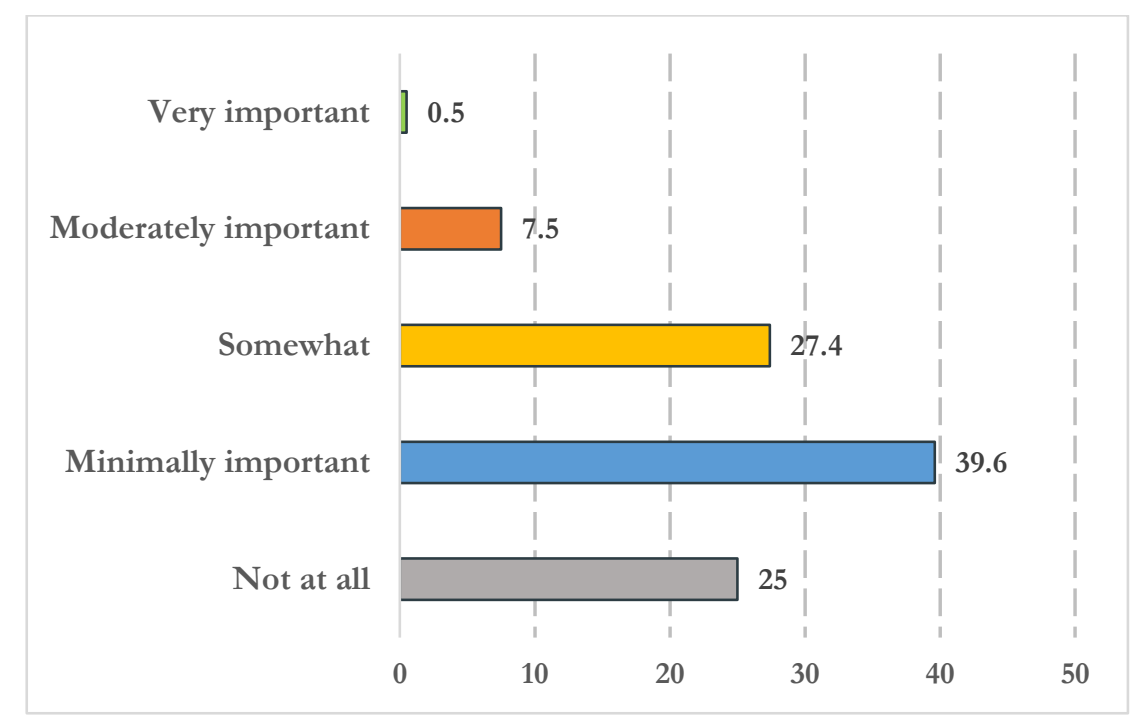

Figure 5-25: Respondents' ratings on perception of bright colours in the room for recovery

As Figure 5-26 shows, the result was slightly positively skewed towards the few respondents that liked a bright coloured room. However, the largest group seems indifferent to bright colours for postnatal rooms. Indeed as birth is an emotional event with attendant physiological and psychological impacts on women, having a room with bright colours might serve to trigger or increase anxiety. 


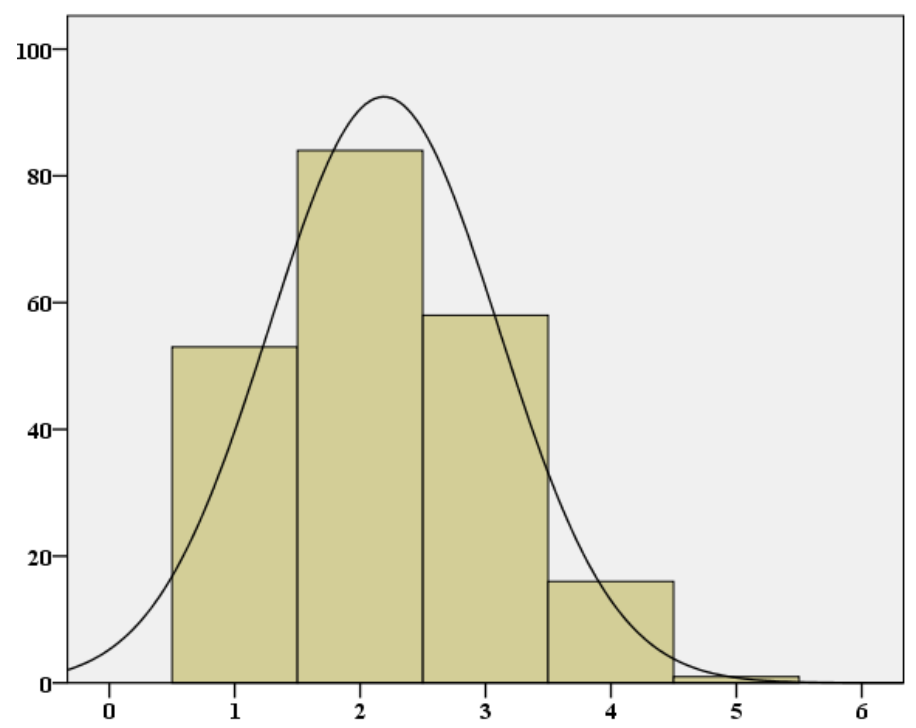

Figure 5-26: A somewhat negatively skewed distribution of ratings of bright colours for recovery

The choice made by especially the 137 respondents who reported not at all and minimally important, indicates a need for further research to better understand what colours could be appropriate for the postnatal hospital environment.

\section{Q3.11: In addition, how important do you think it is to:}

\section{Q6 Have a clean room}

Most of the respondents thought having a clean room would impact positively on the recovery process. One hundred and ninety-nine (93.9\%) respondents ranked a clean room as very important, twelve $(5.7 \%)$ respondents ranked it as moderately important and one $(0.5 \%)$ respondent reported somewhat important (see Figure 5-27).

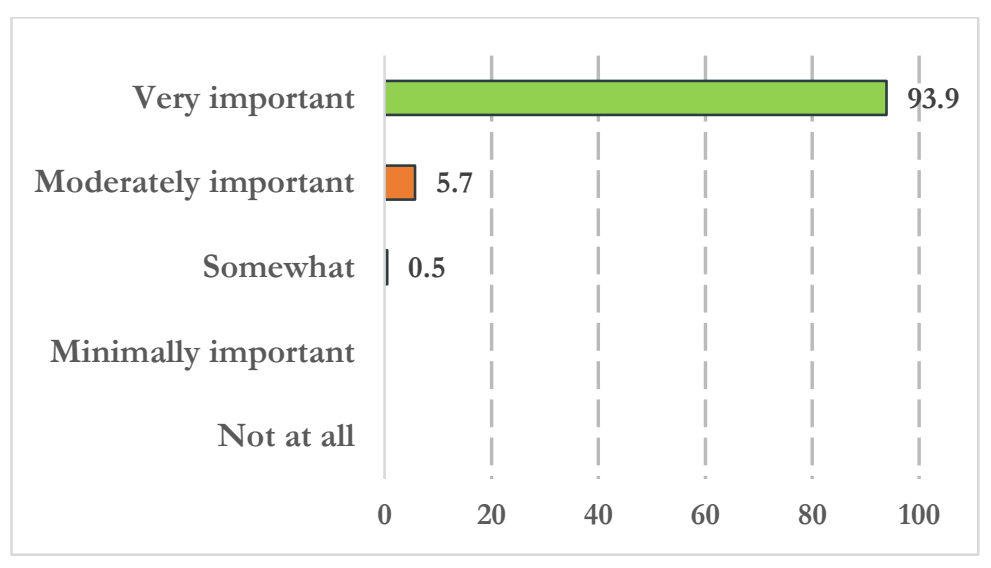

Figure 5-27: Respondents' ratings of a clean room for recovery 
The result indicated the great perceived importance of a hygienic environment in the postnatal recovery process.

\section{Q7 Have a room with furniture made of natural materials}

Respondents' perception of a room made of natural materials was rated moderately. The perception of a room with furniture made of natural materials was rated as not all important by twenty $(9.4 \%)$ respondents, by fifty-six $(26.4 \%)$ as minimally important, and as somewhat important by seventy-seven (36.3\%) respondents. On the other hand, forty-four $(20.8 \%)$ respondents ranked it as moderately important and it was seen as very important by fifteen $(7.1 \%)$ respondents (see Figure 5-28).

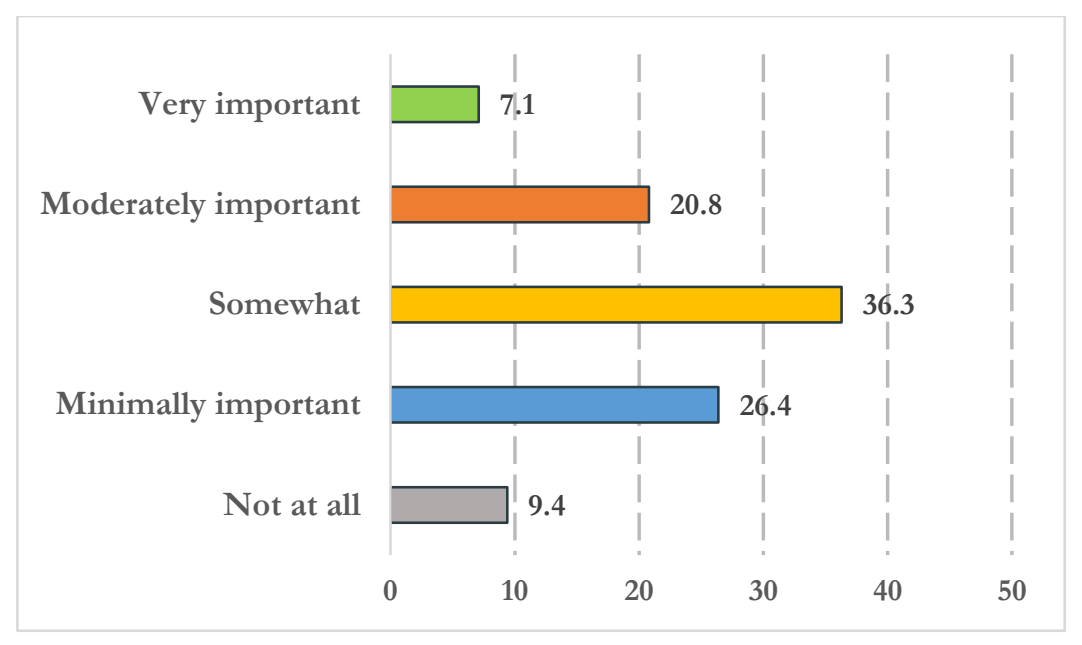

Figure 5-28: Respondents' ratings of a room with furniture made of natural materials

The result reflected a uniform distribution of opinions and is very similar to the question on perception of decoration with natural art and images (see question number 4). It is reasonable to assume that respondents may not totally reject having a room with furniture made of natural materials. However, since this question was not specific about whether respondents would like furniture in the room for certain tasks it is reasonable to assume specificity of furniture may need to be explored in future research.

Q8 Know there is a protective corner in the room that you can withdraw into with your baby

Most of the respondents thought a protective corner in the room which one can withdraw into would support recovery and wellbeing. Eighty six (40.6\%) respondents rated this aspect of space as very important, fifty-nine $(27.8 \%)$ rated it as moderately important while forty-two $(19.8 \%)$ 
respondents reported somewhat important. Five $(2.4 \%)$ and twenty $(9.4 \%)$ respondents said not all important and minimally important respectively (see Figure 5-29).

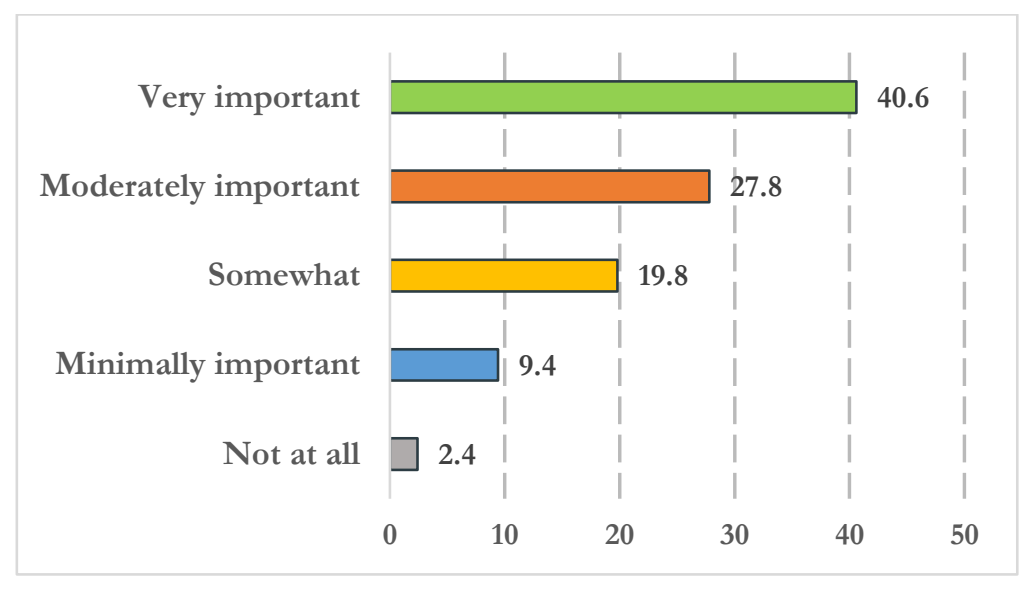

Figure 5-29: Respondents' ratings of a room regarding a protective corner

The result indicated the value of having a designed space within the larger room into which a woman and baby could go at times to find a heightened sense of security and being away from things.

\section{Q9 Be in a room where the door is closed except when visitors are allowed in}

Most of the respondents thought a room where the door is kept closed would support privacy and wellbeing. One hundred and twenty eight $(60.4 \%)$ respondents ranked a room that had door closed for privacy as very important, fifty-seven $(26.9 \%)$ ranked it moderately important while eighteen $(8.5 \%)$ respondents said somewhat important. On the other hand, one $(0.5 \%)$ respondent and eight respondents reported not at all important or minimally important respectively (see Figure 530).

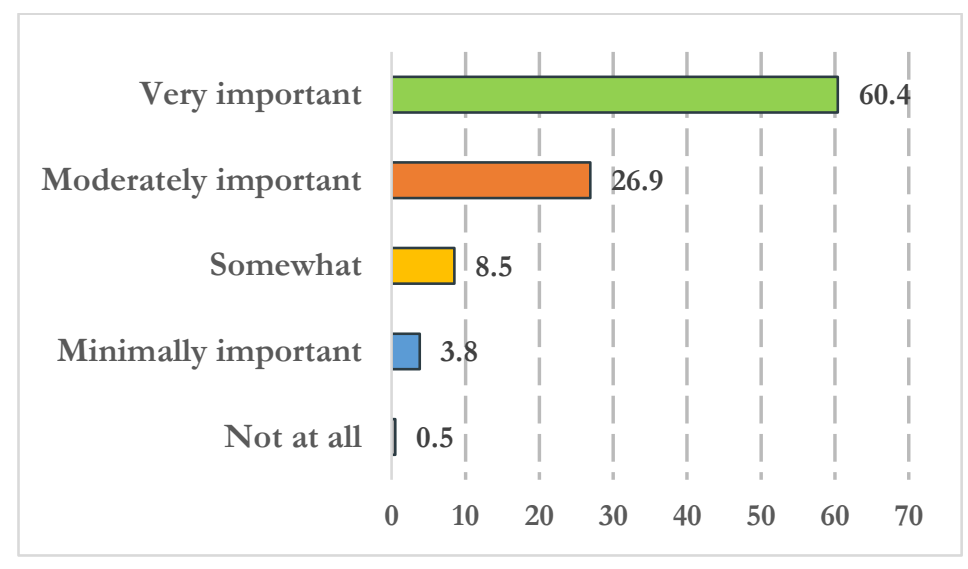

Figure 5-30: Respondents' ratings of a room with door closed for privacy 
The result reflected the behavioural importance of a physical healthcare environment that supports privacy and confidentiality for women's feeling of wellbeing.

The results of the descriptive statistics for the interior environment features are shown in Table 54.

Table 5-4: Preference (mean and standard deviation) of respondents on perception of interior environment features influencing women's recovery and wellbeing $(\mathrm{n}=212)$

\begin{tabular}{llc}
\hline Survey question & M & $\boldsymbol{S D}$ \\
\hline Have a clean room & 4.93 & 0.23 \\
Have a restful room & 4.89 & 0.37 \\
Be in a room where the door is closed except when visitors are allowed in(privacy) & 4.43 & 0.84 \\
Know there is a protective corner in the room that you can withdraw into with & 3.95 & 1.09 \\
your baby & & \\
Be looked after in a room which has a combination of clinical and familiar home & 3.60 & 1.12 \\
features & & \\
Be in a room or ward that is decorated with natural art, images or paintings & 2.96 & 1.05 \\
Have a room with furniture made of natural materials & 2.90 & 1.09 \\
Be looked after in a clinical-looking postnatal space & 2.34 & 1.17 \\
Have bright colours in the room & 2.19 & 0.91 \\
\hline
\end{tabular}

Note: $M=$ mean; $S D=$ standard deviation

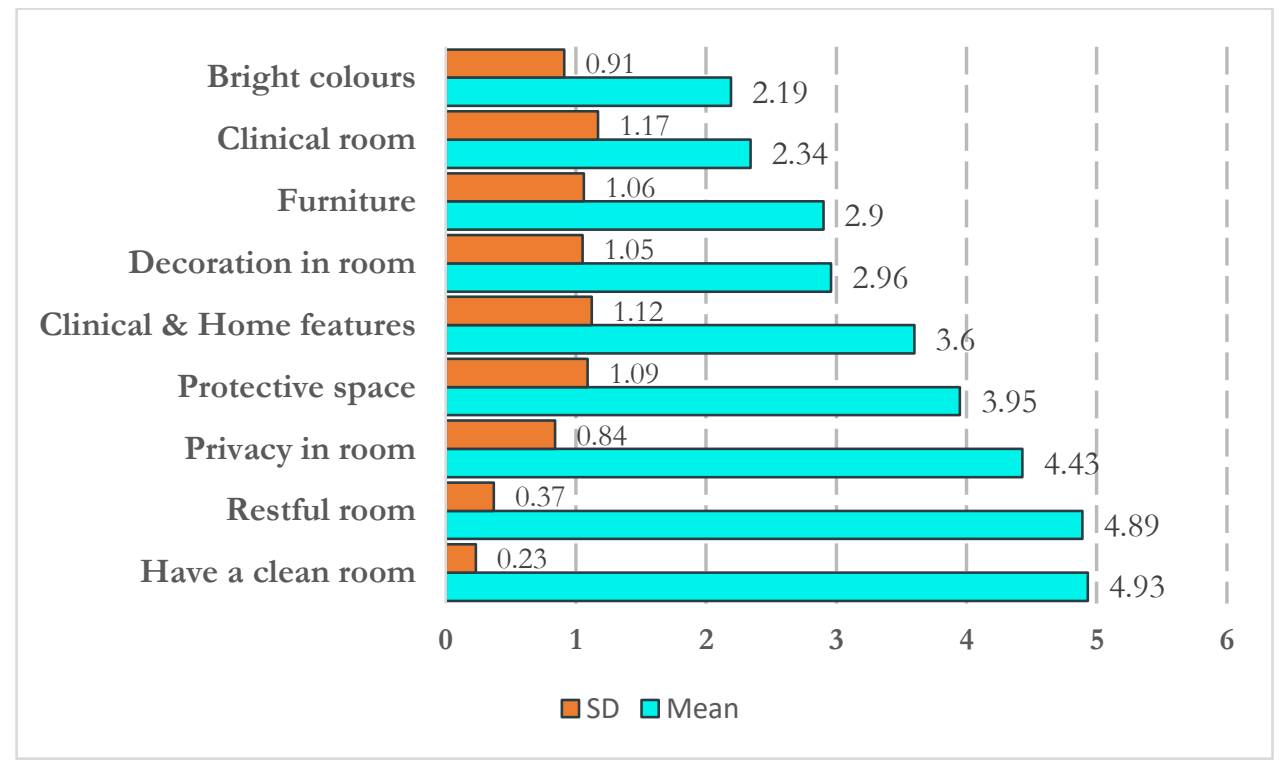

Figure 5-31: Respondents' ratings of interior environment design factors for recovery experience 


\section{Summary}

This section of the analysis has documented the results of descriptive statistics of nine interior environment features. As can be seen from Figure 5-31, the results show that room cleanliness (mean $=4.98, S D=0.14)$ received the highest mean score. Two other design factors, a restful room (mean $=4.93, S D=0.23)$ and closing of door for privacy (mean $=4.43, S D=0.84)$ also received mean scores higher than 4 . In addition, having a protective corner in the room and a combination of clinical-looking and home-like room/ward had mean scores above 3 indicative of their acceptance (neutral). The lower ranked items are decoration in the room with natural art and images, a room with furniture made of natural materials and a clinical-looking room, with bright colours being the lowest rated.

\subsection{Perception of sensory comfort environment}

This section addresses questions focused on a range of questions about comfort provided by the physical environment such as daylight, temperature, air quality and room freshness, and environmental noise (see questions in the heading or in the women's questionnaire in Appendix B) to see how each of these design factors helped respondents in their restoration and wellbeing experience while in postnatal hospital stays.

\section{Q3.12 How important were these features to your feeling of wellbeing during the postnatal period?}

\section{Q1. Having adequate daylighting in your room or ward}

Most of the respondents thought having adequate daylighting in the room or ward supported the feeling of wellbeing. One hundred and sixteen (54.7\%) rated having adequate daylight for wellbeing as very important, seventy-four (34.9\%) ranked it moderately important, and nineteen $(9 \%)$ respondents reported somewhat important. Only three respondents $(1.4 \%)$ ranked it either not at all or minimally important (see Figure 5-32).

The result indicates that daylighting is considered of value for the feeling of wellbeing in postnatal hospital stays. The few respondents who reported somewhat important or not at all may have had limited contact with the windows and therefore, could not be able to experience daylighting access or maybe had given birth in the night and spent little time in hospital before the effect of daylight could be discerned. 


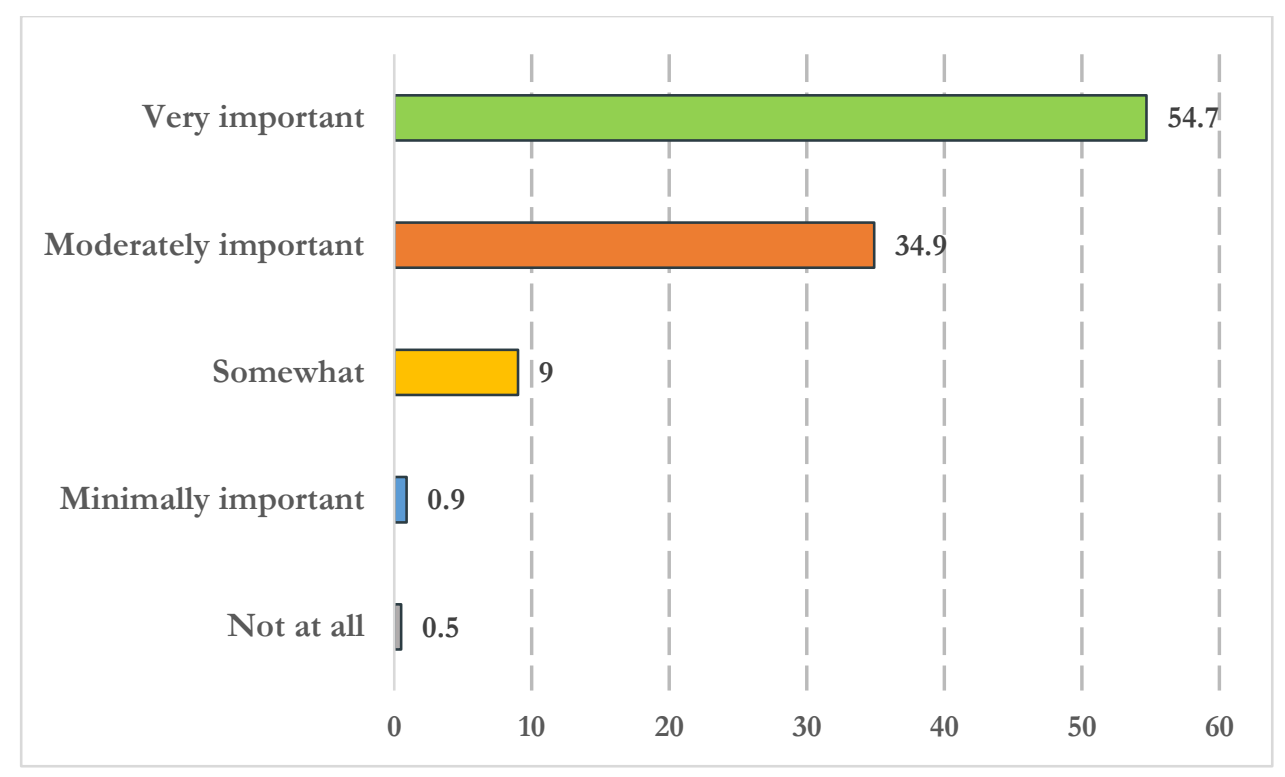

Figure 5-32: Respondents' ratings of adequate daylighting for wellbeing

\section{Q2. Being able to control the temperature}

Most of the respondents perceived being able to control the temperature would impact their feeling of wellbeing. One hundred (47.2\%) rated ability to control the temperature in the physical environment's support for wellbeing as very important, seventy-six (35.8\%) ranked it moderately important while twenty-six $(12.3 \%)$ respondents ranked it somewhat important. One $(0.5 \%)$ respondent, and nine respondents $(4.2 \%)$ ranked it either not at all or minimally important respectively (see Figure 5-33).

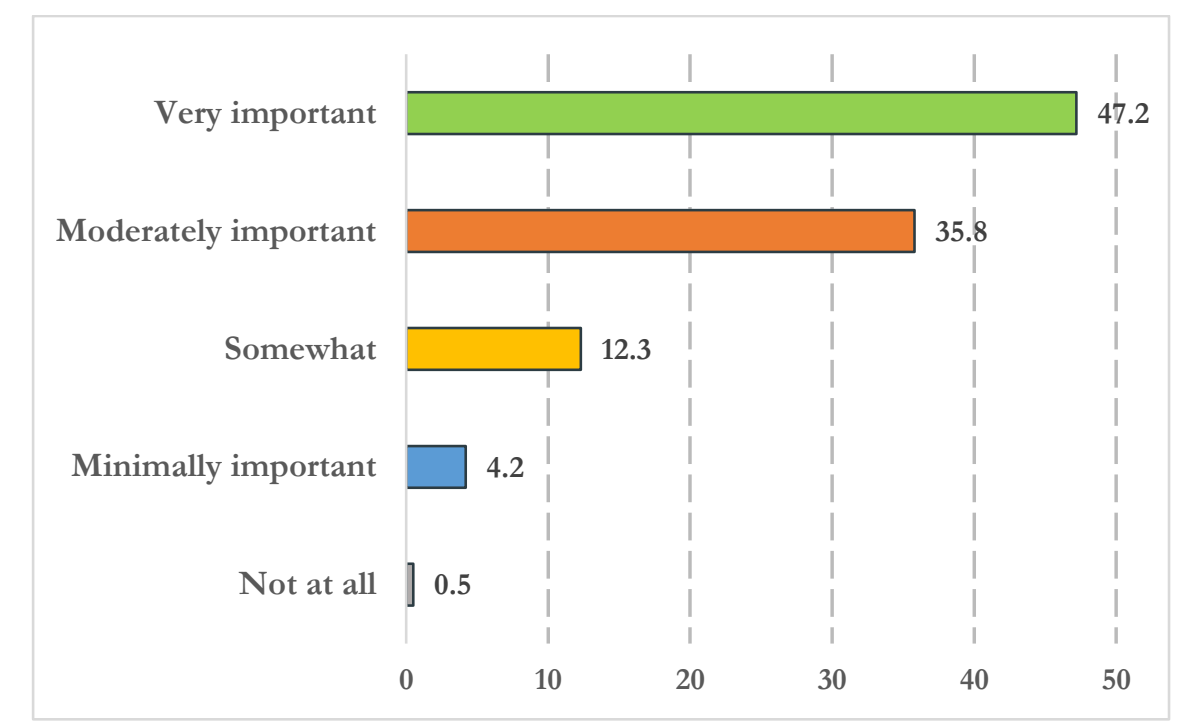

Figure 5-33: Respondents' ratings of temperature control for wellbeing 
In many respects, there are similarities in the results on temperature control with adequate daylighting. The result reflects the value of providing opportunities to support personal control and independence in the postnatal hospital environment. The large majority seem to appreciate the ability to be in control of the physical environment.

\section{Q3. Being able to control noise from mechanical things like ventilation, medical equipment etc.}

Respondents' perception of being able to control noise from mechanical and ventilation equipment was ranked highly. Eighty six respondents (40.6\%) rated the opportunity to control noise as very important, seventy-three (34.4\%) reported it moderately important, and thirty-five $(16.5 \%)$ respondents said somewhat important. On the other hand, eighteen (8.5\%) respondents ranked it not at all or minimally important (see Figure 5-34).

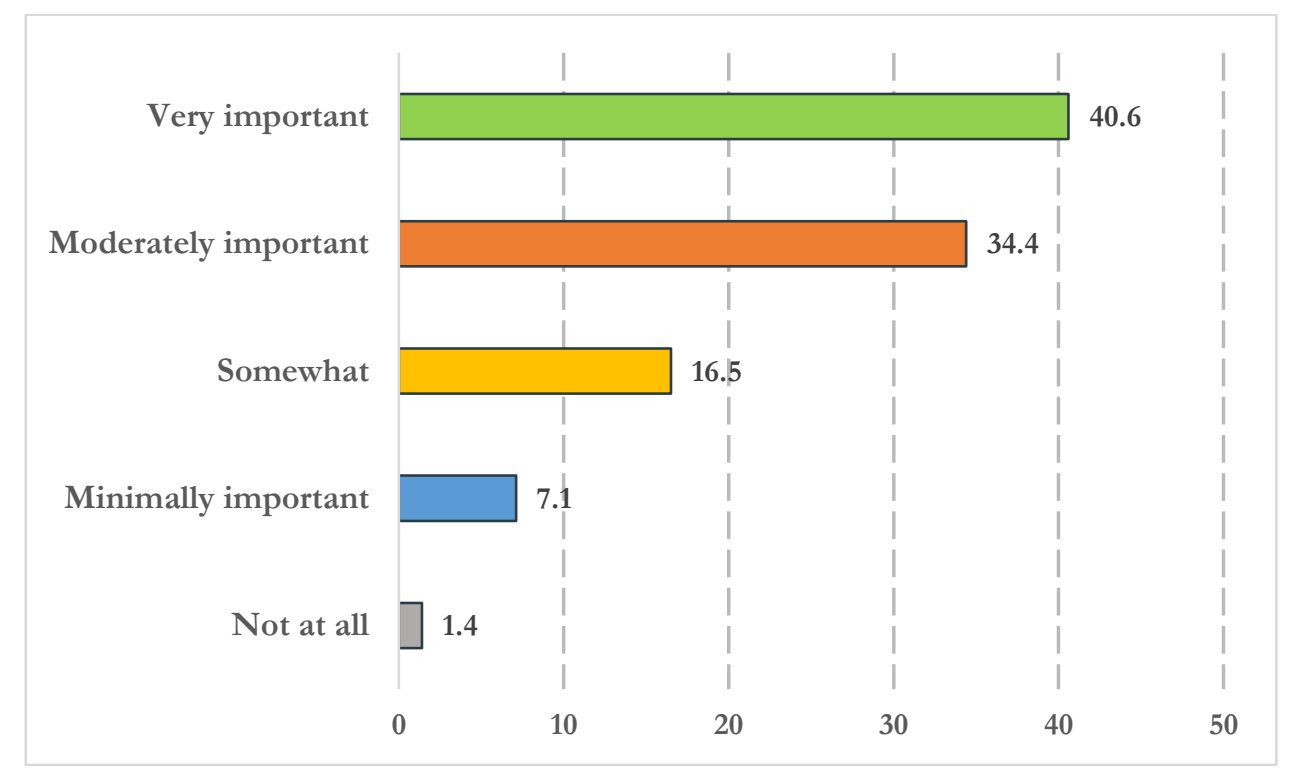

Figure 5-34: Respondents' ratings of ability to control noise for wellbeing

The result reflected the need for a restful environment for postnatal wellbeing. The respondents who reported somewhat, minimally important or not at all important suggest there may be inherent limitations with regard to controlling noise in a hospital environment, which perhaps lies more in the purview of the organisational context.

\section{Q4. Having a room that feels quiet}

Most of the respondents thought a room that feels quiet supported their feeling of wellbeing. One hundred and seventy-two $(81.1 \%)$ respondents rated a room that feels quiet as very important and 
thirty-one $(14.6 \%)$ rated it as moderately important. Just nine $(4.3 \%)$ respondents combined reported somewhat, minimally important, and not at all important (see Figure 5-35).

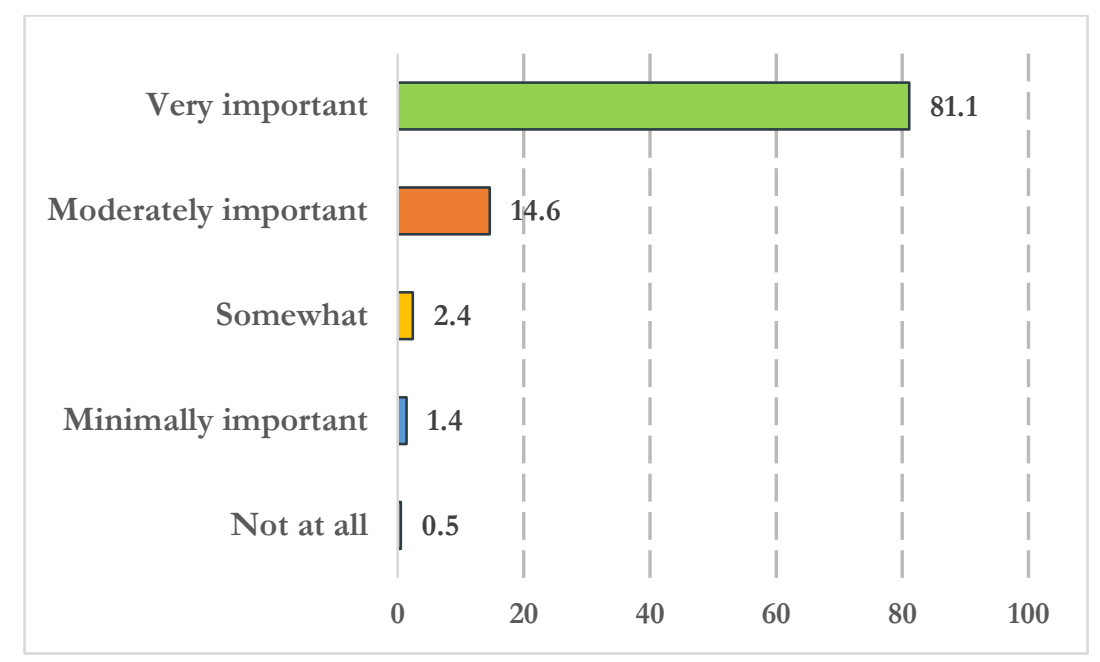

Figure 5-35: Respondents' ratings of quiet room for wellbeing

The result indicated the importance of the sensory impact of the environment in recovery experience and wellbeing. The largest group $(n=203,95.7 \%)$ put a high premium on having a quiet room.

\section{Q5. Having good air quality and room freshness}

Most of the respondents thought the quality of fresh air in the environment impacted on their feeling of wellbeing. One hundred and sixty-four (77.4\%) ranked air quality and room freshness as very important, forty-one as moderately important, and seven $(3.3 \%)$ respondents selected somewhat important (see Figure 5-36).

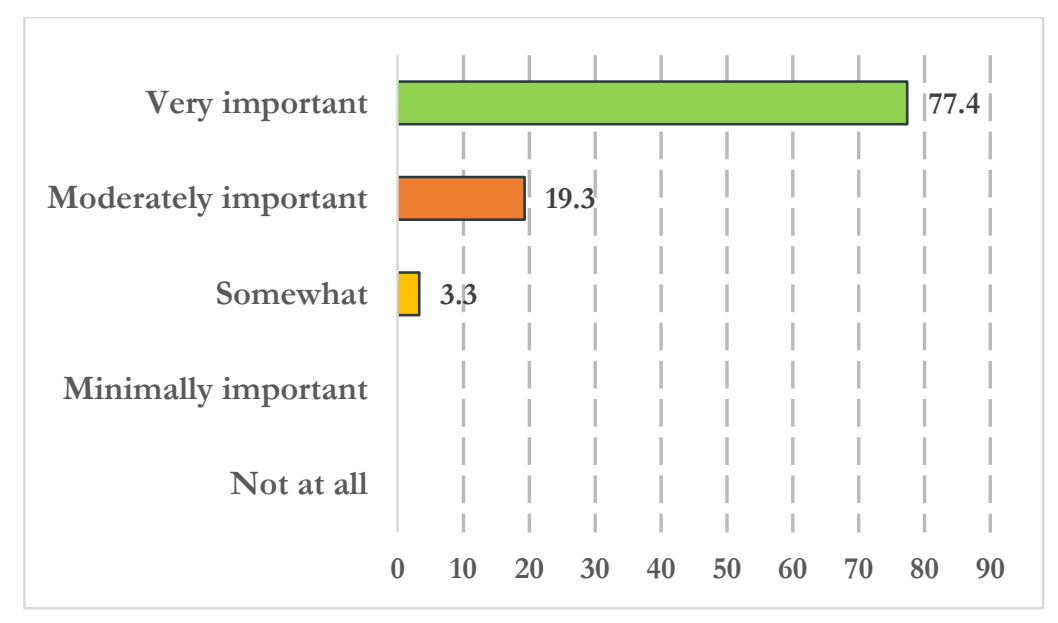

Figure 5-36: Respondents' ratings of air quality and room freshness for wellbeing 
The result reflected awareness of the value of proper ventilation. Air quality is a vital aspect for infection control while air freshness affects how people may feel in the space. The large number of respondents who gave positive responses shows the high priority given to air quality.

\section{Q6. Being able to have additional heating for mother and baby}

Most of the respondents thought being able to have additional heating would support their feeling of wellbeing. One hundred and three $(48.6 \%)$ respondents rated the provision of additional heating as very important, and eighty-five (40.1\%) reported it moderately important. On the other hand, nineteen $(9 \%)$ rated it somewhat important, one $(0.5 \%)$ respondent ranked not at all and four $(1.9 \%)$ respondents selected minimally important (see Figure 5-37).

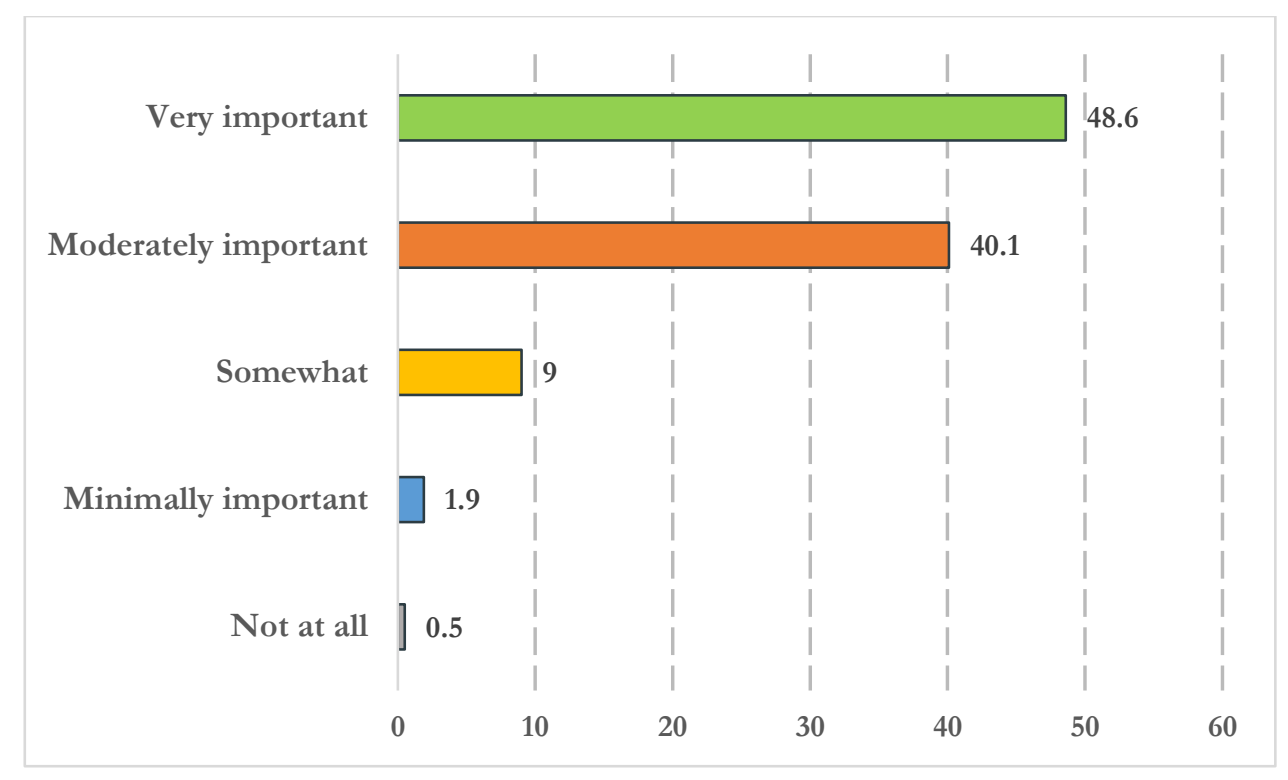

Figure 5-37: Respondents' ratings of additional heating in the room for wellbeing

The result indicated the desire for keeping a warm temperature in the postnatal environment particularly for neonates who have to adapt from the in utero environment to the temperature experienced by the adults.

\section{Q7. Knowing you can open or close the windows to feel comfortable}

Eighty two $(38.7 \%)$ respondents ranked operable windows for comfort as very important, and seventy-eight $(36.8 \%)$ respondents reported it as moderately important. In addition, thirty-five $(16.5 \%)$ ranked it as somewhat important, eleven $(5.2 \%)$ selected minimally important and six $(2.8 \%)$ respondents rated as not at all important (see Figure 5-38). 
The result indicated the need for personal control in the physical healthcare environment by occupants encouraging women-centred care. Just over three quarters $(n=160,75.5 \%)$ of the respondents considered this aspect was important for their sense of wellbeing.

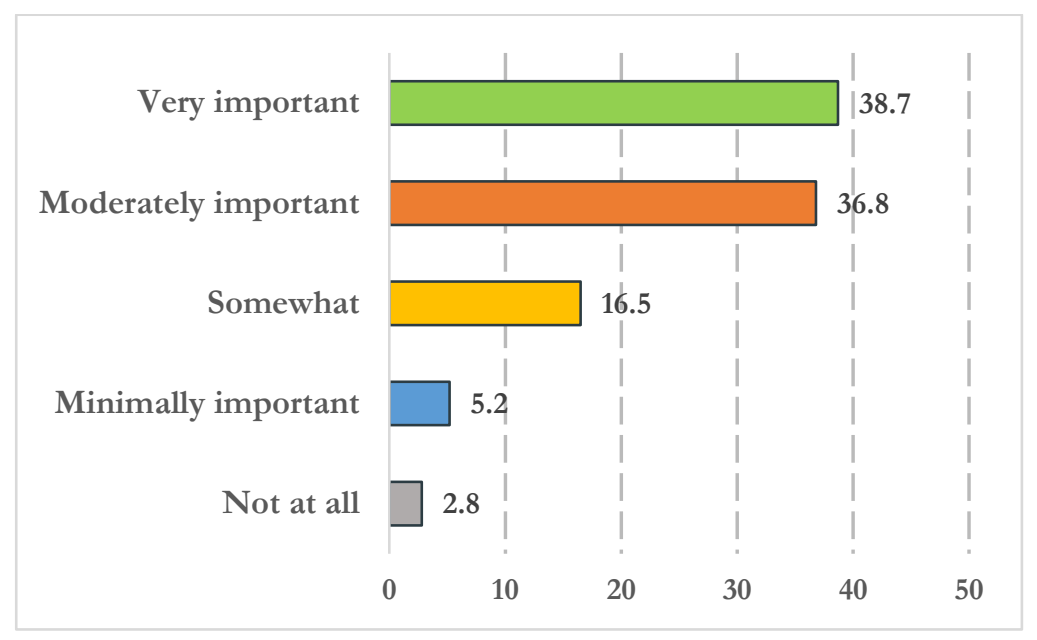

Figure 5-38: Respondents' ratings of operable windows for comfort and wellbeing

Q8. Having a number of natural things in your room such as aquariums, nature sounds of birds, water, scents and images

Respondents' perception of coordinated natural features in the room was ranked less favourably. The perception of natural things for wellbeing was rated as not at all important by thirty-five $(16.5 \%)$ respondents, seventy-three $(34.4 \%)$ rated it as minimally important and it was rated as somewhat important by sixty-nine (32.5\%) respondents. By contrast, it was reported as moderately important by twenty-six (12.3\%) respondents, and as very important by nine (4.2\%) respondents (see Figure 5-39).

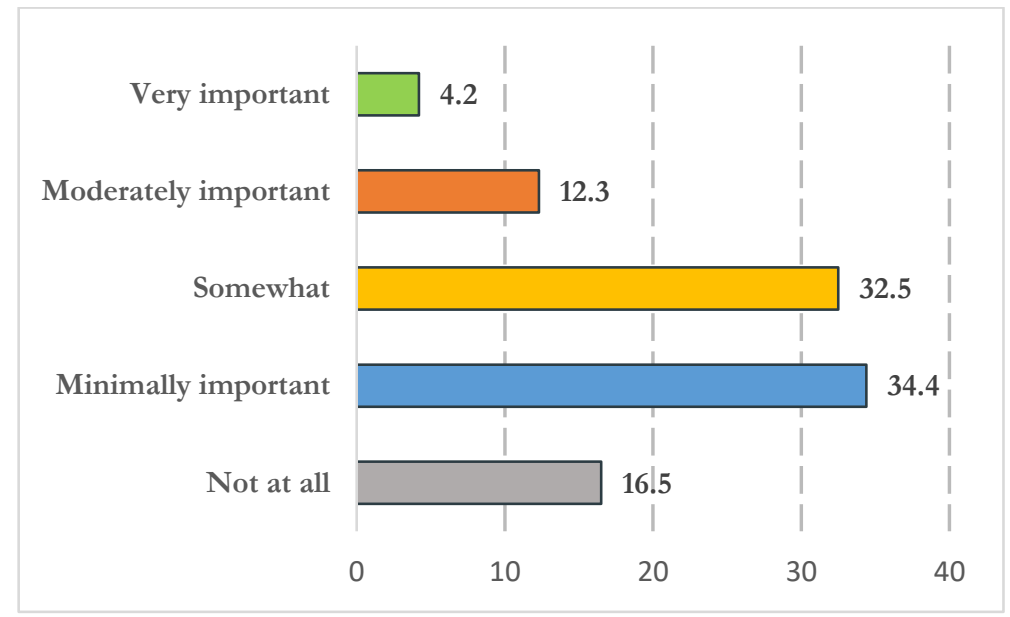

Figure 5-39: Respondents' ratings of natural things in the room for wellbeing 


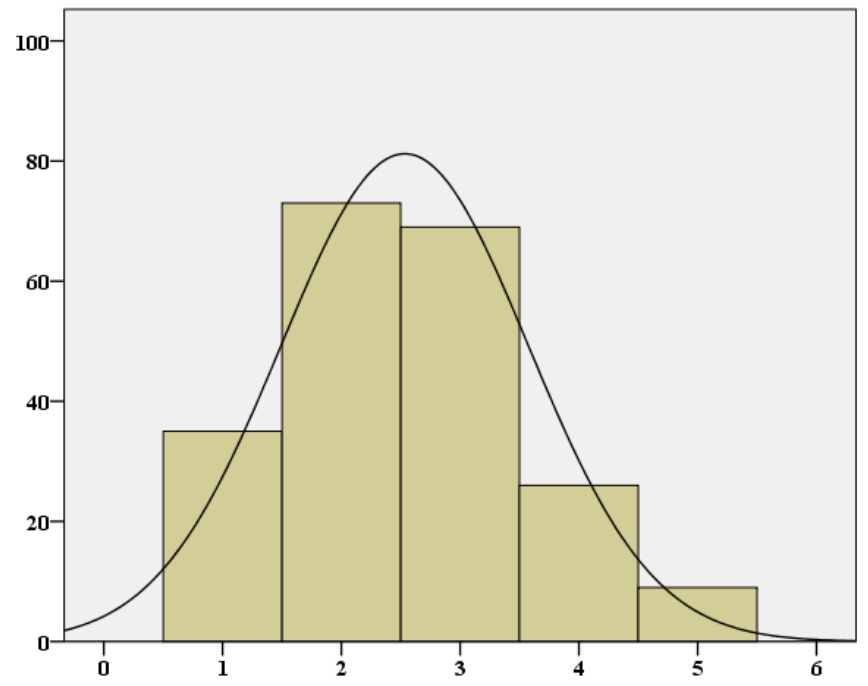

Figure 5-40: A slightly positive skewed distribution of ratings of natural things such as aquariums and images for wellbeing

As Figure 5-40 indicates, the distribution is slightly skewed with fewer respondents who thought natural things in the room were important for their wellbeing. These responses could suggest that listening to nature sounds or having images in the room may not be one of the immediate needs of the respondents.

\section{Q9. Having a hygienic/clean environment}

Nearly all respondents thought a hygienic environment would be positive for wellbeing. One hundred and ninety-seven (92.9\%) respondents rated a hygienic and clean environment for feeling of wellbeing as very important, twelve (5.7\%) rated it as moderately important, and three (1.4\%) respondents reported somewhat important (see Figure 5-41).

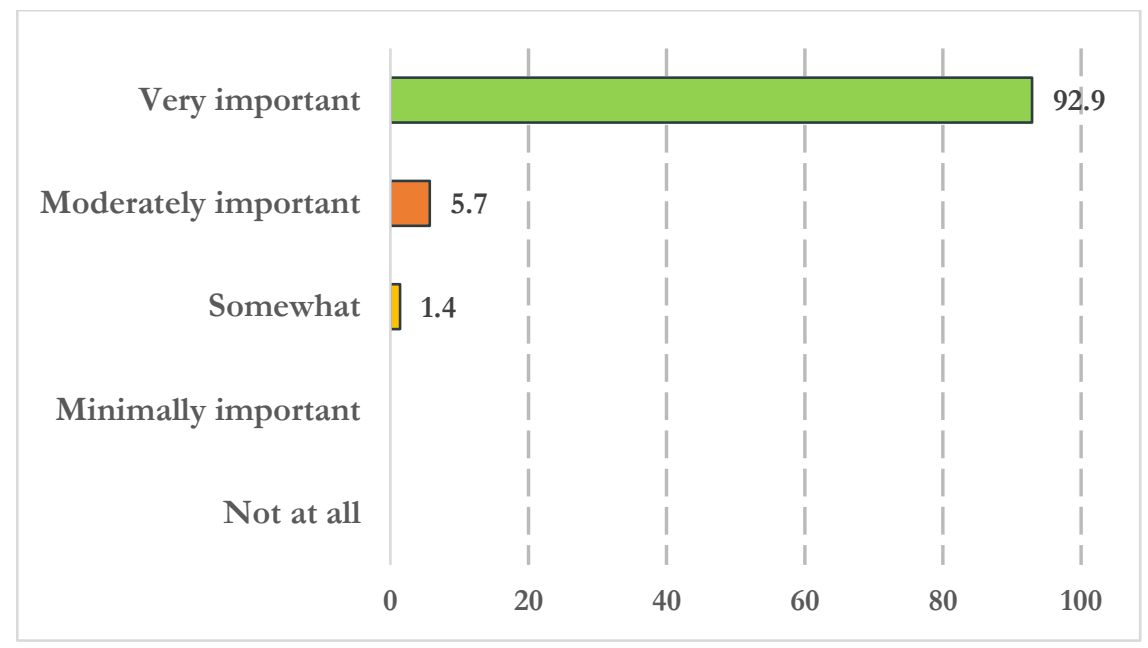

Figure 5-41: Respondents' ratings of hygiene/cleanliness in the room for wellbeing 
Maintenance of high quality standards of hygiene is a mainstream goal by healthcare organisations, and such standards in turn have a large impact on the feeling of wellbeing of those using the facilities.

Table 5-5 summarises the results of the descriptive statistics analyses for all the questions in this section.

Table 5-5: Preference (mean and standard deviation) of respondents on perception of sensory comfort environment features influencing women's recovery and wellbeing $(\mathrm{n}=212)$

\begin{tabular}{lll}
\hline Survey question & M & $\boldsymbol{S D}$ \\
\hline Having a hygienic/clean environment & 4.92 & 0.33 \\
Having a room that feels quiet & 4.75 & 0.62 \\
Having good air quality and room freshness & 4.74 & 0.51 \\
Having adequate daylighting in the room/ward & 4.42 & 0.73 \\
Being a able to have additional heating for mother and baby & 4.34 & 0.76 \\
Being able to control the temperature & 4.25 & 0.87 \\
Being able to control noise from mechanical things like ventilation, medical & 4.06 & 0.99 \\
equipment etc. & & \\
Knowing you can open or close the windows to feel comfortable & 4.03 & 1.01 \\
Pleasantness of the room (such as aquariums, nature sounds of birds, water, scents & 2.53 & 1.04 \\
and images) & & \\
\hline Not & &
\end{tabular}

Note: $M=$ mean; $S D=$ standard deviation

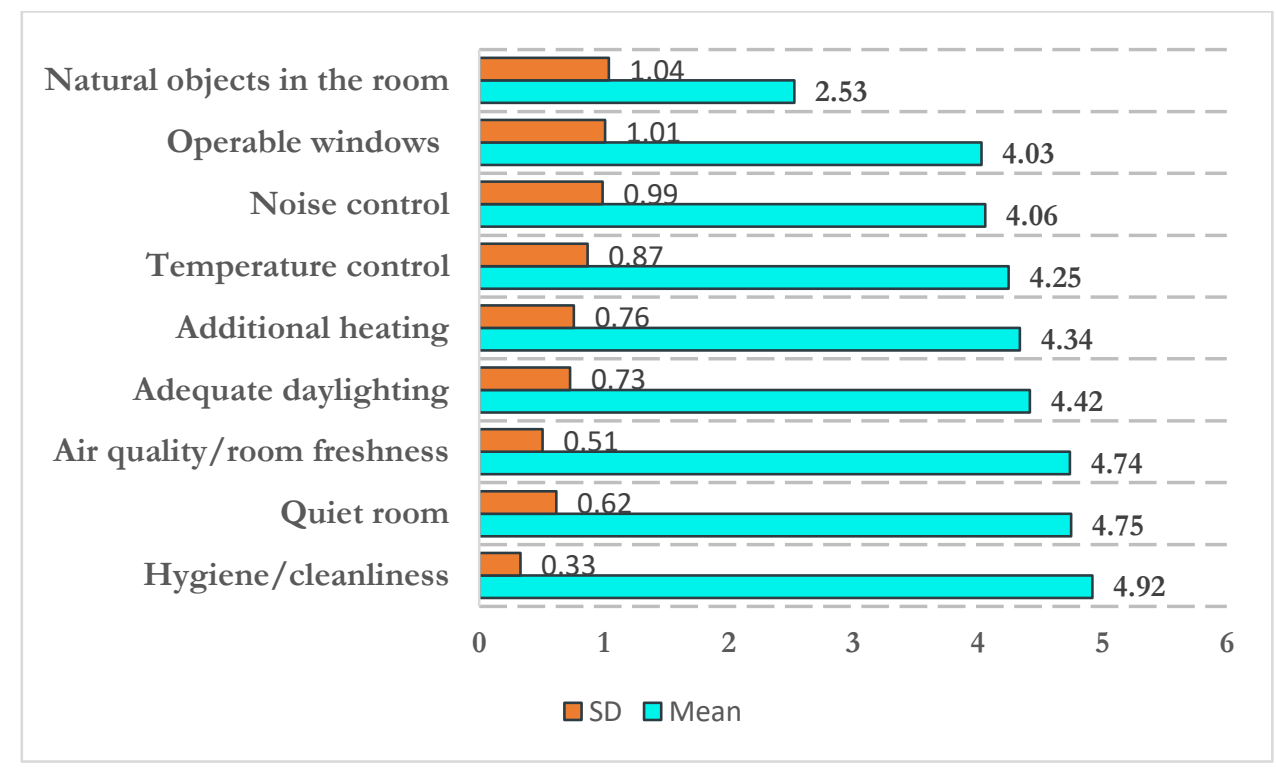

Figure 5-42: Respondents' ratings of sensory environment design factors for wellbeing

\section{Summary}

This section of the survey questionnaire has discussed the descriptive statistics analyses and results of nine sensory environment design factors. The item hygienic and clean environment topped the 
list of design factors with the highest mean score of 4.92. The two closely ranked design features of "having a room that feels quiet" and "air quality and room freshness" were ranked next. All the sensory environment design factors, with one exception, the presence of natural things in the room, had mean scores higher than 4 on a scale of 5 (Figure 5-42). The next section presents the results of the descriptive statistics on perception of social comfort environment.

\subsection{Perception on social comfort environment}

This aim of this section is to see how the physical environment contributes to the goal of social comfort. A range of questions related to social and psychological needs were asked of respondents to see how the physical environment facilitates social wellbeing and interaction. The questions in this section also aim to understand the needs and activities of birth supporters, family and friends in the immediate postnatal phase.

Descriptive statistics analyses of eight aspects (see questions in the heading or in the women's questionnaire in Appendix B) of social comfort environment are discussed in this section.

\section{Q3.25 How often visitors came in to see you in your postnatal room after the birth of your} baby?

Most of the respondents reported multiple visitation during postnatal hospital stays. Sixty-nine respondents had a frequency of visitation of three times, sixteen (7.5\%) four times, and seventyfour $(34.9 \%)$ reported a frequency of five or more times. Twenty-five $(11.8 \%)$ and twenty-eight $(13.2 \%)$ respondents reported once and twice respectively during postnatal hospital stays (see Figure 5-43).

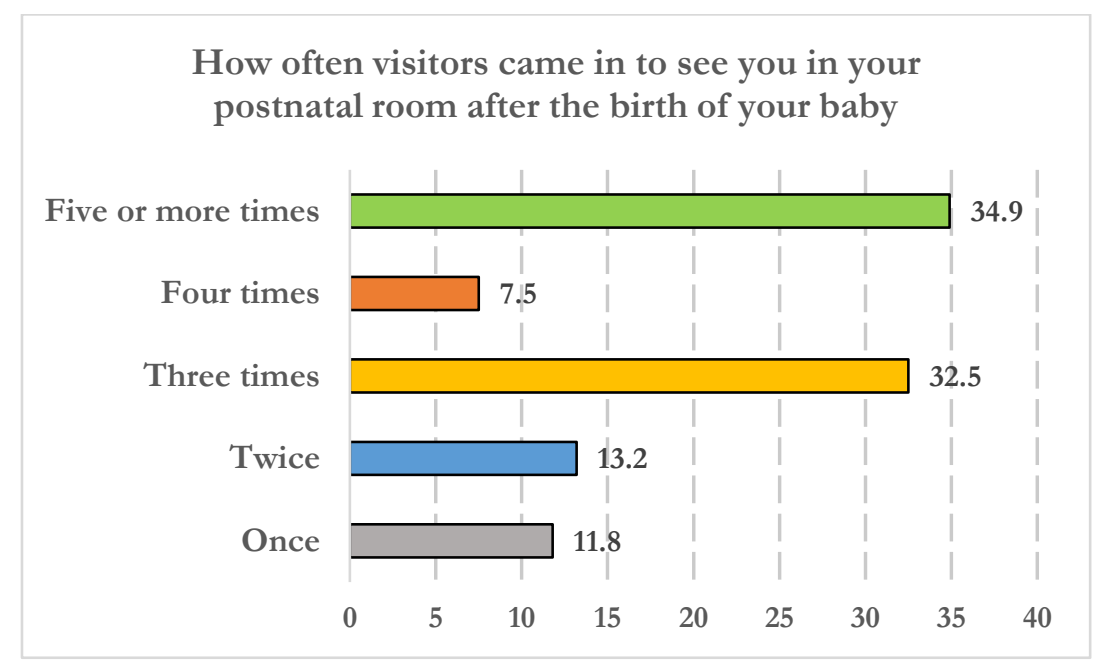

Figure 5-43: Respondents' ratings of frequency of visitation 
Overall, one hundred and fifty nine $(75 \%)$ respondents received visitors three or more times, while 53 respondents $(25 \%)$ had visitors one or two times. Although this question did not ask the number of people who visited and the duration of their postnatal stays, it is reasonable to assume that the situation suggests a busy or crowded environment, particularly if family members and partners arrived at the same time. It is likely that visitors would arrive during hospital visiting hours. This situation may likely have consequences on restfulness while the positive aspects of visitation seem valuable for emotional support and wellbeing signaling a need for well-designed familycentered space.

\section{Q3.26 Women are offered the opportunity to choose the room they would prefer for their postnatal stay}

Being offered the opportunity to choose the room was rated as strongly disagree by sixty respondents (28.3\%), disagree by thirty-nine (18.4\%), and neither agree nor disagree by fifty-seven $(26.9 \%)$ respondents. On the other hand, opportunity to choose the room was rated as strongly agree by sixteen respondents and agree by forty $(18.9 \%$ ) respondents (see Figure 5-44).

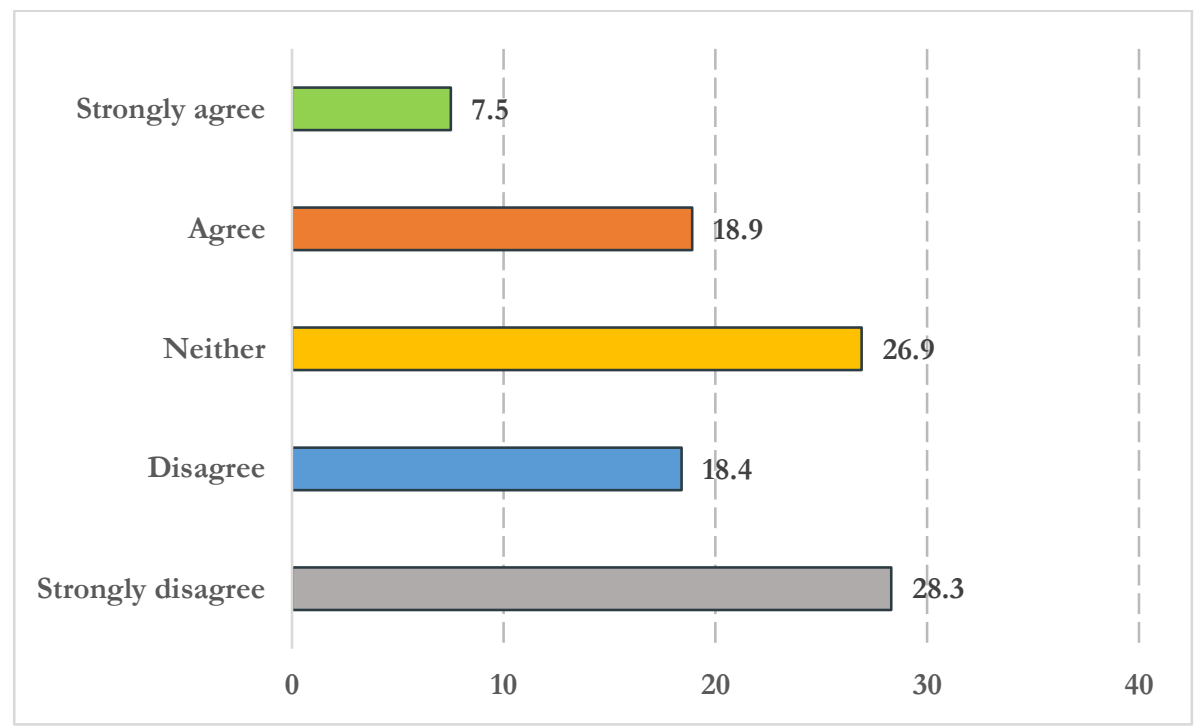

Figure 5-44: Respondents' ratings of opportunity to choose room for social comfort

Nearly half of the respondents $(n=99,46.7 \%)$ disagreed they had the opportunity to choose aroom of their preference. Just over one quarter of respondents $(n=56,26.4 \%)$ agree that they were given the opportunity to choose the kind of room they liked. The result suggests that there may be underlying factors in hospitals regarding allocation of rooms to women, which is entirely an organisational decision. 
Q3.27 From the postnatal room it is good to see the activity of the maternity department

This question asked about having a spatial link between the birth rooms and the postnatal space. For most of the respondents, being able to see the activity in the birth/delivery rooms was rated less favourably. Forty-five (21.2\%) reported this feature as strongly disagree, one hundred and eight $(50.9 \%)$ rated as disagree and fifty-four $(25.5 \%)$ respondents selected neither agree nor disagree. Only five (2.4\%) respondents reported agree (see Figure 5-45).

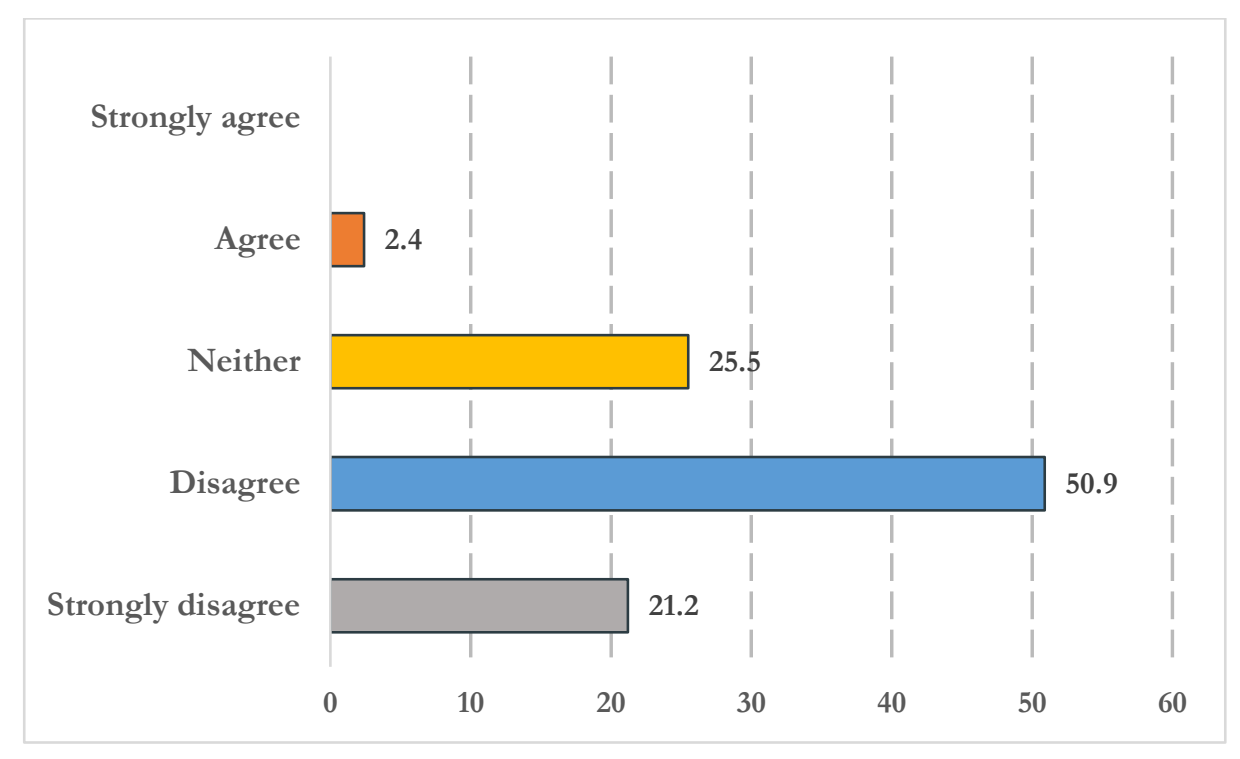

Figure 5-45: Respondents' ratings of spatial connection of postnatal room

Once women reach the postnatal rooms, they may no longer be interested in what is going on in the rest of the maternity department or they may want to rest and recover away from the activity.

Q3.28 Positive distractions such as soft music, natural images etc. in a postnatal room are better for a woman's recovery

Positive distractions were ranked agree by seventy-three (34.4\%) respondents, and as strongly agree by eight $(3.8 \%)$ respondents while fifteen (7.1\%) respondents and three (1.4\%) reported disagree and strongly disagree and one hundred and thirteen $(53.3 \%)$ said neither agree nor disagree (see Figure 5-46). 


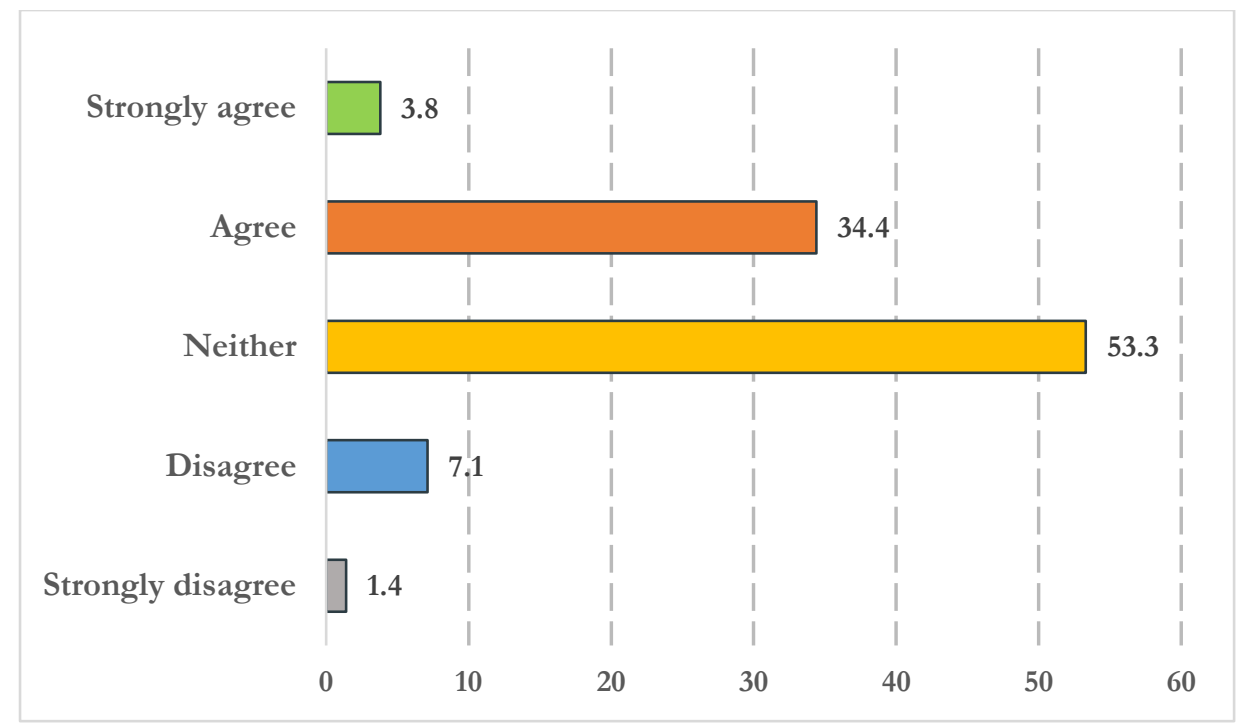

Figure 5-46: Respondents' ratings of positive distractions for social comfort

The result revealed that positive distractions may be likely beneficial for the recovery comfort of women. The few respondents who disagreed are not numerically up to those who gave positive or neutral responses.

\section{Q3.29 Having a communal space near the postnatal space is good for counselling and parental information support}

Most of the respondents thought a communal space close to the postnatal facility would support the learning of parental skills. Thirty-eight $(17.9 \%)$ respondents ranked a communal space as strongly agree, and one hundred and thirty-six (64.2\%) reported agree. By contrast, thirty-one $(14.6 \%)$ respondents stated neither agree nor disagree, five $(2.4 \%)$ and two $(0.9 \%)$ respondents selected disagree and strongly disagree respectively (see Figure 5-47).

The result indicated the value of a communal space, near to but separate from the postnatal ward/space, to encourage opportunities for learning parental skills. This design feature is a key factor to think about in the planning and design of postnatal hospital environments. 


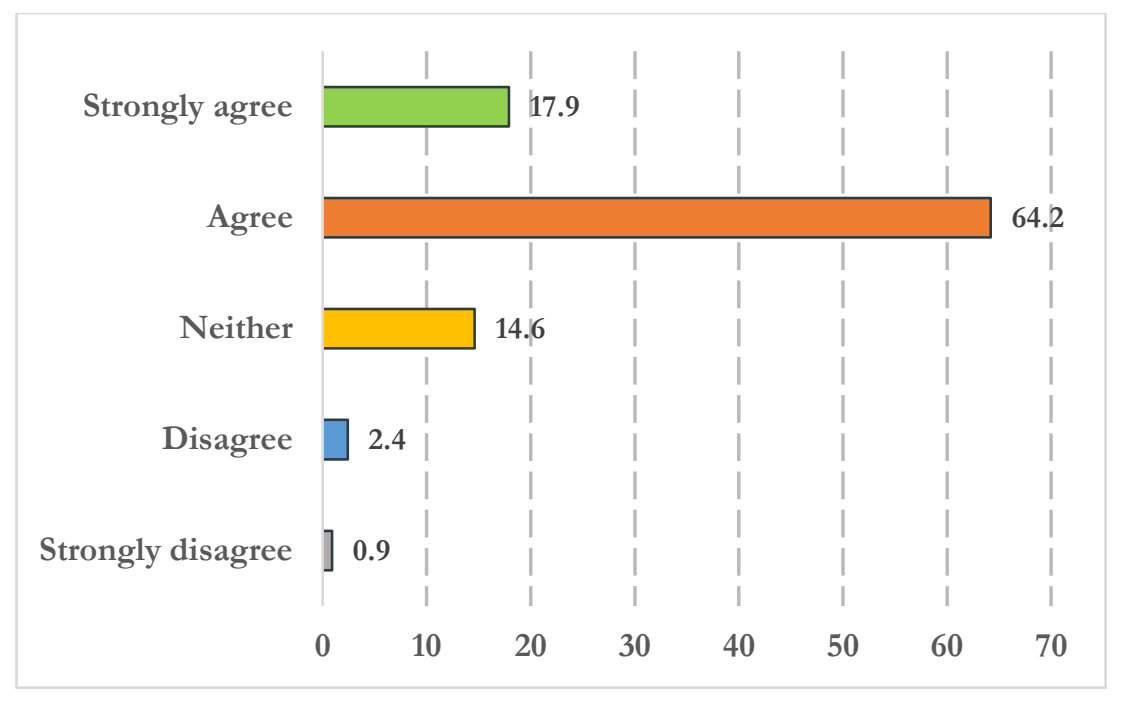

Figure 5-47: Respondents' ratings of communal space for information support

\section{Q3.30 The arrangement of furniture and décor in a communal space helps group learning of parental skills}

Most of the respondents perceived the arrangement of furniture in a communal space would help to support group learning of parental skills. Thirty five (16.5\%) respondents rated the arrangement of furniture for learning support as strongly agree, and one hundred and twenty-two $(57.5 \%)$ selected agree. Fifty-one $(24.1 \%)$ and four (1.8\%, two, in both cases) respondents reported neither agree nor disagree, disagree and strongly disagree (see Figure 5-48).

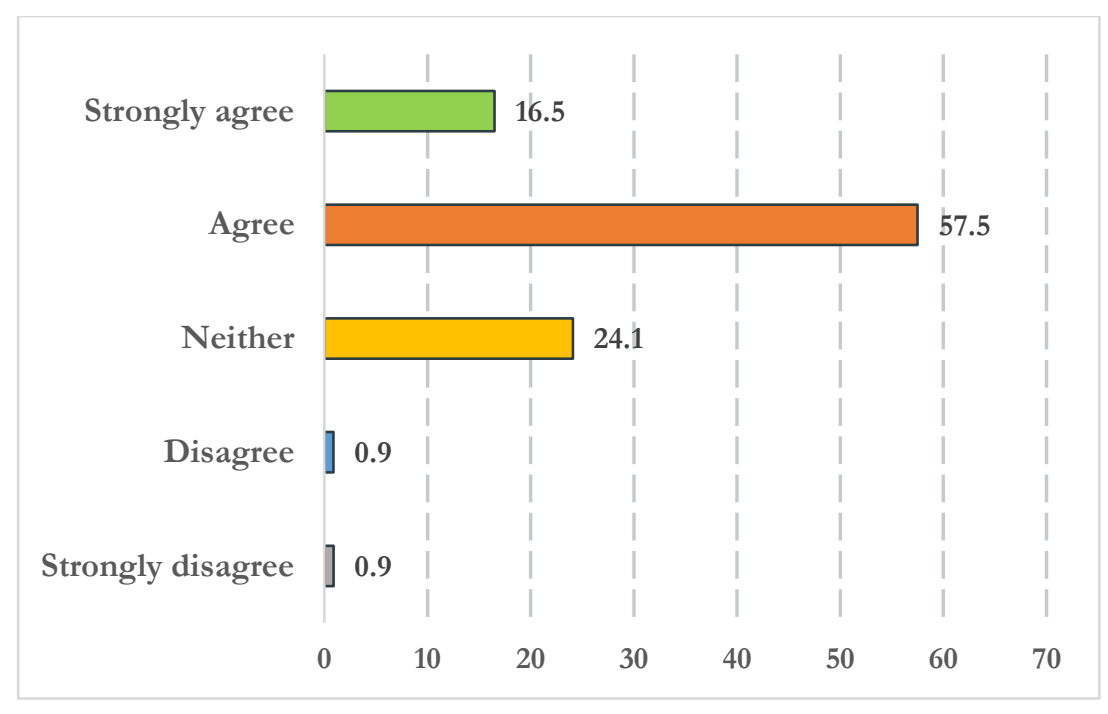

Figure 5-48: Respondents' ratings of furniture and décor layout for group learning 
The result revealed the importance of furniture layouts and arrangements in interiors of healthcare environments. Opportunities for different seating approaches, for example, could foster support which might be beneficial in the learning of parental skills and information.

Although the significant number of the respondents who reported agree outweighs the other groups of respondents, the neither agree nor disagree group may merit further exploration. The effect of furniture arrangement may be ranked below the value of educative lessons on parental needs from helpful medical staff, while respondents may not regard a hospital environment as an ideal setting for learning as it is sterile and busy which may likely generate rather the desire to leave the environment.

\section{Q3.31 Openness of postnatal room decreases the opportunity for discussions with midwives}

Respondents' perception on whether the openness of the room or ward decreases the opportunity for discussions was assessed. Sixteen (7.5\%) respondents reported strongly disagree, and fiftyseven $(26.9 \%)$ respondents selected disagree. On the other hand, eight $(3.8 \%)$, and twenty-seven $(12.7 \%)$ respondents reported strongly disagree and disagree. One hundred and four $(49.1 \%)$ respondents ranked neither agree nor disagree (see Figure 5-49).

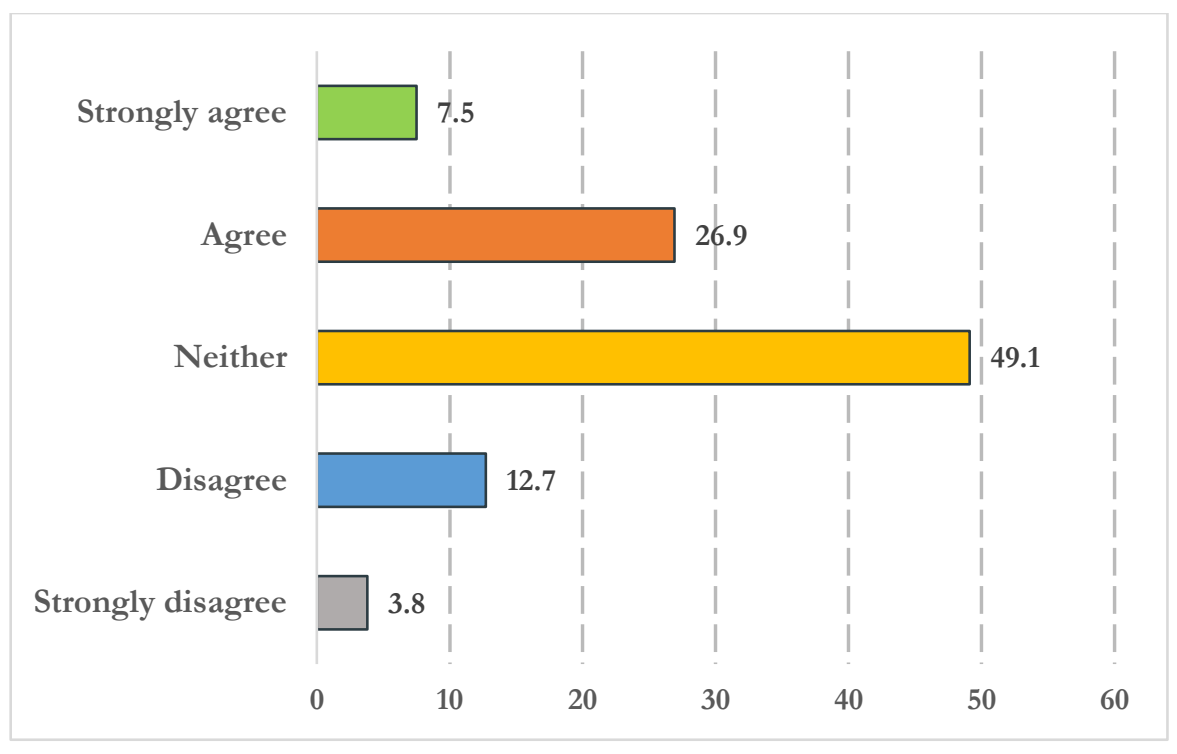

Figure 5-49: Respondents' ratings of open ward and less discussions 
The result indicates that respondents tend to feel that an open-type ward results in less opportunity for personal discussions. The result suggests the need for a balance of privacy and confidentiality with the room occupancy types.

\section{Q3.32 Entering from a passage or corridor to a postnatal room is better for privacy}

The perception of a corridor for providing better privacy was ranked as strongly agree by twenty (9.4\%) respondents, and rated agree by one hundred and seven (50.5\%) respondents. On the other hand, one $(0.5 \%$ respondent and six $(2.8 \%)$ respondents ranked strongly disagree and disagree; while seventy-eight (36.8\%) respondents reported neither agree nor disagree (see Figure 5-50).

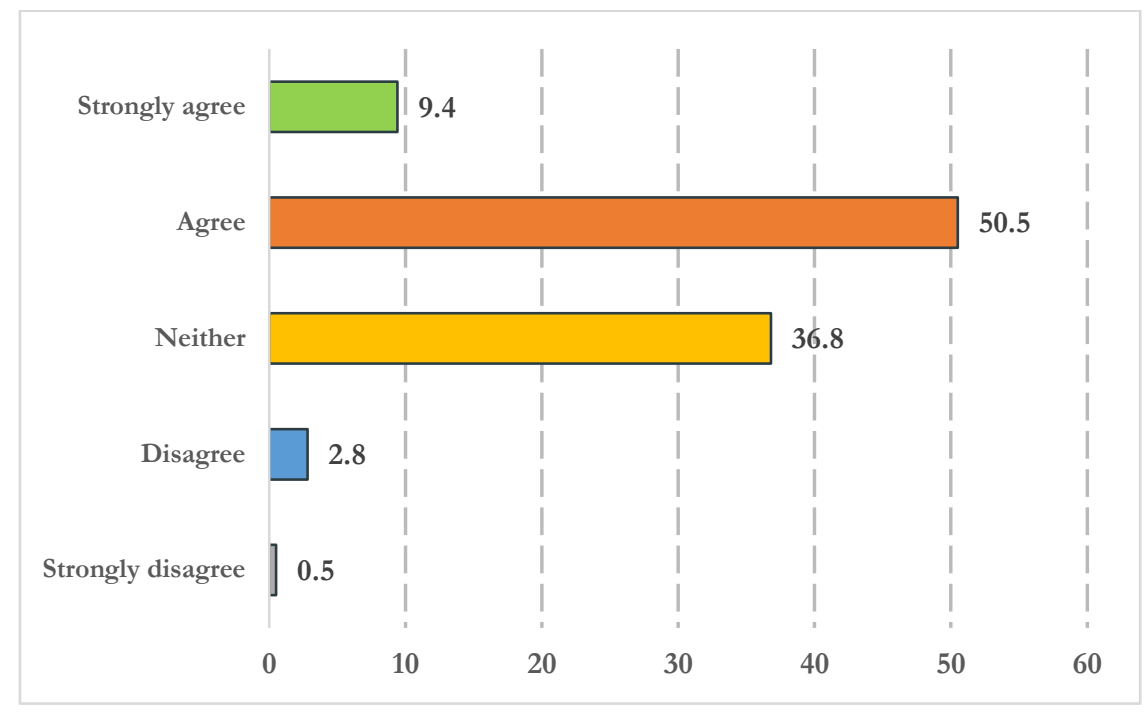

Figure 5-50: Respondents' ratings of a corridor for privacy comfort

The result indicated that spatial arrangements could be better designed to regulate movement into the space (in this case a postnatal ward) to promote privacy providing greater social comfort in the postnatal environment.

Q3.33 Having family members and partner present during postnatal care increases the need for a large space

The effect of having family members and partners present on the need for space was ranked highly. Sixty-four (30.2\%) respondents rated it as strongly agree, and ninety (42.5\%) respondents selected agree. On the other hand, twenty-one $(9.9 \%)$ and thirty-seven $(17.5 \%)$ respondents rated it disagree and neither agree nor disagree respectively (see Figure 5-51). 
The result shows the perception of the spatial effect of social inclusion and interaction via partners, family and friends in the healthcare environment, which could support women's emotional and psychological wellbeing balance.

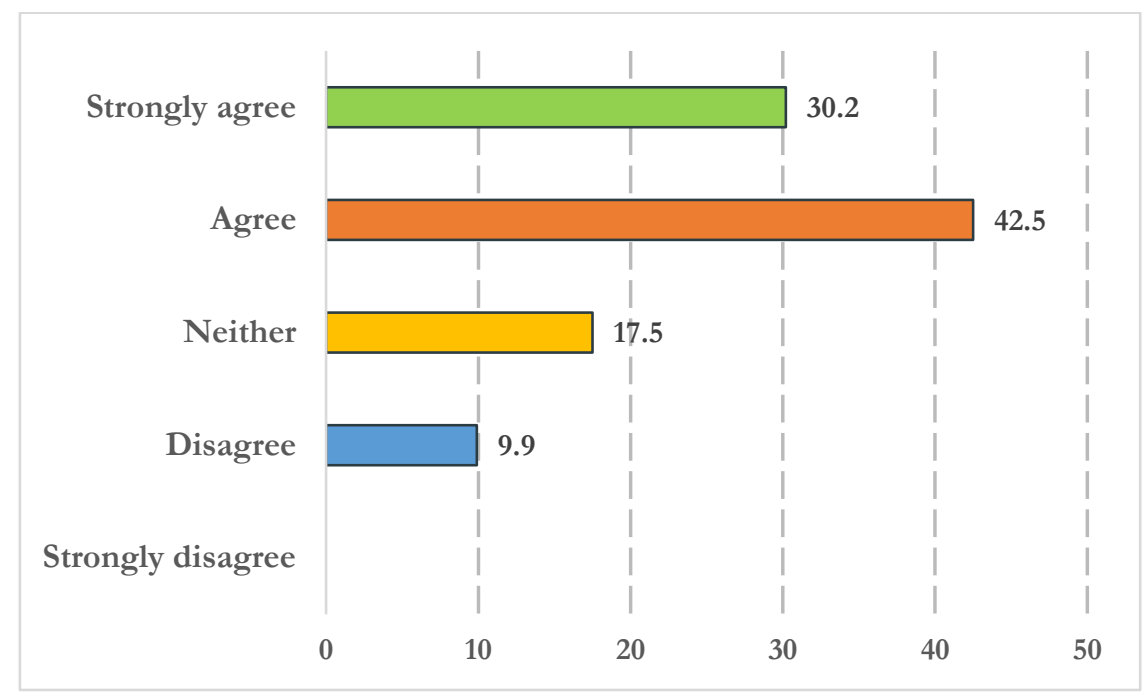

Figure 5-51: Respondents' ratings of family support and space need

\section{Q3.34 Women find a large open postnatal ward or room is better for interactions with other women}

Open ward support for better interactions was ranked by sixty-two $(29.2 \%)$ respondents as strongly disagree, as disagree by fifty-nine $(27.8 \%)$ and as neither agree nor disagree by seventyfour respondents. Only seventeen (9\%) respondents ranked it as agree or strongly agree (see Figure $5-52)$.

The result reflected the less favourable disposition of the respondents towards an open postnatal ward for better interactions. The few respondents who ranked an open ward positively maybe suggesting a preference to be in other women's company rather than being in isolation in singlebed rooms. 


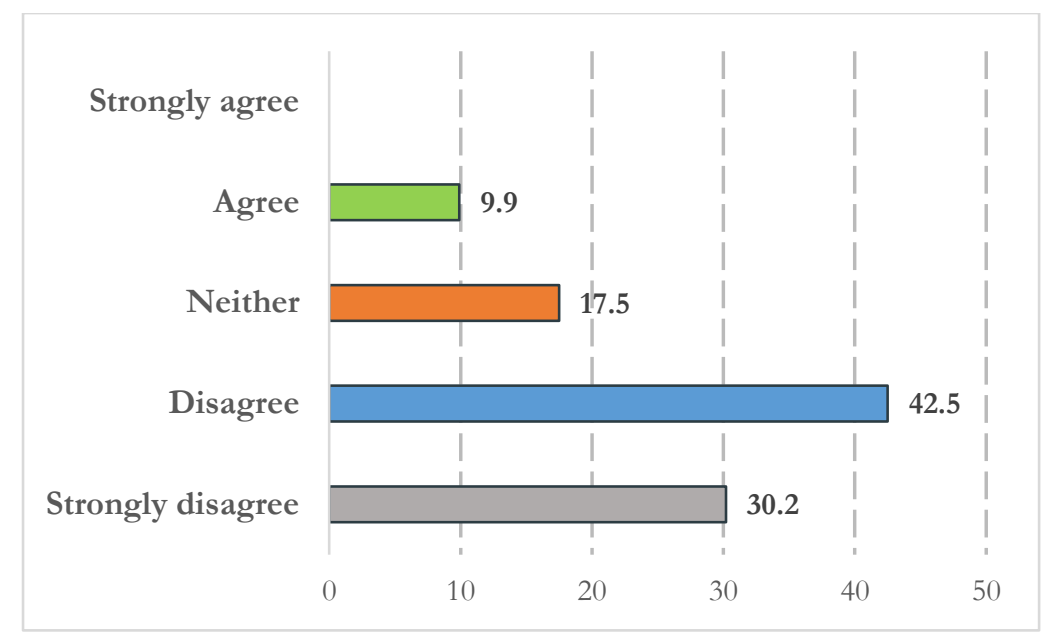

Figure 5-52: Respondents' ratings of open postnatal ward and interactions

Table 5-6 summarises the results of analyses of the nine design features in this section. Figure 553 provides the graphic illustration of all the design aspects with their mean score and standard deviation. None of the mean scores is over 4.

Table 5-6: Preference (mean and standard deviation) of respondents on perception of social comfort features influencing wellbeing $(\mathrm{n}=212)$

\begin{tabular}{|c|c|c|}
\hline Survey question & $\mathbf{M}$ & $S D$ \\
\hline $\begin{array}{l}\text { Having a communal space near the postnatal space is good for counselling } \\
\text { support }\end{array}$ & 3.96 & 0.711 \\
\hline $\begin{array}{l}\text { Having family members and partner present during postnatal care increases the } \\
\text { need for large space }\end{array}$ & 3.93 & 0.934 \\
\hline of furniture and décor in a communal space helps group learning of & 3.88 & 0.718 \\
\hline a corridor to a postnatal room is better for privacy comfort & 3.66 & 0.708 \\
\hline d the opportunity to choose a room that they prefer for their & 3.41 & 1.283 \\
\hline $\begin{array}{l}\text { actions such as soft music, natural images etc. are better for a } \\
\text { very }\end{array}$ & 3. & 0.7 \\
\hline & 3.22 & 0.898 \\
\hline & 2.24 & 1.003 \\
\hline $\begin{array}{l}\text { Spatial connection between postnatal room and birth room (see activity on the } \\
\text { ward) }\end{array}$ & 2.09 & 0.746 \\
\hline
\end{tabular}

Note: $M=$ mean; $S D=$ standard deviation 


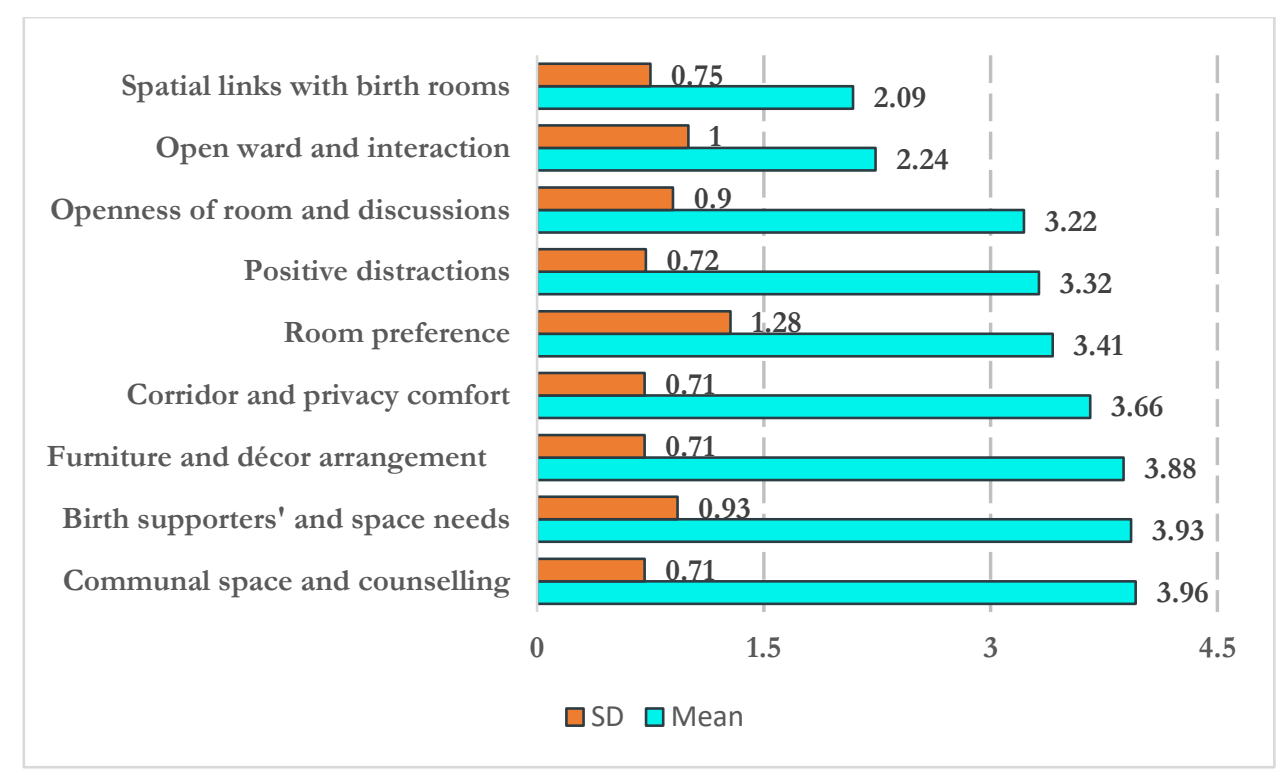

Figure 5-53: Respondents' ratings of social comfort environment features

\subsubsection{Perception of alternative postnatal environments}

This section discusses the analyses and results of the responses to preceding questions (see questions Q3.33, 3.34 and 3.35 in the women's questionnaire in Appendix B) about the choice of postnatal room followed by an alternative setting for postnatal care. Then answers for the openended question are presented.

\subsubsection{Choice of postnatal room}

Most of the respondents reported preference for a single-bed occupancy room. Two hundred and six respondents $(97.2 \%)$ ranked a single-bed room as the best for postnatal care, one $(0.5 \%$, in both cases) respondent chose either an open bay room/ward and or a 2 person room. In addition, four $(1.9 \%)$ fell under the "other" category (see Figure 5-54).

Analysed answers by the four respondents who reported "other", shows a preference for a singlebed room with option to include partners. Personal control of the environment related to privacy in the room was also alluded to. The text of the four respondents is as follows:

A room with a door I can lock if I want to (other 1); It can be a single room but have the capacity for partners to stay also (other 2); Single room but with communal areas to learn breastfeeding as a group with other mums (other 3); and Single room where father and baby can stay with me (other 4). 


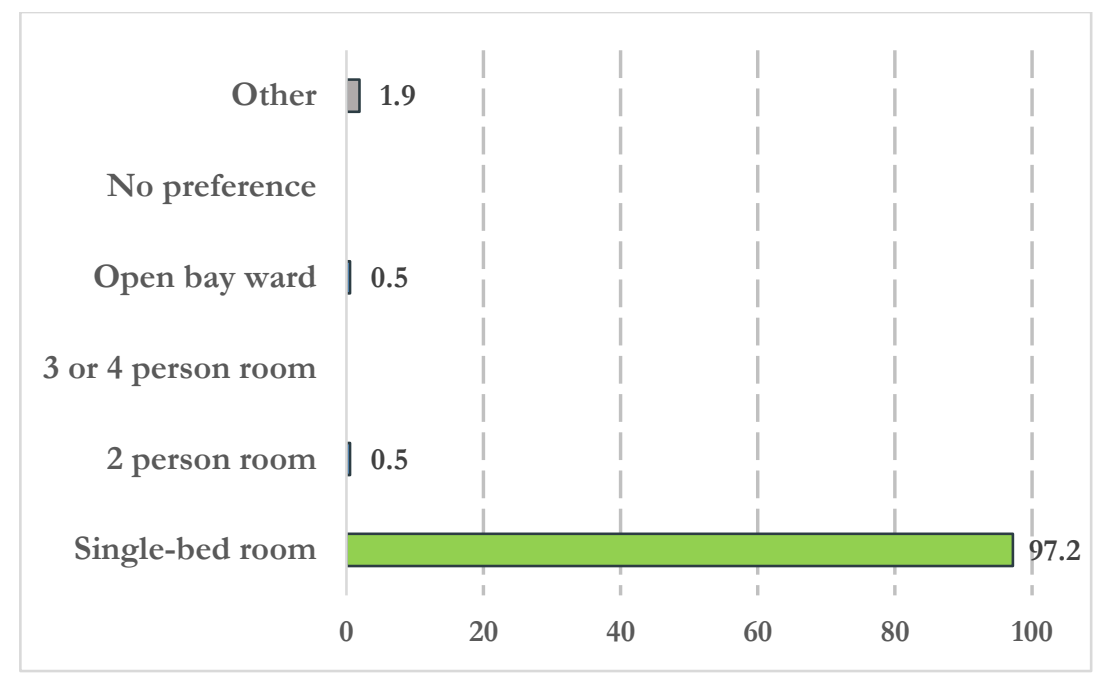

Figure 5-54: Respondents' ratings of choice of room care

The result reflected inclination towards privacy, confidentiality in the room and the ability to rest in comfort including bonding in privacy with partners and family members.

\subsubsection{Rethinking alternative settings for postnatal care}

Respondents' perception of alternative postnatal settings was rated, which revealed interesting results. Eighty-four $(39.6 \%)$ respondents expressed a preference for a family suite, and forty-nine $(23.1 \%$, in both cases) preferred home birth or hospital delivery followed by a postnatal ward. In addition, eighteen $(8.5 \%)$ respondents preferred hotel care while three $(1.4 \%)$ and nine $(4.2 \%)$ respondents respectively ranked no preference and the "other" label (see Figure 5-55). The result reflected a preference for a family suite implying inclusiveness of family and partners in the care and the preference to stay close to a hospital environment.

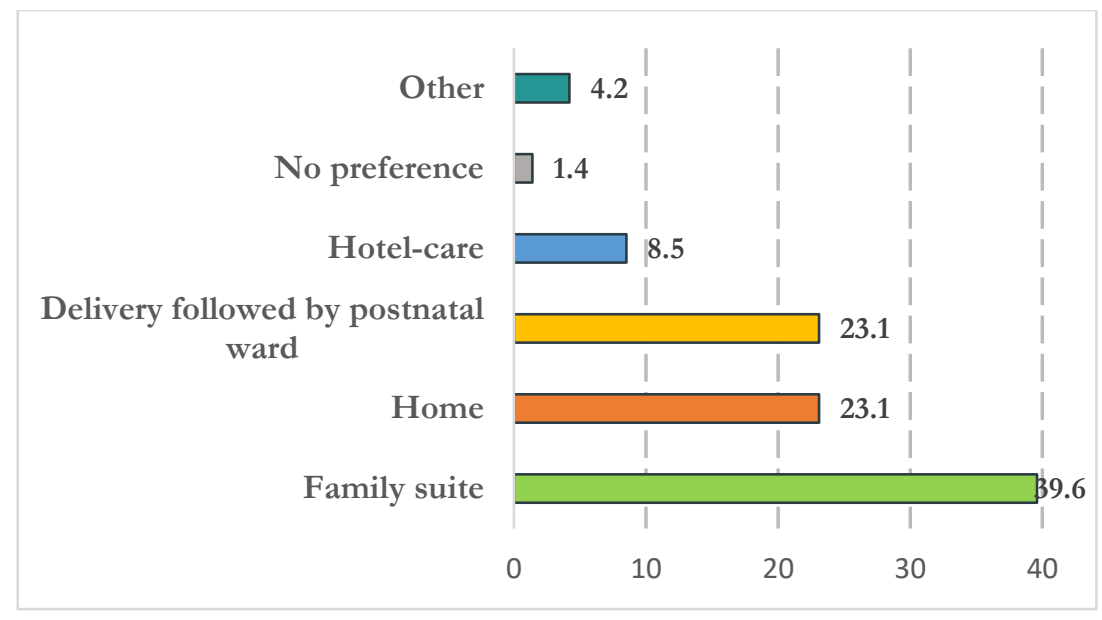

Figure 5-55: Respondents' ratings of alternative settings for postnatal care 
Lastly, 1.6\% (n=3) reported they have no preference; and 4.8\% $(\mathrm{n}=9)$ are not able to say or wanting other choice not listed.

Although postnatal care away from hospital to a hotel care situation is increasingly becoming fashionable especially in Nordic countries (Hildingsson \& Sandin-Bojö, 2011) the result in this current study is mixed. It differs from a Canadian study which found that women liked the choice of a hotel for postnatal care. The result is consistent with an Australian study which found women preferred to go home rather than to the hotel which was the least preferred location for care (McLachlan et al., 2009). The result is also in correspondence with a Swedish study which found that using a hotel for postnatal care was seen as an isolated and inappropriate environment for the new born baby (Rudman \& Waldenström, 2007).

\subsubsection{Open-ended question}

Eighty-one respondents expressed their opinions in the open-ended question. Comments included factors from general to personal, and with more minute details but very useful information. Direct quotations have been selected to describe the issues raised by the respondents and are indicative of the range of responses.

Some of the respondents expressed comments that indicate how hospitals can constitute a burden for rest and sleep and affect psychological wellbeing.

The hospital experience was horrible and made rest and recovery from a traumatic birth much more difficult. (Postnatal woman)

The type of birth plays a big part in post recovery. A caesarean-section means you are confined to your bed for 12 hours and very dependent on midwives. (Postnatal woman)

Most of the respondents expressed the difficulty in coping alone in hospitals especially without their partners to provide emotional and psychological support.

...partners are not allowed at night and this was very hard as I needed help looking after baby since I was very sore. Ideally my partner would have been able to stay the night in a comfortable couch. Partner support is crucial for mother and baby. (Postnatal woman) 
The biggest challenge is being alone with your brand new baby and your partner having to leave. It's unfair and overwhelming. Everything else is just minor detail, but being alone sucked. I also think open wards should be banned - how on earth are you supposed to bond with your baby and get much needed rest with someone else right next to you? (Postnatal woman)

Some respondents thought that allowing the partners to stay with new mothers may be likely to help to reduce the workload of staff who appear already stressed due to understaffing of postnatal wards.

So important to give partners the ability to stay overnight to help the new, and recovering mother out overnight. Eases workload from hospital staff and aids emotional state of mother. (Postnatal woman)

Many comments indicate un-conduciveness of the postnatal environment regarding noise from within the rooms and from other sources, which had a huge impact on rest, relaxation and the wellbeing of the new mothers.

We also found that the noise from the other room was pretty loud and clear. We would have appreciated mumbled sounds and slight noise. Too quiet a place is also disturbing. It's good to know there are people around if we need help. (Postnatal woman)

I think this is such an important topic. From being in a sterile hospital environment and a busy noisy environment, it would've made a huge difference to me ... have a comfortable relaxing environment whereby I had time alone to rest but also time to interact with other mums, learn breastfeeding together. (Postnatal woman)

I found it very difficult to sleep and relax after giving birth as I had to share a room. You and your baby are not on the same routine as the lady and baby next to you. I felt embarrassed when my baby cried, worried I'd wake up the lady next to me. (Postnatal woman)

The constant noise of a buzzer going off in the hallway outside [the] room was a huge barrier to being able to sleep. Why can't the buzzers be located at the nurses' station so women can't hear them and can get some much needed rest! Rooms need much better sound proofing. (Postnatal woman) 
[1] strongly felt better sound proofing [is] needed. Significantly reduced my already minimal chances of sleep. (Postnatal woman)

The aspect of sanitariness and hygiene of the environment was also emphasised as making a large impact postnatally. This alludes to having preference for single rooms with ensuite bathrooms that are easily accessible for women's recovery, comfort and wellbeing.

Many women have difficulty after giving birth and access to maintain hygiene and being able to walk those distances in some privacy lead to an overall postnatal recovery. (Postnatal woman)

Single rooms with your own bathroom is the ideal for postnatal care. Accessibility to a toilet/bathroom [is] very important for them to be close by and preferably private. Especially because not every mother is very mobile. I certainly wasn't. (Postnatal woman)

Some respondents also commented on proper maintenance of hygiene and cleanliness, particularly cleaning systems for the toilets. One participant mentioned that it would be "good to have some diaper disposal mechanisms" which may be particularly suitable whenever women have an extended postnatal stay in hospitals. Many respondents feel strongly that supports from midwives are valued especially in the recovery phase. Some expressed disappointments about not getting any support from medical staff as there was no one to help them out, leading to the decision to go home.

The advice from nurses and midwives is also very important. Access to midwives support...was severely lacking during my stay in hospital...I was left alone, in pain, and struggling to cope during the first night... I discharged myself the next day. To me, the environment and surrounds was immaterial. What I needed was support and guidance, but I didn't get it. (Postnatal woman)

The changeover of midwives every few hours is difficult-they are the key source of information, and each midwife has a totally different approach and gives different advice. (Postnatal woman)

Participants also expressed issues related to privacy. Provision of a single room or private rooms in which women can bond with their new baby and share private moments with their partners were discussed. 
I can't express enough how inadequate it is to share a room, separated by a curtain, with another person after giving birth. Having to listen to their phone calls, have their families constantly visiting- including toddlers who don't understand the meaning of privacy. (Postnatal woman)

You could ask about feelings of personal privacy or lack of privacy in each type of room. (Postnatal woman)

Privacy/access into the room...a window in the door and is there a curtain covering the door? (Postnatal woman)

\section{Summary}

In this section, descriptive statistics analyses and results of nine social comfort design factors have been discussed. It also presented the findings on two additional questions on alternative postnatal settings and one open-ended question. The descriptive statistics results have been summarised in Table 5-6 and Figure 5-53.

\subsection{Discussion of the results}

The purpose of phase one was to investigate perception of the design factors that influence/contribute to women's recovery experience in the immediate postnatal phase. This section describes the summary of major findings of the design factors perception survey focused on 1) perceptions of postnatal room features, 2) interior environment factors, 3) sensory environment factors, and 4) social comfort features.

\subsubsection{Summary of the results}

The evidence gathered in this study provides an understanding about the range of physical design factors which could support women's emotional and physical recovery experiences and their wellbeing. The first three of the four physical design factors categories listed above received overall highest mean scores of $(\mathrm{M}=4.93,4.92$, and 4.88) on a scale of 1 to 5 , in the order of interior, sensory comfort environments, and postnatal room design factors (see Tables 5-3, 5-4 and 5.5). On the other hand, the social comfort environment received mean scores higher than 3 (but not up to 4 ) in most of the design features with the exceptions of two items that had a rating of 2 and above. Table 5-7 shows the top fifteen design factors and features considered to influence women's 
postnatal recovery experiences and wellbeing in hospitals (see also Tables C1 and C2 for the mean scores and standard deviations for all the survey items in Appendix C).

The overall results indicated that a need to "have a clean room" and "hygiene/clean environment" as well as to "have a restful room" topped the list of the most important design factors in the postnatal environment. A "single-occupancy room", "a room or ward that is quiet is good for sleep and helps recovery process", and "having a room that feels quiet" were the three next best ranked designed features. "A room/ward that is quiet" and "a room that feels quiet" were asked of respondents under two different categories, however they both had the same mean scores of $(\mathrm{M}=4.75)$. Similarly, the item of "having good air quality and room freshness" was ranked close to the situation of room quietness with a mean score of $\mathrm{M}=4.74$.

The preliminary results suggest support for hygiene/clean environment, a restful and tranquil environment, a single-occupancy room and sensory comfort (visual environment - natural daylighting and temperature control etc.) were considered mostly important. The 15 top-rated physical design factors for women's recovery and physical wellbeing are illustrated in descending order of higher to lower importance in Figure 5-56.

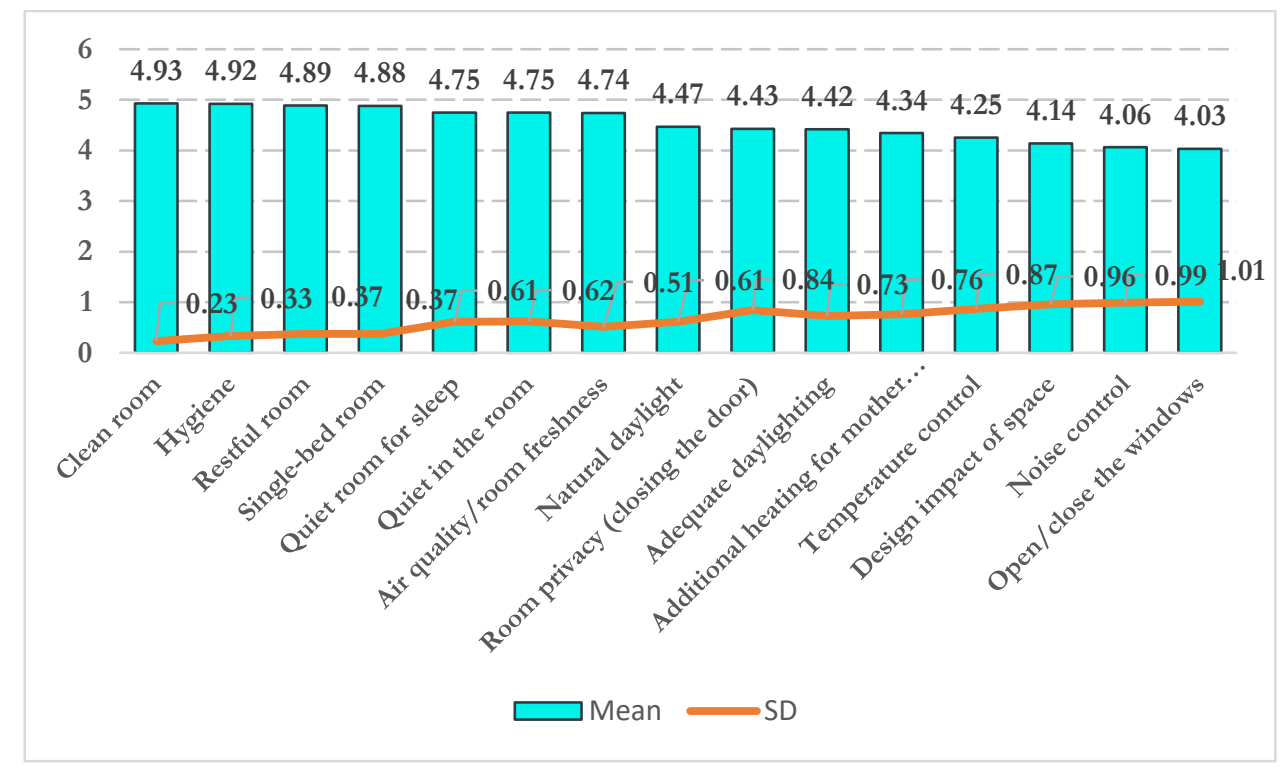

Figure 5-56: Top fifteen design features influencing women's recovery experience

Aside from hygiene/cleanliness which topped the list of design factors, issues regarding rest, sleep and quiet in the postnatal environment seemed to resolve into preference for a single-bed room occupancy by respondents. Evidence in support of this can be found from the three questions asking women what types of room they would like for care where answers were in support of a 
single-bed room. In addition, analysis of the respondents' comments on the items that need to be included for physical environment conducive to restoration gave support to single-bed rooms for privacy, and as relaxing and comfortable spaces.

Although social comfort environment aspects received lower ratings relative to the other design factors, the findings demonstrated a need for spaces to support the inclusion of partners and family members in the postnatal experience.

Pragmatically, the findings have clear implications in four major areas. First, the results have a direct implication on the role of organisational culture in the provision and maintenance of hygiene and cleanliness in the postnatal hospital environment. That women consider this as their top-most priority is arguably not in doubt - it affects the baby and new mothers health and wellbeing.

Second, the results confirm the need for a calm and comfortable environment to promote sleep and restfulness. This suggests the pressing need for devising management strategies that might promote/enhance a restful and restorative environment for women to have adequate sleep to assist the recovery process.

Third, opportunities for personal control of the physical environment and improving the design of the postnatal space to help women get benefits of daylighting and views could promote emotional and psychological wellbeing of women.

Fourth, promotion of person-centred care in the postnatal hospital environment through support facilities for social interaction is also seen as important for their overall wellbeing and health during hospital stays. 
Table 5-7: The 15 most important items in the Design Factors influencing the women's postnatal recovery experiences and wellbeing of the design perception survey (women, $\mathrm{n}=212$ )

\begin{tabular}{lll}
\hline Item & M & SD
\end{tabular}

Have a clean room

$4.93 \quad 0.23$

Having a hygienic/clean environment

$4.92 \quad 0.33$

Have a restful room

$4.89 \quad 0.37$

A single room is better for a woman's postnatal recovery care

$4.88 \quad 0.37$

A room or ward that is quiet is good for sleep and generally helps in the

$4.75 \quad 0.61$

recovery process

Having a room that feels quiet

$4.75 \quad 0.62$

Having good air quality and room freshness

$4.74 \quad 0.51$

Women appreciate a postnatal room in which natural daylight can easily enter

$4.47 \quad 0.61$

Be in a room where the door is closed except when visitors are allowed in(privacy)

Having adequate daylighting in the room/ward

Being a able to have additional heating for mother and baby

Being able to control the temperature

The postnatal room generally has less impact on how postnatal care is experienced by women (reverse coded)

Being able to control noise from mechanical things like ventilation, medical

$4.06 \quad 0.99$ equipment etc.

Knowing you can open or close the windows to feel comfortable

4.031 .01

Note: Items were ranked on a scale from 1 to 5 , where 1 indicated not at all important, and 5 indicated very important; $M=$ mean; $S D=$ standard deviation

\subsection{Conclusion}

This Chapter has presented the descriptive analyses and results of perception of design factors from the phase one survey for women. A number of design factors that could influence recovery experiences of women and wellbeing have been identified. In Chapter 6 the descriptive analyses and results for midwives on perception of design factors are discussed. 


\section{CHAPTER 6}

\section{PHASE ONE: MIDWIVES’ PERCEPTION OF DESIGN FACTORS SURVEY - RESULTS}

The second group of respondents in the phase one survey are the midwives. Data collection was carried out simultaneously with the postnatal participants. The midwives responded to the same questions as did women to see similarities or differences with regard to their perceptions of the physical design factors in the postnatal environment. The first section of this chapter discusses the demographic and work-related information of the respondents including their education levels and backgrounds. The details of the results are presented in the following part of the chapter.

\subsection{Demographic profile}

Of the 58 participants who participated in the on-line survey of midwives, the majority ( $\mathrm{n}=26$, $48.1 \%$ ) were within the age range of $46-64$ years; $33.3 \%$ of the participants $(n=18)$ were in the age range of 36-45. Additionally, 9.3\% of the participants $(n=5)$ fell between 25 and 35 years of age and $9.3 \%$ of participants $(\mathrm{n}=5)$ were greater than 65 years of age.

The other $(n=4,7.3 \%)$ respondents had completed a Nursing and Midwifery degree, and one person had an additional Diploma in Law. The vast majority were from NZ/Pakeha ( $\mathrm{n}=34,61.8 \%)$ and European $(\mathrm{n}=19,34.6 \%)$ backgrounds. $3.6 \%$ of the participants were from a Maori background. A greater number of the respondents are well educated with nearly half who had completed university $(n=25,45.5 \%)$ and nearly half with postgraduate education $(n=26,47.3 \%)$ as shown in Figure 6-1. At the time of the survey $60 \%$ and $40 \%$ were hospital-based and community midwives respectively. 


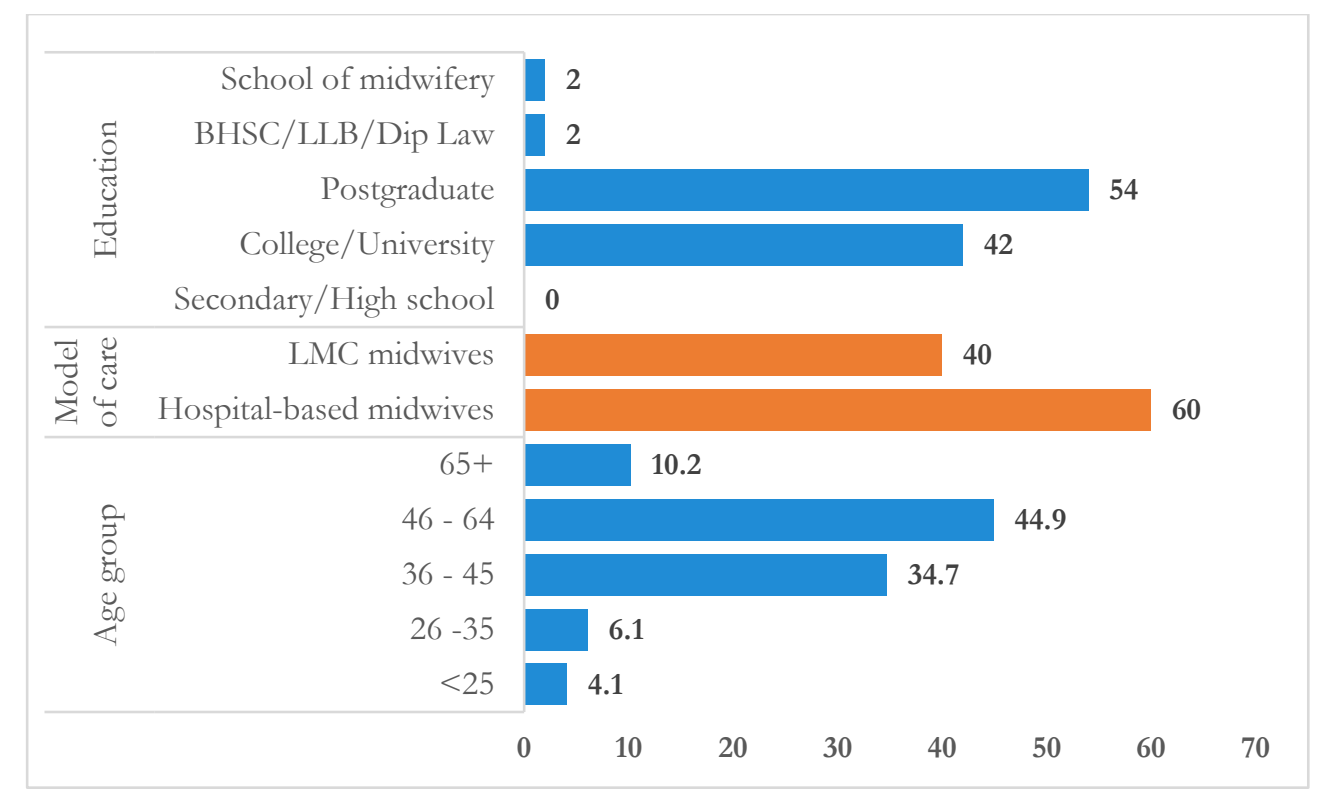

Figure 6-1: Percentage of respondents' model of care, by age group and education level

Of these, $41.8 \%(n=23)$ and $32.7 \%(n=18)$ had been working in hospitals for periods of 5 to15 and 15 to 25 years respectively. $14.5 \%(n=8)$ had work experience of less than 5 years and 3.6\% $(n=2)$ have had experience for periods of 26 to 35 years while $7.3 \%$ had been working for more than thirty-five years. The diversity of different working areas and experience ensured that a wide range of respondents was represented in this study. Figure 6-2 shows the distribution of respondents by years of experience.

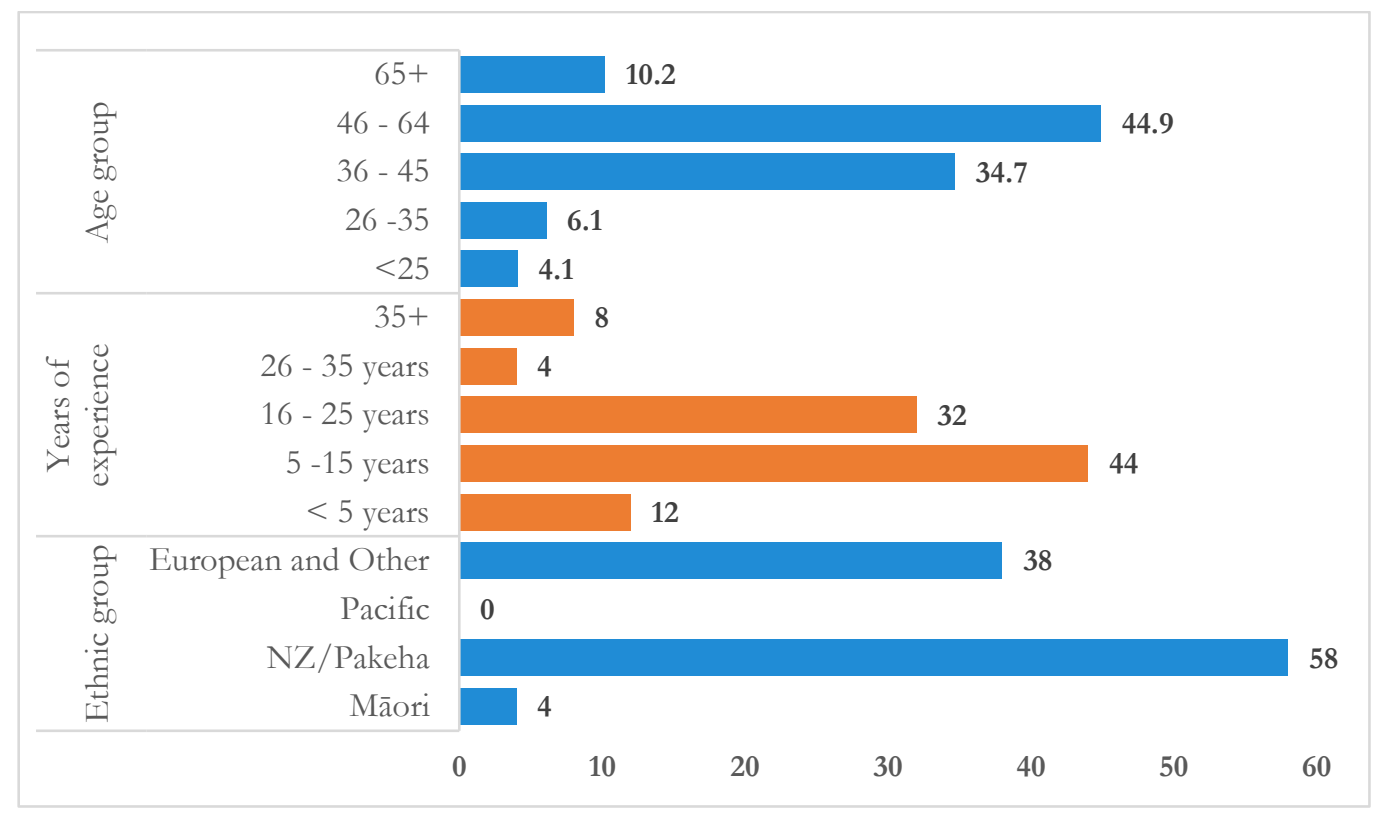

Figure 6-2: Percentage of respondents' (midwives) years of experience, by age group and ethnic group 
Table 6-1: Demographic details of sample participant midwives $(n=50)$ in phase one

Characteristics

\begin{tabular}{|c|c|c|c|}
\hline & & \\
\hline \multirow[t]{2}{*}{ Model of care } & Hospital-based midwives & 30 & 60 \\
\hline & LMC midwives & 20 & 40 \\
\hline \multirow{2}{*}{$\begin{array}{l}\text { Any postnatal ward } \\
\text { experience? }\end{array}$} & Yes & 48 & 96 \\
\hline & No & 2 & 4 \\
\hline \multirow[t]{5}{*}{ Years of experience } & Less than 5 years & 6 & 12 \\
\hline & $5-15$ years & 22 & 44 \\
\hline & $16-25$ years & 16 & 32 \\
\hline & $26-35$ years & 2 & 4 \\
\hline & More than 35 years & 4 & 8 \\
\hline \multirow[t]{5}{*}{ Age (yr) } & $<25$ & 2 & 4.1 \\
\hline & $26-35$ & 3 & 6.1 \\
\hline & $36-45$ & 17 & 34.7 \\
\hline & $46-64$ & 22 & 44.9 \\
\hline & 65 and above & 5 & 10.2 \\
\hline \multirow{7}{*}{$\begin{array}{l}\text { Highest level of education } \\
\text { completed }\end{array}$} & Elementary school/Primary school & - & - \\
\hline & Secondary/High school & - & - \\
\hline & College/University & 21 & 42 \\
\hline & Postgraduate & 27 & 54 \\
\hline & $\mathrm{PhD}$ & - & - \\
\hline & BHSC/LLB/Dip Law & 1 & 2 \\
\hline & School of Midwifery & 1 & 2 \\
\hline \multirow{8}{*}{ Ethnicity } & African & - & - \\
\hline & Asian & - & - \\
\hline & European & 18 & 36 \\
\hline & Māori & 2 & 4 \\
\hline & Middle Eastern & - & - \\
\hline & NZ/Pakeha & 29 & 58 \\
\hline & Pacific Island & - & - \\
\hline & Other (British) & 1 & 2 \\
\hline
\end{tabular}

Category N $\quad \%$

$$
\mathrm{N}
$$

$*$ LMC $=$ Lead Maternity Carer

Q1.3 When was the last time improvements were carried out to the postnatal ward in your hospital?

More than half of the respondents $(n=29,58 \%)$ reported that improvements of the postnatal ward were carried out in the last 5 to 10 years. Five respondents $(10 \%)$ reported 5 years; and six percent $(n=3)$ thought it was done in the last 11 to 15 years while fourteen respondents $(26 \%)$ indicated they had no idea. Further analysis (of both core or hospital-based, and LMC midwives) reveals that of participants who were employed between 5-10 years ago, twelve work as hospitalbased midwives and have experiences ranging from five to fifteen years. Ten of the fourteen participants who had no idea of last improvements in the postnatal wards were also hospital-based midwives, six of whom had experience which ranged from five to fifteen years; three with $16-25$ years' experience and five with work experience of less than 5 years as midwives. 
In other words, the participants could not give the exact period of last improvements in the postnatal ward/unit regardless of their experience. However, many respondents indicated 5- 10 years (see Figure 6-3).

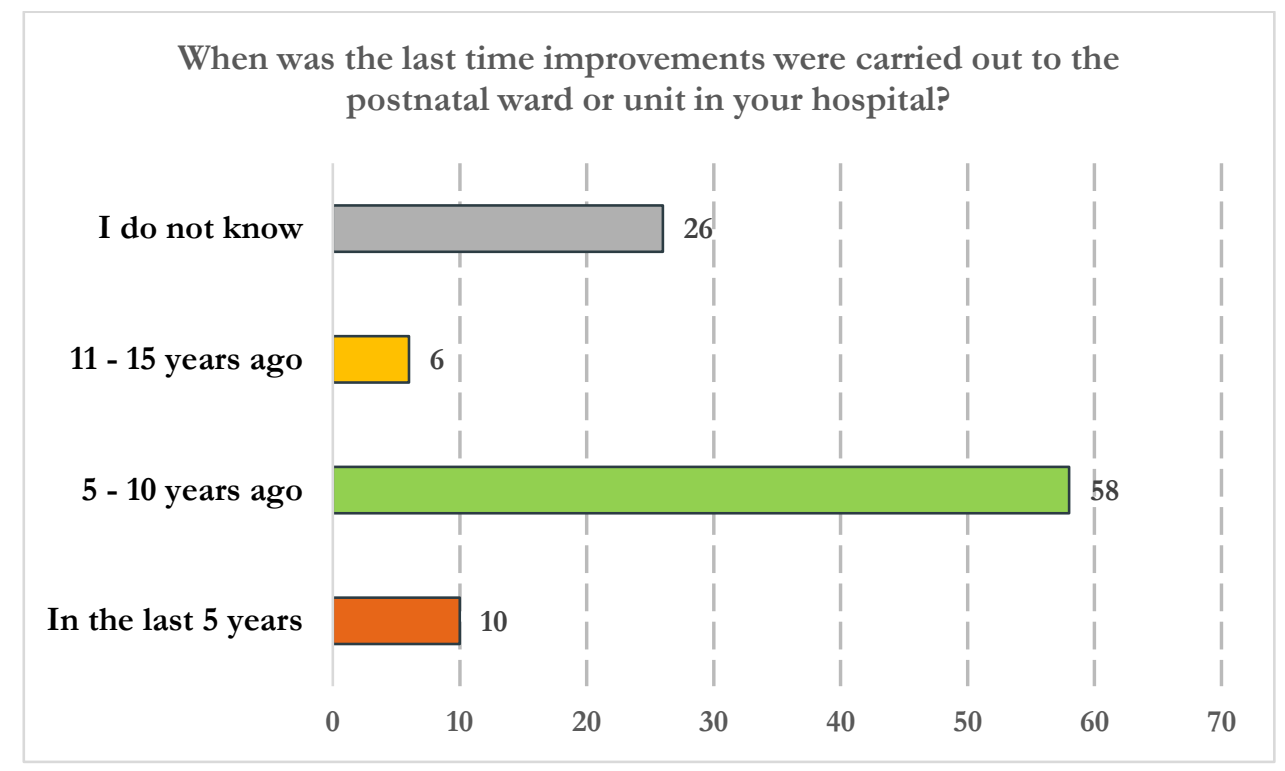

Figure 6-3: Time of last improvements in the postnatal ward

Q2 Have you worked across the maternity model of care that includes working in a postnatal room?

Q2.1 Do you provide care away from the birth room in a designated postnatal ward or other separate room?

Two sequential questions were asked of the respondents to understand the levels of familiarity with postnatal environments. Most of the respondents $(n=48,96 \%)$ reported they have been working across the model of care attending to women in postnatal wards. Only two respondents $(4 \%)$ indicated they have not worked in postnatal wards. With regards to providing care in a designated postnatal ward, participants reported the same response (see Figure 6-4). The results reflected a clear familiarity with postnatal hospital environments. 


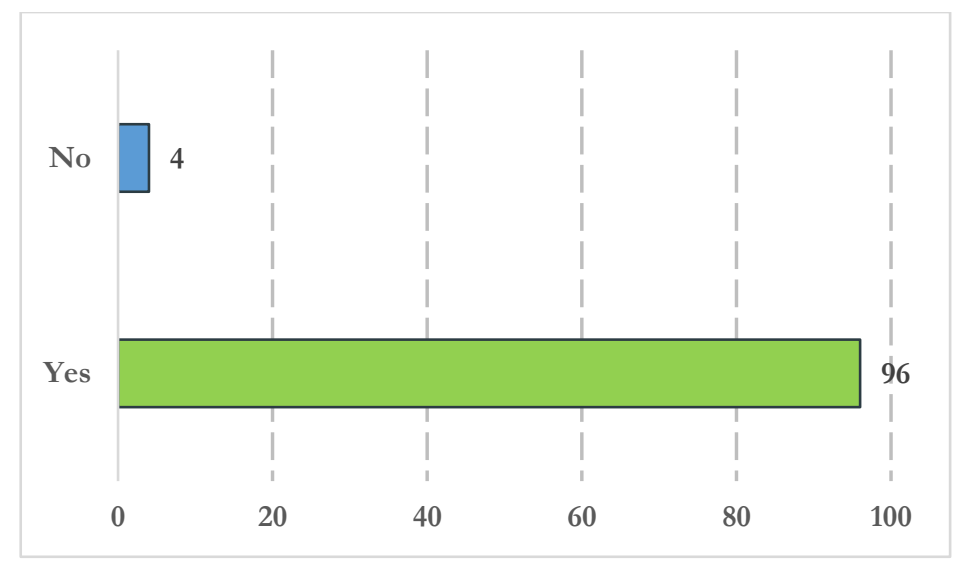

Figure 6-4: Respondents' experience across model of care in the postnatal ward

\subsection{Perception of postnatal physical environment}

Most respondents thought the physical environment would impact on postnatal care and women's experiences, $24 \%$ of the respondents stated agree $(n=12)$ and $74 \%(n=37)$ said strongly agree. Only one respondent (2\%) said neither (see Figure 6-5). Nine out of $10(\mathrm{n}=49)$ respondents indicated that the physical environment would impact on the quality of postnatal caregiving and by extension on women's recovery experiences. The result is consistent with the women's result in which $93.9 \%(\mathrm{n}=199)$ indicated that the physical environment would impact on their postnatal recovery experiences. The result reflected the importance of quality of the environment in care and is consistent with the findings on labour and delivery rooms (Newburn \& Singh, 2003).

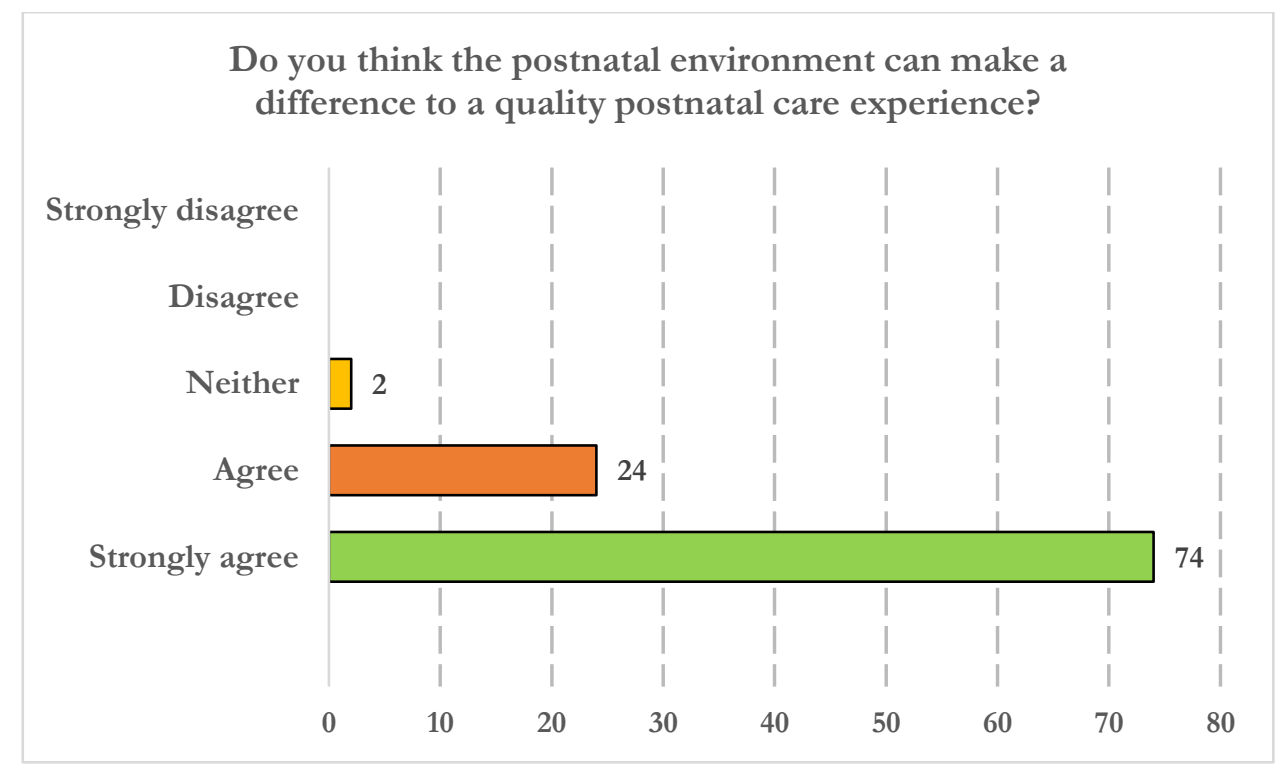

Figure 6-5: Respondents' rating of impact of physical environment on care 


\subsection{Perception of postnatal room features}

A total of nine design factors/features on the survey questionnaire (see midwives survey questionnaire in Appendix B) were rated by the respondents. The results of descriptive analysis are presented next in this section.

\section{What did you think about the room?}

In section 6.2, respondents were asked about perception of the physical environment in general. This section focuses on specific aspects of postnatal room features.

\section{Q3.1: A single room is better for a woman's postnatal recovery care}

Most of the respondents thought that a single room supported a woman's recovery. Perception of a single room was ranked as strongly agree by twenty-six respondents $(52 \%)$, eleven $(22 \%)$ rated agree and ten respondents (20\%) ranked neither agree nor disagree. Three (6\%) stated disagree (see Figure 6-6). Nearly three-quarters $(\mathrm{n}=37,74 \%)$ of the respondents are lending support for single-bed occupancy room.

The result reflected that a single-bed room is of value in women's recovery experiences and wellbeing. The respondents who stated neither agree nor disagree and disagree as a percentage of the whole are relatively small compared to those in overall agreement.

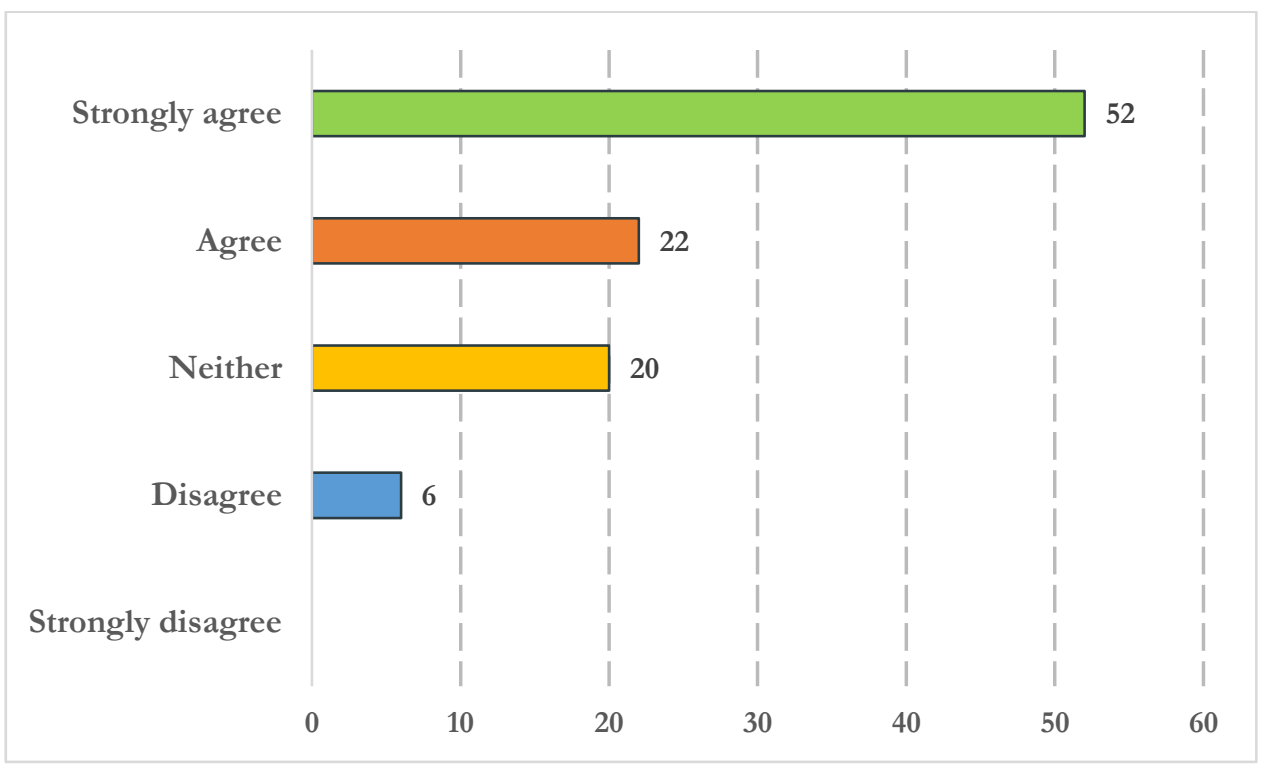

Figure 6-6: Respondents' ratings of a single-bed room's impact on recovery 
Q3.2: A room or ward that is quiet is good for sleep and generally helps in the recovery process

Most of the respondents reported that a room that is quiet supported sleep and helped the recovery process. The majority ( $\mathrm{n}=47,94 \%)$ ranked this as agree and strongly agree, and only three respondents $(6 \%)$ thought neither agree nor disagree (see Figure 6-7).

The result indicated that the sensory quality of the physical environment has a significant contribution to make for sleep and wellbeing.

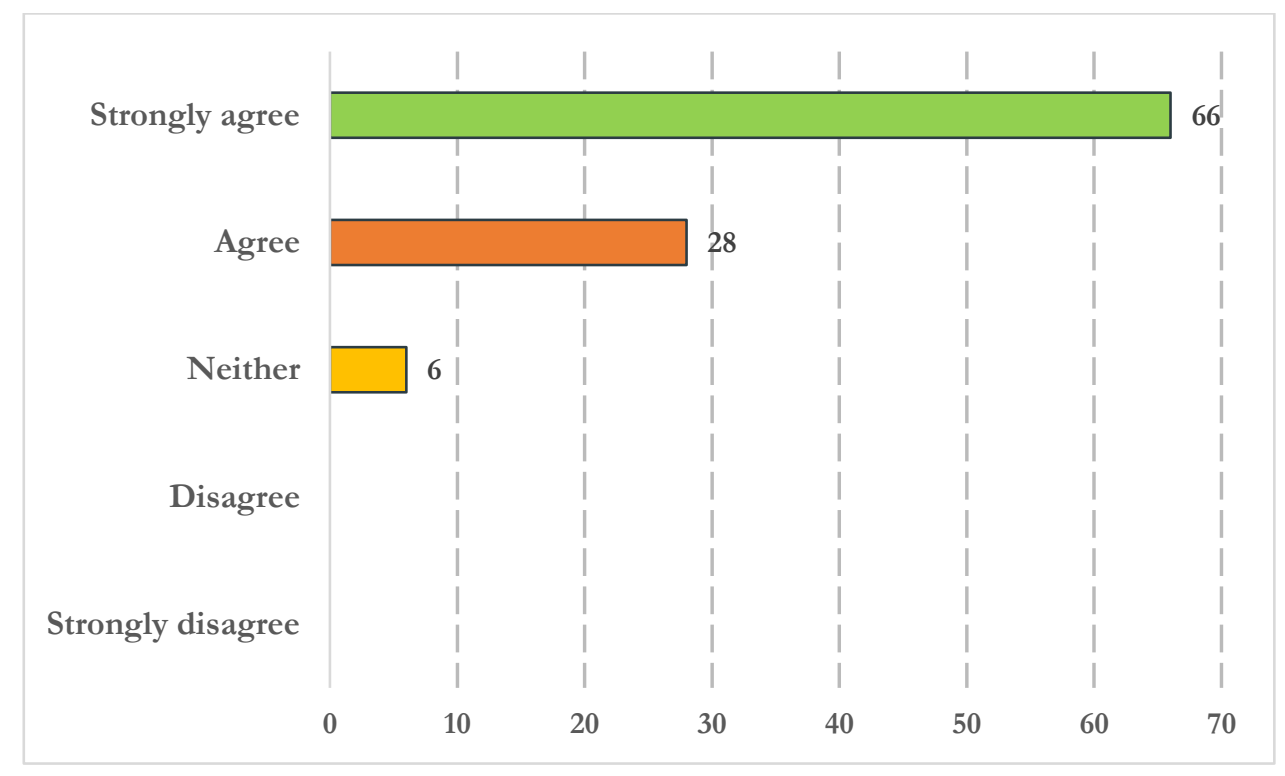

Figure 6-7: Respondents' ratings of a room/ward's impact on sleep and recovery

The large majority of the respondents thought that a quiet room is important for recovery of women in the postnatal hospital environment.

\section{Q3.3: The postnatal room generally has less impact on how postnatal care is experienced by women}

This question was reverse coded so those who thought design of postnatal room/space was important rated it in the negative. The impact of design was reported as strongly disagree by sixteen $(32 \%)$ respondents, twenty-two (44\%) as disagree, and ten respondents ranked neither agree nor disagree. Two (4\%) of the respondents reported agree (see Figure 6-8).

The result indicated that the quality of postnatal room/space is strongly perceived to have impact on postnatal care and women's recovery experiences. 


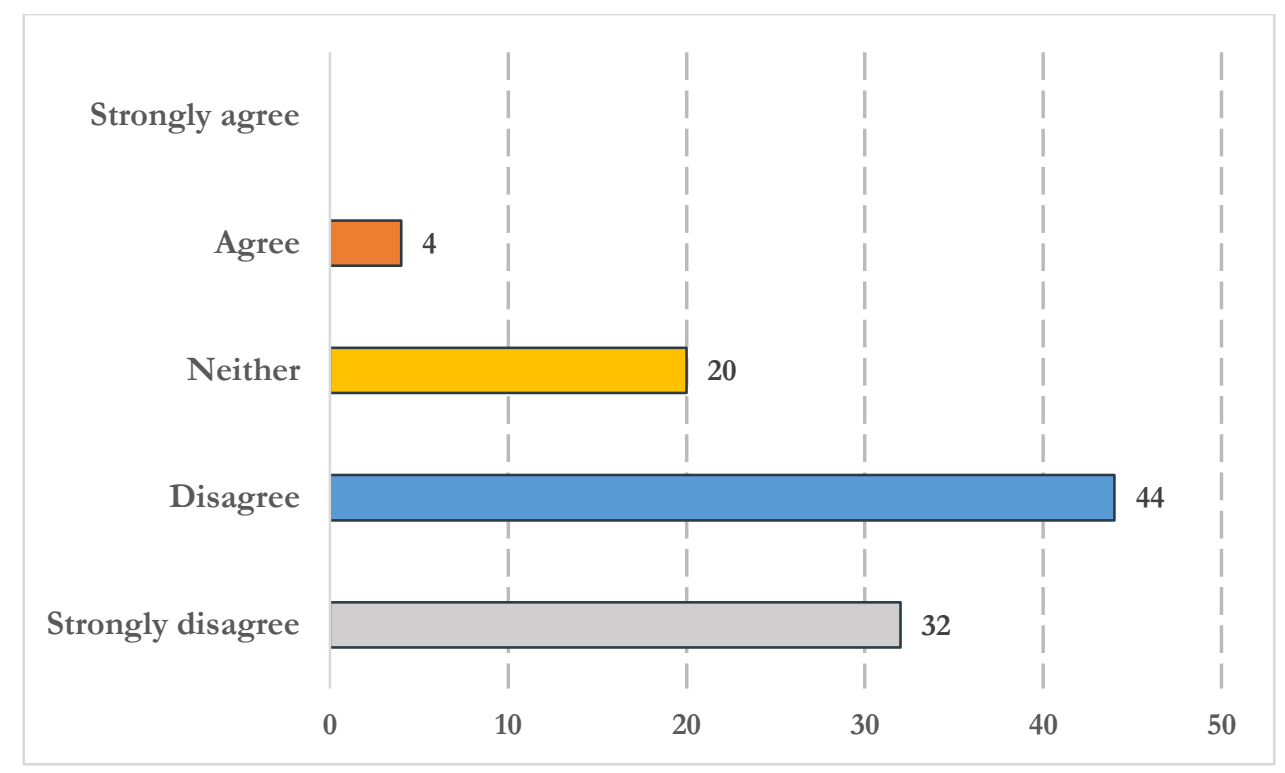

Figure 6-8: Respondents' ratings of impact of space on care

\section{Q3.4: A room with colours is better for birth recovery than a very plain room}

There was a uniform distribution of opinions generally. A room with colours versus a plain room was ranked agree for better birth recovery was ranked agree by nineteen $(38 \%)$ respondents, and twenty-five (50\%) reported neither agree or nor disagree. Another $6 \%$ ( $\mathrm{n}=3$ in both cases) either disagree or strongly agree (see Figure 6-9). The result is not dissimilar to postnatal participants for the same question (see Figure 5-10 in the previous chapter).

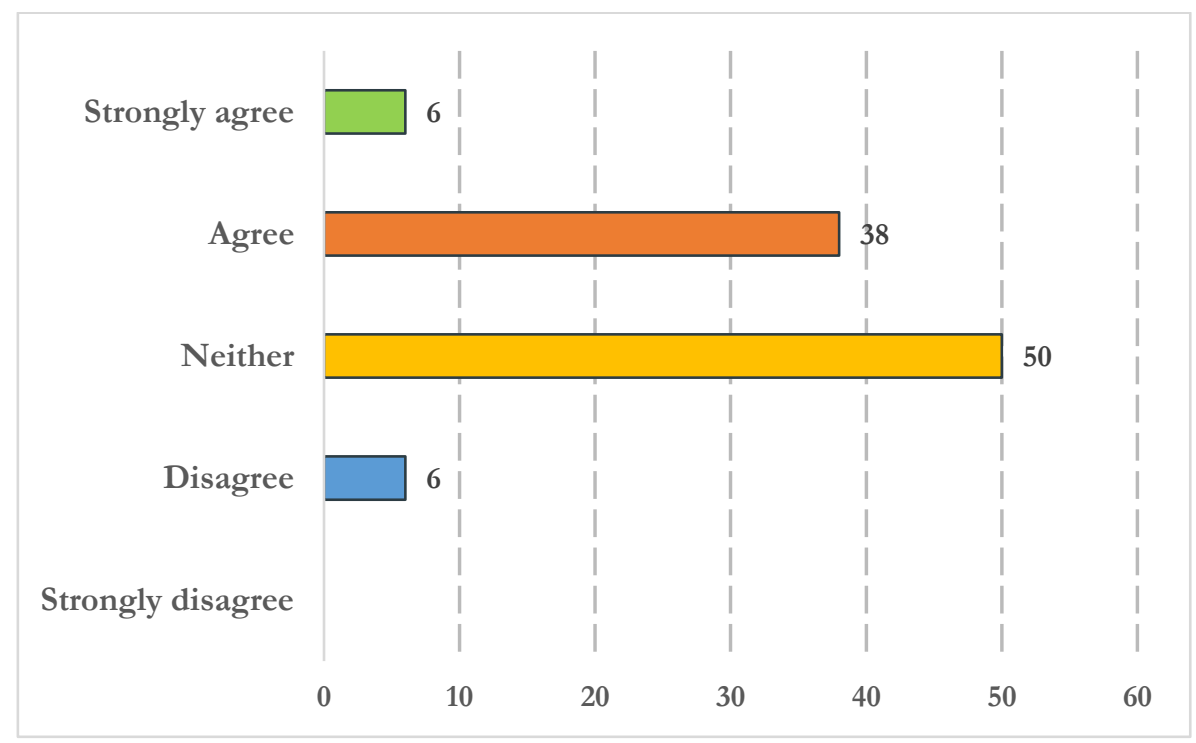

Figure 6-9: Respondents' ratings of room with colours versus plain room for birth recovery 
A close examination of the histogram shows that the distribution was normal. The quantity of respondents $(44 \%, \mathrm{n}=22)$ giving a positive response is nearly equal to the respondents who stated neither agree nor disagree (see Figure 6-10).

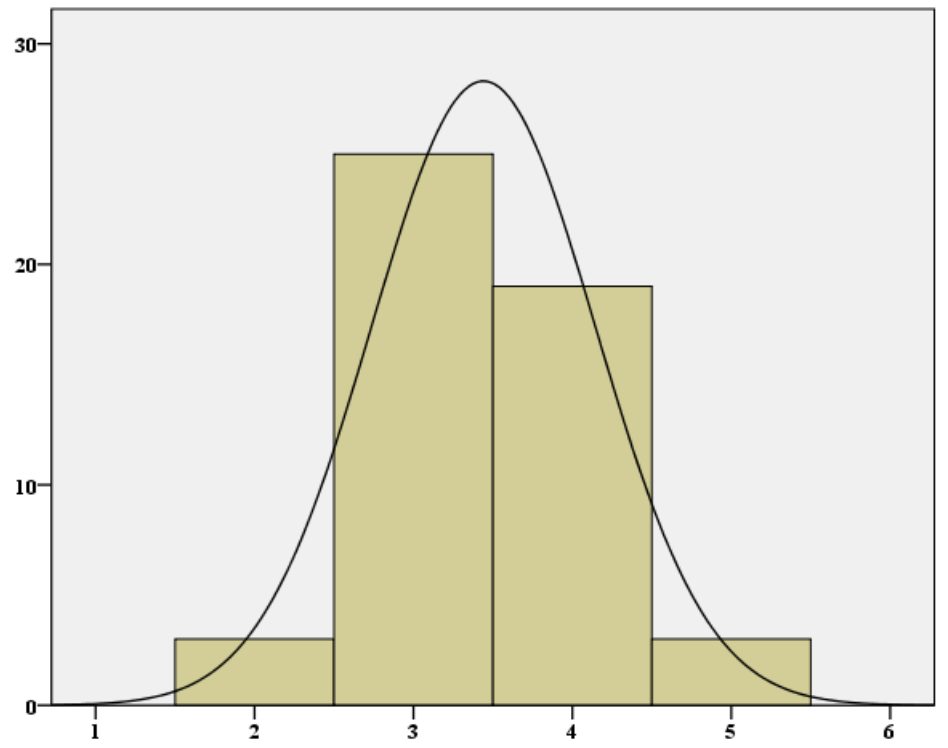

Figure 6-10: A normal distribution of ratings of room with colours and plain room

This suggests that people have varying views regarding choice of colours. It may be more effective to argue that a postnatal room (and other healthcare settings that focus on rehabilitation) should have a specific set of colours, similar to the approach often used in children's hospitals. Further research would be needed to indicate how the interiors of postnatal rooms should look to optimise for recovery since hospital environments conventionally tend to have an apperarance of sterile offwhite colours. Architects, designers, and environmental psychologists would have a role to play. An alternative would be to use more colours in things that could be changed relatively easily such as curtains or bedding.

\section{Q3.5: Most women prefer to stay in a postnatal room that overlooks a garden, or has pictures of natural scenery on the walls}

Most of the respondents thought that women would prefer to stay in a room with views of nature or natural scenery on the walls. Thirty-two respondents (64\%) rated agree, and ten (24\%) strongly agree. Eight respondents (16\%) reported neither agree nor disagree. None disagreed or strongly disagreed (see Figure $6-11)$. Just over 8 out of $10(n=84 \%)$ indicated views of nature were important in women's recovery experience. Although a small proportion of respondents $(n=8)$ did 
not express a view either way, a view of the natural environment can be assumed to have an effect on women's psychological and wellbeing in postnatal hospital stays.

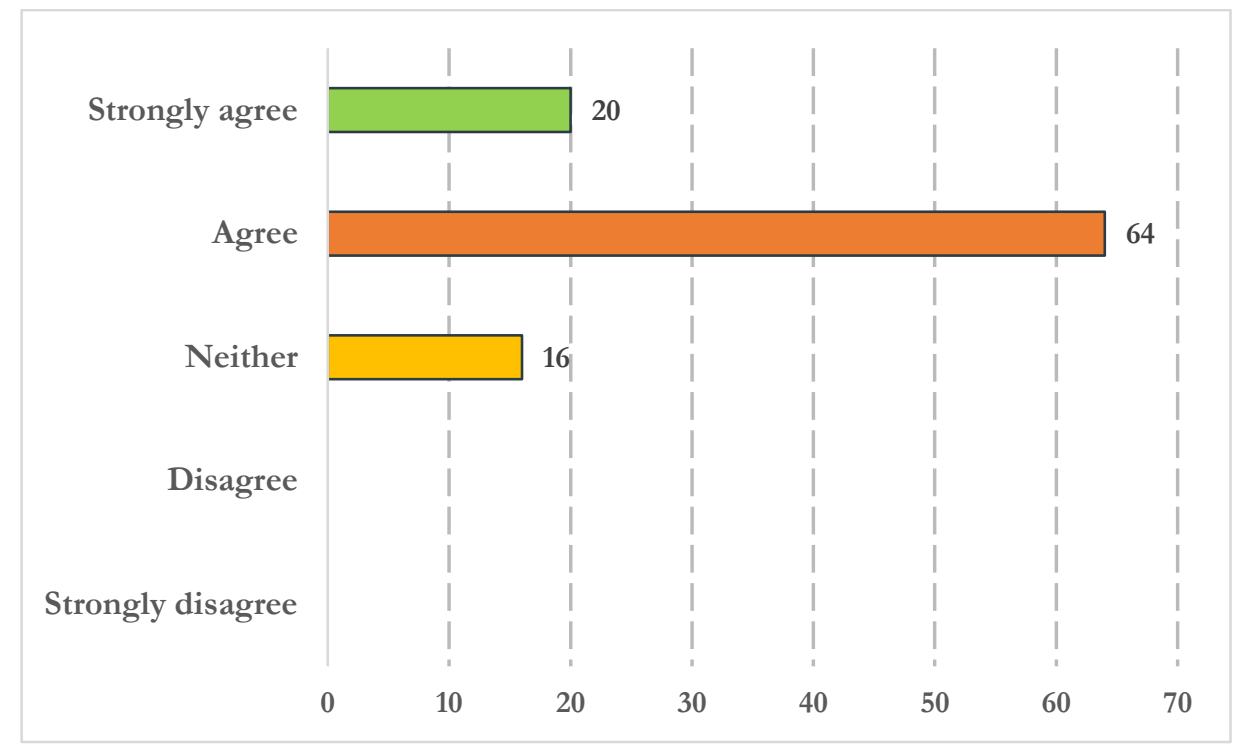

Figure 6-11: Respondents' ratings of room with views of nature for care

\section{Q3.6: A postnatal room with access to outdoor surroundings is better for recovery care}

Most of the respondents thought access to outdoor surroundings would support recovery. Twenty-eight $(56 \%)$ reported agree, fifteen respondents $(30 \%)$ strongly agree, and six $(12 \%)$ reported neither agree nor disagree. Only one respondent $(n=1,2 \%)$ rated disagree (see Figure 612).

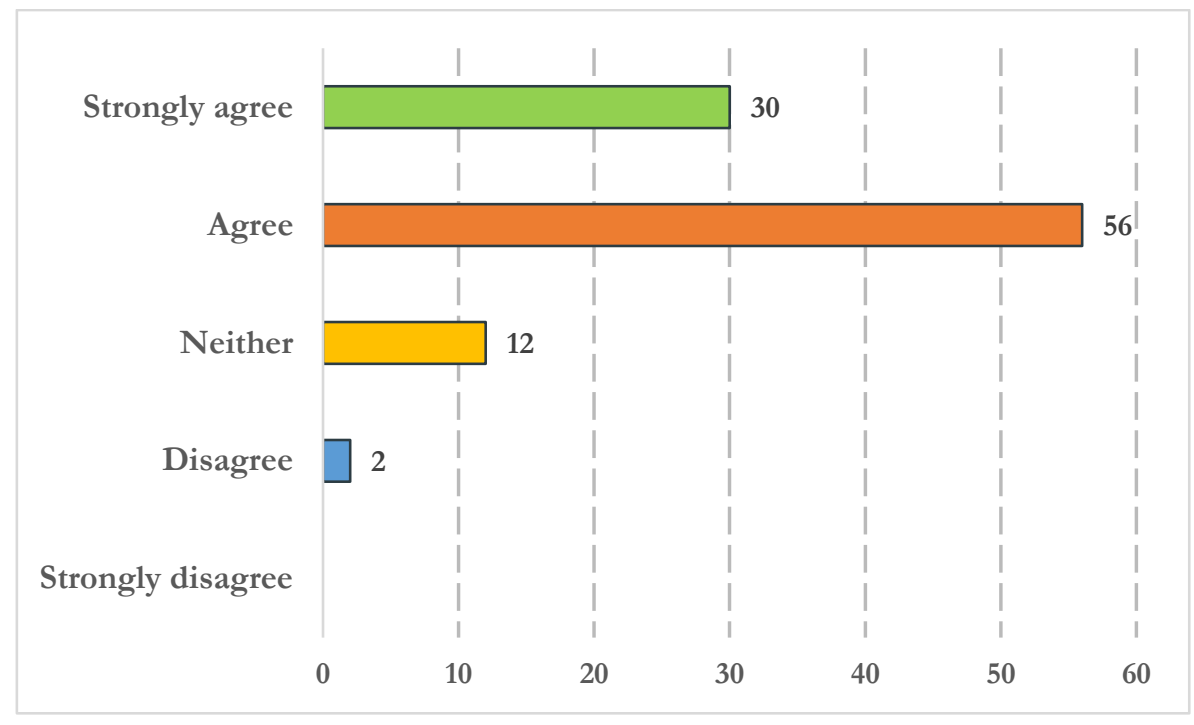

Figure 6-12: Respondents' ratings of a room with access to the outdoors for recovery 
The result reflected the importance of having outdoor surroundings as an integral design of the postnatal environment particularly where women can retreat into the outdoors if they considered it appropriate to do so. The six respondents who reported neither agree nor disagree may be thinking that some women may not choose going outdoors after the birth, perhaps due to stress and pain following childbirth. There could also be a possibility that an outside area was nonexistent in their situation

\section{Q3.7: A postnatal room that lets in natural daylight is better for recovery care}

Most of the respondents reported that allowing natural daylight into the room was better for recovery. A postnatal room with access to natural daylight was ranked agree by twenty $(40 \%)$ respondents and twenty-eight (56\%) respondents ranked strongly agree. Two respondents (4\%) ranked neither agree nor disagree (see Figure 6-13). No respondent rated this question negatively, indicative that daylight may be likely to have a strong link to the quality of postnatal recovery.

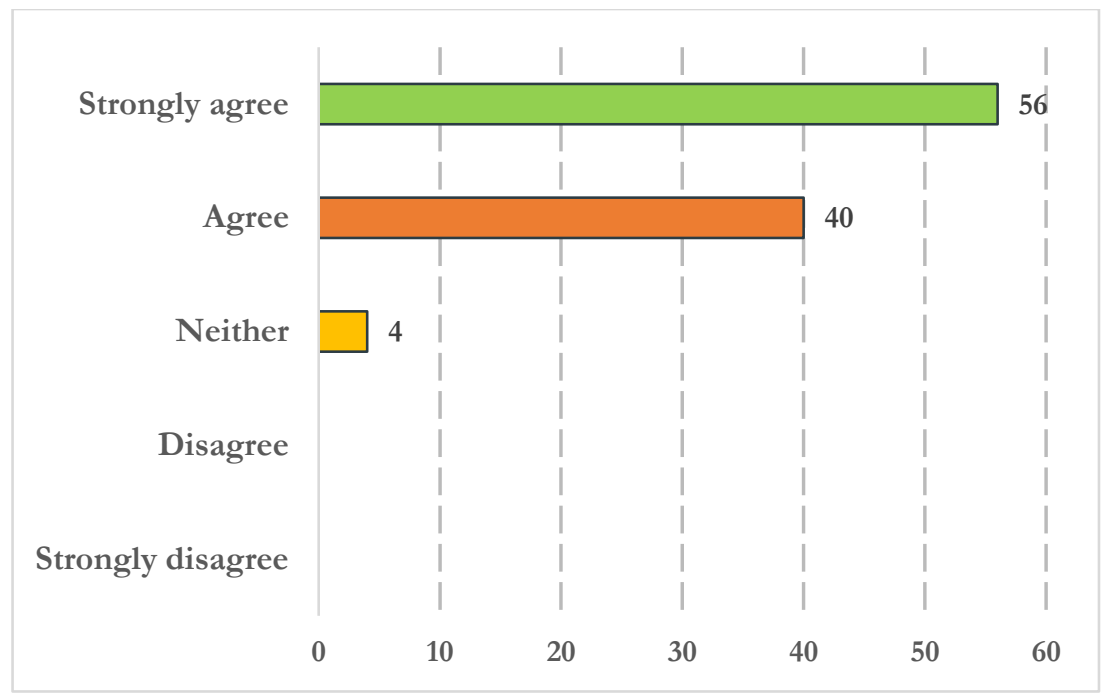

Figure 6-13: Respondents' ratings of a room with access to daylight for recovery

The result indicated the importance of the restorative value of daylight in the healthcare environment. Daylight in the room can have beneficial effects for women to adjust to rhythmic sensations of day and night. The midwives have likely seen benefits as well as for staff who have long working hours.

\section{Q3.8: A postnatal room that has natural finishes such as wood is better for recovery}

The perception of natural finishes in the room was ranked fairly moderately. The quality of a room with natural finishes was rated agree $(24 \%)$ by twelve respondents and seven respondents $(14 \%)$ 
rated strongly agree. Twenty-eight $(56 \%)$ respondents reported neither agree nor disagree while three $(6 \%)$ of the respondents ranked disagree (see Figure 6-14).

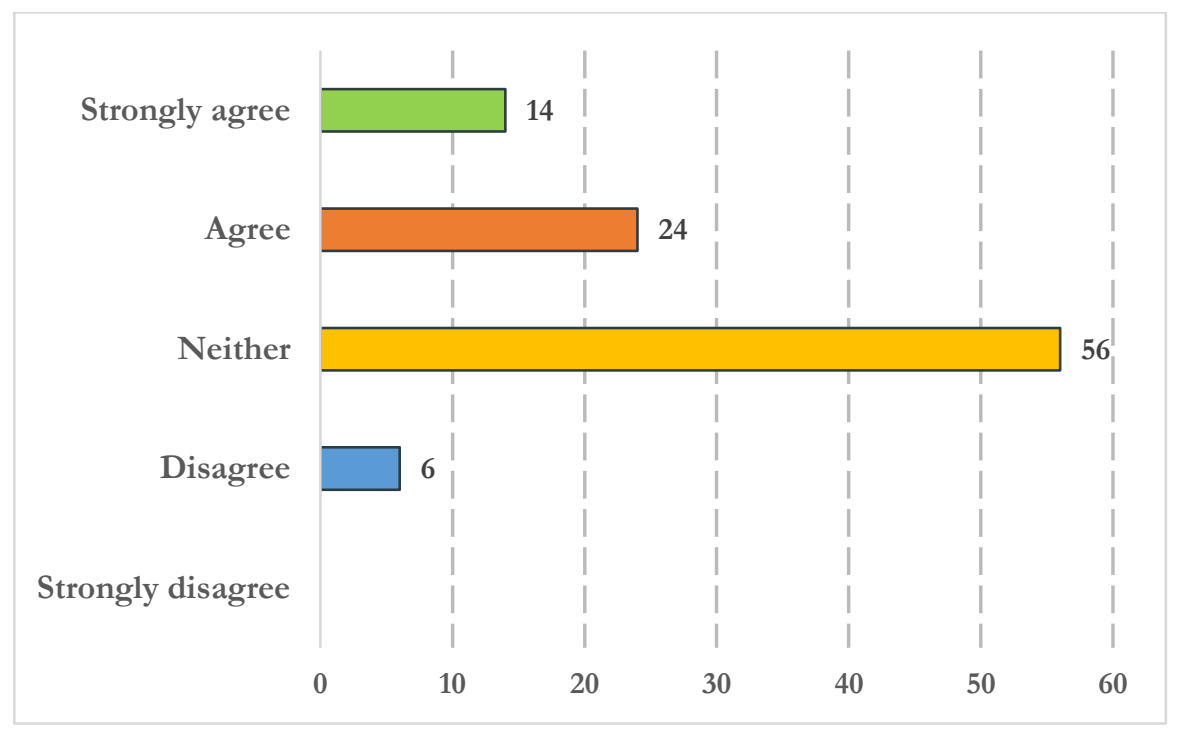

Figure 6-14: Respondents' ratings of natural finishes in the room for recovery

The result reflected largely uniformly distributed opinions among respondents (see Figure 6-15). Just above one-third were positively in favour compared to the respondents who either reported disagree or neither categories.

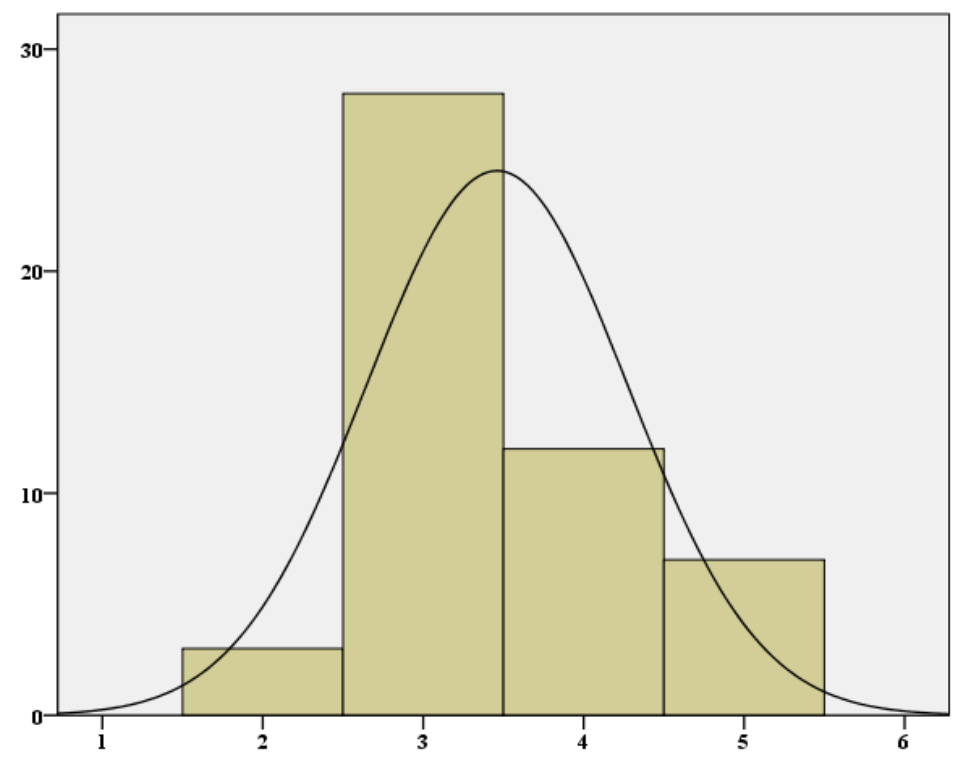

Figure 6-15: A slightly normal distribution of ratings of natural finishes in the room 
Q3.9: Shapes and forms like those of natural objects such as flowers, flowing water etc can make for a more beautiful postnatal room design

Most respondents thought natural forms and shapes of objects such as flowing water or flowers would improve the design of the postnatal environment. Twenty nine $(58 \%)$ respondents, ranked agree and six $(12 \%)$ ranked strongly agree while fifteen $(30 \%)$ respondents selected neither (see Figure 6-16).

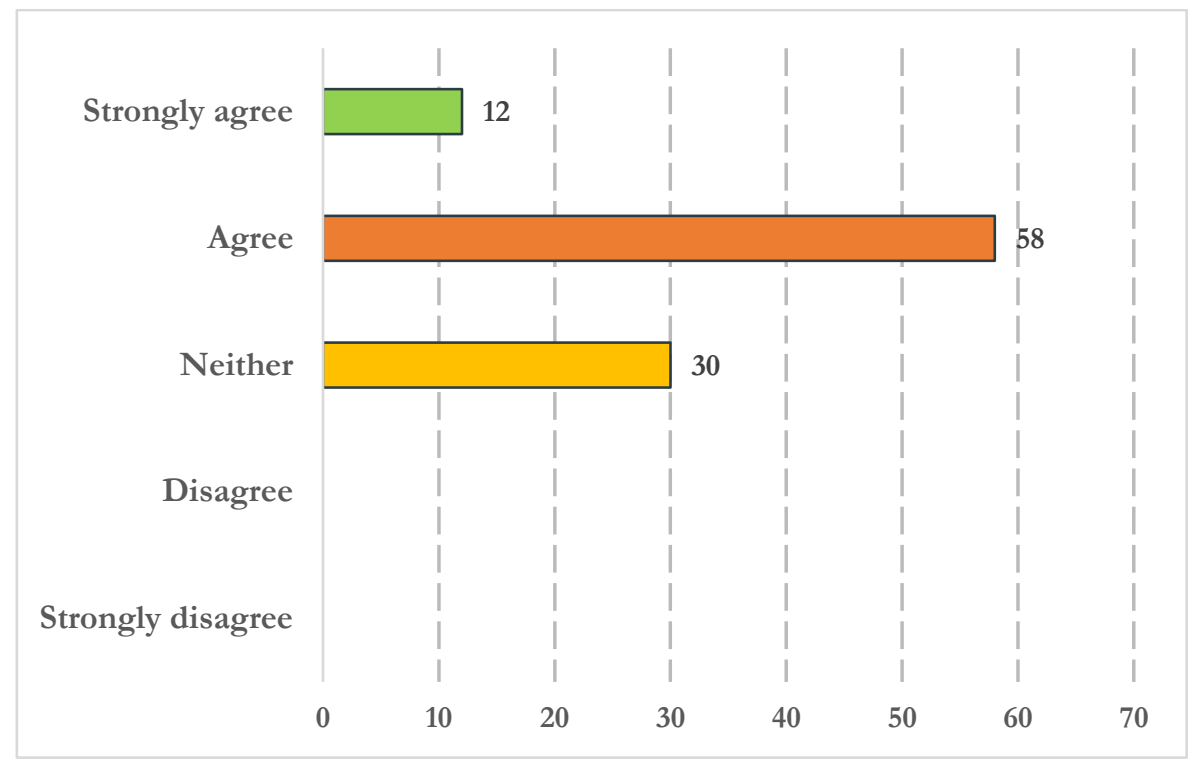

Figure 6-16: Respondents' ratings of shape and natural form for postnatal room design

The result reflected the importance of a well-designed environment for quality care. Shapes and forms are important elements of the built space that applied well and effectively, can create a sense of physical and psychological calm. The large number of positive respondents indicated that these elements are considered to have impact on the experience of both women and caregivers inhabiting the postnatal environment.

In summary, Table 6-2 shows the results of descriptive statistics on perceptions of postnatal room features. 
Table 6-2: Preference (mean and standard deviation) of respondents on perception of postnatal room features contributing to recovery care and women's wellbeing (midwives, $\mathrm{n}=50$ )

\begin{tabular}{llc}
\hline Survey question & M & SD \\
\hline A room or ward that is quiet is good for sleep and generally helps women recover & 4.60 & 0.61 \\
in comfort & & \\
A postnatal room that lets in natural daylight is better for recovery care & 4.52 & 0.58 \\
A single room is better for a woman's recovery care & 4.20 & 0.97 \\
A postnatal room with access to outdoor surroundings is better for care & 4.14 & 0.70 \\
Most women prefer to stay in a postnatal room that overlooks a garden, or has & 4.04 & 0.61 \\
pictures of natural scenery on the walls & & \\
The postnatal room generally has less impact on how postnatal care is experienced & 4.04 & 0.83 \\
by women (reverse coded) & & \\
Shapes and forms (natural objects such as flowers, flowing waters, etc.) for & 3.82 & 0.63 \\
postnatal room design/layout & & \\
A room with natural finishes such as wood is better for recovery & 3.46 & 0.81 \\
A room with colours is better for recovery than a very plain room & 3.44 & 0.71 \\
\hline
\end{tabular}

Note: $M=$ mean; $S D=$ standard deviation

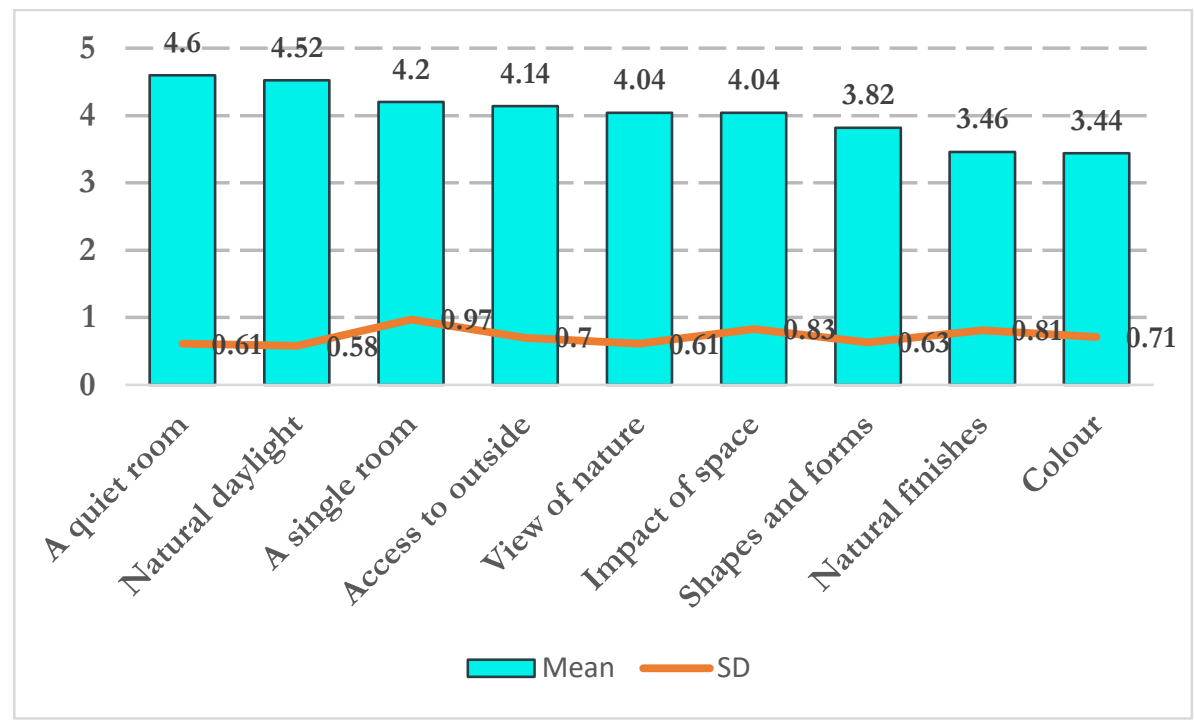

Figure 6-17: Respondents' ratings of postnatal room features

\section{Summary}

Descriptive statistics results comprising perceptions of nine design features of postnatal room features have been presented. A "room or ward that is quiet", "natural daylight" and "a single-bed room" had the highest mean scores of $(M=4.60)$; $(M=4.52)$; and $(M=4.20)$ respectively. Several other design features also had mean scores higher than 4 (= acceptance). The shapes and form design feature had a mean score of $(M=3.82)$ higher than the remaining design features such as colours and natural finishes in the room, which both had mean scores higher than 3 corresponding to neutral as shown in Figure 6-17. The results on perception of interior environment are discussed next. 


\subsection{Perception of postnatal interior environment factors}

This section of the survey comprises nine questions to understand aspects of the interior environment influencing recovery care. As lead-in to the main survey questions, respondents were asked what they thought about the spaces in which postnatal care was provided.

Do you think the interior environment of postnatal spaces in which you work or have worked in the past affects how easy you found it to provide care?

Most of the respondents thought the interior environment affected the ease or difficulty of providing care. Twenty-three respondents $(46 \%)$ ranked this as strongly agree, and twenty-two $(44 \%)$ as agree. By comparison, four respondents stated neither agree nor disagree, and one rated it as strongly disagree while no respondent selected the disagree option (see Figure 6-18). The result reflected the perceived importance of the interior environment in postnatal care giving.

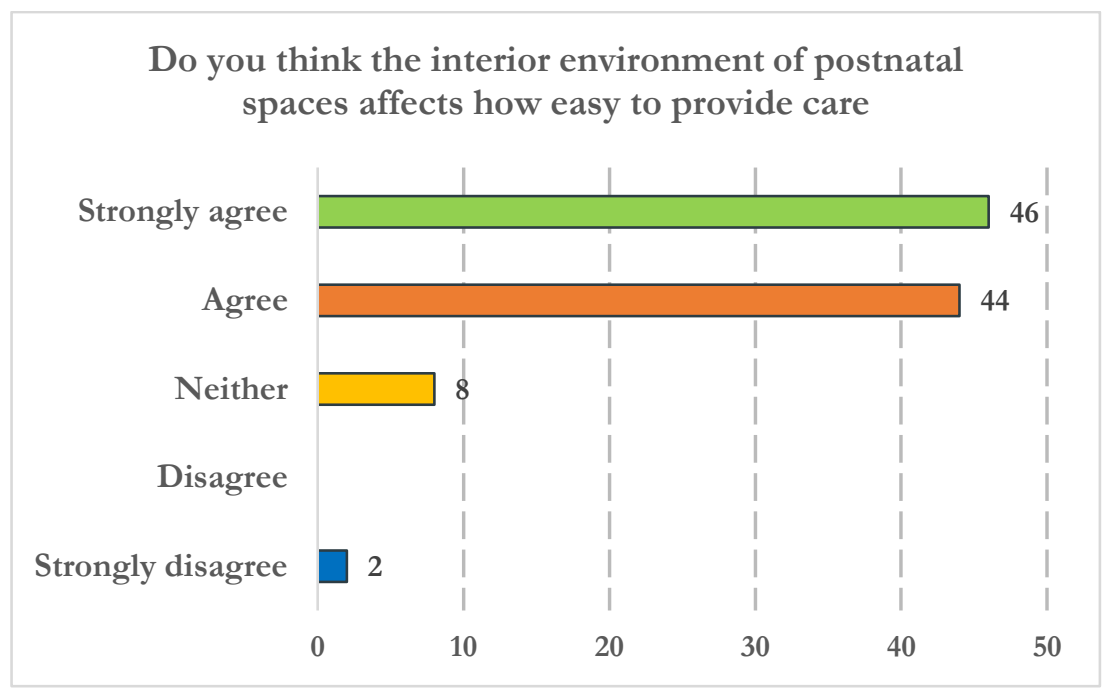

Figure 6-18: Respondents' ratings of interior environment on staff job performance

Responses to the two open-ended questions asking respondents to identify the factors in the postnatal spaces that made their work easy or difficult (see questions 3.11 and 3.12 in the midwives' questionnaire in Appendix B) were analysed to see if midwives would report whether they were satisfied with the spaces or would report where improvements were required.

\subsubsection{Satisfaction and difficulties with space}

45 responses were retrieved from the completed questionnaires and entered into the computer as a Word document. 
Respondents made copious notes about a wide range of design features and facilities that they felt were important to their work as well as those that do not work for them. A close examination of the text led to the coding of the design features identified which were organised into categories of elemental factors including spatial, architectural characteristics, hygiene and maintenance, sound/acoustics, ergonomics and feeling of safety. From the standpoint of the midwives, these design characteristics and factors are perceived important for recovery.

Aspects of the features that support midwives in their work and provide support for women in recovery include space, privacy, tranquility (quiet time) in the environment and natural lighting for visibility. Other factors reported by respondents were cleanliness, fresh air or ventilation, spacious environment and accommodation, usually a single room, where the partner would be able to stay the night, floor layout with reduced travel time for midwives and comfortable furniture for learning of parental skills.

Additionally, factors that made the work difficult in the interior postnatal environment includes poor sound engineering of spaces, noisy and busy situations in rooms, lighting problems (artificial and natural) shared rooms and/or multi-bed accommodation. Other areas hindering work and postnatal care include lack of functional equipment, and poor ergonomics (such as computer, work-surfaces for documentations), as well as safety of mothers and babies. Other repeated factors such as clutter in the way, may be due possibly to narrow spaces and lack of cubicles for personal belongings.

As an example, many participants described the impact of the room on privacy such as the fact that having shared rooms affects routine intimate physical checks.

Double rooms are not private, so it can be difficult to have frank conversations around things like blood loss, incontinence... and increases anxiety when physical checks needs to be performed on postnatal women... when one only has a curtain as "privacy" these checks can be more invasive.

Another respondent described her experience of working with a range of spaces both in birth centres and in hospital-based postnatal wards.

I personally feel that maternity care generally should be kept out of the hospital environments for well, low-risk women and babies and I like caring for postnatal women in their own homes where they seem most relaxed and content. 
As can be seen in Table 6-3, comments from the open-ended questions highlight many important design factors and challenges in postnatal healthcare environments pointing to some of the factors in the design of postnatal hospital environments where there could be potential in addressing future postnatal space needs (Schmied, Cooke, Gutwein, Steinlein, \& Homer, 2008; Schmied et al., 2009). Improvements in both environmental and facilities needs of women could be complementary to views for a new look for postnatal care provision (Morrow et al., 2013).

Table 6-3: Descriptive factors in interior environment of postnatal spaces that contribute to midwives' work in postnatal care

\begin{tabular}{|c|c|c|c|}
\hline Item & Survey question & Code & Category \\
\hline \multirow[t]{13}{*}{ Q3.11 } & $\begin{array}{l}\text { What factors made your } \\
\text { work easy }\end{array}$ & Spaciousness & Spatial \\
\hline & & $\begin{array}{l}\text { Good space- to move easily around } \\
\text { to support women }\end{array}$ & $\begin{array}{l}\text { Safe and accessible } \\
\text { space }\end{array}$ \\
\hline & & Larger space & $\begin{array}{l}\text { Preference for single } \\
\text { rooms }\end{array}$ \\
\hline & & Having own space & \\
\hline & & Single bedrooms & \\
\hline & & Sizeable bathrooms & \\
\hline & & $\begin{array}{l}\text { Rooms for partners to stay the } \\
\text { night }\end{array}$ & \\
\hline & & Floor plan/layout & $\begin{array}{l}\text { Spatial design and } \\
\text { Wayfinding }\end{array}$ \\
\hline & & Fresh air & Hygiene/ cleanliness \\
\hline & & Cleanliness/waste disposal & Maintenance \\
\hline & & Quiet/peace & Tranquil environment \\
\hline & & Natural surroundings & Positive distraction \\
\hline & & Comfortable furniture & Spatial and furnishings \\
\hline \multirow[t]{9}{*}{ Q3.12 } & $\begin{array}{l}\text { What factors made your } \\
\text { work difficult }\end{array}$ & & \\
\hline & & Lack of privacy & Privacy \\
\hline & & $\begin{array}{l}\text { Lack of space (such as for } \\
\text { conversations for parenting issues) }\end{array}$ & Spatial \\
\hline & & $\begin{array}{l}\text { Noisy and busyness in postnatal } \\
\text { rooms }\end{array}$ & $\begin{array}{l}\text { Audio } \\
\text { environment/Acoustics }\end{array}$ \\
\hline & & Functional space/equipment & $\begin{array}{l}\text { Ergonomics } \\
\text { Staff support spaces }\end{array}$ \\
\hline & & Storage & $\begin{array}{l}\text { Fittings/fixtures, } \\
\text { Spatial }\end{array}$ \\
\hline & & Clutter & \\
\hline & & Harsh/Poor lighting & $\begin{array}{l}\text { Personal control and } \\
\text { lighting }\end{array}$ \\
\hline & & $\begin{array}{l}\text { Poor visibility of women and } \\
\text { babies }\end{array}$ & Feeling of safety \\
\hline
\end{tabular}


The next few sections will address the remainder of the survey questions.

\section{Q3.13: During postnatal care how important do you think it is to?}

\section{1) Have a restful room}

Most of the respondents thought a restful room was very important to support women in recovery. Forty-one respondents $(82 \%)$ ranked a restful room as very important, seven (14\%) ranked it moderately important, while just two respondents (4\%) ranked it somewhat. No respondent ranked a restful room not at all important or minimally important (see Figure 6-19).

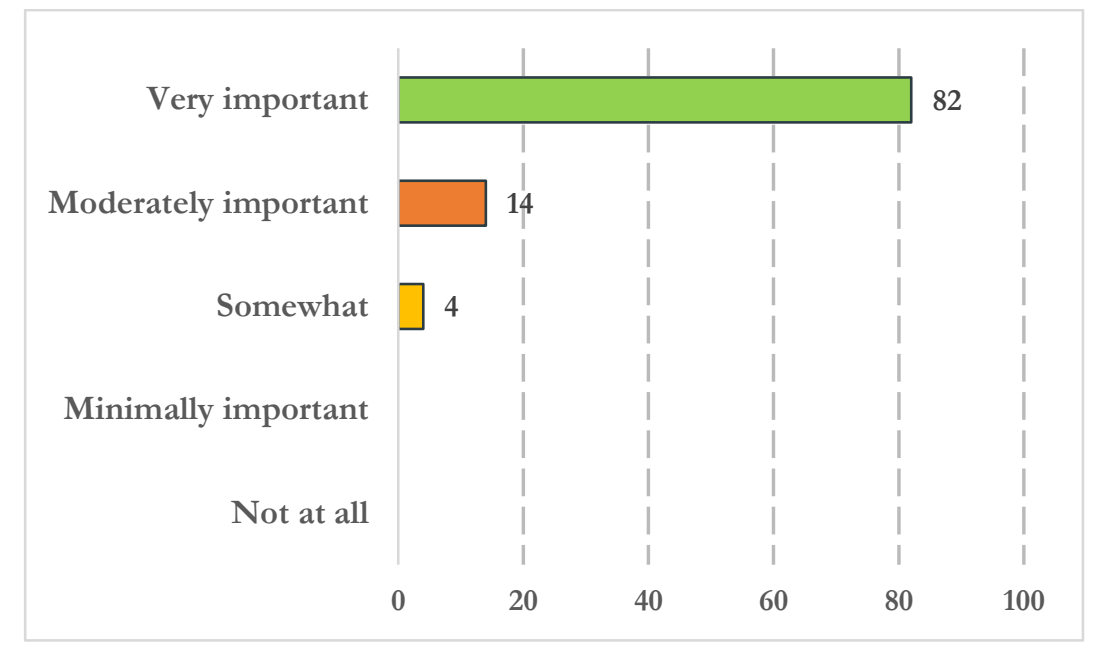

Figure 6-19: Respondents' ratings of care in a restful room

The result indicated clear recognition of the benefit of a restful room and its importance as perceived by healthcare providers. This implicitly hinges on the type of rooms (single-bed or multibed) allocated to women postnatally. It also points out the needs for a quiet and peaceful overall environment during postnatal hospital stays.

\section{2) Care for women in a clinical-looking postnatal space}

Most of the respondents thought a clinical-looking space was not necessarily important during postnatal recovery care. Twenty-one $(42 \%)$ respondents rated a clinical room for caregiving as not at all important, and eighteen (36\%) respondents as minimally important. Nine (18\%) and two respondents (4\%) ranked it somewhat and moderately important respectively (see Figure 6-20). 


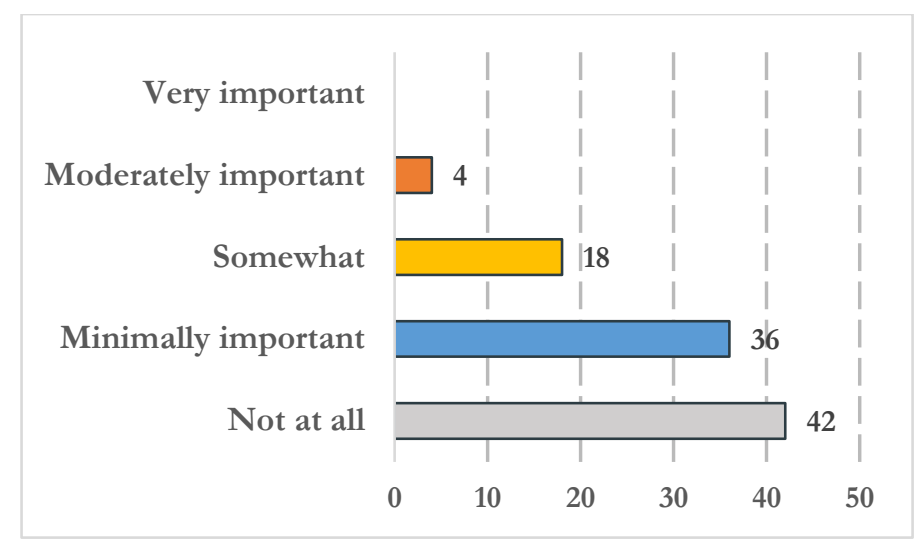

Figure 6-20: Respondents' ratings of clinical space

Eleven $(22 \%)$ respondents combined considered that a clinical environment was somewhat or moderately important, considerations of technocratic birth versus normal physiologic birth are assumed at play to some extent. While safety is essential, it is reasonable to assume that a less clinical environment was being suggested by roughly two-thirds of respondents ( $\mathrm{n}=39 / 50,78 \%$ ) A blend of ideologies in room design may likely impact positively on recovery experiences of new mothers.

\section{3) Provide care in a room which has a combination of clinical and familiar home features}

Most of the respondents reported that a combination of clinical and familiar home features in a room was important in caregiving. Eight respondents (16\%) ranked it as very important, twentyone $(42 \%)$ ranked it as moderately important. Fifteen respondents $(30 \%)$ ranked it somewhat important. Only two (4\%) and four (8\%) respondents ranked it as not at all and minimally important respectively (see Figure 6-21).

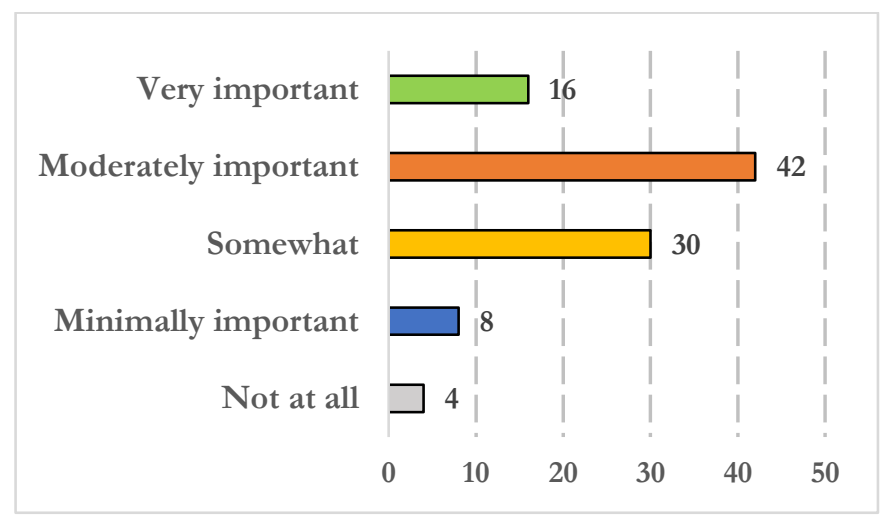

Figure 6-21: Respondents' ratings of care in a room with clinical and familiar home environments 
Just as in the previous question, the result reflected a preference for combining clinical and familiar home environments, because respondents are likely to view birth as more of a natural process than a medical procedure. Of interest are the eight respondents who thought it was very important to have familiar home features in the postnatal space. This is amply supported in the findings from studies on hospital birth room images and home-like environments (Bowden et al., 2016; Homer, 2013; Shin et al., 2004).

\section{4) Give care in a room or ward with natural art, images or paintings}

Respondents rated having art in the room quite postively. Eleven respondents $(22 \%)$ rated art as very important, fifteen (30\%) respondents rated it as moderately important. One-third (34\%) reported somewhat important. Five $(10 \%)$ and two respondents rated it minimally important and not at all important (see Figure 6-22).

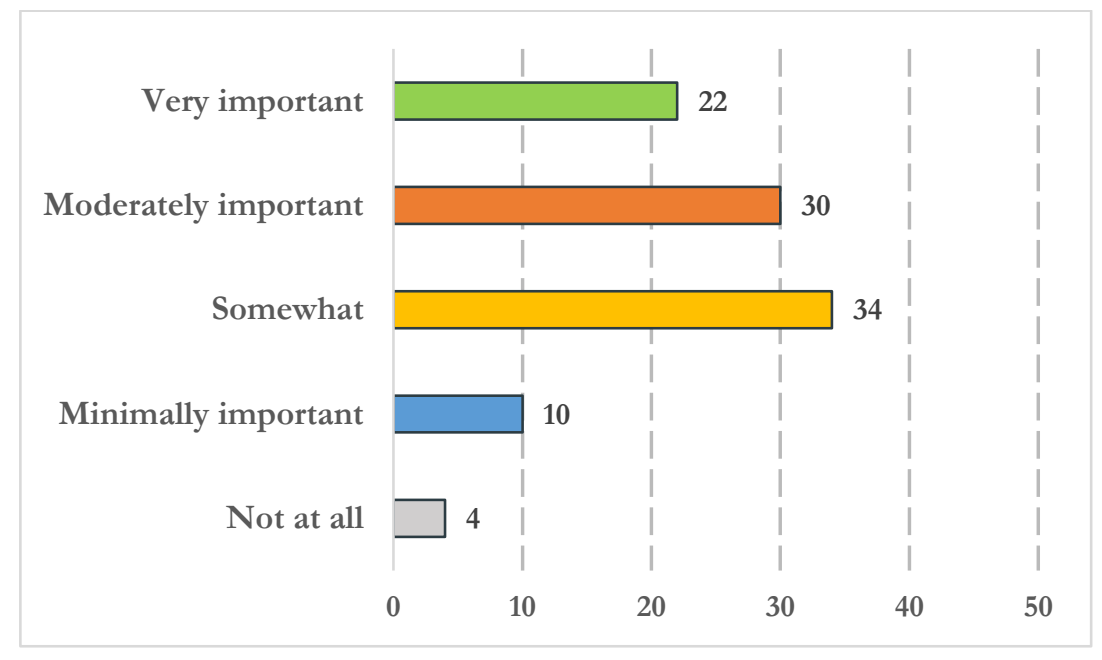

Figure 6-22: Respondents' ratings of care in a room with art or images

The result reflected that the atmosphere of the room was considered important during care.

\section{5) Have bright colours in the room}

Care giving in a bright coloured room was ranked as minimally important by fifteen respondents (30\%), eighteen respondents $(36 \%)$ ranked it as somewhat while ten respondents ranked it as moderately important. Four respondents $(8 \%)$ reported not at all, and 3 respondents $(6 \%)$ reported very important (see Figure 6-23). 


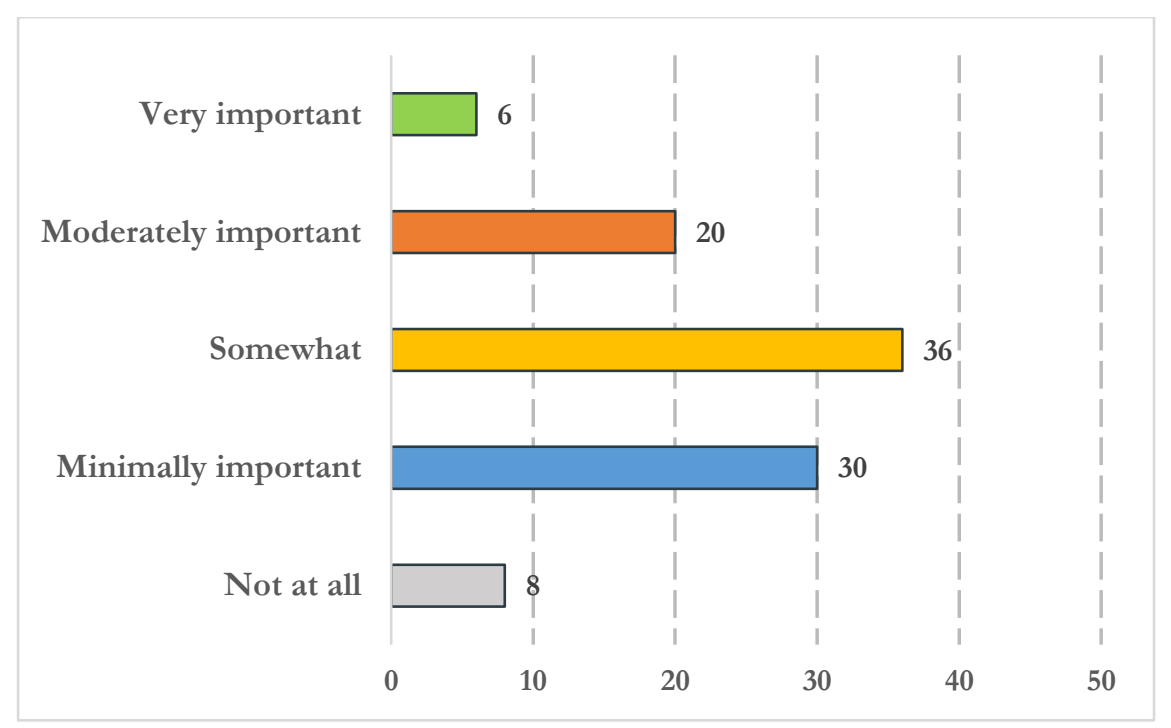

Figure 6-23: Respondents' ratings of care in a room with bright colours

The result reflected a broad spread of views on the perception of bright colours in the room. Although colours may well have a visual appeal in the room, bright colours, which may tend to raise stress appear to be considered less suitable for a postnatal space. A similar result was reported in women's survey responses (see chapter 5). Colour may need to be investigated in more detail to see its effects on physiological, emotional, and psychological aspects of women's recovery experience and wellbeing.

\section{6) Provide care in a room that is clean}

Most of the respondents thought hygiene and cleanliness of the room supported care giving. Interestingly nearly all respondents $(\mathrm{n}=49,98 \%)$ rated a clean room as very important, only one $(\mathrm{n}=1,2 \%)$ rated it as moderately important (see Figure 6-24).

The result indicated the importance of hygiene and cleanliness in a postnatal hospital environment. This result shows that cleanliness is a clinical goal that is accorded higher priority, in return for wellbeing. 


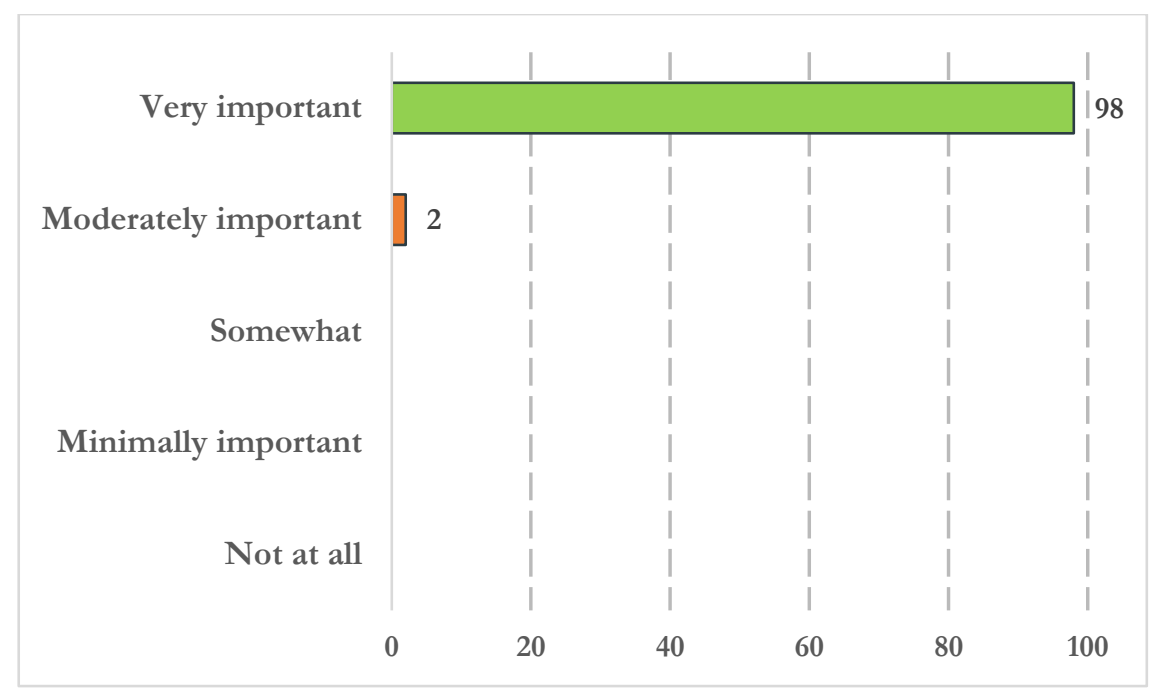

Figure 6-24: Respondents' ratings of care in hygienic and clean room

Cleanliness has links with reduction in transmission of infections if a clean healthcare environment is maintained. In the postnatal phase, a clean environment is important for hygiene of mothers and babies for prevention of infections. Spaces accommodating toilets and shower facilities are important aspects subsumed under maintenance and cleanliness.

\section{7) Attend women in a room with furniture made of natural materials}

Respondents rated the perception of a room with furniture made of natural materials more or less favourably. The choice of furniture made of natural materials in the room was reported as moderately important by nine respondents (18\%), nineteen respondents $(38 \%)$ reported it minimally important while seventeen $(34 \%)$ rated it as somewhat. Two $(4 \%)$ and three $(6 \%)$ respondents ranked it as very important and not at all important (see Figure 6 -25).

Compared with room colours in survey question 3.4, the results are similar. Furniture and colours constitute aspects of hospitals' interior aesthetics which may be considered likely to enhance care but opinions on this do not appear to be strongly held compared with cleanliness, for example. 


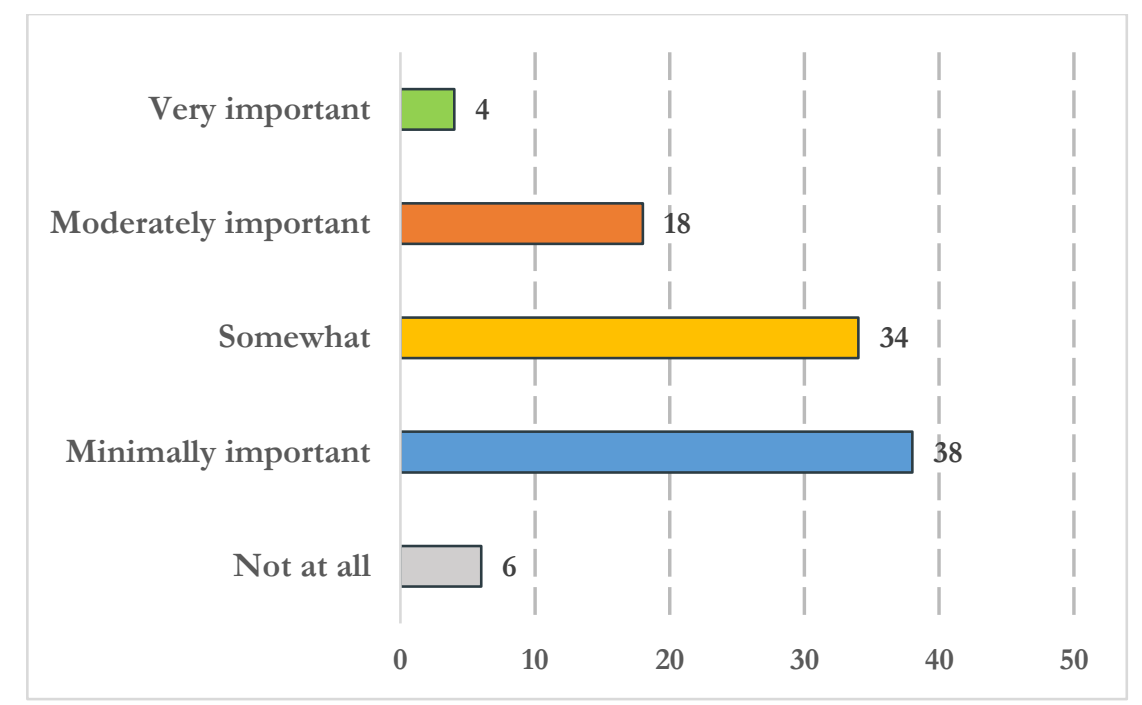

Figure 6-25: Respondents' ratings of care in a room with furniture made of natural materials

\section{8) Give care in postnatal room where door will be closed except when visitors are allowed in}

Most of the respondents thought giving care in a room where the door was closed supported privacy and dignity of women. Twenty respondents (40\%) ranked this feature as very important, and twelve $(24 \%)$ ranked it as moderately important. About one-third $(32 \%)$ reported it as somewhat important. Only two $(4 \%)$ of respondents reported minimally important (see Figure 626).

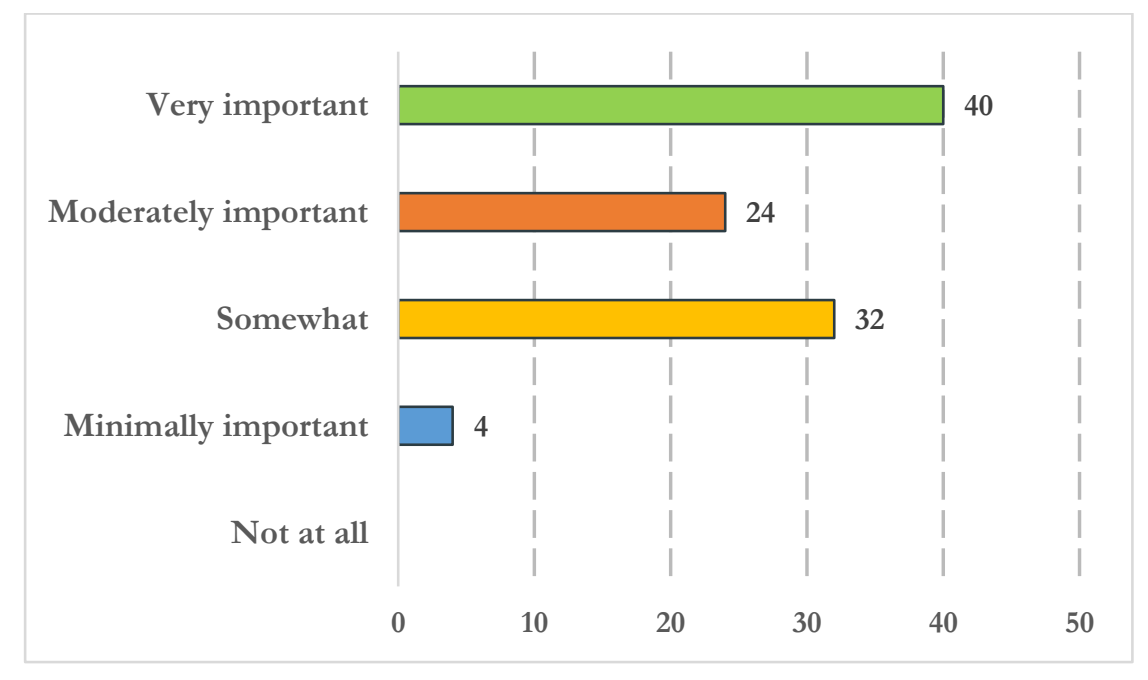

Figure 6-26: Respondents' ratings of privacy in the room with closing of doors 
The result reflected concern for confidentiality and privacy in the immediate postnatal phase. The larger number (about 64\%) of respondents combined thought a room where the door will be closed (for privacy) was very or moderately important. The group that rated somewhat perhaps also considered privacy. However, routine medical checks on women will require occasional opening and closing of the door by staff which may interfere with privacy of women. This position needs further investigation to ascertain what approaches can be beneficial to women during the recovery phase while also allowing midwives to access the room without breaching the confidentiality and privacy of women. This will be discussed in greater detail in the discussion of focus groups in Chapter 9.

\section{9) Provide care in a room in which a variety of materials are used}

Respondents rated the perception of having a variety of materials in the room less favourably. The perception of a variety of materials was reported as moderately important by ten respondents $(20 \%)$ and three respondents reported it as very important. Twenty-seven respondents reported somewhat important while eight respondents (16\%) reported minimally important. Only three $(6 \%)$ and two (4\%) respondents reported very important and not at all important (see Figure 627).

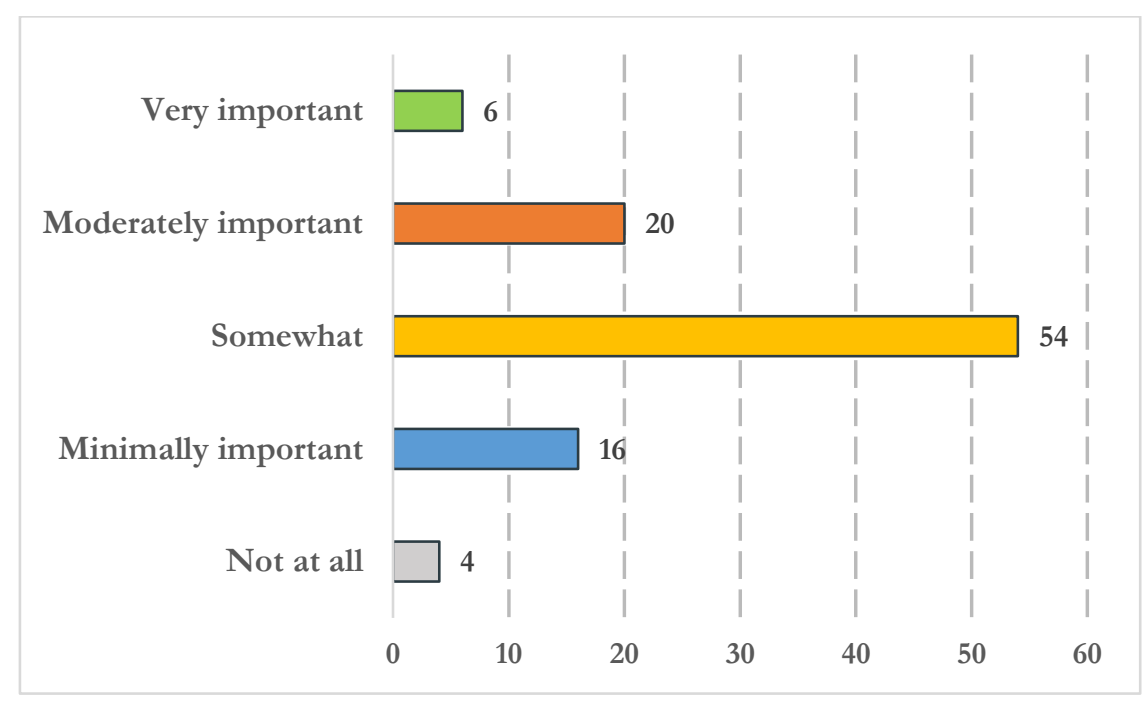

Figure 6-27: Respondents' ratings of room with variety of materials

The result is not dissimilar to those of colours and furniture as they all point to the visual and aesthetic aspects of postnatal hospital environments. In the survey questions for women, this question was accounted for under "have a room with furniture made of natural materials" and "natural things in the room" (see questions number 3.11-2 and 3.12-8 in the women's 
questionnaire in Appendix B). Subsequent analyses of these questions using inferential statistics will compare responses of women and midwives in Chapter 7.

Detailed descriptive statistics on perceptions of interior environment design factors and their corresponding mean scores and standard deviations are shown in Table 6-4.

Table 6-4: Preference (mean and standard deviation) of respondents on perception of interior environment features contributing to recovery care and women's wellbeing ( $\mathrm{n}=50)$

\begin{tabular}{lll}
\hline Survey question & M & SD \\
\hline Provide care in a room that is clean & 4.98 & 0.14 \\
Have a restful room & 4.78 & 0.51 \\
Be in a room where the door is closed except when visitors are allowed in & 4.00 & 0.95 \\
Provide care in a room which has a combination of clinical and familiar home & 3.58 & 0.99 \\
features & & \\
Decorations in a room (ward with natural art, images and paintings) & 3.56 & 1.07 \\
Provide care in a room in which a variety of materials are used & 3.08 & 0.88 \\
Have bright colours in the room & 2.86 & 1.03 \\
Attend women in a room with furniture made of natural materials & 2.76 & 0.96 \\
Clinical room & 1.84 & 0.87 \\
\hline
\end{tabular}

Note: $M=$ mean; $S D=$ standard deviation

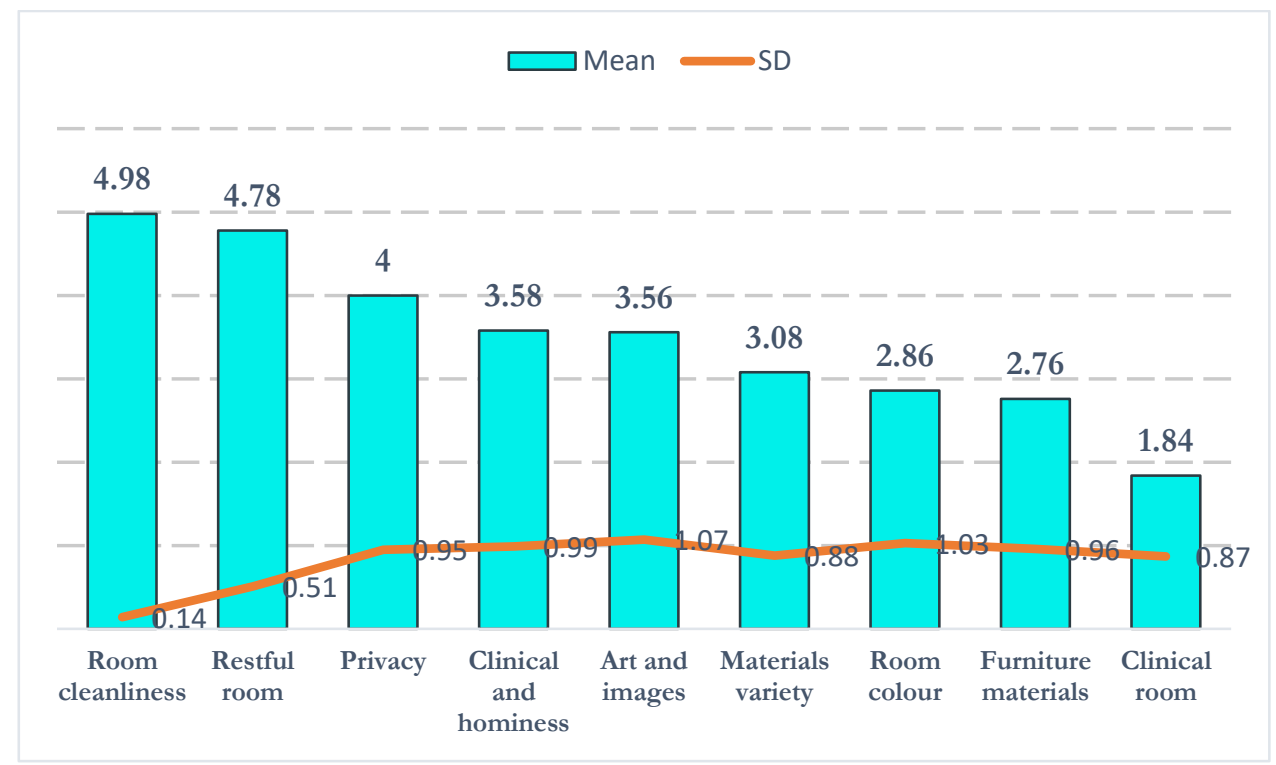

Figure 6-28: Respondents' ratings of interior environment room features

\section{Summary}

This segment of the survey questionnaire has described the results of descriptive statistics on perception of interior environment factors. The results show room cleanliness had the highest mean score $($ mean $=4.98, S D=0.14)$. Two other interior environment factors, room restfulness 
and closing door for privacy also had higher mean scores (mean $=4.77, S D=0.52$ ) and (mean = 4.00, $S D=0.97)$ respectively. Three other items had mean scores higher than 3 indicative of their level of acceptance (neutral). The order of least ranked items are bright colours, having furniture made of natural materials in the room and a clinical room. Figure 6-28 illustrates the distribution of results based on the mean scores and standard deviations.

\subsection{Perception of sensory comfort environment}

This segment of survey questions discusses the results of eight sensory comfort environment questions (see questions as follows or in Appendix B).

Q3.14 How important are these features to the sense of wellbeing in postnatal rooms or wards where you work?

\section{1) Having adequate daylighting}

Most of the respondents thought presence of adequate daylighting in the room would impact on wellbeing of women and medical staff. Forty respondents $(80 \%)$ ranked adequate daylighting as very important, nine ranked $(18 \%)$ ranked it as moderately important. One respondent $(2 \%)$ ranked this design factor as somewhat important (see Figure 6-29).

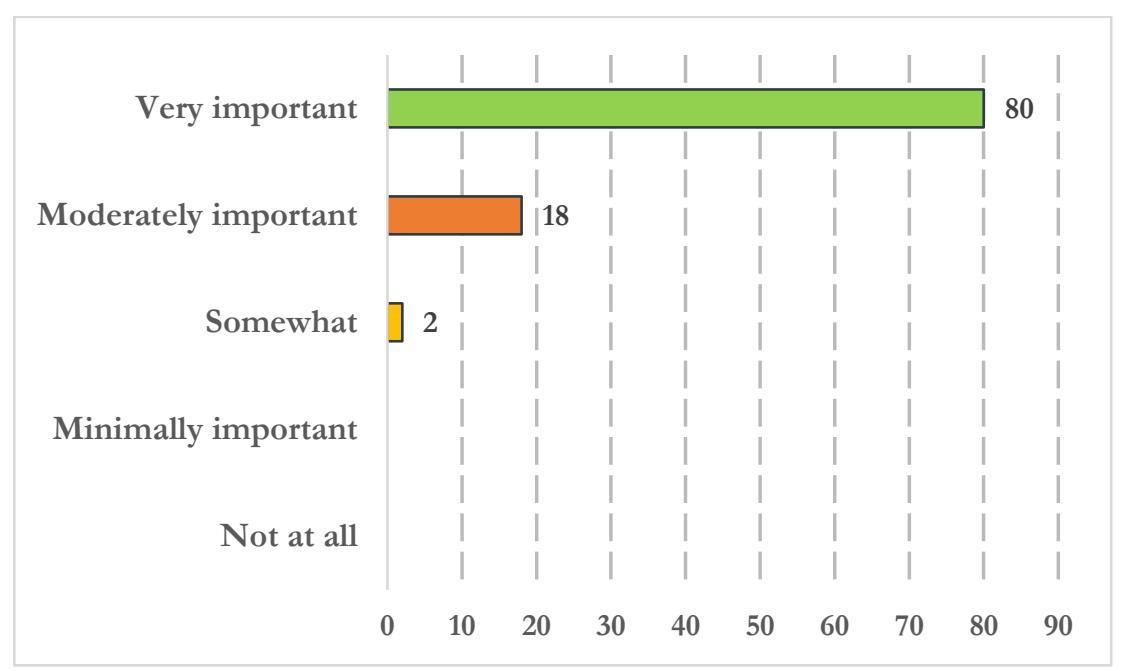

Figure 6-29: Respondents' ratings of adequate lighting for sense of wellbeing

The result reflected the importance of adequate daylighting for wellbeing of postnatal women and staff. Attention also needs to focus on operable windows which may increase autonomy for postnatal women and their family members in postnatal hospital settings. 


\section{2) Being able to control the temperature}

Most of the respondents thought controlling temperature supported the sense of wellbeing for women and staff. Thirty-four respondents $(68 \%)$ rated temperature control for sense of wellbeing as very important, fifteen rated it as $(30 \%)$ moderately important and one $(2 \%)$ rated it somewhat important (see Figure 6-30).

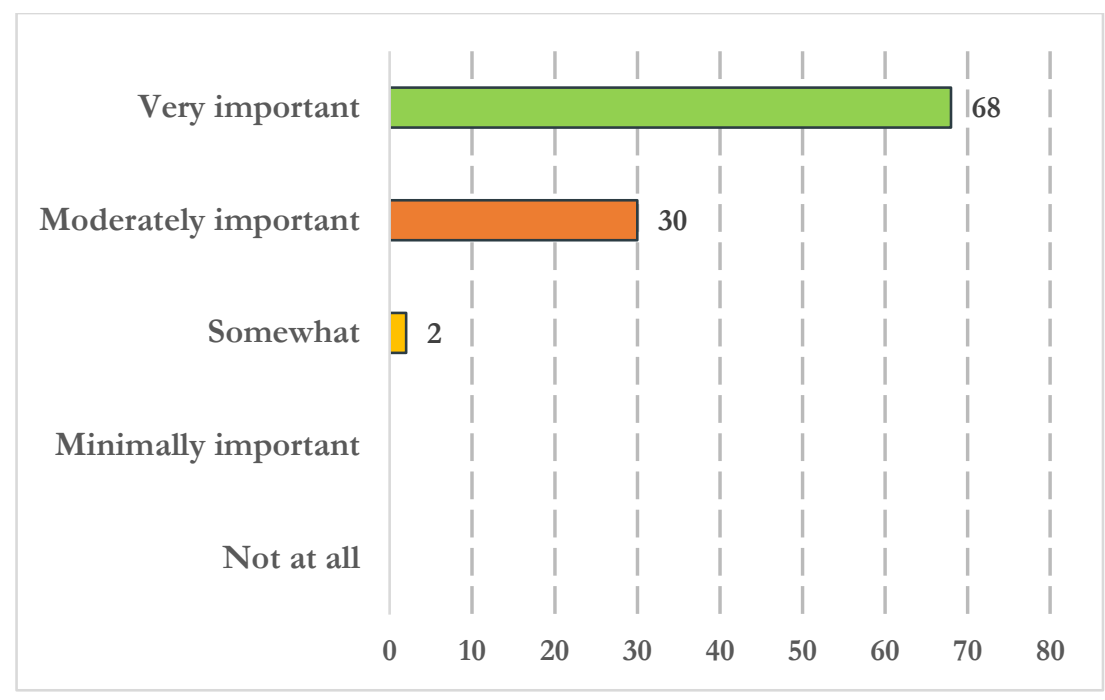

Figure 6-30: Respondents' ratings of temperature control for sense of wellbeing

The result indicated the value placed on a degree of autonomy over the physical environment. Having the means and opportunity to regulate the temperature suitable for a woman's body is vital for their physical and physiological wellbeing.

\section{3) Being able to control noise from mechanical things like ventilation and medical equipment}

Respondents' perception of noise control in the physical environment was rated very highly. Thirty-four $(68 \%)$ respondents rated it as very important and sixteen $(32 \%)$ respondents chose moderately important (see Figure 6-31).

The result reflected the importance of noise control for wellbeing of occupants and staff. Hospital buildings through their design and environmental strategies have the potential to alleviate the consequences of noise, in particular in the postnatal environment, for adequate sleep and rest for new mothers. 


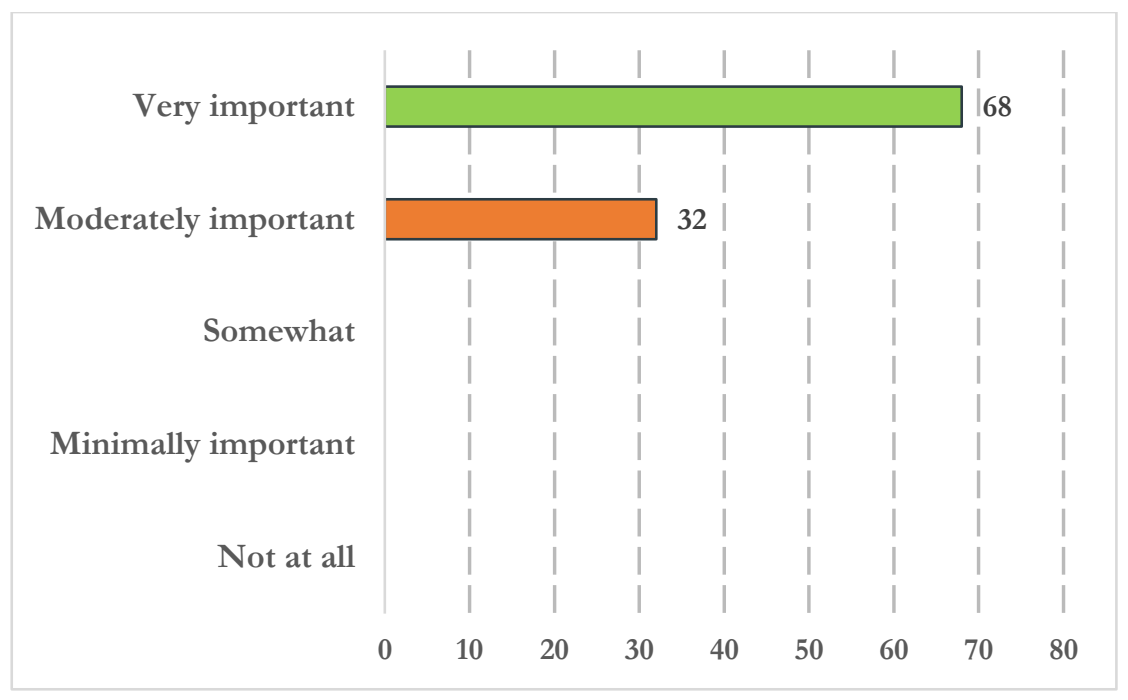

Figure 6-31: Respondents' ratings of noise control for sense of wellbeing

\section{4) Providing care in a room that feels quiet}

Most of the respondents thought a room that feels quiet would impact on wellbeing. Forty-three respondents $(86 \%)$ reported very important and another seven (14\%) respondents reported moderately important (see Figure 6-32).

The result reflected the quality of a quiet room for the sense of wellbeing and in the recovery process in the immediate postnatal phase. Designing spaces that "feel quiet" may need to respond to senses other than auditory for enhanced comfort for occupants of the spaces.

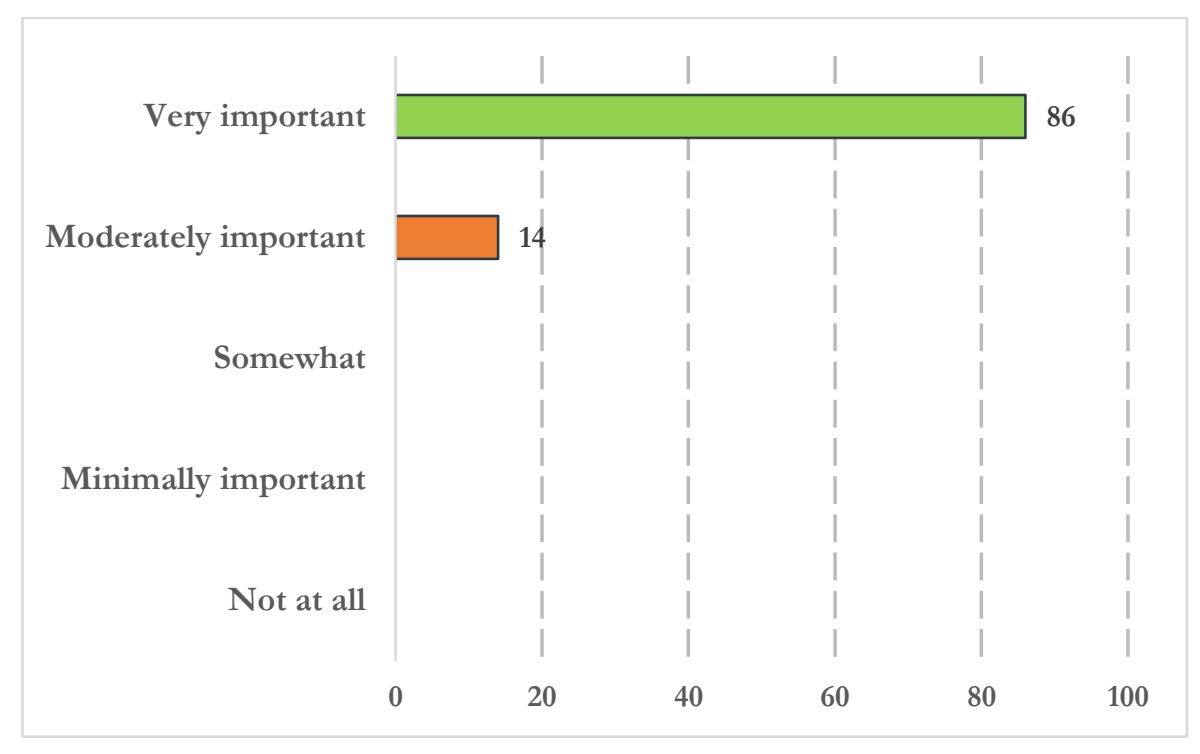

Figure 6-32: Respondents' ratings of a quiet room for sense of wellbeing 
Q3.15 How much do each of the following features contribute to the sense of wellbeing in postnatal rooms or wards where you work?

\section{1) Having good air quality and room freshness}

Most of the respondents thought the quality of indoor air and room freshness have impact on the feelings of wellbeing. Thirty-eight $(76 \%)$ respondents ranked indoor air and room freshness very important and 18\% reported moderately important. Only three $(6 \%)$ of the respondents reported somewhat important (see Figure 6-33).

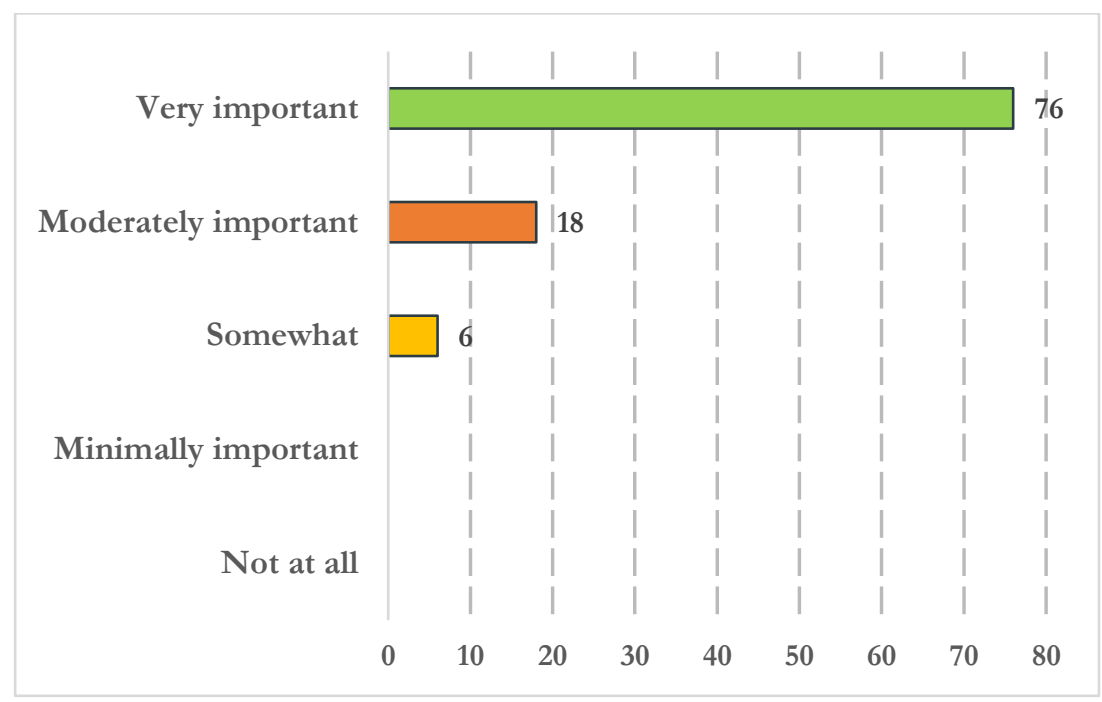

Figure 6-33: Respondents' ratings of good indoor air quality and room freshness for sense of wellbeing

The result reflected that quality of indoor air in the healthcare physical environment was important for achieving the sense of wellbeing of inpatients, staff and others. Air quality and room freshness are also linked to prevention of air-borne infections.

\section{2) Having a hygienic/clean environment}

Respondents' perception of a hygienic/clean environment was ranked very highly. The quality of the rooms regarding their hygiene and cleanliness was ranked as very important by forty-six $(92 \%)$ respondents, and three and one respondents $(8 \%)$ combined ranked it as somewhat and moderately important (see Figure 6-34). 


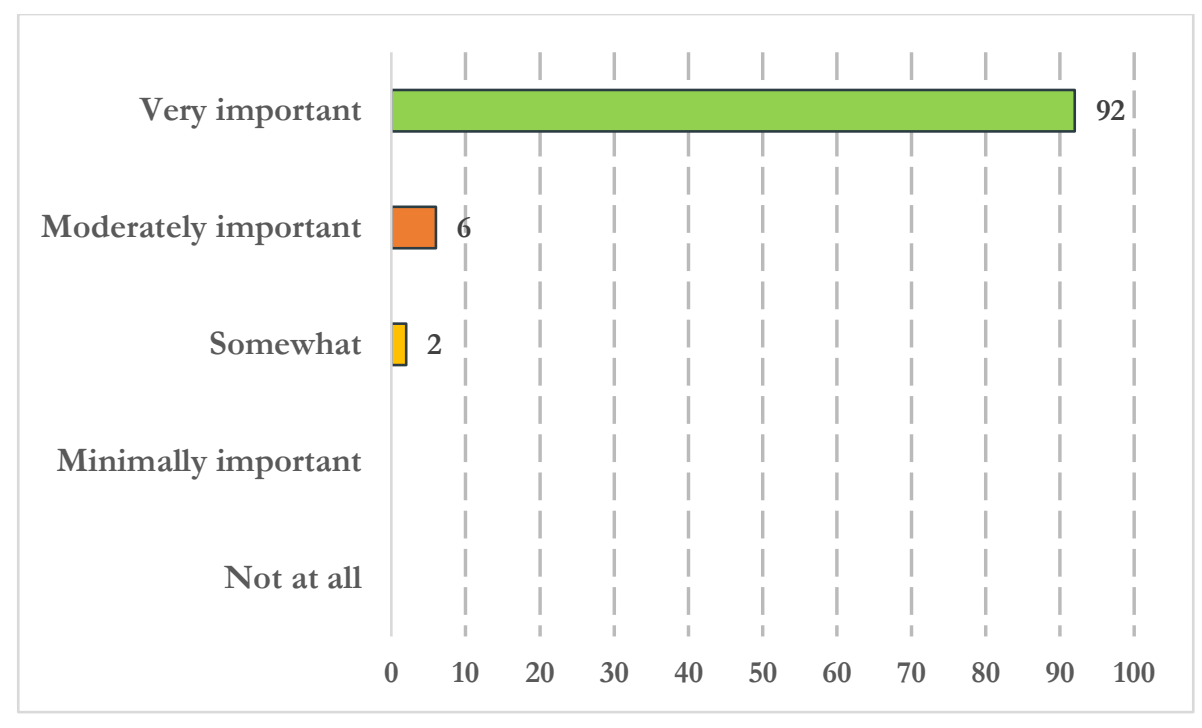

Figure 6-34: Respondents' ratings of hygienic/clean environment on sense for wellbeing

The result suggested that hygiene and cleanliness was a fundamental mainstream goal in healthcare settings.

\section{3) Being able to adjust lighting to the activity in the room}

Most of the respondents thought being able to adjust lighting (artificial and natural) levels to the activity in the room was important for the sense of wellbeing. Forty-one respondents (82\%) rated this as very important, and as moderately $(12 \%)$ and somewhat important $(6 \%)$ by nine respondents respectively (see Figure 6-35).

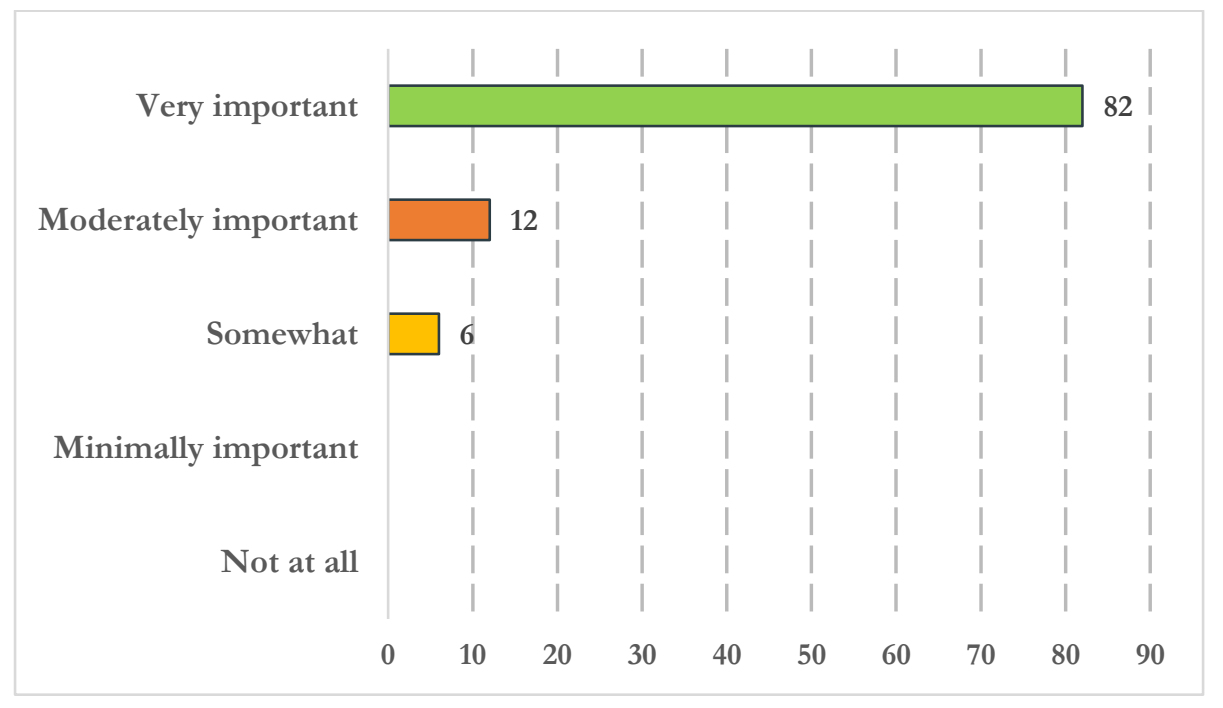

Figure 6-35: Respondents' ratings of lighting control on sense for wellbeing 
The result reflected the quality of the visual environment in performing tasks. Routines in the postnatal environment which involve medical checks at particular intervals need adequate illumination (both artificial and natural) for staff to perform the task. The postnatal environment should also take into account aspects of illumination necessary for women's parental tasks such as breastfeeding.

\section{4) Having a number of natural objects in the room such as aquariums, sounds of birds, water, scents and images}

The quality of the room with natural stimuli was rated as very important by three $(6 \%)$ respondents, and as moderately (18\%) important by nine respondents. Twenty-one (42\%) respondents selected somewhat important. In addition, thirteen $(26 \%)$ and four $(8 \%)$ respondents reported minimally and not at all important (see Figure 6-36).

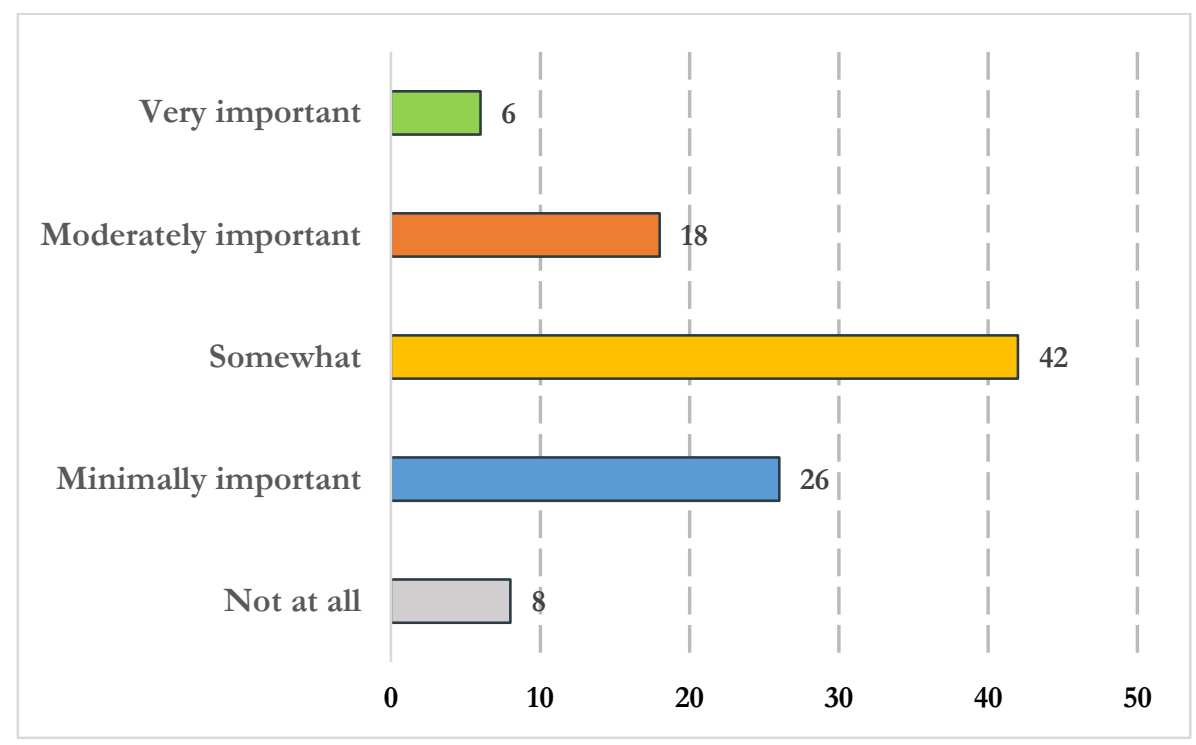

Figure 6-36: Respondents' ratings of natural stimuli in the room on sense for wellbeing

The result reflected a broad distribution in the opinions. When grouped under two headings, 24\% (about one quarter) rated it as important and 34\% (one third) by comparison reported not at all and minimally important. Additionally, the larger majority $(n=21,42 \%)$ were neutral. The result questions the value of natural stimuli or "soft elements" as features in postnatal hospital spaces.

Table 6-5 highlights the results of the descriptive statistics analyses for each question, mean scores and corresponding standard deviations. 
Table 6-5: Preference (mean and standard deviation) of respondents on perception of sensory comfort features contributing to recovery and women's wellbeing $(\mathrm{n}=50)$

\begin{tabular}{lll}
\hline Survey question & M & SD \\
\hline Having a hygienic/clean environment & 4.90 & 0.36 \\
Providing care in a room that feels quiet & 4.86 & 0.35 \\
Having adequate daylighting & 4.78 & 0.47 \\
Adequate lighting (to the activity in the room) and operable windows & 4.76 & 0.56 \\
Air quality and room freshness & 4.70 & 0.58 \\
Being able to control noise from mechanical things like ventilation, medical & 4.68 & 0.47 \\
equipment etc. & 4.66 & 0.52 \\
Being able to control the temperature & & \\
Pleasantness of the room (such as aquariums, nature sounds of birds, water, scents & 2.88 & 1.00 \\
and images) & & \\
\hline
\end{tabular}

Note: $M=$ mean; $S D=$ standard deviation

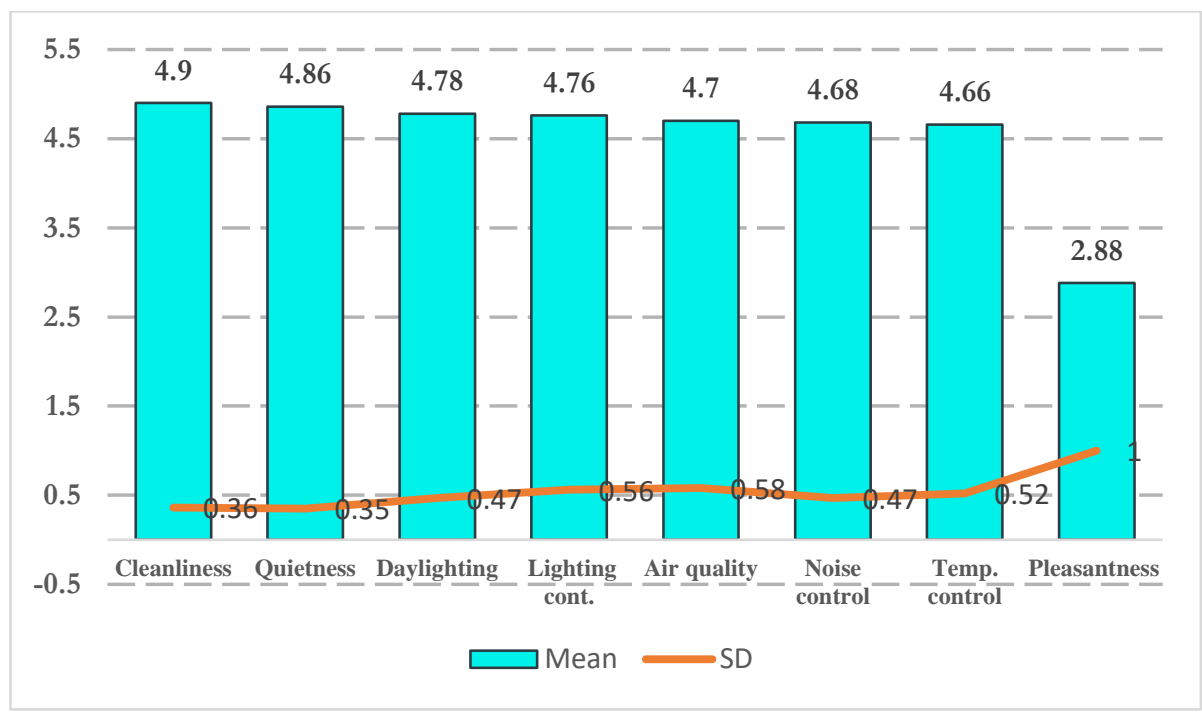

Figure 6-37: Respondents' ratings of sensory comfort environment features

\section{Summary}

The descriptive statistics on perceptions of sensory comfort features have been discussed. Results show that the most important sensory environment feature was hygiene/clean environment with the highest mean score of $(M=4.90, S D=0.36)$. Six other items received mean scores higher than 4 which reflect the importance of those design factors. The item measuring pleasantness of "natural stimuli in the room", had the lowest mean score of $(\mathrm{M}=2.88, S D=1.00)$. Figure 6-37 illustrates the results based on the mean scores and standard deviations. 


\subsection{Perception of social comfort environment}

This section of the survey questionnaire measures the perception of care providers regarding space for social interaction and family support. Eight questions (as listed in the midwives' questionnaire in Appendix B) are discussed.

Two separate questions asked respondents about the levels of involvement in education and information support available to women during the postnatal recovery phase.

\section{Q3.16: In which situation did you find the women in your most recent advice session or} talk about parenting skills?

Respondents reported they met women in various situations during advice sessions on parental skills. The common situations included breastfeeding reported by thirty-one respondents, twentynine in bed, and twenty sitting in a chair. Thirteen reported having a meal, and five respondents reported standing position. Five respondents reported other places such as in the office (see Figure 6-38).

The result reflected different activities of women in the spaces during education and information sessions. The quality of interior furnishings and fixtures is implicated.

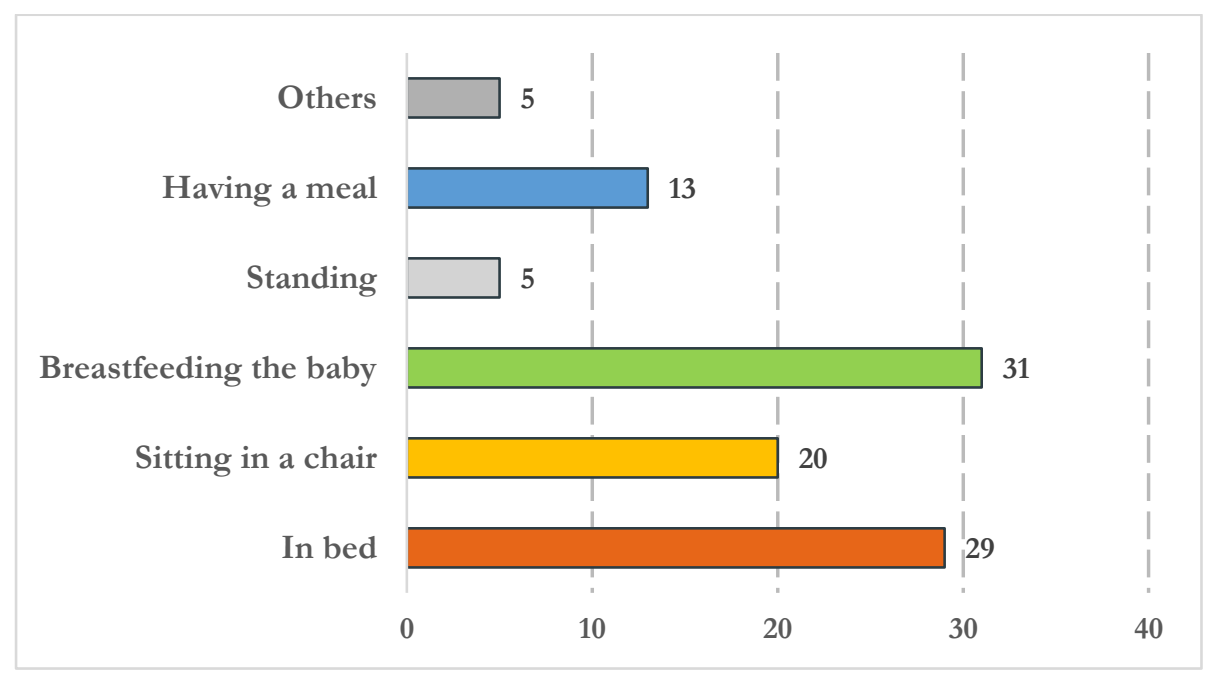

Figure 6-38: Respondents' ratings of women situations during advice sessions

\section{Q3.17: Which ways have you used to communicate parenting skills to women?}

Respondents reported communication of parenting skills through one or more different approaches. Five respondents (in both cases 10\%) reported using all of the approaches or group 
sessions, thirty-three of the respondents $(66 \%)$ used one-on-one talk, and thirty-four $(68 \%)$ individually including the partners. Eight (16\%) and six respondents $(12 \%)$ discussed with women in a group (inside a room or in an open bay ward). Only three respondents (6\%) communicated to women during breastfeeding classes (see Figure 6-39).

The result reflected strong family visitation and support for parental learning skills.

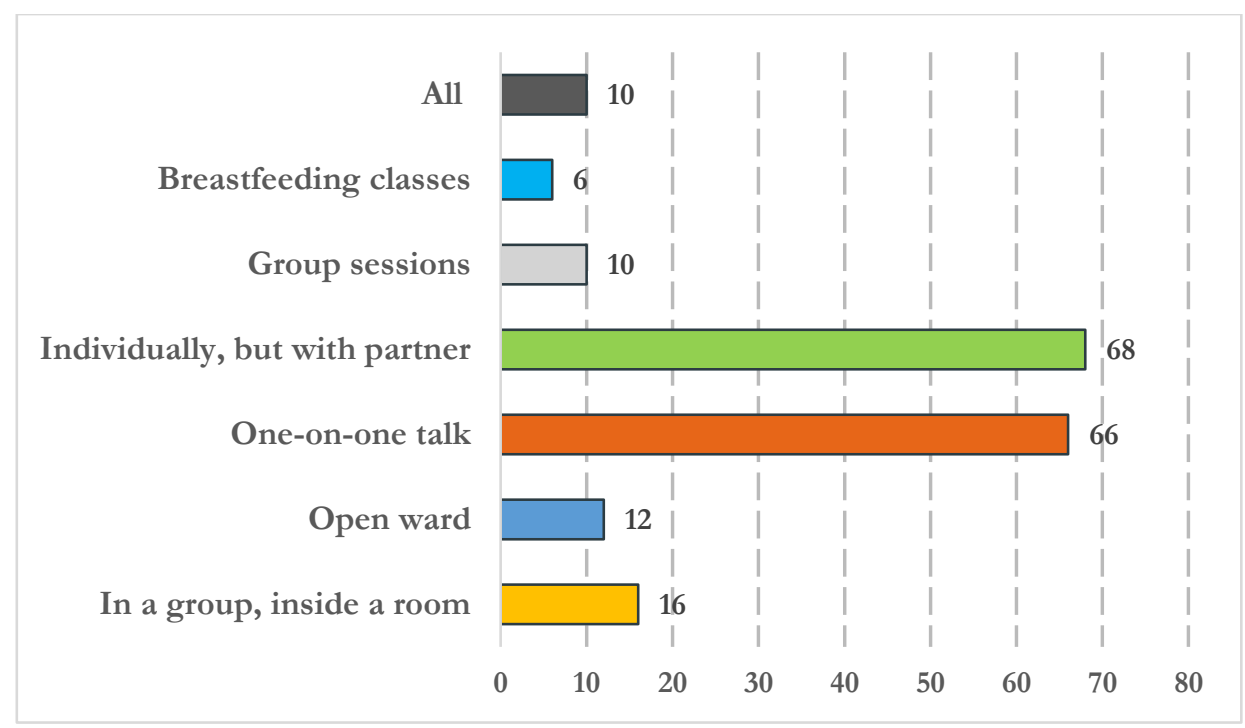

Figure 6-39: Respondents' methods of communication for parental skills

\section{Q3.18: How do you feel about the following statements regarding aspects of postnatal care giving?}

\section{1) Women have the opportunity to choose a room that they prefer}

Respondents' perception of women being able to choose a room of their choice was positively skewed, meaning that few respondents agree women could choose the room they prefer. Nine $(18 \%)$ respondents ranked strongly disagree and ten $(20 \%)$ respondents ranked disagree. Twentytwo respondents (44\%) reported neither agree nor disagree. Seven $(14 \%)$ of the respondents reported agree, and two (4\%) reported strongly agree (see Figure 6-40). 




Figure 6-40: Respondents' ratings of opportunity to choose room for preference

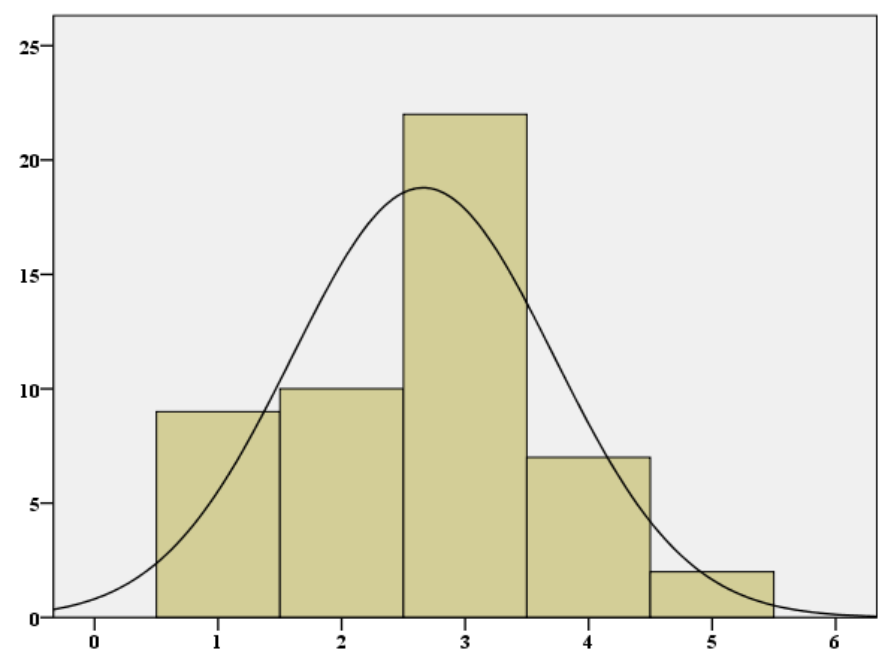

Figure 6-41: A slightly positive skew distribution of ratings of preference to choose room

A scrutiny of the histogram indicated there is a broad range of opinions regarding opportunity for women to choose the room (see Figure 6-41). There may be an inadequate number of single-bed rooms compared with double-bed rooms and the factor of economics is likely to be at play, which depends on the length of stays (LOS) as well as model of care.

\section{2) A postnatal room with several beds decreases opportunity for discussions about personal issues}

Most of the respondents thought open bay-rooms decreased opportunity for intimate personal discussions with women. Twenty-eight respondents $(56 \%)$ rated a room with several beds for reduced discussion as strongly agree, sixteen (32\%) reported agree and four (8\%) respondents 
reported neither agree nor disagree. Only two respondents (4\%) reported disagree (see Figure 6$42)$.

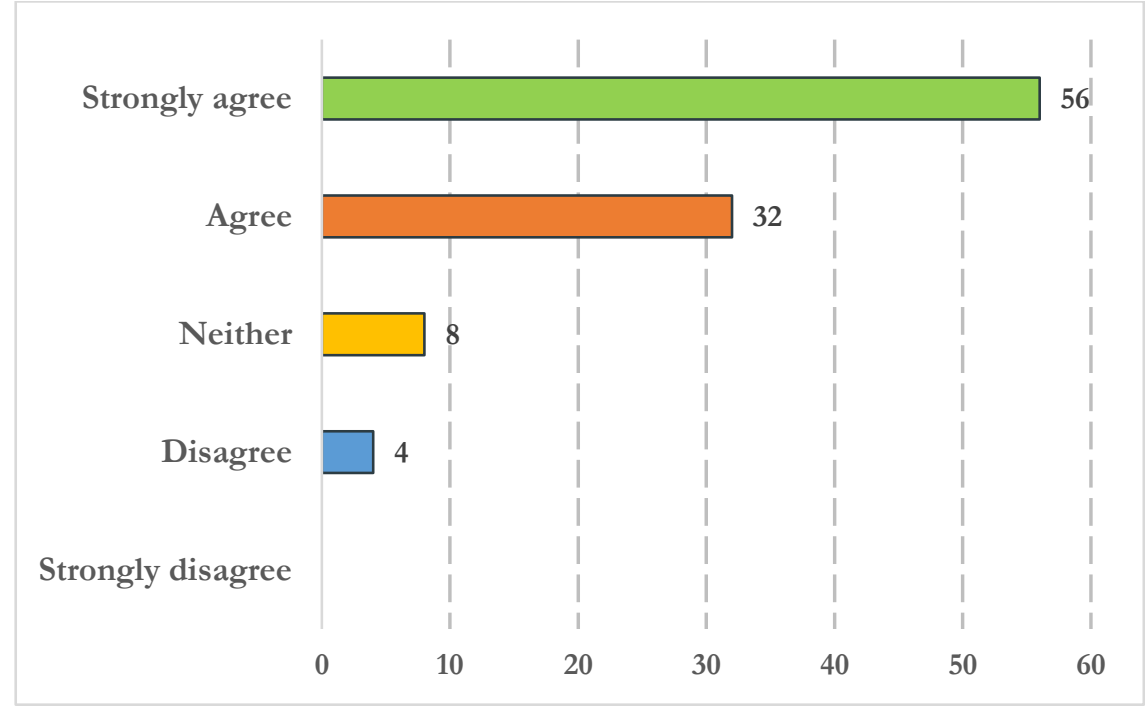

Figure 6-42: Respondents' ratings of open bay wards decrease opportunity for discussion

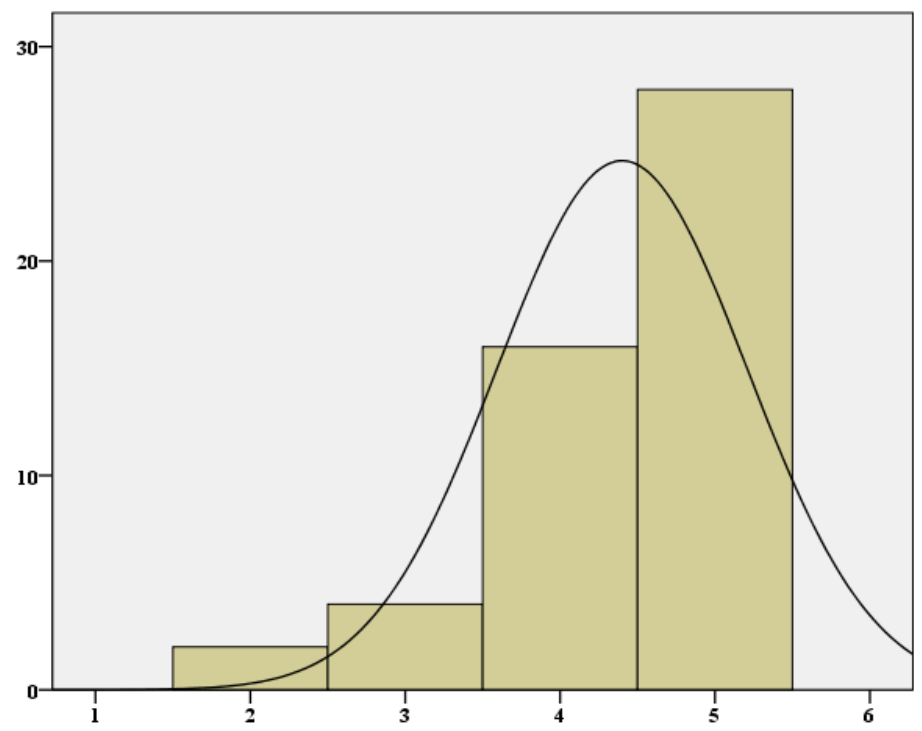

Figure 6-43: A negative skew distribution of ratings of open bay wards on discussion

An examination of the histogram (Figure 6-43) for rating of open bay wards for discussion ratings by the participants revealed the distribution is not normal. This suggests that multi-bed occupancy rooms or open bay wards might not promote discussion. Logically, the more women attended to in an open ward, the more the loss of privacy and dignity and potentially the less the resulting levels of interaction. This is consistent with the findings that multi-bed rooms put patients at disadvantage regarding privacy and dignity compared to single bed-rooms in healthcare environments (see Chaudhury et al., 2005). 


\section{3) Having a close link between postnatal room and birth room is good for social comfort}

Respondents' perception of the benefit of postnatal wards closely linked with birth and delivery rooms had a uniform distribution in opinions. It was rated as strongly agree and agree by eight $(16 \%)$ respondents in total, and as strongly disagree and disagree $(24 \%)$ by twelve respondents. Thirty (60\%) respondents rated neither agree nor disagree (see Figure 6-44).

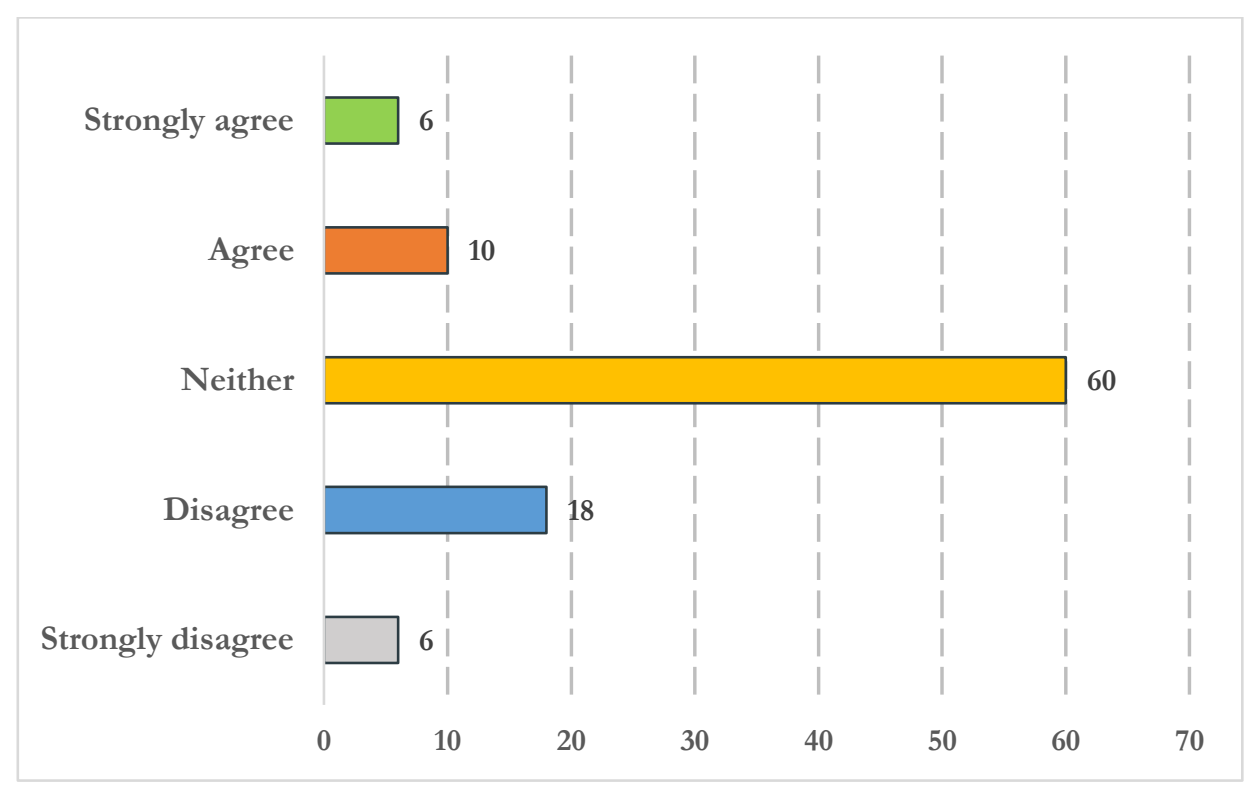

Figure 6-44: Respondents' ratings of spatial connection of postnatal ward with birth rooms

The result reflected a wide range of opinions regarding spatial connectivity of birthing rooms with postnatal wards. The aim here was to examine impact of spatial layouts on ease of circulation, travel time and comfort for midwives and women.

It is possible that the design and location of existing postnatal environments maybe responsible for the result. Some of the surveyed staff work in a large tertiary hospital where the postnatal ward does not have any direct linkage with the birthing areas. It is also possible that those who responded neither agree nor disagree were thinking of a separate postnatal unit away from the birth environment or in the extreme, canvassing a home option for social comfort and wellbeing. The number of respondents who gave a positive response, although slightly less are not too different from those who disagree. 


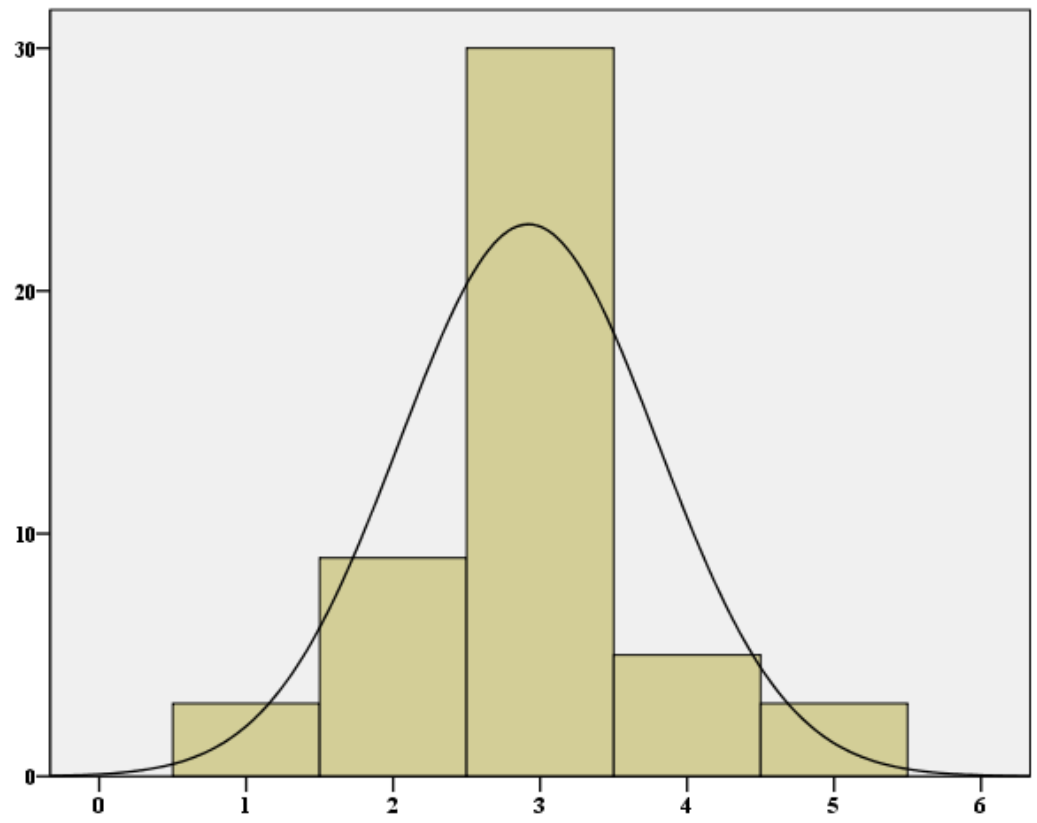

Figure 6-45: A normal distribution of ratings of spatial connection of postnatal ward with birth rooms

\section{4) Having a corridor to enter a postnatal room helps control visitors}

Most of the respondents thought a corridor would help control visitors for privacy. Twenty-three respondents $(46 \%)$ rated agree, four $(8 \%)$ as strongly agree while twenty-one $(42 \%)$ reported neither agree nor disagree. Only 2 respondents stated disagree (see Figure 6-46).

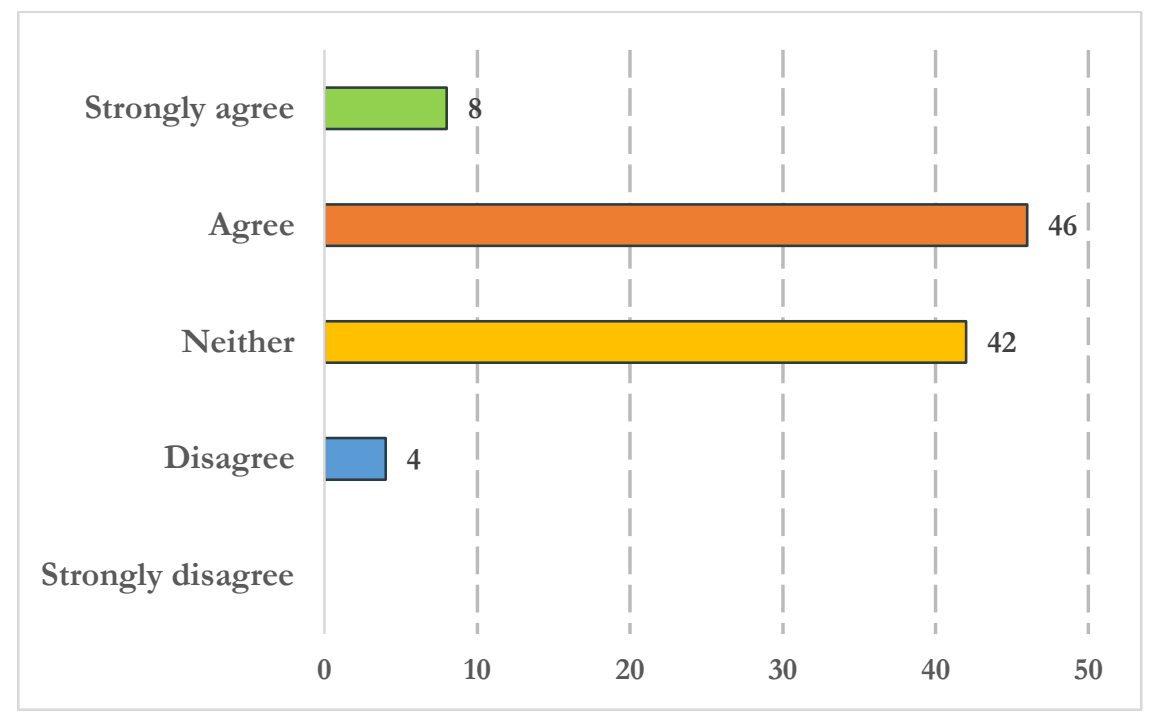

Figure 6-46: Respondents' ratings of a corridor for privacy in postnatal space

The result reflected that a well-designed layout could be used to manage visitors from having direct access to the postnatal space, thus serving as a means of privacy and control. The design of space can be contributory to policy on visiting hours. This raises a question about designing a postnatal 
environment that reduces crowding particularly during the sensitive times when women need to recover from the birth.

\section{5) Proximity of communal space near the postnatal space is good for counselling purposes}

Most of the respondents thought the provision of a communal space on the postnatal ward would impact well on parental information support. Thirty-three respondents $(66 \%)$ reported agree, and seven $14 \%$ ) reported strongly agree. Ten $(20 \%)$ of the respondents combined either ranked disagree or neither agree nor disagree (see Figure 6-47). The result indicated that communal space located near to postnatal space was considered to support new mothers and caregivers for the learning of parental skills.

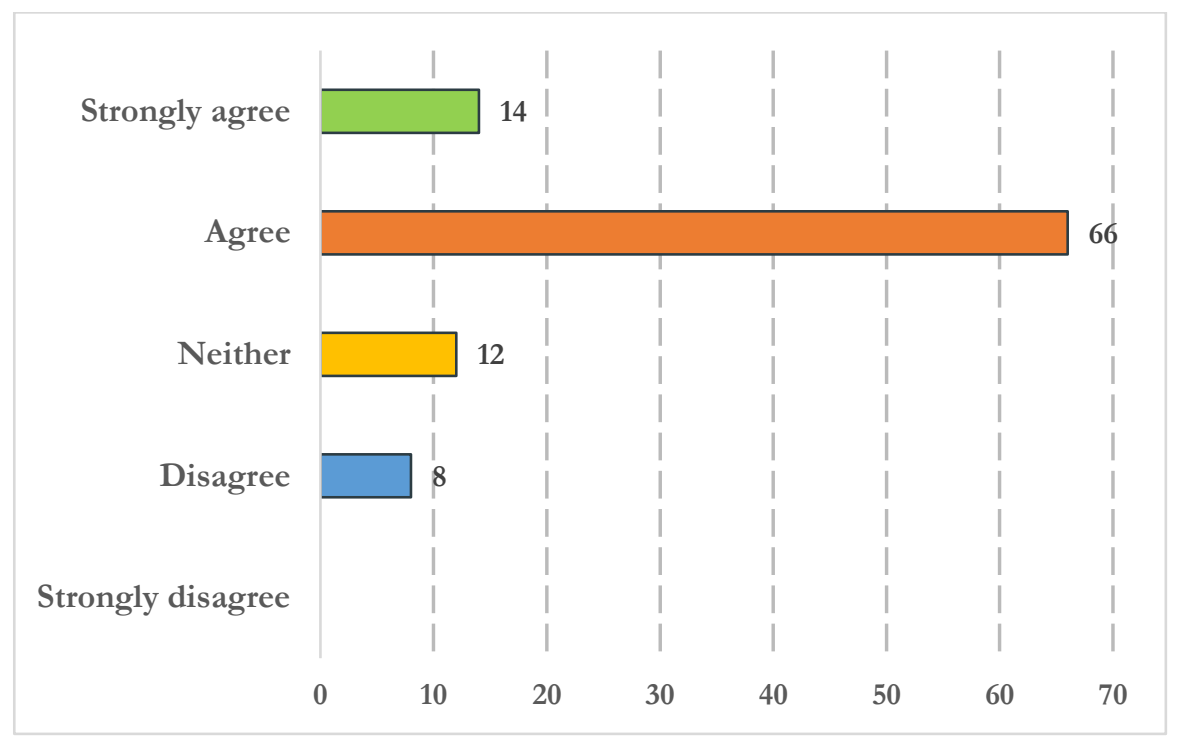

Figure 6-47: Respondents' ratings of communal space for education support

\section{6) Furniture and décor in a communal space can help group learning of parental skills}

Most of the respondents reported that furniture and décor in a communal space could support group learning of parental skills. Thirteen (26\%) ranked strongly agree, thirty-three $(66 \%)$ ranked agree, and three respondents reported neither agree nor disagree. One ranked $(2 \%)$ disagree (see Figure 6-48). 


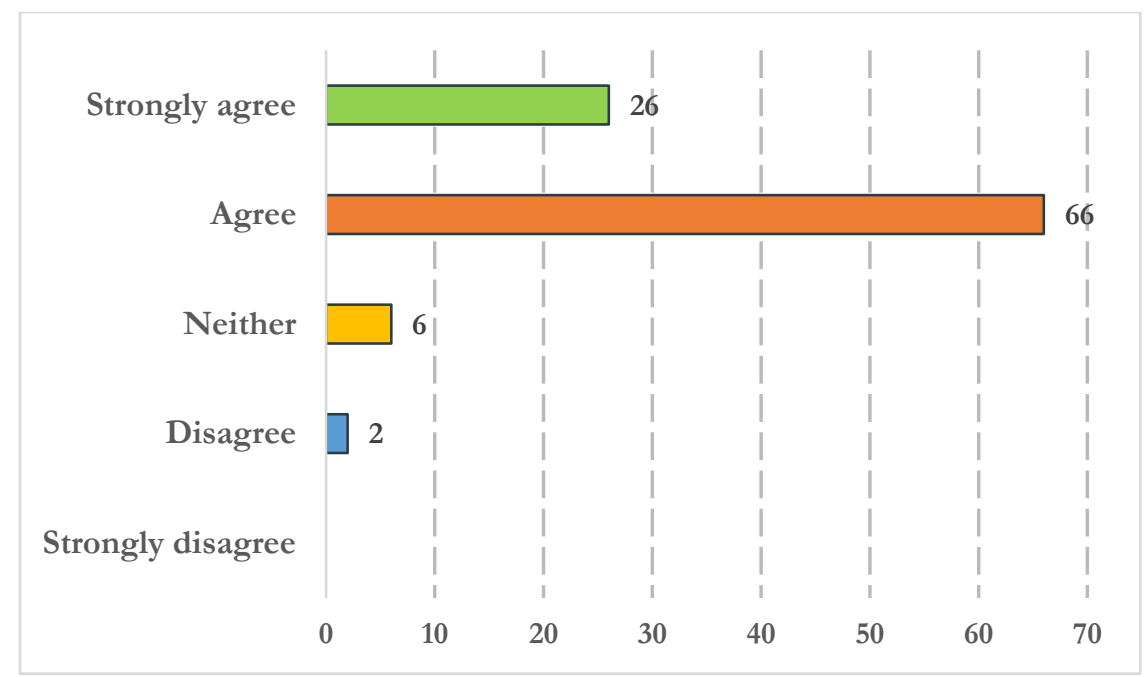

Figure 6-48: Respondents' ratings of furniture and décor for group learning support

The result reflected the importance of furnishings and décor specifically to encourage the learning of parental skills and education in postnatal hospital environments.

\section{7) Positive distractions such as soft music, natural images are better for recovery}

Respondents' perceptions of positive distractions focused on soft music and natural images were rated fairly high. Opportunity for positive distractions was ranked as strongly agree by six respondents, twenty-four (48\%) reported agree, and 36\% reported neither agree nor disagree. Only two respondents $(\mathrm{n}=2,4 \%)$ ranked in the disagree category (see Figure 6-49).

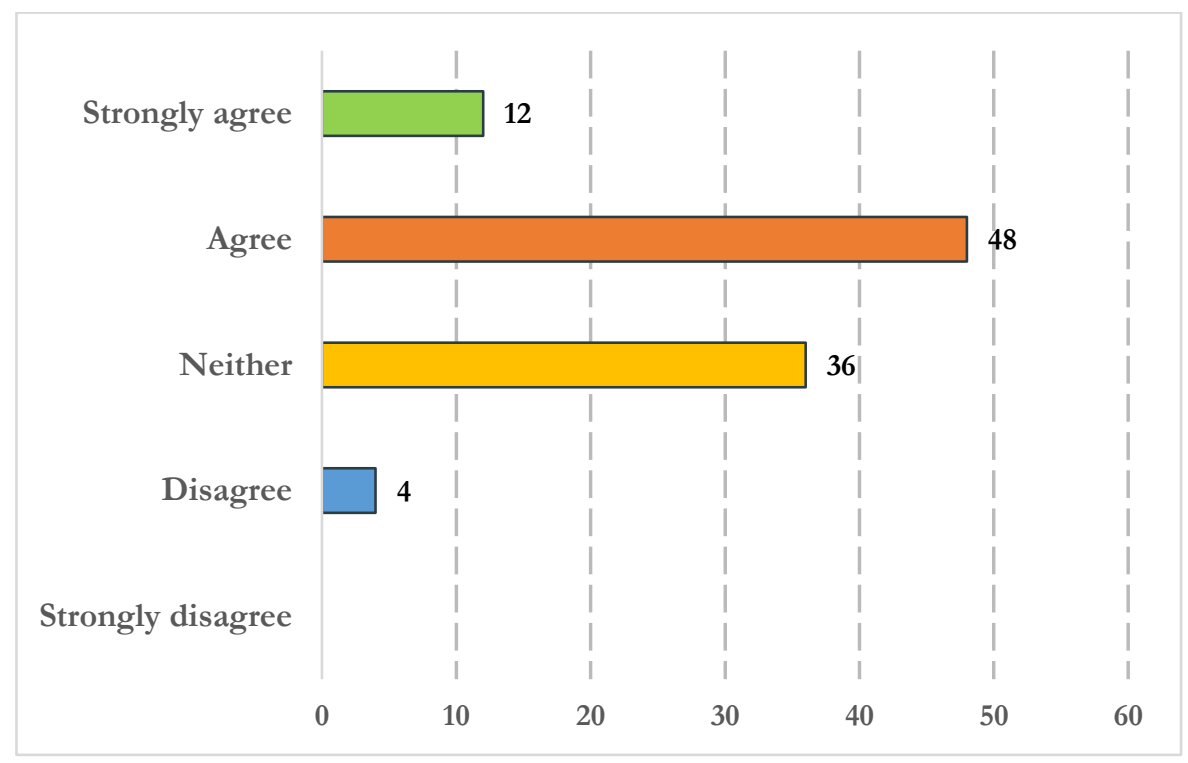

Figure 6-49: Respondents' ratings of positive distractions 
The result reflected that positive distractions were thought to offer benefits to new mothers in the immediate postnatal hospital stays. Plausible explanation for the respondents who were neutral may be due in part to thinking that even soft music contributes to noise and may induce stress for new mothers who are needing to sleep or rest. Another point might be that positive distractions like soft music could be suitable only if care was individualised or personalised where each woman has a private space for herself, baby and family. In spite of this, $60 \%$ of the respondents thought that positive distractions are valuable and capable of improving women's emotional and spiritual wellbeing during the recovery and restoration process.

\section{8) Women interact less in a single private room rather than in a postnatal ward}

Respondents with perceptions on the amount of interaction in a single private room in relation to an open-bay ward was ranked moderately high. Fourteen $(28 \%)$ respondents reported strongly agree, and fifteen (30\%) reported agree. Ten respondents (20\% in both cases) either reported neither agree nor disagree or disagree. One respondent $(2 \%)$ stated strongly disagree (see Figure 6-50).

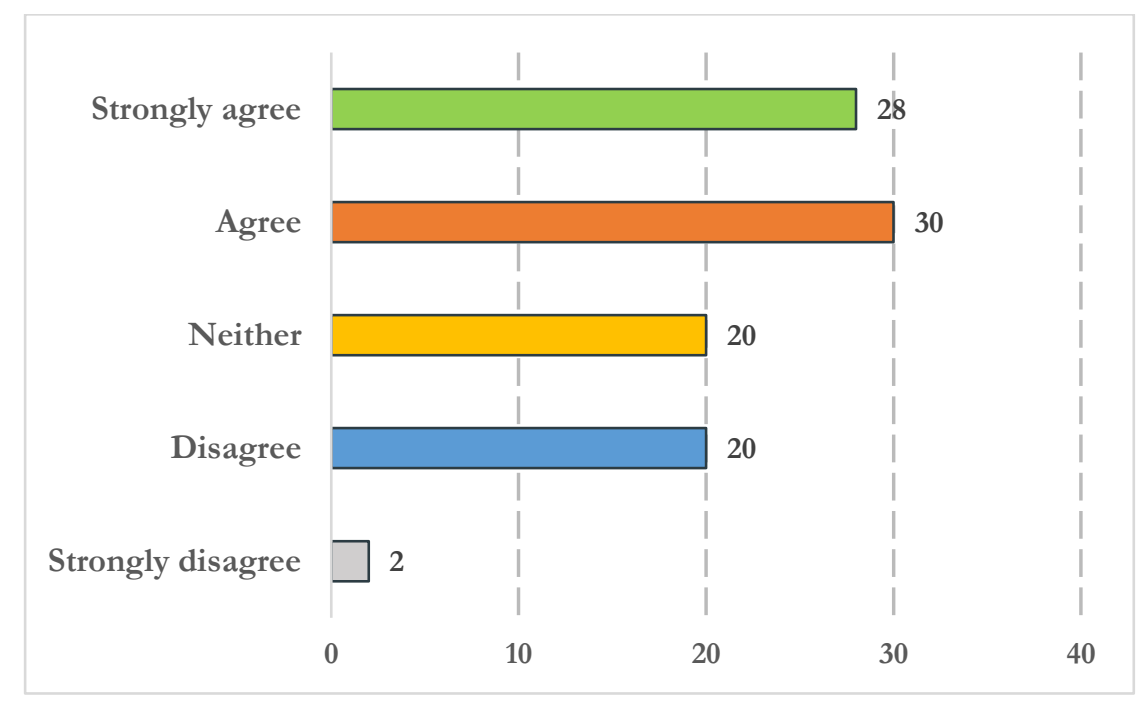

Figure 6-50: Respondents' ratings of interaction in single-bed rooms versus open wards

The result reflected that from midwives' point of view, a single private room offers less opportunity for interaction than a postnatal ward. Assuming that women had no visitors, a single private room could equate to fewer or no interactions at all. More than half of the respondents ( $\mathrm{n}$ $=29,58 \%$ ) thought this affected social interaction which could have other related health and emotional benefits, suggesting that they saw value in multi-bed occupancy as a few women maybe in need of other women for interaction. 
A close examination of the histogram for ratings of interaction in single-bed rooms in Figure 6-51 revealed the distribution is negatively skewed. The respondents valuing one type of room occupancy over another may also be related to how it impacts nursing effectiveness, communication and teamwork.

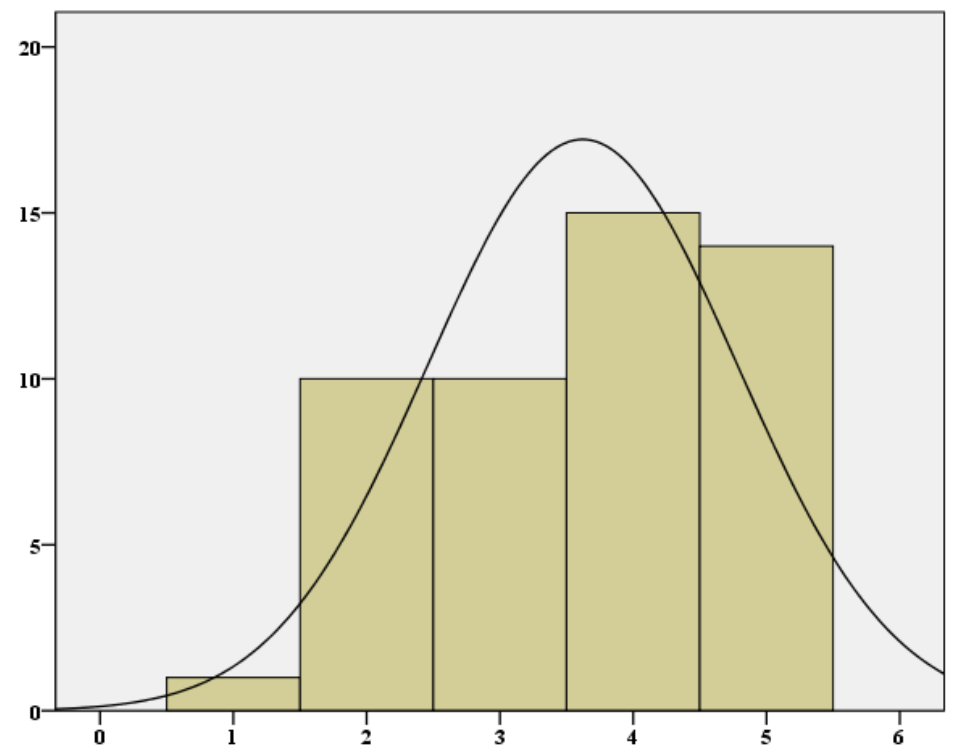

Figure 6-51: A negative skew distribution of ratings of interaction in single versus open ward

However, the $40 \%$ who responded "disagree" and "neither" seem to suggest that there was a lack of a clear-cut preference for the effect of a single room over a shared/multi-bed room.

Table 6-6 summarises the results on descriptive statistics for each question and standard deviations.

Table 6-6: Preference (mean and standard deviation) of respondents on perception of social comfort environment contributing to information support and women's wellbeing $(\mathrm{n}=50)$

\begin{tabular}{llc}
\hline Survey question & M & SD \\
\hline $\begin{array}{l}\text { A postnatal room with several beds decreases the opportunity for discussions } \\
\text { about personal issues }\end{array}$ & 4.43 & 0.83 \\
$\begin{array}{l}\text { Furniture and décor layouts in a communal space can help group learning of } \\
\text { parental skills }\end{array}$ & 4.17 & 0.64 \\
Communal space near the postnatal space is good for counselling purposes & 3.85 & 0.78 \\
Positive distractions such as soft music, natural images etc. are better for recovery & 3.66 & 0.76 \\
Women interact less in a single private room rather than in a postnatal ward & 3.60 & 1.19 \\
Having a passage or corridor to enter a postnatal room or ward helps to control & 3.55 & 0.72 \\
visitors & & \\
Women have the opportunity to choose a room that they prefer & 3.34 & 1.06 \\
Spatial connection between postnatal room and birth room & 2.91 & 0.91 \\
\hline
\end{tabular}

Note: $M=$ mean; $S D=$ standard deviation 


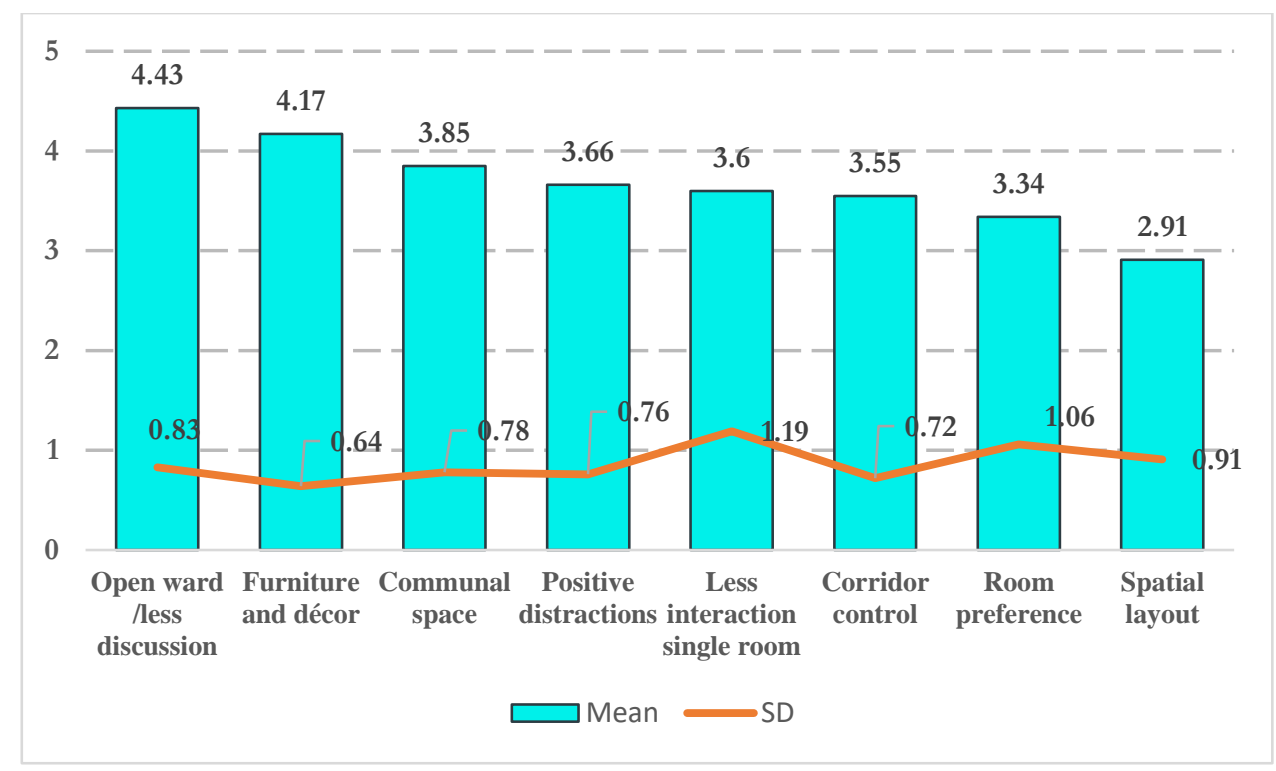

Figure 6-52: Respondents ratings of social comfort environment features

\subsubsection{Open-ended question}

The respondents were asked to give additional comments about anything which might be missed out which they would like to be added to the postnatal room hospital environment (see question 3.20 in the midwives' questionnaire in Appendix B). Twenty-six out of fifty respondents' text information was evaluated using word-frequency analysis. The responses indicated that the words “space” (11), "rooms” (11), "partners” (6), “communal areas” (5), "garden” (4), "postnatal” (3) were most frequently mentioned. In addition, other words that related to comfortable environment, were mentioned such as "music/tv/videos" (3), "natural features/plants", (3), beautiful colour (2), "minimise noise" (1), “access to fresh air" (1), "natural light", (1), and “comforting" (1).

In terms of preference for a restorative environment, the respondents mentioned that ideally a single room option was the best to allow the partners or support persons to stay together with the baby. The need to have comfortable beds, or fold out bed space for partner, cots that are adjustable to mother's position either in or out of bed and comfortable chairs is also stressed. Furthermore, a central communal space outside the rooms, with a sofa, was thought to enhance the opportunity for family to interact and socialise in a familiar setting while women admire each other's babies and share their experiences. The respondents also agreed that communal space would promote group activities such as breastfeeding and other parental skills.

Importantly, respondents felt there is the need for adequate space to move freely in the rooms for safety and ease of attending to women and their babies. The feeling of safety also related to a 
woman being attended in a single room which may explain why postnatal women could not have preferences for rooms of their choice.

Women do not like sharing unless they feel unsafe in the public environment...At times women want a single room and there is no one watching them and that can be unsafe. (Midwife)

People actually want their own room and don't seem to want to socialise. The curtains are always closed in a double room making the space seem smaller and cramped. I don't agree that people should choose their own postnatal room here as it's allocated on a needs basis. (Midwife)

Finally, an idea of a comfortable postnatal environment emphasised inclusion of a more relaxing setting that encourages women for an optimum recovery experience and wellbeing.

I think it would be nice to have some access to outside space, and to have some natural features, e.g. water features, garden. The ability to provide a more homelike environment rather than a hospital room would be nice. The ability for women to choose rooms would be good and for there to be a choice of rooms. (Midwife)

Plants. Something beautiful. I know cost is always a factor but what if each room had one wall of some beautiful colour/wallpaper. All women at my hospital would like to have a rooftop garden to look at rather than the gravel that currently exists. I know they can't walk out there, but even a line of topiaries would be nicer to look at. (Midwife)

\subsection{Discussion of the results}

This section focuses on the discussion of results with regard to the midwives' survey. It will briefly discuss the major findings of the design factors perception survey focused on 1) perceptions of postnatal room features, 2) interior environment factors, 3) sensory environment factors, and 4) social comfort features.

\subsubsection{Summary of the results}

The ranking of perception of design factors revealed that some items of interior environment features and sensory comfort had the overall highest mean scores in the following order $(M=4.98$, 4.90, and 4.86) as can be seen in Tables 6-4 and 6-5. These aspects speak to the hygiene and 
maintenance and audio environment (a room that feels quiet) design variables in built healthcare environment. In addition, two of the three highest mean scores were sensory comfort features, which suggests their level of importance for wellbeing. Items such as "have a restful room" (M = 4.78), "having adequate daylighting" and "adequate lighting and operable windows" received very similar mean scores $(\mathrm{M}=4.78$ and 4.76) respectively. The two items concerning daylighting received similar ratings demonstrating their significant and consistent pattern in the postnatal environment.

Comfort-related aspects regarding control of noise in the postnatal environment had higher ratings including "being able to control noise from mechanical things" $(M=4.68)$ and "a room or ward that is quiet is good for sleep and help women to recover in comfort" $(M=4.60)$.

Regarding social comfort environment, the likelihood of "a room with several beds" limiting discussion had a mean score of $(M=4.43)$ which suggested that the respondents thought open bay wards were not likely to be beneficial for private conversations with women. Table 6-7 shows the list of the top fifteen design features influencing recovery experience and wellbeing of postnatal women (see Tables 11.1 and 11.2 for the rating for all the survey questions in Appendix C).

As was found for women's results, the preliminary findings suggest support for hygiene/clean environment, a restful and tranquil environment, sensory requirements (visual environment such as daylighting, noise and temperature control) were identified as most important. The top 15 ratings for perception of the physical design factors are illustrated in descending order of importance in the midwives' survey (see Figure 6-53).

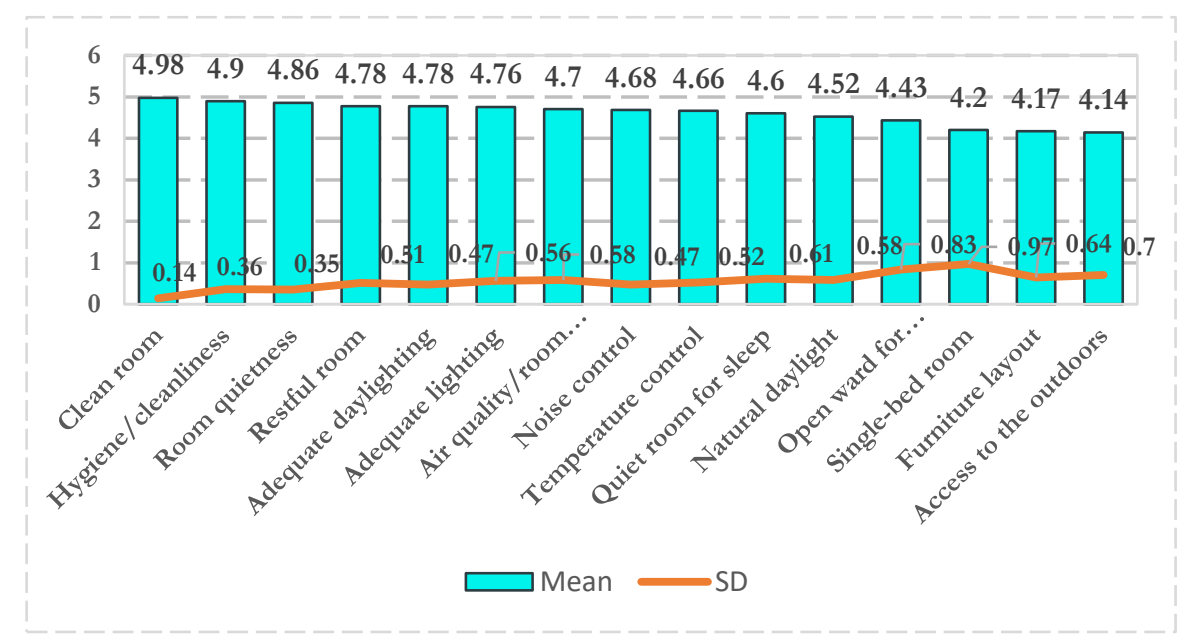

Figure 6-53: Top fifteen design features influencing women's recovery experience (midwives) 
Table 6-7: The 15 most important items in the Design Factors influencing the women's postnatal recovery experiences and wellbeing of the design perception survey (midwives, $\mathrm{n}=50$ )

\begin{tabular}{llc}
\hline Item & M & $\boldsymbol{S D}$ \\
\hline Provide care in a room that is clean & 4.98 & 0.14 \\
Having a hygienic/clean environment & 4.90 & 0.36 \\
Providing care in a room that feels quiet & 4.86 & 0.35 \\
Have a restful room & 4.78 & 0.51 \\
Having adequate daylighting & 4.78 & 0.47 \\
Adequate lighting (to the activity in the room) and operable windows & 4.76 & 0.56 \\
Air quality and room freshness & 4.70 & 0.58 \\
Being able to control noise from mechanical things like ventilation, medical & 4.68 & 0.47 \\
equipment etc. & 4.66 & 0.52 \\
Being able to control the temperature & 4.60 & 0.61 \\
A room or ward that is quiet is good for sleep and generally helps women recover & & \\
in comfort & 4.52 & 0.58 \\
A postnatal room that lets in natural daylight is better for recovery care & 4.40 & 0.83 \\
A postnatal room with several beds decreases the opportunity for discussions \\
about personal issues
\end{tabular}

Note: Items were ranked on a scale from 1 to 5 , where 1 indicated not at all important, and 5 indicated very important; $M=$ mean; $S D=$ standard deviation

\subsection{Conclusion}

This chapter has presented the concluding section of the results on perceptions of the design factors surveys. In this chapter, both descriptive statistics and analyses of the results for midwives were presented. A number of design factors have been identified which have commonalities and differences with the results found in the women's survey and their implications for wellbeing are discussed. The next chapter will present the results of analyses of the inferential statistics among the two user groups where both results were compared. 


\section{CHAPTER 7}

\section{RESULTS OF INFERENTIAL ANALYSIS ON PERCEPTION OF DESIGN FACTORS SURVEYS}

This Chapter presents the inferential analysis of perceptions of design factors. It focuses on comparisons of the results in the questionnaire surveys of postnatal women and midwives and discusses the implications of the findings for the phase two qualitative focus groups. The next sections present the results of the four categories of design factors.

\subsection{Users' perceptions of postnatal room features}

Independent samples $t$ - tests were conducted to compare postnatal room feature mean scores for women and midwives. Five analyses indicated no significant differences in the mean scores between women and midwives. This shows that the variances of the two groups are assumed to be approximately equal. Respondents' perceptions of the postnatal room features were rated significantly higher in seven out of eight factors with the exception of "room colours" which received the lower scores. Accordingly, two design factors including single-bed accommodation and room quietness stand out. A single room accommodation had the highest mean scores reported from women in contrast to midwives (4.88 vs. 4.22) (see Table 7-1 and Figure 7-1).

In general, the responses were found to be normally distributed and equal variances are assumed based upon results of Levene's tests as follows: Natural daylight, $(F(260)=0.30, p=0.58)$. There was no significance difference in the mean scores for women $(M=4.47, S D=0.61)$ and midwives $(\mathrm{M}=4.52, S D=0.58 ; t(260)=-0.56, p=0.58$, two-tailed). The magnitude of the difference in the means (mean difference $=-0.053$ ). The $95 \%$ confidence interval CI was -0.24 to 0.13 . The difference on perception of natural daylight represents a very small effect size, (Cohen $d=.08$ ). This means that the respondents appreciate the value of natural daylight in the postnatal rooms and this appears to contribute to wellbeing of women during recovery. It is also important for staff due in part to making a more conducive working environment. Shapes and forms for postnatal room design, $(F(260)=0.16, p=0.69)$. There was little difference in the mean scores for women $(\mathrm{M}=3.88, S D=0.70)$ and midwives $(\mathrm{M}=3.82, S D=0.63 ; t(260)=0.57, p=0.57$, two-tailed $)$. The effect size, Cohen's $d=0.09$, was very small. 


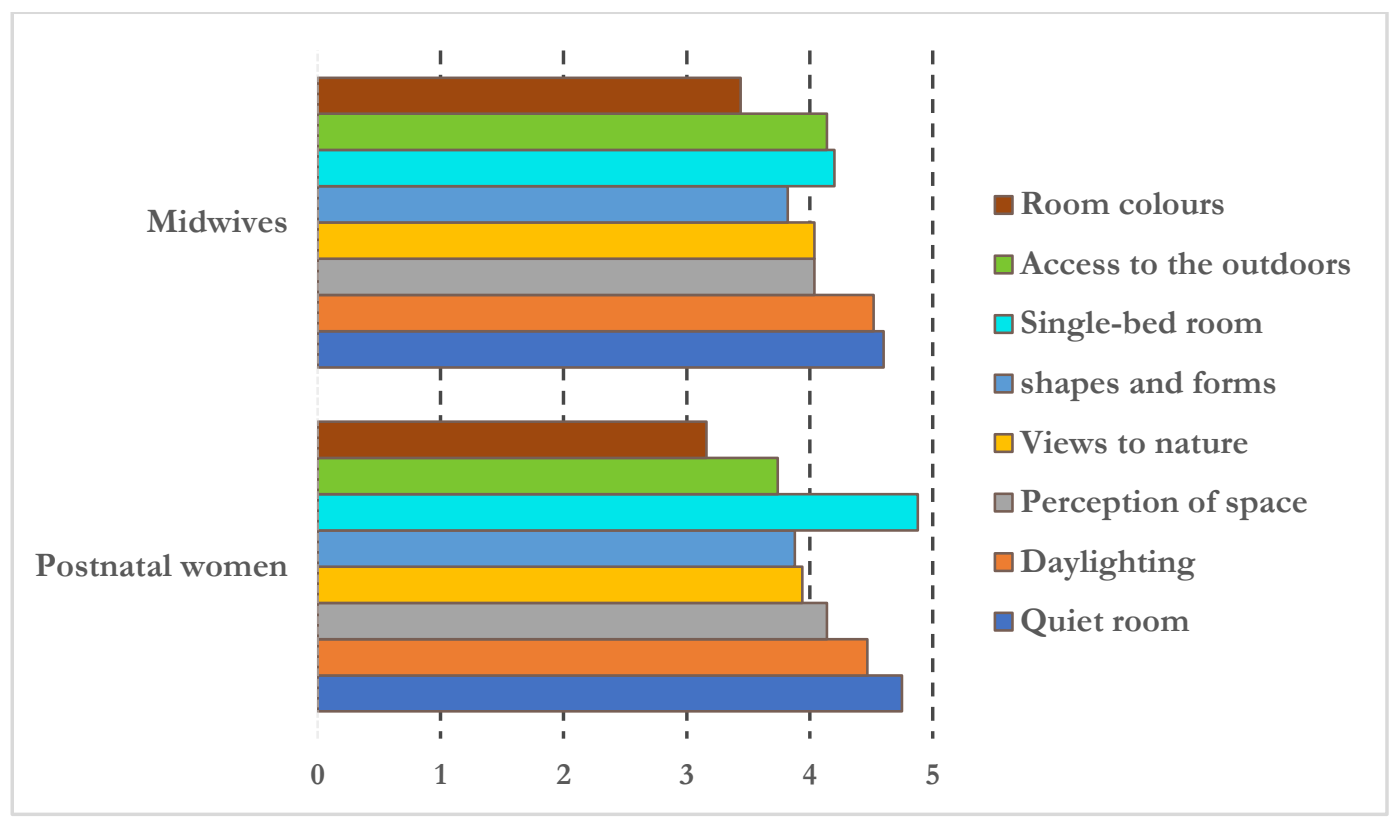

Figure 7-1: Respondents' perception on levels of importance regarding postnatal room features

Similarly, for Room quietness, $(F(260)=3.38, p=0.07)$ there was no significant difference between the mean scores of women $(\mathrm{M}=4.75, S D=0.61)$ and midwives $(\mathrm{M}=4.60, S D=0.61$; $t$ $(260)=1.61, p=0.11$, two-tailed). The mean difference was 0.155 and CI: $-0.04 ; 0.34$. The effect size of 0.2 was small. Design impact of space, $(F(260)=0.73, p=0.39)$. For women $(M=4.14$, $S D=0.96)$ and midwives $(\mathrm{M}=4.04, S D=0.83 ; t(260)=0.66, p=0.51$, two-tailed $)$. Views to nature, $(F(260)=2.87, p=.09)$. There was no significance difference in the mean scores for women $(\mathrm{M}$ $=3.94, S D=0.73)$ and midwives $(M=4.04, S D=0.61 ; t(260)=-0.865, p=0.39$, two-tailed $)$. The difference in the perception of views to nature represents a very small effect size, (Cohen's $d=$ $0.15)$.

On the other hand, three of the analyses conducted show significant differences in the mean scores between women and midwives. For a single room for recovery, equal variances are not assumed based upon the result of Levene's test, $(F(260)=135.20, p<0.001$ hence Welch t' test was used; $\mathrm{t}(52.31)=4.90, p<0.001$, two-tailed $)$. Difference in the mean scores for women $(\mathrm{M}=4.88, S D=$ $0.37)$ and midwives $(M=4.20, S D=0.97)$. Mean difference was 0.68 . The $95 \%$ confidence interval CI was 0.40 to 0.96 . The single-bed room accommodation was perceived by women as positively more higher than the midwives $(\mathrm{p}<0.001)$. The difference in single-bed room shows a large effect size, (Cohen $d=0.92)$. Access/Walk to the outdoors, $(F(260)=19.9, p<0.001)$. There were statistically significant differences in the mean scores between women $(M=3.74, S D=1.13)$ and 
midwives $(M=4.14, S D=0.70 ; t(260)=-3.213, p=0.002$, two-tailed $)$. The difference in access to the outdoors represents a medium effect size, (Cohen's $d=0.42)$. Midwives perceived the ratings of access/walk to the outdoors more than the postnatal women. One probable reason could be that midwives wanted women to start getting used to return to normal after the birth. colours, $(F(260)=1.34, p=0.25)$. Differences between the mean scores for women $(\mathrm{M}=3.16$, $S D=0.72)$ and midwives $(\mathrm{M}=3.44, S D=0.71 ; t(260)=-2.52, p=0.012$, two-tailed $)$. The magnitude of the difference in the means $(=-0.28,95 \%$ CI: $-0.51,-0.06)$. The difference in colours represents a medium effect size (Cohen's $d=0.4$ ). Presumably, calming comfortable colours in the interior of postnatal environment might contribute to psychological recovery experiences of women. Reasons for possible differences in scores of women and midwives are explained later under the discussion in section 8.2.5.5.

Table 7-1 shows the dimensions of postnatal room features that achieved consensus and those aspects without, as well as their corresponding mean scores and standard deviations.

Table 7-1: Preference (Independent Samples $t$-tests) of respondents on items in postnatal room features comparing women and midwives responses

\begin{tabular}{|c|c|c|c|c|c|c|c|c|}
\hline \multirow[t]{2}{*}{ Questionnaire item } & \multicolumn{2}{|c|}{$\begin{array}{l}\text { Women } \\
(n=212)\end{array}$} & \multicolumn{2}{|c|}{$\begin{array}{l}\text { Midwives } \\
(\mathrm{n}=50)\end{array}$} & \multirow{2}{*}{$\begin{array}{l}\text { Differenc } \\
\text { e between } \\
\text { the means }\end{array}$} & \multirow[t]{2}{*}{$\begin{array}{l}\text { Std. } \\
\text { error }\end{array}$} & \multirow[t]{2}{*}{$\begin{array}{l}\text { T - } \\
\text { value }\end{array}$} & \multirow{2}{*}{$\begin{array}{l}p \text {-value } \\
\text { (Two- } \\
\text { tailed) }\end{array}$} \\
\hline & $M$ & $S D$ & $M$ & $S D$ & & & & \\
\hline $\begin{array}{l}\text { Perception of } \\
\text { quietness in rooms }\end{array}$ & 4.75 & .613 & 4.60 & .606 & 0.155 & 0.096 & 1.61 & $.11 \dagger$ \\
\hline Natural daylight & 4.47 & .611 & 4.52 & .580 & -0.053 & 0.095 & -0.56 & $.58 \dagger$ \\
\hline $\begin{array}{l}\text { Design impact of } \\
\text { space }\end{array}$ & 4.14 & .961 & 4.04 & .832 & 0.097 & 0.148 & 0.66 & $.51 \dagger$ \\
\hline Views to nature & 3.94 & .733 & 4.04 & .605 & -0.097 & 0.112 & -0.865 & $.39 \dagger$ \\
\hline $\begin{array}{l}\text { Shapes and form for } \\
\text { postnatal room } \\
\text { design }\end{array}$ & 3.88 & .702 & 3.82 & .629 & 0.062 & 0.108 & 0.573 & $.57 \dagger$ \\
\hline $\begin{array}{l}\text { A single room } \\
\text { recovery }\end{array}$ & 4.88 & .365 & 4.20 & .969 & 0.682 & 0.139 & 4.90 & $<.001 * *$ \\
\hline $\begin{array}{l}\text { Accessibility of room } \\
\text { to the outdoors }\end{array}$ & 3.74 & 1.13 & 4.14 & .700 & -0.404 & 0.126 & -3.213 & $.002^{* *}$ \\
\hline $\begin{array}{l}\text { Room colours versus } \\
\text { plain room }\end{array}$ & 3.16 & .722 & 3.44 & .705 & -0.284 & 0.113 & -2.518 & $.012^{*}$ \\
\hline
\end{tabular}

Note: $\nmid$ means are not significantly different at $p>0.05 ; *$ statistically significant at $p \leq 0.05$; ** statistically significant with Bonferroni adjustment at $p<(0.05 / 4=0.0125)$ 


\subsection{Users' perceptions of interior environment features}

Analysis for each of the interior environment features was conducted using independent samples $\mathrm{t}$-tests. Two of the factors showed no differences or effects $(\mathrm{P}>0.05)$ for both groups. In addition, perception of postnatal women was significantly higher than midwives in three out of eight factors of interior environment. These include provision of a restful room, door and privacy and clinical room. Significant differences were also found in other two interior environment including decoration in the room (natural art and images) and bright colours but were not rated higher by the postnatal women. Both the clinical-looking room and bright colours received the lowest mean scores (see Table 7-2 and Figure 7-2).

The results of the analyses were found to be normally distributed and equal variances assumed or not assumed (where applicable as the case may be) based upon results of Levene's tests were as follows: $\underline{\text { room restfulness, }}$ ( $F(260)=10.66, p<0.001$, (equal variances not assumed). There was no significant difference in the mean scores for women $(\mathrm{M}=4.89, S D=0.37)$ and midwives $(\mathrm{M}$ $=4.78, S D=0.51 ; t(61.70)=1.47, p=0.15$, two-tailed $)$. The magnitude of the difference in the means $($ mean difference $=0.11$ ). The $95 \%$ confidence interval CI was -0.01 to 0.24 and Cohen's $d$ $=0.2$ was established to be a small effect. Combined clinical and familiar home features, $(F(260)$ $=2.56, \mathrm{p}=0.11)$. Difference in the mean scores for women $(\mathrm{M}=3.60, S D=1.12)$ and midwives $(\mathrm{M}=3.58, S D=0.99 ; t(260)=0.11, p=0.91$, two-tailed $)$.

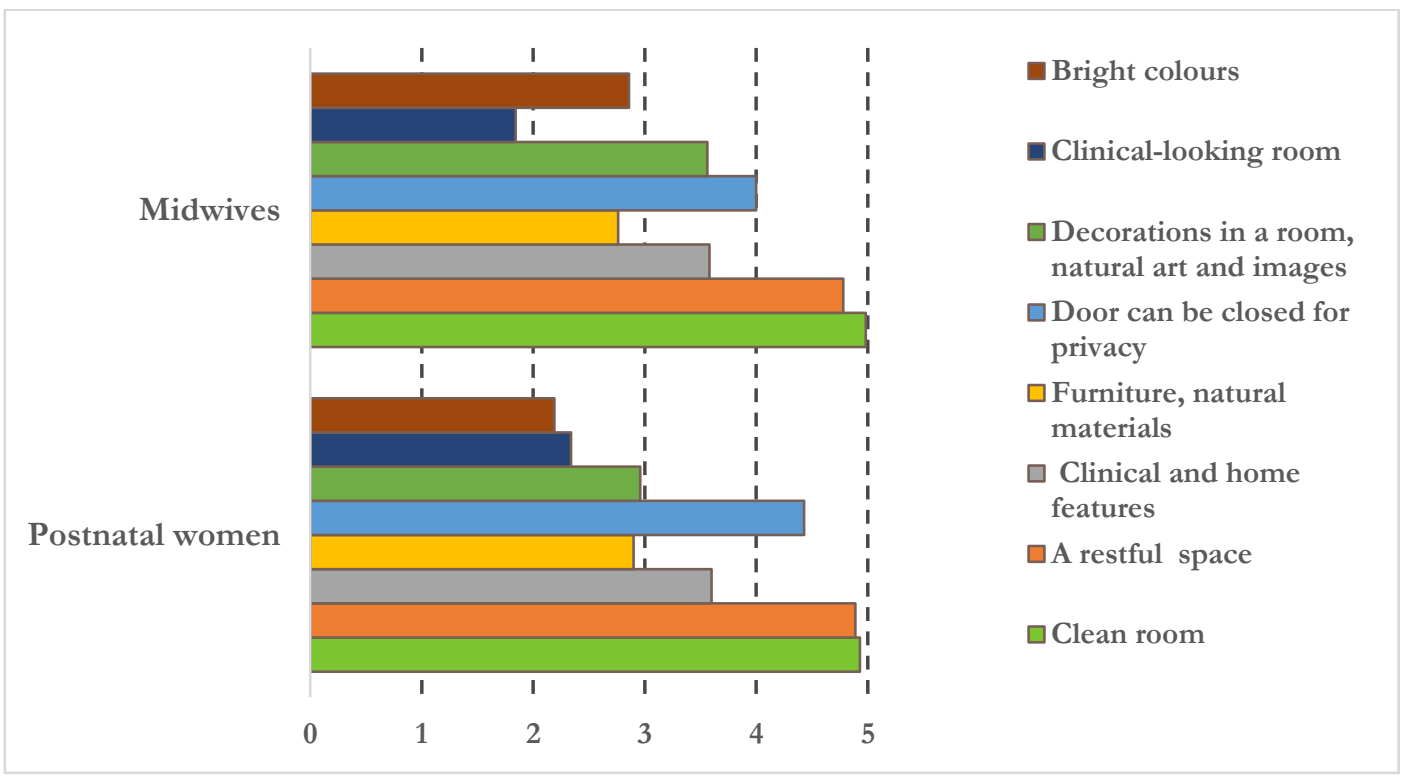

Figure 7-2: Respondents' perception on levels of importance regarding interior environment features 
Conversely, there were significant differences in the mean scores of the factors "room cleanliness", "furniture made of natural materials", and "closing of door for privacy." Others are "decorations in a room i.e. natural art, images etc.", "bright colours", and "clinical-looking postnatal space". For the aspect of Room cleanliness, equal variances are not assumed based on the result of Levene's test of equality, therefore the Welch t' test portion of result was used. $(F(260)=5.94, p=0.02)$. Difference in the mean scores for women $(\mathrm{M}=4.93, S D=0.27)$ and midwives $(\mathrm{M}=4.98, S D=$ $0.14 ; t(142.81)=-1.70, p=0.09)$. Mean difference was -0.05 . The $95 \%$ confidence interval CI was -0.10 to 0.08 . The difference in room cleanliness represents a small effect size, (Cohen $d=$ 0.2). Similarly for Furniture made of natural materials, $(F(260)=0.18, p=0.67)$ difference in the mean scores for women $(\mathrm{M}=2.90, S D=1.06)$ and midwives $(\mathrm{M}=2.76, S D=0.96 ; t(260)=$ $0.83, p=0.41)$. Average responses suggest that postnatal women and midwives seem not to support the use of natural materials for furniture in interior postnatal spaces, with midwives rating it lower than women.

Furthermore, analyses show significant differences in the following. Door closed for privacy, ( $F$ $(260)=2.18, p=0.14)$. Difference in the mean scores for women $(M=4.43, S D=0.84)$ and midwives $(M=4.00, S D=0.95 ; t(260)=3.18, p=0.002$, two-tailed $)$. The difference in the factor of closing door for privacy indicates a medium effect size, (Cohen $d=0.5)$. Aspect of decorations in the room, $(F(260)=0.95, \mathrm{p}=0.33)$. Difference in the mean scores for women $(\mathrm{M}=2.96, S D$ $=1.05)$ and midwives $(M=3.56, S D=1.07 ; t(260)=-3.61, p<0.001$, two-tailed $)$. The mean difference ( $=, 95 \%$ CI: -0.92 to -0.27$)$. The difference in decorations in the room (attractiveness) indicates a moderate effect size, (Cohen's $d=0.5)$. Bright colours, $(F(260)=0.66, p=0.42)$. Difference in the mean scores for women $(\mathrm{M}=2.19, S D=0.91)$ and midwives $(\mathrm{M}=2.86, S D=$ 1.03; $t(260)=-4.56, p<0.001$, two-tailed). The effect size (Cohen's $d=0.7)$ was established to be a fairly large effect.

In terms of clinical-looking postnatal space, the results of Levene's test indicates equal variances not assumed thus Welch t' test result applies: $(F(260)=5.18, p=0.02)$. Difference in the mean scores for women $(\mathrm{M}=2.34, S D=1.17)$ and midwives $(\mathrm{M}=1.84, S D=0.87 ; t(96.35)=3.44, p$ $=0.001$, two-tailed). The difference in clinical-looking postnatal space represents a medium effect size (Cohen $d=0.5)$. Table 7-2 shows the aspects of postnatal interior environment that achieved consensus and those aspects without. 
Table 7-2: Preference (Independent Samples t-tests) of respondents on items in interior environment features comparing women and midwives responses

\begin{tabular}{|c|c|c|c|c|c|c|c|c|}
\hline \multirow[t]{2}{*}{ Questionnaire item } & \multicolumn{2}{|c|}{$\begin{array}{l}\text { Women } \\
(n=212)\end{array}$} & \multicolumn{2}{|c|}{$\begin{array}{l}\text { Midwives } \\
(\mathrm{n}=50)\end{array}$} & \multirow{2}{*}{$\begin{array}{l}\text { Differenc } \\
\text { e between } \\
\text { the means }\end{array}$} & \multirow[t]{2}{*}{$\begin{array}{l}\text { Std. } \\
\text { error }\end{array}$} & \multirow[t]{2}{*}{$\begin{array}{l}\mathrm{T}- \\
\text { value }\end{array}$} & \multirow{2}{*}{$\begin{array}{l}p \text {-value } \\
\text { (Two- } \\
\text { tailed) }\end{array}$} \\
\hline & $M$ & $S D$ & $M$ & $S D$ & & & & \\
\hline $\begin{array}{l}\text { Provision of a clean } \\
\text { room }\end{array}$ & 4.93 & .267 & 4.98 & .141 & -0.046 & 0.027 & -1.70 & $.09 *$ \\
\hline $\begin{array}{l}\text { Provision of a restful } \\
\text { room }\end{array}$ & 4.89 & .368 & 4.78 & .507 & 0.112 & 0.062 & 1.78 & $.08 *$ \\
\hline $\begin{array}{l}\text { Combined clinical } \\
\text { and familiar home } \\
\text { features }\end{array}$ & 3.60 & 1.12 & 3.58 & .992 & 0.019 & 0.172 & 0.11 & $.91 \dagger$ \\
\hline $\begin{array}{l}\text { Furniture made of } \\
\text { natural materials }\end{array}$ & 2.90 & 1.06 & 2.76 & .960 & 0.136 & 0.164 & 0.83 & $.41 \dagger$ \\
\hline Door and privacy & 4.43 & .837 & 4.00 & .948 & 0.429 & 0.135 & 3.18 & $.002 *$ \\
\hline $\begin{array}{l}\text { Decoration in a room } \\
\text { (natural art and } \\
\text { images) }\end{array}$ & 2.96 & 1.05 & 3.56 & 1.07 & -0.598 & 0.165 & -3.61 & $<.001 * *$ \\
\hline Clinical room & 2.34 & 1.17 & 1.84 & .866 & 0.504 & 0.147 & 3.44 & $.001 *$ \\
\hline Bright colours & 2.19 & .914 & 2.86 & 1.03 & -0.671 & 0.147 & -4.56 & $<.001 * *$ \\
\hline
\end{tabular}

Note: $\nmid$ means are not significantly different at $p>0.05 ; *$ statistically significant at $p \leq 0.05 ; * *$ statistically significant with Bonferroni adjustment at $p<(0.05 / 4=0.0125)$

\subsection{Users' perceptions of sensory environment features}

Independent samples $t$ - tests were conducted to compare sensory environment features mean scores for women and midwives. Two analyses indicated no significant differences in the mean scores between women and midwives. However, five design factors comprising daylighting, temperature control, noise control, adjusting lighting and operable windows were rated significantly higher by the midwives than the postnatal women. The least mean score was reported on perception of coordinated natural things (pleasantness) in the room.

Among the two analyses in which there were no significant differences, for hygienic and clean environment the response was found to be normally distributed and equal variances are assumed based upon results of Levene's tests as follows: $(F(260)=0.33, p=0.57)$. No difference was found in the mean scores for women $(\mathrm{M}=4.92, S D=0.33)$ and midwives $(\mathrm{M}=4.90, S D=0.36 ; t(260)$ $=0.29, p=0.77$, two-tailed). Mean difference was established to be 0.01 and the $95 \%$ confidence interval CI was -0.09 to 0.12 . The difference in hygiene and cleanliness represents a very small effect size (Cohen $d=0.06)$. 


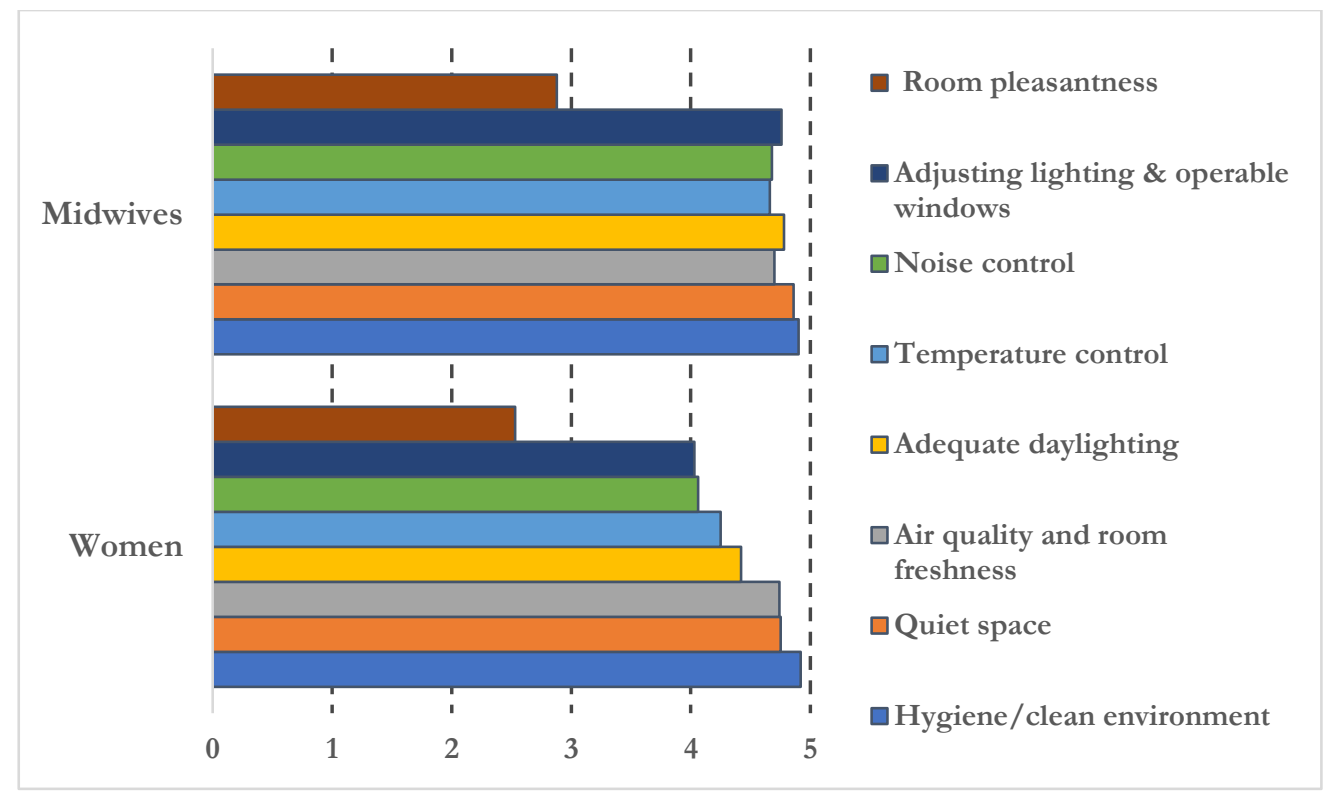

Figure 7-3: Respondents' perception on levels of importance regarding sensory comfort environment

For room quietness, it was found that equal variances are not assumed based upon Levene's tests, thus Welch t' tests result was used. Difference in the mean scores for women $(\mathrm{M}=4.75, S D=$ $0.62)$ and midwives $(M=4.86, S D=0.35 ; t(130.51)=-1.76, p=0.08$, two-tailed $)$. In addition, mean difference was -0.12 and the $95 \%$ confidence interval CI was -0.29 to 0.06 and the effect size, (Cohen $d=0.2)$ was small. Similarly, for air quality and room freshness, $(F(260)=1.18, p=$ $0.28)$ difference in the mean scores for women $(\mathrm{M}=4.74, S D=0.51)$ and midwives $(\mathrm{M}=4.70$, $S D=0.58 ; t(260)=0.49, p=0.62$, two-tailed). The difference in air quality and room freshness indicates a very small effect size (Cohen's $d=0.07)$ was very small.

As expected, mean scores were generally higher for hygiene and clean environment for both the postnatal women and midwives (mean score of 4.92 vs 4.90 ) respectively. This is an interesting result that echoes a similar study (Mourshed \& Zhao, 2012) suggesting the importance of hygiene in healthcare settings. However, the differences in the factor of hygiene and clean environment are not statistically significant, possibly because of the sample size especially for the midwives in the study. Next is room quietness which received higher mean score by midwives compared to women (mean score of 4.84 vs. 4.74). The result shows that midwives are more likely interested in the tranquility of postnatal hospital environments than women.

Additionally, higher mean scores were achieved for air quality and room freshness, with higher mean score for women and slightly lower mean score of (4.74 vs 4.70$)$ for midwives respectively. 
As previously noted under "perceptions on room preference", mean scores on room quietness by women show no difference $(=4.75$ vs 4.75$)$. However, mean scores on room quietness for midwives increased from (4.60) to (4.86). This indicates midwives are more sensitive to hospital noise than women which could be attributed to the fact that midwives stay long hours in hospitals experiencing the prevalent busyness and noisiness (McLachlan et al., 2009; Rayner et al., 2008).

On the other hand, five analyses indicated that there were significant differences among the mean scores. The results of the five analyses are as follows: Adequate daylighting, $(F(260)=23.8, p<$ $0.001)$ difference in the mean scores for women $(M=4.42, S D=0.73)$ and midwives $(M=4.78$, $S D=0.47, t(114.51)=-4.29, p<0.001$, two-tailed). Mean difference was established to be -0.36 and the $95 \%$ confidence interval CI was -0.05 to -0.14 and the effect size, (Cohen $d=0.6$ ) was of medium effect. Temperature control, $(F(260)=11.7, p<0.001)$. Difference in the mean scores for women $(\mathrm{M}=4.25, S D=0.87)$ and midwives $(\mathrm{M}=4.66, S D=0.52, t(121.89)=-4.34, p<0.001$, two-tailed). Noise control, $(F(260)=13.6, p<0.001)$. Mean scores for women $(\mathrm{M}=4.06, S D=$ $0.99)$ and midwives $(M=4.68, S D=0.47, t(163.34)=-6.54, p<0.001$, two-tailed $)$.

Adjusting lighting and operable windows, $(F(260)=12.8, p<0.001)$ also revealed a statistically significant difference between the two groups of respondents. Mean scores for women $(M=4.03$, $S D=1.00)$ and midwives $(M=4.76, S D=0.56, t(260)=-6.94, p<.001$, two-tailed). For Natural things in the room, $(F(260)=1.58, p=0.21)$ initial analysis shows there was a small difference in the mean scores for women $(\mathrm{M}=2.53, S D=1.04)$ and midwives $(\mathrm{M}=2.88, S D=1.00, t(260)=$ $-2.13, p=0.03$, two-tailed), which suggested that respondents did not seem to agree that having natural things in the postnatal space was important However, further analysis revealed there was a significant difference regarding the magnitude of the difference in means (mean difference = 0.35, 95\% CI: $-0.67,-0.03)$. The difference in natural things in the room represents a small-tomedium effect size (Cohen's $d=0.3$ ). Table 7-3 summarises the results of the independent sample t-tests conducted for each of the sensory environment design factors. 
Table 7-3: Preference (Independent Samples t-tests) of respondents on items in sensory environment features comparing women and midwives responses

\begin{tabular}{|c|c|c|c|c|c|c|c|c|}
\hline \multirow[t]{2}{*}{ Questionnaire item } & \multicolumn{2}{|c|}{$\begin{array}{l}\text { Women } \\
(n=212)\end{array}$} & \multicolumn{2}{|c|}{$\begin{array}{l}\text { Midwives } \\
(\mathrm{n}=50)\end{array}$} & \multirow{2}{*}{$\begin{array}{l}\text { Differenc } \\
\text { e between } \\
\text { the means }\end{array}$} & \multirow[t]{2}{*}{$\begin{array}{l}\text { Std. } \\
\text { error }\end{array}$} & \multirow[t]{2}{*}{$\begin{array}{l}\mathrm{T} \text { - } \\
\text { value }\end{array}$} & \multirow{2}{*}{$\begin{array}{l}p \text {-value } \\
\text { (Two- } \\
\text { tailed) }\end{array}$} \\
\hline & $M$ & $S D$ & $M$ & $S D$ & & & & \\
\hline $\begin{array}{l}\text { Hygienic/clean } \\
\text { environment }\end{array}$ & 4.92 & .326 & 4.90 & .364 & 0.015 & 0.052 & 0.29 & $.77 \dagger$ \\
\hline $\begin{array}{l}\text { Provision of a quiet } \\
\text { room }\end{array}$ & 4.75 & .617 & 4.86 & .351 & -0.115 & 0.065 & -1.76 & $.08^{*}$ \\
\hline $\begin{array}{l}\text { Air quality and room } \\
\text { freshness }\end{array}$ & 4.74 & .509 & 4.70 & .580 & 0.041 & 0.082 & 0.49 & $.62 \dagger$ \\
\hline Adequate daylighting & 4.42 & .734 & 4.78 & .465 & -0.355 & 0.083 & -4.29 & $<.001 * *$ \\
\hline Temperature control & 4.25 & .865 & 4.66 & .519 & -0.410 & 0.094 & -4.34 & $<.001 * *$ \\
\hline Noise control & 4.06 & .991 & 4.68 & .471 & -0.623 & 0.095 & -6.54 & $<.001 * *$ \\
\hline $\begin{array}{l}\text { Adjusting lighting \& } \\
\text { operable windows }\end{array}$ & 4.03 & 1.01 & 4.76 & .555 & -0.727 & 0.105 & -6.94 & $<.001 * *$ \\
\hline $\begin{array}{l}\text { Coordinated natural } \\
\text { objects in the room }\end{array}$ & 2.53 & 1.04 & 2.88 & 1.00 & -0.347 & 0.163 & -2.13 & $.034 *$ \\
\hline
\end{tabular}

Note: $\nmid$ means are not significantly different at $p>0.05 ; *$ statistically significant at $p \leq 0.05$; ** statistically significant with Bonferroni adjustment at $p<(0.05 / 4=0.0125)$

\subsection{Users' perceptions of social comfort environment}

Independent samples t-tests were performed to compare the mean scores for women and midwives for the category of social comfort features. Among seven design factors, three analyses reveal there are no significant differences in the mean scores of women and midwives. In addition, perception of midwives was significantly higher than the postnatal women in three out of seven social comfort environment features with a statistically significant difference; open-bay ward for discussions, furniture and décor, and positive distractions. The scale mean for spatial connections (birth and postnatal ward) was the least ranked (see Table 7-4 and Figure 7-4).

For communal space and parental skills, $(F(260)=0.73, \mathrm{p}=0.39)$ there was no difference in the mean scores for women $(\mathrm{M}=3.96, S D=0.71)$ and midwives $(\mathrm{M}=3.86, S D=0.76, t(260)=$ $0.86, p=0.39$, two-tailed). Mean difference was established to be 0.10 and the $95 \%$ confidence interval CI was from -0.13 to 0.32 and the effect size, (Cohen $d=0.14$ ) was small. Next is Corridor and visitors' control, $(F(260)=0.046, p=0.83)$. Mean scores for the postnatal women $(\mathrm{M}=3.66$, $S D=0.71)$ and midwives $(\mathrm{M}=3.58, S D=0.70, t(260)=0.68, p=0.50$, two-tailed $)$. The effect size, (Cohen $d=0.1)$ was small. 


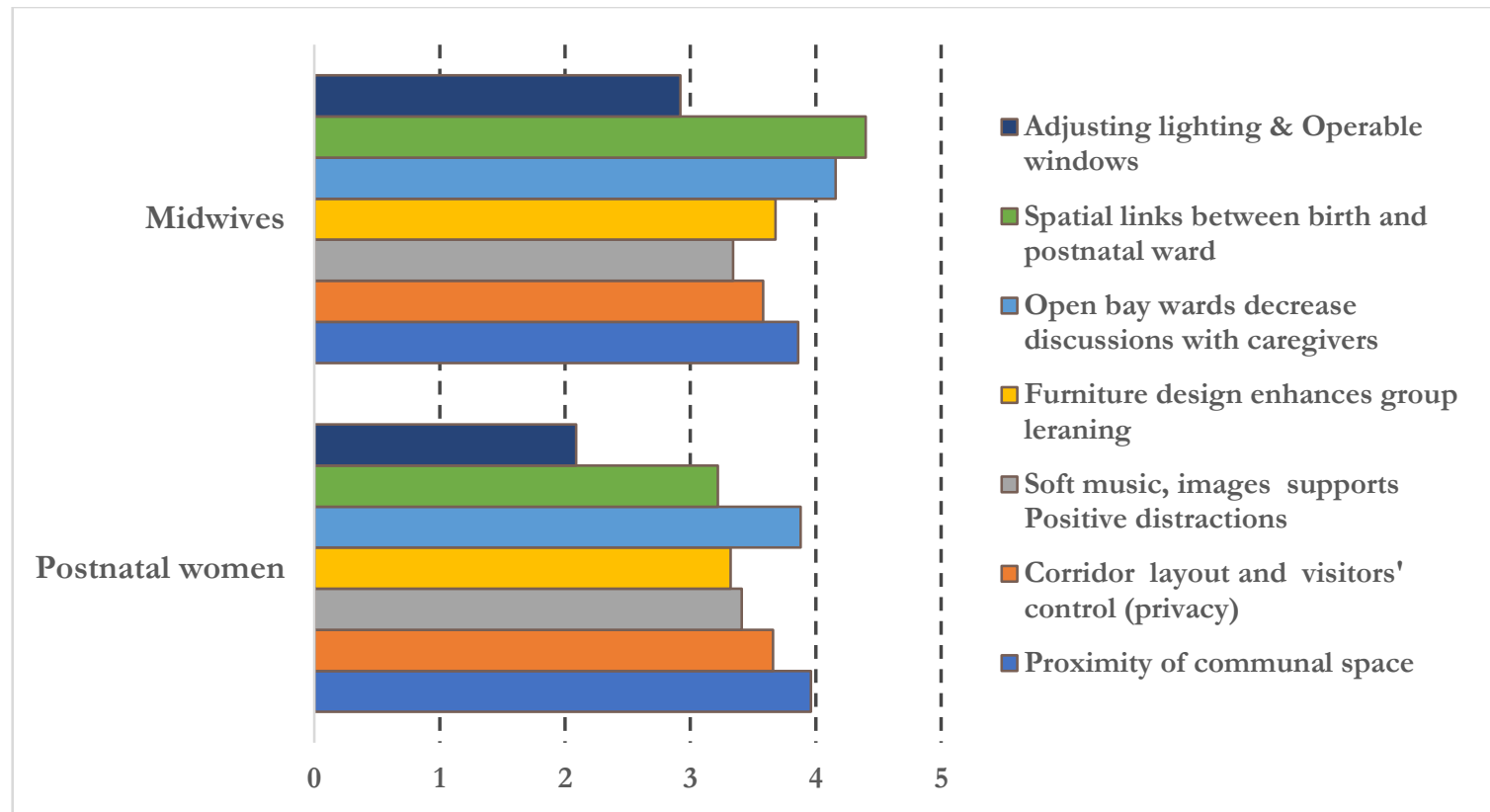

Figure 7-4: Respondents' perception on levels of importance regarding social comfort environment

Although there was a significant difference among respondents based upon the results of $\mathrm{F}$ test, in the case of preference for choice of room, $(F(260)=6.78, p=0.01)$, further analysis shows preference to choose the room was not significant with no difference in the mean scores for women $(\mathrm{M}=3.41, S D=1.28)$ and midwives $(\mathrm{M}=3.34, S D=1.06, t(86.2)=-0.40, p=0.69$, twotailed). The $95 \%$ confidence interval CI was from -0.28 to 0.42 and effect size, (Cohen's $d=0.06$ ) was very small as shown in Table 7-4

As Table 7-4 revealed, four analyses showed significant differences in the mean scores as follows: open-bay ward and discussions, $(F(260)=0.044, p=0.83)$ difference in the mean scores for women $(\mathrm{M}=3.22, S D=0.90)$ and midwives $(\mathrm{M}=4.40, S D=0.81, t(260)=-8.54, p<0.001$, two-tailed). The magnitude of the difference in the means was calculated to be -1.183 , and (CI: 1.46, -0.91). The difference in open ward represents a very large effect size, (Cohen $d=1.4$ ). Respondents' perceived the factor of open-bay ward would interfere with discussions especially regarding issues of privacy and confendiality of the postnatal women. However, midwives scores were significantly higher than the women. One probable reason could be that midwives perceived open-bay ward reduces their workload and they maybe having fewer staff in the postnatal ward. In addition, furniture and décor layouts, $(F(260)=0.948, p=0.33)$ difference in the mean scores for women $(\mathrm{M}=3.96, S D=0.71)$ and midwives $(\mathrm{M}=3.86, S D=0.76, t(260)=-2.57, p=0.01$, two-tailed). In terms of positive distractions, $(F(260)=0.214, p=.64)$. Difference in the mean scores for women $(M=3.32, S D=0.72)$ and midwives $(M=3.68, S D=0.74, t(260)=-3.15, p$ 
$=0.002$, two-tailed). The difference in positive distractions represents a medium effect size, (Cohen $d=0.5$ ). Positive distractions was slightly higher for midwives than postnatal women. One plausible reason for this occurrence would be that an aesthetically pleasant-looking environment is important for both the postnatal women and caregivers and could alleviate stress. Spatial connections between postnatal and birth rooms, $(F(260)=0.008, p=.93)$. Difference in the mean scores for women $(\mathrm{M}=2.09, S D=0.75)$ and midwives $(\mathrm{M}=2.92, S D=0.88, t(260)=-6.84, p$ $<0.001$, two-tailed). Mean difference was found to be -0.83 , and CI: -1.07 to -0.59 . The difference in spatial connections represents a very large effect size, (Cohen $d=1.02)$. Table 7-4 shows the full results of the independent samples $t$-tests for social comfort environment.

Table 7-4: Preference (Independent samples $t$-tests) of respondents on items in social comfort environment features comparing women and midwives responses

\begin{tabular}{|c|c|c|c|c|c|c|c|c|}
\hline \multirow[t]{2}{*}{ Questionnaire item } & \multicolumn{2}{|c|}{$\begin{array}{l}\text { Women } \\
(\mathrm{n}=212)\end{array}$} & \multicolumn{2}{|c|}{$\begin{array}{l}\text { Midwives } \\
(\mathrm{n}=50)\end{array}$} & \multirow{2}{*}{$\begin{array}{l}\text { Difference } \\
\text { between } \\
\text { the means }\end{array}$} & \multirow[t]{2}{*}{$\begin{array}{l}\text { Std. } \\
\text { error }\end{array}$} & \multirow[t]{2}{*}{$\begin{array}{l}\mathrm{T}- \\
\text { value }\end{array}$} & \multirow{2}{*}{$\begin{array}{l}p \text {-value } \\
\text { (Two- } \\
\text { tailed) }\end{array}$} \\
\hline & $M$ & $S D$ & $M$ & $S D$ & & & & \\
\hline $\begin{array}{l}\text { Proximity of } \\
\text { communal space }\end{array}$ & 3.96 & .711 & 3.86 & .756 & 0.098 & 0.111 & 0.86 & $.39 \dagger$ \\
\hline $\begin{array}{l}\text { Corridor and visitors' } \\
\text { control (privacy) }\end{array}$ & 3.66 & .708 & 3.58 & .702 & 0.076 & 0.111 & 0.68 & $.50 \dagger$ \\
\hline $\begin{array}{l}\text { Preference for room } \\
\text { of choice }\end{array}$ & 3.41 & 1.28 & 3.34 & 1.06 & 0.070 & 0.174 & 0.40 & $.69 \dagger$ \\
\hline $\begin{array}{l}\text { Positive distractions } \\
\text { (e.g. soft music, } \\
\text { images) }\end{array}$ & 3.32 & .723 & 3.68 & .741 & -0.283 & 0.114 & -3.15 & $.002^{* *}$ \\
\hline $\begin{array}{l}\text { Furniture and décor } \\
\text { layouts }\end{array}$ & 3.88 & .718 & 4.16 & .618 & -0.283 & 0.110 & -2.57 & $.01 *$ \\
\hline $\begin{array}{l}\text { Open ward and } \\
\text { discussions }\end{array}$ & 3.22 & .898 & 4.40 & .808 & -1.183 & 0.139 & -8.54 & $<.001 * *$ \\
\hline $\begin{array}{l}\text { Spatial connections } \\
\text { (birth and postnatal } \\
\text { rooms) }\end{array}$ & 2.09 & .746 & 2.92 & .877 & -0.83 & 0.121 & -6.84 & $<.001 * *$ \\
\hline
\end{tabular}

Note: $\nmid$ means are not significantly different at $p>0.05 ; *$ statistically significant at $p \leq 0.05$; ** statistically significant with Bonferroni adjustment at $p<(0.05 / 4=0.0125)$

\subsection{Exploratory factor analysis}

Because a few variances were found in the inferential statistics results among women and midwives, it was necessary to conduct an exploratory factor analysis (EFA) in order to extract the components that best fit the constructs of restoration and wellbeing. A principal component analysis (PCA) was performed on the 30-item questionnaire using orthogonal varimax rotation. A two-step approach was adopted. Firstly, all the questionnaire items were loaded to determine the Kaiser-Meyer-Olkin (KMO) (Kaiser, 1974) sampling adequacy for each item, which was 
established as $\mathrm{KMO}=0.747$. Additionally, Bartlett's test of sphericity showed statistically significant correlations among the items (Chi-square $=1915.056 ; p=0.001)$. The extraction values also ranged from 0.528 to 0.799 which were considered high. This initial process produced ten Factors that had eigenvalues greater than 1 . To reduce the large initial number of factors the PCA analysis was performed for the second time using a four-factor solution that yielded good combination of the items with $60.9 \%$ accounted for in the total variance. These four scales were categorised subsequently as interior restorative, environmental, spatial-sensitivity and maintenance.

Factors 1 and 2 had nine and five items while Factors 3 and 4 each had six items. The internal reliability measures also indicated that Factors 1 had a Cronbach's alpha coefficient of 0.818 and Factor 2 a value of 0.745 . Factors 3 and 4 had somewhat lower Cronbach's alpha values of 0.563 and 0.450. A study of female students' attitudes toward science utilised Cronbach's alpha coefficients above the 0.5 level (Buck, Cook, Quigley, Eastwood, \& Lucas, 2009) and in this study, items with a lower Cronbach's alpha of 0.450 or lower may be likely to be of lower priority to women's recovery experiences as well as less important to midwives in care provision. Table 7-5 below shows the factor loadings for each of the components including the percentage of the variance explained.

\subsection{Descriptive and inferential statistics for the PCA}

Following the newly developed scales, both descriptive and inferential statistics were performed in order to obtain the new mean PCA scores and to test which among the questionnaire items had significant diffrences between women and midwives or not.

Independent T-tests were also undertaken on the questionnaire items - the summated components that fully loaded together following the PCA analysis - which were grouped under interior restorative, environmental, spatial-sensitivity and maintenance. The results (see Tables 76 and 7-7) found a significant difference between postnatal women and midwives in the perception of several items.

Overall, 16 out of 25 design factors in the questionnaires showed statistically significant differences in the interior restorative component (six); environmental (five); three from spatial-sensitivity and lastly two from maintenance components. 
Table 7-4: Rotated component matrix of the questionnaire items (women and midwives)

\section{Component}

\begin{tabular}{|c|c|c|c|c|}
\hline & $\begin{array}{l}\text { Interior } \\
\text { restorati } \\
\text { ve }\end{array}$ & Environmental & Spatial & Maintenance \\
\hline $\begin{array}{l}\text { Decoration in the room (natural art and } \\
\text { images or paintings) }\end{array}$ & 0.784 & - & - & - \\
\hline $\begin{array}{l}\text { Pleasantness of rooms (natural things } \\
\text { such as aquariums, images etc.) }\end{array}$ & 0.752 & - & - & - \\
\hline Bright colours & 0.683 & - & - & - \\
\hline Positive distractions & 0.641 & - & - & - \\
\hline $\begin{array}{l}\text { Shapes and forms for postnatal room } \\
\text { design }\end{array}$ & 0.634 & - & - & - \\
\hline Provision of colours in rooms & 0.562 & - & - & - \\
\hline Furniture made of natural materials & 0.538 & - & - & - \\
\hline View to nature preference & 0.497 & - & - & - \\
\hline Furniture and décor layout & 0.418 & - & - & - \\
\hline Temperature control & - & 0.597 & - & - \\
\hline $\begin{array}{l}\text { Adequate illumination/operable } \\
\text { windows }\end{array}$ & - & 0.583 & - & - \\
\hline Adequate daylighting & - & 0.582 & - & - \\
\hline Noise control & - & 0.575 & - & - \\
\hline Accessibility to the outdoors & - & 0.489 & - & - \\
\hline Perception of quietness in rooms & - & - & 0.687 & - \\
\hline Single-bed room occupancy & - & - & 0.599 & - \\
\hline Design impact of space & - & - & 0.512 & - \\
\hline Provision of a restful room & - & - & 0.465 & - \\
\hline Natural daylight & - & - & 0.449 & - \\
\hline Provision of a quiet room & - & - & 0.402 & \\
\hline Hygiene/cleanliness & - & - & - & 0.734 \\
\hline Clean room & - & - & - & 0.671 \\
\hline Clinical room & - & - & - & 0.518 \\
\hline $\begin{array}{l}\text { Combination of clinical and familiar } \\
\text { home features }\end{array}$ & - & - & - & 0.493 \\
\hline Air quality and room freshness & - & - & - & 0.469 \\
\hline Percentage of explained variance (53.9) & 40.1 & 15.6 & 10.4 & 7.74 \\
\hline Cronbach's alpha & 0.818 & 0.745 & 0.563 & 0.450 \\
\hline
\end{tabular}

The inferential statistics results are useful in gaining an overview of the perceptions of users of the postnatal facilities of the design factors, however in phase two, these design factors are further explored in the focus groups to gain in-depth insights in those aspects where variances have been found in the perception of the design factors. 
Table 7-5: Independent samples T-tests results (women and midwives, $\mathrm{n}=262$ )

\section{Interior restorative}

\begin{tabular}{|c|c|c|c|c|c|}
\hline Questionnaire item & F-Value & $\mathrm{M}$ & $S D$ & $\mathrm{CI}$ & $P$-value \\
\hline $\begin{array}{l}\text { Decoration in natural art, images or } \\
\text { paintings }\end{array}$ & 0.95 & 3.08 & 1.08 & $-0.92,-0.27$ & $<0.001 *$ \\
\hline $\begin{array}{l}\text { Pleasantness of rooms (natural } \\
\text { things such as aquariums, images } \\
\text { etc.) }\end{array}$ & 1.58 & 2.60 & 1.04 & $-0.67,-0.03$ & $0.03 *$ \\
\hline Bright colours & 0.66 & 2.32 & 0.97 & $-0.96,-0.38$ & $<0.001 *$ \\
\hline Positive distractions & 0.21 & 3.39 & 0.74 & $-0.58,-0.13$ & 0.002 \\
\hline Spatial room design & 0.16 & 3.87 & 0.69 & $-0.15,0.28$ & 0.57 \\
\hline Provision of colours in rooms & 1.34 & 3.21 & 0.73 & $-0.51,-0.06$ & $0.01 *$ \\
\hline Room furniture in natural materials & 0.18 & 2.87 & 1.04 & $-0.19,0.46$ & 0.41 \\
\hline Views to nature preference & 2.87 & 3.96 & 0.71 & $-0.32,0.12$ & 0.38 \\
\hline $\begin{array}{l}\text { Furniture and décor in communal } \\
\text { space }\end{array}$ & 0.95 & 3.93 & 0.71 & $-0.50,-0.07$ & $0.01 *$ \\
\hline \multicolumn{6}{|c|}{ Environmental } \\
\hline & F-Value & $\mathrm{M}$ & $S D$ & $\mathrm{CI}$ & $P$-value \\
\hline Temperature control & 11.7 & 4.33 & 0.83 & $-0.66,-0.16$ & $<0.001^{*}$ \\
\hline $\begin{array}{l}\text { Adequate illumination and operable } \\
\text { windows }\end{array}$ & 12.8 & 3.81 & 1.07 & $-1.02,-0.44$ & $<0.001 *$ \\
\hline Adequate daylighting & 23.8 & 4.18 & 0.95 & $-0.57,-0.14$ & $<0.001^{*}$ \\
\hline Noise control & 13.6 & 4.49 & 0.70 & $-0.91,-0.34$ & $<0.001^{*}$ \\
\hline $\begin{array}{l}\text { Accessibility of rooms to the } \\
\text { outdoors }\end{array}$ & 19.9 & 4.17 & 0.98 & $-0.65,-0.16$ & $0.02 *$ \\
\hline
\end{tabular}

Note: $*$ Statistically significant at $p \leq 0.05 ; \mathrm{CI}=95 \%$ Confidence intervals (lower and upper bounds

The close agreements in some other areas also need to be accounted for by providing explanations to assist in placing the results in the overall perception and preferences of design features and building spaces for postnatal hospital design including optimisation for recovery experience and wellbeing.

Taken together, the four categories and the mean scores and selection process were used as the basis for further investigation in phase two of this research (see Figure 7-1). 
Table 7-6: Independent samples T-tests results (women and midwives $\mathrm{n}=262$ )

\begin{tabular}{|c|c|c|c|c|c|}
\hline \multirow[b]{2}{*}{ Questionnaire item } & \multicolumn{5}{|c|}{ Spatial-sensitivy } \\
\hline & F-Value & $\mathrm{M}$ & $S D$ & $\mathrm{CI}$ & $P$-value \\
\hline $\begin{array}{l}\text { Perception of quietness in } \\
\text { rooms }\end{array}$ & 3.38 & 4.73 & 0.61 & $-0.35,0.34$ & 0.10 \\
\hline Single-bed room occupancy & 135.20 & 4.75 & 0.60 & $0.52,0.85$ & $<0.001 *$ \\
\hline Design impact of space & 0.73 & 4.12 & 0.94 & $-0.19,0.39$ & 0.51 \\
\hline Provision of a restful room & 10.7 & 4.87 & 0.40 & $-0.01,0.24$ & 0.08 \\
\hline Natural daylight in the room & 0.30 & 4.48 & 0.60 & $-0.24,0.13$ & 0.58 \\
\hline \multirow[t]{3}{*}{ Provision of a quiet room } & 6.64 & 4.77 & 0.58 & $-0.24,0.01$ & 0.08 \\
\hline & \multicolumn{2}{|c|}{ Maintenance } & & & \\
\hline & F-Value & M & $S D$ & $\mathrm{CI}$ & $P$-value \\
\hline Hygienic and clean environment & 0.33 & 4.91 & 0.33 & $-0.09,0.12$ & 0.77 \\
\hline Provision of a clean room & 5.94 & 4.94 & 0.25 & $-0.10,0.08$ & 0.09 \\
\hline Clinical room & 5.18 & 2.25 & 1.14 & $0.21,0.80$ & $<0.001 *$ \\
\hline $\begin{array}{l}\text { Combination of clinical and } \\
\text { familiar home features }\end{array}$ & 2.56 & 3.60 & 1.09 & $-0.32,0.36$ & 0.91 \\
\hline Air quality and room freshness & 1.18 & 4.73 & 0.52 & $-0.12,0.20$ & 0.62 \\
\hline
\end{tabular}

Note: $*$ Statistically significant at $p \leq 0.05 ; \mathrm{CI}=95 \%$ Confidence intervals (lower and upper bounds 
Figure 7-5: Flow chart of selection of design factors and architectural features in phase two

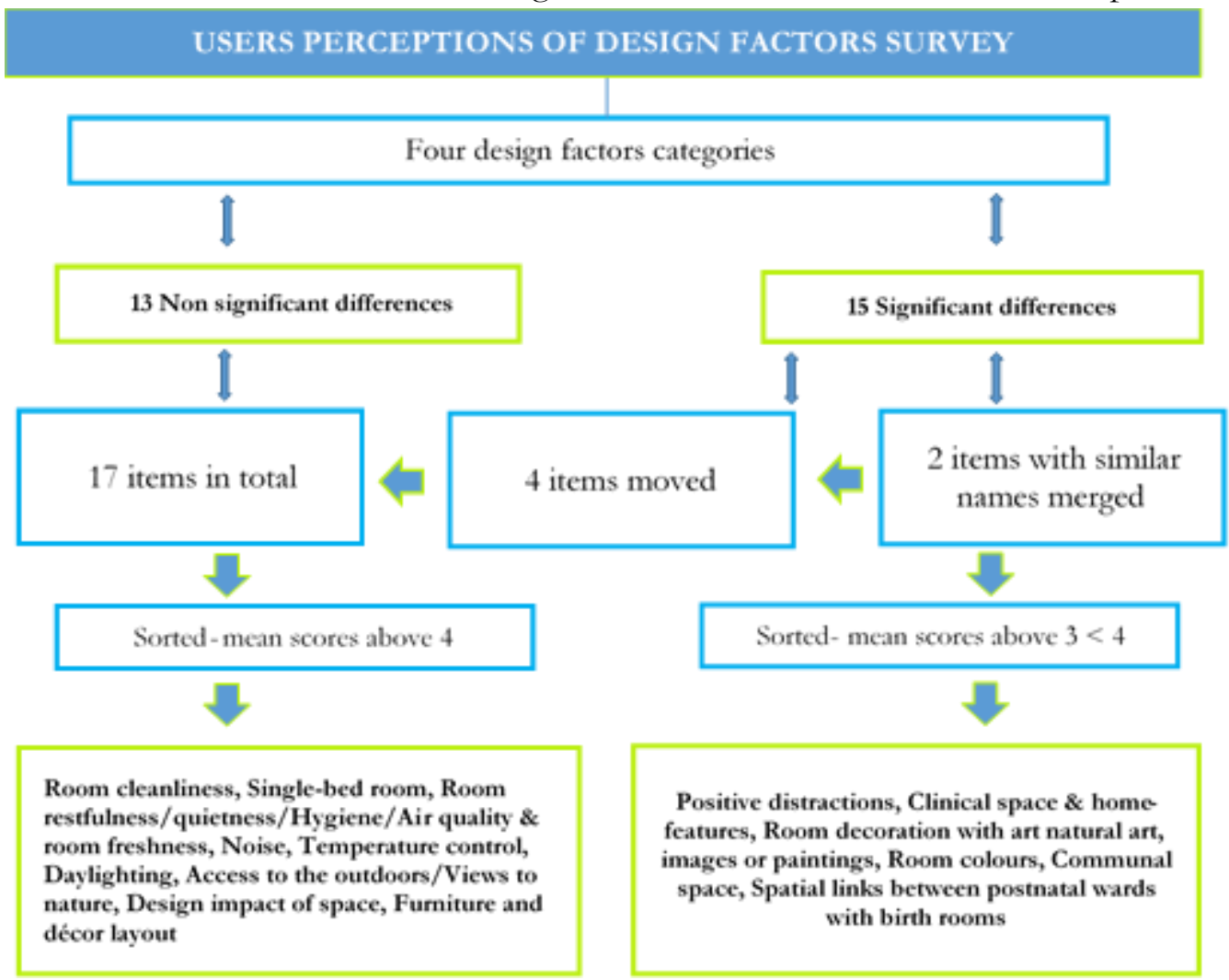

Note: Non-significant factors $=$ No statistically significant differences found in mean scores between women and midwives

\subsection{Conclusion}

The aim of this chapter was to identify the design factors and architectural characteristics influencing women's experiences of recovery in the immediate postnatal hospital stays. Inferential statistics, performed with Independent samples $t$-tests, established design features/factors which have a common set of perceptions and differences among the postnatal women and midwives. In addition, an exploratory factor analysis was also performed for the 25-item questionnaire to identify which combination of the design factors grouped into four categories: interior restorative, environmental, spatial-sensitivity and maintenance could be more influential in the women's recovery and wellbeing. In the next chapter, a discussion on the perceptions of design factors considered important by the respondents in the Phase 1 perception survey is presented. Finally, by relating the evidence found in the surveys to the existing evidence in the healthcare design literature and elsewhere, insights about the important environmental variables for postnatal environment can be established as the primary objectives of phase 1 of the current study. 


\section{CHAPTER 8}

\section{DISCUSSION ON PERCEPTION OF DESIGN FACTORS SURVEYS}

This chapter details the critical insights from the results of both descriptive and inferential analyses presented in Chapters 5, 6, and 7. This research found some similarities and differences in the responses of the participants. Independent samples $t$-tests have been performed to identify significant associations among the two user groups based on their responses, which largely could be attributed to demographic profile, age and education backgrounds of the participants(Mahmood \& Tayib, 2019). As is the case with other researchers (Alvaro et al., 2016; LaVela, Etingen, Hill, \& Miskevics, 2016; Schreuder et al., 2016) who have examined the physical environments and healthcare facility design on patient experience and wellbeing, this current research found many aspects of the physical design and environmental variables had stronger ratings influencing wellbeing and experience while a few others did not.

The discussion section is divided into three parts. Firstly, the results of design factors that found no significant differences among postnatal women and midwives are presented. Secondly, aspects with statistically significant differences between the two user groups are discussed. Thirdly, a few of the environmental indicators that had received lower mean scores but the differences were not statistically different are presented as other similarities in the results.

Table 8-1 gives a summary of the most important physical design factors and architectural indicators on the perception of design factors surveys. It should be noted that the findings are not limited to the ones in Table 8-1. The results listed in this table are only the most important findings. At the same time, a few overlapping findings are also repeated because some questions have been asked with different expressions. For example, hygiene and cleanliness tops the list of most important findings but the differences were not statistically different between the two groups possibly because hygiene and cleanliness are the best standard practices for healthcare institutions. While this is very important, the questions and findings regarding the kinds of space for the rest of women, views to nature and positive distractions are reprised to understand the importance of these design factors in women's recovery experience and contribution to quality of care. While all the results have been presented in previous chapters mentioned above, only the important 
Table 8-1: The summary of most important Physical Design Factors influencing the women's postnatal recovery experiences and wellbeing from perception of design surveys $(n=262)$

\begin{tabular}{|c|c|c|c|}
\hline Item & $\begin{array}{l}\text { Women } \\
\text { Mean }\end{array}$ & $\begin{array}{l}\text { Midwives } \\
\text { Mean }\end{array}$ & p-value \\
\hline Have a clean room & 4.93 & 4.98 & $0.09 *$ \\
\hline Having a hygienic/clean environment & 4.92 & 4.90 & 0.77 \\
\hline Have a restful room & 4.89 & 4.78 & $0.08 *$ \\
\hline Provision of a room that feels quiet & 4.75 & 4.86 & $0.08 *$ \\
\hline $\begin{array}{l}\text { A room or ward that is quiet is good for sleep and } \\
\text { generally helps in the recovery process }\end{array}$ & 4.75 & 4.60 & 0.11 \\
\hline Having good air quality and room freshness & 4.74 & 4.70 & 0.62 \\
\hline A postnatal room in which natural daylight can easily enter & 4.47 & 4.52 & 0.58 \\
\hline Having adequate daylighting in the room/ward & 4.42 & 4.78 & $<0.001 * *$ \\
\hline $\begin{array}{l}\text { Be in a room where the door is closed except when visitors } \\
\text { are allowed in (privacy) }\end{array}$ & 4.43 & 4.00 & $0.002 * *$ \\
\hline $\begin{array}{l}\text { A single room is better for a woman's postnatal recovery } \\
\text { care }\end{array}$ & 4.88 & 4.20 & $<0.001 * *$ \\
\hline $\begin{array}{l}\text { Openness of postnatal room (several beds) decreases the } \\
\text { opportunity for discussions }\end{array}$ & 3.22 & 4.40 & $<0.001 * *$ \\
\hline Being able to control the temperature & 4.25 & 4.66 & $<0.001 * *$ \\
\hline $\begin{array}{l}\text { The postnatal room generally has less impact on how } \\
\text { postnatal care is experienced by women (reverse coded) }\end{array}$ & 4.14 & 4.04 & 0.51 \\
\hline Noise control from ventilation and medical equipment & 4.06 & 4.68 & $<0.001 * *$ \\
\hline $\begin{array}{l}\text { Knowing you can open or close the windows/adequate } \\
\text { lighting and operable windows }\end{array}$ & 4.03 & 4.76 & $<0.001 * *$ \\
\hline $\begin{array}{l}\text { Communal space near postnatal room for counselling } \\
\text { purposes }\end{array}$ & 3.96 & 3.86 & 0.39 \\
\hline $\begin{array}{l}\text { Being cared for in a postnatal room that overlooks a } \\
\text { garden or pictures of nature on the walls }\end{array}$ & 3.94 & 4.04 & 0.39 \\
\hline Furniture and décor layouts & 3.88 & 4.17 & $0.01 *$ \\
\hline $\begin{array}{l}\text { Shapes and forms like those of natural objects can make } \\
\text { for a more beautiful postnatal room design }\end{array}$ & 3.88 & 3.82 & 0.57 \\
\hline Accessibility of room to experience outdoor surroundings & 3.74 & 4.14 & $0.002 * *$ \\
\hline $\begin{array}{l}\text { Entering from a passage to a postnatal ward is better for } \\
\text { privacy control (postnatal layout) }\end{array}$ & 3.66 & 3.58 & 0.50 \\
\hline Combination of clinical and familiar home features & 3.60 & 3.58 & 0.91 \\
\hline $\begin{array}{l}\text { Positive distractions such as soft music, natural images are } \\
\text { better for a woman's recovery }\end{array}$ & 3.32 & 3.68 & $0.002 * *$ \\
\hline Decorations with natural art, images or paintings & 2.96 & 3.56 & $<0.001 * *$ \\
\hline $\begin{array}{l}\text { Have/attend women in a room with furniture made of } \\
\text { natural materials }\end{array}$ & 2.90 & 2.76 & 0.41 \\
\hline Have opportunity to choose a room & 3.41 & 3.34 & 0.69 \\
\hline $\begin{array}{l}\text { A room with colours is better for recovery than a very } \\
\text { plain room (aesthetics) }\end{array}$ & 3.16 & 3.44 & $0.01 *$ \\
\hline $\begin{array}{l}\text { Natural things in the room such as aquariums, nature } \\
\text { sounds, and images (pleasantness of rooms) }\end{array}$ & 2.53 & 2.88 & $0.03 *$ \\
\hline Having bright colours in the room & 2.19 & 2.86 & $<0.001 * *$ \\
\hline
\end{tabular}

Note: Items were ranked on a scale from 1 to 5 , where 1 indicated not at all important, and 5 indicated very important; $\mathrm{M}=$ mean; $S D=$ standard deviation. $*$ Statistically significant at $p \leq 0.05$; ${ }^{*}$ statistically significant with Bonferroni adjustment at $p<(0.05 / 4=0.0125)$ 
findings are discussed. The overall findings are listed in the Tables of descriptive survey in Appendixes C-1 to C-5.

\subsection{The similarities: what design factors influence postnatal recovery experiences and wellbeing}

Independent samples $t$-tests revealed no significant differences between postnatal women and midwives on many design factors ( $p$ s $>0.05)$ influencing women's recovery experinces of hospital stay and their wellbeing. As can been seen in Tables 7-1 through 7-4, some of the design factors that found no significant differences between the two user groups cut across the four categories measured in this current research, ranging from perceptions of postnatal room features, interior environment, to social comfort environment. A detailed discussion on these design factors is presented in the order of their highest to lowest importance based on their mean scores recorded from both respondents to the questionnaires.

\subsubsection{Maintenance and cleanliness (attractiveness)}

Phase 1 findings reveal that cleanliness received the highest score for the perception of design factors surveys. According to Johansson, Oleni and Fridlund (2002) cleanliness (attractiveness) of healthcare physical environment constitutes an overall dimension in which patients' expectation of satisfaction resides and is a vital aspect of healthcare quality. It is well established in healthcare literature that physical facilities and ward environments are a component of patients' satisfaction and can serve as a means of evaluating healthcare services and quality (Stevens, Reininga, Boss, \& van Horn, 2006; Thomas et al., 1995).

Possibly one of the important questions to understand is the respondents' perceptions on cleanliness of the physical environment on wellbeing and caregiving. Although two questions were asked to the postnatal women and midwives under the categories: interior features and sensory environment, perception of cleanliness in the postnatal rooms was found to be generally superior compared to other design factors among the postnatal women and midwives. Both study groups have scored "room cleanliness" very positively; midwives $(M=4.98)$ and women $(M=4.93)$, with a significant positive preference $(p=0.09)$. In another question focused on "hygiene and cleanliness", the results reveal that the perceptions of the postnatal women and midwives are overwhelmingly similar, women $(\mathrm{M}=4.92)$ and midwives $(\mathrm{M}=4.90)$ although not statistically significant $(p=0.77)$. The differences can be that cleanliness was taken as given and evinces the primary responsibility of the healthcare organisation's goal. Despite the occurrence of differences, 
the fact of mean rankings which are very high show the importance of the design factor of hygienic/clean environment.

In mainstream healthcare service delivery, cleanliness/hygiene is an optimal clinical goal and has the potential to improve care quality, patients' wellbeing and staff satisfaction. Cleanliness is indirectly linked to sources of air flow and ventilation systems in hospitals which can be effective in the prevention of hospital-acquired infection in patient rooms (Anderson, Bonner, Scheifele, \& Schneider, 1985). There is well documented evidence that links healthcare settings as potential source of infection through contaminated hospital surfaces (Allen, 2014; Weber, Anderson, \& Rutala, 2013) making cleanliness an essential touchstone in healthcare environments generally and in postnatal settings particularly.

Although cleanliness is crucial for many hospital departments such as operating theatres and intensive care units where patients are at risk of infections largely from contamination of medical instruments (Smith et al., 2018), it is also very important in the birthing environment where women routinely present bodily exposure possibly in contact with hospital surfaces and equipment. Essentially, risk factors with contaminated or untidy environments may have psychological effects on the postnatal women and staff in the hospital settings. In one study, King, Noakes, Sleigh and Camargo-Valero (2013) used simulation to evaluate bioaerosol deposit in single and two-bed inpatient rooms and found small deposits of bioaerosol particles on surfaces in the two layouts, which suggests environmental contamination of hospital rooms and risk of contact transmission. While the study was not conducted in hospital settings, paying attention to cleanliness seems to be the reasons why this design factor was both ranked very highly, which reflects the sensitivity and priority accorded to cleanliness. Pragmatically, cleanliness is a multi-faceted attribute which embodies neatness of surroundings, and more generally the ward ambience. A recent study found cleanliness has been significantly related to pleasantness of room décor and room temperature (MacAllister et al., 2019) . Earlier, Swan, Richardson and Hutton (2003) in a study in environmental psychology found that patients in "appealing” rooms evaluated most dimensions of their hospital experience more favourably than did patients in "typical" rooms.

\subsubsection{Acoustic and restorative environment}

Joseph and Ulrich (2007) suggest that a well-designed acoustical environment is critical to addressing the negative impacts of noise for patients and staff outcomes respectively. It is reasonable that tranquility in the healthcare environment may extend beyond high noise levels to be inclusive of natural forms to provide tranquility and restorativeness in the environment. Watts, 
Khan and Pheasant (2016) claim that soundscapes comprising natural sounds - such as water sounds - and images of natural landscapes in the interior waiting room were associated with positive, quality of hospital experience and reduction in anxiety levels and could provide for restorative environment with tranquility An earlier study (Long \& Stover, 2014) showed that an absence of a quiet, peaceful environment is linked to noisy and busy hospital environments, with noise as the most singular factor contributing to sleep loss and restlessness in patients.

Pleasant natural soundscape could be used as a source of restorative experience in hospital ward settings. One study used a series of laboratory tests of different soundscapes and found that the soundscape interventions were effective in emotional-cognitive response with significant effect on the restoration dimension (Mackrill, Jennings, \& Cain, 2014). The researchers concluded that exploring different ways to improve sounds of hospitals is important rather than a focus on reduction in noise levels especially when natural sound is associated with a more positive relaxation of patients in healthcare settings. In another study by the same authors, it was found that soundscapes utilising natural sounds were much associated with terms including perception of sound in the physical environment, emotional feeling, restoration, behaviour of people towards sound and patient interaction that suggest soundscapes are a diverse mix of sound sources with perception dependent not only on specific sounds, but also the physical, temporal and social context in which they are heard and may offer potential for consideration as interventions in hospital wards (Mackrill, Cain, \& Jennings, 2013).

In the current research, the perception on provision of a quiet room was the most important design factor with the highest levels of agreement under perceptions of room features. Mean scores were higher for both groups with women responding more positively compared to their midwives counterparts $(M=4.75$ vs. 4.60). Although both groups scored this design factor above 4, no statistically significant difference was found $(p=0.11)$ in their perceptions of a quiet room. One probable explanation for this occurrence could be that both women and midwives had the notion that the hospital environment was busy and noisy and hence not conducive for rest. Lei et al. (2009) found differences in the perception of the ratings of sleep disturbing factors between the patients and nurses Frisk and Nordström (2003) also found, in similarity to the finding in the present study, that the patients' and nurses' perceptions of sleep in a 2- bedroom ICU environment were not different. Logically, the provision of a quiet room may be a salient contributor for enhanced sleep and recovery. The results from this study revealed the importance of a tranquil environment in the postnatal hospital environment. Birth routines and processes lend themselves to exhaustion, pain and stress requiring rest and sleep for physical recovery of women. 
Several scientific studies on the impact of noise and noise/sound levels are prevalent in intensive care units (Waye, Elmenhorst, Croy, \& Pedersen, 2013), in PICU (Kol et al., 2015) and in other hospital settings (Brown et al., 2015). Patients' sleep outcomes and wellbeing in the ICU are found to be disrupted by higher noise levels and are judged poorer in the ICU than at home (Freedman, Kotzer, \& Schwab, 1999). Similarly, another study also found that presence of the hospital personnel was strongly associated with noise levels in a hospital setting (Christensen, 2005; Tsiou et al., 2008).

Adverse effects of noise or unwanted sound can be disturbing for both patients and healthcare staff. Higher noise levels affect staff performance and researchers perceived this also to affect patients' wellbeing and recovery (Bayo, García, \& García, 1995). The negative long-term effect on nurses and other healthcare providers, in part, is why fostering healthy work and healing environments should be pursued (Choiniere, 2010). In addition, an increasing number of studies have documented higher noise levels in hospital rooms (Park et al., 2014; Waye et al., 2013), which clearly exceed the recommendations of $30 \mathrm{dBA}$ in patient ward rooms or $35 \mathrm{dBA}$ in treatment and observation rooms put forward by the World Health Organisation (Berglund et al., 1999) with consequences on patients' outcomes, staff and family (Busch-Vishniac et al., 2005).

The detrimental impacts of excessive noise have also been documented as affecting critically ill patients both in physical and psychological wellbeing (Kamdar et al., 2012), being disturbing to patients (Akansel \& Kaymakçi, 2008), as well as having negative health consequences on recovery, and harmful effects on staff (Biley, 1994). Although these studies were limited to mainly ICUs, one study found noisy construction work in the postnatal ward correlated with increased maternal anxiety in postpartum women hospitalised during construction compared to women hospitalised after construction (Mantz \& Britton, 2007), meaning that noise disturbances have negative consequences not only increasing anxiety but also hindering the rest and the wellbeing of the patients including the staff's health possibly worsening the healing of the patients (Loupa, 2020) and in this context, the recovery of the postnatal women, during hospital stays.

Because noise also affects healthcare providers and staff, implementing and observing a quiet time in healthcare environments has been advocated for a restful, healing environment with increased satisfaction for women and staff (Driver \& Colbert, 2010). In a non-randomised controlled trial involving a quiet time intervention in an acute care setting, Gardner et al. (2009, p. 781) demonstrated that differences in mean measured sound levels between the two environments was $10.3 \mathrm{dBA}$, indicative of a "perceived difference of 2 to 1 " meaning the experimental group had half the noise level of the control group, which correlated to average decibel level and number of 
patients awake and asleep. The study reinforces the notion that a scheduled quiet time has positive outcomes on patients including therapeutic benefit (Gardner et al., 2009).

In a three year multi-site study conducted in the UK focusing specifically on environmental factors in the maternity unit design, noise negatively affected women much more on the postnatal ward than on the labour ward, and was a contributory factor to stress of new mothers (Symon et al., 2008b). This finding is not novel; a previous study found that nearly a quarter of background noise affected women in the postnatal environment with an improvement shown for a quiet space (Redshaw, Rowe, Hockley, \& Brocklehurst, 2007). Conventional wisdom suggests expectations of a certain amount of noise in this environment such as crying babies and presence of other women in the postnatal wards. Pervasive crying of babies in the postnatal environment is a point mentioned in the discussion of focus group contributing to wakefulness, sleeplessness and sleep disruptions for the postnatal women. A detailed discussion is provided in Chapter 9.

The crying of babies largely differentiates postnatal hospital environments from the labour and delivery rooms necessitating an evidence-based design approach for improved restorative experience for women. The desire for a quiet room and quiet time has been found to assist recovery and wellbeing (Long \& Stover, 2014) and the lack of quietness and tranquility in postnatal wards signals the need for improvements (Adatia et al., 2014).

\subsubsection{Air quality - fresh air and ventilation}

Air quality and room freshness also recorded very positive responses after perceptions of "room quietness for recovery" and "provision of a room that feels quiet." The mean scores indicate that both the postnatal women and midwives did perceive that air quality and room freshness have influence on wellbeing and recovery in the postnatal environment. Comparatively, the mean score for women was (4.74) whereas for midwives it was (4.70). The results did not find statistically significant differences between the two groups on the factor of air quality and room freshness $(p=$ $0.62)$.

Provision and access to good quality fresh air in healthcare environments is an essential component for comfort and wellbeing of patients and medical staff. Fresh uncontaminated air is key to proper functioning of human vital organs. This requires that 'stale' indoor air is exchanged for 'fresh' outdoor air through ventilation, which can be natural or through mechanical systems. Ventilation may improve an individual's health in a number of ways: by providing air for breathing, removing and dissolving indoor pollutants, and by heating or cooling the indoor environment (Van den 
Berg, 2005). In healthcare environments, ventilation has been recognised as being crucial for both patients and staff as it provides thermal comfort and prevents harmful emissions of airborne pathogenic materials (De Giuli, Zecchin, Salmaso, Corain, \& De Carli, 2013).

Proper indoor comfort condition and indoor air quality (IAQ) are prerequisites for a safe and suitable environment for hospital operating theatres as they are criticial work areas which depend largely on heating, ventilating and air-conditioning (HVAC) installations for indoor environmental quality (IEQ) for both the patients and medical staff (Dascalaki, Lagoudi, Balaras, \& Gaglia, 2008; Ho, Rosario, \& Rahman, 2009). Indeed, Nimlyat and Kandar (2015) argued that an indoor environment with proper ventilation system can provide better air quality to building occupants. In this regard, understanding and "designing" for how fresh air and ventilation work in healthcare spaces could contribute to patient wellbeing.

In a multidisciplinary review, Li et al. (2007) demonstrated a correlation between ventilation, airmovements and transmission of infection. The presence of carbon monoxide in excess of $1500 \mathrm{ppm}$ in indoor air could be dangerous to the occupants (Ramaswamy, Al-Jahwari, \& Al-Rajhi, 2010) and central air conditioning is found ineffective in controlling of carbon monoxide in hospitals (Jung, Wu, Tseng, \& Su, 2015). This could be compensated for through a proper indoor and outdoor airflow exchange. Although Salonen et al. (2013) noted the wider acceptability of mechanical ventilation systems for the purpose of compliance with ventilation standards and guidelines (World Health Organisation, 2009), use of natural ventilation is also common to reduce the risk of spreading infections and to provide acceptable indoor environmental quality.

Baurès et al. (2018) measured chemical and microbiological contaminants in two French hospitals and claimed that indoor air contains a complex mixture of chemical, physical and microbiological compounds with certain concentrations of magnitude found in the two hospitals. The researchers suggested that environments equipped with central air-conditioning systems can effectively remove aerosol pollutants and maybe decrease indoor aerosols compared to non-central airconditioning system. Many hospital wards (with exceptions of Nightingale wards) particularly in many developed countries have been designed and built to be dependent on HVAC systems for the supply of indoor air, restricting window openings for both safety and energy saving strategies (Gilkeson, Camargo-Valero, Pickin, \& Noakes, 2013) while natural ventilation system operate peripherally.

Although natural ventilation is shown to be effective in open wards with an even spread of airborne infection risk in patient areas, however physical partitions can be effective measures to reduce 
infection risk for patients located behind partition walls (Gilkeson et al., 2013). Furthermore, poor air quality emerged as the chief source of environmental dissatisfaction during an assessment of indoor environmental quality thus, efforts should be drawn to the building envelope (De Giuli et al., 2013) and more importantly in the birth and postnatal environments, location of air delivery ducts should be given attention during design and construction for the prevention of cold stress in newborns (Stichler, 2007) as well as for new mothers.

\subsubsection{Visual environment and experience}

Other design factors that reveal no significant differences between the two groups of respondents $(p=>0.05)$ have been divided into three general classifications that include: visual environment and experience; physical layout of postnatal environment; and social comfort. This means that both respondent groups have similar perceptions on these aspects and they can be taken for levels of acceptance and importance in postnatal environments.

\subsubsection{Windows as medium/visual quality}

As articulated by Verderber "humans are biologically predisposed to crave visual information about their environmental surroundings"(Verderber, 1986, p. 452). Visual goals in the physical environment in general are better enhanced through the provision of windows for transmission of daylighting as opposed to roof lights or indirect daylight. The impact of windows and daylighting both on patients and medical staff is studied and supported in available literature in healthcare environments. Studies show that a close relationship exists between human behaviour and daylighting (Gharaveis, Shepley, \& Gaines, 2016). In their study, Gharaveis et al. (2016) found that roughly half of the sampled patients preferred to have the window next to the bed to allow for a direct view outside and they can change their position whenever they needed to view the outside. A previous study by Keep and colleagues collected information among two groups of patients who had a stay of minimum of 48 hours in the Intensive Therapy Unit; one group was in a unit without windows, and the second group in a unit with windows. Keep, James and Inman (1980) identified that subjects reported a less accurate memory of their stay and were less well orientated including reports of negative disorders in the windowless unit.

Natural daylight in the room revealed mean scores of 4 and above from the respondents particularly under the label of postnatal room features. It should be noted that this question was specifically focused on the patient's room windows. Although both the postnatal women ranked the postnatal room that lets in natural daylight higher $(M=4.47)$ and midwives $(M=4.52)$, no 
statistically significant differences between the two groups were found $(p=0.58$ ). The other question about daylight addressed daylight presence in the room and how it affected wellbeing, which revealed stastistically significant differences between the two groups. This is discussed in Section 8-2.

Verderber (1986) pointed out that patients have preferences for informative views of the external environment as opposed to insufficiently windowed areas with high sills and views blocked by nearby walls. This author concluded that lack of meaningful contact with the external surroundings by windows constitutes an undue architectural barrier (Verderber, 1986). One study also reported a possible micro-restorative impact of windows and daylight, which could contribute to positive health outcomes through lowering blood pressure and higher oxygen saturation (Zadeh, Shepley, Williams, \& Chung, 2014). However, the presence of a window in an intensive care unit did not lead to improved outcomes for critically ill patients, although light exposure was limited in the ICU (Wunsch et al., 2011). The size and location of windows were also found to have impact on stress levels and activities of patients (Gharaveis et al., 2016). Very recently, a study found consistency with existing evidence that impacts of windows and daylighting enhance nurses' perceptions of satisfaction and concluded that patient rooms and work stations are important areas to provide daylighting (Gharaveis, Yekita, \& Shamloo, 2020).

Furthermore, a study of end-user perceptions and experiences in a new ICU adopting evidencebased design, abundant natural daylight was found to be associated with a pleasant atmosphere in which the presence of many windows had positive outcomes on mood of users (Ferri, Zygun, Harrison, \& Stelfox, 2015).

Turning to the childbirth environment, diminished sunlight due to installation of exterior overhangs was associated with an increase in the rate of neonatal jaundice from $0.5 \%$ to $17 \%$ of infants in the obstetric ward of a tropical hospital. Recommendation of many windows and reduction in the width of roof overhangs were necessary to allow for passage of adequate indirect sunlight, as well as high intensity of illumination for prevention of jaundice (Barss \& Comfort, 1985). However, current advances in science and differing climates have limited the application of this architectural recommendation in NICUs as infants are sometimes exposed to a spectrum of light known as phototherapy for the treatment of jaundice (Maisels, 2001; Maisels \& McDonagh, 2008). A proper well-lit environment in the NICUs is crucial to fostering infant development and can be achieved with little economic investments (Rodríguez \& Pattini, 2016) including in the postnatal environment. 
Despite the reported benefits of daylighting, a few women in the surveyed hospital reported they did not know where they were and lacked the "connection to the outside world." A plausible explanation could be that those women had been cared for in the rooms either at a distance from the windows or were accommodated in postnatal rooms which obscure events on the outside. The finding in this research corresponds to a previous research review that found hospital occupants in general are commonly disorientated and lack visual-spatial sensory engagement with the external environment (Jiang \& Verderber, 2017).

\subsubsection{Experience of nature}

Views to nature is an environmental feature that reduces patient stress and improves other health and behavioural outcomes (Ulrich, 2001). Nature and natural views is a multidisciplinary area of research. One previous study found that the students who stayed in a dormitory with more natural views from their windows significantly performed better on attentional measures than the students whose dormitory windows have less natural views (Tennessen \& Cimprich, 1995). Furthermore, studies linking views of nature to reduced stress and pain in healthcare environments are widespread. This idea is based on the writing of Kaplan and Kaplan (1989) and others, Kaplan, Kaplan and Ryan (1998) have discussed the potential of nature for enhanced restorative environments that ameliorate stress by creating wellness in individuals. It is established that rooms that provide views of nature to the outside world have positive restorative impacts. One classic study positively associated post-surgical patients' exposure to nature with faster recovery time. Patients who had a view of nature through room windows were significantly discharged from hospitals faster and received fewer pain-reducing medications than patients who did not have a view of nature (Ulrich, 1984).

Mahmood and Tayib (2019) state that the design factor of vistas is regarded as having windows, seeing the sky and the grounds, and a calming and interesting exterior. In many respects, being admitted to hospitals can be stressful, boring and isolating for their occupants. The surroundings and quality of their aesthetics may serve as a resource for respite for occupants and as a means of coping against stress, pain and anxiety.

A sterile hospital which lacks visual qualities may increase stress and pain for postnatal women who may have minimal or no control over the physical environment. Although pain and stress are common with medical procedures such as surgery in patients (Kincey \& Saltmore, 1990), birth labour including caesarean section (C-section) births are pain factors for women (Green \& Baston, 2003; Kabakian-Khasholian, 2013; Kealy, Small, \& Liamputtong, 2010). 
The results from this study revealed that women and midwives expressed the importance of pleasant, natural environments in the questionnaire surveys. The mean scores for views of nature obtained from the postnatal women and midwives were high from both sets of respondents (3.94 vs 4.04). Independent samples $t$ - test comparison for perception on care in a room that overlooks a garden or nature view found no significant differences between both groups of respondents $(p$ $=0.39)$. The reason why women ranked view to nature lower was possibly because of their short hospital stay and it is likely there is a lack of pleasing surroundings/gardens, although they liked this attribute for their recovery experience, and also the fact that midwives rated this design feature higher was because they continuously work in this environment. Thus the postnatal environment has an important contribution to make in terms of comfortable working.

The results from the present study indicate participants' preferences for views to nature and nature images are important, and are considered to form part of a welcoming atmosphere. Although it differs in some respects to this current research in terms of demographic and setting, (Goto, Park, Tsunetsugu, Herrup, \& Miyazaki, 2013) found that exposure of an elderly population to landscaped areas such as natural gardens was associated with improved mood and experience and reduced signs of stress. In the childbirth environment context, Aburas et al. (2017) conducted an experiment using four experimental rooms with visual nature images and four other rooms without images. It was found that use of nature imagery had positive impacts and enhanced women's experience of labour.

Viewing nature or inhabiting natural surroundings can be traced to the ideas of evolutionary psychology and that innate responses influence preference for a particular setting (Nassauer, 1995). Patients fare better in healthcare environments that support them in and align with home-like normal lifestyle including establishments of homelike hospital birth rooms and surroundings mimicking domesticity and naturalness of non-medicalised settings (Douglas \& Douglas, 2005; Fannin, 2003) Such environments are those that incorporate gardens, trees and ornamental plants as well as nature images where views of the outside are impossible. This indicates a consideration of improvements in both the birth rooms and the postnatal spaces. A similar study on the effects of nature images found that some nature images had potential to reduce pain and may have more therapeutic potentials (Vincent et al., 2010). 


\subsubsection{Physical layout of postnatal environment}

\subsubsection{Perception of physical space}

Possibly one of the most important questions under the category of room preferences in the questionnaire survey was to ask respondents about what they thought the effect of a well-designed postnatal space could be on their perceptions of recovery experiences and wellbeing including quality of care in such an environment. However, maybe women are emotionally overwhelmed because of the birth of their babies, which may take the focus away from the design and impact of the postnatal space?

The birth process lends itself to emotions, moods changes and anxiety. Emotion is defined as an interrelated changes in affective mental, short-term states in response to an external or internal stimuli event as pertains to the organism (Scherer, 2005). The physical structure and design of childbirth environment have an increasingly influential role in women's birth experience and outcomes. Dazkir and Read (2012) argue that features of emotions manifest in the influence about how people react to, identify with, and avoid their near environments. Correspondingly, a pleasant postnatal setting could influence whether or not women affiliate more readily within the postnatal spaces that they judged comfortable rather than unfamiliar.

Critics hold that it is a waste of time and effort to ask unwell patients in hospital about their perceptions of architecture. This is not the case with women who give birth in hospitals as they are generally in good health. For clarification about unwell patients and healthy women, either with low-risk or high-risk pregnancies (see Coxon et al., 2014; Jackson, Dahlen, \& Schmied, 2012; Schott et al., 2014; Stark et al., 2016; Steel, Adams, Frawley, Broom, \& Sibbritt, 2015) although the two groups may have different perceptions of healthcare physical environments.

Perception of the design of space and its impact on care and wellbeing was positively ranked high by both groups. The mean score for received from women was $(M=4.14)$ and midwives $(M=$ 4.04) respectively. Although mean scores were 4 or higher for both groups, postnatal women tend to score the perception of space higher than midwives. The results reveal no significant differences $(p=0.51)$. This indicates that both the postnatal women and midwives have the convictions that well-designed spaces can serve as useful tools to lessen fears of birth and emotions.

There is similarity in the results between the postnatal women and midwives in the surveyed hospital about the impact of good design for their recovery experiences. Indeed, the question about the physical environment for wellbeing and recovery was asked at two separate instances to 
test whether or not women and midwives would see value in the design of space. In other instance, mean scores were high for women $(=4.46)$ and midwives $=4.72)$ respectively (see appendix C-3 and C-5 for detailed results).

Lawson, Phiri, and Wells-Thorpe's study on architectural environment and its effects on patients not only found that patients are sensitive to design but also can discriminate between poor and good environments (Lawson, Phiri, \& Wells-Thorpe, 2003). Other research has shown that poor design is related to adverse health outcomes such as anxiety and higher rates of delirium and hinders effectiveness of clinical intervention (Keep et al., 1980; Rubin et al., 1998). Architects and designers need to acknowledge the reported findings by creating physical surroundings that are "psychologically supportive" (Ruga, 1989) in promoting wellbeing thereby serving as a resource and resort to help patients to cope in times of stress (Ulrich, 1991). There is a study of postnatal women in which the physical environment and its organisation influenced their hospital experience and parenting goals (Gaboury et al., 2017).

Research has established that the qualities of the healthcare physical environment may reduce stress and promote patients' satisfaction and recovery (Ulrich et al., 2008). A study which examined the impact of architectural design on psychosocial wellbeing outcomes on patients and staff across a range of facilities comprising a newly built complex, an old rehabilitation facility, and a similar facility as a comparison, reported perceptions of positive mental health, mobility, and satisfaction were greatly enhanced at the new hospital compared to in the former facility (Alvaro et al., 2016).

However, Symon and colleagues note that women have both positive or negative responses when it comes to perceptions on design of the physical environment, with spaciousness strongly linked to satisfaction with care and vice versa (Symon, Paul, Butchart, Carr, \& Dugard, 2008a). A small group of researchers in childbirth environment discussed the relationship between space, neurobiology and midwifery work and argued that the space may trigger oxytocin release, which may have a role in quality service care by midwives (Hammond et al., 2013). Although focused on birth space, a few authors contend that physical space/environment while having the potential to contribute marginally to optimal experience particularly in large, obstetric-led units (OLUs) in hospitals, has less effect on clinical risk management (Seibold et al., 2010).

\subsubsection{Social comfort environment}

Social comfort plays a crucial role in the event of birth and in the immediate postnatal hospital stay. Ulrich (1991) argued that patients benefit from passive or frequent contact with family and 
friends who are helpful and resourceful to provide supports. Theory of supportive design (TSD) proposes that healthcare environments will overcome stress and promote wellness if they are designed to foster access to social support in conjunction with other factors such as a sense of control with physical-social surroundings and access to positive distractions (Ulrich, 1991). Indeed, researchers have recently tested this theory. Andrade et al. (2013) studied the effects of physical and social environment and found that satisfaction with care is mediated by perceptions of quality of physical and social environments. They found that for in-patients, it is perceptions of quality of the social environment that mediate the relationship between objective environmental quality and satisfaction whereas for out-patients it is perception of physical environment.

\subsubsection{Communal facilities}

The results in this research revealed that the design attribute of communal space near the postnatal unit received more positive responses for psychosocial wellbeing. Unsurprisingly, women have ranked this aspect of social environment higher than midwives $(\mathrm{M}=3.96$ vs. 3.86). Further analysis of the results performed with Independent samples $t$-tests revealed no statistically significant diffrences between the postnatal women and midwives $(p=0.39)$. This indicates that the opinions of the respondents showed communal space could play a supportive role in the recovery process during postnatal hospital stay. Possible explanation why postnatal women ranked this aspect of social comfort highly, above 3 (approx. 4) was the perceived opportunity that this social environment could facilitate interpersonal relationship with the caregivers, family and friends in a space that was not the birth and postnatal settings and also to the fact that midwives' work entails continuity of care, therefore the building (social environment) has an important role to play in terms of comfortable environment for the teaching and learning of parenting skills.

Despite the need for communal space, as reflected in the mean scores, few hospitals in New Zealand had provision for communal spaces in spite of indications that such a space could foster social interactions, enhance learning, and improve women's psychosocial wellbeing. As one woman states during focus group discussion:

Having a mix of shared spaces where other mums can give you a hug; where you can go and bring your baby;...I think the big thing about having a baby is that you need experience... with other people who have just had babies is a nice thing (FGD4W3). 
This result speaks to a need for birth supporters' space for and recovery care in the postnatal hospital environment with implications for design spaces, as reported in the preliminary findings of this research (Lawal \& Vale, 2018).

\subsubsection{Combination of clinical space and familiar home features}

Leydecker (2017) suggests that interior design with therapeutic intentions leads to a space which influences behaviour positively, inspiring mindsets of positive thought thereby fostering emotional wellbeing. Much earlier, Swan et al. (2003) found appealing rooms were correlated with the patients positive evaluation of hospital services. In another study, Quan et al. (2012) found that both staff and patients perceived the rooms with less-sophisticated medical equipment were significantly correlated with being more pleasant-looking than the control room.

The analyses of descriptive and inferential statistics revealed that both the postnatal women and midwives on average gave favourable answers to the factor of a "combination of clinical and familiar home features." Both mean scores recorded for postnatal women $(M=3.60)$ and midwives $(\mathrm{M}=3.58)$ were perceived higher $(3.60>3$ the midpoint of a 5 -point Likert scale). The results did not find statistically significant differences between the postnatal women and midives on the factor of a combination of clinical and familiar home features $(p=0.91)$. This indicates that the two groups shared similar views on perceptions on clinical environment postnatally for wellbeing. A study of impact of bed-side technology found that placing medical equipment out of sight assists wellbeing and stress reduction in patients (Tanja-Dijkstra, 2011).

With respect to relating room layout, fixtures and equipment to perceptions of comfortable work environment, Hammond et al. (2014) stressed that modern birth room design and by extension, postnatal spaces are fraught with congestion and clutter, which impact on the midwifery job, creating good ambience may foster a safer work environment However, the result in the current study also suggests caution in providing a completely de-medicalised childbirth environment. In addition, balance of normal home-like features with appropriate clinical settings would be a more realistic approach (Zwelling, 2008). Ideally, a safe environment was thought beneficial leading to a normal and positive birth experiences (Aune et al., 2015). A childbirth facility that promotes options for physiologic birth tailored to human centred design suggests opportunity for improvements for inpatient hospital birth (Breedlove \& Rathbun, 2019) particularly also in the postnatal environment. Additional research would be needed to build on this current result and explore the specific relationships between the combinations of clinical and familiar home features and their impacts on women's sense of safety and wellbeing. 


\subsubsection{Postnatal spatial unit (corridor design/privacy)}

MacAllister, Zimring and Ryherd (2016) and MacAllister et al. (2019) present four spatial layout measures to promote interactions between hospital staff and the patients. Average distance to the nurse station and location of bed are two key measures related to perception of patients' privacy. MacAllister et al. (2019) conclude there are links between spatial layout and patient satisfaction. For example, they reported that rooms with the head of the bed located at furthest distance from the room entry door (corridor) were regarded as more functional as they afforded more privacy compared with rooms with the head of beds at the door, and rooms farthest from the nurse station may be less noisy but indirectly afforded privacy. In a similar line of enquiry, Wang et al. (2013) found that a dedicated service corridor in the ICU turned out to be an effective architectural design strategy to lessen the impacts of noise on staff stress and promotes satisfaction.

In the current research, the results revealed that measures to promote privacy (corridor and visitors' control) were favourably ranked by the postnatal women and midwives. The results did not find a significant difference between women and midwives for the factor of a corridor for privacy $(p=$ 0.50). Interestingly, postnatal women were more positive towards this aspect of design factor than the midwives. ( $M=3.66$ vs 3.58). This may be due to the sensitive period of birth, in which women's needs for rest and privacy were crucial, and midwives' beliefs regarding privacy may be due to the busy and chaotic postnatal ward spaces and other medical staff needing to see women for clinical assessments of both the mothers and babies. Although the result was not found to have statistically significant relationships, the findings indicate both the postnatal women and midwives perceived that practical design measures that can potentially improve women's perception of privacy can facilitate rest after the birth.

\subsubsection{Building form/room layout}

Another important question that relates to the perception of architectural space is the notion of "shapes and forms" for postnatal room design, which reprised the earlier question about perception of the physical environment. As mentioned in Chapter 3, interior design elements and well-designed hospital birth and postnatal facilities could contribute to the perception of hominess (Shin et al., 2004). The impact of contour on aesthetic judgements was investigated focused on aspects of neurobiological responses by (Vartanian et al., 2013), and reported participants possibly judge spaces as beautiful if they were curvilinear rather than rectilinear and curvilinear contour (beauty) is strongly linked to a portion of the brain, with pleasantness playing a major role in it. A within-study experimental design by Pati, O’Boyle, Hou, Nanda and Ghamari (2016) that examines 
exposure to curves versus sharp contours in the built healthcare setting on the patterns of amygdala activation and behavioural response in healthy adults, concludes that objects' forms do have impact on fear response and human perception.

The results in the current research reveal that overall ranking scores are quite high for postnatal women with a mean score of $(=3.88)$ and a mean score of $(=3.82)$ to midwives. When comparing the responses of women and midwives on perceptions of shapes and forms for postnatal room design, no significant difference was found $(p=0.57)$. This means that both groups of respondents shared similar opinions regarding whether shapes of natural objects can make a better postnatal room design. The fact that the mean scores for the spatial design of postnatal environment averaged 3.85 for both respondent groups combined, almost reaching 4.00, indicates that both women and midwives considered it not only an important aspect in hospital birth and postnatal spaces/rooms, but the result is in line with friendliness, flexibility (design and spatial layout) and functionality (choice) which can foster wellbeing for the postnatal women and enhance effectiveness of midwives (Hammond et al., 2017).

Furthermore, the result resonates with the theoretical concept of "mindbodyspirit" architecture, described as a shift towards integration of biology, engineering and spirit/soul in creating birth space (Lepori et al., 2008). Earlier work by the first author, an Italian architect, concludes the design of birth rooms and in this present study, postnatal room design, needs to align with, and support the diversity of activities and interactions of each individual with the place (Lepori, 1994). Additionally, it is argued that design focused on "body" supports wide ranging activities of people in terms of their needs and desires (Franck \& Lepori, 2007, p. 66). Focused on design of birthing environment, Buckley (2015) said that low-stressed environments, differing from biomedical hospital settings, had potential to alleviate hormonal disruptions providing positive outcomes for women.

Birth is considered a natural physiologic process for a woman's body. In their book " $A$ Pattern Language," Alexander and colleagues state that:

It seems unlikely that any process which treats childbirth as a sickness could possibly be a healthy part of a healthy society (Alexander et al., 1977, p. 328).

Alexander and colleagues suggested a tailored design approach in creating spaces for childbirth as a natural and eventful moment for family to bond and where midwives can give utmost support (Alexander et al., 1977, p. 330). Therefore aspects of architecture, both in material and forms 
including shapes which mimic the flows and the ebbs of birth and postnatal feel of emotions could be useful in the creation of space for positive birth and restorative outcomes. An example of this is the reported use of Snoezelen and ambient rooms which found an array of benefits for women including positive distractions from pain, control of environment to women's preferences, and a feel of home-like atmosphere (Hauck et al., 2008; Hodnett et al., 2009).

The physical design of hospital birth environments has been routinely reported in childbirth literature as an aspect that is unsupportive of the birthing and postnatal process and has the potential to influence the quality of care and users' experiences. The physiology of birth including the sensitive time of the recovery phase in a biomedical environment could inform the design with introduction of more flexibility for women-centred spaces (Franck, 2007).

\subsection{Differences}

The analysis from the current study also revealed a number of interesting findings because they highlight some significant relationships among the two user groups suggesting that these important design factors might be contributing to recovery experiences of women and wellbeing. This section is a discussion of design factors found to have statistically significant differences.

\subsubsection{Ambient / sensory environment}

In Table 7-3 four of the five design factors of ambient environment had higher mean scores above 4. The only exception was presence of coordinated natural objects with mean scores below 3 discussed later in the section of interior appearance. These aspects include daylighting, noise control, temperature control, and adjusting lighting and operable windows. In terms of interior room comfort, the factor of acoustic comfort also revealed significant differences, a discussion of which is presented alongside other results of perceptions on sensory/ambient environment features. The segment presents a discussion of each of the highlighted ambient/sensory environment factors.

\subsubsection{Acoustic comfort (quiet rooms)}

Next to the factor of hygiene and cleanliness, higher mean scores were reported for the perceptions of having a room that is restful (room restfulness), with mean score for women $(\mathrm{M}=4.89)$ higher than that of midwives $(M=4.78)$. The results also revealed significant differences $(p=0.08)$ among the two classes of respondents. To understand further the importance and relevance of quiet space, a very similar question focused on provision of a room that feels quiet was asked to both 
respondents, a similar trend in the results was observed. The mean scores differed between the postnatal women $(M=4.75)$ compared to midwives $(M=4.86)$. On comparing, the results did show significant differences between the groups on the factor of room quietness $(p=0.08)$.

These higher mean responses indicate that the respondents perceived that room quietness was very important for the recovery process and wellbing particularly in the first few days after childbirth. Interestingly, the results reveal the same p-values in both instances while there is a reverse of order of responses by closely observing the two answers. The reasons why women gave very positive answers are possibly that they spend more hours in the rooms, particularly if they have had intervention births and the fact they need the rest and sleep Midwives are aware that rest was important in the immediate postnatal phase, however the ambience and quietness of the postnatal spaces are greater concerns for women than they are for midwives.

A restful room equates both to the type of a patient's room, and reduction of noise levels from other sources such as ventilation and medical equipment in a hospital environment. Research has established a strong link between noise and staff stress, job satisfaction and turnover intention (Applebaum, Fowler, Fiedler, Osinubi, \& Robson, 2010). A peaceful, noise-free healing environment has the potential for improving both patients' recovery and staff outcomes. Noise has been found to have negative physiological and psychological effects on patients wellbeing (Cunha \& Silva, 2015b). A significant body of research in the healthcare field has documented the impact of noise on patients and staff outcomes making it the most frequently studied environmental factor in health care (Ulrich et al., 2008).

Numerous studies on room quietness (absence/reduction of noise levels) have been linked to single-bed or multiple bed accommodation. A study on single rooms and patient choice found that improved sleep and peace/quiet constitute the first and second major reasons patients preferred single rooms (Jolley, 2005; Kirk, 2002). In addition, in a review of empirical sources that investigated the links between providing single-bed rooms and noise and quality of sleep, van de Glind et al. (2007) noted single-bed rooms have effect on reduction of noise and quality of sleep. Mixed results have been found with some studies of single-bed patient rooms (Howard, Gleeson, \& Higgins, 2014). While Williams and Gardiner (2015) conclude that both single and shared rooms guarantee social benefits without infringing on privacy and dignity, a recent study found substantial evidence that linked reduced noise/perceived sleep or "room quietness" to single-bed patient rooms (Taylor et al., 2018). The fact that room restfulness has been ranked highly by both women and midwives indicates the importance of rest, sleep, and physical restoration of women, therefore 
the postnatal building/environment has a crucial role to play in ensuring a more therapeutic environment. This links to the importance of acoustic comfort in healthcare settings.

\subsubsection{Adequate daylighting}

According to Aries, Aarts and van Hoof (2015), humans evolution regarding daylight and its lightdark cycle could be a factor that links daylight to human health. Daylight is a vital element for providing natural illumination for patients and staff members in healthcare environments. Potentially, daylight tends to be brighter and has a more balanced spectrum of colours than most of the artificial light sources (Van den Berg, 2005) and has the tendency to visually affect human behaviour (Dianat, Sedghi, Bagherzade, Jafarabadi, \& Stedmon, 2013). In addition, the amount of daylight is an important attribute influencing sensory comfort.(Schreuder et al., 2016).

The current results reveal that the perceptions on the ratings of adequate daylighting in the room/ward from both the postnatal women and midwives were generally high. On comparing the perceptions of postnatal women to midwives, a statistically significant difference was observed $(p$ $=<0.001)$. The mean score received from midwives $(M=4.78)$ was generally higher than from women $(M=4.42)$. Unexpectedly, the result on perception of adequate daylighting under the sensory environment category slightly differs from that of "natural daylight" under postnatal room features discussed in Section 8.1.5.1 above. In the current results, the mean score on perceptions of adequate daylight recorded for the postnatal women $(M=4.47 ; 95 \%)$ and midwives $(M=4.52$; $96 \%$ ) responses did not differ statistically between the two groups.

The reasons why the postnatal women gave positive responses could be related to the use of daylighting for circadian rythms especially for women who have had surgical births and are more likely to have longer hospital stays (Sahlin, Carlander-Klint, Hildingsson, \& Wiklund, 2013) and also the fact that midwives work in the environment, a well-lit environment is important in performance of their job. An empirical evidence-based report concludes that looking out at natural (day)light leads to mixed beneficial health outcomes, including improvements in mood, sleep, and fewer disruptions to circadian rest activity rhythms (Peter, Hunter, \& Howlett, 2003) and some similar instances could be attributed to the results in this present study. Additional research is needed about the relationships between adequate daylighting and recovery in future studies.

Interestingly, it may be that because the questions regarding daylighting focused on the windows (medium) first and the intensity of daylight in the room second could be the reason why the responses on both questions differed even although the both questions were about daylighting. It 
should be noted that daylight in the survey focused solely on natural daylight as opposed to artificial lighting. To understand better the impacts of daylight on recovery and postnatal experience of hospital stay, the trend in the results is probed in detail in the focus group discussions (see chapter 9).

In terms of satisfaction with physical environment for patients and their family, for patients reported improvement of the ward was associated with room layout, natural light and appealing environment whereas for family, quiet space and natural light were found beneficial as a healing environment (Kotzer et al., 2011). Given these findings, close attention is required for design aspects of natural/adequate lighting in the postnatal spaces.

\subsubsection{Adjusting lighting and operable windows}

In addition, a question about whether perception of adjusting lighting and operable windows to feel comfortable was important for wellbeing was asked to the respondents. The mean scores on the factor of adjusting lighting and operable windows were significantly positively high for midwives and women (4.76 vs. 4.03), above 4 , indicative of their value and importance. Mean score on adjusting lighting and operable windows recorded for midwives was 4.76, representing a $27 \%$ increase from the mean score recorded for women $(M=4.03)$. The results showed significant differences between the ranking of the perception of adjusting lighting and operable windows ( $p$ $=<0.001)$. This finding confirms a more recent study on the effect of adjustable bedroom lighting in the maternity ward (Canazei et al., 2019). Although Symon et al. (2008b) found that most women said they are able to control lighting in their rooms, few knew if they could adjust temperature or ventilation, which could be achieved by opening a window. It should be noted that the survey questionnaires did not ask women and midwives if they could adjust lighting or open/close the windows in their rooms, however, the results indicate that postnatal women especially would value hospital environments that allow greater empowerment and control as important aspect of wellbeing.

\subsubsection{Temperature control}

Desire to maintain control in the healthcare physical environment is considerably important for patients' wellbeing. Environmental factors such as light, sound and temperature in hospital settings are generally above the recommended values (Oliveira et al., 2015; Pugh, Jones, \& Griffiths, 2007), suggesting measures for creating a comfortable thermal environment for patients and assisting in their healing (Hwang, Lin, Cheng, \& Chien, 2007). Linder and Christian (2011) investigating 
temperature in a peadiatric ward found that the average nighttime temperature was close to the upper threshold for recommended room temperature for healthy sleep although no fluctuations were observed.

The results from the perceptions on temperature control revealed that midwives scored the factor of temperature control $(M=4.66)$ higher than did the postnatal women $(M=4.25)$. The results also showed a statistically significant difference between the ratings of this aspect $(\mathrm{p}=<0.001)$. A study of design factors in the physical environment of four hospital locations, (Schreuder et al., 2016) found that temperature is considered more important than scent and fresh air in the regression analysis; but also less important in other analysis. The fact that both women and midwives positively ranked temperature control highly indicates that regulating temperature (hot or cold) is important to the wellbeing of new mothers and babies.

\subsubsection{Noise control}

Research has demonstrated the detrimental effects of noise on patients and staff in healthcare environments (see Chapter 2 for details). Hospital environment is generally a place where a patient/client finds it difficult to maintain control once admitted. Studies in the childbirth environment have linked noise with recovery of new mothers. For examples, noise on a maternity ward (Adatia et al., 2014), postpartum women's perception of hospital environment in which noise was a barrier for sleeping (Martell, 2003), Symon's maternity unit design (Symon et al., 2008b) and Doering (2013) who concluded that modifications to physical and social sleep environment have the potential to improve sleep for postnatal women.

The results on perception of noise control reveal that the responses obtained from the postnatal women and midwives were positive. The strongest mean was reported for midwives who thought to control noise from medical equipment was very important $(M=4.68)$. Comparatively, women also felt strongly about the perception of noise control with a positive mean score of $(M=4.06)$. When comparing the responses of women and midwives on perceptions of noise control, significant differences was found $(p<0.001)$. A possible explanation why women's responses were lower could be due to their not being able to control noise even if they had wanted to do so. Likewise, midwives' very positive rating of noise control may be related to their concerns about avoidance of errors as a result of lack of concentration.

In terms of all environmental control related questions, there are identifiable trends in the very positive ratings (mostly $>4$ on the five-point Likert scale) of these design factors, suggesting that 
both the postnatal women and midwives showed a strong emotional commitment for personal control in the hospital birth environment. Unfortunately, the design factors of noise, temperature, lighting controls are all personal control-related to which research has highlighted that the hospital environment is a potential impediment (Devlin \& Arneill, 2003). The results in this present research align with Symon et al. (2008b) who found that midwives complained more about a perceived lack of control over environmental factors, a finding that suggests why midwives gave more positive answers regarding the factor of noise control factor in this study. Kerfoot and Neumann (1992) argued that patients, which also include women giving birth, should feel a sense of control over the environment that relates to lighting, temperature and personal belongings. Efforts need to address how physical healthcare environments can support personal control and autonomy over the environment. Additional research is needed to determine whether and in what ways does having control over environmental factors, especially noise contributes to recovery experiences of postnatal women while in hospitalisation.

\subsubsection{Perception of space (room-type)}

Space affects patients' experiences in multiple dimensions: through altered sensory awareness material and social aspects (Annemans et al., 2018). O'Connor et al. (2012) argue that the environment of care is fundamental to and could affect care especially when individual privacy or a sense of personal identity while receiving care is compromised. A poorly designed health building has been identified as a contributory factor that limits families' access due to insufficient space in patient rooms and compromises confidentiality (Schweitzer et al., 2004). How a user/patient perceives their hospital room can have impact on experience of care and wellbeing.

\subsubsection{Single versus multiple beds accommodation}

Choice of hospital bed accommodation plays a crucial in the care and wellbeing of patients and their families. As noted in Chapter 2, single-bed accommodation has several benefits such as increased privacy and safety and satisfaction (Young \& Yarandipour, 2007), reduction of noise levels and medical errors (van de Glind et al., 2007), and permitting increased number of family members for emotional and psychological support (Choi \& Bosch, 2013).In the childbirth settings, the Labour, Delivery, Recovery and Postpartum (LDRP) version of single-bed room, rather than the Labour, Delivery and Recovery (LDR) room facilitates the mother and infant bonding as they need not change rooms after the birth (Breedlove \& Rathbun, 2019). 
Significant differences were found between the postnatal women and midwives on the type of room accommodation. The results in this current study indicate that an overwhelmingly $98.6 \%$ (n $=212)$ of postnatal women $(\mathrm{M}=4.88)$ responded significantly more positively than did the midwives $74 \%(n=50)$ regarding the perception on single-bed rooms for enhanced recovery. The results also revealed a statistically significant difference $(\mathrm{P}=<0.001)$ between the study groups. This indicates that the respondents strongly considered a single room desirable for women's recovery. This is an important result in the sense that single- patient accommodation has been trending in hospital design across the world (Lawson et al., 2003; Verderber \& Todd, 2012). Women who were allocated to single-occupancy rooms reported this to be important during their postnatal hospital stays. Empirical findings have also shown that single private rooms are linked to a patient's psychological and physical recovery and the environment (Stall, 2012). A review focused on benefits from single rooms found them linked to patient satisfaction, noise reduction and quality of sleep as well as privacy and dignity (van de Glind et al., 2007). Women's preference for single rooms in this study is expressed in the opportunity it affords them to bond with their baby, partners and family members.

Midwives differing in their response, may be due to desires for an inclusive environment for groupcaring rather than caring for an individual in their own space, which impacts on their workload and more importantly, may be due to inadequate numbers of single-bed rooms available to all women in the postnatal ward. Donetto, Penfold, Anderson, Robert and Maben (2017) affirm that all-single room accommodation may offer staff an impoverished experience of caring for patients and reduction of inter-collegiate experience. These concerns have been similarly expressed in previous research about nursing staff attitudes toward a single-bed room as it is more challenging and stressful in performing their duties (Harris, Shepley, White, Kolberg, \& Harrell, 2006). However, there exists evidence that single patient rooms could reduce staff workload from the standpoints of task and physical movement due to reduction in patient transfers (Young \& Yarandipour, 2007). As Williams and Gardiner (2015) explain, sometimes patients preferred to stay in multi-bed/shared rooms rather than in single-bed rooms for company with others and fear of isolation.

Bobrow and Thomas (2008) argue that single-patent rooms are cost effective in the long run particularly in reducing patient transfers/moves. A more recent study found that single rooms compared to shared rooms enhanced family-centered care (Curtis \& Northcott, 2017), which aligns with past research which found single-bed rooms are more advantageous than multi-bed rooms in healthcare environments (Chaudhury et al., 2005). The most recent review on this topic concluded 
with the same finding, emphasising considerations of the patient population in decisions regarding the implementation of single patient rooms (Taylor et al., 2018).

Although debates about single-bed versus multi-bed rooms are far from being conclusive, there are indications they foster socialization and encouragement of patient-family transactions (Verderber \& Todd, 2012). Respecting the postnatal phase as both a private time and a vulnerable time may require having own private space for emotional and psychological wellbeing.

Another factor that found significant differences is the perceptions of open-bay ward (shared rooms) for discussions with nurses/midwives. Midwives gave more positive responses on the aspect of the open bay ward for discussions compared to postnatal women. Midwives thought they were more comfortable for caring for the women in a postnatal ward with several beds while women differed on this. The mean scores for both groups were above 3 and higher $(\mathrm{M}=3.22 \mathrm{vs}$ 4.40) for the postnatal women and midwives respectively, which revealed a statistically significant difference between the two groups $(p=<0.001)$. One probable reason why postnatal women scored open bay ward quite low could be that open-bay wards/rooms may affect privacy and confidentiality more than if they were cared for in single-bed rooms. For midwives, it might be more about reduction of their workloads and shortage of staff in the postnatal environment..

Being attended in an open-bay ward possibly impacts on interactions in the postnatal hospital environment. Postnatal ward routine, birth volumes and policies regarding hospital visitations are factors that possibly have a role to play in interactions in the childbirth environments. In fact Wiegers (2006) argued that the postnatal phase is generally an avenue for interactions in which nursing staff support the mother and couple in establishing a new routine and boost their confidence in parenting, which will be not be achieved in a short hospital stay or a stay in a certain type of accommodation.. In New Zealand, one on-line survey of consumers asking women to evaluate their postnatal care experience in hospitals found that a shared room was inappropriate for a new mother's needs (Maternity Services Consumer Council, 2018) In brief, both results on perceptions of the types of hospital bed-room occupancy indicate that single-bed rooms are important and could foster restoration and recovery experiences of women and their wellbeing.

\subsubsection{Privacy control}

Ensuring privacy of patients is an important consideration in healthcare service provision. Being admitted to or going into hospital to give birth generally involves some certain levels of conversation, which must be respected and protected during care. Mendelson and Wolf (2017) 
stress that the substance of conversations between patient and doctor in the context of a therapeutic relationship is inherently personal. The hospital physical layout, enclosures and elements such as doors are vital tools to enhancing privacy and confidentiality of patients. It can be reasoned that hospital rooms are important areas for health care and can impact patient wellbeing. Olsen and Sabin (2003) conclude that the physical layout, with small size, creates congestion and is a great impediment for privacy of the patients. In addition, Karro et al. (2005) found frequent breaches of patient privacy in an Emergency Department (ED), as many of the patients agreed to hearing other patients' conversations rather than perceiving they were being overheard. The situation was attributed to the types of cubicles/spaces patients were admitted to and length of hospital stay.

The results from the present study revealed that the postnatal women perceived that they were significantly more likely to have privacy in a room where the door is closed ( $M=4.43,87.3 \%)$ compared with midwives $(M=4.00,64 \%)$. The mean score for women (4.43), represented a 57\% increase from the mean score of midwives (4.00). The results found statistically significant differences on the factor of "door and privacy" $(P=0.002)$, which indicates the perceived importance of this design aspect. As previously noted, patients' privacy and dignity have been closely associated with the types of accommodation. Privacy was a major concern for staff in healthcare departments and units has been reported in a previous study (De Giuli et al., 2013), a finding that is consistent with midwives' in this study although a relatively positive score of ( = 4.00 on a scale of $1-5$, where $1=$ not at all important and $5=$ very important ) may be due to the need to maintain an awareness of women in their rooms and to encourage interactions with other women. Perhaps not suprisingly, women responded significantly more positively on perception of privacy indicating the ubiquitous importance of rest for physical restoration following the birth. A common finding in the healthcare literature focused on privacy and confidentiality of patients is that it is strongly linked to single-bed accommodation (Ulrich et al., 2004). However, careful planning can be used to leverage the social benefits of shared rooms without compromising patients' privacy and dignity (Williams \& Gardiner, 2015).

\subsubsection{Quality of outdoors and appearance}

\subsubsection{Experiencing the outdoors}

Spending time outdoors in hospital environments is a means by which the patient can take a break from the sick bed for recovery opportunities while engaging in positive exercise and psychosocial benefits of being with other people. There is robust evidence-based support that outdoor spaces 
in the form of gardens promote health outcomes (Ulrich, 1999). Mean scores differed between women and midwives on the aspect of walking/access to experience the outdoors. The mean score recorded for midwives was $(M=4.14)$ while for the women it was $(M=3.74)$, representing a $60 \%$ increase from the mean score recorded for women (3.74). The results showed significant differences for the perception of ratings of access to the outdoors $(p=0.002)$. The results revealed that a trend of interests to engage with outdoor environments, however, it is limited by a number of factors. First, the spatial layouts and location of postnatal spaces can be a barrier to accessible outdoor space. Low accessibility of a rooftop garden was reported for patients and staff in contrast to designers and administrative staff who reported higher accessibility (Davis, 2011). In addition, a study which addressed barriers to garden visitation in children's hospitals found that limited seating provision, shading and greenery are frequently mentioned factors that discourage garden visitation in healthcare settings (Pasha, 2013). In the surveyed hospital, both the birth delivery suites and the postnatal wards are situated on upper levels, which makes outdoor accessibility difficult if not impossible. Another factor is the overall lack of hospital outdoor spaces which may account for the result. The result suggests a need to consider in planning accessibility to facilities in a hospital's birth and postnatal environments especially for more effective outdoor space use.

Freedom to move around in hospital birth environments has been identified as problematic (Lepori, 1994). The results in this current study are consistent with an earlier study that reported access to outside space provides a link to a wider world and for providing people with a safe place to walk, reflect, talk to other patients or be alone (Walsh \& Boyle, 2009). Kerfoot and Neumann (1992) argued that the lure of an interesting view in the hall (outside of a room) can be a motivational factor for patients, and in this context, women to ambulate. Given the results, the generally higher mean scores on their own are indications of the levels of acceptance.

The feasibility of contact to the outdoor spaces during the postnatal period hinges on reduced mobility of women after the birth, which depends on the nature of each woman's birth and parity level and physical recovery after C-section (Kealy et al., 2010), going outdoors may be the last thing as they need to spend days in bed for the recovery process. Evidence from this current study suggests that first time mothers often find that the whole experience of giving birth and the new challenge of tending a newborn baby mean that they do not think of accessing the outdoors. On the contrary, evidence indicates that midwives prioritise women going to the outdoors as an attestation of independence while hospitalised to help them at home, although patients and family members frequently were not aware of existence of gardens in the hospital environment (Whitehouse et al., 2001). 


\subsubsection{Interior room aesthetics}

\subsubsection{Positive distractions}

Swan et al. (2003) found that patients who stayed in appealing rooms reported positive evaluation of their hospital experience compared to patients in typical hospital rooms. The investigators state appealing rooms are well-decorated hotel-like rooms with wood furniture, art, carpeted floors and vinyl tiles in bathrooms. A visit to a hospital requires an amount of wait times and concerns for patients and their family members. Nanda et al. (2012) investigating visual art on behaviour of patients in an emergency department (ED) conclude that visual art has positive impacts on the waiting experience of patients. In addition, Suess and Mody (2017) conclude that infusion of the hospitality model into healthcare spaces emphasising the "patient as customer" is important for enhancing the patient hospital experience.

Regarding positive distractions, (soft music and images), researchers have established a link between specific audio alongside visual and natural images contributing to patients' outcomes and a potential consideration for healing environment (Diette et al., 2003; Laursen et al., 2014). Thorgaard, Ertmann, Hansen, Noerregaard and Spanggaard (2005) found that a designed music environment correlated with a positive attitude, relaxation and improved satisfaction in the stay period. A detailed discussion of positive distractions on patient and staff wellbeing is discussed in chapter 2. Soft music, perhaps personalised to each woman, can be more relaxing and increase positive mood of a new mother in the postnatal phase. In this research, the results of the perceptions on positive distractions revealed very moderate responses from the postnatal women and midwives. On comparing the postnatal women and the midwives the results showed significant differences on perceptions of positive distractions $(p=0.002)$ between the postnatal women $(\mathrm{M}=3.32)$ and midwives $(\mathrm{M}=3.68)$ with midwives rating positive distractions more than women.

The moderate ratings of this design attribute by women and midwives could be related to possibly a lack of distraction elements in the postnatal ward, which are more likely to assist in improving mood and satisfaction of hospital stay, whereas midwives possibly preferred a comfortable environment since most times they work indoors. Other research that focused on distraction in healthcare unit settings found that positive distraction such as window views of pleasant outside, soothing artwork and calming music could be introduced into the NICU setting (Shepley, 2006) and this may apply or include the hospital postnatal wards. 


\subsubsection{Decorations in the room}

Another design feature under the banner of positive distractions is "decorations in the room" focused on natural art, images and paintings. Of interest is the consistent pattern in the present results under the interior environment with that of positive distractions (social comfort environment), which revealed that midwives continue to perceive these elements of interior environment relatively higher than do women. The mean score from midwives was $(M=3.56)$, representing a $40 \%$ increase from the mean score belonging to $(M=2.96)$ women. The results also showed statistically significant differences between both groups on the ratings of decorations with natural art, images and paintings $(p=<0.001)$, meaning that both groups did not think similarly about decorations in the room. A qualitative study of next of kin in the ICUs (Olausson, Ekebergh, \& Lindahl, 2012) affirmed that the design, interiors and furnishings in the patient room are crucial for the next of kin's experiences and impact on wellbeing. The researchers suggested that simple design interventions can make the patient room a more healing and recovery environments.

The reasons for this occurrence appear not different from the ones mentioned above, meaning that even though postnatal women preferred a conducive atmosphere (interior and exterior), there is a belief about hospital interiors having a certain standard for the interior environment. In addition, the short length of stay by women in hospitalisation following the birth, could create limited avenues to explore and experience the interiors.

\subsubsection{Coordinated natural objects (pleasantness) in the room}

In terms of pleasantness in the room (things such as aquariums, nature sounds of birds, and nature images, see Table 7-3), mean scores differed between the postnatal women and midwives and was the least ranked under the sensory environment category. Just as other questions focused on distraction, nearly $50 \%$ of women $(n=212)$ perceived coordinated natural objects in the room as not important compared to $16.5 \%$ who thought it was important, and almost one-third $(32.5 \%, \mathrm{n}$ $=212$ ) were neutral. By comparison, $34 \%$ of midwives judged the perception of coordinated natural objects as not important, as against $24 \%$ who said it was important. Suprisingly, a larger number $(42 \%)$, almost half $(\mathrm{n}=50)$ of midwives were undecided. On comparing, mean score recorded for midwives was $(M=2.88)$ compared to women, which was $(M=2.53)$. The results did find significant differences between the two groups $(p=0.03)$ Quan et al. found although pleasantness of the room showed significant differences, the item was the least rated among other environmental aspects (Quan et al., 2012), which is in consonance with this study. However, 
Quan's findings showed that pleasantness did receive higher scores from patients than staff, a reverse of what was seen in this current study.

As can be seen, the design factor of coordinated natural objects was moderately low $(<3$ on a scale of $1-5$, where $1=$ not at all important and $5=$ very important). However, based on two earlier results focused on design features of positive distractions in which mean scores were above 3 indicates a moderate number of respondents still preferred a more aesthetically appealing postnatal environment with positive distractions.

The stark appearance of traditional healthcare environments and lack of control by patients have been major factors for stress, dissatisfaction and complaints (Quan et al., 2012). The latest review of research by Jiang (2020) regarding positive distractions in the healthcare settings concluded several beneficial health outcomes for patients. This broad conclusion supports the results in this current study for further research. Correspondingly, as the results have shown, focusing on positive distractions in the postnatal environment could improve women's positive hospital experiences since the findings have indicated a lack of adequate interior features in the spaces, a situation which possibly contributed to less adequate and clear-cut opinions from the respondents.

\subsubsection{Clinical room}

Being cared for in a clinical-looking postnatal space also revealed significant differences between the postnatal women and midwives $(p=0.001)$. Interestingly, a clinical room was an issue for the two groups but less so for the postnatal women in which the mean score was 2.34 , representing a $50 \%$ increase from the mean score of midwives $(M=1.84)$. For example, clinical space was not important for $78 \%$ of midwives compared to $22 \%$ who said it was important. On the other hand, $65.5 \%$ of women did not find a clinical-looking postnatal space important, against $17.9 \%$ ( $\mathrm{n}=212$, approx.. 18\%) who were undecided and an additional 16.5\% who considered it important.

A plausible explanation for postnatal women's more positive answers is that safety of life and health is paramount in the minds of women in event of emergency - avaiilablilty of necessary equipment and tools when needed. Comparatively, it may be that midwives are promoting normal birth where women should be fine without the use of medical intervention and technology. DavisFloyd (2001) stressed the balance of three models of technocratic, humanistic, and holistic relating to mind-body-spirit relationships for childbirth in hospitals for women's wellbeing including how these translate to childbirth spaces focused on the models. It is effective to assume that a totally "high-tech" clinical setting may not be suitable, especially in the postnatal setting. 


\subsubsection{Colours}

In terms of room colours in the postnatal environment, respondents perceived neutrally about room with colours rather than a very plain room for recovery experiences. The mean score reported by postnatal women was lower ( $\mathrm{M}=3.16$ ) compared to midwives $(\mathrm{M}=3.44)$. When comparing the responses further, significant differences were found between the respondents on perceptions of a room with colour versus a plain room $(p=0.01)$. This result supports the claim that women focused less on colours in the room but more on space that permits freedom for movement (Franck, 2007). However, a slightly higher mean score by midwives suggests a preference for a pleasant, visual environment. This may be the case because staff spend long working hours experiencing the place on a day-to-day basis.

Another survey question asked to respondents specifically on perception of having "bright colours" in the room. The mean scores from both respondent groups indicate they did not perceive having bright colours in the postnatal room was important for recovery process and wellbeing. Mean score by midwives was comparatively lower $(\mathrm{M}=2.86)$ compared to the postnatal women $(\mathrm{M}=$ 2.19). Although both groups ranked bright colours very low, statistically significant differences were found between the two groups $(\phi=<0.001)$. Possible reasons why postnatal women ranked colours relatively lower maybe due to a lack of appealing colours for positive sensory awareness and experience in the postnatal environment compared to a panoply of appealing birth rooms available in hospitals elsewhere (Bowden et al., 2016) and secondly, because they seemed less bothered about colours in the rooms rather were focused on their care and babies.

It should be noted that the aim was to test whether and if colours at all were important for women's recovery rather than assuming that they would like colours by presenting a schema of colours to women/midwives during the surveys. Hence, this may possibly account for the low rating of colours in this study. Interestingly, the question about a plain room versus a room with colours showed the mean scale was moderately high (neutral) for women and midwives (3.16 vs 3.44) respectively.

Lack of empirical studies on which to base colour application in room design has been a challenge for designers (Dalke et al., 2006), which may prevent much use in healthcare settings targeted at specific populations. A 1988 review by Beech states that the ability to discriminate between colours is an innate expression that is influenced by personality and cultural background. The richness of the social functions embedded in objects also "affect colour preference and are capable of change" (Tofle, Schwarz, Yoon, \& Max-Royale, 2004, p. 32). 
Human response to colour is a complex task that involves interactions between light, eye and the brain (Park \& Park, 2013). Numerous studies on the impact of colours have been linked with lighting and visual art in hospitals and healthcare design. One study found that a poor environment can have negative effect on recovery outcomes and affect staff morale (Dalke et al., 2006). Colours with high levels of pleasure with low levels of arousal are most likely to induce a state of calm whereas colours causing displeasure and high levels of arousal are likely to provoke anxiety (Lankston, Cusack, Fremantle, \& Isles, 2010). Much existing research on the impact of colours was studied in children and paediatric hospital patients (Park \& Park, 2013) however, colour preferences specific to the childbirth environment appear to be very little researched particularly in postnatal environments. An attractive healthcare environment providing a variety of beauty in colour of objects had earlier been postulated by Florence Nightingale to promote recovery and healing for patients. With regards to healing and restorative environment, Biley (1996) discussed both positive and negative effects of colours including greens and claimed that green symbolises balance, harmony, growth, healing and love and could impact brain functioning.

Mazuch and Stephen (2005) suggested that the principles of emotional mapping which aligns colours to a range of emotions (vulnerable, relaxed and fearful) may be a useful approach to colour research in healthcare environments. Further research needs to understand women's preferences regarding room colours that are psychologically suitable for postnatal recovery experience and across different cultural contexts.

\subsection{Other similarities}

No significant differences were found in two other design features, preference of room choice and furniture made of natural materials (Table 8-1). The mean scores for these design features are generally between low or moderate, with the lowest rating score recorded for furniture made of natural materials (2.90 vs 2.76) recorded for women and midwives respectively. Just as for other design elements in section 8-1, these two aspects did not find significant differences $(p=>0.05)$.

\subsubsection{Preference for room of choice (personal space)}

Possibly one of the most important questions under social comfort environment was whether giving women the opportunity to choose a room of their preference could make a difference to their postnatal hospital stay experience and better satisfaction with care. Preference for choice of room was ranked as third most agreed features under the social comfort category. $(M=3.41)$ and midwives $(\mathrm{M}=3.34) \mathrm{p}=0.69$ 
One study reported that while many patients had preferred a single room, in practice patients are not given a choice, citing that allocation to beds is predicated on convenience rather than patient choice (Jolley, 2005). As far back as 2000, women had expressed increased satisfaction with single room maternity care (Janssen et al., 2000) as it enabled the mother and baby to stay in the same room throughout the intra- and post-partum periods and accommodated staff hence permitted couplet care (Spradlin, 2009). Studies investigating single room maternity care, LDRP also known as couplet model of care, and impacts on experience of care on childbearing women and staff have been discussed in the Chapter 3.

\subsubsection{Furniture/finish materials}

Although perceptions on "furniture made of natural materials" were generally ranked lower by the two groups, women's mean score was still relatively higher $(\mathrm{M}=2.90)$ compared to midwives with a mean score of $(\mathrm{M}=2.76)$. No significant differences were found between the two groups $(p=$ 0.41). The lower mean scores raise an important question about the appropriateness of natural finishes such as wood for postnatal room furnishings. The reason for midwives' relatively low rating of furniture made of natural materials could be related to fears of contamination of the furniture surfaces, possibly leading to infection. Karpanen et al. (2012) examine the effectiveness of copper alloy furnishing in clinical environments and observe traces of hospital-associated pathogens on the ward furnishings and fittings despite best standards practice on cleaning.

Appearance of furniture and its form create a strong desire for relaxation and comfort. It should be noted that the questionnaires were only focused on materials rather than preference for shape of furniture. In one study of furniture forms and influence on emotional responses, it was found that curvilinear forms elicited more pleasant emotions compared to rectangular forms. The investigators concluded that the former can guide designers in faciliatating for welcoming and pleasant interior environments (Dazkir \& Read, 2012). Previous literature focused on furniture had been for mental health facilities rather than in healthcare facilities (Shepley et al., 2013), such as furniture arrangement for behavioural outcomes (Corey, Wallace, Harris, \& Casey, 1986) not choice of finishes. Therefore, necessary preventive and precautionary measures will have to guide the adoption of appropriate furnishings in postnatal spaces. Additional research would be needed to explore the lower rating for furniture and material finishes as aspects of hospital ward furnishings.

Overall, the results reveal that women and midwives are concerned about spatial factors (additional spaces), confidentiality and dignity and appropriate furniture design. Consequently, the results are 
in line with previous studies which contend that environmental perception of users is strongly influenced by the quality of humanisation of the healthcare environment (Biley, 1996; Mazuch \& Stephen, 2005).

\subsection{Conclusion}

This Chapter has presented a discussion of analysis of both the descriptive and inferential statistics for the perception of design factors surveys reported in Chapters 5, 6, and 7 for Phase one. Several design factors and environmental indicators for wellbeing and hospital experience were found to be considered of higher importance and a few aspects showed low levels of importance. In general, Phase 1 outcomes also reveal a greater correspondence with similar research regarding the physical environment factors for wellbeing and hospital experience in healthcare research settings and other environment-related fields. Because the outcomes in this study can be taken as exploratory due to very limited research in the postnatal environments regarding the physical design factors for wellbeing and hospital experience, more research will be needed to more confirm all the outcomes in this research. Nevertheless, even though there is little precedent to turn to, and even with a few questions not very effective, the overall generally positive perceptions of the factors in the postnatal environment are enough to draw useful conclusions.

One important finding from this research is the very positive rating of single-bed accommodation aside from hygiene/cleanliness which can be regarded as the standard best practice. Additionally, one very interesting finding is the perception on shapes and forms for postnatal room design which received high mean scores. In fact, the opinions of both respondents are very similar (3.88 vs 3.82), almost 4 on a 1-5 Likert scale. Although a previous study was generally focused on patients' perception of architectural space (Lawson et al., 2003) however, in this study, women and midwives perceived natural form and shapes recorded a moderately-high importance for design of postanatal spaces. This suggests that designing a postnatal environment unlike the traditional rectilinear shape, with soothing form could contribute to the wellbeing and postnatal recovery experiences of new mothers. One study focused on angular and curved shapes found that curved shapes were associated with safe and positive concepts and female names compared to angular shapes with danger and negative concepts and male names (Palumbo, Ruta, \& Bertamini, 2015).

Although low mean scores were observed, design factor of room colours is another interesting find, indicating that many respondents perceived a calming and positive environment for postnatal recovery important. Although limited research focused on perception of colour for the postnatal environment, respondents felt that their impacts could contribute to improving wellbeing by 
providing a comfortable and appealing environment. Overall, further research is needed to specifically examine different types of shapes and forms, natural and manmade, to clarify the influence of shapes and design on wellbeing and recovery experiences of new mothers during postnatal hospital stays. Moreover, the same applies to assessing the effects of a range of room colours such that research could provide for their guidance and possible application in postanatal environments.

With conclusion of phase one and key factors identified, these were used to inform the focus group protocol for phase two. The next chapter describes the findings of the focus group discussions (FGDs) as the qualitative strand in this current study. 


\section{CHAPTER 9}

\section{PHASE TWO: FOCUS GROUP FINDINGS AND DISCUSSION}

This chapter presents the findings of the focus groups. Participants' characteristics in the focus groups are first presented. The rest of the chapter documents the qualitative themes and categories that were generated based on the results from the four focus groups which took place in May, June and August 2018.

\subsection{Participants' characteristics}

Nine women and five senior registered midwives participated in the focus groups in this present study. "Women" in this study are nursing mothers with babies aged between 1 month and 4 months at the time of the focus group discussions. Women in the focus groups were aged between 25 and 40 years old; with midwives being generally older, aged between 35 and 55 years old. A larger percentage of the focus group members have postgraduate versus undergraduate degrees (79\% vs. 21\%) one participant had a secondary school qualification (see Table 9-1). The focus groups had a mix of African and Filipino ethnic nationalities (21.4\%) and European/NZ Pakeha (78.6\%). Aside from the women who had their babies with them, one woman was accompanied by a family member.

As mentioned in Chapter 4, three different venues were used for the (FGDs) with session interactions mostly lasting longer than an hour. The summary of each FGD session and venue is shown in Table 9-2. 
Table 9-1: Demographic profile and characteristics of focus group participants in the study $\mathrm{n}=$ 14

\begin{tabular}{|c|c|c|c|c|c|c|c|}
\hline Item & Status & $\begin{array}{l}\text { Age } \\
\text { range }\end{array}$ & Education & Parity & $\begin{array}{l}\text { Site for } \\
\text { FGD }\end{array}$ & $\begin{array}{c}\text { Date of } \\
\text { FGD }\end{array}$ & Code \\
\hline 1 & Woman & $25-29$ & Postgraduate & $\begin{array}{l}\text { New } \\
\text { mother }\end{array}$ & $\begin{array}{l}\text { Meeting } \\
\text { Rm }\end{array}$ & $29 / 5 / 18$ & FGD1W1 \\
\hline 2 & Woman & $30-34$ & Postgraduate & $\begin{array}{l}\text { New } \\
\text { mother }\end{array}$ & $\begin{array}{c}\text { Meeting } \\
\text { Rm }\end{array}$ & $29 / 5 / 18$ & FGD1W2 \\
\hline 3 & Woman & $30-34$ & Postgraduate & $\begin{array}{l}\text { New } \\
\text { mother }\end{array}$ & $\begin{array}{c}\text { Meeting } \\
\text { Rm }\end{array}$ & $29 / 5 / 18$ & FGD1W3 \\
\hline 4 & Midwife & $36-45$ & Postgraduate & $\mathrm{N} / \mathrm{A}$ & $\begin{array}{l}\text { Hospital } \\
\text { site }\end{array}$ & $20 / 6 / 18$ & FGD2MW1 \\
\hline 5 & Midwife & $46-64$ & Postgraduate & $\mathrm{N} / \mathrm{A}$ & $\begin{array}{l}\text { Hospital } \\
\text { site }\end{array}$ & $20 / 6 / 18$ & FGD2MW2 \\
\hline 6 & Midwife & $30-34$ & First degree & $\mathrm{N} / \mathrm{A}$ & $\begin{array}{l}\text { Hospital } \\
\text { site }\end{array}$ & $20 / 6 / 18$ & FGD2MW3 \\
\hline 7 & Midwife & $46-64$ & Postgraduate & $\mathrm{N} / \mathrm{A}$ & $\begin{array}{l}\text { Hospital } \\
\text { site }\end{array}$ & $20 / 6 / 18$ & FGD2MW4 \\
\hline 8 & Midwife & $36-45$ & Postgraduate & $\mathrm{N} / \mathrm{A}$ & $\begin{array}{l}\text { Meeting } \\
\text { Rm }\end{array}$ & $21 / 6 / 18$ & FGD3MW1 \\
\hline 9 & Woman & $30-34$ & High school & $\begin{array}{l}\text { New } \\
\text { mother }\end{array}$ & $\begin{array}{c}\text { Meeting } \\
\text { Rm }\end{array}$ & $21 / 6 / 18$ & FGD3W1 \\
\hline 10 & Woman & $25-29$ & First degree & $\begin{array}{l}\text { New } \\
\text { mother }\end{array}$ & $\begin{array}{c}\text { Meeting } \\
\text { Rm }\end{array}$ & $21 / 6 / 18$ & FGD3W2 \\
\hline 11 & Woman & $35-39$ & High school & $\begin{array}{l}\text { New } \\
\text { mother }\end{array}$ & $\begin{array}{c}\text { Meeting } \\
\text { Rm }\end{array}$ & $16 / 8 / 18$ & FGD4W1 \\
\hline 12 & Woman & $30-34$ & Postgraduate & $\begin{array}{l}\text { New } \\
\text { mother }\end{array}$ & $\begin{array}{c}\text { Meeting } \\
\text { Rm }\end{array}$ & $16 / 8 / 18$ & FGD4W2 \\
\hline 13 & Woman & $30-34$ & Postgraduate & $\begin{array}{l}\text { New } \\
\text { mother }\end{array}$ & $\begin{array}{c}\text { Meeting } \\
\text { Rm }\end{array}$ & $16 / 8 / 18$ & FGD4W3 \\
\hline 14 & Woman & $30-34$ & Postgraduate & $\begin{array}{l}\text { Mother } \\
\text { of } 2\end{array}$ & $\begin{array}{c}\text { Meeting } \\
\text { Rm }\end{array}$ & $16 / 8 / 18$ & FGD4W4 \\
\hline
\end{tabular}

Note: FGD1W1 = Focus Group Discussion 1, Women 1; FGD2MW1 = Focus Group Discussion 2, Midwife 2 and so forth.

During the focus group discussions (FGDs), the participants were requested to look back and comment about their positive experiences and disappointments while being attended to or working in postnatal hospital environments. The researcher requested the focus group participants to confine the discussions mainly to aspects of the physical environments and the building spaces, and the impacts they think these have had on the recovery experiences, quality of postnatal care and effects on wellbeing. 
Table 9-2: Characteristics of settings and duration of the FGDs

\begin{tabular}{|cccccc} 
Session & Date & $\begin{array}{c}\text { Room } \\
\text { type }\end{array}$ & Participants & $\begin{array}{c}\text { Duration } \\
\text { (minutes) }\end{array}$ & Debriefing \\
\hline FGD 1 & $29 / 05 / 2018$ & $\begin{array}{c}\text { Office } \\
\text { space }\end{array}$ & 3 women & 52 & Yes \\
\hline FGD 2 & $20 / 06 / 2018$ & $\begin{array}{c}\text { Seminar } \\
\text { room }\end{array}$ & 4 midwives & 83 & Yes \\
\hline FGD 3 & $21 / 06 / 2018$ & $\begin{array}{c}\text { Meeting } \\
\text { room }\end{array}$ & $\begin{array}{c}1 \text { midwife, } 2 \\
\text { women }\end{array}$ & 95 & Yes \\
\hline FGD 4 & $16 / 08 / 2018$ & Meeting & 4 women & 75 & Yes \\
\hline
\end{tabular}

Figures 9-1 and 9-2 show the settings for the focus group discussions. In addition, Figure 9-3 depicts the preliminary solution based on the results of the perceptions of design factors surveys from phase 1. As noted previously, these results of the questionnaire surveys were used to inform the focus group protocol, which serves as the talking point for the FGDs.
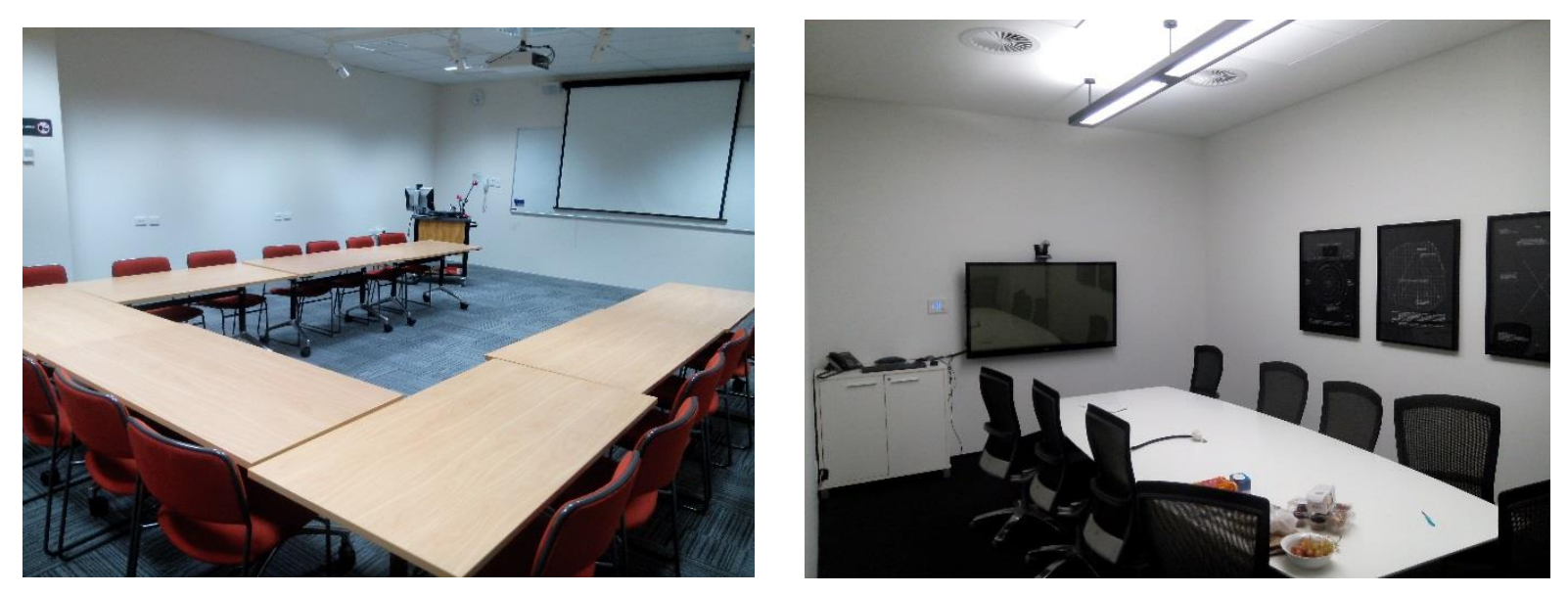

Figure 9-1: Two of three settings for the focus group discussions 


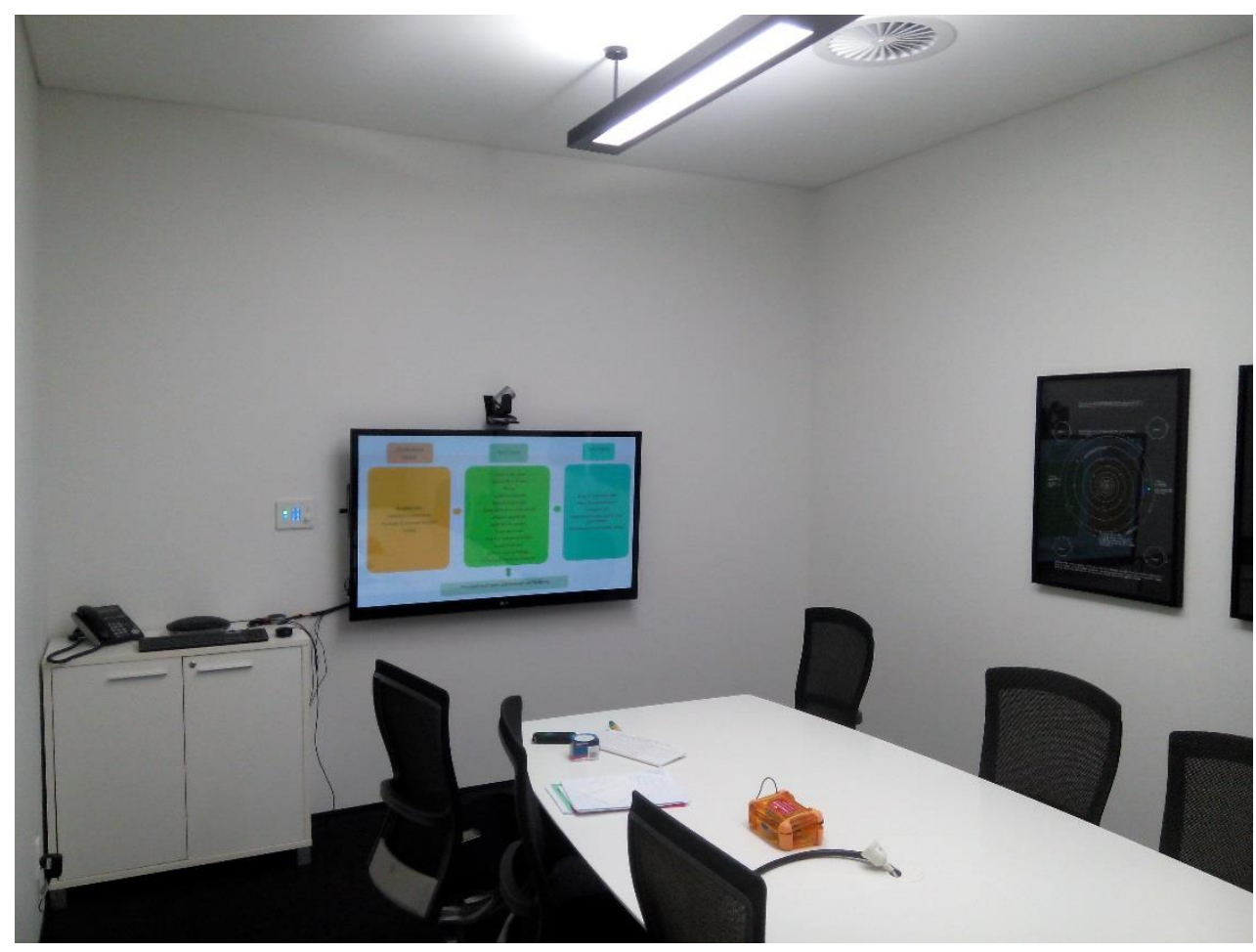

Figure 9-2: Preliminary solution developed from findings of phase 1 surveys, presented to focus group participants

Table 9-3 shows the key findings of the FGDs, grouped into three main categories, with detailed discussion of each sub-category in the following sections, illustrated by verbatim transcribed quotations from the FGDs where appropriate. The derivation of the categories and sub-categories used below was explained in Chapter 4. 
Table 9-3: Key findings of phase two focus groups

Phase two Research question: What were the ways in which the design factors and the postnatal spaces affected women's recovery experiences and wellbeing during hospital stays?

Main category

Sub-category

\section{Recovery experiences in spatial units}

Single-bed rooms for emotional support

Privacy/Valuing dignity

Noise and harsh lighting

Mixed sensory stimulation

Optimising daylight

Wayfinding

\section{Elusive restfulness}

Adapting to familiar noise

Modifying/Sensing the space

Materials and technology

Behavioural

\section{Mutual role integration}

It feels like a hospital

Humanising the space so that we feel we belong here

Lack of space for family experience

Space inhibition for activities of daily life

Organisational support, resources and staffing

\subsection{Category: Recovery experiences in spatial units}

The first category developed from the FGDs was recovery experiences in spatial units. This reflected the types of room occupancy as experienced by women and how they impacted on recovery experiences and wellbeing. Women participants described the postnatal period as a very vulnerable and private time, which involved emotional, physical and psychological occurences that needed a quiet time and a comfortable environment to be with partners and family members. 


\subsubsection{Single-bed room for emotional support}

Women participants expressed powerful emotions and feelings as they recounted a variety of experiences in single and double-bed occupancy rooms. The participants raised concerns for emotional support in the hospital environments through the recognition of the importance of a private space both for women and partners or family members to support sleep and restfulness and for peaceful hospital experiences.

It was a very vulnerable time for me. It was a very vulnerable time for all three of us: me, my partner, and my baby. Also it's a very private time... If we have our own space, where we could [start] bonding together as a three; me, my partner and my baby that was so important. (FGD3W2)

Yeah, not a long a great experience I found. Partly because they [rooms] were shared... And I mean at the time, you're still struggling to like walk, incontinence stuff. So it's very personal time and very vulnerable. So being like having this little was almost delivery room-like hospital bed and a chair like breastfeeding so like touchy or something. And then there is a curtain. And then there is someone else. (FGD4W2)

As a new parent, imagine sharing a room with another parent maybe when you just finally gets your baby to sleep and you want to [have] some sleep and the next baby starts to cry, all of you are now awake. So you just don't get any sleep at all...I had to tell them - can I get a private room. That experience is something I wouldn't recommend. If possible they should scrap that [double-rooms]. (FGD1W2)

Some participants were anxious about birth pain, which according to them, could interfere with the ease to use toilets in a shared room. They explained that birth pain was normal irrespective of modes of birth of a woman. As one participant notes:

No matter the process how even the birth was, there is a process, you'll still feel the pain of birth. No matter what, it might not be as intense as some other women but you still feel the pain especially the first 3 days it's very crucial. (FGD1W2)

Most of the participants affirmed that provision of single-bed rooms with ensuite toilet facilities rather than a double/multi-bed room (with toilets shared) was ideal to reduce emotional and psychological stress encountered in a double room. 
The first one [toilet] was very okay. But the last room before I was discharged, the toilet was not in the room. You had to go out to use the toilet and the bathroom. Every other person, walking past would see you. Even to add to that, for a woman that has been stitched, you will appreciate having your personal toilet. (FGD1W3)

Haven't you got one where you are sharing bathrooms when you've got to lock the door? If you're going to use the bathroom, you have to lock the door so other people don't walk in. It is not very nice. So you can't go taller. You just going to wait. And you are not able to shower at all. That must be really annoying... but I can imagine I would find that quite awkward if she had a baby and bleeding. Feeling about vulnerable. (FGD2MW4)

However, some participants identified a few benefits with shared rooms regardless of their perceived inadequacies with noise and visitors. This also included sharing of toilets if the occupants were not more than two in in a double room.

...There are some advantages to having 4 in a room. I am fairly new midwife. And the one four bedder room is my day's job. And it was quite communal. To know each other and often on occasion they would chat. They share something the others do hear and so there are some advantages to that. It's probably more advantageous to the single room. Of course there could be visitors there, noise and the other things. (FGD2MW1)

Having just two people sharing one bathroom was good. The shower - it was an open shower - so you're just open to the thing. So it was good. (FGD4W2)

\subsubsection{Privacy and valuing dignity}

In the postnatal hospital environments, the participants expressed challenges in privacy in shared or multi-bed occupancy rooms. The need for privacy and dignity was indicated, in part, due to physical complications (such as bleeding), emotional and psychological stress by women following birth, making the need for a calming environment for recuperation. The women also described needs for privacy arising from the emotional and physical stress if babies could not feed or they were not able to express or breastfeed appropriately.

...you've got all the physical complications...you're still bleeding; you're probably not properly dressed; you're struggling to move around... But typically, you are 
exhausted. You are really emotional. You're going through all the stuff for the first time. There is a whole lot to learn. If it's hard to feed your baby, then you get really anxious...they really need to feed for their health ...It's just really challenging. I think from my perspective that is where that sort of desire for privacy comes from. (FGD4W2)

Within a certain period of time going on in your body and your baby. Your milk won't start coming in, and your baby would not sleep all night. So there is a night that your baby doesn't generally sleep. And so you're like trying to wait for your milk to come until you have the food to feed her. And I think that is the other stressful part. Like you are exhausted. (FGD4W3)

Conceptualisation of privacy was also related to having an own space but with a defined boundary with two occupants in a shared room, although curtain partitions and walking past women close to the doors by other women's visitors heavily negate this makeshift privacy. However some participants felt that using solid partitions and arguably rearranging or moving a number of things around in shared spaces could be beneficial to prevent invasion of privacy and provide more comfort for women's wellbeing.

So the both sections of the room was curtained off. Curtains were all around. So we could close ourselves off when and if we wanted to. And that was an illusion of privacy I guess rather than the actual privacy. And people to see me came and see me. The other families had to walk past where I was to see me. (FGD4W1)

In terms of shared rooms, if you place the door in a different way, and if you place the sink in a different way, we might never met or never met or had to walk past to get to her space. And where you had to walk through her space to get to the basin. And then the bathroom was at the door, so both of us are walking out of the room ... to use the bathroom. (FGD3W2)

Women attached great values to privacy whether they were attended in single-bed rooms or double/shared rooms. The invasion of privacy has the tendency to cause feelings of un-safety in a room and to cause withholding of information in the presence of others who may be strangers to women. As one midwife described:

I have heard some of my clients say if they are in shared room and there is male family members in that room, if the midwife is asking them about really personal 
things they might not want to answer or might give a bit incorrect information. But I don't know how much that would affect recovery. (FGD2MW3)

This position was corroborated by a woman participant:

... I think having that little certain more to not have that intrusion quite frequently and not knowing who was coming in and out and people not really explaining who they were - who are you, why are you here? ...And it's not a practical thing to get dress up all the time, it can get used to the kind of breastfeeding, which is if you are first-time mum and there are people walking in and out... And being in your home is probably the ideal. But if you can be in hospital it would be an ideals to have a great respect for privacy. (FGD3W2)

Invasion of personal privacy of new mothers in the rooms through unscheduled visits from caregivers was challenging. Some participants used terms such as "annoying" and "frustrating"to describe such visits, which disturbed the opportunity to sleep.

Sometimes, I would say it's a nuisance. Because maybe for me there are some times that I just want to start sleeping and then they would like we've come for checkup. But knowing that that's their duties, some of them kind of give you notice. Their own invasion of privacy cannot be compromised. (FGD1W3)

Nevertheless, women agreed that it was normal for care providers to pay visits as it was crucial for their health and wellbeing and as such did not amount to invasion of privacy. It was suggested that adequate indication and information prior to visits would prepare women to be psychologically ready and allow then to manage their time for sleep or rest prior to such visits.

But I think that's just normal. If you're in hospital, you just have to give up some of that privacy for people coming in and out and checking on you. And I think when you're in hospital, you don't mind the nurses or midwives. (FGD4W3)

The time issue is really frustrating and I thought as a patient but for some of us, things could be invading could be negated by maybe someone to knock on. This just require: "Hi, can I just expect to check on you". (FGD3W2)

In all the FGDs it became apparent that privacy could aid recovery experiences and satisfaction with care, with a single-room occupancy largely contributing to it. From the standpoint of some midwives (though some midwives agreed on a single-bed room), the responsibility and trust of 
care and also ascertaining health and wellbeing of women and babies while in hospitals overrides other considerations.

There's a lot of people who are sick or physically need help with things. People who need pain relief. Like I guess, it's tricky, you can’t just give a person a paper and a pen in one go just make sure you are only taking that in every 4 to 6 hours. You have to take that responsibility. Because it's a midwife's job I suppose... You need to make sure that you are on top of the pain relief. You have done all your checks you are required to do on that baby or that woman. (FGD3MW1)

\subsubsection{Noise and harsh lighting}

A preponderance of comments during focus groups indicate the busyness and noisy situations in the postnatal wards which contributed to lack of sleep or inadequate rest for women. Some participants described crying of babies in the postnatal environment that often leads to confusion about how to differentiate which baby was crying from other babies. The restorative benefits of sleep should inform the thinking of the spaces on how to better manage the babies to enable new mothers to have adequate sleep and rest while in hospital prior to home.

I was tired and with children. I was actually unable to tell whether or not - my baby or is it the other baby was it. So all the time, but often as far I do tell the nurses, these babies need to be moved into the family room as people need to walk or people in the way. Two babies on by my side. Six babies in this whole little space. I was really conscious of if he was crying at night and keeping other people awake. For me I just can't say definitely whether or not that is quite good for recovery. I definitely know that it's about time we are going to change a shared room. How pleasant is sound that needs to be at the baby in the room. (FGD4W1)

Bright lighting and noise from other mothers' visitors in the postnatal environment largely contributed to discomforts for the participants. While personal control of lighting fixtures in hospitals can benefit women in terms of sleep or enable them to rest, spaces were not designed for adjusting the lighting levels to suit each woman's needs.

So, maternity rooms were busy and bright. I felt we are in a shared room. That was really unpleasant. But there was a lot of noise, busyness in the maternity suite. And people everywhere. And I said is that allowed to having too much of headlight. (FGD4W1) 
But if I share the space with another person, the light can affect me. If they turn on the light. I don't want it dark to sleep but if there is a separate lighting system. When they have something to do, I can relax. (FGD1W1)

\subsubsection{Mixed sensory stimulation}

Sensory stimulation refers to the extent to which natural daylighting via windows can contribute to achieving visual stimulation for new mothers who need to recuperate (Malkin, 1992). Sensory stimulation can be affected by the layout of rooms, orientation and position of beds, which can impact on daylighting into rooms or results in poor visibility to the outside. Women used expressions "to see the sky," and "to see the outside" interchangeably to describe experiences of limited, or lack of, views to the outside and lack of natural lighting in the spaces. A feeling expressed was that shared or multi-bed rooms deprived women of daylighting which could have negative behavioural consequences.

Even if to look out of window... But if we were spending a couple of days in that spot, which either you're totally curtained in or you're absolutely public. To get any sense of daylight, you have to be absolutely public. That would drive you crazy if you are going to spend more half a day. (FGD3W2)

At least we just want to see the sky, we don't even want the windows opened we just want to see the sky. That is going to brighten the day. (FGD1W3)

Sensory stimulation also relates to visual qualities of nearby physical environments that shift the focus not only from daylighting in a room but also to the qualities of surrounding landscapes. Some women were displeased with views of repetitive blocks of buildings and car parks in hospitals.

Well at times, you are there for long, you can't see outside. When you are looking through the windows, you are just seeing the roof of another building. A patient would want to see outside...You don't know what's going on the outside. (FGD1W2)

Conversely, a woman who stayed in a two-person shared room did enjoy natural lighting and had opportunity of a positive view of the outside.

So it is very useful that we get one of shared rooms instead of had to wake other people up. But I think the thing that was challenging was that the room was half as 
nice. Had a big window looking onto the out. I can see the hues. It was very comfortable. (FGD4W3)

\subsubsection{Optimising daylight}

The daylighting in interior postnatal spaces was identified as a potential environmental factor to tackling incidents of jaundice in neonates (Barss \& Comfort, 1985). Exposing newborns to natural light has the capacity of breaking down the bilirubin in neonates. A promising design approach is creating spaces for daylighting penetration and optimisation in postnatal environments for newborns wellbeing, which in turn can have positive impact on wellbeing of new mothers.

The sunlight, UV light, through window is fine. They could have designed a small area where people could take their babies to show them some sun and there is nothing. That has always driven me crazy. (FGD3MW1)

\subsubsection{Layout and wayfinding}

Some women recounted challenges faced in locating or navigating safely from their rooms to other parts of the hospital in spite of previous facility tour experiences. Motherhood is a period for physical and emotional adjustments (Smith, 1999) and hospital layout may contribute to how difficult or easy it is to find one's way around in the physical environment. A postnatal women recounted her experience when she needed assistance from the midwife which could lead to anxiety and emotional distress for women.

I think the ward was quite confusing. The layout was confusing. I wouldn't have known where to go to find a midwife if I needed one. I would just have to wander in to the corridor, in my dressing gown, including walk around for a while to find who I need.... So it's not a place designed to encourage you to seek out what you need. (FGD3W2)

The current room layouts and ease of access to major service routes such as elevators and exits posed serious problems for women especially after the birth with limited mobility. The midwives captured the hospital layouts and described the scenarios this way:

You meet so many women getting into the lift. It's very hard getting into the lift.

(FGD2MW2) 
The whole maternity should be on ground level. These women would come and labour and they've got to come out to the fourth floor and they've got to walk along a massive big long corridor. Whereas in other buildings, you drove out to the door. You walked into the delivery suites. So access is really not good. (FGD2MW4)

\subsection{Category: Elusive restfulness}

The "elusive restfulness" category represents the degree to which women were affected by busy and noisy situations in postnatal environments. The category included four subcategories which are discussed in detail below.

\subsubsection{Adapting/sensitive to familiar noise}

Adapting to or being sensitive to familiar noise mostly related to persistent noise from other persons in the rooms (both other women and babies) who made noise while sleeping. This situation affected some women's sleep and contributed to anxiety and fear for other women who stayed awake. Some participants used the language that their body became "wired" against sleep partly because of hormonal changes in the body or they had the mentality of a hospital being a busy place and they would not expect it to be restful.

And also they make a lot of noise when they're sleeping. You are like so attuned to this little noise maker in your room. You're like Oh are they okay, are they okay? You can be tired but your body can be kind of wired. (FGD4W3)

Cumulatively, I don't think I got 6 hours of sleep. It's just like my body just got used to no sleep. During the day, that's when the baby is asleep, and you want to sleep and the midwives are coming and visitors and food staff. So you can't send them away. At night, you cannot still sleep because that's when the baby begins its own stuff. (FGD1W3)

\subsubsection{Modifying/Sensing the space}

Participants in the focus groups felt that defective acoustic design of the postnatal environment made it feel unrelaxing and uncomfortable. Most women said they found it difficult to have peaceful conversations with their partners and to rest especially in shared rooms, without other 
women overhearing their conversations. The walls and doors were considered to be the weak points allowing distractiing external noise into the postnatal environment.

\subsubsection{Materials and technology}

Participants in the study described interventions to promote restfulness and wellbeing of women. Interventions to engender a peaceful and comfortable environment including appropriate materials to reduce negative noise impact were explicitly mentioned especially for shared rooms. Most of the participants agreed that sound proofed walls, acoustic doors, and solid materials for partitions (for curtain units) had potentials to reduce negative impact of noise.

Different to other walls where you need acoustics and you have the normal doors. And you have normal walls. The noise is so much high, really you need soundproof walls. And you need soundproof doors. (FGD4W3)

A participant spoke about using visual technique of assessing rooms as opposed to using bells to indicate to medical staff and significant others whenever women are resting or sleeping. The nursing station could monitor rooms through light sensors on the doors to detect whether a woman is sleeping or not. This could allow women increased opportunity for rest.

In my head, I was imagining there is a little button that you can push the light outside the room, so let see if this person is sleeping. Just a sign you can put on the wall because they do, every woman if they are going to do that, and is not all that common. I think, not difficult and useful. (FGD3MW1)

\subsubsection{Behavioural}

Some women might stay longer than the usual one or two days postnatally and defintely need sleep for their wellbeing. One participant reported that she used eye masks and earplugs before she could sleep. And she was able to sleep for about two hours while the partner and a family member watched the baby.

But the thing is like you just have to sleep whenever you can - day or night when you have a little baby. Because she [baby] was on a 3-hour cycle and at 3 hour, I had to feed her. Change her. Express, specifically like an hour and a half of looking after her. And half an hour of breastfeeding. So they were like you gonna sleep 
now. Put on the eye mask. Put on the earplugs. Try and get some sleep. Just to recover a bit. (FGD4W3)

\subsection{Category: Mutual role integration}

Mutual role integration explains the development of a synergy between space and care. It envisions the extent to which the participants - the women and midwives - expressed optimism for improvements in postnatal hospital environments. Although most of the participants agreed that biomedical settings offered the assurance of back up medical support for the safety of new mothers' and newborns' health, it also undermined their wellness situations in attainment of wellbeing.

Being in a hospital environment impacts on some women psychologically and behaviourally manifesting in them acting like a sick person.

For me being in hospital, I am kind of acting like a sick person, if you like. That was kind of opting out of society. (FGD3W2)

Two midwives in the FGDs also agreed with the impact of hospitals on women:

It lends themselves to patients. Like behaving like a patient I think. In your room, there might be/would be an armchair usually for most of the spaces and bed. But the bed is at the centre of the room and that is where and so it lends itself to women behaving as if they are sick. As if they need to be in bed. Where else do you go, you can't sit in your armchair or you can't lie in your bed. FGD3MW1

If you are home, you're on the couch. You're not in your bed all the time. You would have some naps, of course. But you will be a lot more likely to be behaving like well person. My personal bias is that I can't ask well women who are having complications with pregnancies and labours shouldn't be in hospital. (FGD3MW1)

Mutual role integration acknowledges and demonstrates the value of supportive environments for women's wellness situations.

\subsubsection{It felt like a hospital with hospital wards}

During the discussions "It felt like a hospital" was frequently repeated by women, which conveyed deep messages about biomedical settings and their impacts on psychological wellbeing. This description was perceived to be incompatible with women's wellness situations of birth in hospital. 
While some women had the belief that hospital environments always have a ubiquitous institutional character, they expected a more homely atmosphere and relaxing environment.

[The] environment felt like a hospital. It didn't feel like a comfortable space for me to relax. It felt like a hospital with hospital wards. And you kind of couldn't get away that from where you were. The thoughts of good place where there were linoleum. So, more comfort. Less function combined would be nice. (FGD4W1)

Some participants in the FGDs regarded hospital as a strange, unpleasant setting. Some participants felt that after a successful birth, going into the comfort of the home environment was the ideal place to relax and recover and have control over what they wanted for themselves.

I would always say to people: get the hell out of hospital. Because it's like you're in a train station. It was always ringing all the time. And people coming and out without announcing themselves. (FGD3MW1)

A home-like environment was expressed as a potential means of reducing the strangeness with hospitals. Women believed introducing features of domesticity had the potential to make the spaces more relaxing and comfortable for recovery experience and wellbeing.

I am just thinking everything in the hospital is white. It is hospital. It is not the house. Some decorations like balloons or such things to make it feel like a house. (FGD1W1)

... The maternity unit is still attached to hospital, but is more homely-based. It's got a more homely feel. It doesn't have the beds, high ceilings. The ceilings are low.

The colours (excitement) are okay. They are more homely. (FGD2MW4)

\subsubsection{Humanising the space so that we feel we belong here}

Women described aspects of postnatal environment that did not convey the feeling for a humanised environment of care. Spaces often portrayed an unwelcoming impression rather than sense of belonging. The FGDs suggested a disconnection exists between the environment for care and one which takes into account human concerns and needs. The participants recognised the role of design of spaces to engender positive emotional and psychological wellbeing.

To make you feel for the short time you're, you belong here. This is a safe place versus a comfortable place. I think that would get a good balance between your 
need to decide whether it can be affordable... But also little bit more individualised and a little bit more personalised. (FGD3W2)

The institutional features of healthcare built environments were mentioned as functional settings concerned about process and efficiency, especially in childbirth environments with many births.

It was the same kind of the room with the hospital environment because it was so functional as always expected to have as this same space is. But it is all the way precisely because there has been so much emphasis on function... There is not really, anything that is there that invites people to steady, or invite people to relax or invite people to linger and just enjoy. (FGD4W2)

Well, I get that it's a hospital. The births things are in bulk. It's a horribly sterile environment. For example, the beds, it's great that you have the beds that some of them can raise ups, you can be setting up in the bed that is terrific. But it's not really big enough to cosily put your baby safely, in a way that makes women feel particularly comfortable. (FGD3MW1)

Some participants stressed the issue of the increasing practice of mixing up "potential risk" women with "low-risk" regarding hospital births, while birth philosophies suggest that high-risk pregnancies and births require a medical environment and low-risk births require a primary care setting, with hospitals lacking flexibility to accommodate individual needs (Malkin, 1992). FGDs felt that a calming and comfortable environment that will accommodate the individual and collective needs of women should be the pursuits for positive recovery experience during hospital stays.

When you're talking about the hospital, you are talking about sick people. When you're talking about maternity, majority that you are talking about are well women and well situation. It is a normal physiological part of being a person as having a baby. You just want to go and have a rest, breastfeed ... But you just really need a more homely space. You don't want this medicalised model of care. (FGD2MW4)

Some participants felt that altitudes of women in space can assist to show their inclinations about domesticity which eventually affects behaviour during the postnatal hospital stay. 
A lot of the postnatal midwives would wear normal clothing. We don't wear scrubs. I do for births. Postnatally the nurses wear the uniform but midwives usually don't. (FGD2MW3)

We encourage them to not wear hospital gowns. To wear their own clothes.

(FGD2MW2)

\subsubsection{Lack of space for family experience}

Another sub-category that builds on humanising the space and care was the lack of space for family experience. Traditionally, and in many cultures, family play an important role in birth events. Most women expected that during the events of birth they would have the support and reassurance of partners or family members for emotional, physical and social wellbeing. However, there is a challenge for many large hospitals in meeting the goals of family-centred space that accommodate the immediate needs of women and partners.

Women lamented the lack of spaces for partners and families when they were turned away during the crucial recovery period. Some women explained how it was painful to see their partners leave the hospital when their presence was in sore need; to assist with babies while they rest and sleep to enable recovery and wellbeing.

On the second night, I am was really tired and I thought if my husband was there to help me, It would help me a lot. At first, my staying in hospital that long was good, because I need my family to help me. So I need to return home early so that I can have their support. (FGD1W1)

He felt in the way. That he was unwelcome. Sort of. It wasn't a space for him. And he thought there was no space for him, he has been snuggling for hours. I had to go and search for somewhere to put him. He was in a couch next to the baby. We quite have to go home and be comfortable. (FGD3W2)

One of the reasons for postnatal hospital stays is to help new mothers acquire some parental skills prior to going home. Women's self-esteem increases when they are able to "get it right" learning the skills for motherhood, however learning opportunities may be hampered unless physical needs and, by extension, environments for learning skills, are addressed (Emmanuel, Creedy, \& Fraser, 2001). The lack of spaces to meet for learning of parental skills was antithetical to the roles of midwives regarding support for information and education. As one midwife points out: 
There is an environment that she can use for learning new skills, especially for first time mums. And want to be able to include the partners. Getting the support of education total environment and not medicalised environment. (FGD3MW1)

This environment has the capacity to foster interactions that some women complained were not being encouraged while in their rooms. The design of postnatal environments assumes a singlespace basis with less focus on activities and spaces to support wellbeing. Whereas communal areas and spaces that are different from typical hospital rooms were perceived by women to help them to leave their rooms and interact with other mums if there was opportunity to have access to one.

Probably everyone else is also struggling. So you can't just wanted to stay to your own space because there was no encouragement from anyone else. If there is a communal space that we could go to, if you want to. Then I think that would might have been different. (FGD4W1)

If they have their space and there is an area that is communal where they could go out with their baby and just sit and talk to other moms would be easily perfect. It would be like a big home, with the big lounge and you can ring the bell and go and see who is in charge. And say hi, I need help with this. But I can just retreat to my own space if I need to. (FGD2MW4)

My thinking is not so much like a hospital with some medical facilities as this is like a big shared flat. So you have the rooms. And I think this kind of pretty much individual rooms that's fine. But you do have common areas. And you do have a lounge, where you can have people coming to pick vegetables. (FGD4W2)

\subsubsection{Space inhibition for activities of daily life}

After an eventful moment of birth women felt it was essential to return to normal activities of daily life. Some women who have had extended hospital stays due to medical complications as well as increasing trends for flexible postnatal hospital stay (Cuncarr \& Skinner, 2011) felt outdoor environments offer valuable opportunities to walk around or a move to a garden rather than stay in their hospital beds. Many of the participants in the FGDs described the lack of outdoor environments they can go to for fresh air which resonates with the perceptions of "it felt like a hospital with hospital wards". The provision of outdoor spaces enables women to "get away" from the perception of being in a hospital, which could promote confidence building, reassuring women of the things they used to do before pregnancy and birth. 
I think I would have a big open space in the middle which is open to the outside that has grass and greenery and trees where some women could go and just hang out. That would be a nice set of ways of thinking about it. It just kind of make it more open. Less clinical. Somewhere you really want to be. I guess you are not really sick but you have to go the hospital to give birth to a baby and feel better. (FGD4W1)

I would totally put in a rooftop garden. I would make it that at the end of each ward, there was a small deck. You could get out and bathe some fresh air. (FGD3MW1)

It ... would be great to have like the maternity postnatal space connected to the outdoors. Walking out to see gardens where you're still able to have a bit of privacy: it means - it's not open to everyone - but you are able to be in another space outside. Getting fresh air. That sort of thing. (FGD4W3)

\subsubsection{Organisational support, resources and staffing}

The last sub category identified in mutual role integration was organisational support and staffing. The organisation culture and context seemed to exact great influence and had relationships with both physical and social environments. While the participants felt improvements to postnatal hospital environments were important and they expressed optimism regarding those, they were also cautious of the implications on resources, which depended on the priorities accorded by organisations to maternity services as resource-driven rather than woman-centred (Bick, 2008). Some participants described:

I think this kind of comes back to whether or not the DHB prioritises maternity care in terms of funding. And I think probably that's a bit of a challenge. (FGD4W3)

I think we needed a whole new birthing centre with postnatal rooms for low risk women. And probably you need an ICU unit which is high risk [but] Separate from that whole. (FGD2MW2)

Organisational contexts were also of concern regarding the number of staff available to postnatal hospital wards. Midwives in the FGDs complained of burn-outs due to the relatively limited number available to attend to many women. An holistic approach is required to address the 
postnatal workforce deficit which continues to impact on quality postnatal care for recovery women and ultimately wellbeing (Crawford, Lilo, Stone, \& Yates, 2008; Forster et al., 2006).

There is a staffing issue. Not being able to choose because we used to have two midwives in one of the postnatal areas in PodC. And now we've lost 5 beds space. (FGD2MW2)

I guess it makes sense to have it ..specifically...having community maternity homes that kind of thing... when you have a sort of separate staff they don't have to operate on you. But they may be just midwives rather than all the doctors and everything else's as well. (FGD4W2)

\subsection{Conclusion}

This Chapter has detailed the findings of the FGDs comprising 14 informants. The focus groups were assembled as a follow-up to the results of surveys of users' perceptions of design factors in phase one of this study. The findings provided explanations as to why some of the design features and building spaces identified and ranked by users were important for the recovery experiences of women during the postnatal phase. They also provide insights regarding why midwives are increasingly finding it challenging in providing quality postnatal care with respect to inadequate spaces and staffing issues, aligning with findings in the literature. In the next chapter, the phase one and phase two findings are integrated and interpreted and their results presented, leading to formulation of design guidelines for postnatal restorative spaces in urban tertiary hospitals. 


\section{CHAPTER 10}

\section{FACILITATING RESTORATIVE SPACES IN URBAN TERTIARY HOSPITAL ENVIRONMENT}

The purpose of this two-phase research project was to gain an understanding of the design factors and building spaces influencing women's recovery experiences and to develop evidence-based design guidelines for restorative postnatal spaces for urban tertiary hospitals under three key questions: What are the perceptions of design factors influencing women's recovery experience and wellbeing during postnatal hospital stays? What were the ways in which the design factors and the postnatal spaces affected women's recovery experiences and wellbeing during hospital stays? How can the findings be applied in proposing design guidelines to assist quality postnatal care in large, urban tertiary hospitals? This chapter presents the integration of the results of phase one questionnaires and the phase two focus groups findings, followed by a brief discussion. It concludes by proposing design guidelines for postnatal restorative spaces in large hospitals

\subsection{Conclusions on perception of design factors questionnaire surveys and focus groups}

The quantitative data from the users' perception questionnaire surveys revealed a number of significant design factors (Chapters 5, 6, and 7). The results depict the extent to which healthcare physical environments are important for providing settings for postnatal care and in facilitating recovery experiences of women. The fifteen highest-ranked design factors reveal mean scores greater than 4 on a scale of 1 to 5 (see Tables 5-7 and 6-7).

Items highly ranked by both women and midwives include hygienic/clean environment, a restful room, room that feels quiet, adequate natural daylighting, and view to nature, shapes and form for postnatal room design, air quality and room freshness and communal space for the learning of parental skills. The questionnaire surveys also confirmed furniture made of natural materials was relatively important with respect to recovery experiences and caregiving in postnatal environments. However, a few aspects of design factors such as single-bed room for recovery, positive distractions, noise control from medical equipment, temperature control, adequate daylighting in the room and accessibility of room to the outdoors - even though they received mean scores 
higher than 3 and 4 in some instances (see Tables 7-1 to 7-4; and 8-2) revealed statistically significant differences between the postnatal women and midwives. Variations in the results need to be accounted for in terms of how they might inform the overall findings. Because of this, the phase two study was used to follow up and subsequent integration of the focus group findings enabled understanding and insights which are addressed below.

Regarding single-bed rooms, while ninety-eight percent of women $(\mathrm{n}=208 / 212)$ ranked a singlebed room as very important for recovery, only seventy-eight percent $(n=37 / 50)$ of midwives did so. Both results suggest positive acceptance, the difference is that both have rated single-bed rooms to suit their respective needs. The findings from the FGDs gave three plausible explanations.

First, providing space that allows families to stay together supported emotional and social comfort, coping with the stress and tasks of the arrival of new baby and subsequent parenting suggesting single-bed rooms rather than multi-bed have significant impact on how women manage "loneliness" with a new baby obtain emotional and psychological support, and experience satisfaction and wellbeing (Curtis \& Northcott, 2017; Janssen et al., 2000). Lack of privacy is a recurring theme in comments common in healthcare settings (Karro et al., 2005) including postnatal areas making single-bed room most suitable for increased privacy, and reduction in noise levels. Ambient environment factors are important indicators in overall satisfaction (Ferri et al., 2015; Harris et al., 2002).

Secondly, the questionnaires revealed that hospital postnatal environments were perceived as inappropriate settings for recovery largely because of the noise and busyness of the environment. In this study, all participants were very supportive of ability to control noise in the postnatal environment, which had impact on recovery experiences and wellbeing. Existing studies have shown that measures to promote more sleep, enhance restorative environments and facilitate women's and staff hospital experience and wellbeing (Cunha \& Silva, 2015a; Dube et al., 2008; Hinkulow, 2014) as already discussed in Chapter two.

Both questionnaires and FGDs demonstrated the importance of having adequate daylighting in the postnatal room. The virtually unanimous response suggests the value of visual environments and sensory stimulation. Sensory stimulation was stressed in the report of FGDs in Chapter 9 to promote opportunities to "see the sky" and "see the outside". The rhythmic sensation of day and night is an essential element in recuperation, appreciated for recovery experiences in hospital settings. This is a significant finding of this current study distinct from the labour and birth-related environments studies where women desire rooms that are dark and warm, discouraging open 
windows in the room or exposure to the outside world (Aburas et al., 2017; Newburn \& Singh, 2003). While both women and midwives have ranked this feature highly (see Tables 5-3 and 6-2), there is a plausible reason for midwives ranking adequate daylighting higher as they need more natural lighting to perform tasks more than women would need it; hospital is a work environment for care providers whereas women spend relatively short time in hospitals.

The major problem identified was the lack of space for partners or family members to stay the night coupled with a lack of outdoor space in the design of postnatal facilities that women could go to for outdoor activities. A significant theme of spaces for partners dominated the responses to the open questions (see sections 5.8.1.3 and 6.6.1) lending credence to the need for spatial considerations for birth partners and supporters (Lawal \& Vale, 2018). Additionally, outdoor contact is beneficial for physical and psychosocial wellbeing. Providing opportunity of access to a garden and outdoor space could encourage new mothers and other families to come together and share their birth stories (Callister, 2004; Savage, 2001) because birth is a social event, integrating social networks for enhanced wellbeing (Walsh \& Newburn, 2002). While social support is very important for patients and women in this context, staff may not be able to provide this role (Tarkka et al., 2003) because nurses and midwives have tight busy schedules in hospitals.

Recovery experience in hospitals fosters relationships between women and midwives. Regardless of some findings (Tarkka et al., 2003), many midwives supported women with information and learning parental skills in the immediate postnatal period. Although women may require individualised care, dependant on organisations' models of care and contexts, midwives looked after care and wellbeing of women. Midwives wanted home-like features for women and access to natural environments and pleasant atmosphere for recovery experiences and wellbeing of women and outdoor experience after the eventful moment of birth.

Finally, both the survey questionnaires and the FGDs indicated that this current research was successful and the combined user group offered strong insights about design factors in hospital physical environments influencing recovery experiences and wellbeing.

\subsection{Discussion of perception of design factors questionnaire surveys and focus groups}

In the previous section, conclusions of questionnaires and focus group findings were described and possible explanations have been offered regarding the variance in the results found in some design factors. Linking the results of quantitative surveys from phase one and qualitative FGDs in 
phase two enabled greater understanding and insights that could be useful for improvements in postnatal hospital environments and in future research. This section presents the discussion of findings of the two phase research by considering the questions asked at the beginning of the research

\subsubsection{What are the perceptions of design factors influencing women's recovery experience during postnatal hospital stays}

Based on the analysed results in the previous chapters on the four categories of the questionnaires and the four factor solution adopted for the 25-item questionnaire for the principal component analysis (PCA), significant evidence was found under the four factors: interior restorative, environmental, spatial and maintenance. Details are given below.

A brief discussion of the descriptive statistics on the perception of design factors questionnaires is first presented. Overall, the perception of the factors; sensory comfort $(\mathrm{M}=4.21,4.53)$ and postnatal room features $(M=4.12,4.10)$ had the highest mean scores for women and midwives respectively. Average mean scores for interior environment were moderately high $(\mathrm{M}=3.53,3.55)$ and slightly lower for the social comfort environment $(M=3.36,3.71)$ based on the Likert-type scale from 1 to 5 , depicting ranking from not at all important to very important. Interestingly, the mean scores received from women and midwives show marked similarities in all four categories.

The exploratory factor analysis (EFA) was undertaken using the PCA on the 25-item questionnaire as previously discussed in Sections 7.5 and 7.6, average mean scores were also calculated for the four Factors components comprising interior restorative (3.25), environmental (4.19), spatial (4.62) and maintenance (4.09). The overall mean statistics suggest that both women and midwives have higher positive rating of the four new scales or factors. The independent $t$-tests carried out on these new four scales found a significant difference in perception in five out of nine items for the interior restorative factor; five from environmental; and one each from spatial and maintenance attributes. The perception of decoration of room with natural art and images; pleasantness of room with natural things such as aquariums, nature sounds of birds; bright colours in the room; the arrangement of furniture and décor in communal space; and positive distractions were statistically significant for the interior restorative component. Similarly all five items of the environmental attributes revealed a strong level of significance, $\mathrm{p}<0.001$ suggesting the importance of calm, restful and comfortable hospital settings for recovery experiences. Moreover, the perceptions of the factors; single-bed room occupancy and a clinical postnatal room were statistically significant for spatial restorativeness and maintenance components. 
When it comes to things that drive patient/user experience in a hospital, single-bed room occupancy trumps other considerations because it represents a pathway to other environmental support. Many studies have shown the importance of a provision of single room accommodation in hospital settings for both patients and clinicians with benefits including privacy and dignity, satisfaction with care, personal control, reducing adverse clinical errors and infection control (Chaudhury, Mahmood, \& Valente, 2006; NHS Estates, 2005; Ulrich et al., 2008). Questionnaire responses also confirmed that a clinical room was not favourably received, however, differences were statistically significant with women ranking a clinical room or ward $(M=2.34)$ higher than the midwives $(\mathrm{M}=1.84)$ meaning that the presence of medical equipment may need to some degree to be available in event of medical needs for care of postnatal women. The PCA and inferential statistics results and their discussion have been presented in detail in Chapters 7 and 8.

From the qualitative focus group discussions (FGDs) in Chapter 9, three main categories emerged comprising recovery experience with different spatial units; elusive restfulness; and mutual role integration comprised of sub-categories, such as mixed sensory stimulation, adapting to familiar noise, sensing the space, it feels like a hospital and humanising the space.

The integration of the quantitative and qualitative data allowed explanation of the differences and commonalities with the quantitative data from the perception of the design factor surveys. Combining the four factors of the PCA attributes in the perception of design factors questionnaires and linking them with the themes generated in the FGDs provided further explanations and highlighted the importance of the architectural factors and building spaces for the recovery experiences and wellbeing of women in the postnatal phase. The next section presents a discussion of the phase two qualitative FGDs insights and explanation of the quantitative survey from phase one.

\subsubsection{What were the ways in which the design factors and the postnatal spaces affected women's recovery experiences and wellbeing during hospital stays}

From the categories which emerged from the qualitative focus group discussions (FGDs), the perception of the design factors influencing women's recovery experiences in the immediate postnatal phase are depicted and explained in terms of the recovery experiences in the spatial units, elusive restfulness, and mutual role integration - that is to say, the recognition and integration of the restorative space with care giving. Figure 10-1 shows the users' perception and understanding of the healthcare physical environment's role in enhancing the restorative experience including how these aspects may contribute to quality postnatal healthcare. 


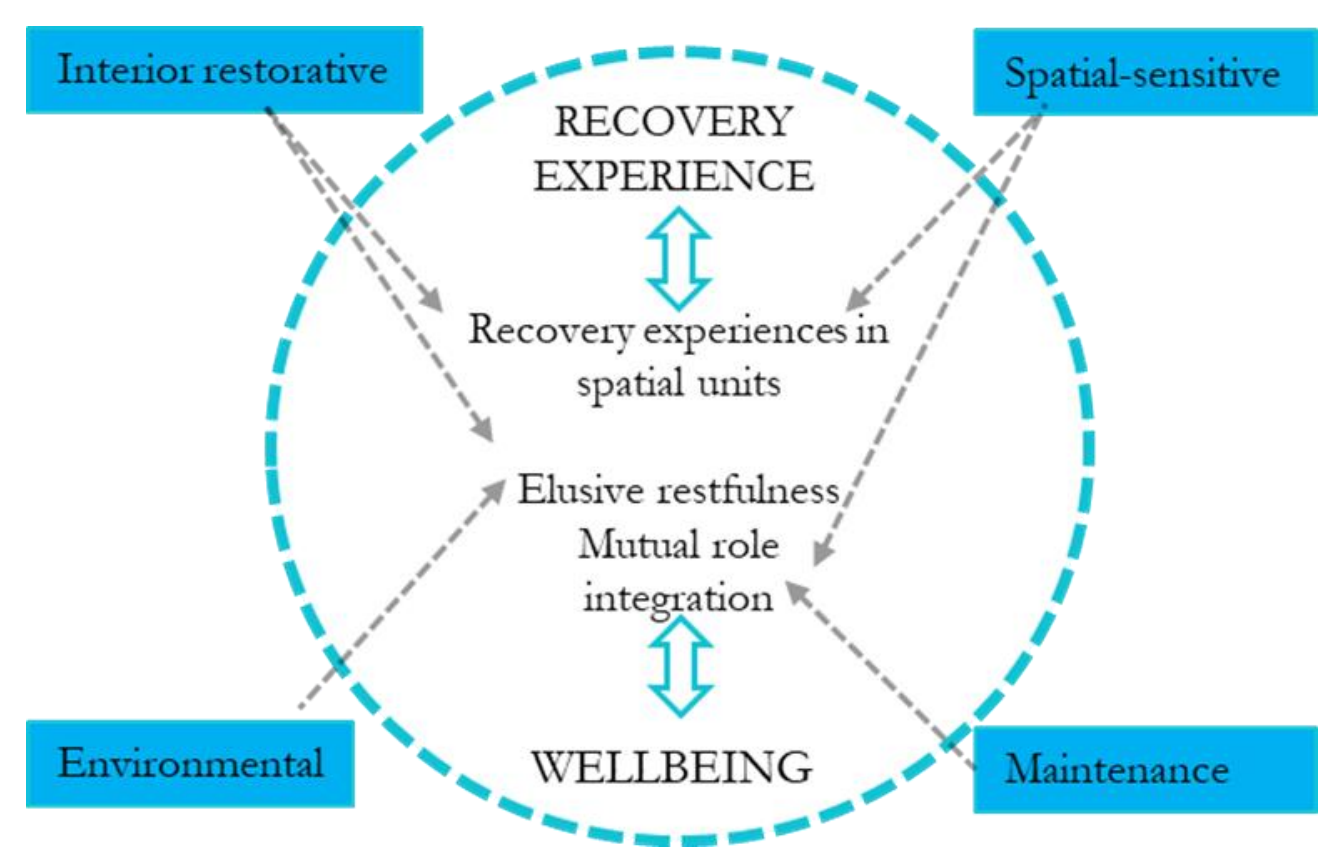

Figure 10-1: Linking of quantitative attributes to qualitative categories, adapted from (Buck et al., 2009)

As Figure 10-1 revealed, the linking of the quantitative survey data on perception of postnatal hospital space with the qualitative focus groups indicates how the qualitative categories from the FGDs are linked with the sub-categories and to the quantitative attributes (see Figure 10-2 for details). The results of the quantitative perception of design factors surveys also revealed that both the postnatal women and midwives had positive ratings of all the four categories apart from a few factors that were not significant.

The major concerns from the findings of the surveys and the FGDs can be summed up as the need for 1 ) restorative atmosphere and spaces; 2 ) direct visual access to the outdoors and adequate daylight in the room; 3) spatial sensitive spaces that optimise for sleep and rest; 4) accommodating partners/family members in the care and 5) outdoor environments to facilitate "normalised" resumption of activity of daily life. 


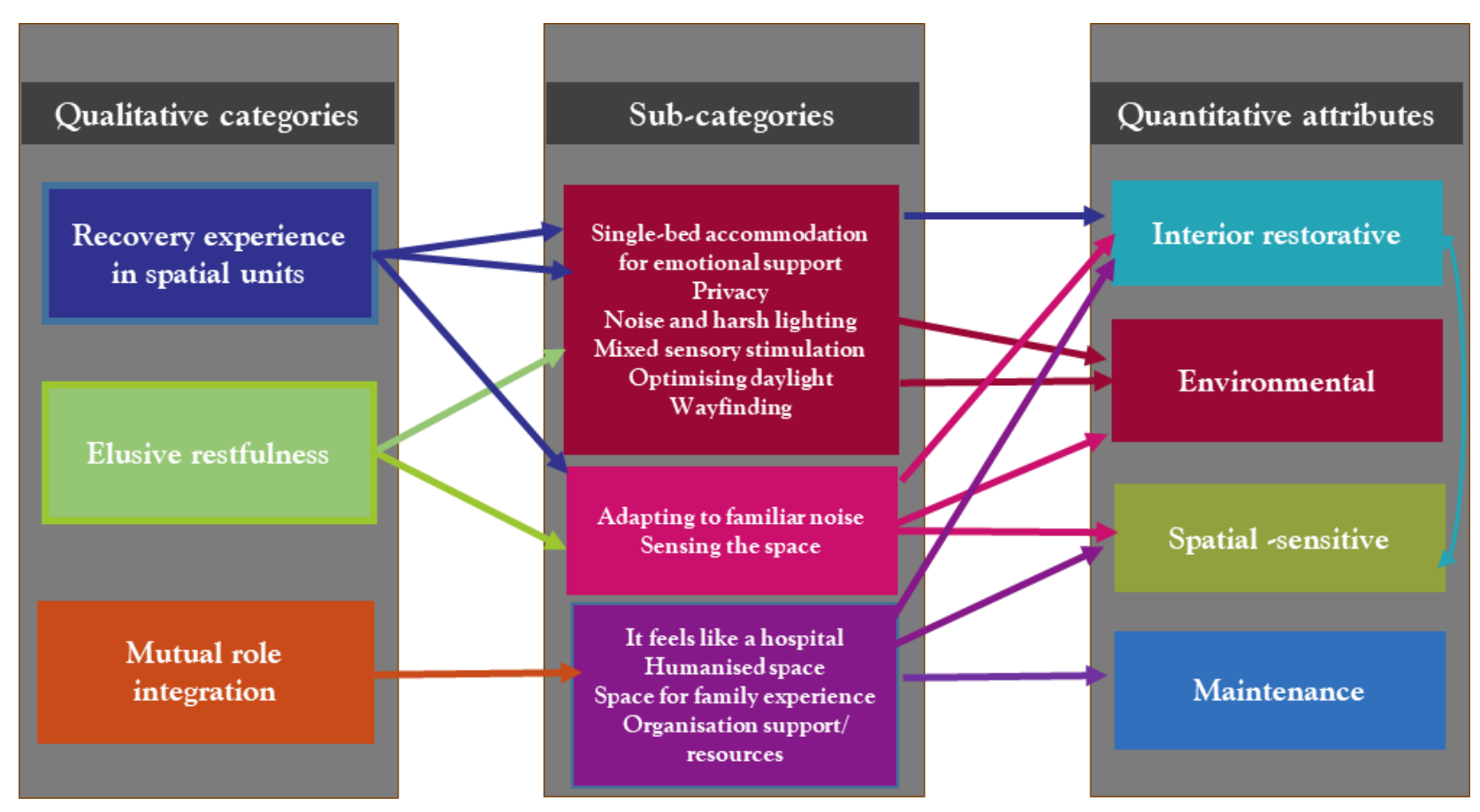

Figure 10-2: Mixed methods findings linking quantitative and qualitative data

\subsection{Implications for design guidelines to assist quality postnatal care in large, tertiary hospitals}

\subsubsection{Woman as patient in wellbeing studies - Single-bedroom allocation in healthcare}

The patient room may be the point that a patient or her family are most concerned about in a hospital next in line to care. Being in the healthcare environment translates to a patient leaving their private comfort zone to a public zone where the patient may be asked for personal information related to medical history and treatments which needs to be kept private. The patient lacking access to a single-bed room may become uneasy and stressful with the presence of other patients. For this reason, single versus multi-bed rooms for the patient hospital experience are widely studied areas in healthcare design research (Ulrich et al., 2008; Young \& Yarandipour, 2007). An exhaustive review of empirical studies on single-patient rooms has found many benefits of single-bed rooms such as reduced hospital acquired infection, reduced noise levels and privacy (Chaudhury et al., 2005; Detsky \& Etchells, 2008; van de Glind et al., 2007).

The findings of this current study have found that a single bed-room option was of utmost concern to many women for their postnatal care and strongly linked to families' involvement in the care of new mothers and their babies. Women desire much sleep and rest following the birth, multi-bed 
rooms could be stressful to supporting the need for rest and limiting involvement of women in patient/woman centred care in maternity hospitals (De Labrusse et al., 2016). Lending credence to single-bed rooms, one of the midwives stated:

... I asked the woman does she want to go home or does she wants to go to double room. She chose...she wants to go home she doesn't want the double room. So if she would have chosen to stay at the hospital without the single room that could affect her care. FGD2MW3

While a new mother said:

As a new parent, imagine sharing a room with another parent maybe when you just finally gets your baby to sleep and you want to [have] some sleep and the next baby starts to cry. All of you are now awake. So you just don't get any sleep at all...I had to tell them - can I get a private room. FGD1W2

This study has revealed that single-bed rooms can offer a supportive environment for the recovery experiences of women and wellbeing in care giving. Previous studies in the childbirth environment have generally focused on the physical environment needs of the labour and birth rooms (Newburn \& Singh, 2005), a "Snoezelen" room (Hauck et al., 2008), standard labour room and "ambient room" (Hodnett et al., 2009), with the exception of a study focused on a single room for both intrapartum and postnatal maternity care (Janssen et al., 2000). In the wider healthcare environments research, numerous studies have examined single-bed versus multi-bed rooms. For example, research conducted in the UK at the University of Sheffield for NHS Estates using three case studies in three different hospitals indicated that patients' satisfaction was associated with privacy and ensuite toilet facilities. Single room provision was considered more important irrespective of the sizes of the rooms (NHS Estates, 2005). In the maternity setting, Janssen et al. (2000) found that single rooms were linked to higher satisfaction of women with maternity services.

In the USA, Verderber and Todd (2012) presented the case for a semiprivate inpatient room against the trends of an all-private room because of the latter's healthcare provision inadequacies set against the consistent calls for privacy and dignity of patients in healthcare settings (Verderber, 2010). Given the strengths and weaknesses attributed to all-private and semi-private rooms, Verderber and Todd's conclusion was that the future design of inpatient rooms should be coordinated aligning with advances in technology, care delivery and policies (Verderber \& Todd, 2012, p. 21). However, there are limited studies that have examined the impact of a single room 
on recovery and restorative experiences and wellbeing. Focusing on this aspect could be beneficial for future decisions about which kind of rooms should be prioritised. A recent exhaustive review of empirical studies on single occupancy patient rooms accorded support regarding their beneficial outcomes for healthcare users in general (Taylor et al., 2018). The findings of this current research are consistent with previous studies indicating patients and families show greater preference for single rooms, while interventions that allow room choices to be made can impact sleep, health and wellbeing (Harris et al., 2006; Jolley, 2005; Persson, Anderberg, \& Kristensson Ekwall, 2015; Pilkington, 2013; Taylor et al., 2018).

\subsubsection{Visual preference and views to the outdoors - Valuing sensory experience}

The study findings also linked the restorative experiences to the extent which the interior environments fostered positive stimulation (Ulrich, 1991). The interior healthcare environment could be contributory in terms of what it affords the occupants of the spaces to "buffer" stress. The recovery phase requires that women shift focus away from the happenings and the impact of unfamiliar physical environment. Access to daylight and windows and contact to the outdoors are vital for experiencing positive stimulation. According to the surveys and FGD responses from women and midwives, decoration (in art, images or paintings), natural things, colours in the rooms, adequate daylight, positive distractions and access to the outdoors can foster the shifting of focus and reconnecting to being in home-like situations. These elements can be potential surrogates in the postnatal spaces in much the same way as "fascination" which the Kaplans have proven to have potential influence in restoring directed attention fatigue (DAF) and stress recovery, thus facilitating restoration process (Kaplan \& Kaplan, 1989; Kaplan, 1995). The arrays of affective and aesthetic meanings in the physical environment, especially when the postnatal women have opportunity for views to the outdoors could result in reduced stress and foster wellbeing (Ulrich, 1983, 1984). Women and midwives in this research alluded to the value of sensory stimulation essentially lacking when women in particular, are limited by the inability to see the sky because:

When you are looking through the window, you are just seeing the roof of another house [building]. A patient would want to see outside. FGD1W3

Even if to look out of window...To get any sense of daylight, you have to be absolutely public. That would drive you crazy if you are going to spend more half a day. FGD3W2 
The use of natural features and images was termed positive distractions both in Ulrich's Theory of Supportive Design (Ulrich, 1991) and by Shepley (2006). Shepley explains that positive distractions will "allow the individual to shift focus from negative foci within the health environment to the more restorative aspects of the non-medical world" (Shepley, 2006, p. 34). Logically, being closer to the windows in healthcare settings is a factor of type of room accommodation as shown in this study. Therefore, the room interiors may then become the only means for the women to have some "respite" and restorativeness in the postnatal environment.

Research suggests that phased and incremental development of healthcare settings may inadvertently alienate the users' visual-spatial sensory engagement with the external environment (Jiang \& Verderber, 2017). Empirical evidence found the visual environment, nature art and images and their links to positive distractions in healthcare settings were found to be beneficial for the physical and emotional wellbeing of patients in rehabilitation facilities such as the Sarah Network of rehabilitation hospitals (Campagnol \& Shepley, 2014) including the Rehab Basel centre (REHAB Basel, n.d.).

In parallel, the complementary use of art, colour, nature and images in interior healthcare environments such as in the Neonatal Intensive Care Units (NICUs), have been suggested for their potential stress-reduction role as positive distractions and may have beneficial physiological health outcomes (Shepley, 2006). Research findings from this current study have indicated that women and midwives have positive preference for a postnatal interior environment that has a blend of natural images, decoration in the rooms and colours including the value of a view to the outdoors for achieving comfortable and relaxing settings for the overall postnatal recovery experiences and wellbeing. The findings of this current research are consistent with Pati and Nanda's (2011) study in a paediatric tertiary healthcare setting which found positive distractions were associated with calmed behaviours of patients in the waiting areas. Devlin et al. (2016) who asked 236 patients to list three aspects of their rooms for quality hospital stay found that positive distractions $(33.2 \%)$ ranked higher for the patient experience above perceived control $(22.4 \%)$ and social support $(6 \%)$ suggesting that the overall ambience was more important. Such exemplars utilising aesthetic qualities of nature images and their surrogates in the interior healthcare environments and direct views to nature in the outdoors confirmed that their application in the postnatal environments could positively impact on the recovery experience of women who spend time or have extended postnatal hospital stays. 


\subsubsection{Spatial sensitivity and tranquility - restorative design of postnatal spaces}

The audio environment is crucial for sleep and rest in the immediate postnatal phase. Most of the respondents, with few exceptions, in this study were frustrated, felt helpless and concerned with the noisy situations of the postnatal environment. Interestingly, all the ambient environment indicators such as noise control show a strong level of significance, $p<0.001$ (see Chapter 7).

As discussed in Chapter two, environmental noise-reduction strategies in healthcare research fall into three broad areas (Ulrich et al., 2010). Beyond these environmental control measures, the findings of this research indicate that a few women exercise a behaviour approach such as the use of ear-plugs and eye masks that act to cushioning the seemingly environmental stressors, such as unwanted noise and light, coming from the spaces. The findings echo previous research that found sound masking as the most effect technique for improving sleep (Xie, Kang, \& Mills, 2009). On the other hand, the lack of respite for sleep in the postnatal environment led some women to return home although to rest in the hospital afterwards had informed their decisions to give birth in hospitals.

... I was so exhausted that my sister said: put in some ear plugs. Wear eye masking. Go to sleep. And that was quite good. Because it brought down all the noising and the lightings. FGD4W3

We didn't see any reason to go to the hospital. The pregnancy was brilliant. We thought: Oh yeah! Let's go somewhere else for change of scene... we would stay [t]here for a lot of sleeping and then go home. It became chaotic. Because it wasn't a comfortable kind of place to rest and especially for my partner as well. FGD3W1

The need for sleep after the birth was considered to be the most essential part for mental, physical and emotional recuperation which women hold very dear, above information and education in parental skills (Emmanuel et al., 2001). It can be argued that when a room or space promotes tranquility with discussions kept at a low ebb, such space can facilitate sleep more readily. Existing literature has identified efforts taken by care providers to improving the audio environment to create a restful and healing hospital environment (Boehm \& Morast, 2009; Hinkulow, 2014). Cunha and colleague found that subjective wellbeing is influenced specifically by the noise from clinical sources (i.e. monitors and medical equipment) including physiological and psychological disturbances in patients (Cunha \& Silva, 2015a). Beyond these findings, the combined results from assessment of the environmental factors and the FGDs indicate that high priority needs to be 
given to the sensitive and private moments during the postnatal phase. However, it may be important to design a postnatal environment that can be restorative for wellbeing of women whilst allowing care providers to do their job effectively.

\subsubsection{Access to nature and daylight - designing for outdoor experience}

Hospital stays may result in boredom. Engaging in outdoor activities may serve as an opportunity of breaking away from the "boredom" of the interior hospital environment to an exterior environment where the users of the health facilities may be immersed in nature for their mental and physical wellbeing. The notion of "person-nature transactions" in healthcare facilities has been identified as a core embodiment of the larger concept of patient-centred design related to health and wellbeing outcomes of the users (Verderber \& Fine, 2000, p. 346). The findings in this research have established that postnatal restorative experience and wellbeing can also be linked to contact to the outdoors/nature and adequate daylight. The postnatal women and midwives stated that optimising daylight was crucial for the newborns' health in preventing jaundice. In addition, the women preferred outdoor spaces that they could go to rather than remaining in bed. Existing research has studied the role of daylighting and healthcare outcomes, with daylight linked to higher satisfaction of patients (Gharaveis et al., 2016). Moreover, a very recent study concluded that daylighting and view to the outside enhances nurses' perceptions with regard to satisfaction and alertness at work (Gharaveis et al., 2020).

The findings from this research also indicated that having an accessible garden in the healthcare setting particularly in the postnatal area clearly demonstrates a positive way for confidence building after the experience of childbirth. One woman describes it this way:

Suddenly it's not just you, you've got a little baby. And it's quite traumatic to your body. So being able to walk around just builds up like inner strength and confidence. ...it would be great to have like the maternity postnatal space connected to the outdoors. Walking out to see gardens where you're still able to have a bit of privacy. FGD4W3

The midwives are unanimuous that the provision of outodoor space and exposure to nature could be beneficial to women for enhanced positive recovery experience and wellbeing.

[Provisions of] natural daylight or artificial lighting, access to outside and access to fresh air. FGD2MW1-4 
In conclusion, findings have revealed that single-bed room spaces, views to the outdoors (sensory experience), tranquility (restorative spaces) and access to nature and daylight (designing for outdoor experience) could be important parameters and aspects to promote recovery experiences and wellbeing of women during postnatal hospital stays. The findings can be summed up as three design factors: spatial-sensitivity; interior restorative spaces; and humanised outdoor spaces.

\subsection{Design guidelines}

At the beginning of this research, the aim was stated to gain an understanding of the design factors and building spaces influencing women's recovery experiences and to develop evidence-based design guidelines for restorative postnatal spaces in urban tertiary hospitals. The questionnaires developed for this current study were focused on design elements/factors informed by existing research into childbirth and general healthcare environments. Potentially they may have limited application in the postnatal environment given the different physiological, emotional and psychosocial needs of women in the immediate postnatal period. Consequently, gaining insights about these design factors in the postnatal physical environment differentiate it from the birthroom/delivery spaces.

As an outcome of the process of this two-phase research a set of guidelines have been generated which can serve to facilitate a supporting environment for the recovery experiences and wellbeing of new mothers during hospitalisation. This section summarises the list of the design guidelines which can be applied in the design and planning of restorative hospital postnatal spaces.

The guidelines are presented in the following sections.

\subsubsection{Women's inpatient postnatal / spatial units}

\section{$\underline{\text { Single-bed room, private space to allow parents-infants bonding }}$}

As much as possible, consider providing an all single-bed units in the postnatal ward to support postnatal recovery experiences of the new mothers, infants and family. The provision of a single room offered during the sensitive period post-birth allows the family not only to bond as a family unit but also allows for privacy and fostering of woman-infant-parent bonding.

- Single rooms are superior to multi-bed/open-bay rooms as enhancers for women's recovery experiences and wellbeing, largely for their role in the emotional, sensitive and vulnerable time as new mothers adjust to the reality of parenthood. 
- Where it is impossible to have an all single-bed rooms, consider a 2-person bedroom (with ensuite bathroom) but not open wards or multi-bed rooms with several beds.

- Support family-centred care approach by providing temporary fold-up/down beds for the birth partners, husbands and family members for overnight sleep. The husband or an assisting family member could make use of this makeshift bed while assisting the new mother to sleep and get the rest especially during the first few hours and days after the birth for her recovery and wellbeing. The makeshift bed could be concealed or hidden into the walls and brought out when there is a need for it without forming clutter in the space.

- Offer dedicated seats in the rooms to allow birth partners and visitors of the postnatal women to have somewhere to sit down and support the new mothers.

- Provide a dedicated storage space for women's belongings and sufficient storage for equipment carts with clearance from door swings for dignity of inhabitants of the space.

- Make the door self-closing in the postnatal wards/room for privacy and fitted with with sound-proofing.

- Create a short, private dedicated corridor before arriving/entering into a woman's room. However, this should not eliminate the normal courtesy requirement to "knock" before entering into a postnatal room.

- Consider the deliberate use of design forms and elements in future design of hospital postnatal wards - curvilinear forms rather than rectilinear forms - that are inviting and can arouse positive feelings, interests and calmness.

\subsubsection{Interior ambience (restorative)}

Regarding the interior ambience, there is a need to pay attention to the space in terms of how relaxing and comfortable for sleep and rest it is, including its overall pleasantness. The following design principles are proposed.

\section{Quiet, private and restorative space}

Consider providing computerised room-sensor light alarms, which can be positioned on the doors with display monitors in the midwives/nurses' stations to show when women are sleeping and should not be disturbed. This will serve to optimise sleep and adequate rest for the new mothers and regulate "interference" from healthcare providers. As noted in Chapter 2, the provision of noise-sensor alarms both in the neonatal intensive care unit and operating theatres has been shown 
to be beneficial for reducing noise levels and support restoration of patients (Chang et al., 2006; Ukegjini et al., 2020) and support for woman-centred care.

- Acoustically insulated wall partitions rather than flexible or curtain partitions and soundproofed doors are recommended for room separation to reduce noise levels and facilitate adequate sleep and restoration.

- Provide women access to temperature controls in the room or have a designated area where temperature can be easily controlled to suit the conditions of each postnatal woman.

- Offer the individual postnatal woman lighting controls adjacent to or near the bed-side wall.

- Provide access to natural light to reach the bedside of the woman

- Women's sense of wellbeing and recovery are linked to visual quality of the room (i.e. material finishes and furnishings, soothing colours), access to the outdoors and proximity to windows; these preferences hold the promise for enhancing visual stimulation, moods, relaxation and lower stress levels. The following guidelines are proposed.

- Provide postnatal women with views outdoors through windows in the rooms for sensory stimulation, to provide a positive psychological effect and emotional wellbeing. In the case of shared rooms, where this is not feasible, natural light can be introduced in the postnatal space by means of skylights, supplemented with rich arrays of natural images, art or paintings as a positive distraction.

- Contextual art, images or painting should be relaxing for the emotional wellbeing of the women and their families. Where possible, introduce images of nature and art for stressreducing effect and relaxation, replenishing women emotionally while lying in bed as an important aspect of providing access to nature as a means of positive distraction. This idea resonates well with a Snoezelen room concept for women in childbirth (Hauck et al., 2008) and also with the finding of the benefits of simulated nature images for improved patients' physiological ratings and health outcomes (Pati, Freier, et al., 2016).

- Access to music which is enjoyed by each woman may serve as positive distraction and may be likely soothing for the new mothers. However, such music should be self-selected and maybe played based on individualised arrangements on private headsets without constituting a disturbance to other parents in the postnatal ward. 
Aesthetically pleasing interior space utilising appropriate colours makes a difference to maternal recovery experience and wellbeing.

Colour use in the healthcare environments is somewhat controversial, with the exception of children's hospital environment where research has found specific types of colours to be effective (Coad \& Coad, 2008; Park \& Park, 2013). Importantly some aspects of colours have positive impact on people's mood and relaxation. Consider applying warm and soothing colours that support recovery in the interiors of postnatal environments. The provision of green colour symbolises healing, recovery and love (Biley, 1996) from which postnatal women could find harmony in the space. Also, the notion of emotional mapping for colours (Mazuch \& Stephen, 2005) is an important approach that could be beneficial for healthcare designers and architects who aim to design the environment for wellbeing support for the recovery of new mothers. Additionally, as noted previously, the types of colours for use in the postnatal environment wasnot explored in the questionnaire surveys, therefore the opinions of postnatal women should be sought in the selection process regarding the choice of colours for the interiors of the postnatal environment.

\subsubsection{Support social and family-centred spaces}

There are two principal ways of fostering togetherness of families and relation with other mothers: 1) internal/interior space; and 2) external/outdoor areas. The first aspect uses a single room concept, which has already been discussed above. The second involves creating an accessible outdoor space that the new mothers and families and possibly staff could go into and socialise and meet other mothers as a way for a return to normal activities of daily living. Outdoor space with a garden allows women to meditate, relax and possibly get insightful moments while suspending other thoughts (momentarily) about catering for babies.

- Consider providing outdoor spaces, gardens with a small deck/patio attached to each room (with physical access from rooms) in the postnatal environment. In addition, provide seats (shaded with trees) where postnatal mothers could take their baby for breastfeeding and to relax in a comfortable environment. This takes the focus away from the institutional environment.

- Outdoor spaces should take into cognisance the qualities of safety and security, to ensure that the mothers and other users would not have anxious feelings while making use of the spaces. 
Furniture and décor layouts

The birth of a baby is a life-time experience for most women and especially to first-time mothers, which means women will have to learn and acquire parenting skills from other women and families. Provide purpose-made furniture and décor designed to support an interactive learning process as a group, on how to manage breastfeeding and information about hygiene, maternal health and wellbeing and newborns. A comfortable seating pattern in a lively atmosphere could also create the chance for women to share their birth and postnatal journeys and recovery experiences.

\subsubsection{Administrative and support spaces}

- Provide space that gives opportunity for practical and informative information and guidance on parental learning skills. In this regard, provision of a total environment for education that includes family support spaces (i.e communal spaces) and amenities for the learning of parental skills and interactions is recommended in large postnatal hospital environments.

- Make provision for kitchen space to prepare food/meals

- Due to fatigue during labour and birth, most women need nourishing food to assist in regaining energy and vitality and ability to have adequate breastfeeding of newborns.

- Support minimal distance walking from the birth suites to the postnatal wards. Ideally both should be on one level.

- One of the drawbacks of hospital design is its inherent focus on functions and efficiency of its workforce with minimal considerations for physical, emotional and humanistic dimensions of wellbeing. Regarding walking distance, a maternity unit should facilitate the ease of the new mother's transition from one room to another (although they may not have to walk themselves, but sometimes they do so). The postnatal environment design as well as its spatial links to the birthing suites should be stress-reducing and not stress-giving.

- Short walking distance between the birth rooms and postnatal spaces is helpful as this research found that using lifts/elevators post birth was stressful for women. Therefore, any form of barriers that may trigger stress and interfere with wellbeing should be avoided.

- Standalone, low-rise, residential-like facilities are preferred in design and planning of future postnatal wards for large, tertiary hospitals. A standalone facility for a special population, in this context women in postnatal care, is acceptable (Shepley \& Wilson, 1999). The facility should incorporate access to a garden or rooftop garden - with green trees and a decktype area for fresh air. The concept of green roofs or more accurately rooftop gardens, can "catalyse" a blending of healthcare with nature (Guenther \& Vittori, 2013, p. 67). 


\section{CHAPTER 11}

\section{CONCLUSIONS AND FUTURE RESEARCH}

This chapter presents the conclusions of a two phase explanatory sequential mixed methods research project extensively covered in this thesis. This study examines the nature of architectural characteristics and physical design factors that could influence recovery experience, physical restoration and wellbeing of women during the postnatal phase in large, tertiary hospitals. This Chapter summarises the research that was conducted and discusses its conclusions based on the findings reported in Chapters 5 to 10.

\subsection{Summary of research findings}

This current study was informed by the gaps in knowledge about physical environments for the recovery of women during a hospital stay. As such, this study has asked the question, how can postnatal hospital environments be restorative and conducive for postnatal recovery care and wellbeing in large, tertiary hospitals? Although evidence-based research studies on healing environments are being carried out and are accumulating over the years, there are limited studies focused on physical environmental needs of women during the postnatal phase. This research is an effort to fill the gaps in knowledge.

The research was conducted in New Zealand with a particular focus on large, tertiary hospitals. An explanatory sequential mixed methods approach was taken to the research comprising two phases each with its own methods. Phase one of the study utilised online questionnaire surveys to examine the users' perceptions of design factors in the postnatal environment. The questionnaires were completed by 212 women and 50 midwives using Qualtrics. In phase two, focus groups of three to four members (fourteen in total), were assembled in four different sessions to discuss the results of phase one and to provide explanations for the physical design factors/features in the physical environment that were considered to support or hinder women's physical restoration and recovery experiences in the postnatal phase.

The findings from this research have moved the discourse from the traditional labour and birth rooms research in the childbirth environment. 


\subsection{Contribution to knowledge}

This research has its uniqueness in the sense that this is the first time research has focused exclusively on the healthcare physical environment related to women's needs in the immediate postnatal phase. Despite limited existing research and increasing concerns regarding physical environment to meet quality postnatal care, no studies have been undertaken to date particularly from the architectural standpoint with an evidence-based design research focus. Nevertheless, there are some limitations in this study which need to be stated so that future research endeavours may consider them worth looking into.

\subsection{Limitations}

A possible limitation of this research, is that self-created survey questionnaires were used. With any survey instrument, issues of validity, reliability and generalisability are bound to arise and the rigour in the data must be considered. However, the survey questionnaire formation and development went through standard procedures and questionnaires were pilot-tested among both experts and postnatal women before being administered to the research participants. Another possible limitation is that only one hospital was used for this research. Taking multiple cases and hospital settings could not only have resulted in larger samples but also in different perspectives to the results. Regardless, the large sample size of women and midwives in this study was adequate for standard statistical analysis for drawing valid conclusions.

Another limitation is that hospital-based nurses and physcians (obstetricians and paediatricians) were not included in this research, so the ratings of the physical environment and design factors for the postnatal environment may have been partial towards the bent of women and midwives and may limit views regarding wellbeing and physical-environment transactions from other healthcare groups.

In addition, this research did not discriminate between the mode of birth for women who are lowrisk with uncomplicated vaginal deliveries compared with high-risk women with intervention births or ceaserean sections, which may show differences, commonalities or similarities regarding their perceptions on the design factors influencing recovery experiences and wellbeing.

Because postnatal women and midwives in the current study sample and their scores were not calculated to know who had rated the physical environment higher or lower to inform those to be invited in phase two focus group discussions (FGDs) it is likely that unmeasured variables may 
have influenced the selection into the focus groups. Needless to say participation in the focus group was based on willingness and self-selecting of participants themselves. This also means that participants who ranked the physical environment more highly for recovery and wellbeing but could not continue with Phase 2 FGDs may have been unintentionally excluded from the small, convenience sample of focus group participants. Notwithstanding, the opinions of the FGD key informants when matched with open-ended responses in the surveys showed that those who were passionate and had important information to contribute attended the focus groups.

Finally, probably the main limitation is the absence of visual cues and representations of the design factors and architectural characteristics in the present research. It is possible that the provision of specific visual cues and design elements may have yielded different results, the routines of the postnatal environment, including the short postnatal hospital stay, were barriers to their use. However, the findings of this mixed methods approach carry much promise for visual materials which could be used in future research to allow rigorous empirical testing using different design prototypes with research subjects in many hospital settings.

Lastly and importantly too, there is a need for studies to devise innovative methodology that might be used to study participants in the postnatal hospital environment.

\subsection{Future research}

The restoration and recovery values in this study identified important architectural factors and building spaces as found by women and midwives that could have potential application in both design of a new building and optimising existing postnatal spaces in large tertiary hospitals.

At the outset of this research was the motivation to examine how restorative spaces could impact recovery experiences of women giving birth in Nigerian and New Zealand hospitals. Generally, hospital architecture reflects a similar international style globally. Different models of care exist from one maternity hospital to another especially in Nigeria and other non-western countries. Although spaces that recognise different cultural norms and practices were alluded to in the findings of this research, they were not accounted for in this present study's scope. However, it could be interesting to investigate the findings in other hospital contexts with postnatal women and nurses-midwives - using the same or similar questionnaires and approach - to see if the findings are universal or have cultural differences in future studies.

For examples, future research could investigate the findings with women of different cultural backgrounds to ascertain if the provision of 
1) restorative atmosphere and spaces;

2) direct visual access to the outdoors and adequate daylight in the room;

3) spatial-sensitivity of spaces that opmitise for sleep and rest;

4) accommodating partners/family members in the care and

5) outdoor environments to facilitate "normalised" resumption of activity of daily life (overall findings - see Section 9.22)

could benefit women not just as users of maternity facilities but could also contribute to improved birth and recovery outcomes in general especially in Sub-Saharan African countries where maternal mortality rate is high (UNICEF \& World Bank, 2015).

In addition, experimental studies in future studies could examine the effect of environmental interventions such as soundproofed single rooms as stress-reducing measures versus traditionally designed hospital rooms on sleep deprivation and sleep quality and wellbeing particularly for women with longer hospital stays in large tertiary hospitals. Additionally, research might investigate the acceptable noise levels for postnatal hospital environment as in a study done for the NICU (Knutson, 2013).

The study also found natural daylight a strong contributor for sensory stimulation in the postnatal environment in promoting recovery experiences as well as a positive mood enhancer. The views of the key informants in this study were that if multi-bed rooms (with limited or lack of natural daylight) could be rearranged, further studies could investigate the impact of such arrangement in the room and impact on women's recovery experiences and wellbeing.

The increasing recognition of women-family centred care and the role of birth partners, family and friends has emerged as being of high value as a form of social support during the sensitive and vulnerable time of recovering from trauma of birth. Further research could investigate which specific design features of communal facilities and interior design might encourage more interaction and the learning of parental skills and increased satisfaction with postnatal recovery care during the postnatal phase. 


\subsection{Concluding thoughts}

Physical built healthcare facilities are increasingly becoming relevant tools for promoting positive hospital experiences and patient-centred care in general healthcare and especially in the maternity care domain. Women and caregivers have clear preferences for design factors and building spaces which could influence physical restoration and wellbeing of women, and potentially facilitate space for healthcare providers in effective caregiving. Knowledge gained from this research may assist women who give birth and undergo the postnatal recovery phase in large, tertiary hospitals. The preliminary guidelines proposed in this research may be applied in postnatal ward design and for optimising existing ones. 


\section{REFERENCES}

Aburas, R. A. (2014). Examining the influence of nature stimulus in enhancing labor experience in LDR units. PhD Thesis. Interior and Environmental Design. Texas Tech University.

Aburas, R. A., Pati, D., Casanova, R., \& Adams, N. G. (2017). The influence of nature stimulus in enhancing the birth experience. HERD: Health Environments Research \& Design Journal, 10(2), 81-100.

Adatia, S., Law, S., \& Haggerty, J. (2014). Room for improvement: Noise on a maternity ward. BMC health services research, 14(1), 604.

Akansel, N., \& Kaymakçi, Ş. (2008). Effects of intensive care unit noise on patients: A study on coronary artery bypass graft surgery patients. Journal of Clinical Nursing, 17(12), 1581-1590.

Alexander, C., Ishikawa, S., Silverstein, M., Jacobson, M., Fiksdahi-King, I., \& Angel, S. (1977). A Pattern Language: Towns, Buildings, Construction. New York: Oxford University Press.

Alexiou, E., Degl' Innocenti, A., Kullgren, A., \& Wijk, H. (2016). The impact of facility relocation on Patients' perceptions of ward atmosphere and quality of received forensic psychiatric care. Journal of Forensic and Legal Medicine, 42, 1-7.

Alimoglu, M. K., \& Donmez, L. (2005). Daylight exposure and the other predictors of burnout among nurses in a University Hospital. International journal of nursing studies, 42(5), 549-555.

Allen, G. (2014). Implementing AORN recommended practices for environmental cleaning. AORN Journal, 99(5), 570-582.

Alvaro, C., Wilkinson, A. J., Gallant, S. N., Kostovski, D., \& Gardner, P. (2016). Evaluating intention and effect: The impact of healthcare facility design on patient and staff well-being. HERD: Health Environments Research \& Design Journal, 9(2), 82-104.

Alzoubi, H., \& Al-Rqaibat, S. M. (2015). The effect of hospital design on indoor daylight quality in children section in King Abdullah University Hospital, Jordan. Sustainable Cities and Society, 14, 449-455.

Alzoubi, H., Al-Rqaibat, S. M., \& Bataineh, R. F. (2010). Pre-versus post-occupancy evaluation of daylight quality in hospitals. Building and environment, 45(12), 2652-2665.

American Academy of Paediatrics, \& American College of Obstetricians and Gynaecologists. (1992). Guidelines for Perinatal Care. Elk Grove Village, II and Washington, DC: American Academy of Paediatrics and American College of Obstetricians and Gynaecologists.

Anderson, J. D., Bonner, M., Scheifele, D. W., \& Schneider, B. C. (1985). Lack of nosocomial spread of Varicella in a pediatric hospital with negative pressure ventilated patient rooms. Infection Control \& Hospital Epidemiology, 6(3), 120-121.

Andrade, C. C., \& Devlin, A. S. (2015). Stress reduction in the hospital room: Applying Ulrich's theory of supportive design. Journal of Environmental Psychology, 41, 125-134. 
Andrade, C. C., \& Devlin, A. S. (2016). Who wants control in the hospital room? Environmental control, desirability of control and stress / ¿Quién desea control en la habitación del hospital? Control ambiental, deseo de control y estrés. Psyecology, 7(3), 236-261.

Andrade, C. C., Lima, M. L., Pereira, C. R., Fornara, F., \& Bonaiuto, M. (2013). Inpatients' and outpatients' satisfaction: The mediating role of perceived quality of physical and social environment. Health \& Place, 21, 122-132.

Annemans, M., Van Audenhove, C., Vermolen, H., \& Heylighen, A. (2018). The role of space in patients' experience of an emergency department: A qualitative study. Journal of Emergency Nursing, 44(2), 139-145.

Antonovsky, A. (1987). Unraveling the Mystery of Health : How People Manage Stress and Stay Well. San Francisco: Jossey-Bass.

Antonovsky, A. (1996). The salutogenic model as a theory to guide health promotion. Health promotion international, 11(1), 11-18.

Applebaum, D., Fowler, S., Fiedler, N., Osinubi, O., \& Robson, M. (2010). The impact of environmental factors on nursing stress, job satisfaction, and turnover intention. The Journal of nursing administration, 40, 323.

Ariadne Lab, \& MASS Design Group. (2017). Designing capacity for high value healthcare: The impact of design on clinical care in childbirth. Retrieved from https://www.ariadnelabs.org/

Aries, M., Aarts, M., \& van Hoof, J. (2015). Daylight and health: A review of the evidence and consequences for the built environment. Lighting Research \& Technology, 47(1), 6-27.

Askelsdottir, B., Lam-de Jonge, W., Edman, G., \& Wiklund, I. (2013). Home care after early discharge: Impact on healthy mothers and newborns. Midwifery, 29(8), 927-934.

Atkinson, R., \& Flint, J. (2001). Accessing hidden and hard-to-reach populations: Snowball research strategies. Social research update, 33(1), 1-4.

Aune, I., Torvik, H. M., Selboe, S.-T., Skogås, A.-K., Persen, J., \& Dahlberg, U. (2015). Promoting a normal birth and a positive birth experience-Norwegian women' s perspectives. Midwifery, 31(7), 721-727.

Bäckström, C., \& Wahn, E. H. (2011). Support during labour: First-time fathers' descriptions of requested and received support during the birth of their child. Midwifery, 27(1), 67-73.

BaHammam, A. (2006). Sleep in acute care units. Sleep and Breatbing, 10(1), 6-15.

Bailey, E., \& Timmons, S. (2005). Noise levels in PICU: An evaluative study. Paediatric Nursing, 17(10), 22.

Bailey, S., Wilson, G., \& Yoong, W. (2015). What factors affect documentation by midwives? A prospective study assessing relationship between length of shift, workload and quality of note keeping. Midwifery, 31(8), 787-792.

Bailey, S. J., \& Howe, J. K. (1993). High-volume obstetrics in a combined LDR/LDRP program. Nursing Management, 24(12), 42. 
Baillie, L. (2009). Patient dignity in an acute hospital setting: A case study. International journal of nursing studies, 46(1), 23-37.

Barss, P., \& Comfort, K. (1985). Ward design and neonatal jaundice in the tropics: Report of an epidemic. British Medical Journal (Clin Res Ed), 291(6492), 400-401.

Baurès, E., Blanchard, O., Mercier, F., Surget, E., Le Cann, P., Rivier, A., . . Florentin, A. (2018). Indoor air quality in two French hospitals: Measurement of chemical and microbiological contaminants. Science of the total environment, 642, 168-179.

Bayo, M. V., García, A. M., \& García, A. (1995). Noise levels in an urban hospital and workers' subjective responses. Archives of Environmental Health: An International Journal, 50(3), 247-251.

Beake, S., McCourt, C., \& Bick, D. (2005). Women's views of hospital and community-based postnatal care: The good, the bad and the indifferent. Evidence-Based Midwifery, 3(2), 80-86.

Beake, S., Rose, V., Bick, D., Weavers, A., \& Wray, J. (2010). A qualitative study of the experiences and expectations of women receiving in-patient postnatal care in one English maternity unit. BMC pregnancy and childbirth, 10(1), 1-9.

Beam, G. (2012). The Problem with Survey Research New Brunswick: Transaction Publishers.

Beauchemin, K. M., \& Hays, P. (1996). Sunny hospital rooms expedite recovery from severe and refractory depressions. Journal of Affective Disorders, 40(1), 49-51.

Bediako, S. M., Lattimer, L., Haywood, C., Ratanawongsa, N., Lanzkron, S., \& Beach, M. C. (2011). Religious coping and hospital admissions among adults with sickle cell disease. Journal of Behavioral Medicine, 34(2), 120-127.

Berglund, B., Lindvall, T., Schwela, D. H., \& World Health Organization. (1999). Guidelines for community noise.

Berkman, L. F., Glass, T., Brissette, I., \& Seeman, T. E. (2000). From social integration to health: Durkheim in the new millennium. Social Science \& Medicine, 51(6), 843-857.

Bhavnani, V., \& Newburn, M. (2010). Left to your own devices: The postnatal care experiences of 1260 firsttime mothers: National Childbirth Trust.

Bick, D. E. (2008). Best evidence for best maternal health? The conundrum of maternity service policy for postnatal care. Midwives, 11(5), 42.

Biley, F. C. (1994). Effects of noise in hospitals. British journal of nursing, 3(3), 110-113.

Biley, F. C. (1996). Hospitals: Healing environments? Complementary Therapies in Nursing and Midwifery, 2(4), 110-115.

Birks, M. (2014). Practical philosophy. In J. Mills \& M. Birks (Eds.), Qualitative Methodology: A practical Guide (pp. 17-30). London: Sage Publications. Retrieved from https://dx.doi.org/10.4135/9781473920163.

Bishop, K. (2012). The role of art in a paediatric healthcare environment from children's and young people's perspectives. Procedia-Social and Behavioral Sciences, 38, 81-88. 
Blix, E., Kumle, M. H., Ingversen, K., Huitfeldt, A. S., Hegaard, H. K., Ólafsdóttir, Ó. Á., . . . Lindgren, H. (2016). Transfers to hospital in planned home birth in four Nordic countriesA prospective cohort study. Acta obstetricia et gynecologica Scandinavica, 95(4), 420-428.

Blomkvist, V., Eriksen, C. A., Theorell, T., Ulrich, R. S., \& Rasmanis, G. (2005). Acoustics and psychosocial environment in intensive coronary care. Occupational and environmental medicine, 62(3), e1-e1.

Bobrow, M., \& Thomas, J. (2008). Inpatient care facilities. In S. A. Kliment (Ed.), Building Type Basics for Healthcare Facilities (2nd ed., pp. 175-239). New York: John Wiley \& Sons.

Boehm, H., \& Morast, S. (2009). Quiet time. AJN The American Journal of Nursing, 109(11), 29-32.

Bolten, N., De Jonge, A., Zwagerman, E., Zwagerman, P., Klomp, T., Zwart, J., \& Geerts, C. (2016). Effect of planned place of birth on obstetric interventions and maternal outcomes among low-risk women: A cohort study in the Netherlands. BMC pregnancy and childbirth, 16(1), 329.

Boucher, D., Bennett, C., McFarlin, B., \& Freeze, R. (2009). Staying home to give birth: Why women in the United States choose home birth. Journal of midwifery \& women's health, 54(2), 119-126.

Bowden, C., Sheehan, A., \& Foureur, M. J. (2016). Birth room images: What they tell us about childbirth. A discourse analysis of birth rooms in developed countries. Midwifery, 35, 71-77.

Braga, L. (2018). The Sarah Network of rehabilitation hospitals. Retrieved from https://ipbis.org/the sarah network.html

Bravo, P., Uribe, C., \& Contreras, A. (2011). Early postnatal hospital discharge: The consequences of reducing length of stay for women and newborns. Revista da Escola de Enfermagem da USP, 45(3), 758-763.

Breedlove, G., \& Rathbun, L. (2019). Facility design: Reimagining approaches to childbirth in hospital and birth center settings. The Journal of perinatal \& neonatal nursing, 33(1), 26-34.

Brodribb, W., Zadoroznyj, M., \& Dane, A. (2012). Evaluating the implementation of the universal postnatal contact services in Queensland: The experiences of health care providers and mothers. Brisbane, Australia: Queensland Centre for Mothers \& Babies.

Bromley, E. (2012). Building patient-centeredness: Hospital design as an interpretive act. Social Science \& Medicine, 75(6), 1057-1066.

Brooks, A. (2007). Feminist standpoint epistemology: Building knowledge and empowerment through women's lived experience. In B. SharleneNagyHesse \& PatriciaLinaLeavy (Eds.), Feminist Research Practice (pp. 53-82). Thousand Oaks, California: Sage Publications, Inc.

Brown, B., Rutherford, P., \& Crawford, P. (2015). The role of noise in clinical environments with particular reference to mental health care: A narrative review. International journal of nursing studies, 52(9), 1514-1524.

Brown, K. K., \& Gallant, D. (2006). Impacting patient outcomes through design: Acuity adaptable care/universal room design. Critical Care Nursing Quarterly, 29(4), 326-341. 
Brown, S., Small, R., Argus, B., Davis, P. G., \& Krastev, A. (2002). Early postnatal discharge from hospital for healthy mothers and term infants. Cochrane Database of Systematic Reviews(3).

Buck, G., Cook, K., Quigley, C., Eastwood, J., \& Lucas, Y. (2009). Profiles of urban, low SES, African American girls' attitudes toward science: A sequential explanatory mixed methods study. Journal of Mixed Methods Research, 3(4), 386-410.

Buckley, S. J. (2015). Executive summary of hormonal physiology of childbearing: Evidence and implications for women, babies, and maternity care. The Journal of perinatal education, 24(3), 145-153.

Burnard, P., Gill, P., Stewart, K., Treasure, E., \& Chadwick, B. (2008). Analysing and presenting qualitative data. British dental journal, 204(8), 429.

Burns, E. E., Boulton, M. G., Cluett, E., Cornelius, V. R., \& Smith, L. A. (2012). Characteristics, interventions, and outcomes of women who used a birthing pool: A prospective observational study. Birth, 39(3), 192-202.

Busch-Vishniac, I. J., West, J. E., Barnhill, C., Hunter, T., Orellana, D., \& Chivukula, R. (2005). Noise levels in Johns Hopkins hospital. The Journal of the Acoustical Society of America, 118(6), 3629-3645.

Callister, L. C. (2004). Making meaning: Women's birth narratives. Journal of Obstetric, Gynecologic \& Neonatal Nursing, 33(4), 508-518.

Campagnol, G., \& Shepley, M. M. (2014). Positive distraction and the rehabilitation hospitals of João Filgueiras Lima. HERD: Health Environments Research \& Design Journal, 8(1), 199-227.

Canazei, M., Pohl, W., Weninger, J., Bliem, H., Weiss, E. M., Koch, C., . . . Marth, C. (2019). Effects of adjustable dynamic bedroom lighting in a maternity ward. Journal of Environmental Psychology, 62, 59-66.

Carling, S. J., Demment, M. M., Kjolhede, C. L., \& Olson, C. M. (2015). Breastfeeding duration and weight gain trajectory in infancy. Pediatrics, 135(1), 111-119.

Carolan-Olah, M., Kruger, G., \& Garvey-Graham, A. (2015). Midwives' experiences of the factors that facilitate normal birth among low risk women at a public hospital in Australia. Midwifery, 31(1), 112-121.

Carpman, J. R., Grant, M. A., \& Simmons, D. A. (1985). Hospital design and wayfinding: A video simulation study. Environment and Behavior, 17(3), 296-314.

Carr, K. C. (1994). Characteristics of the supportive and non-supportive childbirth environment. International Journal of Childbirth Education, 9(3), 10-13.

Cattrell, R., Lavender, T., Wallymahmed, A., Kingdon, C., \& Riley, J. (2005). Postnatal care: What matters to midwives. British Journal of Midwifery, 13(4), 206-213.

Chang, Y.-J., Pan, Y.-J., Lin, Y.-J., Chang, Y.-Z., \& Lin, C.-H. (2006). A noise-sensor light alarm reduces noise in the newborn intensive care unit. American journal of perinatology, 23(05), 265272.

Chatterjee, A., \& Vartanian, O. (2014). Neuroaesthetics. Trends in cognitive sciences, 18(7), 370-375. 
Chaudhury, H., Mahmood, A., \& Valente, M. (2005). Advantages and disadvantages of singleversus multiple-occupancy rooms in acute care environments: A review and analysis of the literature. Environment and Behavior, 37(6), 760-786.

Chaudhury, H., Mahmood, A., \& Valente, M. (2006). Nurses' perception of single-occupancy versus multioccupancy rooms in acute care environments: An exploratory comparative assessment. Applied Nursing Research, 19(3), 118-125.

Chi, P., Gutberg, J., \& Berta, W. (2020). The conceptualization of the natural environment in healthcare facilities: A scoping review. HERD: Health Environments Research \& Design Journal, 13(1), 30-47.

Choi, Y.-S., \& Bosch, S. J. (2013). Environmental affordances: Designing for family presence and involvement in patient care. HERD: Health Environments Research \& Design Journal, 6(4), 5375.

Choiniere, D. B. (2010). The effects of hospital noise. Nursing administration quarterly, 34(4), 327 333.

Christensen, M. (2005). Noise levels in a general surgical ward: A descriptive study. Journal of Clinical Nursing, 14(2), 156-164.

Cleveland, A. M. (1994). ICU visitation policies. Nursing Management, 25(9), 3.

Coad, J., \& Coad, N. (2008). Children and young people's preference of thematic design and colour for their hospital environment. Journal of Child Health Care, 12(1), 33-48.

Codinhoto, R., Tzortzopoulos, P., Kagioglou, M., Aouad, G., \& Cooper, R. (2008). The effects of the built environment on bealth outcomes: University of Salford.

Cohen, S. (1988). Psychosocial models of the role of social support in the etiology of physical disease. Health psychology, 7(3), 269.

Collier, J. (1957). Photography in anthropology: A report on two experiments. American anthropologist, 59(5), 843-859.

Connellan, K., Gaardboe, M., Riggs, D., Due, C., Reinschmidt, A., \& Mustillo, L. (2013). Stressed spaces: Mental health and architecture. HERD: Health Environments Research \& Design Journal, 6(4), 127-168.

Corbin, J. M., \& Strauss, A. L. (2008). Basics of Qualitative Research : Techniques and Procedures for Developing Grounded Theory (3rd ed.). Thousand Oaks, California: Sage Publications.

Corbin, J. M., \& Strauss, A. L. (2015). Basics of Qualitative Research : Techniques and Procedures for Developing Grounded Theory (4th ed.). Thousand Oaks, California: Sage Publications.

Corey, L. J., Wallace, M. A., Harris, S. H., \& Casey, B. (1986). Psychiatric ward atmosphere. Journal of Psychosocial Nursing and Mental Health Services, 24(10), 10-16.

Coxon, K., Sandall, J., \& Fulop, N. J. (2014). To what extent are women free to choose where to give birth? How discourses of risk, blame and responsibility influence birth place decisions. Health, Risk \& Society, 16(1), 51-67. 
Crawford, B., Lilo, S., Stone, S., \& Yates, A. (2008). Review of the quality, safety and management of maternity services in the Wellington area. Wellington: Ministry of Health, New Zealand.

Creswell, J. W. (2010). Mapping the developing landscape of mixed methods research. In T. Abbas \& T. Charles (Eds.), SAGE Handbook of mixed methods in social \& behavioral research (Vol. 2, pp. 45-68). Thousand Oaks, California: Sage Publications, Inc.

Creswell, J. W. (2011a). Controversies in mixed methods research. In N. K. Denzin \& Y. S. Lincoln (Eds.), The Sage handbook of qualitative research (4 ed., pp. 269-284). Thousand Oaks, California: Sage Publications, Inc.

Creswell, J. W. (2012). Educational Research: Planning, Conducting, and Evaluating Quantitative and Qualitative Research (4th ed.). Boston, MA: Pearson Education, Inc.

Creswell, J. W. (2013). Qualitative Inquiry and Research Design: Choosing Among Five Approaches (3rd ed.). Los Angeles: Sage Publications.

Creswell, J. W. (2015). A Concise Introduction to Mixed Methods Research. Thousand Oaks, California: Sage Publications.

Creswell, J. W., \& Plano Clark, V. L. (2018). Designing and Conducting Mixed Methods Research (3rd ed.). Thousand Oaks, California: Sage Publications.

Cuncarr, C., \& Skinner, J. (2011). Quantity or quality of postnatal length of stay? A literature review examining the issues and the evidence. New Zealand College of Midwives Journal(44), 12-16.

Cunha, M., \& Silva, N. (2015a). Hospital noise and patients' wellbeing. Procedia-Social and Behavioral Sciences, 171, 246-251.

Cunha, M., \& Silva, N. (2015b). Hospital noise and patients' wellbeing. Procedia-Social and Behavioral Sciences, 171, 246-251.

Curtis, E., \& Redmond, R. (2007). Focus groups in nursing research. Nurse Researcher, 14(2).

Curtis, P., \& Northcott, A. (2017). The impact of single and shared rooms on family-centred care in children's hospitals. Journal of Clinical Nursing, 26(11-12), 1584-1596.

Dahlberg, U., \& Aune, I. (2013). The woman's birth experience-The effect of interpersonal relationships and continuity of care. Midwifery, 29(4), 407-415.

Dahlberg, U., Persen, J., Skogås, A.-K., Selboe, S.-T., Torvik, H. M., \& Aune, I. (2016). How can midwives promote a normal birth and a positive birth experience? The experience of firsttime Norwegian mothers. Sexual \& Reproductive Healthcare, 7, 2-7.

Dalke, H., Little, J., Niemann, E., Camgoz, N., Steadman, G., Hill, S., \& Stott, L. (2006). Colour and lighting in hospital design. Optics and Laser Technology, 38(4), 343-365.

Danbjørg, D. B., Wagner, L., \& Clemensen, J. (2014). Do families after early postnatal discharge need new ways to communicate with the hospital? A feasibilility study. Midwifery, 30(6), 725-732.

Dancey, C. P., Reidy, J. G., \& Rowe, R. (2012). Statistics for the Health Sciences : A Non-Mathematical Introduction. London: Sage Publications. 
Danel, I., Johnson, C., Berg, C., Flowers, L., \& Atrash, H. (1997). Length of maternal hospital stay for uncomplicated deliveries, 1988-1995: The impact of maternal and hospital characteristics. Maternal and child health journal, 1(4), 237-242.

Dascalaki, E. G., Lagoudi, A., Balaras, C. A., \& Gaglia, A. G. (2008). Air quality in hospital operating rooms. Building and environment, 43(11), 1945-1952.

Datar, A., \& Sood, N. (2006). Impact of postpartum hospital-stay legislation on newborn length of stay, readmission, and mortality in California. Pediatrics, 118(1), 63-72.

Davey, J. D. (2011). A theoretical model of learning employing constructivism, neuroscience, and phenomenology: Constructivist neurophenomenology. (PhD Thesis), Southern Illinois University at Carbondale, Ann Arbor. ProQuest Dissertations \& Theses Global database.

Davis-Floyd, R. (2001). The technocratic, humanistic, and holistic paradigms of childbirth. International Journal of Gynecology \& Obstetrics, 75, S5-S23.

Davis, B. E. (2011). Rooftop hospital gardens for physical therapy: A post-occupancy evaluation. HERD: Health Environments Research \& Design Journal, 4(3), 14-43.

Davis, D. L., Baddock, S., Pairman, S., Hunter, M., Benn, C., Wilson, D., . . Herbison, P. (2011). Planned place of birth in New Zealand: Does it affect mode of birth and intervention rates among low-risk women? Birth, 38(2), 111-119.

Davis, D. L., \& Homer, C. S. (2016). Birthplace as the midwife's work place: How does place of birth impact on midwives? Women and Birth, 29(5), 407-415.

Davis, D. L., \& Walker, K. (2010). Case-loading midwifery in New Zealand: Making space for childbirth. Midwifery, 26(6), 603-608.

Dazkir, S. S., \& Read, M. A. (2012). Furniture forms and their influence on our emotional responses toward interior environments. Environment and Behavior, 44(5), 722-732.

De Giuli, V., Zecchin, R., Salmaso, L., Corain, L., \& De Carli, M. (2013). Measured and perceived indoor environmental quality: Padua hospital case study. Building and environment, 59, 211226.

De Labrusse, C., Ramelet, A.-S., Humphrey, T., \& Maclennan, S. J. (2016). Patient-centered care in maternity services: A critical appraisal and synthesis of the literature. Women's Health Issues, 26(1), 100-109.

del Rosario Ruiz, M., \& Limonero, J. T. (2014). Professional attitudes towards normal childbirth in a shared care unit. Midwifery, 30(7), 817-824.

Department of Health. (2001). The NHS Plan: A Plan for Investment, a Plan for Reform. London: Stationery Office.

Detsky, M. E., \& Etchells, E. (2008). Single-patient rooms for safe patient-centered hospitals. Jama, 300(8), 954-956.

Devlin, A. S. (2014). Wayfinding in healthcare facilities : Contributions from environmental psychology. Behavioral sciences, 4(4), 423-436. 
Devlin, A. S., Andrade, C. C., \& Carvalho, D. (2016). Qualities of inpatient hospital rooms: Patients' perspectives. HERD: Health Environments Research \& Design Journal, 9(3), 190-211.

Devlin, A. S., \& Arneill, A. B. (2003). Health care environments and patient outcomes: A review of the literature. Environment and Behavior, 35(5), 665-694.

Dianat, I., Sedghi, A., Bagherzade, J., Jafarabadi, M. A., \& Stedmon, A. W. (2013). Objective and subjective assessments of lighting in a hospital setting: Implications for health, safety and performance. Ergonomics, 56(10), 1535-1545.

Diette, G. B., Lechtzin, N., Haponik, E., Devrotes, A., \& Rubin, H. R. (2003). Distraction therapy with nature sights and sounds reduces pain during flexible bronchoscopy: A complementary approach to routine analgesia. Chest, 123(3), 941-948.

Dijkstra, K., Pieterse, M., \& Pruyn, A. (2006). Physical environmental stimuli that turn healthcare facilities into healing environments through psychologically mediated effects: Systematic review. Journal of advanced nursing, 56(2), 166-181.

Dijkstra, K., Pieterse, M., \& Pruyn, A. (2008). Stress-reducing effects of indoor plants in the built healthcare environment: The mediating role of perceived attractiveness. Preventive medicine, 47(3), 279-283.

Dilani, A. (2015). The beneficial health outcomes of salutogenic design. Design for Health.

Dillman, D. A., Smyth, J. D., \& Christian, L. M. (2014). Internet, Phone, Mail, and Mixed-Mode Surveys: The Tailored Design Method (4th ed.). Hoboken, New Jersey: John Wiley \& Sons.

Dixon, L., Prileszky, G., Guilliland, K., Hendry, C., Miller, S., \& Anderson, J. (2012). What evidence supports the use of free-standing midwifery led units (primary units) in New Zealand/Aotearoa? New Zealand College of Midwives Journal(46), 13.

Doering, J. J. (2013). The physical and social environment of sleep in socioeconomically disadvantaged postpartum women. Journal of Obstetric, Gynecologic \& Neonatal Nursing, 42(1), E33-E43.

Donetto, S., Penfold, C., Anderson, J., Robert, G., \& Maben, J. (2017). Nursing work and sensory experiences of hospital design: A before and after qualitative study following a move to all-single room inpatient accommodation. Health \& Place, 46, 121-129.

Douglas, C. H., \& Douglas, M. R. (2005). Patient-centred improvements in health-care built environments: Perspectives and design indicators. Health expectations, 8(3), 264-276.

Douglas, L. (2014). Review of the book Towards an Articulated Phenomenological Interpretation of Architecture - Phenomenal Phenomenology. Architecture New Zealand(5), 85-85.

Driver, R. L., \& Colbert, T. (2010). Shhh... implementation of quiet time on the mother/infant unit. Journal of Obstetric, Gynecologic, \& Neonatal Nursing, 39, S23-S23.

Dube, J. A. O., Barth, M. M., Cmiel, C. A., Cutshall, S. M., Olson, S. M., Sulla, S. J., . . Holland, D. E. (2008). Environmental noise sources and interventions to minimize them: A tale of 2 hospitals. Journal of nursing care quality, 23(3), 216-224. 
Duncan, J. (2011). The effect of colour and design in labour and delivery: A scientific approach. Optics \& Laser Technology, 43(2), 420-424.

Dunne, C. L., Fraser, J., \& Gardner, G. E. (2014). Women's perceptions of social support during labour: Development, reliability and validity of the birth companion support questionnaire. Midwifery, 30(7), 847-852.

Duysburgh, E., Kerstens, B., Kouanda, S., Kaboré, C. P., Yugbare, D. B., Gichangi, P., ... Osman, N. B. (2015). Opportunities to improve postpartum care for mothers and infants: Design of context-specific packages of postpartum interventions in rural districts in four subSaharan African countries. BMC pregnancy and childbirth, 15(1), 131.

Eberhard, J. P. (2009a). Applying neuroscience to architecture. Neuron, 62(6), 753-756.

Eberhard, J. P. (2009b). Brain Landscape : The Coexistence of Neuroscience and Architecture. Oxford: Oxford University Press.

Edelstein, E. A. (2015). Neuroscience and architecture. In M. Kanaani \& D. Kopec (Eds.), The Routledge Companion for Architecture Design and Practice: Established and Emerging trends (pp. 269287): Routledge.

Eisen, S. L., Ulrich, R. S., Shepley, M. M., Varni, J. W., \& Sherman, S. (2008). The stress-reducing effects of art in pediatric health care: Art preferences of healthy children and hospitalized children. Journal of Child Health Care, 12(3), 173-190.

Ellis, M. R., Thomlinson, P., Gemmill, C., \& Harris, W. (2013). The spiritual needs and resources of hospitalized primary care patients. Journal of Religion and Health, 52(4), 1306-1318.

Emmanuel, E., Creedy, D., \& Fraser, J. (2001). What mothers want: A postnatal survey. Australian Journal of Midwifery, 14(4), 16-20.

Evans, G. W., \& McCoy, J. M. (1998). When buildings don't work: The role of architecture in human health. Journal of Environmental Psychology, 18(1), 85-94.

Fahy, K., Foureur, M. J., \& Hastie, C. (2008). Birth Territory and Midwifery Guardianship: Theory for Practice, Education and Research: Elsevier Health Sciences.

Fahy, K., Parratt, J., Foureur, M., \& Hastie, C. (2011). Birth territory: A theory for midwifery practice. In R. Bryan \& M. Sinclair (Eds.), Theory for midwifery practice (2nd ed., pp. 215-240). Hampshire, UK: Palgrave Macmillan Press.

Fahy, K., \& Parratt, J. A. (2006). Birth territory: A theory for midwifery practice. Women and Birth, 19(2), 45-50.

Fannin, M. (2003). Domesticating birth in the hospital:"Family-centered" birth and the emergence of "homelike" birthing rooms. Antipode, 35(3), 513-535.

Felippe, M. L., Kuhnen, A., da Silveira, B. B., \& Lelli, G. (2017). What is a restorative hospital environment? Environmental meaning, affective stress restoration and physical attributes in pediatric inpatient rooms. Children, Youth and Environments, 27(1), 17-46. 
Fenko, A., \& Loock, C. (2014). The influence of ambient scent and music on patients' anxiety in a waiting room of a plastic surgeon. HERD: Health Environments Research \& Design Journal, 7(3), 38-59.

Ferri, M., Zygun, D. A., Harrison, A., \& Stelfox, H. T. (2015). Evidence-based design in an intensive care unit: End-user perceptions. BMC anesthesiology, 15(1), 57.

Fink, A. M. (2011). Early hospital discharge in maternal and newborn care. Journal of Obstetric, Gynecologic \& Neonatal Nursing, 40(2), 149-156.

Forbes, I., Foureur, M. J., Leap, N., \& Homer, C. (2008). Birthing unit design: Researching new principles. Design \& Health Scientific Review, 47-53.

Ford, E., \& Ayers, S. (2009). Stressful events and support during birth: The effect on anxiety, mood and perceived control. Journal of Anxiety Disorders, 23(2), 260-268.

Forster, D. A., McLachlan, H. L., Yelland, J., Rayner, J., Lumley, J., \& Davey, M.-A. (2006). Staffing in postnatal units: is it adequate for the provision of quality care? Staff perspectives from a state-wide review of postnatal care in Victoria, Australia. BMC health services research, 6(1), 83.

Foureur, M. J., Davis, D., Fenwick, J., Leap, N., Iedema, R., Forbes, I., \& Homer, C. S. (2010). The relationship between birth unit design and safe, satisfying birth: Developing a hypothetical model. Midwifery, 26(5), 520-525.

Foureur, M. J., \& Harte, J. D. (2017). Salutogenic design for birth. In Health and Well-being for Interior Architecture (pp. 108-122): Routledge.

Foureur, M. J., Leap, N., Davis, D. L., Forbes, I., \& Homer, C. S. (2010). Developing the birth unit design spatial evaluation tool (BUDSET) in Australia: A qualitative study. HERD: Health Environments Research \& Design Journal, 3(4), 43-57.

Foureur, M. J., Leap, N., Davis, D. L., Forbes, I. F., \& Homer, C. S. (2011). Testing the birth unit design spatial evaluation tool (BUDSET) in Australia: A pilot study. HERD: Health Environments Research \& Design Journal, 4(2), 36-60.

France, D., Throop, P., Joers, B., Allen, L., Parekh, A., Rickard, D., \& Deshpande, J. K. (2009). Adapting to family-centered hospital design: Changes in providers' attitudes over a twoyear period. HERD: Health Environments Research \& Design Journal, 3(1), 79-96.

Franck, K. A. (2007). From the body. In K. A. Franck \& R. B. Lepori (Eds.), Architecture from the Inside Out: From the Body, the Senses, the Site, and the Community (2nd ed.). Chichester Wiley.

Franck, K. A., \& Lepori, R. B. (2007). Architecture from the Inside Out: From the Body, the Senses, the Site, and the Community (2nd ed.). Chichester: Wiley.

Fredriksson, A.-C., Hellström, L., \& Nilsson, U. (2009). Patients' perception of music versus ordinary sound in a postanaesthesia care unit: A randomised crossover trial. Intensive and Critical Care Nursing, 25(4), 208-213.

Freedman, N. S., Kotzer, N., \& Schwab, R. J. (1999). Patient perception of sleep quality and etiology of sleep disruption in the intensive care unit. American journal of respiratory and critical care medicine, 159(4), 1155-1162. 
Frisk, U., \& Nordström, G. (2003). Patients' sleep in an intensive care unit-patients' and nurses' perception. Intensive and Critical Care Nursing, 19(6), 342-349.

Gabor, J. Y., Cooper, A. B., Crombach, S. A., Lee, B., Kadikar, N., Bettger, H. E., \& Hanly, P. J. (2003). Contribution of the intensive care unit environment to sleep disruption in mechanically ventilated patients and healthy subjects. American journal of respiratory and critical care medicine, 167(5), 708-715.

Gaboury, J., Capaday, S., Somera, J., \& Purden, M. (2017). Effect of the postpartum hospital environment on the attainment of mothers' and fathers' goals. Journal of Obstetric, Gynecologic \& Neonatal Nursing, 46(1), 40-50.

Gappell, M. (1995). Psychoneuroimmunology. In S. O. Marberry (Ed.), Innovations in Healthcare Design: Selected Presentations from the First Five Symposia on Healthcare Design (pp. 115-120). New York: Van Nostrand Renhold.

García Marcos, R., Martí-Bonmatí, L., Martínez, J. J., Vilar, J., Katic, N., Lemercier, P., \& Díaz Dhó, R. (2014). Decorative elements in the medical imaging area improve patients' perception of pleasantness. Radiología (English Edition), 56(2), 129-135.

Gardiner, C., Brereton, L., Gott, M., Ingleton, C., \& Barnes, S. (2011). Exploring health professionals' views regarding the optimum physical environment for palliative and end of life care in the acute hospital setting: A qualitative study. BMJ supportive \& palliative care, 1(2), 162-166.

Gardner, G., Collins, C., Osborne, S., Henderson, A., \& Eastwood, M. (2009). Creating a therapeutic environment: A non-randomised controlled trial of a quiet time intervention for patients in acute care. International journal of nursing studies, 46(6), 778-786.

Geraghty, S., Speelman, C., \& Bayes, S. (2019). Fighting a losing battle: Midwives experiences of workplace stress. Women and Birth, 32(3), e297-e306.

Gerlach-Spriggs, N., Kaufman, R. E., \& Warner, S. B. (1998). Restorative Gardens : The Healing Landscape. New Haven, CT: Yale University Press.

Geue, K., Goetze, H., Buttstaedt, M., Kleinert, E., Richter, D., \& Singer, S. (2010). An overview of art therapy interventions for cancer patients and the results of research. Complementary therapies in medicine, 18(3-4), 160-170.

Gharaveis, A., Shepley, M. M., \& Gaines, K. (2016). The role of daylighting in skilled nursing shortterm rehabilitation facilities. HERD: Health Environments Research \& Design Journal, 9(2), 105118.

Gharaveis, A., Yekita, H., \& Shamloo, G. (2020). The perceptions of nurses about the behavioral needs for daylighting and view to the outside in inpatient facilities. HERD: Health Environments Research \& Design Journal, 13(1), 191-205.

Gilkeson, C., Camargo-Valero, M., Pickin, L., \& Noakes, C. (2013). Measurement of ventilation and airborne infection risk in large naturally ventilated hospital wards. Building and environment, 65, 35-48.

Giorgi, A. (1997). The theory, practice, and evaluation of the phenomenological method as a qualitative research procedure. Journal of phenomenological psychology, 28(2), 235-260. 
Glaser, B. G., \& Strauss, A. L. (1967). The Discovery of Grounded Theory: Strategies for Qualitative research. Chicago: Aldine Pub. Co.

Go"kcay, D., \& Yildirim, G. (2011). A scientific look at the design of aesthetically and emotionally engaging interactive entertainment experiences. In Affective Computing and Interaction: Psychological, Cognitive and Neuroscientific Perspectives (pp. 281-307): IGI Global.

Goldhagen, S. W. (2017). Welcome to Your World: How the Built Environment Shapes Our Lives (First ed.). New York: HarperCollins.

Golembiewski, J. A. (2010). Start making sense: Applying a salutogenic model to architectural design for psychiatric care. Facilities, 28(3/4), 100-117.

Goodman, P., Mackey, M. C., \& Tavakoli, A. S. (2004). Factors related to childbirth satisfaction. Journal of advanced nursing, 46(2), 212-219.

Goodwin, L., Taylor, B., Kokab, F., \& Kenyon, S. (2018). Postnatal care in the context of decreasing length of stay in hospital after birth: The perspectives of community midwives. Midwifery, 60, 36-40.

Goto, S., Park, B.-J., Tsunetsugu, Y., Herrup, K., \& Miyazaki, Y. (2013). The effect of garden designs on mood and heart output in older adults residing in an assisted living facility. HERD: Health Environments Research \& Design Journal, 6(2), 27-42.

Gould, D. (2002). Subliminal medicalisation. British Journal of Midwifery, 10(7), 418-418.

Gravetter, F. J., \& Wallnau, L. B. (2011). Essentials of Statistics for the Behavioral Sciences (8th ed.). Belmont, CA: Wadsworth Cengage Learning.

Green, J. M., \& Baston, H. A. (2003). Feeling in control during labor: Concepts, correlates, and consequences. Birth, 30(4), 235-247.

Grigg, C. P., Tracy, S. K., Daellenbach, R., Kensington, M., Tracy, M., Monk, A., \& Schmied, V. (2017). Women's birthplace decision-making, the role of confidence: Part of the evaluating maternity units study, Aotearoa New Zealand. Paper presented at the 31st ICM Triennial COngress, Midwives making a difference in the world.

Grigg, C. P., Tracy, S. K., Schmied, V., Daellenbach, R., \& Kensington, M. (2015). Women's birthplace decision-making, the role of confidence: Part of the evaluating maternity units study, New Zealand. Midwifery, 31(6), 597-605.

Groat, L. N., \& Wang, D. (2002). Architectural Research Methods. New York: John Wiley \& Sons.

Groat, L. N., \& Wang, D. (2013). Architectural Research Methods: John Wiley \& Sons.

Guba, E. G., \& Lincoln, Y. S. (1994). Competing paradigms in qualitative research. In N. K. Denzin \& Y. S. Lincoln (Eds.), Handbook of qualitative research (pp. 105-117). Thousand Oaks, California: Sage Publications.

Guenther, R., \& Vittori, G. (2013). Sustainable Healthcare Architecture (2nd ed.). Hoboken, New Jersey: John Wiley \& Sons, Inc. 
Gutmann, D. (2011). Rehabilitation. In P. Meuser (Ed.), Construction and Design Manual: Hospitals and Health Centres (Vol. 2, pp. 035-040): DOM publisher.

Hallowell, S. G., Froh, E. B., \& Spatz, D. L. (2017). Human milk and breastfeeding: An intervention to mitigate toxic stress. Nursing outlook, 65(1), 58-67.

Halm, M. A., \& Titler, M. G. (1990). Appropriateness of critical care visitation: Perceptions of patients, families, nurses, and physicians. Journal of nursing care quality, 5(1), 25-37.

Hamilton, J. B., Sandelowski, M., Moore, A. D., Agarwal, M., \& Koenig, H. G. (2012). "You need a song to bring you through": The use of religious songs to manage stressful life events. The Gerontologist, 53(1), 26-38.

Hammersley, M. (1987). Some notes on the terms 'validity'and 'reliability'. British Educational Research Journal, 13(1), 73-82.

Hammond, A., Foureur, M. J., Homer, C. S., \& Davis, D. A. (2013). Space, place and the midwife: Exploring the relationship between the birth environment, neurobiology and midwifery practice. Women and Birth, 26(4), 277-281.

Hammond, A., Homer, C. S., \& Foureur, M. J. (2014). Messages from space: An exploration of the relationship between hospital birth environments and midwifery practice. HERD: Health Environments Research \& Design Journal, 7(4), 81-95.

Hammond, A., Homer, C. S., \& Foureur, M. J. (2017). Friendliness, functionality and freedom: Design characteristics that support midwifery practice in the hospital setting. Midwifery, 50, 133-138.

Haran, C., Van Driel, M., Mitchell, B. L., \& Brodribb, W. E. (2014). Clinical guidelines for postpartum women and infants in primary care-A systematic review. BMC pregnancy and childbirth, 14(1), 51.

Harper, D. (2002). Talking about pictures: A case for photo elicitation. Visual studies, 17(1), 13-26.

Harris, D. D., Shepley, M. M., White, R. D., Kolberg, K. J. S., \& Harrell, J. W. (2006). The impact of single family room design on patients and caregivers: Executive summary. Journal of Perinatology, 26(S3), S38-48.

Harris, P. B., McBride, G., Ross, C., \& Curtis, L. (2002). A place to heal: Environmental sources of satisfaction among hospital patients Journal of Applied Social Psychology, 32(6), 1276-1299.

Harte, J. D., Leap, N., Fenwick, J., Homer, C. S., \& Foureur, M. J. (2014). Methodological insights from a study using video-ethnography to conduct interdisciplinary research in the study of birth unit design. International Journal of Multiple Research Approaches, 8(1), 36-48.

Harte, J. D., Sheehan, A., Stewart, S. C., \& Foureur, M. J. (2016). Childbirth supporters' experiences in a built hospital birth environment: Exploring inhibiting and facilitating factors in negotiating the supporter role. HERD: Health Environments Research \& Design Journal, 9(3), 135-161.

Hastie, C. (2011). The birthing environment: A sustainable approach. In L. Davies, R. Daellenbach, \& M. Kensington (Eds.), Sustainable, Midwifery and Birth. London: Routledge 
Hauck, Y., Rivers, C., \& Doherty, K. (2008). Women's experiences of using a Snoezelen room during labour in Western Australia. Midwifery, 24(4), 460-470.

Hauptmann, D., \& Neidich, W. (2010). Cognitive Architecture: From Bio-politics to Noo-politics; Architecture \& Mind in the Age of Communication and Information. Rotterdam: 010 Publishers.

Havill, N. L. (2012). Therapeutic landscapes for birth: A research synthesis. (PhD Thesis), University of North Carolina at Chapel Hill, Ann Arbor.

Health and Disability Ethics Committees. (2016). Health and Disability Ethics Committees: Annual Report 2016. Retrieved from https://ethics.health.govt.nz/.

Henderson, J., Burns, E. E., Regalia, A. L., Casarico, G., Boulton, M. G., \& Smith, L. A. (2014). Labouring women who used a birthing pool in obsteric units in Italy: Prospective observational study. BMC pregnancy and childbirth, 14(1), 17.

Hermus, M. A., Wiegers, T. A., Hitzert, M. F., Boesveld, I. C., van den Akker-van, M. E., Akkermans, H. A., . . R Rijnders, M. E. (2015). The Dutch birth centre study: Study design of a programmatic evaluation of the effect of birth centre care in the Netherlands. BMC pregnancy and childbirth, 15(1), 148.

Hilbers, J., Haynes, A. S., \& Kivikko, J. G. (2010). Spirituality and health: An exploratory study of hospital patients' perspectives. Australian Health Review, 34(1), 3-10.

Hildingsson, I., Cederlöf, L., \& Widén, S. (2011). Fathers' birth experience in relation to midwifery care. Women and Birth, 24(3), 129-136.

Hildingsson, I., Johansson, M., Karlström, A., \& Fenwick, J. (2013). Factors associated with a positive birth experience: An exploration of swedish women's experiences. International journal of childbirth, 3(3), 153-164.

Hildingsson, I., \& Sandin-Bojö, A.-K. (2011). 'What is could indeed be better'-Swedish women's perceptions of early postnatal care. Midwifery, 27(5), 737-744.

Hinkulow, M. B. (2014). Evidence to change practice: Creating a restful hospital environment for nurses and patients. Archives of psychiatric nursing, 28(1), 74-75.

Ho, S. H., Rosario, L., \& Rahman, M. M. (2009). Three-dimensional analysis for hospital operating room thermal comfort and contaminant removal. Applied Thermal Engineering, 29(10), 20802092.

Hodnett, E. D. (1989). Personal control and the birth environment: Comparisons between home and hospital settings. Journal of Environmental Psychology, 9(3), 207-216.

Hodnett, E. D., Stremler, R., Weston, J. A., \& McKeever, P. (2009). Re-conceptualizing the hospital labor room: The PLACE (pregnant and laboring in an ambient clinical environment) pilot trial. Birth, 36(2), 159-166.

Hofmann, B. (2002). Respect for patients' dignity in primary health care: A critical appraisal. Scandinavian journal of primary health care, 20(2), 88-91. 
Hollander, J. B., \& Foster, V. (2016). Brain responses to architecture and planning: A preliminary neuro-assessment of the pedestrian experience in Boston, Massachusetts. Architectural science review, 59(6), 474-481.

Holmberg, K. S., Peterson, U. M., \& Oscarsson, M. G. (2014). A two-decade perspective on mothers' experiences and feelings related to breastfeeding initiation in Sweden. Sexual \& Reproductive Healthcare, 5(3), 125-130.

Homer, C. S. (2013). Home-like environments for labour and birth: Benefits for women and babies. BMJ Evidence-Based Medicine, 18(4), e32-e32.

Horsburgh, R. C. (1995). Occasional notes: Healing by design. THE NEW ENGLAND JOURNAL OF MEDICINE, 333(11), 735-740.

Howard, M. B., Gleeson, A., \& Higgins, S. (2014). Hospice patients' and families' preference for shared versus single rooms. Palliative medicine, 28(1), 94.

Huisman, E. R., Morales, E., van Hoof, J., \& Kort, H. (2012). Healing environment: A review of the impact of physical environmental factors on users. Building and environment, 58, 70-80.

Huynh, T.-G., Owens, R. L., \& Davidson, J. E. (2019). Impact of built design on nighttime family presence in the intensive care unit. HERD: Health Environments Research \& Design Journal, 18.

Hwang, R.-L., Lin, T.-P., Cheng, M.-J., \& Chien, J.-H. (2007). Patient thermal comfort requirement for hospital environments in Taiwan. Building and environment, 42(8), 2980-2987.

Igarashi, T., Wakita, M., Miyazaki, K., \& Nakayama, T. (2014). Birth environment facilitation by midwives assisting in non-hospital births: A qualitative interview study. Midwifery, 30(7), 877-884.

Ivankova, N. V., \& Stick, S. L. (2007). Students' persistence in a distributed doctoral program in educational leadership in higher education: A mixed methods study. Research in Higher Education, 48(1), 93-135.

Jackson, M., Dahlen, H., \& Schmied, V. (2012). Birthing outside the system: Perceptions of risk amongst Australian women who have freebirths and high risk homebirths. Midwifery, 28(5), 561-567.

Jackson, M. E., \& Bailes, A. J. (1995). Home birth with certified nurse-midwife attendants in the United States: An overview. Journal of Nurse-Midwifery, 40(6), 493-507.

Janssen, P. A., Carty, E. A., \& Reime, B. (2006). Satisfaction with planned place of birth among midwifery clients in British Columbia. The Journal of Midwifery \& Women's Health, 51(2), 9197.

Janssen, P. A., Klein, M. C., Harris, S. J., Soolsma, J., \& Seymour, L. C. (2000). Single room maternity care and client satisfaction. Birth, 27(4), 235-243.

Jenkinson, B., Josey, N., \& Kruske, S. (2014). BirthSpace: An evidence-based guide to birth environment design. Queensland Centre for Mothers \& Babies, The University of Queensland. 
Jiang, S. (2020). Positive distractions and play in the public spaces of pediatric healthcare environments: A literature review. HERD: Health Environments Research and Design Journal.

Jiang, S., \& Verderber, S. (2017). On the planning and design of hospital circulation zones: A review of the evidence-based literature. HERD: Health Environments Research \& Design Journal, 10(2), 124-146.

Johansson, K., Aarts, C., \& Darj, E. (2010). First-time parents' experiences of home-based postnatal care in Sweden. Upsala Journal of Medical Sciences, 115(2), 131-137.

Johansson, L., Bergbom, I., Waye, K. P., Ryherd, E., \& Lindahl, B. (2012). The sound environment in an ICU patient room-A content analysis of sound levels and patient experiences. Intensive and Critical Care Nursing, 28(5), 269-279.

Johansson, L., Lindahl, B., Knutsson, S., Ögren, M., Persson Waye, K., \& Ringdal, M. (2018). Evaluation of a sound environment intervention in an ICU: A feasibility study. Australian Critical Care, 31(2), 59-70.

Johansson, M., Rubertsson, C., Rådestad, I., \& Hildingsson, I. (2012). Childbirth-an emotionally demanding experience for fathers. Sexual \& Reproductive Healthcare, 3(1), 11-20.

Johansson, P., Oleni, M., \& Fridlund, B. (2002). Patient satisfaction with nursing care in the context of health care: A literature study. Scandinavian journal of caring sciences, 16(4), 337-344.

Johnson, P. (1996). Birth under water-to breathe or not to breathe. BJOG: An International Journal of Obstetrics \& Gynaecology, 103(3), 202-208.

Johnson, R. B., \& Onwuegbuzie, A. J. (2004). Mixed methods research: A research paradigm whose time has come. Educational researcher, 33(7), 14-26.

Johnson, R. B., Onwuegbuzie, A. J., \& Turner, L. A. (2007). Toward a definition of mixed methods research. Journal of Mixed Methods Research, 1(2), 112-133.

Jolley, S. (2005). Single rooms and patient choice. Nursing Standard, 20(9), 41 - 48.

Jongerden, I. P., Slooter, A. J., Peelen, L. M., Wessels, H., Ram, C. M., Kesecioglu, J., . . van Dijk, D. (2013). Effect of intensive care environment on family and patient satisfaction: A before-after study. Intensive Care Medicine, 39(9), 1626-1634.

Joroff, M. L., \& Morse, S. J. (1984). A proposed framework for the emerging field of architectural research. In J. C. Synder (Ed.), Architectural Research (pp. 15-28). New York: Van Nostrand.

Joseph, A., \& Ulrich, R. S. (2007). Sound control for improved outcomes in healthcare settings. In: The Robert Wood Johnson Foundation.

Jouhki, M.-R. (2012). Choosing homebirth-The women's perspective. Women and Birth, 25(4), e56e61.

Jung, C.-C., Wu, P.-C., Tseng, C.-H., \& Su, H.-J. (2015). Indoor air quality varies with ventilation types and working areas in hospitals. Building and environment, 85, 190-195.

Kabakian-Khasholian, T. (2013). 'My pain was stronger than my happiness': Experiences of caesarean births from Lebanon. Midwifery, 29(11), 1251-1256. 
Kaiser, H. F. (1974). An index of factorial simplicity. Psychometrika, 39(1), 31-36.

Kamdar, B. B., Needham, D. M., \& Collop, N. A. (2012). Sleep deprivation in critical illness: Its role in physical and psychological recovery. Journal of intensive care medicine, 27(2), 97-111.

Kaplan, R., \& Kaplan, S. (1989). The Experience of Nature: A Psychological Perspective: Cambridge University Press.

Kaplan, R., Kaplan, S., \& Ryan, R. (1998). With People in Mind: Design and Management of Everyday Nature: Island Press.

Kaplan, S. (1995). The restorative benefits of nature: Toward an integrative framework. Journal of Environmental Psychology, 15(3), 169-182.

Kaplowitz, M. D., Hadlock, T. D., \& Levine, R. (2004). A comparison of web and mail survey response rates. Public opinion quarterly, 68(1), 94-101.

Karakas, T., \& Yildiz, D. (2020). Exploring the influence of the built environment on human experience through a neuroscience approach: A systematic review. Frontiers of Architectural Research.

Karnik, M., Printz, B., \& Finkel, J. (2014). A hospital's contemporary art collection: Effects on patient mood, stress, comfort, and expectations. HERD: Health Environments Research \& Design Journal, 7(3), 60-77.

Karpanen, T. J., Casey, A., Lambert, P., Cookson, B., Nightingale, P., Miruszenko, L., \& Elliott, T. S. (2012). The antimicrobial efficacy of copper alloy furnishing in the clinical environment: A crossover study. Infection Control \& Hospital Epidemiology, 33(1), 3-9.

Karro, J., Dent, A. W., \& Farish, S. (2005). Patient perceptions of privacy infringements in an emergency department. Emergency Medicine Australasia, 17(2), 117-123.

Kealy, M. A., Small, R. E., \& Liamputtong, P. (2010). Recovery after caesarean birth: A qualitative study of women's accounts in Victoria, Australia. BMC pregnancy and childbirth, 10(1), 47.

Keating, A., \& Fleming, V. E. (2009). Midwives' experiences of facilitating normal birth in an obstetric-led unit: A feminist perspective. Midwifery, 25(5), 518-527.

Keep, P., James, J., \& Inman, M. (1980). Windows in the intensive therapy unit. Anaesthesia, 35(3), 257-262.

Kerfoot, K., \& Neumann, T. (1992). Creating a healing environment: The nurse manager's challenge. Nursing Economics, 10(6), 423-425.

Kesecioglu, J. (2015). Improving the patient's environment: The ideal intensive care unit. Réanimation, 24(2), 341-343.

Kincey, J., \& Saltmore, S. (1990). Surgical treatments. In M. Johnston \& L. Wallace (Eds.), Stress and Medical Procedures (pp. 120-137). Oxford, UK: Oxford University Press.

King, M.-F., Noakes, C., Sleigh, P., \& Camargo-Valero, M. (2013). Bioaerosol deposition in single and two-bed hospital rooms: A numerical and experimental study. Building and environment, $59,436-447$. 
Kirk, S. (2002). Patient preferences for a single or shared room in a hospice. Nursing times, 98(50), $39-41$.

Knutson, A. J. (2013). Acceptable noise levels for neonates in the neonatal intensive care unit. (Doctor of Audiology Doctoral), Washington University of Medicine, Independent Studies and Capstones. Retrieved from http://digitalcommons.wustl.edu/pacs capstones/643

Kol, E., Aydın, P., \& Dursun, O. (2015). The effectiveness of environmental strategies on noise reduction in a pediatric intensive care unit: Creation of single-patient bedrooms and reducing noise sources. Journal for Specialists in Pediatric nursing, 20(3), 210-217.

Kopec, D. A. (2006). Environmental Psychology for Design. New York: Fairchild.

Kotzer, A. M., Zacharakis, S. K., Raynolds, M., \& Buenning, F. (2011). Evaluation of the built environment: Staff and family satisfaction pre- and post-occupancy of the children's hospital. HERD: Health Environments Research \& Design Journal, 4(4), 60-78.

Kozub, E., Scheler, S., Necoechea, G., \& O’Byrne, N. (2017). Improving nurse satisfaction with open visitation in an adult intensive care unit. Critical Care Nursing Quarterly, 40(2), 144-154.

Krueger, R. A. (1994). Focus Groups : A Practical Guide for Applied Research (2nd ed.). Thousand Oaks, California: Sage Publications.

Krueger, R. A., \& Casey, M. A. (2000). Focus Groups: A Practical Guide for Applied Research (3rd ed.). Thousand Oaks, California: Sage Publications.

Krueger, R. A., \& Casey, M. A. (2002) Designing and conducting focus group interviews. In. Social Analysis: Selected Tools and Techniques. Minnesota, USA: Social Development Family of the World Bank.

Krueger, R. A., \& Casey, M. A. (2009). Focus Groups: A Practical Guide for Applied Research (4th ed.). Los Angeles: SAGE.

Kruger, G. B., \& McCann, T. V. (2018). Challenges to midwives' scope of practice in providing women's birthing care in an Australian hospital setting: A grounded theory study. Sexual \&eproductive Healthcare, 18, 37-42.

Kuck, M. (1972). The modern maternity unit. Public health, 86(4), 169-173.

Kurth, E., Spichiger, E., Stutz, E. Z., Biedermann, J., Hösli, I., \& Kennedy, H. P. (2010). Crying babies, tired mothers-challenges of the postnatal hospital stay: An interpretive phenomenological study. BMC pregnancy and childbirth, 10(1), 21.

Kutash, M., \& Northrop, L. (2007). Family members' experiences of the intensive care unit waiting room. Journal of advanced nursing, 60(4), 384-388.

Lankston, L., Cusack, P., Fremantle, C., \& Isles, C. (2010). Visual art in hospitals: Case studies and review of the evidence. Journal of the Royal Society of Medicine, 103(12), 490-499.

Larkin, P., Begley, C. M., \& Devane, D. (2012). 'Not enough people to look after you': An exploration of women's experiences of childbirth in the Republic of Ireland. Midwifery, 28(1), 98-105. 
Larsen, L. S., Larsen, B. H., \& Birkelund, R. (2014). A companionship between strangers-the hospital environment as a challenge in patient-patient interaction in oncology wards. Journal of advanced nursing, 70(2), 395-404.

Larsson, C., Saltvedt, S., Edman, G., Wiklund, I., \& Andolf, E. (2011). Factors independently related to a negative birth experience in first-time mothers. Sexual \& Reproductive Healthcare, 2(2), 83-89.

Laursen, J., Danielsen, A., \& Rosenberg, J. (2014). Effects of environmental design on patient outcome: A systematic review. HERD: Health Environments Research \& Design Journal, 7(4), 108-119.

LaVela, S. L., Etingen, B., Hill, J. N., \& Miskevics, S. (2016). Patient perceptions of the environment of care in which their healthcare is delivered. HERD: Health Environments Research \& Design Journal, 9(3), 31-46.

LaVela, S. L., \& Gallan, A. (2014). Evaluation and measurement of patient experience. Patient Experience Journal, 1(1), 28-36.

Laverty, S. M. (2003). Hermeneutic phenomenology and phenomenology: A comparison of historical and methodological considerations. International journal of qualitative methods, 2(3), 21-35.

Lawal, L. A., \& Vale, R. (2018). Inclusive birth supporters' space and recovery care in the postnatal hospital environment. Paper presented at the ASEAN PostGraduate Conference (APGC), University of Malaya, Kuala Lumpur.

Lawson, B., \& Phiri, M. (2000). Room for improvement. The Health service journal, 110(5688), 2426.

Lawson, B., Phiri, M., \& Wells-Thorpe, J. (2003). The architectural healthcare environment and its effects on patient health outcomes: A report on an NHS Estates funded research project. London: Stationery Office.

Lee, C. F., Lee, J. C., \& Lee, A. C. (2013). Statistics for Business and Financial Economics (3rd ed.). New York: Springer.

Leech, N. L., \& Onwuegbuzie, A. J. (2008). Qualitative data analysis: A compendium of techniques and a framework for selection for school psychology research and beyond. School Psychology Quarterly, 23(4), 587.

Leech, N. L., \& Onwuegbuzie, A. J. (2009). A typology of mixed methods research designs. Quality \& quantity, 43(2), 265-275.

Lei, Z., Qiongjing, Y., Qiuli, W., Sabrina, K., Xiaojing, L., \& Changli, W. (2009). Sleep quality and sleep disturbing factors of inpatients in a Chinese general hospital. Journal of Clinical Nursing, 18(17), 2521-2529.

Lepori, R. B. (1994). Freedom of movement in birth places. Children's Environments, 11(2), 81-87.

Lepori, R. B., Foureur, M. J., \& Hastie, C. (2008). Mindbodyspirit architecture: Creating birth space. In K. Fahy, M. Foureur, \& C. Hastie (Eds.), Birth Territory and Midwifery Guardianship: Theory for Practice, Education and Research. Edinburgh: Elsevier Limited. 
Leydecker, S. (2017). Healthy patient rooms in hospitals: Emotional wellbeing naturally. Architectural Design, 87(2), 76-81.

Li, Y., Leung, G. M., Tang, J., Yang, X., Chao, C., Lin, J. Z., .. . Qian, H. (2007). Role of ventilation in airborne transmission of infectious agents in the built environment-A multidisciplinary systematic review. Indoor air, 17(1), 2-18.

Lim, R. (2018). Benefits of quiet time interventions in the intensive care unit: A literature review. Nursing Standard, 32(30), 41.

Lindberg, C. M., Tran, D. T., \& Banasiak, M. A. (2016). Individual differences in the office: Personality factors and work-space enclosure. Journal of Architectural and Planning Research, 33(2), 105-120.

Linder, L. A., \& Christian, B. J. (2011). Characteristics of the nighttime hospital bedside care environment (sound, light, and temperature) for children with cancer. Cancer Nursing, 34(3), 176.

Lindgren, H. E., \& Erlandsson, K. (2010). Women's experiences of empowerment in a planned home birth: A Swedish population-based study. Birth, 37(4), 309-317.

Lindgren, H. E., Rådestad, I. J., \& Hildingsson, I. (2011). Transfer in planned home births in Sweden-effects on the experience of birth: A nationwide population-based study. Sexual \& Reproductive Healthcare, 2(3), 101-105.

Lohr, V. I., \& Pearson-Mims, C. H. (2000). Physical discomfort may be reduced in the presence of interior plants. HortTechnology, 10(1), 53-58.

Long, T., \& Stover, P. (2014). A culture of quiet: Caring for patients by creating an environment for healing. International Journal of Human Caring, 18(2), 46-46.

Lorenz, S. G. (2007). The potential of the patient room to promote healing and well-being in patients and nurses: An integrative review of the research. Holistic nursing practice, 21(5), 263277.

Loupa, G. (2020). Influence of noise on patient recovery. Current Pollution Reports, 1-7.

Lundgren, I. (2010). Women's experiences of giving birth and making decisions whether to give birth at home when professional care at home is not an option in public health care. Sexual \& Reproductive Healthcare, 1(2), 61-66.

MacAllister, L., Zimring, C., \& Ryherd, E. (2016). Environmental variables that influence patient satisfaction: A review of the literature. HERD: Health Environments Research \& Design Journal, 10(1), 155-169.

MacAllister, L., Zimring, C., \& Ryherd, E. (2019). Exploring the relationships between patient room layout and patient satisfaction. HERD: Health Environments Research and Design Journal, 12(1), 91-107.

MacArthur, C., Winter, H., Bick, D., Knowles, H., Lilford, R., Henderson, C., . . Gee, H. (2002). Effects of redesigned community postnatal care on womens' health 4 months after birth: A cluster randomised controlled trial. The Lancet, 359(9304), 378-385. 
Mackrill, J., Cain, R., \& Jennings, P. (2013). Experiencing the hospital ward soundscape: Towards a model. Journal of Environmental Psychology, 36, 1-8.

Mackrill, J., Jennings, P., \& Cain, R. (2014). Exploring positive hospital ward soundscape interventions. Applied ergonomics, 45(6), 1454-1460.

Madriz, E. (2000). Focus groups in feminist research. In N. K. Denzin \& Y. S. Lincoln (Eds.), Handbook of qualitative research (2nd ed., pp. 835-850). Thousands Oaks, California: Sage Publications.

Maguire, D. J., Burger, K. J., O'Donnell, P. A., \& Parnell, L. (2013). Clinician perceptions of a changing hospital environment. HERD: Health Environments Research \& Design Journal, 6(3), 69-79.

Mahmood, F. J., \& Tayib, A. Y. (2019). Healing environment correlated with patients' psychological comfort: Post-occupancy evaluation of general hospitals. Indoor and Built Environment.

Maisels, M. J. (2001). Phototherapy—traditional and nontraditional. Journal of Perinatology, 21(1), S93-S97.

Maisels, M. J., \& McDonagh, A. F. (2008). Phototherapy for neonatal jaundice. New England Journal of Medicine, $358(9)$, 920-928.

Malkin, J. (1992). Hospital Interior Architecture: Creating Healing Environments for Special Patient Populations: Van Nostrand Reinhold Company.

Malkin, J. (2003). The business case for creating a healing environment. Center for Health Design Business Briefing: Hospital Engineering \& Facilities Management, 1.

Malkin, J. (2008). A Visual Reference for Evidence-Based Design: Center for Health Design.

Manary, M. P., Boulding, W., Staelin, R., \& Glickman, S. W. (2013). The patient experience and health outcomes. New England Journal of Medicine, 368(3), 201-203.

Mantz, R., \& Britton, J. R. (2007). Hospital construction and postpartum anxiety. General hospital psychiatry, 29(6), 562-566.

Marcus, C. C. (2007). Healing gardens in hospitals. Interdisciplinary Design and Research e-Journal, 1(1), $1-27$.

Marcus, C. C., \& Sachs, N. A. (2014). Therapeutic Landscapes: An Evidence-Based Approach to Designing Healing Gardens and Restorative Outdoor Spaces. Hoboken, New Jersey: John Wiley \& Sons.

Martell, L. K. (2000). The hospital and the postpartum experience: A historical analysis. Journal of Obstetric, Gynecologic, \& Neonatal Nursing, 29(1), 65-72.

Martell, L. K. (2001). Heading toward the new normal: A contemporay postpartum experience. Journal of Obstetric, Gynecologic, \& Neonatal Nursing, 30(5), 496-506.

Martell, L. K. (2003). Postpartum women's perceptions of the hospital environment. Journal of Obstetric, Gynecologic, \& Neonatal Nursing, 32(4), 478-485. 
Martin, C. (2000). Putting patients first: Integrating hospital design and care. The Lancet, 356(9228), 518.

Maternity Services Consumer Council. (2018). Women's assessments of their 'inpatient' postnatal care. In TENA KOUTOU KATOA, KIA ORANA, TALOFA LAVA, MALO LELEI, FAKAALOFA ATU (Vol. 110).

Mathews, J. J., \& Zadak, K. (1991). The alternative birth movement in the United States: History and current status. Women \& bealth, 17(1), 39-56.

Matiti, M., Cotrel-Gibbons, E., \& Teasdale, K. (2007). Promoting patient dignity in healthcare settings. Nursing Standard (through 2013), 21(45), 46.

Maude, R. M., \& Foureur, M. J. (2007). It's beyond water: Stories of women's experience of using water for labour and birth. Women and Birth, 20(1), 17-24.

Mazuch, R., \& Stephen, R. (2005). Creating healing environments: Humanistic architecture and therapeutic design. Journal of Public Mental Health, 4(4), 48-52.

Mbekenga, C. K., Lugina, H. I., Christensson, K., \& Olsson, P. (2011). Postpartum experiences of first-time fathers in a Tanzanian suburb: A qualitative interview study. Midwifery, 27(2), 174180.

McCourt, C., Rayment, J., Rance, S., \& Sandall, J. (2016). Place of birth and concepts of wellbeing: An analysis from two ethnographic studies of midwifery units in England. Anthropology in Action, 23(3), 17-29.

McDermott, K., Elixhauser, A., \& Sun, R. (2017). Trends in hospital inpatient stays in the United States, 2005-2014. Statistical Brief, 225.

McLachlan, H. L., Gold, L., Forster, D. A., Yelland, J., Rayner, J., \& Rayner, S. (2009). Women's views of postnatal care in the context of the increasing pressure on postnatal beds in Australia. Women and Birth, 22(4), 128-133.

Mendelson, D., \& Wolf, G. (2017). Health privacy and confidentiality. In I. Freckelton \& K. Peterson (Eds.), Tensions and Traumas in Health Laws: Federation Press.

Menke, S., Jenkinson, B., Foureur, M. J., \& Kildea, S. (2019). Is the birthing unit design spatial evaluation tool valid for diverse groups? Women and Birth, 32(4), 372-379.

Mercer, R. T., \& Ferketich, S. L. (1988). Stress and social support as predictors of anxiety and depression during pregnancy. Advances in Nursing Science, 10(2), 26-39.

Mingers, J. (2004). Critical realism and information systems: Brief responses to Monod and Klein. Information and Organization, 14(2), 145-153.

Ministry of Health New Zealand. (2019). Report on maternity 2017. Wellington Retrieved from https://www.health.govt.nz/publication/report-maternity-2017.

Mondy, T., Fenwick, J., Leap, N., \& Foureur, M. J. (2016). How domesticity dictates behaviour in the birth space: Lessons for designing birth environments in institutions wanting to promote a positive experience of birth. Midwifery, 43, 37-47. 
Monsen, M. G., \& Edéll-Gustafsson, U. M. (2005). Noise and sleep disturbance factors before and after implementation of a behavioural modification programme. Intensive and Critical Care Nursing, 21(4), 208-219.

Monti, F., Agostini, F., Dellabartola, S., Neri, E., Bozicevic, L., \& Pocecco, M. (2012). Pictorial intervention in a pediatric hospital environment: Effects on parental affective perception of the unit. Journal of Environmental Psychology, 32(3), 216-224.

Moore, G. T. (1984). New directions for environment-behavior research in architecture. In J. C. Snyder (Ed.), Architectural Research (Vol. 6, pp. 95-112). New York: Van Nostrand Reinhold.

Moore, M. L. (2003). Current studies on two separate topics: Breastfeeding postpartum length of hospital stay. The Journal of perinatal education, 12(2), 44-46.

Moran, D., \& Mooney, T. (2002). The Phenomenology Reader: Psychology Press.

Morgan, D. L. (1996). Focus groups. Annual review of sociology, 22(1), 129-152.

Morgan, D. L. (1998). Practical strategies for combining qualitative and quantitative methods: Applications to health research. Qualitative health research, 8(3), 362-376.

Morgan, D. L. (2007). Paradigms lost and pragmatism regained: Methodological implications of combining qualitative and quantitative methods. Journal of Mixed Methods Research, 1(1), 4876.

Morrison, B., \& Ludington-Hoe, S. (2012). Interruptions to breastfeeding dyads in an LDRP Unit. The American Journal of Maternal/ Child Nursing, 37(1), 36-41.

Morrison, W. E., Haas, E. C., Shaffner, D. H., Garrett, E. S., \& Fackler, J. C. (2003). Noise, stress, and annoyance in a pediatric intensive care unit. Critical care medicine, 31(1), 113-119.

Morrow, J., McLachlan, H., Forster, D., Davey, M.-A., \& Newton, M. (2013). Redesigning postnatal care: Exploring the views and experiences of midwives. Midwifery, 29(2), 159-166.

Morse, J. M., \& Niehaus, L. (2009). Mixed Method Design : Principles and Procedures. Walnut Creek, California: Left Coast Press.

Mourshed, M., \& Zhao, Y. (2012). Healthcare providers' perception of design factors related to physical environments in hospitals. Journal of Environmental Psychology, 32(4), 362-370.

Moustakas, C. E. (1994). Phenomenological Research Methods. Thousand Oaks, California: Sage Publications.

Murphy, M., \& Mansfield, J. (2017). Can architecture heal? Building as instruments of health. Architectural Design, 87(2), 82-89.

Murthy, V. S., Malhotra, S., Bala, I., \& Raghunathan, M. (1995). Detrimental effects of noise on anaesthetists. Canadian Journal of Anaesthesia, 42(7), 608-611.

Nanda, U., Chanaud, C., Nelson, M., Zhu, X., Bajema, R., \& Jansen, B. H. (2012). Impact of visual art on patient behavior in the emergency department waiting room. The Journal of emergency medicine, 43(1), 172-181. 
Nassauer, J. I. (1995). Culture and changing landscape structure. Landscape ecology, 10(4), 229-237.

Nastasi, B. K., Hitchcock, J. H., \& Brown, L. M. (2010). An inclusive framework for conceptualizing mixed methods design typologies: Moving toward fully integrated synergistic research models. Handbook of mixed methods in social \& behavioral research, 2, 305338.

Negron, R., Martin, A., Almog, M., Balbierz, A., \& Howell, E. A. (2013). Social support during the postpartum period: Mothers' views on needs, expectations, and mobilization of support. Maternal and child health journal, 17(4), 616-623.

Nejati, A., Rodiek, S., \& Shepley, M. (2016). The implications of high-quality staff break areas for nurses' health, performance, job satisfaction and retention. Journal of nursing management, 24(4), 512-523.

Nejati, A., Shepley, M., Rodiek, S., Lee, C., \& Varni, J. (2016). Restorative design features for hospital staff break areas: A multi-method study. HERD: Health Environments Research \& Design Journal, 9(2), 16-35.

Newburn, M., \& Singh, D. (2003). Creating a better birth environment: Women's views about the design and facilities in maternity units. London: National Childbirth Trust.

Newburn, M., \& Singh, D. (2005). Are women getting the birth environment they need: Report of a national survey of women's experiences. London: National Childbirth Trust.

NHS Estates. (2005). W ard layouts with single rooms and space for flexibility. London: The Stationery Office.

Nickel, H. (2011). Obstetrics. In P. Meuser (Ed.), Construction and Design Manual: Hospitals and Health Centres: Specialist Clinics and Medical Departments (Vol. 2, pp. 15-24): DOM publishers.

Nightingale, F. (1863). Notes on hospitals: Longman, Green, Longman, Roberts, and Green.

Nimlyat, P. S., \& Kandar, M. Z. (2015). Appraisal of indoor environmental quality (IEQ) in healthcare facilities: a literature review. Sustainable Cities and Society, 17, 61-68.

Notelovitz, M. (1978). The single-unit delivery system-A safe alternative to home deliveries. American Journal of Obstetrics \& Gynecology, 132(8), 889-894.

Nunnally, J. C. (1978). Psychometric Theory (2nd ed.). New York: McGraw-Hill.

O'Cathain, A. (2009). Mixed methods research in the health sciences: A quiet revolution. Journal of Mixed Methods Research, 3(1), 3-6.

O'Cathain, A., Murphy, E., \& Nicholl, J. (2007). Why, and how, mixed methods research is undertaken in health services research in England: A mixed methods study. BMC health services research, 7(1), 85.

O'Connor, M., O'Brien, A., Bloomer, M., Morphett, J., Peters, L., Hall, H., . . Munro, I. (2012). The environment of inpatient healthcare delivery and its influence on the outcome of care. HERD: Health Environments Research \& Design Journal, 6(1), 104-116. 
Olausson, S., Ekebergh, M., \& Lindahl, B. (2012). The ICU patient room: Views and meanings as experienced by the next of kin: A phenomenological hermeneutical study. Intensive and Critical Care Nursing, 28(3), 176-184.

Oliveira, L., Gomes, C., Nicolau, L. B., Ferreira, L., \& Ferreira, R. (2015). Environment in pediatric wards: Light, sound, and temperature. Sleep medicine, 16(9), 1041-1048.

Olsen, J. C., \& Sabin, B. R. (2003). Emergency department patient perceptions of privacy and confidentiality. The Journal of emergency medicine, 25(3), 329-333.

Olsson, A., Lundqvist, M., Faxelid, E., \& Nissen, E. (2005). Women's thoughts about sexual life after childbirth: Focus group discussions with women after childbirth. Scandinavian journal of caring sciences, 19(4), 381-387.

Onwuegbuzie, A. J., Dickinson, W. B., Leech, N. L., \& Zoran, A. G. (2009). A qualitative framework for collecting and analyzing data in focus group research. International journal of qualitative methods, 8(3), 1-21.

Orr, R. (1995). The planetree philosophy. In S. O. Marberry (Ed.), Innovations in Healthcare Design: Selected Presentations from the First Five Symposia on Healthcare Design. New York: Van Nostrand Reinhold.

Overgaard, C., Fenger-Grøn, M., \& Sandall, J. (2012). Freestanding midwifery units versus obstetric units: Does the effect of place of birth differ with level of social disadvantage? BMC Public Health, 12(1), 478.

Pallasmaa, J. (2005). Lived space: Embodied experience and sensory thought. Encounters: architectural essays.

Pallasmaa, J., Mallgrave, H. F., \& Arbib, M. A. (2013). Architecture and Neuroscience: Tapio WirkkalaRut Bryk Foundation.

Palumbo, L., Ruta, N., \& Bertamini, M. (2015). Comparing angular and curved shapes in terms of implicit associations and approach/avoidance responses. PloS one, 10(10).

Park, J. G., \& Park, C. (2013). Color perception in pediatric patient room design: American versus Korean pediatric patients. HERD: Health Environments Research \& Design Journal, 6(4), 10 26.

Park, K.-O. (2007). Social support for stress prevention in hospital settings. The journal of the Royal Society for the Promotion of Health, 127(6), 260-264.

Park, M. J., Yoo, J. H., Cho, B. W., Kim, K. T., Jeong, W.-C., \& Ha, M. (2014). Noise in hospital rooms and sleep disturbance in hospitalized medical patients. Environmental health and toxicology, 29.

Park, S.-H., \& Mattson, R. H. (2009). Ornamental indoor plants in hospital rooms enhanced health outcomes of patients recovering from surgery. The journal of alternative and complementary medicine, 15(9), 975-980.

Parsons, M. T., Mahoney, C., \& Weathers, L. S. (1999). Family suite: An innovative method to provide inexpensive postpartum care. Health Care Management Review, 24(4), 65-69. 
Parvin, A., Jones, C. E., \& Hull, S. A. (2004). Experiences and understandings of social and emotional distress in the postnatal period among Bangladeshi women living in Tower Hamlets. Family Practice, 21 (3), 254-260.

Pascali-Bonaro, D., \& Kroeger, M. (2004). Continuous female companionship during childbirth: A crucial resource in times of stress or calm. The Journal of Midwifery \& Women's Health, 49(S1), 19-27.

Pasha, S. (2013). Barriers to garden visitation in children's hospitals. HERD: Health Environments Research \& Design Journal, 6(4), 76-96.

Passini, R., Rainville, C., Marchand, N., \& Joanette, Y. (1995). Wayfinding in dementia of the Alzheimer type: Planning abilities. Journal of clinical and experimental neuropsychology, 17(6), 820832.

Pati, D., Freier, P., O’Boyle, M., Amor, C., \& Valipoor, S. (2016). The impact of simulated nature on patient outcomes: A study of photographic sky compositions. HERD: Health Environments Research \& Design Journal, 9(2), 36-51.

Pati, D., Harvey Jr, T. E., \& Barach, P. (2008). Relationships between exterior views and nurse stress: An exploratory examination. HERD: Health Environments Research \& Design Journal, 1(2), 27-38.

Pati, D., \& Nanda, U. (2011). Influence of positive distractions on children in two clinic waiting areas. HERD: Health Environments Research \& Design Journal, 4(3), 124-140.

Pati, D., O’Boyle, M., Hou, J., Nanda, U., \& Ghamari, H. (2016). Can hospital form trigger fear response? HERD: Health Environments Research \& Design Journal, 9(3), 162-175.

Patterson, A. H., \& Passini, R. (1974). The evaluation of physical settings: To measure attitudes, behavior, or both? Paper presented at the EDRA.

Patterson, E. T. (1998). Early postpartum discharge: How are mothers managing? MCN: The American Journal of Maternal/Child Nursing, 23(5), 278.

Pease, N., \& Finlay, I. G. (2002). Do patients and their relatives prefer single cubicles or shared wards? Palliative medicine, 16(5), 445-446.

Pennington, H., \& Isles, C. (2013). Should hospitals provide all patients with single rooms? BMJ, 347, f5695.

Peri Bader, A. (2015). A model for everyday experience of the built environment: The embodied perception of architecture. The Journal of Architecture, 20(2), 244-267.

Persson, E., Anderberg, P., \& Kristensson Ekwall, A. (2015). A room of one' s own-Being cared for in a hospital with a single-bed room design. Scandinavian journal of caring sciences, 29(2), 340-346.

Peter, B., Hunter, C., \& Howlett, O. (2003). The benefits of daylight through windows. Troy, New York: Rensselaer Polytechnic Institute. 
Phillips, C. R. (1999). Family-centered maternity care: Past, present, future. International Journal of Childbirth Education, 14(4), 6.

Pilkington, S. (2013). Causes and consequences of sleep deprivation in hospitalised patients. Nursing Standard, 27(49).

Pineda, R. G., Stransky, K. E., Rogers, C., Duncan, M. H., Smith, G. C., Neil, J., \& Inder, T. (2012). The single-patient room in the NICU: Maternal and family effects. Journal of Perinatology, 32(7), 545-551.

Pinette, M. G., Wax, J., \& Wilson, E. (2004). The risks of underwater birth. American journal of obstetrics and gynecology, 190(5), 1211-1215.

Plough, A., Polzin-Rosenberg, D., Galvin, G., Shao, A., Sullivan, B., Henrich, N., \& Shah, N. T. (2019). An exploratory study of the relationship between facility design and the provision of childbirth care. Journal of midwifery \& women's health, 64(1), 12-17.

PMMRC. (2018). Twelfth annual report of the perinatal and maternal mortality review committee: Reporting mortality 2016. Wellington: Health Quality \& Safety Commission. Retrieved from http://www.hqsc.govt.nz/our-programmes/mrc/pmmrc.

Poh, H. L., Koh, S. S. L., Seow, H. C. L., \& He, H.-G. (2014). First-time fathers' experiences and needs during pregnancy and childbirth: A descriptive qualitative study. Midwifery, 30(6), 779-787.

Polit, D. F., \& Beck, C. T. (2017). Nursing Research: Generating and Assessing Evidence for Nursing Practice (10th ed.). Philadelphia: Wolters Kluwer.

Pope, D. (2010). Decibel levels and noise generators on four medical/surgical nursing units. Journal of Clinical Nursing, 19(17-18), 2463-2470.

Powell, R. A., \& Single, H. M. (1996). Focus groups. International Journal for Quality in Health Care, $8(5), 499-504$.

Powell, R. A., Single, H. M., \& Lloyd, K. R. (1996). Focus groups in mental health research: Enhancing the validity of user and provider questionnaires. International Journal of Social Psychiatry, 42(3), 193-206.

Public Health Agency of Canada. (2009). What mothers say: The Canadian maternity experiences survey. Retrieved from www.publichealth.gc.ca/mes.

Pugh, R. J., Jones, C., \& Griffiths, R. (2007). The impact of noise in the intensive care unit. In Intensive Care Medicine (pp. 942-949): Springer.

Qualtrics, L. (2017). Qualtrics [software]. Retrieved from https://vuw.qualtrics.com

Quan, X., Joseph, A., \& Ensign, J. C. (2012). Impact of imaging room environment: Staff job stress and satisfaction, patient satisfaction, and willingness to recommend. HERD: Health Environments Research \& Design Journal, 5(2), 61-79. 
Rados, M., Kovács, E., \& Mészáros, J. (2015). Intimacy and privacy during childbirth. A pilotstudy testing a new self-developed questionnaire: The childbirth intimacy and privacy scale (CIPS). New Medicine, 19(1), 16-24.

Ramaswamy, M., Al-Jahwari, F., \& Al-Rajhi, S. M. M. (2010). LAQ in Hospitals-Better Health through Indoor Air Quality Awareness. Paper presented at the Proceedings of the Tenth International Conference Enhanced Building Operations, Kuwait.

Rashid, M. (2013). The question of knowledge in evidence-based design for healthcare facilities: Limitations and suggestions. HERD: Health Environments Research \& Design Journal, 6(4), 101-126.

Rayner, J.-A., Forster, D., McLachlan, H., Yelland, J., \& Davey, M.-A. (2008). A state-wide review of hospital postnatal care in Victoria, Australia: The views and experiences of midwives. Midwifery, 24(3), 310-320.

Rayner, J.-A., McLachlan, H. L., Peters, L., \& Forster, D. A. (2013). Care providers' views and experiences of postnatal care in private hospitals in Victoria, Australia. Midwifery, 29(6), 622-627.

Razurel, C., Bruchon-Schweitzer, M., Dupanloup, A., Irion, O., \& Epiney, M. (2011). Stressful events, social support and coping strategies of primiparous women during the postpartum period: A qualitative study. Midwifery, 27(2), 237-242.

Redshaw, M., Rowe, R., Hockley, C., \& Brocklehurst, P. (2007). Recorded delivery: A national survey of women's experience of maternity care 2006. Retrieved from NPEU, Oxford: http://www.npeu.ox.ac.uk/recorded-delivery

Redshaw, M., Rowe, R., Schroeder, L., Puddicombe, D., Macfarlane, A., Newburn, M., . . Marlow, N. (2011). Mapping maternity care: the configuration of maternity care in England. Birthplace in England research programme.

Reed, R. A. (1995). Creating a healing environment by design. The Journal of ambulatory care management, 18(4), 16-31.

REHAB Basel. (n.d.). Welcome to REHAB Basel. Retrieved from https://www.rehab.ch/en/home.html

Riggs, E., Yelland, J., Szwarc, J., Wahidi, S., Casey, S., Chesters, D., . . . Brown, S. (2016). Fatherhood in a new country: A qualitative study exploring the experiences of Afghan men and implications for health services. Birth, 43(1), 86-92.

Rippin, A. S., Zimring, C., Samuels, O., \& Denham, M. E. (2015). Finding a middle ground: Exploring the impact of patient- and family-centered design on nurse-family interactions in the neuro ICU. HERD : Health Environments Research \& Design Journal, 9(1), 80-98.

Robinson, J. W. (1990). Architectural research: Incorporating myth and science. Journal of Architectural Education, 44(1), 20-32.

Rodríguez, R. G., \& Pattini, A. E. (2016). Neonatal intensive care unit lighting: Update and recommendations. Arch Argent Pediatr, 114(4), 361-367. 
Roland, P., Russell, J., Kathy Culpepper, R., \& Sheila Cox, S. (2001). Visitation in critical care: Processes and outcomes of a performance improvement initiative. Journal of nursing care quality, 15(2), 18-26.

Rubin, H. R., Owens, A. J., \& Golden, G. (1998). Status report (1998): An investigation to determine whether the built environment affects patients' medical outcomes: Center for Health Design Martinez, CA.

Rudman, A., \& Waldenström, U. (2007). Critical views on postpartum care expressed by new mothers. BMC health services research, 7(1), 178.

Ruga, W. (1989). Designing for the six senses. Journal of health care interior design: proceedings from the . Annual National Symposium on Health Care Interior Design. National Symposium on Health Care Interior Design (U. S.), 1, 29-34.

Ruppenthal, L., Tuck, J., \& Gagnon, A. J. (2005). Enhancing research with migrant women through focus groups. Western Journal of Nursing Research, 27(6), 735-754.

Saad, M., \& de Medeiros, R. (2016). Programs of religious/spiritual support in hospitals-five "Whies" and five "Hows". Philosophy, Ethics, and Humanities in Medicine, 11(1), 5.

Sadler, G. R., Lee, H. C., Lim, R. S. H., \& Fullerton, J. (2010). Recruitment of hard-to-reach population subgroups via adaptations of the snowball sampling strategy. Nursing \& bealth sciences, 12(3), 369-374.

Sahlin, M., Carlander-Klint, A.-K., Hildingsson, I., \& Wiklund, I. (2013). First-time mothers' wish for a planned caesarean section: Deeply rooted emotions. Midwifery, 29(5), 447-452.

Salonen, A. H., Oommen, H., \& Kaunonen, M. (2014). Primiparous and multiparous mothers' perceptions of social support from nursing professionals in postnatal wards. Midwifery, 30(4), 476-485.

Salonen, H., Lahtinen, M., Lappalainen, S., Nevala, N., Knibbs, L. D., Morawska, L., \& Reijula, K. (2013). Design approaches for promoting beneficial indoor environments in healthcare facilities: A review. Intelligent Buildings International, 5(1), 26-50.

Sapkota, S., Kobayashi, T., \& Takase, M. (2012). Husbands' experiences of supporting their wives during childbirth in Nepal. Midwifery, 28(1), 45-51.

Savage, J. S. (2001). Birth stories: A way of knowing in childbirth education. The Journal of perinatal education, 10(2), 3.

Scherer, K. R. (2005). What are emotions? And how can they be measured? Social science information, 44(4), 695-729.

Schmied, V., \& Bick, D. (2014). Postnatal care - Current issues and future challenges. Midwifery, 30(6), 571-574.

Schmied, V., Cooke, M., Gutwein, R., Steinlein, E., \& Homer, C. (2008). Time to listen: Strategies to improve hospital-based postnatal care. Women and Birth, 21(3), 99-105. 
Schmied, V., Cooke, M., Gutwein, R., Steinlein, E., \& Homer, C. (2009). An evaluation of strategies to improve the quality and content of hospital-based postnatal care in a metropolitan Australian hospital. Journal of Clinical Nursing, 18(13), 1850-1861.

Schott, S., Avoort, I., Descamps, P., Richmond, D., Adams, T., Oei, G., . . Friese, K. (2014). Four countries, four ways of discussing low-risk pregnancy and normal delivery: In France, Germany, The Netherlands, and the United Kingdom. Archives of Gynecology and Obstetrics, 289(2), 451-456.

Schreuder, E., Lebesque, L., \& Bottenheft, C. (2016). Healing environments: What design factors really matter according to patients? an exploratory analysis. HERD: Health Environments Research \& Design Journal, 10(1), 87-105.

Schwandt, T. A. (2007). The Sage Dictionary of Qualitative Inquiry (3rd ed.): Sage publications.

Schweitzer, M., Gilpin, L., \& Frampton, S. (2004). Healing spaces: Elements of environmental design that make an impact on health. Journal of Alternative \& Complementary Medicine, 10(Supplement 1), S-71-S-83.

Seamon, D. (2000). A way of seeing people and place: Phenomenology in environment-behavior research. In Theoretical Perspectives in Environment-Behavior Research: Underlying Assumptions, Research Problems, and Methodologies. (pp. 157-178). Dordrecht, Netherlands: Kluwer Academic Publishers.

Seibold, C., Licqurish, S., Rolls, C., \& Hopkins, F. (2010). 'Lending the space': Midwives perceptions of birth space and clinical risk management. Midwifery, 26(5), 526-531.

Selman, L., Speck, P., Gysels, M., Agupio, G., Dinat, N., Downing, J., . . . Harding, R. (2013). 'Peace' and 'life worthwhile' as measures of spiritual well-being in African palliative care: A mixed-methods study. Health and Quality of Life Outcomes, 11, 94.

Setola, N., Naldi, E., Cocina, G. G., Eide, L. B., Iannuzzi, L., \& Daly, D. (2019). The impact of the physical environment on intrapartum maternity care: Identification of eight crucial building spaces. HERD: Health Environments Research \& Design Journal, 12(4), 67-98.

Shattell, M., Bartlett, R., Beres, K., Southard, K., Bell, C., Judge, C. A., \& Duke, P. (2015). How patients and nurses experience an open versus an enclosed nursing station on an inpatient psychiatric unit. Journal of the American Psychiatric Nurses Association, 21(6), 398-405.

Sheehy, A., Foureur, M. J., Catling-Paull, C., \& Homer, C. S. (2011). Examining the content validity of the birthing unit design spatial evaluation tool within a woman-centered framework. The Journal of Midwifery \& Women's Health, 56(5), 494-502.

Shepley, M. M. (2006). The role of positive distraction in neonatal intensive care unit settings. Journal of Perinatology, 26(S3), S34.

Shepley, M. M. (2012). The on-again off-again romance between nature and technology in healthcare settings. Paper presented at the ACSA Proceedings, annual conference, Boston, MA. 
Shepley, M. M., Bryant, C., \& Frohman, B. (1995). Validating a building prototype: A postoccupancy evaluation of a women's medical center. Journal of Interior Design, 21(2), 15-29.

Shepley, M. M., Pasha, S., Ferguson, P., Huffcut, J. C., Kiyokawa, G., \& Martere, J. (2013). Design research and behavioral health facilities. The Center for Health Design.

Shepley, M. M., \& Wilson, P. (1999). Designing for persons with AIDS: A post-occupancy study at the Bailey-Boushay House. Journal of Architectural and Planning Research, 16(1), 17-32.

Shin, J. H., Maxwell, L. E., \& Eshelman, P. (2004). Hospital birthing room design: A study of mothers' perception of hominess. Journal of Interior Design, 30(1), 23-36.

Shiovitz-Ezra, S., \& Litwin, H. (2012). Social network type and health-related behaviors: Evidence from an American national survey. Social Science \& Medicine, 75(5), 901-904.

Shirazi, M. R. (2014). Towards an Articulated Phenomenological Interpretation of Architecture: Phenomenal Phenomenology: Routledge

Short, A. E., Short, K. T., Holdgate, A., Ahern, N., \& Morris, J. (2011). Noise levels in an Australian emergency department. Australasian Emergency Nursing Journal, 14(1), 26-31.

Shulkin, D., O'Keefe, T., Visconi, D., Robinson, A., Rooke, A. S., \& Neigher, W. (2014). Eliminating visiting hour restrictions in hospitals. Journal for Healthcare Quality, 36(6), 54-57.

Siddiqui, Z. K., Zuccarelli, R., Durkin, N., Wu, A. W., \& Brotman, D. J. (2015). Changes in patient satisfaction related to hospital renovation: Experience with a new clinical building. Journal of hospital medicine, 10(3), 165-171.

Silverman, D. (2013). Doing Qualitative Research (4th ed.). London: Sage Publications

Simkin, P. (1986). Stress, pain, and catecholamines in labor: Part 2. stress associated with childbirth events: A pilot survey of new mothers. Birth, 13(4), 234-240.

Singh, D., \& Newburn, M. (2006). Feathering the nest: What women want from the birth environment. RCM midwives: the official journal of the Royal College of Midwives, 9(7), 266-269.

Smith, A., Winter, S., Lappin, D., Sherriff, A., McIvor, I., Philp, P., .. Stewart, A. (2018). Reducing the risk of iatrogenic Creutzfeldt-Jakob disease by improving the cleaning of neurosurgical instruments. Journal of Hospital Infection, 100(3), e70-e76.

Smith, J. A. (1999). Identity development during the transition to motherhood: An interpretative phenomenological analysis. Journal of reproductive and infant psychology, 17(3), 281-299.

Smith, J. A., Flowers, P., \& Larkin, M. (2009). Interpretative Phenomenological Analysis : Theory, Method and Research. London: Sage Publications.

Sommer, R. (1983). Social Design: Creating Building with People in Mind. New Jersey: Prentice Hall.

Spradlin, L. R. (2009). Implementation of a couplet care program for families after a cesarean birth. AORN Journal, 89(3), 553-562.

Stall, N. (2012). Private rooms: Evidence-based design in hospitals. Canadian Medical Association, 184(2), 162-163. 
Stark, M. A. (1999). The effect of a restorative intervention on directed attentional capacity in the third trimester of pregnancy and labor pain. (PhD Thesis), The University of Michigan, Retrieved from https://search.proquest.com/docview/304521472? accountid=14782 ProQuest Central database. (9929960)

Stark, M. A. (2000). Is it difficult to concentrate during the 3rd trimester and postpartum? Journal of Obstetric, Gynecologic, \& Neonatal Nursing, 29(4), 378-389.

Stark, M. A., Remynse, M., \& Zwelling, E. (2016). Importance of the birth environment to support physiologic birth. Journal of Obstetric, Gynecologic \& Neonatal Nursing, 45(2), 285-294.

Steel, A., Adams, J., Frawley, J., Broom, A., \& Sibbritt, D. (2015). The characteristics of women who birth at home, in a birth centre or in a hospital labour ward: A study of a nationallyrepresentative sample of 1835 pregnant women. Sexual \& Reproductive Healthcare, 6(3), 132137.

Steele, J. R., Jones, A. K., Clarke, R. K., \& Shoemaker, S. (2015). Health care delivery meets hospitality: A pilot study in radiology. Journal of the American College of Radiology, 12(6), 587593.

Stenglin, M., \& Foureur, M. J. (2013). Designing out the fear cascade to increase the likelihood of normal birth. Midwifery, 29(8), 819-825.

Sternberg, E. M., \& Wilson, M. A. (2006). Neuroscience and architecture: Seeking common ground. Cell, 127(2), 239-242.

Stevens, M., Reininga, I. H., Boss, N. A., \& van Horn, J. R. (2006). Patient satisfaction at and after discharge. Effect of a time lag. Patient education and counseling, 60(2), 241-245.

Stichler, J. F. (2001). Creating healing environments in critical care units. Critical Care Nursing Quarterly, 24(3), 1-20.

Stichler, J. F. (2007). Is your hospital hospitable?: How physical environment influences patient safety. Nursing for women's bealth, 11(5), 506-511.

Stichler, J. F. (2011). Patient-centered healthcare design. Journal of Nursing Administration, 41(12), 503-506.

Stichler, J. F. (2018). Ensuring shared understanding: Defining and analyzing concepts. Health Environments Research \& Design Journal, 11(3), 6-10.

Stover, L. (2002). Interventions to decrease family anxiety. Critical Care Nurse, 22(6), 61-65.

Strauss, A. L. (1987). Qualitative Analysis for Social Scientists. Cambridge: University of Cambridge Press.

Strauss, A. L., \& Corbin, J. M. (1998). Basics of Qualitative Research : Techniques and Procedures for Developing Grounded Theory (2nd ed.). Thousand Oaks: Sage Publications.

Suess, C., \& Mody, M. (2017). Hospitality healthscapes: A conjoint analysis approach to understanding patient responses to hotel-like hospital rooms. International Journal of Hospitality Management, 61, 59-72. 
Sussman, A., \& Hollander, J. B. (2014). Cognitive Architecture: Designing for How We Respond to the Built Environment: Routledge.

Swan, J. E., Richardson, L. D., \& Hutton, J. D. (2003). Do appealing hospital rooms increase patient evaluations of physicians, nurses, and hospital services? Health Care Management Review, 28(3), 254-264.

Symon, A. G., Paul, J., Butchart, M., Carr, V., \& Dugard, P. (2008a). Maternity unit design study part 2: Perceptions of space and layout. British Journal of Midwifery, 16(2), 110-114.

Symon, A. G., Paul, J., Butchart, M., Carr, V., \& Dugard, P. (2008b). Maternity unit design study part 3: Environmental comfort and control. British Journal of Midwifery, 16(3), 167-171.

Taavoni, S., Sheikhan, F., Abdolahian, S., \& Ghavi, F. (2016). Birth ball or heat therapy? A randomized controlled trial to compare the effectiveness of birth ball usage with sacrumperineal heat therapy in labor pain management. Complementary Therapies in Clinical Practice, 24, 99-102.

Tanja-Dijkstra, K. (2011). The impact of bedside technology on patients' well-being. HERD: Health Environments Research \& Design Journal, 5(1), 43-51.

Tarkka, M. T., Paavilainen, E., Lehti, K., \& Åstedt-Kurki, P. (2003). In-hospital social support for families of heart patients. Journal of Clinical Nursing, 12(5), 736-743.

Tashakkori, A., \& Teddlie, C. (2010a). Putting the human back in "human research methodology": The researcher in mixed methods research. Journal of Mixed Methods Research, 4(4), 271-277.

Tashakkori, A., \& Teddlie, C. (2010b). Sage Handbook of Mixed Methods in Social \& Behavioral Research (2nd ed.). Thousand Oaks, California: Sage Publications.

Taylor, E., Card, A. J., \& Piatkowski, M. (2018). Single-occupancy patient rooms: A systematic review of the literature since 2006. HERD: Health Environments Research \& Design Journal, 11(1), 85-100.

Teddlie, C., \& Tashakkori, A. (2006). A general typology of research designs featuring mixed methods. Research in the Schools, 13(1), 12-28.

Teddlie, C., \& Tashakkori, A. (2010). Overview of contemporary issues in mixed methods research. In A. Tashakkori \& C. Teddlie (Eds.), SAGE Handbook of mixed methods in social \& behavioral research (2nd ed.). Thousands Oaks, California: Sage Publications.

Teddlie, C., \& Tashakkori, A. (2012). Common "core" characteristics of mixed methods research: A review of critical issues and call for greater convergence. American Behavioral Scientist, 56(6), 774-788.

Tembo, A. C., \& Parker, V. (2009). Factors that impact on sleep in intensive care patients. Intensive and Critical Care Nursing, 25(6), 314-322.

Tennessen, C. M., \& Cimprich, B. (1995). Views to nature: Effects on attention. Journal of Environmental Psychology, 15(1), 77-85. 
The NHS Information Centre. (2009). NHS Maternity Statistics 2007 - 2008. Retrieved from https://digital.nhs.uk/data-and-information/publications/statistical/nhs-maternitystatistics/2007-08

The Scottish Government. (2017). The best start: A five-year forward plan for maternity and neonatal care in Scotland. Edinburgh, UK: Scottish Government.

The Women's Health Service (WHS), \& Capital \& Coast District Health Board (CCDHB). (2017). The Women's Health Service Annual Clinical Report 2017. Retrieved from Wellington: https://www.ccdhb.org.nz/news-publications/publications-and-consultationdocuments/

Thiis-Evensen, T. (1987). Archetypes in Architecture. Oslo: Norwegian University Press.

Thomas, L., MacMillan, J., McColl, E., Priest, J., Hale, C., \& Bond, S. (1995). Obtaining patients' views of nursing care to inform the development of a patient satisfaction scale. International Journal for Quality in Health Care, 7(2), 153-163.

Thorgaard, P., Ertmann, E., Hansen, V., Noerregaard, A., \& Spanggaard, L. (2005). Designed sound and music environment in postanaesthesia care units-A multicentre study of patients and staff. Intensive and Critical Care Nursing, 21(4), 220-225.

Timmermann, C., Uhrenfeldt, L., \& Birkelund, R. (2015). Room for caring: Patients' experiences of well-being, relief and hope during serious illness. Scandinavian journal of caring sciences, 29(3), 426-434.

Tofle, R. B., Schwarz, B., Yoon, S., \& Max-Royale, A. (2004). Color in healthcare environments. The Coalition for Health Environments Research.

Townsend, B., Fenwick, J., Thomson, V., \& Foureur, M. J. (2016). The birth bed: A qualitative study on the views of midwives regarding the use of the bed in the birth space. Women and Birth, 29(1), 80-84.

Trochelman, K., Albert, N., Spence, J., Murray, T., \& Slifcak, E. (2012). Patients and their families weigh in on evidence-based hospital design. Critical Care Nurse, 32(1), e1-e10.

Tsiou, C., Efthymiatos, G., \& Katostaras, T. (2008). Noise in the operating rooms of Greek hospitals. The Journal of the Acoustical Society of America, 123(2), 757-765.

Ukegjini, K., Kastiunig, T., Widmann, B., Warschkow, R., \& Steffen, T. (2020). Impact of intraoperative noise measurement on the surgeon stress and patient outcomes. A prospective, controlled, single-center clinical trial with 664 patients. Surgery, 167(5), 843851.

Ulrich, R. S. (1983). Aesthetic and affective response to natural environment. In I. Altman \& J. F. Wohlwill (Eds.), Behavior and the Natural Environment (pp. 85-125). Boston, MA: Springer.

Ulrich, R. S. (1984). View through a window may influence recovery from surgery. Science, 224(4647), 420-421.

Ulrich, R. S. (1991). Effects of interior design on wellness: Theory and recent scientific research. Journal of health care interior design, 3(1), 97-109. 
Ulrich, R. S. (1992). How design impacts wellness. Paper presented at the The Healthcare Forum Journal.

Ulrich, R. S. (1999). Effects of gardens on health outcomes: Theory and research. In C. C. Marcus \& M. Barnes (Eds.), Healing Gardens: Therapeutic Benefits and Design Recommendations. New York: John Wiley \& Sons.

Ulrich, R. S. (2001). Effects of healthcare environmental design on medical outcomes. Paper presented at the Design and Health: Proceedings of the Second International Conference on Health and Design. Stockholm, Sweden: Svensk Byggtjanst.

Ulrich, R. S., Berry, L. L., Quan, X., \& Parish, J. T. (2010). A conceptual framework for the domain of evidence-based design. HERD: Health Environments Research \& Design Journal, 4(1), 95114.

Ulrich, R. S., Simons, R. F., Losito, B. D., Fiorito, E., Miles, M. A., \& Zelson, M. (1991). Stress recovery during exposure to natural and urban environments. Journal of Environmental Psychology, 11(3), 201-230.

Ulrich, R. S., Zimring, C., Joseph, A., \& Choudhary, R. (2004). The role of the physical environment in the hospital of the 21st century: A once-in-a-lifetime opportunity. Concord, CA: The Center for Health Design.

Ulrich, R. S., Zimring, C., Zhu, X., DuBose, J., Seo, H.-B., Choi, Y.-S., . . Joseph, A. (2008). A review of the research literature on evidence-based healthcare design. HERD: Health Environments Research \& Design Journal, 1(3), 61-125.

UNICEF, \& World Bank. (2015). Trends in maternal mortality: 1990-2015: Estimates from WHO, UNICEF, UNFPA, World Bank Group and the United Nations Population Division. Retrieved from Geneva, Switzerland: www.who.int

Usher, K., \& Jackson, D. (2014). Phenomenology. In J. Mills \& M. Birks (Eds.), Qualitative Methodology: A Practical Guide: Sage Publications.

van de Glind, I., de Roode, S., \& Goossensen, A. (2007). Do patients in hospitals benefit from single rooms? A literature review. Health Policy, 84(2), 153-161.

Van den Berg, A. E. (2005). Health impacts of healing environments; A review of evidence for benefits of nature, daylight, fresh air, and quiet in healthcare settings. Groningen: UMCG.

Van den Berg, A. E., Hartig, T., \& Staats, H. (2007). Preference for nature in urbanized societies: Stress, restoration, and the pursuit of sustainability. Journal of social issues, 63(1), 79-96.

Van der Gucht, N., \& Lewis, K. (2015). Women' s experiences of coping with pain during childbirth: A critical review of qualitative research. Midwifery, 31(3), 349-358.

van Hoof, J., \& Verkerk, M. J. (2013). Developing an integrated design model incorporating technology philosophy for the design of healthcare environments: A case analysis of facilities for psychogeriatric and psychiatric care in The Netherlands. Technology in Society, 35(1), 1-13.

van Manen, M. (1990). Researching Lived Experience: Human Science for an Action Sensitive Pedagogy. Albany, N.Y: State University of New York Press. 
van Manen, M., \& Adams, C. A. (2010). Phenomenology. In P. Peterson, E. L. Baker, \& B. McGaw (Eds.), International Encyclopedia of Education (3rd ed., pp. 449-455). Oxford: Elsevier.

Vartanian, O., Navarrete, G., Chatterjee, A., Fich, L. B., Leder, H., Modroño, C., . . Skov, M. (2013). Impact of contour on aesthetic judgments and approach-avoidance decisions in architecture. Paper presented at the Proceedings of the National Academy of Sciences.

Verderber, S. (1986). Dimensions of person-window transactions in the hospital environment. Environment and Behavior, 18(4), 450-466.

Verderber, S. (2006). Hospital futures - Humanism versus the machine. In C. Wagenaar (Ed.), The Architecture of Hospitals (pp. 76-87): NAi Publishers.

Verderber, S. (2010). Innovations in Hospital Architecture: Routledge.

Verderber, S., \& Fine, D. J. (2000). Healthcare Architecture in an Era of Radical Transformation. New Haven, CT: Yale University Press.

Verderber, S., \& Todd, L. G. (2012). Reconsidering the semiprivate inpatient room in US hospitals. HERD: Health Environments Research \& Design Journal, 5(2), 7-23.

Vincent, E., Battisto, D., Grimes, L., \& McCubbin, J. (2010). The effects of nature images on pain in a simulated hospital patient room. HERD: Health Environments Research \& Design Journal, $3(3), 42-55$.

Wagenaar, C. (2006). Five revolutions: A short history of hospital architecture. In C. Wagenaar (Ed.), The Architecture of Hospitals (pp. 27-41): NAi Publishers.

Walch, J. M., Rabin, B. S., Day, R., Williams, J. N., Choi, K., \& Kang, J. D. (2005). The effect of sunlight on postoperative analgesic medication use: A prospective study of patients undergoing spinal surgery. Psychosomatic Medicine, 67(1), 156-163.

Waldenström, U., Borg, I. M., Olsson, B., Sköld, M., \& Wall, S. (1996). The childbirth experience: A study of 295 new mothers. Birth, 23(3), 144-153.

Walker, L. O., \& Wilging, S. (2000). Rediscovering the "M" in "MCH": Maternal health promotion after childbirth: THOUGHTS \& OPINIONS. Journal of Obstetric, Gynecologic, \& Neonatal Nursing, 29(3), 229-236.

Walsh, D. J., \& Newburn, M. (2002). Towards a social model of childbirth: Part one. British Journal of Midwifery, 10(9), 540-544.

Walsh, J., \& Boyle, J. (2009). Improving acute psychiatric hospital services according to inpatient experiences. A user-led piece of research as a means to empowerment. Issues in Mental Health Nursing, 30(1), 31-38.

Walsh, W. F., McCullough, K. L., \& White, R. D. (2006). Room for improvement: nurses' perceptions of providing care in a single room newborn intensive care setting. Advances in Neonatal Care, 6(5), 261-270.

Wang, Z., Downs, B., Farell, A., Cook, K., Hourihan, P., \& McCreery, S. (2013). Role of a service corridor in ICU noise control, staff stress, and staff satisfaction: Environmental research 
of an academic medical center. HERD: Health Environments Research \& Design Journal, 6(3), 80-94.

Warren, C., Daly, P., Toure, L., \& Mongi, P. (2006). Postnatal care. In J. Lawn \& K. Kerber (Eds.), Opportunities for Africa's Newborns: Practical data, policy and programmatic support for newborn care in Africa (pp. 79-90). Geneva, Switzerland: WHO on behalf of The Partnership for Maternal, Newborn and Child Health.

Watt, S., Sword, W., \& Krueger, P. (2005). Longer postpartum hospitalization options - Who stays, who leaves, what changes? BMC pregnancy and childbirth, 5(1).

Watts, G., Khan, A., \& Pheasant, R. (2016). Influence of soundscape and interior design on anxiety and perceived tranquillity of patients in a healthcare setting. Applied Acoustics, 104, 135-141.

Waye, K. P., Elmenhorst, E.-M., Croy, I., \& Pedersen, E. (2013). Improvement of intensive care unit sound environment and analyses of consequences on sleep: An experimental study. Sleep medicine, 14(12), 1334-1340.

Weber, D. J., Anderson, D., \& Rutala, W. A. (2013). The role of the surface environment in healthcare-associated infections. Current opinion in infectious diseases, 26(4), 338-344.

Wen, S. W., Liu, S., Marcoux, S., \& Fowler, D. (1998). Trends and variations in length of hospital stay for childbirth in Canada. CMAJ, 158(7), 875-880.

Wertz, R. W., \& Wertz, D. C. (1989). Lying-in: A History of Childbith in America (Expanded ed.). New Haven and London: Yale University Press.

Westreich, R., Spector-Dunsky, L., Klein, M., Papageorgiou, A., Kramer, M., \& Gelfand, M. (1991). The influence of birth setting on the father's behavior toward his partner and infant. Birth, 18(4), 198-202.

Whitehouse, S., Varni, J. W., Seid, M., Cooper-Marcus, C., Ensberg, M. J., Jacobs, J. R., \& Mehlenbeck, R. S. (2001). Evaluating a children's hospital garden environment: Utilisation and consumer satisfaction. Journal of Environmental Psychology, 21(3), 301-314.

Wiegers, T. A. (2006). Adjusting to motherhood: Maternity care assistance during the postpartum period: How to help new mothers cope. Journal of Neonatal Nursing, 12(5), 163-171.

Wiegers, T. A., van der Zee, J., Kerssens, J. J., \& Keirse, M. J. (1998). Home birth or short-stay hospital birth in a low risk population in the Netherlands. Social Science \& Medicine, 46(11), 1505-1511.

Wier, L. M., Pfuntner, A., Maeda, J., Stranges, E., Ryan, K., Jagadish, P., . . Elixhauser, A. (2011). HCUP facts and figures: Statistics on hospital-based care in the United States, 2009. Retrieved from Rockville, MD: http://www.hcup-us.ahrq.gov/reports.jsp

Williams, C., \& Gardiner, C. (2015). Preference for a single or shared room in a UK inpatient hospice: Patient, family and staff perspectives. BMJ supportive \& palliative care, 5(2), 169-174.

Williams, M. A. (1995). Design for therapeutic outcomes. In S. O. Marberry (Ed.), Innovations in Healthcare Design: Selected Presentations From the First Five Symposia on Healthcare Design (pp. 105115). New York: Van Nostrand Reinhold. 
Winkel, G., Saegert, S., \& Evans, G. W. (2009). An ecological perspective on theory, methods, and analysis in environmental psychology: Advances and challenges. Journal of Environmental Psychology, 29(3), 318-328. doi:10.1016/j.jenvp.2009.02.005

Wood, R. J., Mignone, J., Heaman, M. I., Robinson, K. J., \& Roger, K. S. (2016). Choosing an outof-hospital birth centre: Exploring women's decision-making experiences. Midwifery, 39, 12-19.

World Health Organisation. (2009). WHO guidelines on hand hygiene in health care: First global patient safety challenge clean care Is safer care. Retrieved 20 June 2019, from World Health Organisation https://www.ncbi.nlm.nih.gov/pubmed/23805438

Wray, J. (2006). Postnatal care: Is it based on ritual or a purpose? A reflective account. British Journal of Midwifery, 14(9), 520-526.

Wray, J. (2012). Impact of place upon celebration of birth: Experiences of new mothers on a postnatal ward. MIDIRS Midwifery Digest, 22(3).

Wunsch, H., Gershengorn, H., Mayer, S. A., \& Claassen, J. (2011). The effect of window rooms on critically ill patients with subarachnoid hemorrhage admitted to intensive care. Critical Care, 15, R81.

Wurtman, R. J. (1975). The effects of light on man and other mammals. Annual Review of Physiology, 37(1), 467-483.

Xie, H., Kang, J., \& Mills, G. H. (2009). Clinical review: The impact of noise on patients' sleep and the effectiveness of noise reduction strategies in intensive care units. Critical Care, 13(2), 208.

Yelland, J., McLachlan, H., Forster, D., Rayner, J., \& Lumley, J. (2007). How is maternal psychosocial health assessed and promoted in the early postnatal period? Findings from a review of hospital postnatal care in Victoria, Australia. Midwifery, 23(3), 287-297.

Yelland, J., Small, R., Lumley, J., Cotronei, V., Warren, R., \& Rice, P. L. (1998). Support, sensitivity, satisfaction: Filipino, Turkish and Vietnamese women's experiences of postnatal hospital stay. Midwifery, 14(3), 144-154.

Young, P., \& Yarandipour, R. (2007). Examining the case for single rooms. Health estate, 61(8), 85.

Zadeh, R. S., Shepley, M. M., Williams, G., \& Chung, S. S. E. (2014). The impact of windows and daylight on acute-care nurses' physiological, psychological, and behavioral health. HERD: Health Environments Research \& Design Journal, 7(4), 35-61.

Zadoroznyj, M., Brodribb, W. E., Young, K., Kruske, S., \& Miller, Y. D. (2015). 'I really needed help': What mothers say about their post-birth care in Queensland, Australia. Women and Birth, 28(3), 246-251.

Zaharna, M., \& Guilleminault, C. (2010). Sleep, noise and health: Review. Noise and Health, 12(47), 64.

Zborowsky, T. (2014). The legacy of Florence Nightingale's environmental theory: Nursing research focusing on the impact of healthcare environments. HERD: Health Environments Research \& Design Journal, 7(4), 19-34. 
Zeisel, J. (2005). Inquiry by Design: Environment/Behavior/Neuroscience in Architecture, Interiors, Landscape, and Planning. New York: WW Norton \& Co.

Zeisel, J. (2006). Inquiry by Design: Environment/Behavior/Neuroscience in Architecture, Interiors, Landscape, and Planning (Rev ed.). New York: WW Norton \& Co.

Zielinski, R., Ackerson, K., \& Low, L. K. (2015). Planned home birth: Benefits, risks, and opportunities. International journal of women's health, 7, 361.

Zielinski, R. E., Brody, M. G., \& Low, L. K. (2016). The value of the maternity care team in the promotion of physiologic birth. Journal of Obstetric, Gynecologic \& Neonatal Nursing, 45(2), 276-284.

Zwelling, E. (2008). The emergence of high-tech birthing. Journal of Obstetric, Gynecologic \& Neonatal Nursing, 37(1), 85-93.

Zwelling, E., \& Phillips, C. R. (2001). Family-centered maternity care in the new millennium: Is it real or is it imagined? The Journal of perinatal \& neonatal nursing, 15(3), 1-12. 


\section{PUBLICATIONS DURING CANDIDATURE}

\section{Conference presentations}

Lawal, L.A., \& Vale, R. (2018). Inclusive birth supporters' space and recovery care in the postnatal hospital environment. In the proceedings of ASEAN Post Graduate Conference (APGC) 2018, with the theme: Inclusive Built Environment Towards Realising New Urban Agenda. 15 November 2018, Mercu Alam Bina, University of Malaya, Kuala Lumpur, Malaysia.

Lawal, L.A., \& Vale, R. (2018). Design factors related to postpartum environments: Preferences for sense-sensitive spaces. In the proceedings of Academy of Neuroscience for Architecture (ANFA) Conference, with the theme: Shared Behavioural Outcomes. 20 - 22 September 2018, Salk Institute, La Jolla, California.

Lawal, L.A., Vale, R., \& Zari-Pedersen, M. (2020). Restorative design features in the postnatal hospital environment: A wellbeing perspective. Architecture Media Politics Society (Amps Conference), with the theme: Experiential Design- Rethinking relations between people, objects and environments. 20-22 January 2020, Florida State University, William Johnston Building, Tallahassee. 


\section{APPENDIX}

\section{A: ETHICAL APPROVALS}

A1. Out of scope letter from HDECs

Health
and
Disability
Ethics
Committees

Monday, 25 September 2017

Mr Lateef Lawa.

School of Architecture

Victoria University of Wellington New Zealand

Lateet.Lawal@vuw.ac.nz

Dear Mr Lawal,

\author{
Health and Disabi ity Ethics Committees \\ Mrity of liest \\ 133 Useewcen atee: \\ $\mathrm{PO}$ Box 5013 \\ Wellington
S011 \\ 06004 ETHCS \\ nsecogronongovtre
}

\begin{tabular}{|ll|}
\hline Study title: & $\begin{array}{l}\text { Spaces for improvement in chldb rth recovery care: A case study } \\
\text { of postnatal room designs in hospltals }\end{array}$ \\
\hline
\end{tabular}

Thank you for emalling HDEC a completed scope of review form on 18 September 2017. The Secretariat has assessed the information provided in your form and supporting documents against the Standard Operating Procedures.

Your study will not require submission to HDEC, as on the basis of the information you have submitted, it does not appear to be within the scope of HDEC review. This scope is described in section three of the Standard Operating Procedures for Health and Disablity Ethics Commiltees.

HDECs check that proposed health and dlsablity research meets establlshed ethical standards that aim to protect participants. Health and disabilty research is defined in our Standard Operating Procedures as research that alms to generate knowledge for the purpose of Improving health and Independence outcomes. Your scope of revlew form. described a questionnalre and focus groups to examine the design of spaces of postnatal care hospitals. This is not health research and is outside the scope of HDEC review.

If you consider that our advice on your project being out of scope is in incorrect please contact us as soon as possible glving reasons for this.

This letter does not constitute ethical approval or endorsement for the activity described In your application, but may be used as evidence that HDEC revlew is not required for it.

Please note, your localty may have additional ethical revlew policles, please check with your localty. if your study involves a DHB, you must contact the DHB's research oflice before you begin. If your study Involves a university or polytechnic, you must contact its Institutional ethics committee before you begin.

Please don't hesitate to contact us for further informaton.

Yours sincerely,

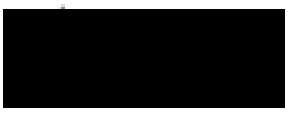

Tom Kent

Advisor

Health and Disablity Ethics Commlttees

hdecsgmoh.govt.nz 


\section{A2: VUW, Human Ethics Committees approval}

TE WHARE WĀNANGA O TE ŨPOKO O TE IKA A MĀUI

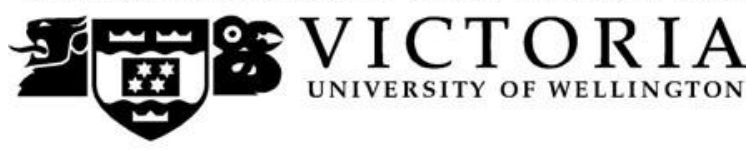

\section{MEMORANDUM}

Phone $\quad 0-4-4635205$

Email_stephen.marshall@vuw.ac.nz

\begin{tabular}{l|l}
\hline TO & Lateef Lawal \\
\hline COPY TO & Prof Robert Vale \\
\hline FROM & Dr Stephen Marshall, Acting Convener, Human Ethics Committee \\
\hline
\end{tabular}

\begin{tabular}{l|l}
\hline DATE & 8 October 2017 \\
\hline PAGES & 1 \\
\hline
\end{tabular}

\begin{tabular}{l|l}
\hline SUBJECT & $\begin{array}{l}\text { Ethics Approval: } 24397 \\
\text { Spaces for improvement in childbirth recovery care: A case study of } \\
\text { postnatal room designs in hospitals }\end{array}$ \\
\hline
\end{tabular}

Thank you for your application for ethical approval, which has now been considered by the Standing Committee of the Human Ethics Committee.

Your application has been approved from the above date and this approval continues until 31 March 2019. If your data collection is not completed by this date you should apply to the Human Ethics Committee for an extension to this approval.

Best wishes with the research.

Stephen Marshall,

Acting Convener, Victoria University Human Ethics Committee 


\section{A3: Letter indicating support for phase one of the study from CCDHB}

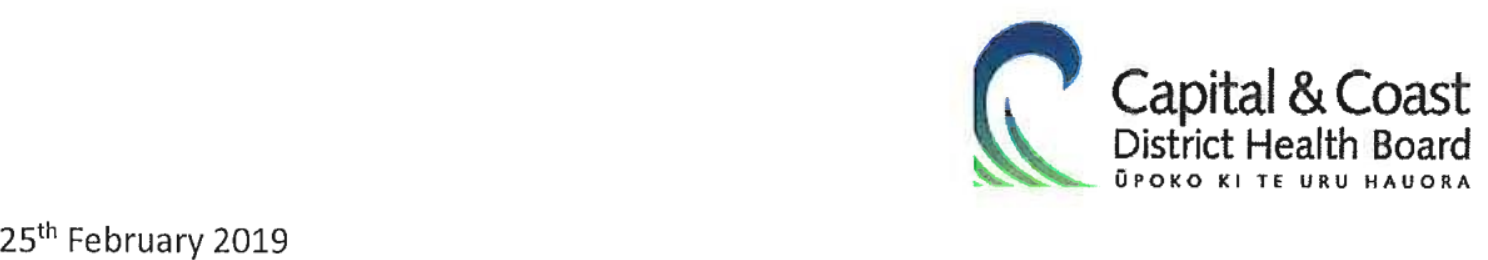

$25^{\text {th }}$ February 2019

Lateef Lawal

lateef.lawal@vuw.ac.nz

Dear Lateef

RE: Letter of Support for PhD candidate's architectural research - phase one

The Womens Health Service, Clinical Audit and Research Committee agreed in principle to support PhD candidate Lateef Lawal's architectural research. His study is entitled: Spaces for improvement in childbirth recovery care: A case study of postnatal room designs in hospitals.

All committee members agreed that this study is of importance and might benefit postpartum women in their recovery.

This letter allows for phase one, an online survey and questionnaire to be developed and disseminated to pregnant and recently postpartum women, self-employed midwives and midwives employed by the DHB.

Support and approval have been obtained through till the end of this study.

Yours sincerely

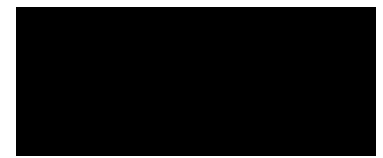

Carolyn Coles

Associate Director of Midwifery 


\section{A4: Letter indicating support for phase two of the study from CCDHB}

$25^{\text {th }}$ February 2019

Lateef Lawal

lateef.lawal@vuw.ac.nz

Dear Lateef

RE: Letter of Support for PhD candidate's architectural research - phase two

The Womens Health Service, Clinical Audit and Research Committee agreed in principle to support PhD candidate Lateef Lawal's architectural research. His study is entitled: Spaces for improvement in childbirth recovery care: A case study of postnatal room designs in hospitals.

All committee members agreed that this study is of importance and might benefit postpartum women in their recovery.

This letter enables phase two of the study to progress, a follow up focus group discussion with women and midwives to elucidate the findings from phase one.

Support and approval have been obtained through till the end of this study.

Yours sincerely

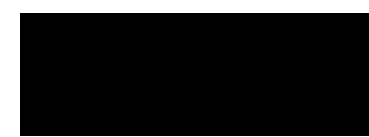

Carolyn Coles

Associate Director of Midwifery 


\section{A5: Phase one information and consent form}

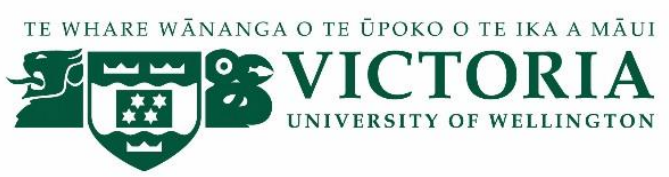

\section{Spaces for improvement in childbirth recovery care: A case study of postnatal room designs in hospitals}

\section{INFORMATION FOR PARTICIPANTS}

\section{Who am I?}

My name is Lateef Ademola Lawal, my email address is Lateef.Lawal@vuw.ac.nz and I am a Doctoral candidate at the School of Architecture, Victoria University of Wellington. I am researching the nature of spaces in recovery after childbirth. This survey is the first part of a twphase research project towards my Ph.D. thesis.

You are invited to take part in this research. Please read this information before deciding whether or not to take part. If you decide to participate, thank you for your contribution. If you decide not to participate, thank you for considering this request.

What is the aim of the project?

This project examines the impact of the design of postnatal space on women's postnatal care in hospitals. The postnatal period is a special time to celebrate childbirth, an opportunity to rest and recover, and to learn important parenting skills. A hospital environment can contribute to the initial recovery process, to the experience and to the attainment of the goals of postnatal care from the point of view of all stakeholders involved.

The purpose of this survey is to assess your views about the physical design factors of the postnatal space that decrease or increase the ability to use the space in enhancing the postnatal care of women in hospitals. This research has been approved by the Victoria University of Wellington Human Ethics Committee with reference number 24397.

\section{How can you help?}

As a woman whose most recent birth was between $\mathbf{1}$ day and $\mathbf{2}$ years ago, in any hospital, in a primary birth unit, or in a birth centre, you are invited to participate to give your views on how the postnatal space/environment impacted on your wellbeing and your experience of postnatal care. You can do this by pointing out the aspects that are good or not good for your experience of postnatal care. Your help to identify these aspects of postnatal environment that make care proceed smoothly from hospitals prior to going home is valuable for this research. This study seeks to understand your opinion as advice for possible improvement to hospitals' postnatal settings. If you agree to take part you will be asked to complete a survey that will ask you questions in the form of a set of general statements about the physical design features of the postnatal room and NOT the birth room. There are no right or wrong answers. You can also change 
your responses anytime you feel like doing so by clicking on the back button. The survey will take you about 15 minutes to complete.

\section{What will happen to the information you give?}

This research is anonymous. This means that nobody, including the researchers, will be aware of your identity. By answering the survey, you are giving consent for us to use your responses in this research. Your answers will remain completely anonymous and unidentifiable. Once you submit the survey, it will not be possible to withdraw your answers. Please do not include any personal identifiable information in your responses.

\section{What will the project produce?}

The information from my research will be used in a $\mathrm{PhD}$ dissertation, in academic publications and in presentations at conferences.

\section{If you have any questions or problems, who can you contact?}

If you have any questions, either now or in the future, please feel free to contact either:

\section{Student:}

Name: Lateef Ademola Lawal

University email address:

Lateef.Lawal@,vuw.ac.nz

\section{Supervisors:}

Name: Professor Robert Vale

Role: Professorial Research Fellow

School: Architecture

Robert.Vale@vuw.ac.nz

Name: Dr Maibritt Pedersen Zari

Role: Deputy Head of School

School: Architecture

Maibritt.Pedersen@,vuw.ac.nz

\section{Human Ethics Committee information}

If you have any concerns about the ethical conduct of the research you may contact the Victoria University HEC Convenor: Associate Professor Susan Corbett. Email susan.corbett@vuw.ac.nz or telephone +64-4-4635480. 


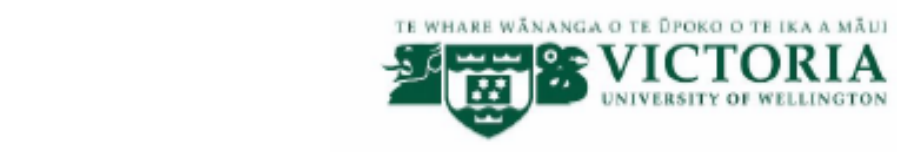

\title{
Spaces for improvement in childbirth recovery care: A case study of postnatal room designs in hospitals
}

\author{
CONSENT fOr PARTICIPATION
}

This consent form will be held for 3 years after the completion date of this project unless the participant withdraws from this study before 30 November, 2017 when it will be destroyed.

Researcher: Lateef Ademola Lawal, School of Architecture, Victoria University of Wellington.

- I have read the Information Sheet and the project has been explained to me. My questions have been answered to my satisfaction. I understand that I can ask further questions at any time.

I understand that:

- I may withdraw from this study at any point before 30 November, 2017, and any information that I have provided will be returned to me or destroyed.

- The identifiable information I have provided will be destroyed three years after the research is finished.

- $\quad$ Any information I provide will be kept confidential to the researcher and the supervisor

- I understand that the results will be used for a PhD dissertation, academic publications and conferences.

- My name will not be used in reports, nor will any information that would identify me.

- I would like to receive a copy of the final report and I have added my email address below.

Yes $\square$ No $\square$

Signature of participant:

Name of participant:

Date:

Contact details: 


\section{A6: Phase one information form (midwives).}

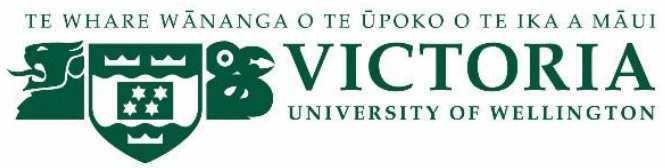

\section{Spaces for improvement in childbirth recovery care: A case study of postnatal room designs in hospitals}

\section{INFORMATION FOR PARTICIPANTS}

\section{Who am I?}

My name is Lateef Ademola Lawal, my email address is Lateef.Lawal@vuw.ac.nz and I am a Doctoral candidate at the School of Architecture, Victoria University of Wellington. I am researching the nature of spaces in recovery after childbirth. This survey is the first part of a TWO PHASE research project towards my Ph.D. thesis.

You are invited to take part in this research. Please read this information before deciding whether or not to take part. If you decide to participate, thank you for your contribution. If you decide not to participate, thank you for considering this request.

\section{What is the aim of the project?}

This project examines the impact of the design of postnatal space on women's postnatal care in hospitals. The postnatal period is a special time to celebrate childbirth, an opportunity to rest and recover, and to learn important parenting skills. A hospital environment can contribute to the initial recovery process, to the experience and to the attainment of the goals of postnatal care from the point of view of all stakeholders involved.

The purpose of this survey is to assess your views about the physical design factors of the postnatal space that decrease or increase the ability to use the space in enhancing the postnatal care of women in hospitals. This research has been approved by the Victoria University of Wellington Human Ethics Committee with reference number 24397.

\section{How can you help?}

As a midwife you are invited to give your views on how the postnatal space/environment impacts on the quality of postnatal care that you can provide. You can do this by pointing out the aspects that are good or not good for the experience of postnatal women. Your help to identify these aspects of postnatal environment that make care proceed smoothly from hospital prior to going home is valuable for this research. This study seeks to understand your opinion as advice for possible improvement to hospitals' postnatal settings. If you agree to take part you will be asked to complete a survey that will ask you questions in the form of a set of general statements about the physical design features of the postnatal room and NOT the birth room. There are no right or wrong answers. You can also change your responses anytime you feel like doing so by clicking on the back button. The survey will take you about 15 minutes to complete.

\section{What will happen to the information you give?}

This research is anonymous. This means that nobody, including the researchers, will be aware of your identity. By answering the survey, you are giving consent for us to use your responses in this research. Your answers will remain completely anonymous and unidentifiable. Once you submit the survey, it will not be 
possible to withdraw your answers. Please do not include any personal identifiable information in your responses.

\section{What will the project produce?}

The information from my research will be used in a $\mathrm{PhD}$ dissertation, in academic publications and in presentations at conferences.

\section{If you have any questions or problems, who can you contact?}

If you have any questions, either now or in the future, please feel free to contact either:

\section{Student:}

Name: Lateef Ademola Lawal

University email address:

Lateef.Lawal@,vuw.ac.nz

\section{Supervisors:}

Name: Professor Robert Vale

Role: Professorial Research Fellow

School: Architecture

Robert.Vale@,vuw.ac.nz

Name: Dr Maibritt Pedersen Zari

Role: Deputy Head of School

School: Architecture

Maibritt.Pedersen@,vuw.ac.nz

\section{Human Ethics Committee information}

If you have any concerns about the ethical conduct of the research you may contact the Victoria University HEC Convenor: Associate Professor Susan Corbett. Email susan.corbett@vuw.ac.nz or telephone +64-4-463 5480 . 
A7: Phase one survey flyer

\section{WANT TO HELP A RESEARCH ABOUT RECOVERY AFTER CHILDBIRTH?}

This content is unavailable.

Please consult the print version for access.

ELIGIBILITY: You can help if your most recent birth was between 1 day and 2 years ago, in any hospital, in a primary birthing unit, or in a birth centre, by taking part in this on-line survey to give your views on how the postnatal space impacted on your well being.

This survey should take about 15 minutes to complete.

Please use the link below to go to the survey

http://vuw.qualtrics.com/ife/form/sV 3 xhUy8othBex4Dr

OR

simply Scan the QR Code

Thank you for your interest in the survey.

\section{CONTACT}

If you have any questions, please contact me:

Lateef.Lawalevuw.ac.nz

Lateef Lawal

school of Architecture victoria University of Wellington

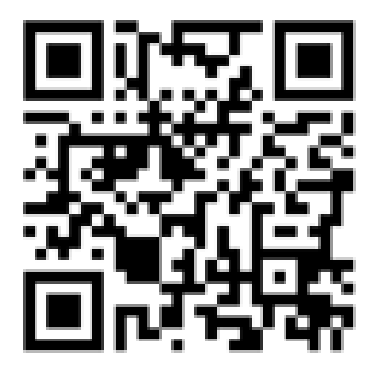




\section{A8: Phase two information and consent form}

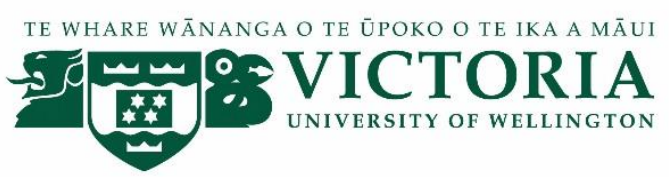

\section{Spaces for improvement in childbirth recovery care: A case study of postnatal room designs in hospitals}

\section{INFORMATION SHEET FOR PARTICIPANTS FOR FOCUS GROUPS}

You are invited to take part in this research. Please read this information before deciding whether or not to take part. If you decide to participate, thank you. If you decide not to participate, thank you for considering this request.

\section{Who am I?}

My name is Lateef Ademola Lawal and I am a Doctoral candidate at the School of Architecture, Victoria University of Wellington. The focus group interview is the second part of a two-phase research project towards my Ph.D. thesis.

\section{What is the aim of the project?}

This research investigates the perceptions of the physical design factors of postnatal space on women's postnatal recovery in hospitals, with the goal of creating evidence-based design framework/guidelines for a restorative hospital postnatal environment. It seeks to understand the links between the physical setting of postnatal space, the social and the organisational contexts. The postnatal environment should be a functional environment and space that can influence recovery care for new mothers especially as the period after the birth is a significant time for rest and recovering from childbirth, and learning important parenting skills. A hospital environment can contribute to the initial recovery process, to the experience and to the attainment of the goals of postnatal care from the point of view of all stakeholders (women, care providers, and designers of hospitals) involved.

The Victoria University of Wellington Human Ethics Committee has approved this research, with reference number 24397.

\section{How can you help?}

You have been invited to participate in this focus groups because you took part in the first on-line survey questionnaire - as first phase of this research - about the physical design factors of the postnatal hospital environment and you also gave consent to participate in the Phase 2 of the research. The focus group interviews are to build up on the first survey, validate the findings and to elicit more information about the postnatal space design indicators. This process would help us to a better understanding of the situation - your experiences of the space, relationships and interactions in the space - as whole components of the event for creating a better recovery and restorative environment for women after the birth.

If you agree to take part, you will be part of a focus group in either of the two venues approved to be used: Clinical Services Block (CSB802) at the Wellington; and Room VS101 at the School of 
Architecture, Victoria University of Wellington. I will ask you and other participants' questions about your general feeling of the postnatal hospital environment and the impact of privacy on recovery in the postnatal hospital environment among other related questions.

The focus group will last for one and a half hours maximum. I will audio record the focus group with your permission and write it up later.

The information shared during the focus group is confidential. That means after the focus group, you may not communicate to anyone, including family members and close friends, any details about the focus group.

You can withdraw from the focus group at any time before the focus group begins.

You can also withdraw while the focus group is in progress. However it will not be possible to withdraw the information you have provided up to that point as it will be part of a discussion with other participants.

\section{What will happen to the information you give?}

This research is confidential. This means that the researchers named below will be aware of your identity but the research data will be combined and your identity will not be revealed in any reports, presentations, or public documentation.

You will not be named in the $\mathrm{PhD}$ thesis but your organisation will be named (provided you have the authority to agree to this on behalf of the organisation).

Only my supervisors and I will read the notes or transcript of the focus group. The focus group transcripts, summaries and any recordings will be kept securely and destroyed on 31 December 2019.

\section{What will the project produce?}

The information from my research will be used in a $\mathrm{PhD}$ dissertation, in academic publications and in presentations at conferences.

\section{If you accept this invitation, what are your rights as a research participant?}

You do not have to accept this invitation if you don't want to. If you do decide to participate, you have the right to:

- choose not to answer any question;

- ask for the recorder to be turned off at any time during the focus group;

- withdraw from the focus group while it is taking part however it will not be possible to withdraw the information you have provided up to that point;

- ask any questions about the study at any time;

- read over and comment on a written summary of the focus group;

- be able to read any reports of this research by emailing the researcher to request a copy.

\section{If you have any questions or problems, who can you contact?}

If you have any questions, either now or in the future, please feel free to contact either:

\section{Student:}

Name: Lateef Ademola Lawal

University email address:

Lateef.Lawal@,vuw.ac.nz

\section{Supervisors:}

Name: Professor Robert Vale

Role: Professorial Research Fellow

School: Architecture

Robert.Vale@vuw.ac.nz

Name: Dr Maibritt Pedersen Zari

Role: Deputy Head of School

School: Architecture

Maibritt.Pedersen@vuw.ac.nz 


\section{Human Ethics Committee information}

If you have any concerns about the ethical conduct of the research you may contact the Victoria University HEC Convenor: Dr Judith Loveridge. Email hec@vuw.ac.nz or telephone +64-4-463 6028 . 
Spaces for improvement in childbirth recovery care: A case study of postnatal room designs in hospitals

\section{CONSENT TO PARTICIPATE IN FOCUS GROUPS}

This consent form will be held for one and a half years after the completion date of this project

Researcher: Lateef Ademola Lawal, School of Architecture, Victoria University of Wellington

- I have read the Information Sheet and the project has been explained to me. My questions have been answered to my satisfaction. I understand that I can ask further questions at any time.

- I agree to take part in an audio-recorded focus group.

I understand that:

- I acknowledge that I am agreeing to keep the information shared during the focus group confidential. I am aware that after the focus group, I must nok communicate to anpone, including family members and close friends, any details about the focus group.

- I can withdraw from the focus group while it is in progress however it will not be possible to withdraw the information I have provided up to that point as it will be part of a discussion with other participants

- The identifiable information I have provided will be destroved on 31 December 2019

- Any information I provide will be kept confidential to the researcher and my supervisor.

- I understand that the results will be used in a $\mathrm{PhD}$ dissertation, in academic publications and in presentations at conferences.

- My name will not be used in reports, nor will anp information that would identify me.

- I consent to information or opinions which I have given being attributed to me in any reports on this research:

- I would like a summary of the focus group:

Yes $\square$ No $\square$

- I would like to receive a copy of the final report and have added my email Yes $\square$ No $\square$ address below.

Signature of participant:

Name of participant:

Date:

Contact details: 
A9: Phase two focus groups flyer

\section{FOCUS GROUP INTERVIEWS ABOUT RECOVERY SPACE AFTER THE BIRTH}

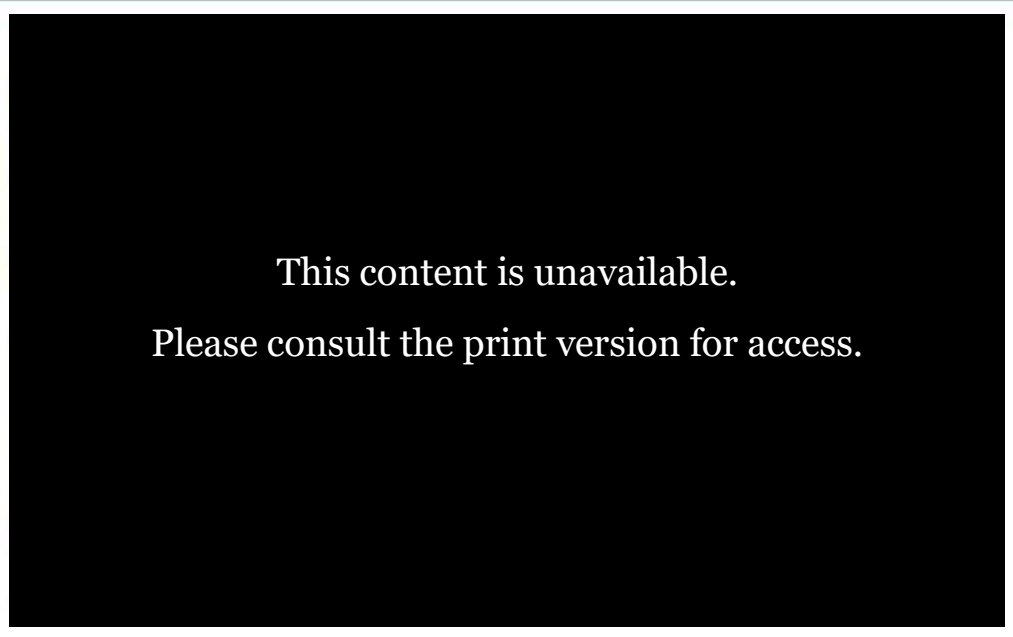

Description: Participants are invited to attend a one-hour focus group interview that seeks to understand the nature of spaces for recovery after the birth.

The interview session will be audiotaped.

Who: Women \& Midwives who filled out the initial survey

Where: 1) Level 8, CSB802 Wellington Regional Hospital, Ridoliford Road Newtown

Time: One hour on June 19, and June 20, 2018 from 11am - 3pm each day

Where: 2) Room VS101, School of Architecture, Victoria University of

Wellington, 139 Vivion Street, TelAvo

Time: One hour on June 21, June 23, and June 25, 2018 from 11 am - 3pm each day

Please respond ASAP to e-mail sent to you, or by June 15, 2018 to:

$$
\text { Lateef Lawal }
$$

E-mail: loteef.lawalervw.ac.nz

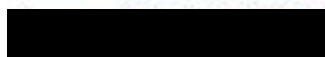

You will be contacted and sent a notification for the interview schedule 


\section{A10: Focus groups protocol}

\section{Focus groups protocol}

\section{Project Topic: Spaces for improvement in childbirth recovery care: A case study of postnatal room designs in hospitals}

Time of focus group discussion: $12-1 \mathrm{pm}$

Date: 16 August 2018

Venue : Meeting Room VS101, School of Architecture, Te Aro campus, Victoria University of Wellington

Researcher/Interviewer: Lateef Lawal

Assistant moderator:

This research investigates the perceptions of the design factors of postnatal space on women's recovery experiences in hospitals. The postnatal environment should be a functional environment and space that can influence recovery care for new mothers especially as the period after the birth is a significant time for rest and recovering from childbirth, and learning important parenting skills.

The phase two of this research follows up on the results of questionnaire surveys on perceptions of the physical design factors influencing women's recovery experiences and wellbeing.

\section{Questions:}

1. What is your general feeling about the postnatal hospital environment when you were there?

2. Tell me about your positive experiences you have had with the postnatal hospital environment where you were attended or worked?

3. Tell me about your disappointments you have had with the postnatal hospital environment where you were attended or worked?

4.What do you think is the impact of privacy on recovery in the postnatal hospital environment?

5. How can the midwives maintain awareness of each woman's needs while maintaining each woman's privacy in the postnatal environment?

6. Describe how you handle disruption to sleep or to rest in the postnatal hospital environment?

7. What do you think is the optimum physical postnatal hospital environment to meet postnatal care?

8. Imagine that the hospital managers can improve the postnatal environment. If you consider three ways this might be done:

0 changing how the existing building is used,

$\bigcirc$ upgrading the existing building, and

- construction of completely new postnatal units,

what would be your suggestions for each of these categories?

9. Of all the things we've discussed, what to you is the most important? 


\section{B: SURVEY QUESTIONNAIRES}

\section{B1: Word version of survey questionnaire, women.}

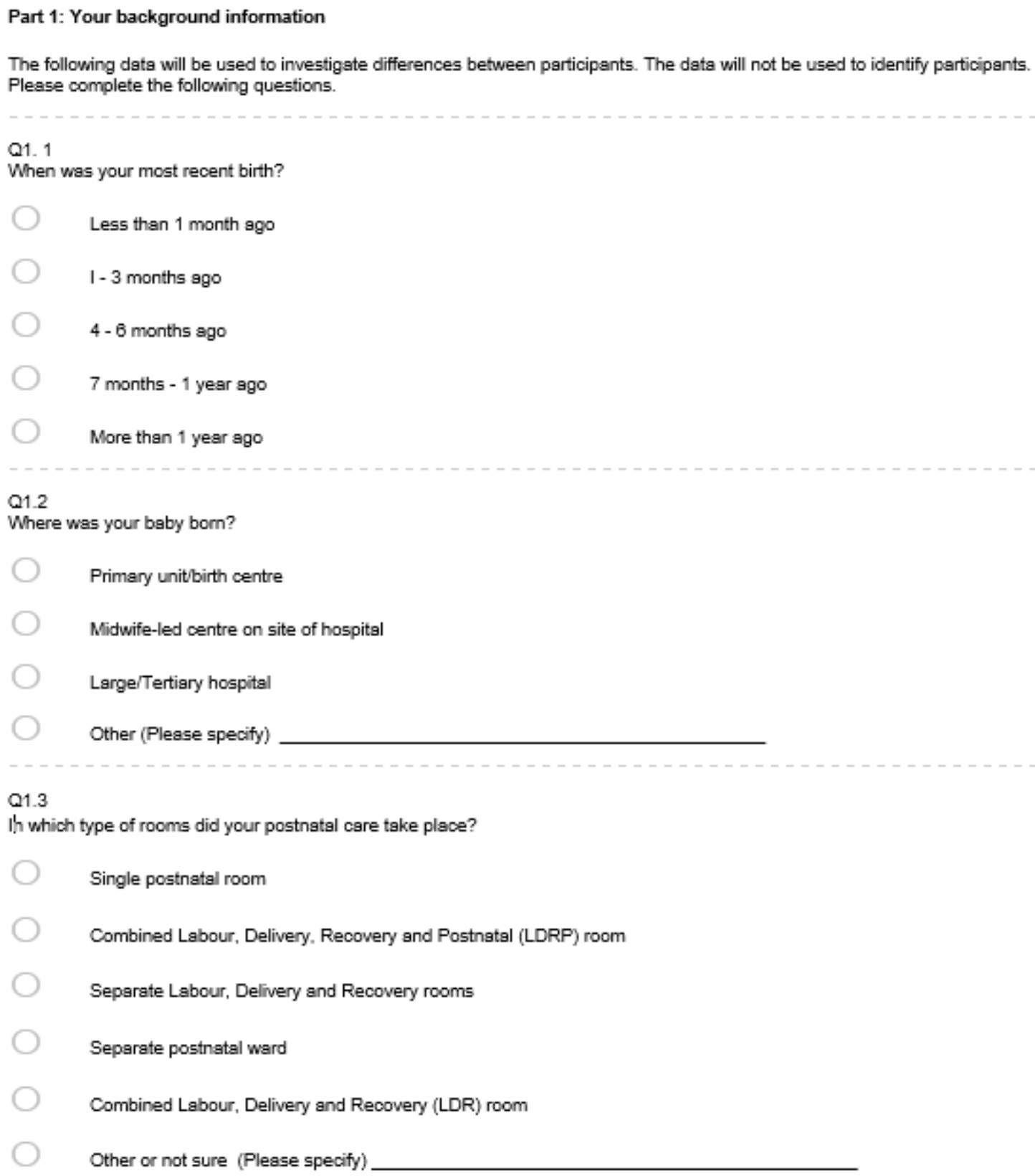


Q1.4

How many time(s) have you given birth?

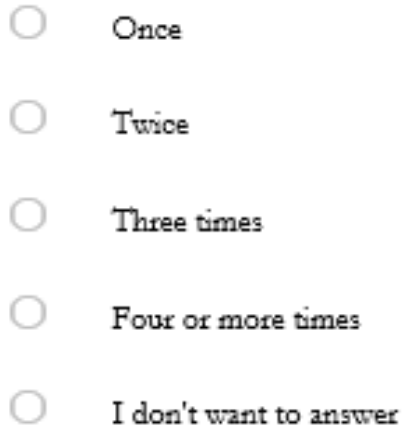

What is your highest level of completed education?

Elementary school/Primary school

Secondary school (High school)

College/University

Postgraduate

Technical/Vocational degree

Other (Please specify)

Q1.6

In which age range do you fall?

Less than 20 years

$20-24$ years

$25-29$ years

$30-34$ years

$35-39$ years

40 years and over 
Q1.7

What is your ethnicity?

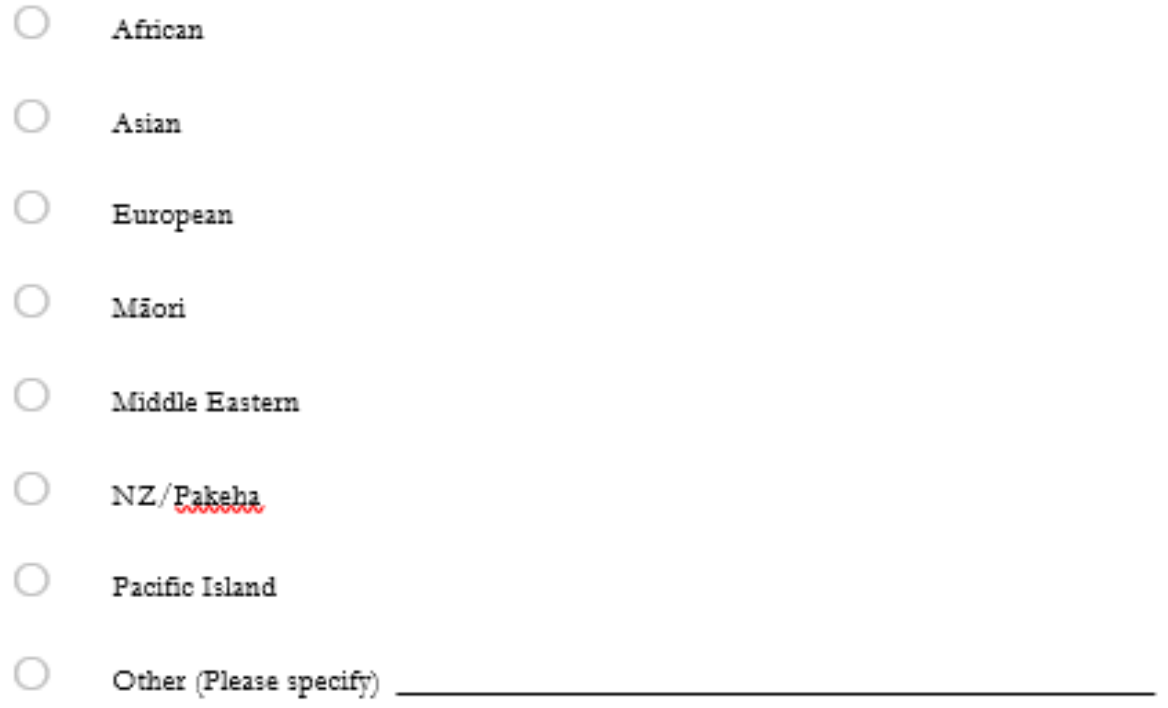

Part 2: How important is the postnatal space for postnatal care giving?

Giving birth is a transformational experience. The next stage involves recovering from the experience and acquiring of key parental skills as part of quality postnatal care. We would like to know the aspects of physical design factors of the postnatal ward or room that you found to be good or not so good during your postnatal care in hospital.

Q2.0

From the list below, select at least three items that best describe your postnatal room or ward in which rou received care.

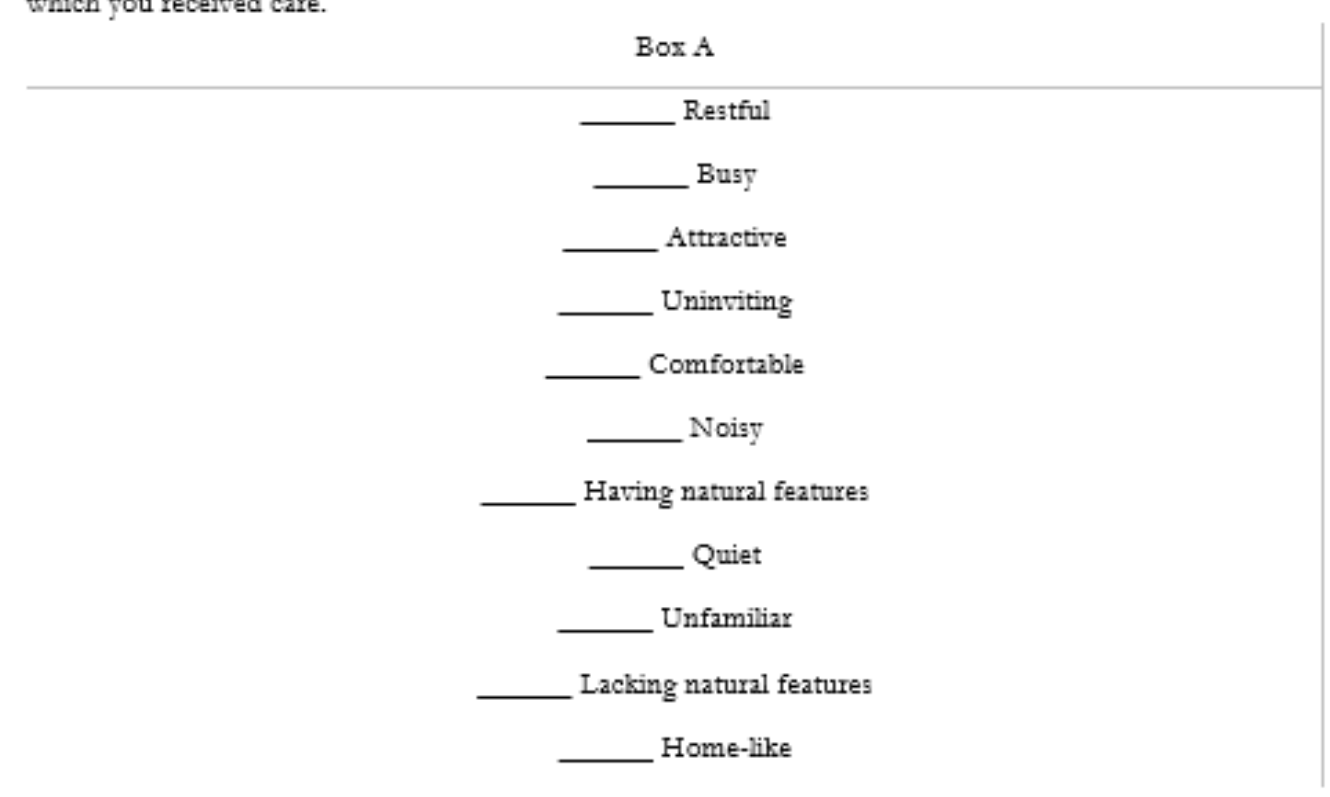


Q2.1

Which of the following features were in your postnatal room or ward during your postnatal care? Please select all that apply.

Potted plants or flowers

Paintings of natural scenery

Other art work

Visual display of natural sights and scenery such as mountains, sea tiews, animals, etc. on TV

Soft music playing in the

Bright colours on walls

Pastel colours on walls

Use of natural materials such as wood, etc.

Views of gardens or outdoors

Other (Please specify)

Part 3: Your experience of postnatal care

Q3.0

Today women normally have a shorter hospital postnatal stay of about two days. Do you think the physical environment in which postnatal care takes place can affect a quality postnatal care experience in terms of women's physical, emotional and psychological wellbeing?

\begin{tabular}{|c|c|c|c|c|c|}
\hline & $\begin{array}{l}\text { No, } \\
\text { strongly } \\
\text { disagree } \\
\text { (1) }\end{array}$ & $\begin{array}{c}\text { No, disagree } \\
\text { (2) }\end{array}$ & $\begin{array}{l}\text { Neither agree } \\
\text { nor disagree } \\
\text { (3) }\end{array}$ & Yes, agree (4) & $\begin{array}{c}\text { Yes, strongly } \\
\text { agree (5) }\end{array}$ \\
\hline A & & & & & \\
\hline
\end{tabular}

What did you think about the room?

After the task of giving birth, the next few hours should aim to help you rest and recover in your room/ward. Below are a get of general statements about the features of the room. 


\begin{tabular}{|c|c|c|c|c|c|}
\hline & $\begin{array}{l}\text { No, } \\
\text { strongly } \\
\text { disagree } \\
\text { (1) }\end{array}$ & $\begin{array}{c}\text { No, disagree } \\
\text { (2) }\end{array}$ & $\begin{array}{c}\text { Neither agree } \\
\text { nor disagree } \\
\text { (3) }\end{array}$ & Yes, agree (4) & $\begin{array}{c}\text { Yes, strongly } \\
\text { agree (5) }\end{array}$ \\
\hline $\begin{array}{l}\text { A single room is } \\
\text { better for a woman's } \\
\text { postnatal recovery } \\
\text { care }\end{array}$ & O & 0 & 0 & O & $\mathrm{O}$ \\
\hline
\end{tabular}

Q3.2

\begin{tabular}{c|ccccc} 
& $\begin{array}{c}\text { No, } \\
\text { strongly } \\
\text { disagree } \\
(1)\end{array}$ & $\begin{array}{c}\text { No, disagree } \\
(2)\end{array}$ & $\begin{array}{c}\text { Neither agree } \\
\text { nor disagree }\end{array}$ & Yes, agree (4) & $\begin{array}{c}\text { Yes, strongly } \\
\text { agree }(5)\end{array}$ \\
\hline $\begin{array}{c}\text { A room or ward that } \\
\text { is quiet is good for } \\
\text { sleep and generally } \\
\text { helps in the recovery } \\
\text { process }\end{array}$ & & 0 & & \\
\hline
\end{tabular}

Q3.3

\begin{tabular}{l|ccccc} 
& $\begin{array}{c}\text { No, } \\
\text { strongly } \\
\text { disagree } \\
(1)\end{array}$ & $\begin{array}{c}\text { No, disagree } \\
(2)\end{array}$ & $\begin{array}{c}\text { Neither agree } \\
\text { nor disagree }\end{array}$ & Yes, agree (4) & $\begin{array}{c}\text { Yes, strongly } \\
\text { agree (5) }\end{array}$ \\
\hline $\begin{array}{l}\text { Women are generally } \\
\text { too emotional about } \\
\text { the birth of their } \\
\text { baby to notice if the } \\
\text { space is good or bad }\end{array}$ & & & & \\
\end{tabular}




\begin{tabular}{|c|c|c|c|c|c|}
\hline & $\begin{array}{l}\text { No, } \\
\text { strongly } \\
\text { disagree } \\
\text { (1) }\end{array}$ & $\begin{array}{c}\text { No, disagree } \\
\text { (2) }\end{array}$ & $\begin{array}{c}\text { Neither agree } \\
\text { nor disagree } \\
\text { (3) }\end{array}$ & Yes, agree (4) & $\begin{array}{c}\text { Yes, strongly } \\
\text { agree (5) }\end{array}$ \\
\hline $\begin{array}{l}\text { A room with colours } \\
\text { is better for recovery } \\
\text { than a very plain } \\
\text { room }\end{array}$ & 0 & O & O & O & O \\
\hline
\end{tabular}

Q3.5

\begin{tabular}{|c|c|c|c|c|c|}
\hline & $\begin{array}{c}\text { No, } \\
\text { strongly } \\
\text { disagree } \\
\text { (1) }\end{array}$ & $\begin{array}{c}\text { No, disagree } \\
\text { (2) }\end{array}$ & $\begin{array}{c}\text { Neither agree } \\
\text { nor disagree } \\
\text { (3) }\end{array}$ & Yes, agree (4) & $\begin{array}{c}\text { Yes, strongly } \\
\text { agree (5) }\end{array}$ \\
\hline $\begin{array}{l}\text { Most women prefer } \\
\text { to stay in a postnatal } \\
\text { room that overlooks } \\
\text { a garden, or has } \\
\text { pictures of natural } \\
\text { scenery }\end{array}$ & 0 & O & $\mathrm{O}$ & O & $\mathrm{O}$ \\
\hline
\end{tabular}

Q3.6

\begin{tabular}{l|ccccc} 
& $\begin{array}{c}\text { No, } \\
\text { strongly } \\
\text { disagree } \\
(1)\end{array}$ & No, disagree & $\begin{array}{c}\text { Neither agree } \\
\text { nor disagree }\end{array}$ & Yes, agree (4) & $\begin{array}{c}\text { Yes, strongly } \\
\text { agree (5) }\end{array}$ \\
\hline $\begin{array}{l}\text { Women appreciate a } \\
\text { postnatal room or } \\
\text { ward in which } \\
\text { natural daylight can } \\
\text { easily enter into the }\end{array}$ & & & & & \\
room
\end{tabular}




\begin{tabular}{|c|c|c|c|c|c|}
\hline & $\begin{array}{c}\text { No, } \\
\text { strongly } \\
\text { disagree } \\
\text { (1) }\end{array}$ & $\begin{array}{c}\text { No, disagree } \\
\text { (2) }\end{array}$ & $\begin{array}{c}\text { Neither agree } \\
\text { nor disagree } \\
\text { (3) }\end{array}$ & Yes, agree (4) & $\begin{array}{l}\text { Yes, strongly } \\
\text { agree (5) }\end{array}$ \\
\hline $\begin{array}{l}\text { Having family } \\
\text { members and } \\
\text { partner present } \\
\text { during postnatal care } \\
\text { increases the need } \\
\text { for a large space }\end{array}$ & 0 & $\mathrm{O}$ & O & $\mathrm{O}$ & $\mathrm{O}$ \\
\hline
\end{tabular}

Q3.8

\begin{tabular}{|c|c|c|c|c|c|}
\hline & $\begin{array}{c}\text { No, } \\
\text { strongly } \\
\text { disagree } \\
\text { (1) }\end{array}$ & $\begin{array}{c}\text { No, disagree } \\
\text { (2) }\end{array}$ & $\begin{array}{c}\text { Neither agree } \\
\text { nor disagree } \\
\text { (3) }\end{array}$ & Yes, agree (4) & $\begin{array}{c}\text { Yes, strongly } \\
\text { agree (5) }\end{array}$ \\
\hline $\begin{array}{l}\text { Shapes and forms } \\
\text { like those of natural } \\
\text { objects such as } \\
\text { flowers, flowing } \\
\text { water, etc. can make } \\
\text { a more beautiful } \\
\text { postnatal room } \\
\text { design }\end{array}$ & $\mathrm{O}$ & O & O & O & O \\
\hline
\end{tabular}


Interior

Some people think that your surroundings can affect how you recover after giving birth.

Q3.9

\begin{tabular}{l|ccccc} 
& $\begin{array}{c}\text { No, } \\
\text { strongly } \\
\text { disagree } \\
(1)\end{array}$ & $\begin{array}{l}\text { No, disagree } \\
(2)\end{array}$ & $\begin{array}{c}\text { Neither agree } \\
\text { nor disagree } \\
(3)\end{array}$ & Yes, agree (4) & $\begin{array}{c}\text { Yes, strongly } \\
\text { agree (5) }\end{array}$ \\
\hline $\begin{array}{l}\text { Do you think the } \\
\text { interior environment } \\
\text { affects postnatal } \\
\text { recovery? }\end{array}$ & 0 & 0 & 0 & & \\
\end{tabular}

Q3.10

During postnatal care how important do you think it is to...

\begin{tabular}{|c|c|c|c|c|}
\hline $\begin{array}{c}\text { Not at all } \\
\text { (1) }\end{array}$ & $\begin{array}{l}\text { Minimally } \\
\text { important } \\
\text { (2) }\end{array}$ & $\begin{array}{l}\text { Somewhat } \\
\text { (3) }\end{array}$ & $\begin{array}{l}\text { Moderately } \\
\text { important (4) }\end{array}$ & $\begin{array}{c}\text { Very } \\
\text { important }(5)\end{array}$ \\
\hline
\end{tabular}

A) Have a restful room

B) Be looked after in a clinical-looking postnatal space

C) Be looked after in 2 room which has a combination of clinical and familiar home features

D) Be in a room or ward that is decorated with natural art, images or paintings

E) Have bright colours in the room 
Q3.11

In addition, how important do you think it is to...

$\begin{array}{ccccc}\begin{array}{c}\text { Not at all } \\ \text { (1) }\end{array} & \text { Minimally } & \text { Somewhat } & \text { Moderately } & \text { Very } \\ \text { important (2) } & \text { (3) } & \text { important (4) } & \text { important (5) }\end{array}$

A) Have a clean room

B) Have a room with furniture made of natural materials

C) Be able to walk outside and experience the outdoor surroundings

D) Know there is 2 protective comer in the room that you can withdraw into with your baby

B) Be in 2 room where the door is closed except when visitors are allowed in 


\section{Sensory comfort}

A warm and comfortable indoor climate (room temperature, adequate fresh air etc.) in your postnatal ward or room can impact on your wellbeing. Below are a set of general statements about the features of a comfortable indoor climate.

Q3.12

How important were these features to your feeling of wellbeing during the postnatal period?

$\begin{array}{ccccc}\text { Not at } & \text { Minimally } & \text { Somewhat } & \text { Moderately } & \text { Very } \\ \text { all (1) } & \text { important } & (3) & \text { important (4) } & \text { important (5) }\end{array}$
A) Haring adequate
daylighting in your room
or ward
B) Being able to control
the temperature
C) Being able to control noise from mechanical things like ventilation, medical equipment, etc.
D) Haring a room that feels quiet
E) Having good air quality and room freshness
F) Being able to have additional heating for mother and baby
G) Knowing you can open or close the windows to feel comfortable
H) Having a number of natural things in your room such as aquariums, nature sounds of birds, water, scents and images
I) Having a hygienic/clean environment 
Safety and security

As 2 mother, being cared for in a safe environment is important. Below are a set of general statements about safety features.

Q3.13

\begin{tabular}{l|ccccc} 
Q3.13 & $\begin{array}{c}\text { No, } \\
\text { strongly } \\
\text { disagree } \\
(1)\end{array}$ & $\begin{array}{c}\text { No, disagree } \\
(2)\end{array}$ & $\begin{array}{c}\text { Neither agree } \\
\text { nor disagree } \\
(3)\end{array}$ & Yes, agree (4) & $\begin{array}{c}\text { Yes, strongly } \\
\text { agree (5) }\end{array}$ \\
\hline $\begin{array}{l}\text { Moring to a separate } \\
\text { postnatal room or } \\
\text { ward after giving } \\
\text { birth increased your } \\
\text { feeling of safety }\end{array}$ & & & & & \\
\end{tabular}

Q3.14

\begin{tabular}{l|ccccc} 
& $\begin{array}{c}\text { No, } \\
\text { strongly } \\
\text { disagree } \\
(1)\end{array}$ & $\begin{array}{l}\text { No, disagree } \\
(2)\end{array}$ & $\begin{array}{c}\text { Neither agree } \\
\text { nor disagree } \\
(3)\end{array}$ & Yes, agree (4) & $\begin{array}{c}\text { Yes, strongly } \\
\text { agree (5) }\end{array}$ \\
\hline $\begin{array}{l}\text { Regardless of the } \\
\text { design of the birth } \\
\text { room, a postnatal } \\
\text { room gives you } \\
\text { freedom and escape } \\
\text { from the birth } \\
\text { process }\end{array}$ & & & & \\
\end{tabular}

Q3.15

\begin{tabular}{|c|c|c|c|c|c|}
\hline & $\begin{array}{c}\text { No, } \\
\text { strongly } \\
\text { disagree } \\
\text { (1) }\end{array}$ & $\begin{array}{c}\text { No, disagree } \\
\text { (2) }\end{array}$ & $\begin{array}{c}\text { Neither agree } \\
\text { nor disagree } \\
\text { (3) }\end{array}$ & Yes, agree (4) & $\begin{array}{c}\text { Yes, strongly } \\
\text { agree (5) }\end{array}$ \\
\hline $\begin{array}{l}\text { Women find a large } \\
\text { open postnatal ward } \\
\text { or room is better for } \\
\text { interactions with } \\
\text { other women }\end{array}$ & O & 0 & $\mathrm{O}$ & $\mathrm{O}$ & O \\
\hline
\end{tabular}


Q3.16

\begin{tabular}{|c|c|c|c|c|c|}
\hline & $\begin{array}{c}\text { No, } \\
\text { strongly } \\
\text { disagree } \\
\text { (1) }\end{array}$ & $\begin{array}{c}\text { No, disagree } \\
\text { (2) }\end{array}$ & $\begin{array}{c}\text { Neither agree } \\
\text { nor disagree } \\
\text { (3) }\end{array}$ & Yes, agree (4) & $\begin{array}{c}\text { Yes, strongly } \\
\text { agree }(5)\end{array}$ \\
\hline $\begin{array}{l}\text { Women can easily } \\
\text { find their way around } \\
\text { if they decide to } \\
\text { move from one part } \\
\text { of the hospital birth } \\
\text { environment to } \\
\text { another part }\end{array}$ & O & ? & 0 & C & $\cap$ \\
\hline
\end{tabular}

Q3.17

No, strongly disagree

(1)

\author{
No, disagree
}

(2)

Neither agree nor disagree

(3)
Yes, strongly

agree (5)
The movement from the birth room to the postnatal room was a long walk

Q3.18
No, strongly disagree

(1)

No, disagree
(2)

(2)

Neither agree

nor disagree Yes, agree (4)

(3)
Yes, strongly

agree (5)

\section{A space that feels} enclosed offers better protection for rest and recovery

Q3.19

No, strongly disagree

(1)

A room with a clear overview of the surroundings feels less gafe for mother and baby

No, disagree
(2)

(2)
Neither agree nor disagree

(3)
Yes, strongly agree (5) 
No, strongly disagree No, disagree

Neither agree (1)

(2) nor disagree

Yes, agree (4) Yes, strongly

(3) agree (5)

Rooming-in care (where mother and baby stay together) is better for the care of mother and baby

Q3.21

\begin{tabular}{l|ccccc} 
& $\begin{array}{c}\text { No, } \\
\text { strongly } \\
\text { disagree } \\
(1)\end{array}$ & $\begin{array}{c}\text { No, disagree } \\
(2)\end{array}$ & $\begin{array}{c}\text { Neither agree } \\
\text { nor disagree }\end{array}$ & Yes, agree (4) & $\begin{array}{c}\text { Yes, strongly } \\
\text { agree (5) }\end{array}$ \\
\hline $\begin{array}{l}\text { Well-baby care in a } \\
\text { separate nursery is } \\
\text { better for both the } \\
\text { mother and the baby }\end{array}$ & & & & & \\
\end{tabular}

Q3.22

\begin{tabular}{l|ccccc} 
& $\begin{array}{c}\text { No, } \\
\text { strongly } \\
\text { disagree } \\
(1)\end{array}$ & $\begin{array}{c}\text { No, disagree } \\
(2)\end{array}$ & $\begin{array}{c}\text { Neither agree } \\
\text { nor disagree }\end{array}$ & Yes, agree (4) & $\begin{array}{c}\text { Yes, strongly } \\
\text { agree (5) }\end{array}$ \\
\hline $\begin{array}{l}\text { Hospital policy } \\
\text { which limits access } \\
\text { for unauthorised } \\
\text { persons to the } \\
\text { postnatal room is } \\
\text { preferable for } \\
\text { women's sense of } \\
\text { wellbeing }\end{array}$ & & & \\
\end{tabular}


Q3.23

\begin{tabular}{l|ccccc} 
& $\begin{array}{c}\text { No, } \\
\text { strongly } \\
\text { disagree } \\
(1)\end{array}$ & $\begin{array}{c}\text { No, disagree } \\
\text { (2) }\end{array}$ & $\begin{array}{c}\text { Neither agree } \\
\text { nor disagree }\end{array}$ & Yes, agree (4) & $\begin{array}{c}\text { Yes, strongly } \\
\text { agree (5) }\end{array}$ \\
\hline $\begin{array}{l}\text { Women prefer a } \\
\text { home-like } \\
\text { environment for } \\
\text { their postmatal care }\end{array}$ & & & & & \\
\end{tabular}

Q3.24

\begin{tabular}{|c|c|c|c|c|c|}
\hline & $\begin{array}{c}\text { No, } \\
\text { strongly } \\
\text { disagree } \\
\text { (1) }\end{array}$ & No, disagree (2) & $\begin{array}{l}\text { Neither agree } \\
\text { nor disagree (3) }\end{array}$ & Yes, agree (4) & $\begin{array}{c}\text { Yes, strongly } \\
\text { agree }(5)\end{array}$ \\
\hline $\begin{array}{l}\text { Women would prefer } \\
\text { an experience similar } \\
\text { to staying in a hotel } \\
\text { for their postnatal } \\
\text { care }\end{array}$ & 0 & 0 & $C$ & O & \\
\hline
\end{tabular}

\section{Social comfort}

Welcoming visitors and being cared for are important for most women after giving birth. Below are a set of general statements about the features in the environment that either support or discourage this.

Q3.25

How often visitors came in to see you in pour postnatal room after the birth of pour baby?

Once

Twice

Three times

Four times

Five or more times 


\begin{tabular}{l|ccccc} 
No, & $\begin{array}{c}\text { Neither agree } \\
\text { strongly } \\
\text { disagree } \\
(1)\end{array}$ & $\begin{array}{c}\text { No, disagree } \\
\text { nor disagree }\end{array}$ & Yes, agree (4) & $\begin{array}{c}\text { Yes, strongly } \\
\text { agree (5) }\end{array}$ \\
\hline $\begin{array}{l}\text { Women are offered } \\
\text { the opportunity to } \\
\text { choose the room } \\
\text { they would prefer for } \\
\text { their postnatal stay }\end{array}$ & & & & \\
\end{tabular}

Q3.27

No, strongly disagree (1)

From the postnatal room it is good to be able to see the activity of the maternity department

Q3.28
Neither agree

nor disagree Yes, agree (4)

(3)

(2)

(2)

Yes, strongly agree (5)

\begin{tabular}{|c|c|c|c|c|c|}
\hline & $\begin{array}{c}\text { No, } \\
\text { strongly } \\
\text { disagree } \\
\text { (1) }\end{array}$ & $\begin{array}{c}\text { No, disagree } \\
\text { (2) }\end{array}$ & $\begin{array}{c}\text { Neither agree } \\
\text { nor disagree } \\
\text { (3) }\end{array}$ & Yes, agree (4) & $\begin{array}{c}\text { Yes, strongly } \\
\text { agree (5) }\end{array}$ \\
\hline $\begin{array}{l}\text { Positive distractions } \\
\text { such as soft music, } \\
\text { natural images etc. in } \\
\text { a postnatal room are } \\
\text { better for a woman's } \\
\text { recovery }\end{array}$ & O & O & $\mathrm{O}$ & O & O \\
\hline
\end{tabular}




\begin{tabular}{l|ccccc} 
& $\begin{array}{c}\text { No, } \\
\text { strongly } \\
\text { disagree } \\
(1)\end{array}$ & $\begin{array}{c}\text { No, disagree } \\
(2)\end{array}$ & $\begin{array}{c}\text { Neither agree } \\
\text { nor disagree } \\
(3)\end{array}$ & Yes, agree (4) & $\begin{array}{c}\text { Yes, strongly } \\
\text { agree (5) }\end{array}$ \\
\hline $\begin{array}{l}\text { Having a communal } \\
\text { space near the } \\
\text { postnatal space is } \\
\text { good for counselling } \\
\text { and parental } \\
\text { information support }\end{array}$ & & & & & \\
\end{tabular}

Q3.30

No, strongly disagree

(1)
Neither agree nor disagree

(3)
Yes, strongly agree (5)

The arrangement of furniture and décor in a communal space helps group learning of breastfeeding and parental skills

Q3.31

\begin{tabular}{|c|c|c|c|c|c|}
\hline & $\begin{array}{c}\text { No, } \\
\text { strongly } \\
\text { disagree } \\
\text { (1) }\end{array}$ & $\begin{array}{c}\text { No, disagree } \\
\text { (2) }\end{array}$ & $\begin{array}{c}\text { Neither agree } \\
\text { nor disagree } \\
\text { (3) }\end{array}$ & Yes, agree (4) & $\begin{array}{c}\text { Yes, strongly } \\
\text { agree (5) }\end{array}$ \\
\hline $\begin{array}{l}\text { Openness of a } \\
\text { postnatal room } \\
\text { decreases the } \\
\text { opportunity for } \\
\text { discussions with } \\
\text { midwives }\end{array}$ & & & & & \\
\hline
\end{tabular}

Q3.32

\begin{tabular}{ccccc} 
No, & \multicolumn{3}{c}{ Neither agree } \\
strongly & No, disagree & $\begin{array}{c}\text { nor disagree } \\
\text { disagree }\end{array}$ & Yes, agree (4) & Yes, strongly \\
(1) & & (3) & & agree (5)
\end{tabular}

Entering from a

passage or corridor

to a postnatal room

is better for privacy 
Q3.33

If you could you choose a postnatal room what type you would choose: (Please select from the options)

Single room 2 person room 3 or 4 person room Open bay room or ward

No preference

Other (Please specify)

Q3.34

If you could you choose your postnatal care which would you would choose for preference: (Please select from the options')

Family suit

No preference

Home

Delivery followed by postnatal ward

Hotel-care

Other (Please specify)

Q3.35

Please add anything you think we have missed out regarding postnatal rooms

Q3.36

If you are willing to participate in Phase 2 of this research, where a set of images of indoor and outdoor surroundings will be shown to a number of participants in a small focus group, please tick below.

Yes, please e-mail me at: Lateef. Lawal@vuw.ac.nz

No 


\section{B2: Word version of survey questionnaire, midwives.}

\section{Part 1: Your background information}

The following data will be used to investigate differences between participants. The data will not be used to identify participants. Please complete the following questions.

Q1.0

How long have you been working as a Core midwife or as a Lead Maternity Carer|midwife?

Less than 5 years

$5-15$ vears

$16-25$ years

$26-35$ years

More than 35 years

Q.1.1

Do you work as a Lead Maternity Carer (LMC) midwife?

Yes (Please add your most recent hospital postnatal care location)

No

Q.1.2

Do you work as a Core midwife employed in a tertiary hospital, primary birth unit, or in the community?

Yes (Please include the hospital or primary birth unit where you work)

No 
Q1.3

When was the last time improvements were carried out to the postnatal ward or unit in your hospital?

In the last 5 years

$5-10$ years ago

11 - 15 years ago

16 - 20 years ago

More than 20 years ago

I do not know

Q1.4

What is your highest level of completed education?

Elementary/Primary school

Secondary school (high school)

College/University

Postgraduate

$\mathrm{PhD}$

Other (Please specify) 
Q1.5

In which age range do you fall?

Less than 25 years

$26-35$ years

$36-45$ years

$46-64$ years

$65+$

Q1.6

What is your ethnicity?

African

Asian

European

Mãori

Middle Eastern

NZ/Pakeha

Pacific Island

Other (Please specify)

Part 2: How important is the postnatal ward/room for postnatal care giving?

Giving birth is transformational for women. As a midvife you are on hand to assist these women to get on well with their lives by giving support and information as part of quality postnatal care. We would like to know the aspects of physical design factors of the postnatal ward or room that you found have helped or hindered postnatal care giving in hospital. 
Have you worked across the maternity model of care that includes working in a postnatal room (Please tick)

Yes

No

Q2.1

Do you provide postnatal care away from the birth room in a designated postnatal ward or other separate room?

Yes

No

Q2.2

What were your experiences of working in such space(s)? (Please add your comments in the box below)

Q2.3

From the list below, select and drag at least three items into Box $\mathrm{A}$ that best describe your most recent postnatal room or ward experience

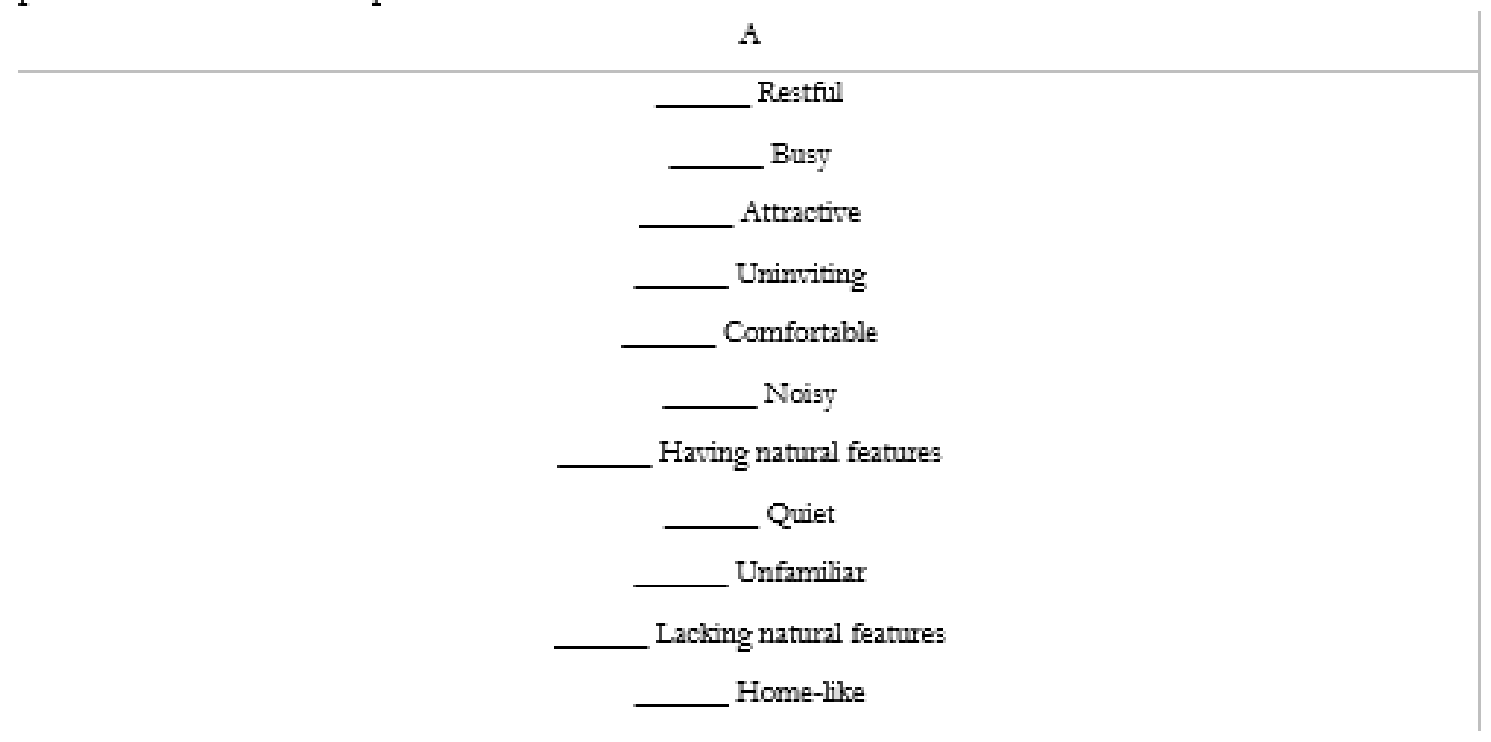


Part 3: Your experience of postnatal care giving

Q3.0

Normally today women have a shorter hospital postnatal stay of about two days. Do you think the postnatal environment can make a difference to a quality postnatal care experience and the satisfaction of women who have recently given birth?

\begin{tabular}{|c|c|c|c|c|c|}
\hline & $\begin{array}{l}\text { No, strongly } \\
\text { disagree (1) }\end{array}$ & No, disagree (2) & $\begin{array}{c}\text { Neither agree } \\
\text { nor disagree (3) }\end{array}$ & Yes, agree (4) & $\begin{array}{c}\text { Yes, strongly } \\
\text { agree (5) }\end{array}$ \\
\hline$A$ & 0 & $\bigcirc$ & & & \\
\hline
\end{tabular}

What do you think about the room?

Space can impact on the quality of care that you give to the women. Some people think that the space that works well for birthing women is also good for midwives to do their work. Below are a get of general statements about the features you think are important in the space.

Q3.1

\begin{tabular}{|c|c|c|c|c|c|}
\hline & $\begin{array}{l}\text { No, strongly } \\
\text { disagree (1) }\end{array}$ & $\begin{array}{c}\text { No, disagree } \\
(2)\end{array}$ & $\begin{array}{c}\text { Neither agree nor } \\
\text { disagree (3) }\end{array}$ & Yes, agree (4) & $\begin{array}{c}\text { Yes, strongly } \\
\text { agree (5) }\end{array}$ \\
\hline $\begin{array}{l}\text { A single room is } \\
\text { better for a } \\
\text { woman's recovery } \\
\text { care }\end{array}$ & O & ) & 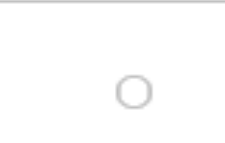 & O & 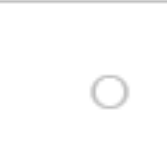 \\
\hline
\end{tabular}

Q3.2

\begin{tabular}{l|ccccc} 
Q3.2 & $\begin{array}{c}\text { No, strongly } \\
\text { disagree (1) }\end{array}$ & $\begin{array}{c}\text { No, disagree } \\
(2)\end{array}$ & $\begin{array}{c}\text { Neither agree nor } \\
\text { disagree (3) }\end{array}$ & $\begin{array}{l}\text { Yes, agree (4) } \\
\text { Yes, strongly } \\
\text { agree (5) }\end{array}$ \\
\hline $\begin{array}{l}\text { A room or ward } \\
\text { that is quiet is good } \\
\text { for sleep and } \\
\text { generally helps }\end{array}$ & & & & \\
women recorrer in & & & & \\
comfort & & & &
\end{tabular}

Q3.3

\begin{tabular}{|c|c|c|c|c|c|}
\hline & $\begin{array}{l}\text { No, strongly } \\
\text { disagree (1) }\end{array}$ & No, disagree (2) & $\begin{array}{c}\text { Neither agree nor } \\
\text { disagree (3) }\end{array}$ & Yes, agree (4) & $\begin{array}{c}\text { Yes, strongly } \\
\text { 2gree (5) }\end{array}$ \\
\hline $\begin{array}{l}\text { The postnatal room } \\
\text { generally has less } \\
\text { impact on how } \\
\text { postnatal care is } \\
\text { experienced by } \\
\text { women }\end{array}$ & O & 0 & 0 & 0 & 0 \\
\hline
\end{tabular}


Q3.4

\begin{tabular}{|c|c|c|c|c|c|}
\hline & $\begin{array}{l}\text { No, strongly } \\
\text { disagree (1) }\end{array}$ & No, disagree (2) & $\begin{array}{c}\text { Neither agree nor } \\
\text { disagree (3) }\end{array}$ & Yes, agree (4) & $\begin{array}{c}\text { Yes, strongly } \\
\text { agree (5) }\end{array}$ \\
\hline $\begin{array}{l}\text { A room with } \\
\text { colours is better for } \\
\text { birth recovery than } \\
\text { a very plain room }\end{array}$ & 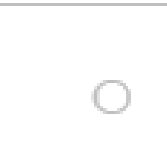 & 7 & 5 & 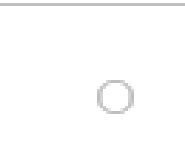 & ( \\
\hline
\end{tabular}

Q3.5

\begin{tabular}{l|cccc} 
No, strongly & No, disagree (2) & $\begin{array}{c}\text { Neither agree nor } \\
\text { disagree (3) }\end{array}$ & Yes, agree (4) & $\begin{array}{c}\text { Yes, strongly } \\
\text { agree }(5)\end{array}$ \\
\hline $\begin{array}{l}\text { Most wromen prefer } \\
\text { to stay in a }\end{array}$ & \\
postratal room that \\
orerlooks a garden, \\
or has pictures of \\
natural scenery on \\
the walls
\end{tabular}

Q3.6

\begin{tabular}{c|ccccc} 
No, strongly & No, disagree (2) & $\begin{array}{c}\text { Neither agree nor } \\
\text { disagree (3) }\end{array}$ & Yes, agree (4) & $\begin{array}{c}\text { Yes, strongly } \\
\text { agree }(5)\end{array}$ \\
\hline $\begin{array}{c}\text { A postmatal room } \\
\text { with access to } \\
\text { outdoor }\end{array}$ & 0 & & & \\
$\begin{array}{c}\text { surroundings is } \\
\text { better for care }\end{array}$ & 0 & 0 &
\end{tabular}

Q3.7

\begin{tabular}{l|ccccc} 
& $\begin{array}{c}\text { No, strongly } \\
\text { disagree (1) }\end{array}$ & No, disagree (2) & $\begin{array}{c}\text { Neither agree nor } \\
\text { disagree (3) }\end{array}$ & $\begin{array}{c}\text { Yes, agree (4) } \\
\text { Yes, strongly } \\
\text { agree (5) }\end{array}$ \\
\hline $\begin{array}{l}\text { A postnatal room } \\
\text { that lets in natural } \\
\text { daylight is better } \\
\text { for recovery care }\end{array}$ & $\bigcirc$ & 0 & &
\end{tabular}

Q3.8

\begin{tabular}{|c|c|c|c|c|c|}
\hline & $\begin{array}{l}\text { No, strongly } \\
\text { disagree (1) }\end{array}$ & No, disagree (2) & $\begin{array}{c}\text { Neither agree nor } \\
\text { disagree (3) }\end{array}$ & Yes, agree (4) & $\begin{array}{l}\text { yes, strongly } \\
\text { agree (5) }\end{array}$ \\
\hline $\begin{array}{l}\text { A postnatal room } \\
\text { that has natural } \\
\text { finishes such } 25 \\
\text { wood is better for } \\
\text { recovery care }\end{array}$ & $\bigcirc$ & 7 & 0 & 0 & $C$ \\
\hline
\end{tabular}


Q3.9

\begin{tabular}{|c|c|c|c|c|c|}
\hline & $\begin{array}{l}\text { No, strongly } \\
\text { disagree (1) }\end{array}$ & No, disagree (2) & $\begin{array}{c}\text { Neither agree nor } \\
\text { disagree (3) }\end{array}$ & Yes, agree (4) & $\begin{array}{l}\text { Yes, strongly } \\
\text { agree (5) }\end{array}$ \\
\hline $\begin{array}{l}\text { Shapes and forms } \\
\text { like those of natural } \\
\text { objects such as } \\
\text { flowers, flowing } \\
\text { water, etc. can } \\
\text { make for a more } \\
\text { beautiful postnatal } \\
\text { room design }\end{array}$ & O & O & O & O & O \\
\hline
\end{tabular}

\section{Interior}

Some people think that your surroundings can affect how you do your work.

Q3.10

Do you think the interior environment of postnatal space(s) in which you work or have worked in the past affects how easy you found it to provide care?

\begin{tabular}{c|ccccc} 
& $\begin{array}{c}\text { No, strongly } \\
\text { disagree (1) }\end{array}$ & No, disagree (2) & $\begin{array}{c}\text { Neither agree nor } \\
\text { disagree (3) }\end{array}$ & Yes, agree (4) & $\begin{array}{c}\text { Yes, strongly } \\
\text { agree (5) }\end{array}$ \\
\hline A & 0 & 0 & 0 & 0
\end{tabular}

Q3.11

What factors made your work easy?

Q3.12

What factors made your work difficult? 
Q3.13

During postnatal care how important do you think it is to...

\begin{tabular}{l|cccc} 
Not at all & $\begin{array}{c}\text { Minimally } \\
\text { important (2) }\end{array}$ & $\begin{array}{c}\text { Somewhat } \\
\text { (3) }\end{array}$ & $\begin{array}{c}\text { Moderately } \\
\text { important (4) }\end{array}$ & $\begin{array}{l}\text { Very important } \\
\text { A) Have a restful room }\end{array}$ \\
B) Care for women in & \\
a clinical-looking \\
postnatal space \\
C) Provide care in a \\
room which has a \\
combination of clinical \\
and familiar home \\
features \\
D) Give care in a \\
room or ward writh \\
natural art, images or \\
paintings \\
E) Have bright colours
\end{tabular}




\section{Sensory comfort}

A wam and comfortable indoor climate (room temperature, fresh air etc.) in the postnatal ward/ room can impact on the comfort and well-being of women who have recently given birth and staff. Below are a set of general statements about the features needed for a comfortable climate.

Q3.14

How important are these features to the sense of wellbeing in postnatal rooms or wards where you work?

\begin{tabular}{|c|c|c|c|c|c|}
\hline & Not at all (1) & $\begin{array}{l}\text { Minimally } \\
\text { important (2) }\end{array}$ & $\begin{array}{l}\text { Somewhat } \\
\text { (3) }\end{array}$ & $\begin{array}{l}\text { Moderately } \\
\text { important (4) }\end{array}$ & $\begin{array}{l}\text { Very important } \\
\text { (5) }\end{array}$ \\
\hline $\begin{array}{l}\text { A) Haring adecquate } \\
\text { daylighting }\end{array}$ & & O & D & 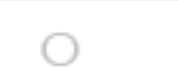 & 0 \\
\hline $\begin{array}{l}\text { B) Being able to control } \\
\text { the temperature }\end{array}$ & & & 0 & & O \\
\hline $\begin{array}{l}\text { C) Being able to control } \\
\text { noise from mechanical } \\
\text { things like ventilation, } \\
\text { medical ecuipment, etc. }\end{array}$ & O & $\Omega$ & & & \\
\hline $\begin{array}{l}\text { D) Providing care in a } \\
\text { room that feels quiet }\end{array}$ & 0 & O & 0 & 0 & 0 \\
\hline
\end{tabular}

Q3.15

How much do each of the follorving features contribute to the sense of wellbeing in postnatal rooms or wards where you work?

\begin{tabular}{|c|c|c|c|c|c|}
\hline & Not at all (1) & $\begin{array}{c}\text { Minimally } \\
\text { important (2) }\end{array}$ & $\begin{array}{l}\text { Somewhat } \\
\text { (3) }\end{array}$ & $\begin{array}{l}\text { Moderately } \\
\text { important (4) }\end{array}$ & $\underset{(5)}{\text { Very important }}$ \\
\hline $\begin{array}{l}\text { A) Having good air } \\
\text { quality and room } \\
\text { freshness }\end{array}$ & & 0 & 0 & 0 & O \\
\hline $\begin{array}{l}\text { B) Having a } \\
\text { hrgienic/clean } \\
\text { environment }\end{array}$ & 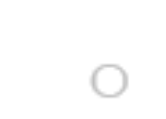 & $\mathrm{O}$ & 0 & 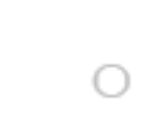 & 0 \\
\hline $\begin{array}{l}\text { C) Being able to adjust } \\
\text { lighting to the activity in } \\
\text { the room }\end{array}$ & 0 & $\mathrm{O}$ & O & 0 & 0 \\
\hline $\begin{array}{l}\text { D) Having a number of } \\
\text { natural things in the } \\
\text { room such as aquariums, } \\
\text { sounds of birds, water, } \\
\text { scents and images }\end{array}$ & $\mathrm{O}$ & O & O & $\cap$ & 0 \\
\hline
\end{tabular}




\section{Social comfort}

Communication of parenting skills with women is an important part of your work in the postnatal period. Below are a set of general statements about social comfort.

Q3.16

In which situations did you find the women in your most recent advice session or talk about parenting skills? Please select all that apply

In bed

Sitting in a chair

Breastfeeding the baby

Standing

Having a meal

Other (Please specify)

Q3.17

Which ways have you used to communicate parenting skills to women? Please select all that apply

In a group, inside a room

Single group session in an open ward

One-on-One talk

Individually but with partner present

Multiple group sessions

Other (Please specify) 
How do you feel about the following statements regarding aspects of postnatal care giving?

\begin{tabular}{|c|c|c|c|c|c|}
\hline & $\begin{array}{l}\text { No, strongly } \\
\text { disagree (1) }\end{array}$ & $\begin{array}{c}\text { No, disagree } \\
\text { (2) }\end{array}$ & $\begin{array}{c}\text { Neither agree nor } \\
\text { disagree (3) }\end{array}$ & Yes, agree (4) & $\begin{array}{l}\text { Yes, strongly } \\
\text { agree (5) }\end{array}$ \\
\hline $\begin{array}{l}\text { Women have the } \\
\text { opportunity to choose a } \\
\text { room that they prefer }\end{array}$ & 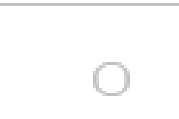 & ( & & & 0 \\
\hline $\begin{array}{l}\text { A postnatal room with } \\
\text { sereral beds decreases } \\
\text { the opportunity for } \\
\text { discussions about } \\
\text { personal issues }\end{array}$ & & & & & 5 \\
\hline $\begin{array}{l}\text { Having a close link } \\
\text { between postnatal room } \\
\text { and birth room is good } \\
\text { for social comfort }\end{array}$ & & & & & 0 \\
\hline $\begin{array}{l}\text { Having a passage or } \\
\text { corridor to enter a } \\
\text { postnatal room or ward } \\
\text { helps to control visitors }\end{array}$ & & & & & \\
\hline $\begin{array}{l}\text { Communal space near } \\
\text { the postnatal space is } \\
\text { good for counselling } \\
\text { purposes }\end{array}$ & ) & & & & 0 \\
\hline $\begin{array}{l}\text { Fumiture and décor in a } \\
\text { communal space can } \\
\text { help group learning of } \\
\text { breastfeeding and } \\
\text { parenting skills }\end{array}$ & & & & & \\
\hline $\begin{array}{l}\text { Positive distractions } \\
\text { such } 2 s \text { soft music, } \\
\text { natural images etc. are } \\
\text { better for recovery }\end{array}$ & 7 & 0 & 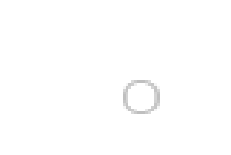 & $\cap$ & ( \\
\hline $\begin{array}{l}\text { Women interact less in a } \\
\text { single private room } \\
\text { rather than in a postnatal } \\
\text { ward }\end{array}$ & 0 & $\cap$ & $\cap$ & 0 & C \\
\hline
\end{tabular}

Q3.19

If you would like to include anything that you think was missed out about the postnatal room please add your comments in the box below.

Q3.20 If you would like to participate in Phase 2 of this research, where 2 set of images of indoor and outdoor surroundings will be shown to a number of participants in a small focus group, please tick below.

Yes, please email me at Lateef Lawal@uw.acnz

No 


\section{C: TABLE OF DESCRIPTIVE SURVEY}

C-1: The mean and standard deviation for all item in the Design Factors influencing women's recovery experiences and wellbeing on perception of design factors survey

(women, $\mathrm{n}=212$ )

$\begin{array}{lll}\text { Item } & \text { M } & S D\end{array}$

Have a clean room

$4.93 \quad 0.23$

Having a hygienic/clean environment

$4.92 \quad 0.33$

Have a restful room

$4.89 \quad 0.37$

A single room is better for a woman's postnatal recovery care

$4.88 \quad 0.37$

A room or ward that is quiet is good for sleep and generally helps in the

$4.75 \quad 0.61$

recovery process

Having a room that feels quiet

$4.75 \quad 0.62$

Having good air quality and room freshness

$4.74 \quad 0.51$

Women appreciate a postnatal room in which natural daylight can easily enter

$4.47 \quad 0.61$

into the room

Be in a room where the door is closed except when visitors are allowed in

$4.43 \quad 0.84$

Having adequate daylighting in the room or ward

$4.42 \quad 0.73$

Being a able to have additional heating for mother and baby

$4.34 \quad 0.76$

Being able to control the temperature

Women are generally too emotional about the birth of their baby to notice if the

$4.14 \quad 0.96$

space is good or bad (reverse coded)

Being able to control noise from mechanical things like ventilation, medical

$4.06 \quad 0.99$ equipment etc.

Knowing you can open or close the windows to feel comfortable

4.031 .01

Having a communal space near the postnatal space is good for counselling and

$3.96 \quad 0.71$ parental information support

Know there is a protective corner in the room that you can withdraw into with your baby

Most women prefer to stay in a postnatal room that overlooks a garden, or has pictures of natural scenery on the walls Having family members and partner present during postnatal care increases the need for large space 


\begin{tabular}{|c|c|c|}
\hline Item & $\mathrm{M}$ & $S D$ \\
\hline $\begin{array}{l}\text { Shapes and forms like those of natural objects such as flowers, flowing waters, } \\
\text { etc. can make a more beautiful postnatal room design }\end{array}$ & 3.88 & 0.70 \\
\hline $\begin{array}{l}\text { The arrangement of furniture and décor in a communal space helps group } \\
\text { learning of breastfeeding and parental skills }\end{array}$ & 3.88 & 0.71 \\
\hline Be able to walk outside and experience the outdoor surroundings & 3.74 & 1.13 \\
\hline Entering from a passage or corridor to a postnatal room is better for privacy & 3.66 & 0.71 \\
\hline $\begin{array}{l}\text { Be looked after in a room which has a combination of clinical and familiar home } \\
\text { features }\end{array}$ & 3.60 & 1.12 \\
\hline $\begin{array}{l}\text { Women are offered the opportunity to choose the room they would prefer for } \\
\text { their postnatal stay (reversed coded) }\end{array}$ & 3.41 & 1.28 \\
\hline $\begin{array}{l}\text { Positive distractions such as soft music, natural images etc. in a postnatal room } \\
\text { are better for a woman's recovery }\end{array}$ & 3.32 & 0.72 \\
\hline $\begin{array}{l}\text { Openness of a postnatal room decreases the opportunity for discussions with } \\
\text { midwives }\end{array}$ & 3.22 & 0.90 \\
\hline A room with colours is better for recovery than a very plain room & 3.16 & 0.72 \\
\hline Be in a room or ward that is decorated with natural art, images or paintings & 2.96 & 1.05 \\
\hline Have a room with furniture made of natural materials & 2.90 & 1.09 \\
\hline $\begin{array}{l}\text { Having a number of natural things in your room such as aquariums, nature } \\
\text { sounds of birds, water, scents and images }\end{array}$ & 2.53 & 1.04 \\
\hline Be looked after in a clinical-looking postnatal space & 2.34 & 1.17 \\
\hline $\begin{array}{l}\text { Women find a large open postnatal ward or room is better for interaction with } \\
\text { other women }\end{array}$ & 2.24 & 1.00 \\
\hline Have bright colours in the room & 2.19 & 0.91 \\
\hline $\begin{array}{l}\text { From the postnatal room it is good to be able to see the activity of the maternity } \\
\text { department }\end{array}$ & 2.09 & 0.75 \\
\hline
\end{tabular}

Note: $M=$ mean; $S D=$ standard deviation 
C-2: The mean and standard deviation for all item in the Design Factors influencing women's recovery care on perception of design factors survey (midwives, $n=50$ )

\begin{tabular}{|c|c|c|}
\hline Item & $\mathbf{M}$ & $S D$ \\
\hline Provide care in a room that is clean & 4.98 & 0.14 \\
\hline Having a hygienic/clean environment & 4.90 & 0.36 \\
\hline Providing care in a room that feels quiet & 4.86 & 0.35 \\
\hline Have a restful room & 4.78 & 0.51 \\
\hline Having adequate daylighting & 4.78 & 0.47 \\
\hline Adequate lighting (to the activity in the room) and operable windows & 4.76 & 0.56 \\
\hline Having good air quality and room freshness & 4.70 & 0.58 \\
\hline $\begin{array}{l}\text { Being able to control noise from mechanical things like ventilation, medical } \\
\text { equipment etc. }\end{array}$ & 4.68 & 0.47 \\
\hline Being able to control the temperature & 4.66 & 0.52 \\
\hline $\begin{array}{l}\text { A room or ward that is quiet is good for sleep and generally helps women recover } \\
\text { in comfort }\end{array}$ & 4.60 & 0.61 \\
\hline A postnatal room that lets in natural daylight is better for recovery care & 4.52 & 0.58 \\
\hline $\begin{array}{l}\text { A postnatal room with several beds decreases the opportunity for discussions } \\
\text { about personal issues }\end{array}$ & 4.43 & 0.83 \\
\hline A single room is better for a woman's postnatal recovery care & 4.20 & 0.97 \\
\hline $\begin{array}{l}\text { Furniture and décor layouts in a communal space can help group learning of } \\
\text { parental skills }\end{array}$ & 4.17 & 0.64 \\
\hline A postnatal room with access to outdoor surroundings is better for care & 4.14 & 0.70 \\
\hline $\begin{array}{l}\text { Most women prefer to stay in a postnatal room that overlooks a garden, or has } \\
\text { pictures of natural scenery on the walls }\end{array}$ & 4.04 & 0.61 \\
\hline $\begin{array}{l}\text { The postnatal room generally has less impact on how postnatal care is experienced } \\
\text { by women (reverse coded) }\end{array}$ & 4.04 & 0.83 \\
\hline Give care in a room where the door is closed except when visitors are allowed in & 4.00 & 0.95 \\
\hline Communal space near the postnatal space is good for counselling purposes & 3.85 & 0.78 \\
\hline $\begin{array}{l}\text { Shapes and forms like those of natural objects such as flowers, flowing waters, } \\
\text { etc. can make for a more beautiful postnatal room design }\end{array}$ & 3.82 & 0.63 \\
\hline Positive distractions such as soft music, natural images etc. are better for recovery & 3.66 & 0.76 \\
\hline Women interact less in a single private room rather than in a postnatal ward & 3.60 & 1.19 \\
\hline $\begin{array}{l}\text { Provide care in a room which has a combination of clinical and familiar home } \\
\text { features }\end{array}$ & 3.58 & 0.99 \\
\hline
\end{tabular}




\begin{tabular}{llc}
\hline Item & $\mathrm{M}$ & $\mathrm{SD}$ \\
\hline Give care in a room or ward with natural art, images and paintings & 3.56 & 1.07 \\
Having a passage or corridor to enter a postnatal room or ward helps to control & 3.55 & 0.72 \\
visitors & & \\
A postnatal room that has natural finishes such as wood is better for recovery & 3.46 & 0.81 \\
care & 3.44 & 0.71 \\
A room with colours is better for birth recovery than a very plain room & 3.34 & 1.06 \\
Women have the opportunity to choose a room that they prefer & 3.08 & 0.88 \\
& 2.91 & 0.91 \\
Provide care in a room in which a variety of materials are used & & \\
Having a close link between postnatal room and birth room is good for social \\
comfort
\end{tabular}

Note: $M=$ mean; $S D=$ standard deviation 
C-3: Participant responses as a percentage of the sample, means and standard deviations for each item of the perceptions of design factors influencing recovery and wellbeing in women's postnatal design survey $(n=212)$

\begin{tabular}{|c|c|c|c|c|c|c|}
\hline Item & NSD & ND & NA/ND & $\mathbf{A}$ & SA & $\mathbf{M}(S D)$ \\
\hline Physical environment can affect a quality postnatal care experience in terms of women's psychological wellbeing & - & 1.4 & 4.7 & 40.6 & 53.3 & $4.46(0.66)$ \\
\hline A single room is better for a woman's postnatal recovery care & - & - & 1.4 & 9 & 89.6 & 4.88(0.37) \\
\hline A room or ward that is quiet is good for sleep and generally helps in the recovery process & 0.5 & 1.4 & 2.4 & 13.7 & 82.1 & 4.75(0.61) \\
\hline Women are generally too emotional about the birth of their baby to notice if the space is good or bad (reverse coded) & 40.6 & 43.4 & 7.5 & 6.1 & 2.4 & $4.14(0.96)$ \\
\hline A room with colours is better for recovery than a very plain room & 0.9 & 13.2 & 58.5 & 24.1 & 3.3 & $3.16(0.72)$ \\
\hline Most women prefer to stay in a postnatal room that overlooks a garden, or has pictures of natural scenery on the walls & & 2.4 & 22.6 & 53.3 & 21.7 & $3.94(0.73)$ \\
\hline Women appreciate a postnatal room in which natural daylight can easily enter & - & 0.5 & 4.7 & 42.5 & 52.4 & $4.47(0.61)$ \\
\hline Be able to walk outside and experience the outdoor surroundings & 4.7 & 9 & 25 & 30.7 & 30.7 & $3.74(1.13)$ \\
\hline $\begin{array}{l}\text { Shapes and forms like those of natural objects such as flowers, flowing waters, etc. can make a more beautiful postnatal } \\
\text { room design }\end{array}$ & - & 1.9 & 25.5 & 55.2 & 17.5 & $3.88(0.70)$ \\
\hline Women are offered the opportunity to choose the room that they prefer for their postnatal stay (reverse coded) & 28.3 & 18.4 & 26.9 & 18.9 & 7.5 & $3.41(1.28)$ \\
\hline $\begin{array}{l}\text { From the postnatal room it is good to be able to see the activity of the maternity department (spatial links between the } \\
\text { birth and postnatal rooms) }\end{array}$ & 21.2 & 50.9 & 25.5 & 2.4 & - & $2.09(0.75)$ \\
\hline Positive distractions such as soft music, natural images etc. in a postnatal room are better for a woman's recovery & 1.4 & 7.1 & 53.3 & 34.4 & 3.8 & $3.32(0.72)$ \\
\hline Having a communal space near the postnatal space is good for counselling and parental information support & 0.9 & 2.4 & 14.6 & 64.2 & 17.9 & $3.96(0.71)$ \\
\hline The arrangement of furniture and décor in a communal space helps group learning of breastfeeding and parental skills & 1.1 & 1.1 & 27.3 & 51.9 & 18.7 & $3.88(0.72)$ \\
\hline Openness of a postnatal room decreases the opportunity for discussions with midwives & 3.8 & 12.7 & 49.1 & 26.9 & 7.5 & $3.22(0.90)$ \\
\hline Entering from a passage or corridor to a postnatal room is better for privacy & 0.5 & 2.8 & 36.8 & 50.5 & 9.4 & $3.66(0.71)$ \\
\hline Having family members and partner present during postnatal care increases the need for large space & - & 9.9 & 17.5 & 42.5 & 30.2 & $3.93(0.93)$ \\
\hline Women find a large open postnatal ward or room is better for interactions with other women & 29.2 & 27.8 & 34.9 & 6.1 & 1.9 & $2.24(1.00)$ \\
\hline Women prefer home-like environment for their postnatal care & - & 4.2 & 22.6 & 49.5 & 23.6 & $3.92(0.79)$ \\
\hline Women would prefer an experience similar to staying in a hotel for their postnatal care & 2.4 & 16 & 38.7 & 32.1 & 10.8 & $3.33(0.95)$ \\
\hline
\end{tabular}

Note: NSD = No, strongly disagree; ND = No, disagree; NA/ND = Neither agree nor disagree; $\mathrm{A}=$ Yes, agree; $\mathrm{SA}=$ Yes, strongly agree; $\mathrm{M}=$ mean; $S D=$ standard deviation. 
C-4: Participant responses as a percentage of the sample, means and standard deviations for each item of the perceptions of design factors influencing recovery and wellbeing in women's postnatal design survey $(n=212)$

\begin{tabular}{|c|c|c|c|c|c|c|}
\hline Item & 1 & 2 & 3 & 4 & 5 & $\mathbf{M}(S D)$ \\
\hline Have a restful room & - & - & 1.9 & 7.1 & 91 & $4.89(0.27)$ \\
\hline Be looked after in a clinical-looking postnatal space & 24.5 & 41 & 17.9 & 8.5 & 8 & 2.34(1.17) \\
\hline Be looked after in a room which has a combination of clinical and familiar home features & 3.3 & 15.6 & 23.6 & 33 & 24.5 & $3.60(1.12)$ \\
\hline Be in a room or ward that is decorated with natural art, images or paintings & 8.5 & 24.1 & 37.3 & 23.1 & 7.1 & $2.96(1.05)$ \\
\hline Have bright colours in the room & 25 & 39.6 & 27.4 & 7.5 & 0.5 & $2.19(0.91)$ \\
\hline Have a clean room & - & - & 0.5 & 5.7 & 93.9 & $4.93(0.27)$ \\
\hline Have a room with furniture made of natural materials & 9.4 & 26.4 & 36.3 & 20.8 & 7.1 & $2.90(1.06)$ \\
\hline Know there is a protective corner in the room that you can withdraw into with your baby & 2.4 & 9.4 & 19.8 & 27.8 & 40.6 & $3.95(1.09)$ \\
\hline Be in a room where the door is closed except when visitors are allowed in (privacy) & 0.5 & 3.8 & 8.5 & 26.9 & 60.4 & $4.43(0.84)$ \\
\hline Having adequate daylighting in your room or ward & 0.5 & 0.9 & 9 & 34.9 & 54.7 & $4.42(0.73)$ \\
\hline Being able to control the temperature & 0.5 & 4.2 & 12.3 & 35.8 & 47.2 & $4.25(0.87)$ \\
\hline Being able to control noise from mechanical things like ventilation, medical equipment, etc. & 1.4 & 7.1 & 16.5 & 34.4 & 40.6 & $4.06(0.99)$ \\
\hline Having a room that feels quiet & 0.5 & 1.4 & 2.4 & 14.6 & 81.1 & $4.75(0.62)$ \\
\hline Having good air quality and room freshness & - & - & 3.3 & 19.3 & 77.4 & $4.74(0.51)$ \\
\hline Being able to have additional heating for mother and baby & 0.5 & 1.9 & 9 & 40.1 & 48.6 & $4.34(0.76)$ \\
\hline Knowing you can open or close the windows to feel comfortable & 2.8 & 5.2 & 16.5 & 36.8 & 38.7 & $4.03(1.01)$ \\
\hline Having a number of natural things in your room such as aquariums, nature sounds of birds, water scents and images & 16.5 & 34.4 & 32.5 & 12.3 & 4.6 & $2.53(1.04)$ \\
\hline Having a hygienic/clean environment & - & - & 1.4 & 5.7 & 92.9 & $4.92(0.33)$ \\
\hline
\end{tabular}

Note: 1 = Not at all important; 2 = Minimally important; 3 = Somewhat; 4 = Moderately important; $5=$ Very important; $\mathrm{M}=$ mean; $S D=$ standard deviation. 
C-5: Participant responses as a percentage of the sample, means and standard deviations for each item of the perceptions of design factors influencing recovery care in midwives postnatal design survey $(n=50)$

\begin{tabular}{|c|c|c|c|c|c|c|}
\hline Item & NSD & ND & NA/ND & $\mathbf{A}$ & SA & $\mathbf{M}(S D)$ \\
\hline Postnatal environment can make a difference to a quality postnatal care experience and satisfaction of women & - & - & 2 & 24 & 74 & $4.72(0.50)$ \\
\hline Do you think the interior environment of postnatal space(s) affects how easy you found it to provide care & 2 & - & 8 & 44 & 46 & $4.32(0.79)$ \\
\hline A single room is better for a woman's postnatal recovery care & - & 6 & 20 & 22 & 52 & $4.20(0.97)$ \\
\hline A room or ward that is quiet is good for sleep and generally helps women recover in comfort & - & - & 6 & 28 & 66 & $4.60(0.61)$ \\
\hline The postnatal room generally has less impact on how postnatal care is experienced by women (reverse coded) & 32 & 44 & 20 & 4 & - & $4.04(0.83)$ \\
\hline A room with colours is better for recovery than a very plain room & - & 6 & 50 & 38 & 6 & $3.44(0.71)$ \\
\hline Most women prefer to stay in a postnatal room that overlooks a garden, or has pictures of natural scenery on the walls & - & - & 16 & 64 & 20 & $4.04(0.61)$ \\
\hline A postnatal room with access to outdoor surroundings is better for care & - & 2 & 12 & 56 & 30 & $4.14(0.70)$ \\
\hline A postnatal room that lets in natural daylight is better for recovery care & - & - & 4 & 40 & 56 & $4.52(0.58)$ \\
\hline A postnatal room that has natural finishes such as wood is better for recovery care & - & 6 & 56 & 24 & 14 & $3.46(0.81)$ \\
\hline $\begin{array}{l}\text { Shapes and forms like those of natural objects such as flowers, flowing waters, etc. can make for a more beautiful } \\
\text { postnatal room design }\end{array}$ & - & - & 20 & 58 & 12 & $3.82(0.63)$ \\
\hline Women have the opportunity to choose a room that they prefer (reverse coded) & 18 & 20 & 44 & 14 & 4 & $3.34(1.06)$ \\
\hline A postnatal room with several beds decreases the opportunity for discussions about personal issues & - & 4 & 8 & 32 & 56 & $4.40(0.81)$ \\
\hline $\begin{array}{l}\text { Having a close link between postnatal room and birth room is good for social comfort (spatial links between the birth } \\
\text { and postnatal rooms) }\end{array}$ & 6 & 18 & 60 & 10 & 6 & $2.92(0.88)$ \\
\hline Having a passage or corridor to enter a postnatal room or ward helps to control visitors & - & 4 & 42 & 46 & 8 & $3.58(0.70)$ \\
\hline Communal space near the postnatal space is good for counselling purposes & - & 8 & 12 & 66 & 14 & $3.86(0.76)$ \\
\hline Furniture and décor in a communal space can help group learning of breastfeeding and parental skills & - & 2 & 6 & 66 & 26 & $4.16(0.62)$ \\
\hline Positive distractions such as soft music, natural images etc. are better for recovery & - & 4 & 36 & 48 & 12 & $3.68(0.74)$ \\
\hline Women interact less in a single private room rather than in a postnatal ward & 2 & 20 & 20 & 30 & 28 & $3.62(1.16)$ \\
\hline
\end{tabular}

Note: NSD = No, strongly disagree; ND = No, disagree; NA/ND = Neither agree nor disagree; A = Yes, agree; SA = Yes, strongly agree; $\mathrm{M}=$ mean; $S D=$ standard deviation. 
C-6: Participant responses as a percentage of the sample, means and standard deviations for each item of the perceptions of design factors influencing recovery care in midwives postnatal design survey $(n=50)$

\begin{tabular}{|c|c|c|c|c|c|c|}
\hline Item & 1 & 2 & 3 & 4 & 5 & $\mathbf{M}(S D)$ \\
\hline Have a restful room & - & - & 4 & 14 & 82 & $4.78(0.51)$ \\
\hline Care for women in a clinical-looking postnatal space & 42 & 36 & 18 & 4 & - & $1.84(0.87)$ \\
\hline Provide care in a room which has a combination of clinical and familiar home features & 4 & 8 & 30 & 42 & 16 & $3.58(0.99)$ \\
\hline Give care in a room or ward with natural art, images or paintings & 4 & 10 & 34 & 30 & 22 & $3.56(1.07)$ \\
\hline Have bright colours in the room & 8 & 30 & 36 & 20 & 6 & $2.86(1.03)$ \\
\hline Provide care in a room that is clean & - & - & - & 2 & 98 & $4.98(0.14)$ \\
\hline Attend women in a room with furniture made of natural materials & 6 & 38 & 34 & 18 & 4 & $2.76(0.96)$ \\
\hline Give care in a postnatal room where the door will be closed except when visitors are allowed in (privacy) & - & 4 & 32 & 24 & 40 & $4.00(0.95)$ \\
\hline Provide care in a room in which a variety of materials are used & 4 & 16 & 54 & 20 & 6 & $3.08(0.88)$ \\
\hline Having adequate daylighting & - & - & 2 & 18 & 80 & $4.78(0.47)$ \\
\hline Being able to control the temperature & - & - & 2 & 30 & 68 & $4.66(0.52)$ \\
\hline Being able to control noise from mechanical things like ventilation, medical equipment, etc. & - & - & - & 32 & 68 & $4.68(0.47)$ \\
\hline Provide care in a room that feels quiet & - & - & - & 14 & 86 & $4.86(0.35)$ \\
\hline Having good air quality and room freshness & - & - & 6 & 18 & 76 & $4.70(0.58)$ \\
\hline Having a hygienic/clean environment & - & - & 2 & 6 & 92 & $4.90(0.36)$ \\
\hline Being able to adjust lighting to the activity in the room/operable windows & - & - & 6 & 12 & 82 & $4.76(0.56)$ \\
\hline Having a number of natural things in your room such as aquariums, nature sounds of birds, water scents and images & 8 & 26 & 42 & 18 & 6 & $2.88(1.00)$ \\
\hline
\end{tabular}

Note: 1 = Not at all important; 2 = Minimally important; 3 = Somewhat; 4 = Moderately important; 5 = Very important; $\mathrm{M}=$ mean; $S D=$ standard deviation. 


\section{Overall Summary of Missing Values}

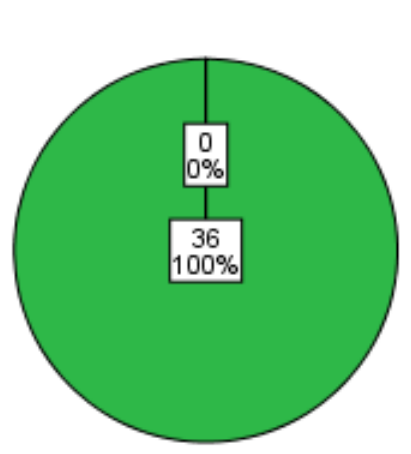

Variables

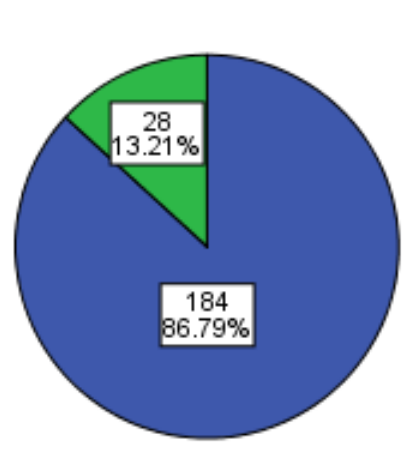

Cases

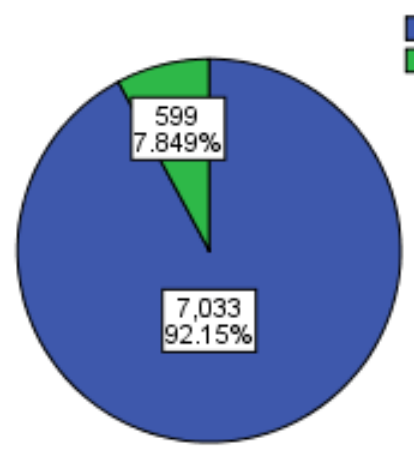

Values

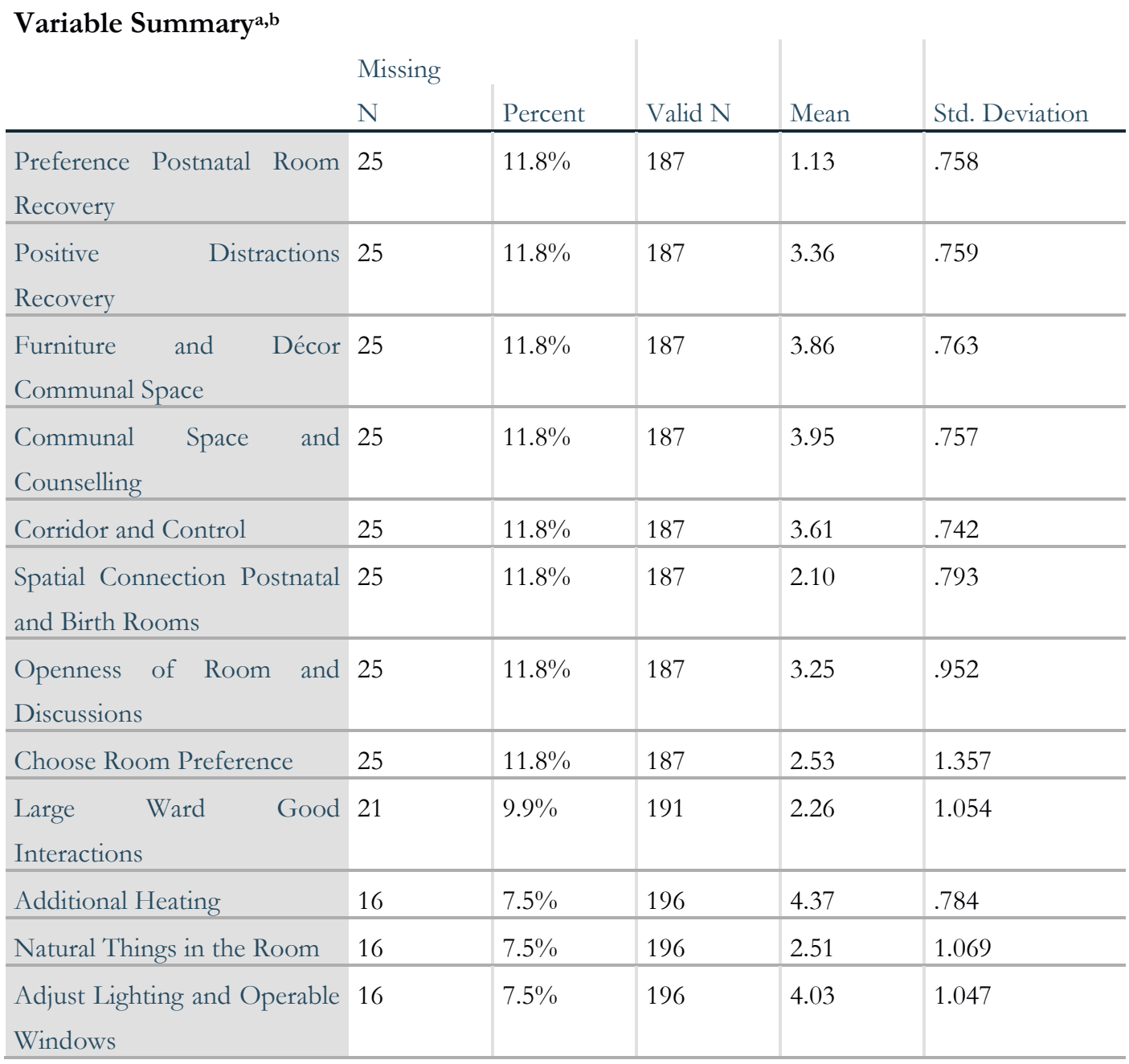




\begin{tabular}{|c|c|c|c|c|c|}
\hline $\begin{array}{l}\text { Hygienic and Clean } \\
\text { Environment }\end{array}$ & 16 & $7.5 \%$ & 196 & 4.91 & .339 \\
\hline $\begin{array}{l}\text { Air Quality and Room } \\
\text { Freshness }\end{array}$ & 16 & $7.5 \%$ & 196 & 4.72 & .524 \\
\hline Room Feel Quiet & 16 & $7.5 \%$ & 196 & 4.72 & .637 \\
\hline Noise Control & 16 & $7.5 \%$ & 196 & 4.06 & 1.031 \\
\hline Temperature Control & 16 & $7.5 \%$ & 196 & 4.27 & .895 \\
\hline Adequate Daylighting & 16 & $7.5 \%$ & 196 & 4.45 & .753 \\
\hline Protective Corner & 15 & $7.1 \%$ & 197 & 3.93 & 1.130 \\
\hline $\begin{array}{l}\text { Combined Clinical and } \\
\text { Familiar }\end{array}$ & 15 & $7.1 \%$ & 197 & 3.57 & 1.143 \\
\hline Door and Privacy & 14 & $6.6 \%$ & 198 & 4.46 & .853 \\
\hline Furniture Natural Materials & 14 & $6.6 \%$ & 198 & 2.89 & 1.096 \\
\hline Clean Room & 14 & $6.6 \%$ & 198 & 4.93 & .276 \\
\hline Bright Colours & 14 & $6.6 \%$ & 198 & 2.21 & .941 \\
\hline $\begin{array}{l}\text { Room Decoration Natural Art } \\
\text { and Images }\end{array}$ & 14 & $6.6 \%$ & 198 & 2.96 & 1.084 \\
\hline
\end{tabular}

a. Maximum number of variables shown: 25

b. Minimum percentage of missing values for variable to be included: $0.0 \%$

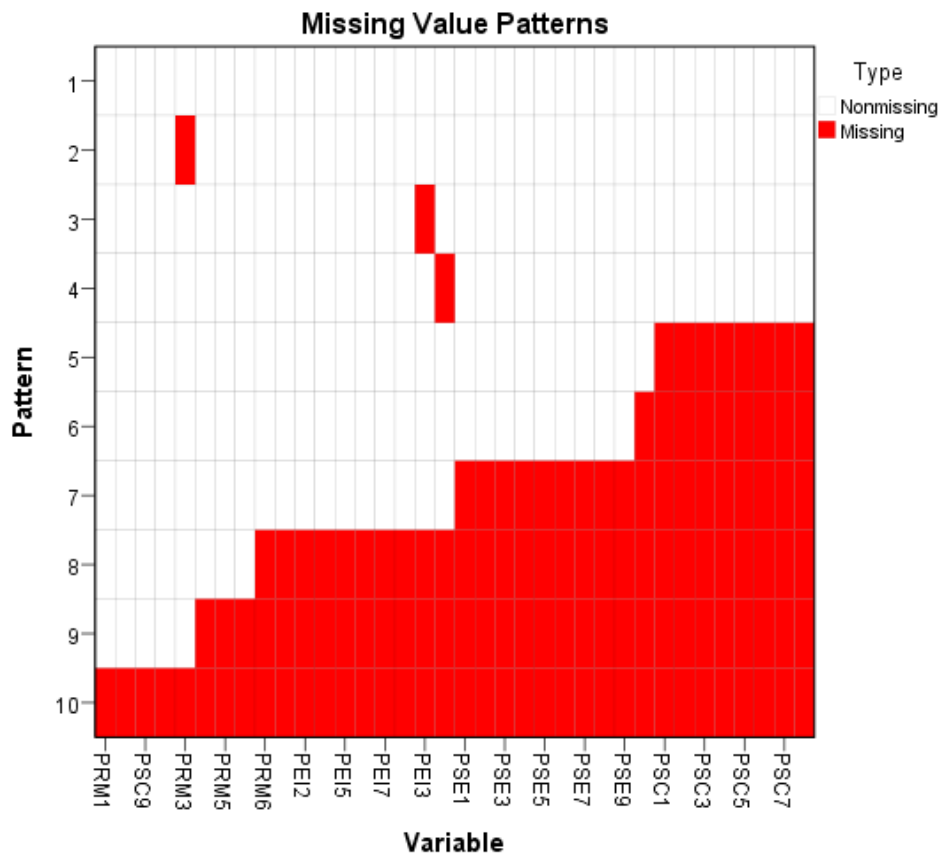




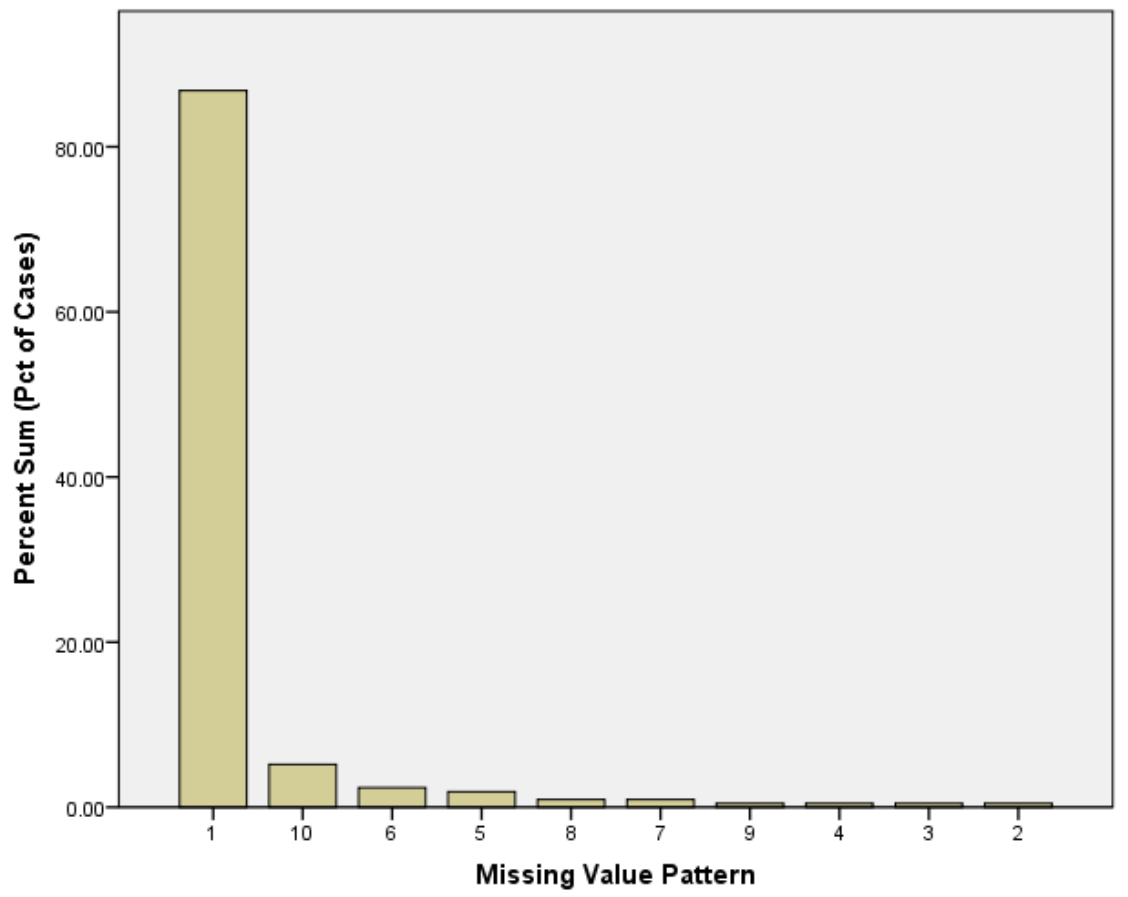

\begin{tabular}{|c|c|c|c|c|c|}
\hline \multicolumn{2}{|c|}{ Missing data } & & & & \\
\hline & & Frequency & Percent & Valid Percent & $\begin{array}{l}\text { Cumulative } \\
\text { Percent }\end{array}$ \\
\hline \multirow[t]{10}{*}{ Valid } & .00 & 182 & 85.8 & 85.8 & 85.8 \\
\hline & 1.00 & 4 & 1.9 & 1.9 & 87.7 \\
\hline & 2.00 & 1 & .5 & .5 & 88.2 \\
\hline & 9.00 & 4 & 1.9 & 1.9 & 90.1 \\
\hline & 21.00 & 5 & 2.4 & 2.4 & 92.5 \\
\hline & 30.00 & 2 & .9 & .9 & 93.4 \\
\hline & 41.00 & 2 & .9 & .9 & 94.3 \\
\hline & 44.00 & 1 & .5 & .5 & 94.8 \\
\hline & 50.00 & 11 & 5.2 & 5.2 & 100.0 \\
\hline & Total & 212 & 100.0 & 100.0 & \\
\hline
\end{tabular}




\section{Overall Summary of Missing Values}
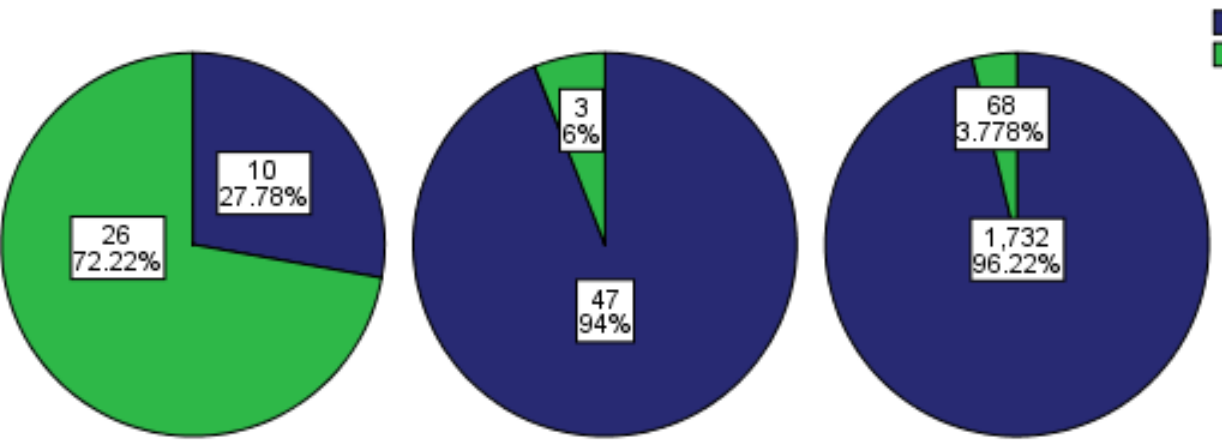

Complete Data

$\square$ Incomplete Data

Variables

Cases

Values

Variable Summary a,b

\begin{tabular}{|c|c|c|c|}
\hline & \multicolumn{2}{|c|}{ Missing } & \multirow[b]{2}{*}{ Valid N } \\
\hline & $\mathrm{N}$ & Percent & \\
\hline Less Interaction Single Room & 3 & $6.0 \%$ & 47 \\
\hline Positive Distractions Recovery & 3 & $6.0 \%$ & 47 \\
\hline $\begin{array}{l}\text { Furniture and Décor Communal } \\
\text { Space }\end{array}$ & 3 & $6.0 \%$ & 47 \\
\hline $\begin{array}{l}\text { Communal Space and } \\
\text { Counselling }\end{array}$ & 3 & $6.0 \%$ & 47 \\
\hline Corridor and Control & 3 & $6.0 \%$ & 47 \\
\hline $\begin{array}{l}\text { Spatial Connections Postnatal } \\
\text { and Birth Rooms }\end{array}$ & 3 & $6.0 \%$ & 47 \\
\hline $\begin{array}{l}\text { Openness of Room and } \\
\text { Discussions }\end{array}$ & 3 & $6.0 \%$ & 47 \\
\hline Choose Room Preference & 3 & $6.0 \%$ & 47 \\
\hline Natural Things & 3 & $6.0 \%$ & 47 \\
\hline $\begin{array}{l}\text { Adjust Lighting and Operable } \\
\text { Windows }\end{array}$ & 3 & $6.0 \%$ & 47 \\
\hline Hygienic and Clean Environment & 3 & $6.0 \%$ & 47 \\
\hline Air Quality and Room Freshness & 3 & $6.0 \%$ & 47 \\
\hline Room Feel Quiet & 3 & $6.0 \%$ & 47 \\
\hline Noise Control & 3 & $6.0 \%$ & 47 \\
\hline Temperature Control & 3 & $6.0 \%$ & 47 \\
\hline Adequate Daylighting & 3 & $6.0 \%$ & 47 \\
\hline Room Variety Materials & 2 & $4.0 \%$ & 48 \\
\hline
\end{tabular}




\begin{tabular}{ll|l|l}
\hline Door and Privacy & 2 & $4.0 \%$ & 48 \\
\hline Furniture Natural Materials & 2 & $4.0 \%$ & 48 \\
\hline Clean Room & 2 & $4.0 \%$ & 48 \\
\hline Bright Colours & 2 & $4.0 \%$ & 48 \\
\hline Natural Art and Images & 2 & $4.0 \%$ & 48 \\
\hline Combined Clinical and Familiar & 2 & $4.0 \%$ & 48 \\
\hline Clinical Room & 2 & $4.0 \%$ & 48 \\
\hline Room Restfulness & 2 & $4.0 \%$ & 48 \\
\hline
\end{tabular}

a. Maximum number of variables shown: 25

b. Minimum percentage of missing values for variable to be included: $0.0 \%$

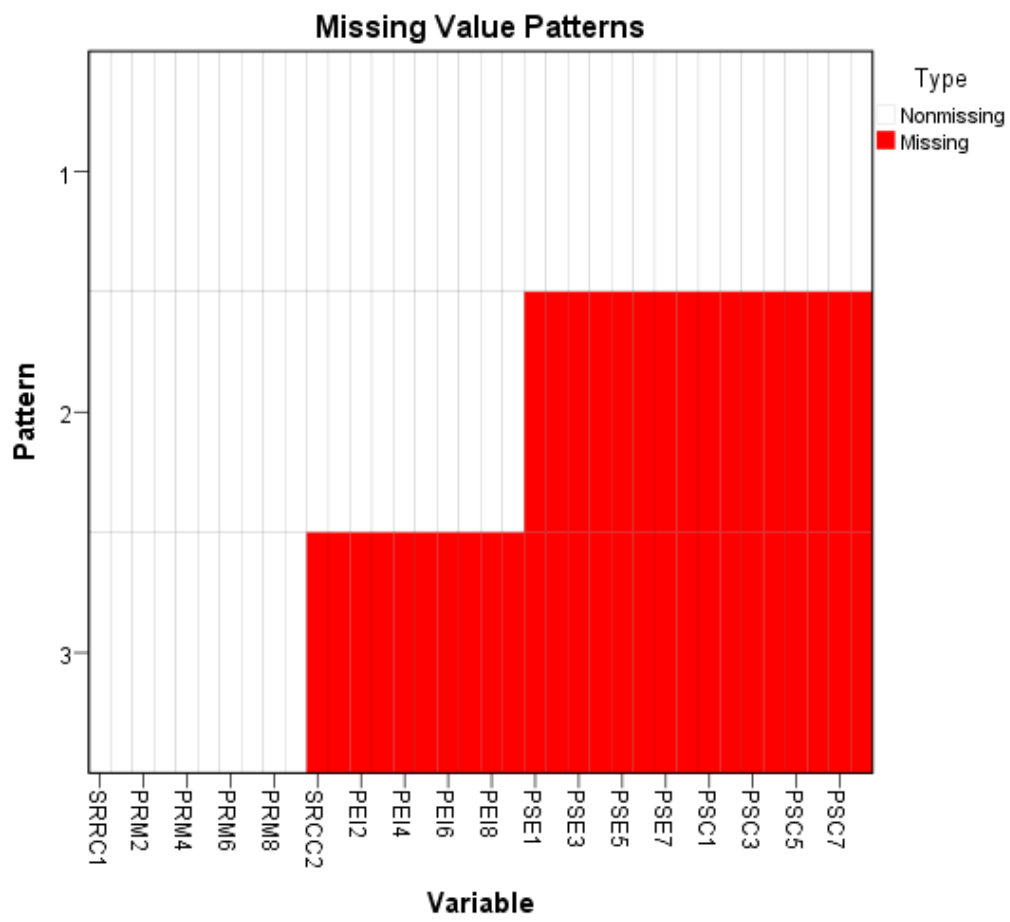




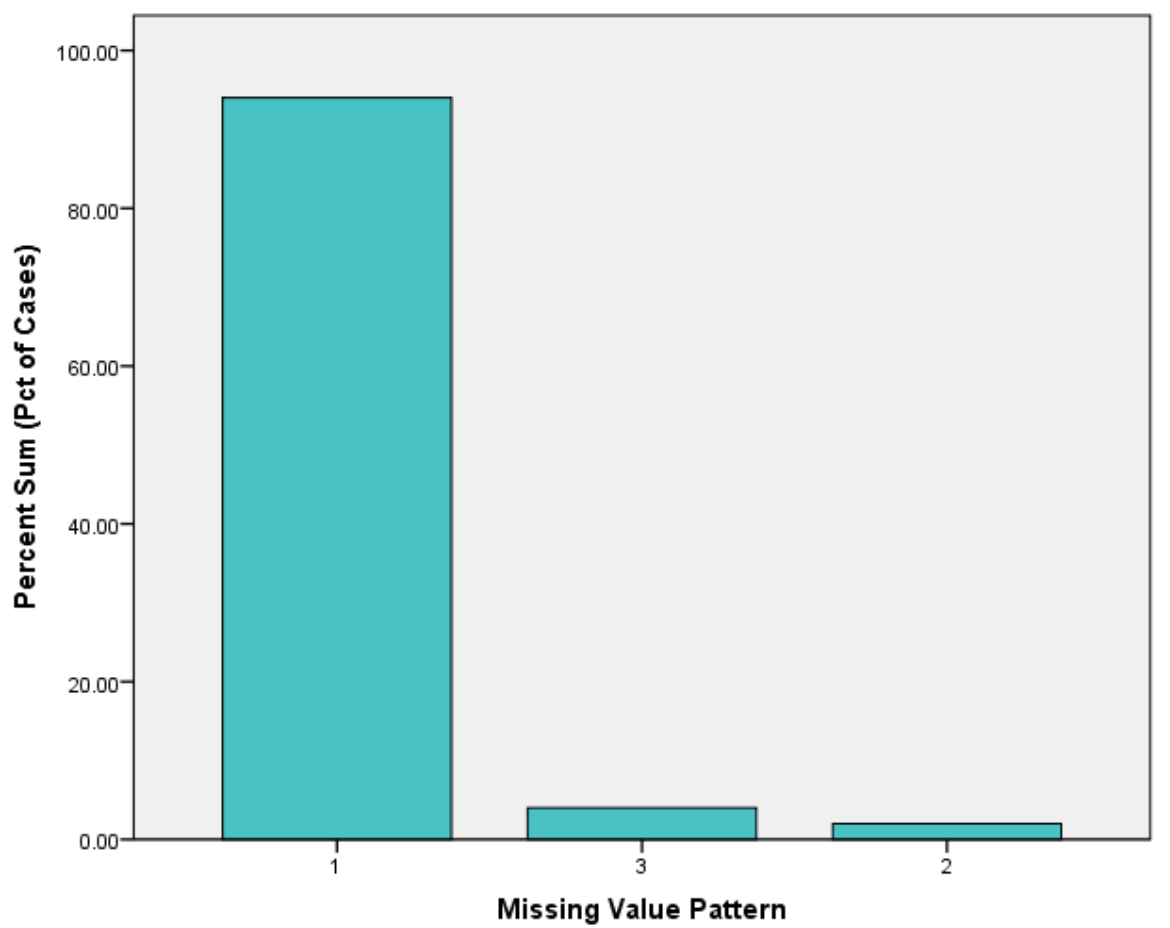

\begin{tabular}{|c|c|c|c|c|c|}
\hline \multicolumn{6}{|c|}{ missingdata } \\
\hline & & Frequency & Percent & Valid Percent & $\begin{array}{c}\text { Cumulative } \\
\text { Percent }\end{array}$ \\
\hline \multirow[t]{4}{*}{ Valid } & .00 & 47 & 94.0 & 94.0 & 94.0 \\
\hline & 16.00 & 1 & 2.0 & 2.0 & 96.0 \\
\hline & 26.00 & 2 & 4.0 & 4.0 & 100.0 \\
\hline & Total & 50 & 100.0 & 100.0 & \\
\hline
\end{tabular}

\begin{tabular}{|c|c|c|c|c|c|c|}
\hline \multicolumn{7}{|c|}{ Result Variables } \\
\hline & \multirow[b]{2}{*}{ Result Variable } & \multirow{2}{*}{$\begin{array}{l}\mathrm{N} \text { of Replaced } \\
\text { Missing Values }\end{array}$} & \multicolumn{2}{|c|}{ Case Number of Non-Missing Values } & \multirow[b]{2}{*}{$\mathrm{N}$ of Valid Cases } & \multirow[b]{2}{*}{ Creating Function } \\
\hline & & & First & Last & & \\
\hline 1 & SRRC2_1 & 2 & 1 & 50 & 50 & SMEAN(SRRC2) \\
\hline 2 & PEI1_1 & 2 & 1 & 50 & 50 & SMEAN(PEI1) \\
\hline 3 & PEI2_1 & 2 & 1 & 50 & 50 & SMEAN(PEI2) \\
\hline 4 & PEI3_1 & 2 & 1 & 50 & 50 & SMEAN(PEI3) \\
\hline 5 & PEI4_1 & 2 & 1 & 50 & 50 & SMEAN(PEI4) \\
\hline 6 & PEI5_1 & 2 & 1 & 50 & 50 & SMEAN(PEI5) \\
\hline 7 & PEI6_1 & 2 & 1 & 50 & 50 & SMEAN(PEI6) \\
\hline 8 & PEI7_1 & 2 & 1 & 50 & 50 & SMEAN(PEI7) \\
\hline 9 & PEI8_1 & 2 & 1 & 50 & 50 & SMEAN(PEI8) \\
\hline 10 & PEI9_1 & 2 & 1 & 50 & 50 & SMEAN(PEI9) \\
\hline 11 & PSE1_1 & 3 & 1 & 50 & 50 & SMEAN(PSE1) \\
\hline 12 & PSE2_1 & 3 & 1 & 50 & 50 & SMEAN(PSE2) \\
\hline
\end{tabular}




\begin{tabular}{ll|l|l|l|l|l}
\hline 13 & PSE3_1 & 3 & 1 & 50 & 50 & SMEAN(PSE3) \\
\hline 14 & PSE4_1 & 3 & 1 & 50 & 50 SMEAN(PSE4) \\
\hline 15 & PSE5_1 & 3 & 1 & 50 & 50 & SMEAN(PSE5) \\
\hline 16 & PSE6_1 & 3 & 1 & 50 & 50 & SMEAN(PSE6) \\
\hline 17 & PSE7_1 & 3 & 1 & 50 & 50 & SMEAN(PSE7) \\
\hline 18 & PSE8_1 & 3 & 1 & 50 & 50 & SMEAN(PSE8) \\
\hline 19 & PSC1_1 & 3 & 1 & 50 & 50 & SMEAN(PSC1) \\
\hline 20 & PSC2_1 & 3 & 1 & 50 & 50 & SMEAN(PSC2) \\
\hline 21 & PSC3_1 & 3 & 1 & 50 & 50 & SMEAN(PSC3) \\
\hline 22 & PSC4_1 & 3 & 1 & 50 & 50 SMEAN(PSC4) \\
\hline 23 & PSC5_1 & 3 & 1 & 50 & 50 & SMEAN(PSC5) \\
\hline 24 & PSC6_1 & 3 & 1 & 50 & 50 & SMEAN(PSC6) \\
\hline 25 & PSC7_1 & 3 & 1 & 50 & 50 & SMEAN(PSC7) \\
\hline 26 & PSC8_1 & 3 & 1 & 50 & SMEAN(PSC8) \\
\hline
\end{tabular}

\section{T-Test}

\section{Warnings}

The Paired Samples Correlations table is not produced.

The Paired Samples Test table is not produced.

\section{Paired Samples Statistics}

\begin{tabular}{llr|r|r|r} 
& Mean & $\mathrm{N}$ & Std. Deviation & \multicolumn{1}{c}{ Std. Error Mean } \\
\hline \multirow{2}{*}{ Pair 1 } & Adequate Daylighting & $4.77 \mathrm{a}$ & 47 & .476 & .069 \\
\cline { 2 - 6 } & SMEAN(PSE1) & $4.77 \mathrm{a}$ & 47 & .476 & .069 \\
\hline
\end{tabular}

a. The correlation and $t$ cannot be computed because the standard error of the difference is 0 .

\section{Warnings}

The Paired Samples Correlations table is not produced.

The Paired Samples Test table is not produced.

\section{Paired Samples Statistics}

\begin{tabular}{llr|r|r|r} 
& & \multicolumn{1}{c}{ Mean } & \multicolumn{1}{c}{ N } & \multicolumn{1}{c}{ Std. Deviation } & \multicolumn{1}{c}{ Std. Error Mean } \\
\hline \multirow{2}{*}{ Pair 1 } & Air Quality and Room Freshness & $4.68^{\mathrm{a}}$ & 47 & .594 & .087 \\
\cline { 2 - 7 } & SMEAN(PSE5) & $4.68^{\mathrm{a}}$ & 47 & .594 & .087 \\
\hline
\end{tabular}

a. The correlation and $t$ cannot be computed because the standard error of the difference is 0 . 